\title{
Optical Fiber High Temperature Sensor Instrumentation for Energy Intensive Industries
}

\section{Final Scientific/Technical Report}

DOE Award Number: DE-FC36-01G011050

Report Issued: November 2006

Recipient Organization: Virginia Polytechnic Institute \& State University Office of Sponsored Programs

460 Turner Street, Suite 306 (0170)

Blacksburg, VA 24061

Principal Investigator:

Dr. Anbo Wang

Director of the Center for Photonics Technology

Phone (540) 231-4355

Fax (540) 231-2158

Email: awang@vt.edu

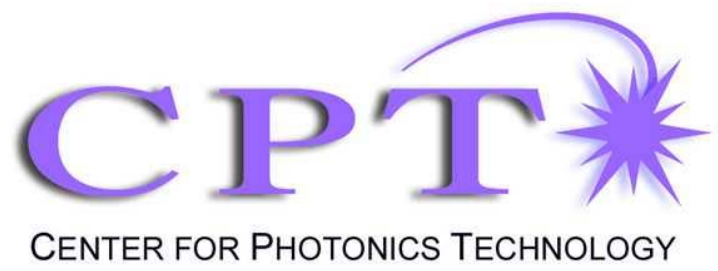




\section{Disclaimer:}

This report was prepared as an account of work sponsored by an agency of the United States Government. Neither the United States Government nor any agency thereof, nor any of their employees, makes any warranty, express or implied, or assumes any legal liability or responsibility for the accuracy, completeness, or usefulness of any information, apparatus, product, or process disclosed, or represents that its use would not infringe privately owned rights. Reference herein to any specific commercial product, process, or service by trade name, trademark, manufacturer, or otherwise does not necessarily constitute or imply its endorsement, recommendation, or favoring by the United States Government or any agency thereof. The views and opinions of authors expressed herein do not necessarily state or reflect those of the United States Government or any agency thereof. 


\section{Executive Summary}

This report summarizes technical progress during the program "Optical Fiber High Temperature Sensor Instrumentation for Energy Intensive Industries", performed by the Center for Photonics Technology of the Bradley Department of Electrical and Computer Engineering at Virginia Tech. The objective of this program was to use technology recently invented at Virginia Tech to develop and demonstrate the application of self-calibrating optical fiber temperature and pressure sensors to several key energy-intensive industries where conventional, commercially available sensors exhibit greatly abbreviated lifetimes due primarily to environmental degradation.

A number of significant technologies were developed under this program, including

- a laser bonded silica high temperature fiber sensor with a high temperature capability up to $700^{\circ} \mathrm{C}$ and a frequency response up to $150 \mathrm{kHz}$,

- the world's smallest fiber Fabry-Perot high temperature pressure sensor (125 x 20 $\mu \mathrm{m})$ with $\mathbf{7 0 0}^{\circ} \mathrm{C}$ capability,

- UV-induced intrinsic Fabry-Perot interferometric sensors for distributed
measurement,

- a single crystal sapphire fiber-based sensor with a temperature capability up to $1600^{\circ} \mathrm{C}$.

These technologies have been well demonstrated and laboratory tested. Our work plan included conducting major field tests of these technologies at EPRI, Corning, Pratt \& Whitney, and Global Energy; field validation of the technology is critical to ensuring its usefulness to U.S. industries. Unfortunately, due to budget cuts, DOE was unable to follow through with its funding commitment to support Energy Efficiency Science Initiative projects and this final phase was eliminated. Accomplishments during the program are summarized briefly here and described in detail in the remainder of the report.

$\rightarrow$ A cleanroom facility was designed and installed at the Center for Photonics Technology for the fabrication of diaphragm-based Fabry-Perot sensors.

$\rightarrow$ Sensor testing methods have been developed and effectively utilized to analyze various aspects of sensor performance. A high temperature, high pressure dynamic test system was designed and assembled to allow sensor evaluation at temperature up to $1200{ }^{\circ} \mathrm{C}$, background pressure up to $200 \mathrm{psi}$, and dynamic pressure more than 140 $\mathrm{dB}$ with a frequency of $47 \mathrm{kHz}$

$\rightarrow$ Borosilicate ring-based engine sensors were designed and fabricated, and their performance characteristics evaluated.

$\rightarrow$ Photolithography procedures were optimized for etched diaphragm-based sensor fabrication.

$\rightarrow$ Direct bonding, hydroxide catalysis bonding, and sol-gel-based bonding techniques were investigated for diaphragm/ferrule bonding requirements. 
$\rightarrow$ Laser bonding-based pressure sensors were successfully demonstrated up to $1800 \mathrm{psi}$.

$\rightarrow$ A novel demodulation technique was developed, called grating-assisted operating point tuning (GA-OPT), by using a diffraction grating with feedback control. The GA-OPT features dynamic operating-point control, high frequency response, insensitivity to light source fluctuation, and real time operation.

$\rightarrow$ A prototype of the digital signal processing system was implemented.

$\rightarrow$ Corrosion rates of single crystal sapphire and zirconia in glass were evaluated.

$\rightarrow$ Thermally bonded engine sensors were fabricated, packaged, and evaluated for sensitivity, dynamic response, and temperature dependence.

$\rightarrow$ A fabrication process for miniature diaphragm-based EFPI sensors was developed that reduces the sensor size to $125 \mu \mathrm{m}$ through a series of processes involving fusionsplicing, cleaving and etching. The fiber tip sensor is fabricated with fused silica fibers only, eliminating the problem of thermal expansion mismatch between different materials.

$\rightarrow$ Hollow fiber-based miniature sensors, which employ a simple and efficient fabrication process, were designed and evaluated in both static and dynamic pressure environments. Sensitivities of about $1.17 \mathrm{~nm} / \mathrm{psi}$ were demonstrated with no apparent hysteresis and very good repeatability and linearity. The sensitivity can be further improved by reducing the thickness of the diaphragm. The measured temperature coefficient was about $0.05 \mathrm{~nm} /{ }^{\circ} \mathrm{C}$.

$\rightarrow$ Sapphire diaphragm-based high temperature sensors were designed. Three types of fibers have been tested using with two types of fused silica wafers.

$\rightarrow$ A novel method, laser ablation on hot substrate, was developed to etch sapphire using excimer laser.

$\rightarrow$ A simple, novel process was developed to fabricate intrinsic Fabry-Perot interferometric sensors for temperature and strain measurements by wet chemical etching and fusion splicing. This process is not only a cost effective technology but also has potential for batch processing.

$\rightarrow$ We have fabricated IFPI sensors on commercially available photosensitive fiber by using UV laser source, and have built an interferogram analysis based measuring system, and have demonstrated their usage for temperature, strain and pressure sensing. We also proposed a spatial frequency division multiplexing scheme, and showed the potential to densely multiplex IFPI sensors.

$\rightarrow$ A thermally tunable filter was demonstrated with a tuning range of $90.8 \mathrm{~nm}$ with a temperature sensitivity of $1.63 \mathrm{~nm} /{ }^{\circ} \mathrm{C}$ and a long-term drift of less than $5.0 \mathrm{pm}$ in the $1550 \mathrm{~nm}$ band. The filter features polarization independence, high repeatability, and inherently low drift and low insertion loss.

$\rightarrow$ The current data analysis system was analyzed and a new fiber sensor spectrum analysis system was designed to be portable, cost effective, with low power consumption, and adaptable to many types of fiber sensors. We are currently programming the USB host driver to achieve control of the spectrometer and writing 
the system application program. The size of a finished system PCB board, with CPU, memory, USB ports, power and LCD circuits on it, will not be larger than the size of the LCD screen.

$\rightarrow$ Three types of fiber optic pressure sensors were successfully field tested near the fan of a jet engine, demonstrating not only good sensor performance, but also that the sensor packaging and installation are suitable for engine operation. 


\section{Table of Contents}

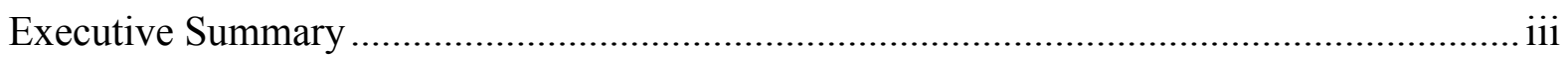

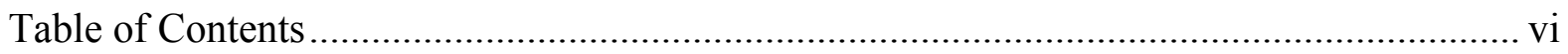

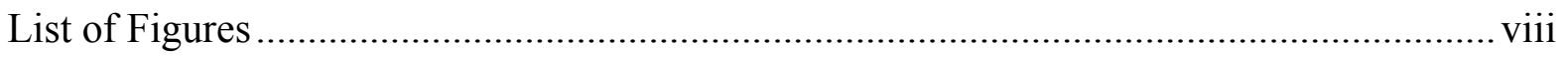

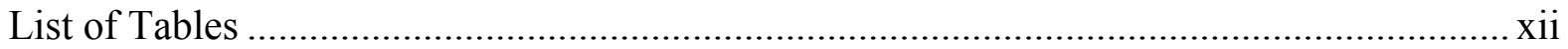

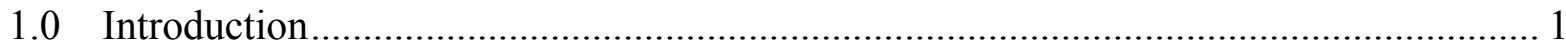

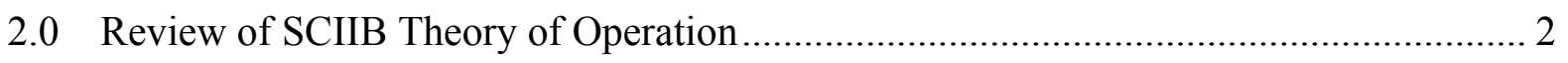

3.0 Pressure Sensor Development.............................................................................. 4

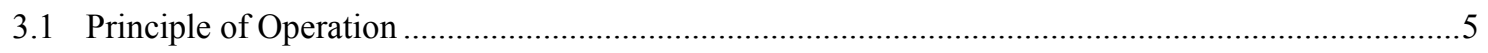

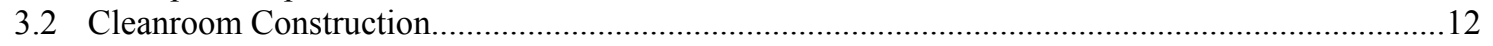

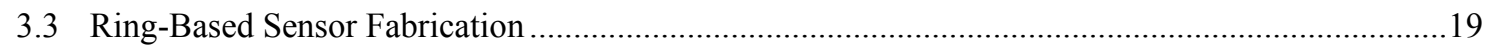

3.4 Etched Diaphragm-Based Pressure Sensors ……..........................................................................

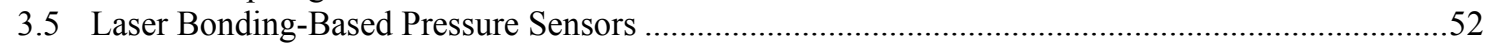

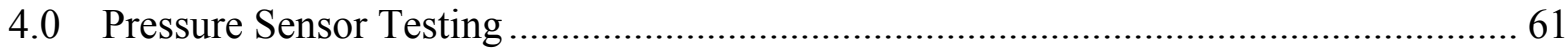

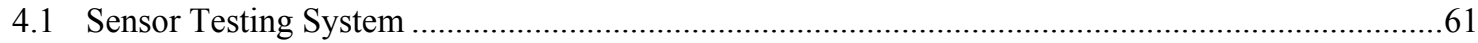

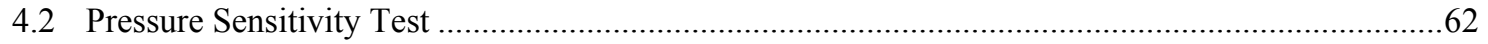

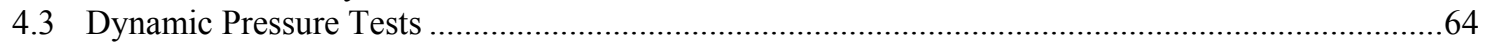

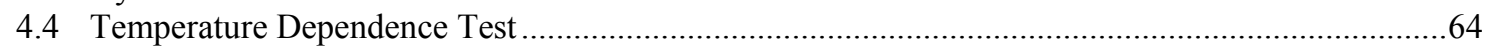

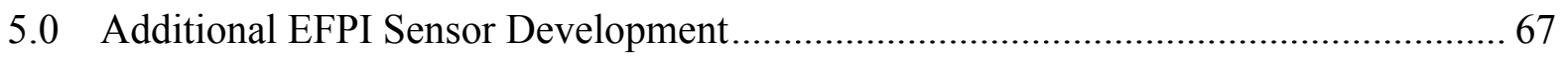

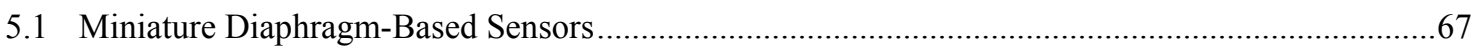

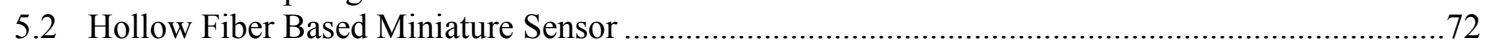

5.3 Diaphragm-Based High Temperature Sensor .............................................................................. 81

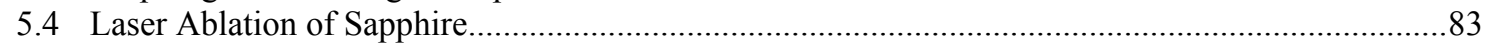

6.0 A Novel Intrinsic Fabry-Perot Fiber Optic Sensor ........................................................ 86

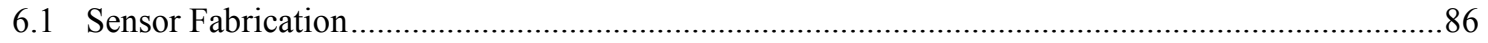

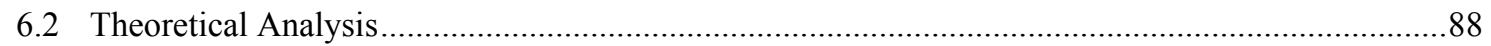

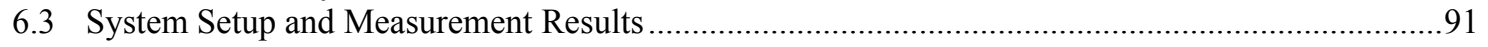

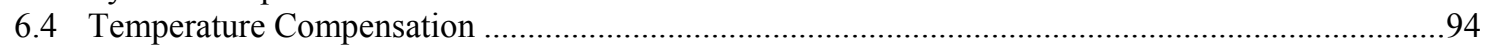

7.0 Multiplexed Intrinsic Fabry-Perot Interferometric (IFPI) Sensors ................................. 98

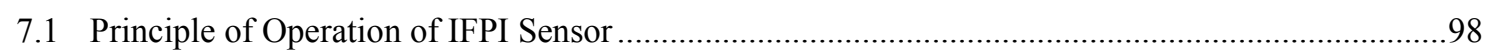

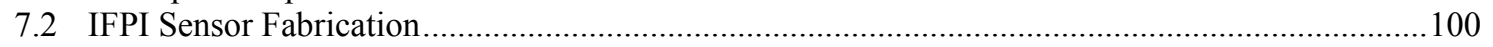

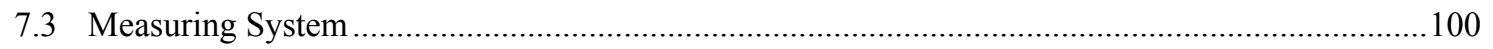

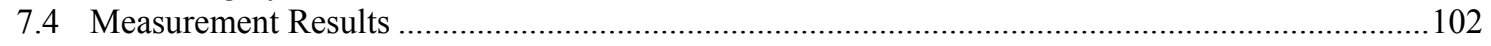

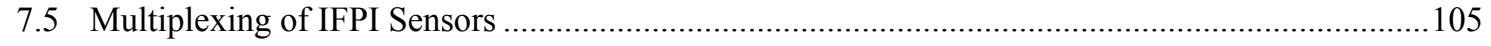

8.0 Testing in Simulated Harsh Environments ............................................................... 108

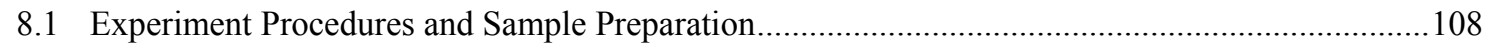

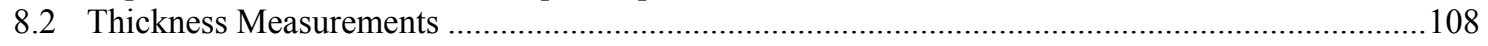

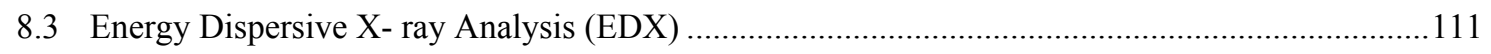

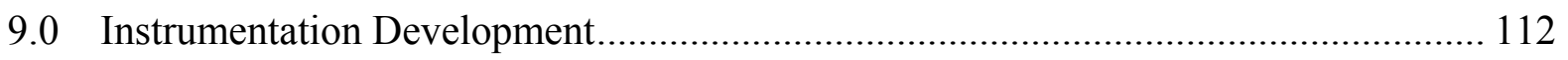

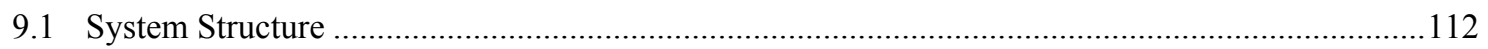

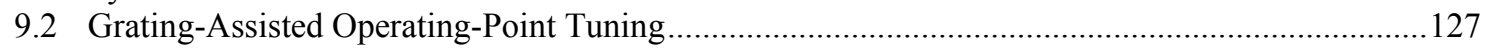




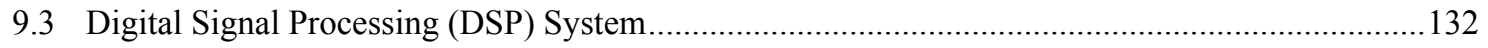

9.4 Thermally Tunable Extrinsic Fabry-Perot Filter ........................................................................137

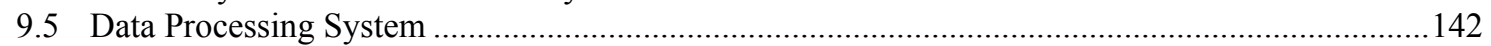

10.0 Fiber Optic Engine Pressure Sensor Field Test ................................................. 153

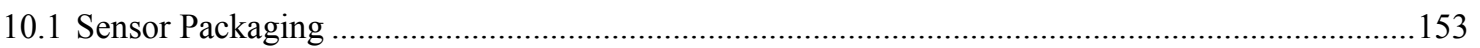

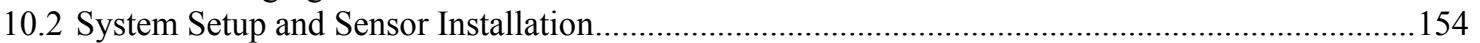

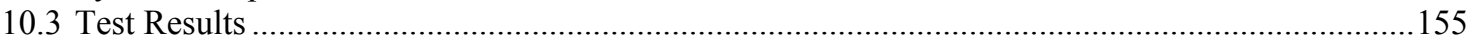

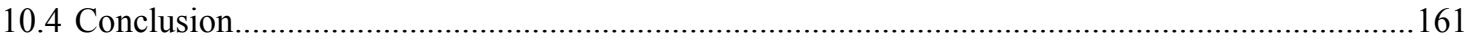

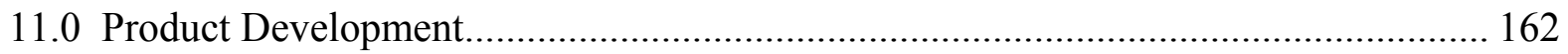

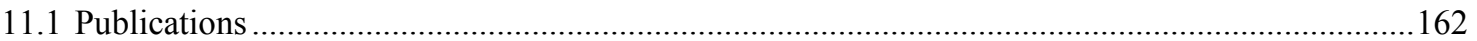

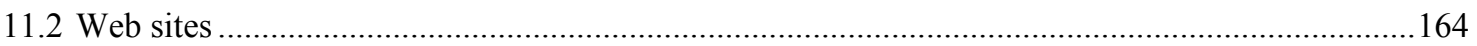

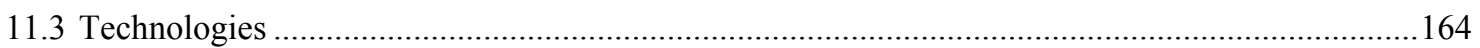

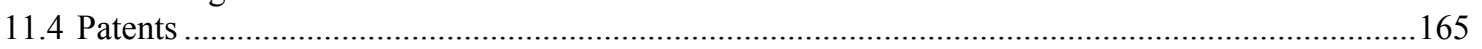

12.0 Conclusions and Future Work ...................................................................... 167

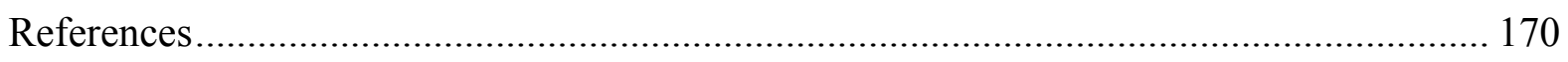

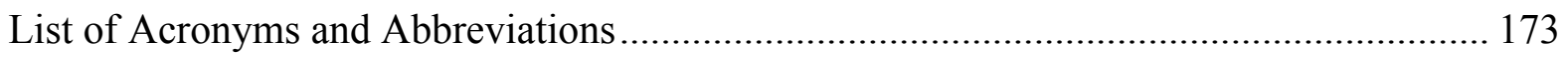

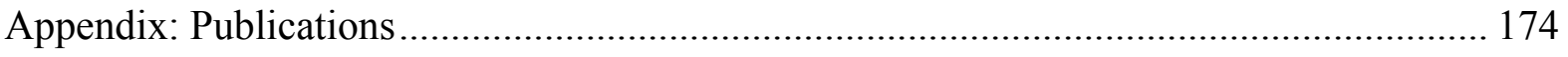

Optical Fiber Sensors for Energy Intensive Industries vii 


\section{List of Figures}

Figure 2.1. Illustration of the principle of the self-calibrated interferometric/intensity-based (SCIIB) fiber sensor.

Figure 3.1. Schematic of basic diaphragm-based SCIIB sensors. ......................................

Figure 3.2. Illustration of the linear operating range of the sensor response curve.................6

Figure 3.3. Structural model for diaphragm-based sensor...............................................8

Figure 3.4. Predicted frequency response of the sensor versus pit diameter. ........................9

Figure 3.5. Predicted diaphragm sensitivity versus pit diameter. ..................................... 10

Figure 3.6. SCIIB (a) wideband signal; (b) narrowband signal; (c) ratio of the two..............13

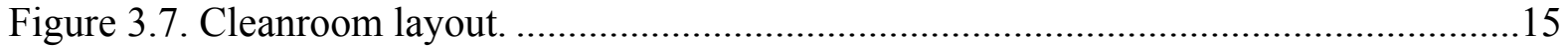

Figure 3.8. A view of the CPT cleanroom from the main door..........................................16

Figure 3.9. Bold Technology wet processing station (left) and Edwards AUTO 500

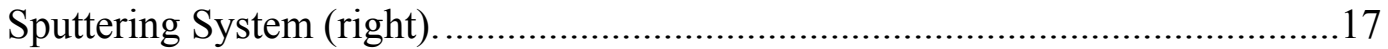

Figure 3.10. DEKTAK3 Profiler (left) and Gaertner Ellipsometer (right) in the cleanroom. .18

Figure 3.11. Structure of ring-based sensor............................................................ 20

Figure 3.12. Borosilicate ring thickness experimental setup. ........................................21

Figure 3.13. Surface inspection of ring before and after etching.....................................22

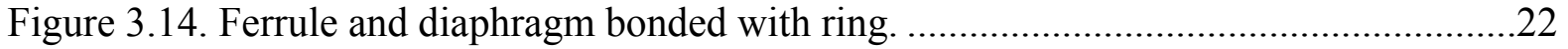

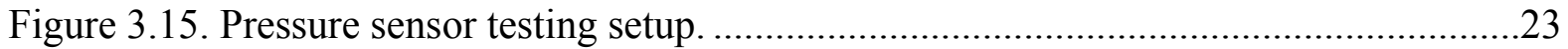

Figure 3.16. Experimental narrowband signal data and Eq. 3-22 curve fit. .........................24

Figure 3.17. Narrowband signal in the $1^{\text {st }}$ test round......................................................26

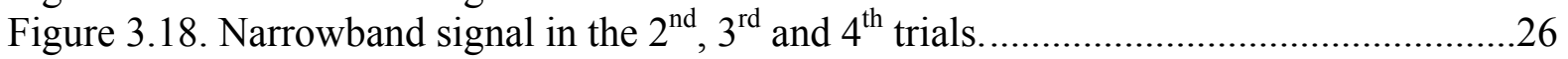

Figure 3.19. Narrowband signals at three operating temperatures. .....................................2

Figure 3.20. Stability of narrowband signal, wideband signal and their ratio during a several day test; total useful testing period was about 68 hours due to data collection

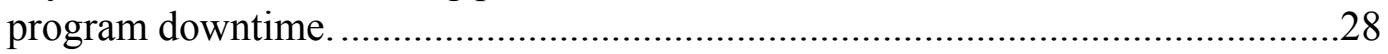

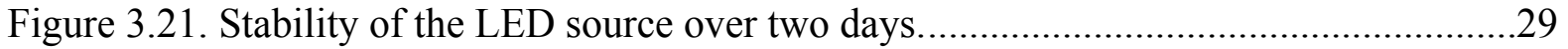

Figure 3.22. Structure of etched diaphragm-based sensor.................................................

Figure 3.23. Wet etched pit depth in a fused silica diaphragm........................................ 31

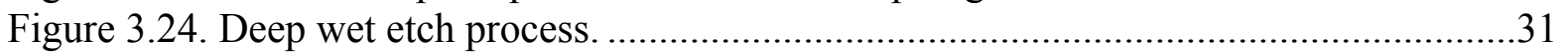

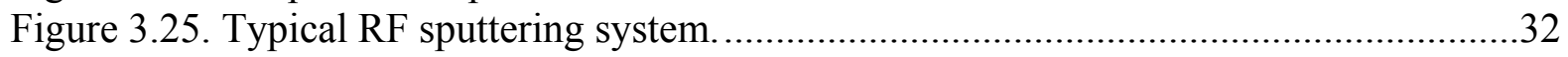

Figure 3.26. Profile of a fused silica diaphragm etched in BHF for 5 hours using the deep wet etching process illustrated in Figure 3.24 ............................................................33

Figure 3.27. Representative etched pit in silica diaphragm.............................................33

Figure 3.28. Computer simulation of fused silica diaphragm deflection...............................34

Figure 3.29. Two step etching process used to assist in diaphragm-ferrule alignment. ..........34

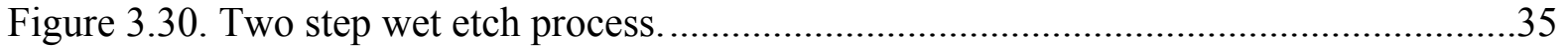

Figure 3.31. Depth profile results of two step wet etch in fused silica (DEKTAK3

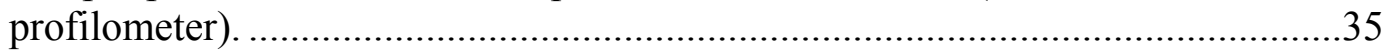

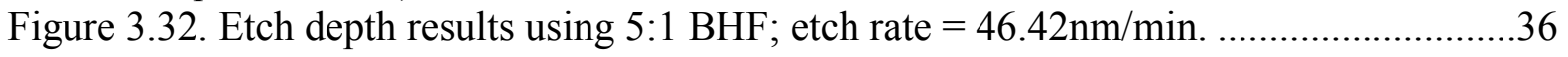

Figure 3.33. Etch depth results using commercial BHF; etch rate $=83.3 \mathrm{~nm} / \mathrm{min} \ldots \ldots \ldots \ldots \ldots . . . .36$

Figure 3.34. Improvements in pit surface quality through changes in BHF composition.......37

Figure 3.35. Impact of UV light/substrate separation distance on exposure uniformity. ........38

Figure 3.36. Improvements in diameter deviation resulting from process improvements. Pits

on the left exhibit a diameter deviation of $73 \%$; those on the right only $9.6 \%$....38 
Figure 3.37. Depth profile results of etched pits from two different fused silica diaphragms

(Veeco DEKTAK3 profilometer)

Figure 3.38. Depth profile results of six etched pits from the same fused silica diaphragm

(Veeco DEKTAK3 profilometer). 39

Figure 3.39. A special chuck has been designed for batch fabrication using direct bonding.

(a) Top view of the chuck with the ferrules inserted in the holes and fixed by adhesive (b) side view (c) side view with the etched diaphragms bonded.

Figure 3.40. (a) Initially pressure is applied at the middle of one wafer to create a single point of contact between the pieces (b) Retracting the mechanical spacers creates a bonding wave that propagates from the middle of the sample toward the periphery. Surface cleanliness and roughness have a strong influence on the quality of the bond.

Figure 3.41. Portions of the interface of two bonded wafer (a) completely bonded (b) not bonded due to presence of void. .44

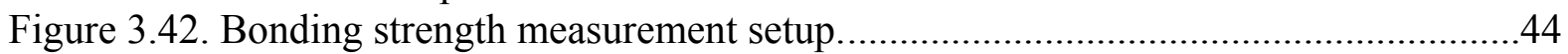

Figure 3.43. Hydroxide-catalysis room temperature fused silica bonding. ...........................45

Figure 3.44. Interface of a wafer bonded with a ferrule bonded using the mechanism of

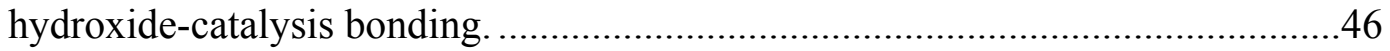

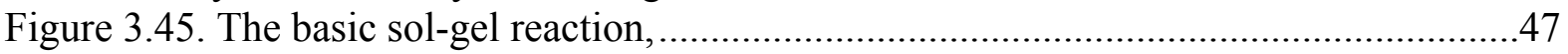

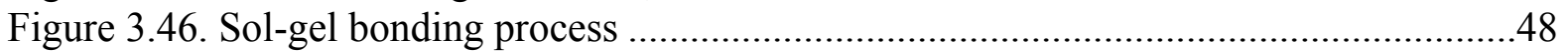

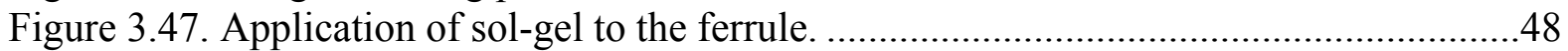

Figure 3.48. Sol-gel bonded diaphragm and ferrule. .........................................................4

Figure 3.49. Interference fringes of a representative diaphragm-based pressure sensor.........49

Figure 3.50. SCIIB pressure sensor test results for 13-200 psi and 200-13 psi......................50

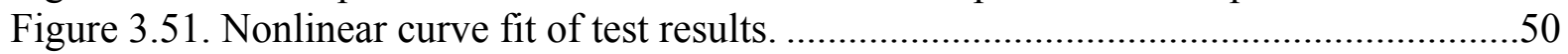

Figure 3.52. Comparison of PSI output (top) to SCIIB test results (bottom)..........................51

Figure 3.53. Diaphragm based fiber optic pressure sensor..................................................52

Figure 3.54. CPT's engine sensor; the sensor head is $8 \mathrm{~mm}$ long and $1.8 \mathrm{~mm}$ in diameter. .....52

Figure 3.55. $\mathrm{CO}_{2}$ laser automatic sensor fabrication system. .............................................53

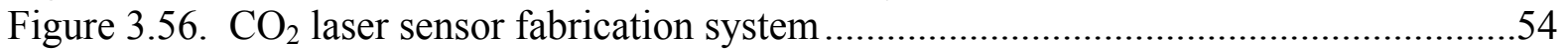

Figure 3.57. Rotary device and laser bonding process. ....................................................5

Figure 3.58. Diaphragm and ferrule thermal bonding. …………….....................................5

Figure 3.59. Micrograph of diaphragm and ferrule laser bonding (top view).......................55

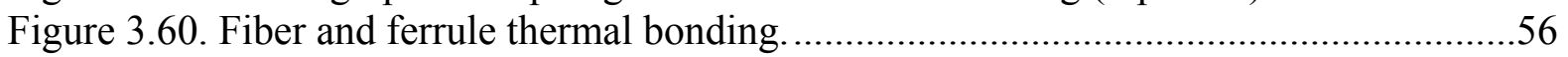

Figure 3.61. Micrograph of the ferrule and fiber laser bonding (side view)..........................56

Figure 3.62. Thermal bonding between an optical fiber and a ferrule...................................57

Figure 3.63. Photo of the fused bonding between an optical fiber and a ferrule.....................57

Figure 3.64. The basic structure of interferometer-based white light system...........................58

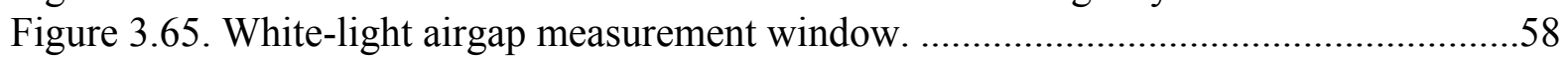

Figure 3.66. MMF engine pressure sensor temperature dependence test...............................59

Figure 3.67. MMF engine pressure sensor pressure sensitivity test. .....................................59

Figure 3.68. MMF engine pressure sensor pressure sensitivity test. .......................................60

Figure 4.1. Schematic of the high temperature dynamic pressure test system. .......................61

Figure 4.2. Diaphragm deflection model. .............................................................................62

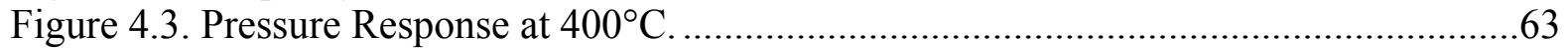

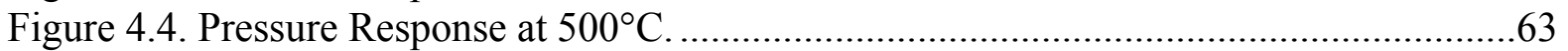




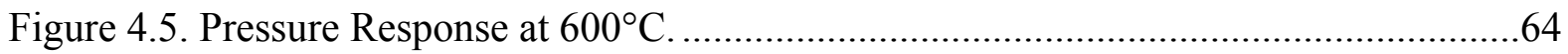

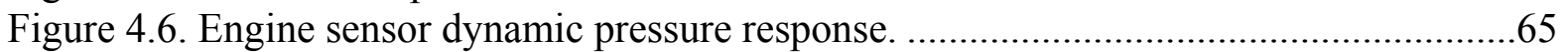

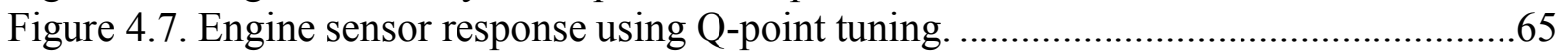

Figure 4.8. Engine sensor temperature dependence test $\left(25^{\circ} \mathrm{C}\right.$ to $\left.600^{\circ} \mathrm{C}\right)$............................66

Figure 5.1. A traditional diaphragm-based EFPI pressure sensor structure. ...........................67

Figure 5.2. $62.5 \mu \mathrm{m}$-core fiber and a $50 \mu \mathrm{m}$-core fiber etched in $50 \% \mathrm{HF}$ solution for 5 minutes. The cores of both fibers are clearly etched off to form a 'hollow tube' in the tip but claddings are nearly intact. ................................................................68

Figure 5.3. Basic structure of a miniature fiber tip pressure sensor. Slightly different interferometric structures are possible; only the simplest is presented here..........69

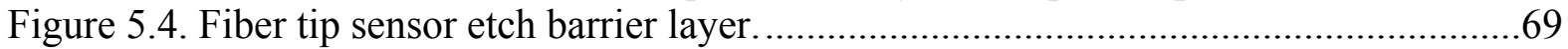

Figure 5.5. Fiber tip sensor cavity formation process.......................................................70

Figure 5.6. Fiber tip sensor diaphragm fabrication process.................................................. 70

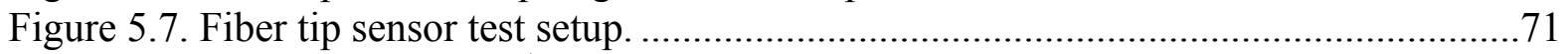

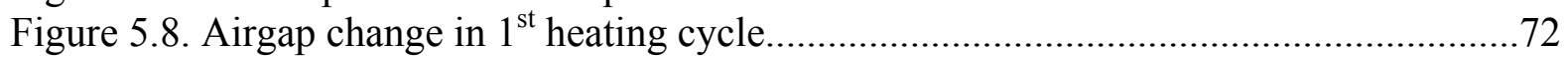

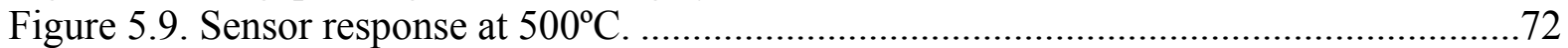

Figure 5.10. Schematic (top) and photograph (bottom) of the hollow fiber-based miniature

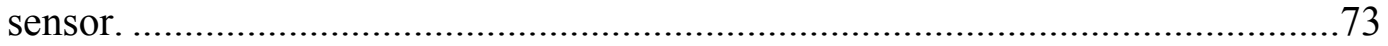

Figure 5.11. Complete setup for miniature sensor fabrication: cleaver, splicer and microscope.

Figure 5.12. Micron Optics Component Test System (CTS), a high resolution swept laser

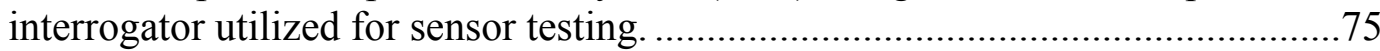

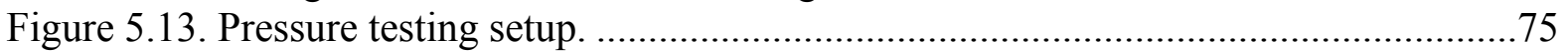

Figure 5.14. Interference fringes of a hollow fiber based pressure sensor. ..............................77

Figure 5.15. Airgap change versus pressure change for a hollow fiber-based pressure sensor.77

Figure 5.16. Repeatability of airgap change, six curves plotted together...............................78

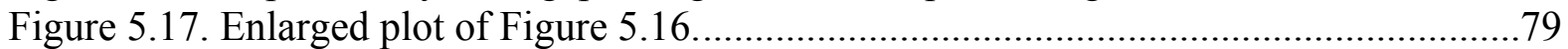

Figure 5.18. Sensor's temperature dependence. ................................................................. 80

Figure 5.19. Relative position between the transducer and the sensor for dynamic testing....80

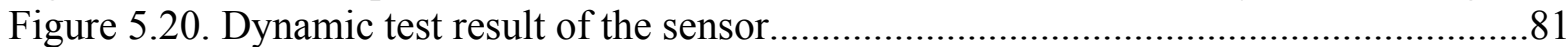

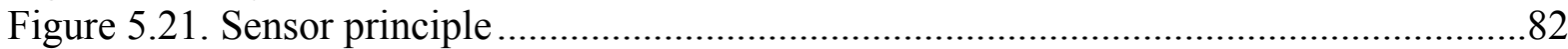

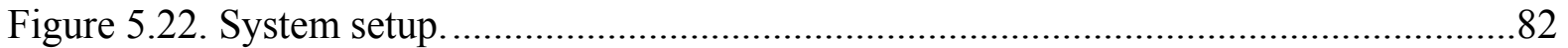

Figure 5.23. Interference signal generated by various types of fibers and wafers...................83

Figure 5.24. Principle of backside heating laser ablation ...................................................... 84

Figure 5.25. Surface quality produced with backside heating laser ablation (left) and direct

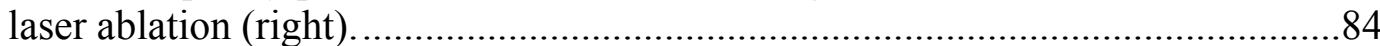

Figure 6.1. Differential wet chemical etching (a) Before etching; (b) After etching; (c) End

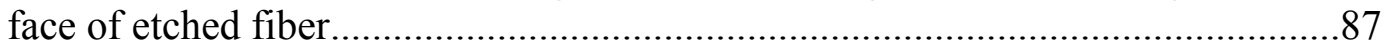

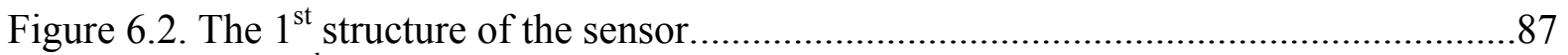

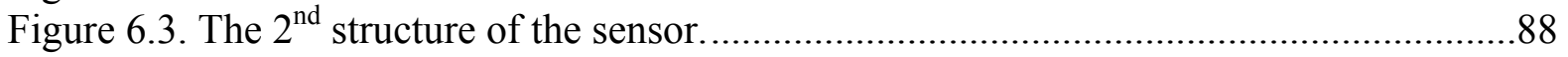

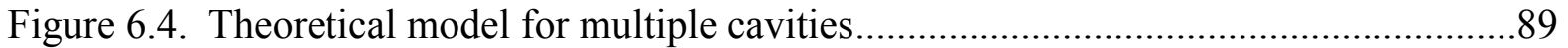

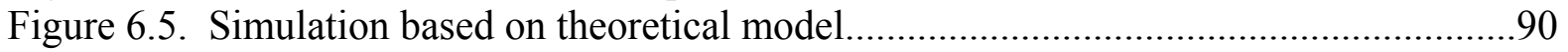

Figure 6.6. Interference fringes captured from testing system .............................................99

Figure 6.7. Principle diagram of the sensor system ..............................................................91

Figure 6.8. The output of OPD when the temperature was increased. ...................................92

Figure 6.9. The output of OPD when the temperature was decreased...................................92 
Figure 6.10. Hysteresis of temperature measurement.................................................93

Figure 6.11. Setup for strain measurement. ..................................................................93

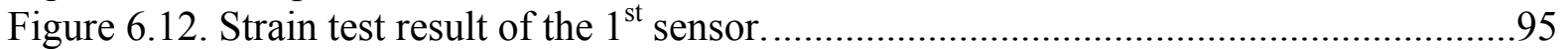

Figure 6.13. Strain test result of the 2 nd sensor..............................................................95

Figure 6.14. Comparison between the optical sensors and the foil strain gauge...................96

Figure 6.15. Spectrum of multiplexed FP sensor and LPG. .........................................96

Figure 6.16. FFT of the spectrum of multiplexed FP and LPG......................................97

Figure 7.1. UV-induced IFPI sensor structure (left) and photograph (right)........................99

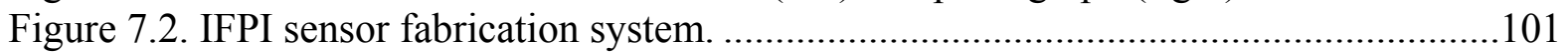

Figure 7.3. Diagram of IFPI measuring system....................................................... 101

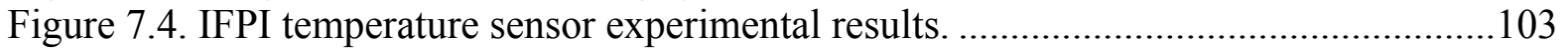

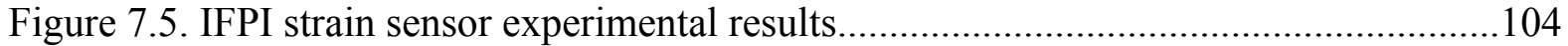

Figure 7.6. Schematic representation of the proposed IFPI pressure sensor. .......................104

Figure 7.7. IFPI pressure sensor experimental results. .................................................. 104

Figure 7.8. Interferogram and FFT result of signals from 11 sensors. ..............................106

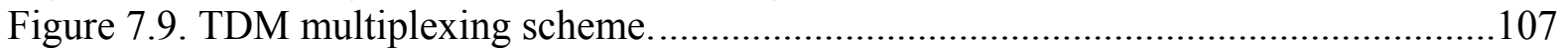

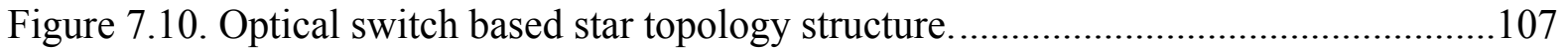

Figure 8.1. Slices of crucibles with sapphire and zirconia in soda lime glass.....................108

Figure 8.2. Cross section of sapphire in soda lime glass. ................................................ 109

Figure 8.3. EDX of zirconia-glass interface for silicon (top left), sodium (top right), zirconium (bottom left) and calcium (bottom right)..........................................111

Figure 9.1. Schematic of the diaphragm based engine sensor system................................113

Figure 9.2. Characteristics of the high power LED. ........................................................114

Figure 9.3. Temperature dependence of the optical power and CWL...............................114

Figure 9.4. Driving current dependence of the optical power and CWL at different

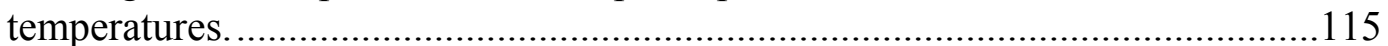

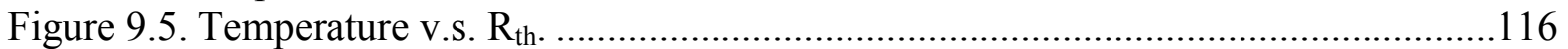

Figure 9.6. Schematic of the HTC-3000 Hybrid Temperature Controller (Wavelength

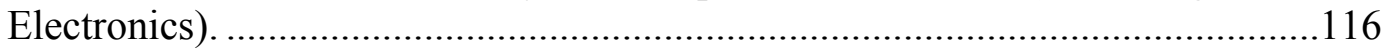

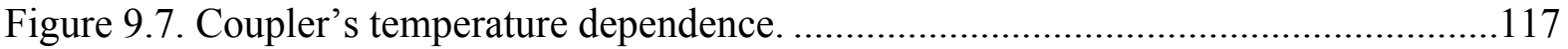

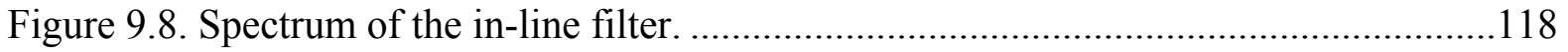

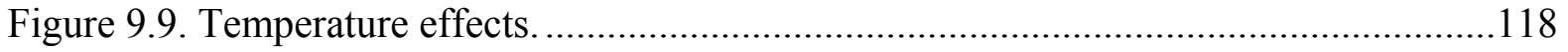

Figure 9.10. Schematics of optoelectronic circuits...................................................... 119

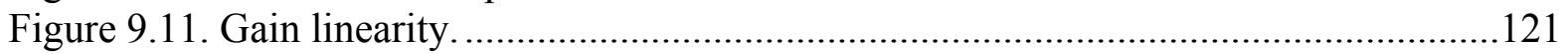

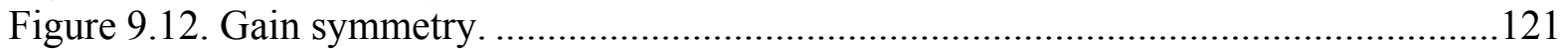

Figure 9.13. Frequency response of the two preamplifiers.........................................121

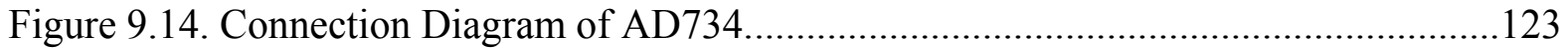

Figure 9.15. Two-Quadrant Divider with Fixed Voltage Scaling. ......................................124

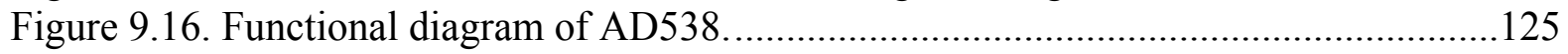

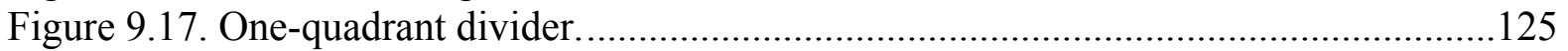

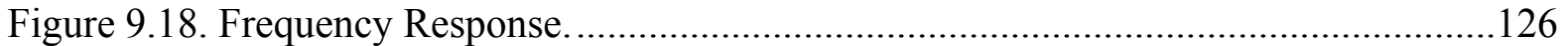

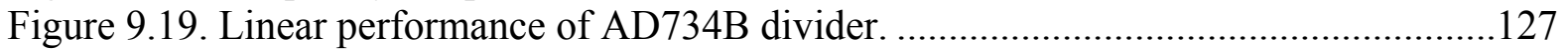

Figure 9.20. Illustration of the principle of an FFPI sensor system based on GA-OPT.......129

Figure 9.21. A theoretical calculation of the wavelength dependence of the operating point, where $2 \Delta \lambda=10 \mathrm{~nm}, \mathrm{R}=3.5 \%$, and F-P cavity length $\mathrm{L} 0$ is in $\mu \mathrm{m} . \ldots \ldots \ldots \ldots \ldots \ldots . . . . . .129$

Figure 9.22. Angular range and receiving bandwidth $(2 \Delta \lambda)$ v.s. wavelength tuning range. .131 
Figure 9.23. Spectral distributions received by the detector at different grating positions and the original spectral distribution of the SLED...............................................132

Figure 9.24. Acoustic wave and its spectrum detected by the fiber sensor without GA-OPT

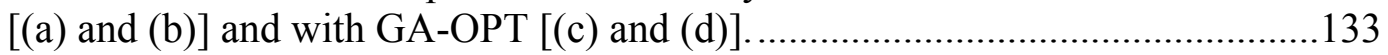

Figure 9.25. Signal flow diagram for the digital system...............................................134

Figure 9.26. Schematic of the thermally tunable EFPI filter. ........................................138

Figure 9.27. Optical micrograph of an EFPI filter..........................................................139

Figure 9.28. Photo of a packaged thermally tunable EFPI filter. ......................................139

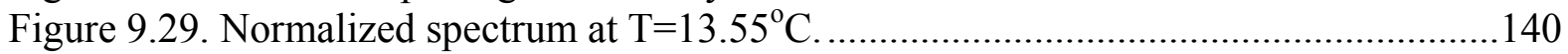

Figure 9.30. Tuning curves of the thermally tunable EFPI filter......................................141

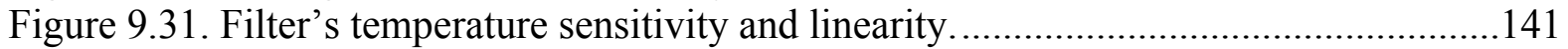

Figure 9.32. Block diagram of current data processing system.....................................143

Figure 9.33. Block diagram of newly designed data processing system. ...........................144

Figure 9.34. i.MX1 Functional Block Diagram............................................................. 147

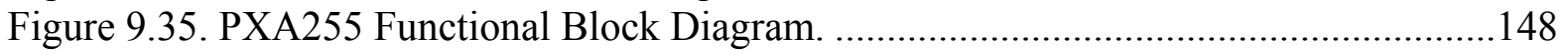

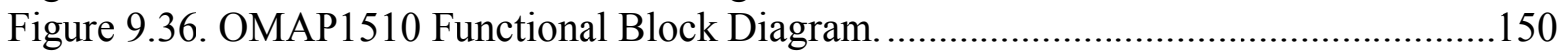

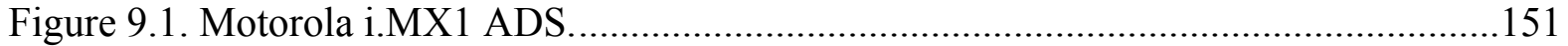

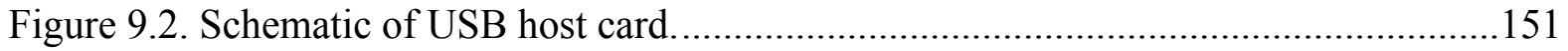

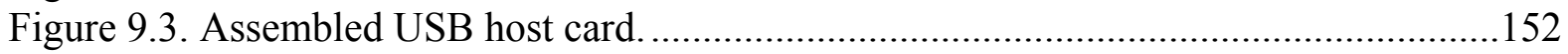

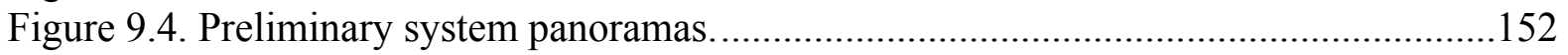

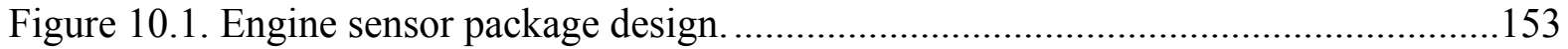

Figure 10.2. Representative packaged diaphragm-ferrule based fiber optic pressure sensor

(left) and miniature sensor (right) .............................................................153

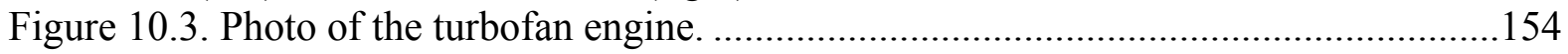

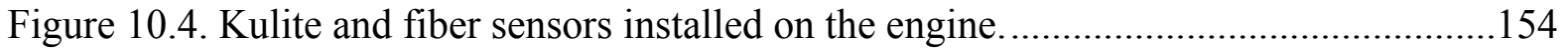

Figure 10.5. System instrumentation in control room. .................................................155

Figure 10.6. Diaphragm-ferrule based fiber sensor and Kulite sensor signals in time domain.156

Figure 10.7. Diaphragm-ferrule based fiber sensor and Kulite sensor signals in frequency

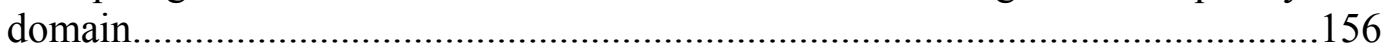

Figure 10.8. Time domain response of the optical sensor compared with that of the Kulite sensor. (The upper plot shows the response of the optical sensor; while the lower one shows the response of the Kulite sensor.).

Figure 10.9. Frequency response of the optical sensor compared with that of the Kulite sensor. (The upper plot shows the response of the optical sensor; while the lower one shows the response of the Kulite sensor.)................................................158

Figure 10.10. Mini-sensor output vs. Kulite sensor output, time domain signal ................159

Figure 10.11. Mini-sensor output vs. Kulite sensor output, FFT spectrum.........................160

\section{List of Tables}

Table 3.1. Values of $\alpha_{\mathrm{mn}}$ (constant related to the vibrating modes of the diaphragm)...........9

Table 3.2. Properties of fused silica $\left(@ 25^{\circ} \mathrm{C}\right)$......................................................................... 9

Table 3.3. Detailed cleanroom specifications...................................................................... 14

Table 3.4. Detailed specifications of DEKTAK3 profilometer......................................... 17 
Table 3.5. Detailed specifications of Gaertner ellipsometer.

Table 3.6. Comparison of thickness measurement using the profile method and calipers......21

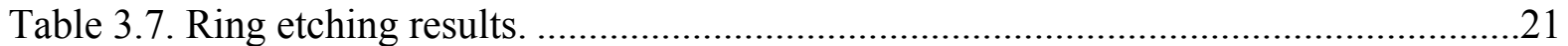

Table 3.8. Fitting parameters from Eq. 3-22 for three operating temperatures. .....................22

Table 3.9. Chromium coating thickness resulting from various processing parameters..........32

Table 8.1. Experimental thickness measurement data employing both methods. ..................110

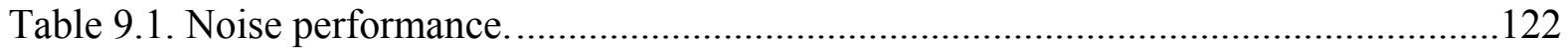

Table 9.2. Component listing for current data processing system......................................143

Table 9.3. Component listing for new data processing system.........................................145

Table 10.1. Main frequencies measured by Kulite and diaphragm-ferrule based sensors. ...157

Table 10.2. Comparison results between the first miniature fiber sensor and the Kulite sensor 158

Table 10.3. Comparison results between the second miniature fiber sensor and the Kulite

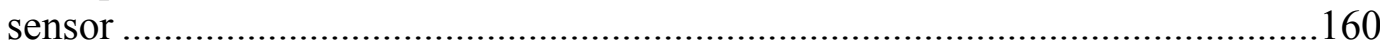




\subsection{Introduction}

This is the final report for the Virginia Tech Center for Photonics Technology (VTCPT) research project entitled "Optical Fiber High Temperature Sensor Instrumentation for Energy Intensive Industries." The objective of this program was to use technology recently invented at Virginia Tech to develop and demonstrate the application of self-calibrating optical fiber temperature and pressure sensors to several key energy-intensive industries where conventional, commercially available sensors exhibit greatly abbreviated lifetimes due primarily to environmental degradation. The developed sensors fulfill documented needs for real-time accurate monitoring of temperatures and pressures in the harsh high-temperature environments typical of several Industries of the Future (IOF), including transportation, power generation, glass, and metal-casting industries. The end goal of this effort is to allow significant energy efficiency gains, while reducing many of the hazardous emissions associated with these industries, through process control optimization with robust, reliable sensors.

The impetus for development of improved sensors in the IOF industries is well documented. In a recent report commissioned by the U.S. Department of Energy on the potential economic impact of sensors with improved performance in harsh environments, one of the major conclusions was that development of improved harsh environment sensor technologies is projected to have a large (several percent) impact on the energy usage and waste production of U.S. industry. This report states that improved harsh environment sensors could save 17 to 45 trillion Btu of energy in the glass industry with corresponding reductions in greenhouse gas emissions; 26 trillion Btu of energy in the steel industry due to avoidance of scrap and rework; and 0.25 quadrillion Btu of energy across all the strategic energy-consuming industries identified as Industries of the Future (IOF) by the Department of Energy.

Pressure and temperature sensors are vitally important for efficient energy use and low environmental emissions in many industrial processes and engineering systems, especially energy-intensive industries such as the power generation, metal casting, glass and transportation sectors. However, many of these processes involve various harsh environmental conditions, such as high temperature, high pressure, chemical corrosion, and strong electromagnetic interference (EMI), which often make conventional sensors and measurement devices difficult to apply.

Instrumentation based on optical fiber sensors has been demonstrated to be especially attractive for the measurement of a wide variety of physical and chemical parameters because of such inherent advantages as 1) an immunity to electromagnetic interference, 2) avoidance of ground loops, 3) capability of responding to a wide variety of measurands, 4) avoidance of electric sparks, 5) resistance to harsh environment, 6) remote operation, 7) capability of multiplexing, and 8) ease of integration into large-scale fiber networking and communication systems. Because of these inherent advantages, fiber sensors are extremely attractive to a wide range of industries, including the energy-intensive IOF industries. 
This program directly leverages recent breakthroughs at VTCPT in self-calibrated interferometric/intensity-based (SCIIB) sensors and single crystal sapphire fibers. The SCIIB technology for the first time successfully combines optical fiber interferometry and intensity-based signal processing into a single sensor system that possesses all the major advantages of both of these optical methods while eliminating or significantly minimizing the disadvantages that have limited industrial applications of optical fiber sensors in the past. Major advantages of the SCIIB system include small size, high resolution and accuracy, high frequency response, high temperature capability, immunity to electromagnetic interference, strong resistance to chemical corrosions, and self-calibrating and absolute measurement.

In addition, this program also leverages research at VTCPT in the development of sensors based on fibers made of single-crystal sapphire for use in extremely harsh environments, particularly where temperatures exceed $1500^{\circ} \mathrm{C}$. Sapphire is an excellent material for the construction of harsh environment sensors, due to its high melting point, excellent transparency, and well-documented resistance to corrosion.

\subsection{Review of SCIIB Theory of Operation}

For clarity, the operation of the SCIIB pressure sensor is reviewed here. The basic SCIIB configuration is shown in Figure 2.1. The light from an optical source is launched into a two-by-two fiber coupler and propagates along the optical fiber to the sensor head. As shown in the inset of Figure 2.1, the input fiber and a reflector are thermally fused to a hollow glass tube with a predetermined air gap separation. By thermally fusing the glass capillary tube to the glass fibers, the use of organic adhesives such as epoxy is avoided, eliminating the temperature limits and temperature-dependent mechanical properties and hysteresis of those adhesives. The incident light is first partially reflected at the endface of the input fiber $\left(\mathrm{R}_{1}\right.$ in Figure 2.1). The remainder of the light propagates across the air gap to the reflector surface where a second reflection $\left(R_{2}\right)$ is generated. The two reflections travel back along the same input fiber through the same coupler to the photodetection end.

In order to overcome problems associated with conventional fiber interferometric sensors, such as relative measurement, low frequency response, and complexity in signal demodulation, a special optoelectronic signal demodulation method is employed that allows self-calibrating absolute measurement with high frequency response. When the source coherence length is longer than the path length difference of the two legs in an interferometer, interference is observed when the two reflections are combined; when it is less than the path length difference, interference does not occur, and the combination of the two beams results in the sum of their intensities. In the SCIIB system, the coherence length of the sensor channel (Channel 2 in Figure 2.1) is engineered to exceed the path length difference in the Fabry-Perot cavity (twice the gap length), resulting in interference fringes as the gap length is changed by the measurand of interest. The coherence length of the reference channel (Channel 1 in Figure 2.1) is adjusted to be much shorter than the path length difference in the Fabry-Perot cavity. Therefore, no interference occurs and the power output of the reference channel is simply the sum of the optical power of the two reflections 
$\mathrm{R}_{1}$ and $\mathrm{R}_{2}$. Both channels are affected by changes in fiber loss, bending, connector loss and fluctuations in source power, while only the sensor channel exhibits changes due to the effect of the desired measurand, e.g. temperature or pressure, on the gap length. The undesired fluctuations can be canceled out by taking the ratio of the two channels. To simplify the processing and interpretation of the sensor output, the sensor probe is designed so that the optical intensity of the output over the expected full range of the measurand remains within the quasi-linear portion of the interferometric output. This is accomplished by controlling the length, diameter, wall thickness, and the modulus of elasticity of the capillary alignment tube during sensor fabrication. In this way complicated signal processing involving fringe counting or spectral decomposition can be avoided, and a simple photodetector used.

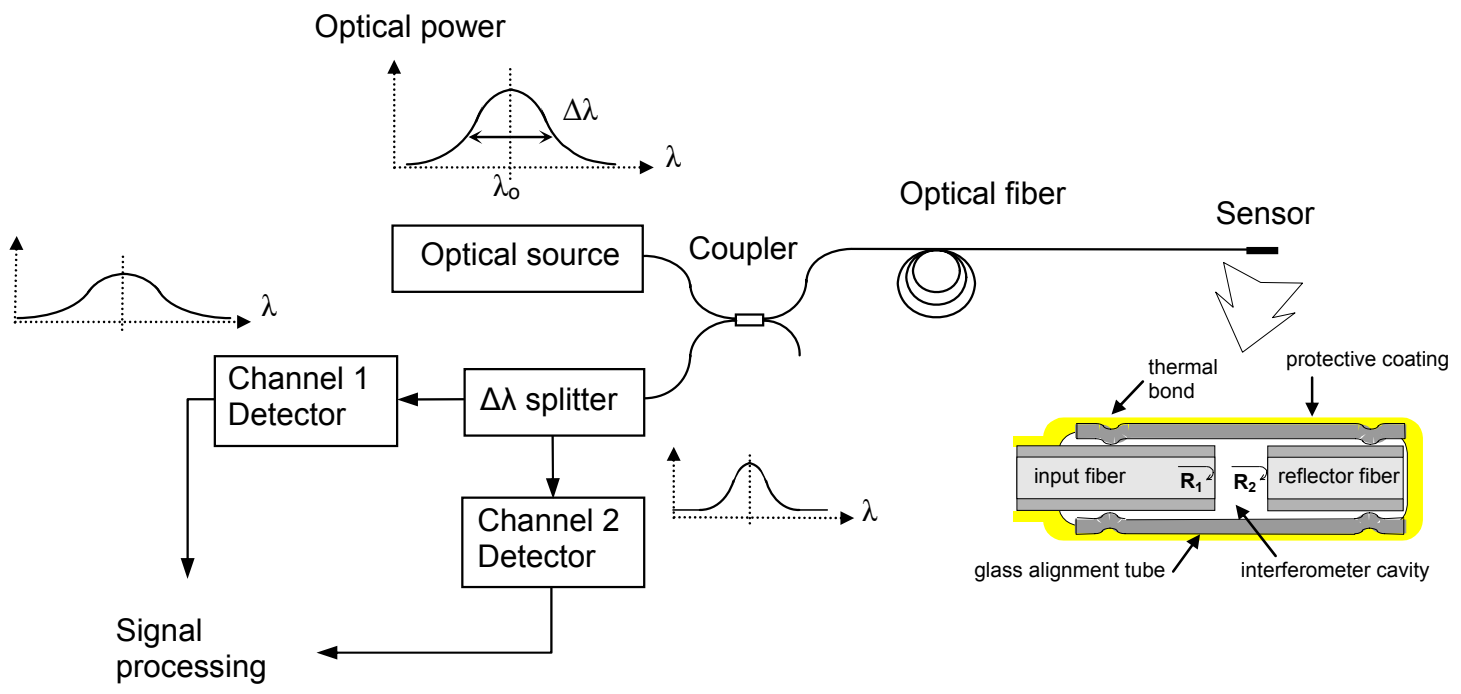

Figure 2.1. Illustration of the principle of the self-calibrated interferometric/intensity-based (SCIIB) fiber sensor.

When a hydrostatic pressure is applied to the sensor head illustrated in Figure 2.1, the airgap length in the sensor will change due to the longitudinal and lateral compression of the glass alignment tube. This variation can be expressed as

$$
\Delta L=\frac{L}{E} \frac{p r_{o}^{2}}{r_{o}^{2}-r_{i}^{2}}(1-2 \mu),
$$

where $p$ is the applied pressure, $E$ is the Young's modulus of the tube material, $\mu$ is the Poisson ratio, $L$ is the distance between the two points where the fibers are fused to the alignment tube, and $r_{o}$ and $r_{i}$ are the outer and inner radii of the glass tube, respectively.

Equation (2-1) indicates that the airgap change $\Delta L$ is linear with respect to the applied pressure. In addition, the equation illustrates the SCIIB design flexibility for different dynamic measurement ranges through the involvement of several parameters, including the inner and outer diameters of the hollow glass tube, the distance between the two fusion points, and the mechanical properties of the hollow glass material. 


\subsection{Pressure Sensor Development}

The first task of the sensor development program was to specify the technical requirements for real time measurement and monitoring of the pressures and temperatures for the power generation, glass melting, metal casting and the transportation industries. This was performed through a collaborative effort with the industrial partners. A trip was conducted to Pratt \& Whitney and to Alstom Power (formerly ABB) to discuss the technology and specify the requirements for the sensors. The parameters for development of the pressure sensors for use in power generation and transportation were determined for the pressure sensors for use in both test and flight engines. In conjunction with Corning, Inc., the parameters for use in crown as well as in glass temperature measurements for a variety of glass compositions have been specified. The specified parameters include crown temperatures, three different representative glass compositions for corrosion testing, in glass thermocouple temperatures, and normal thermocouple lifetimes.

The SCIIB technology described in Section 2.0 has been adapted for the measurement of pressure in both ground-based and in-flight turbine engines. A tube-based SCIIB system is not well suited for the turbine engine requirements; a diaphragm-based SCIIB sensor system (Figure 3.1) was developed during this program. The system is composed of a sensor probe, a broadband light source such as an LED, an optoelectronic signal processing unit and silica glass optical fibers and optical components linking the sensor probe and the signal processing unit. The sensor head is constructed by inserting an optical fiber, with its endface cleaved

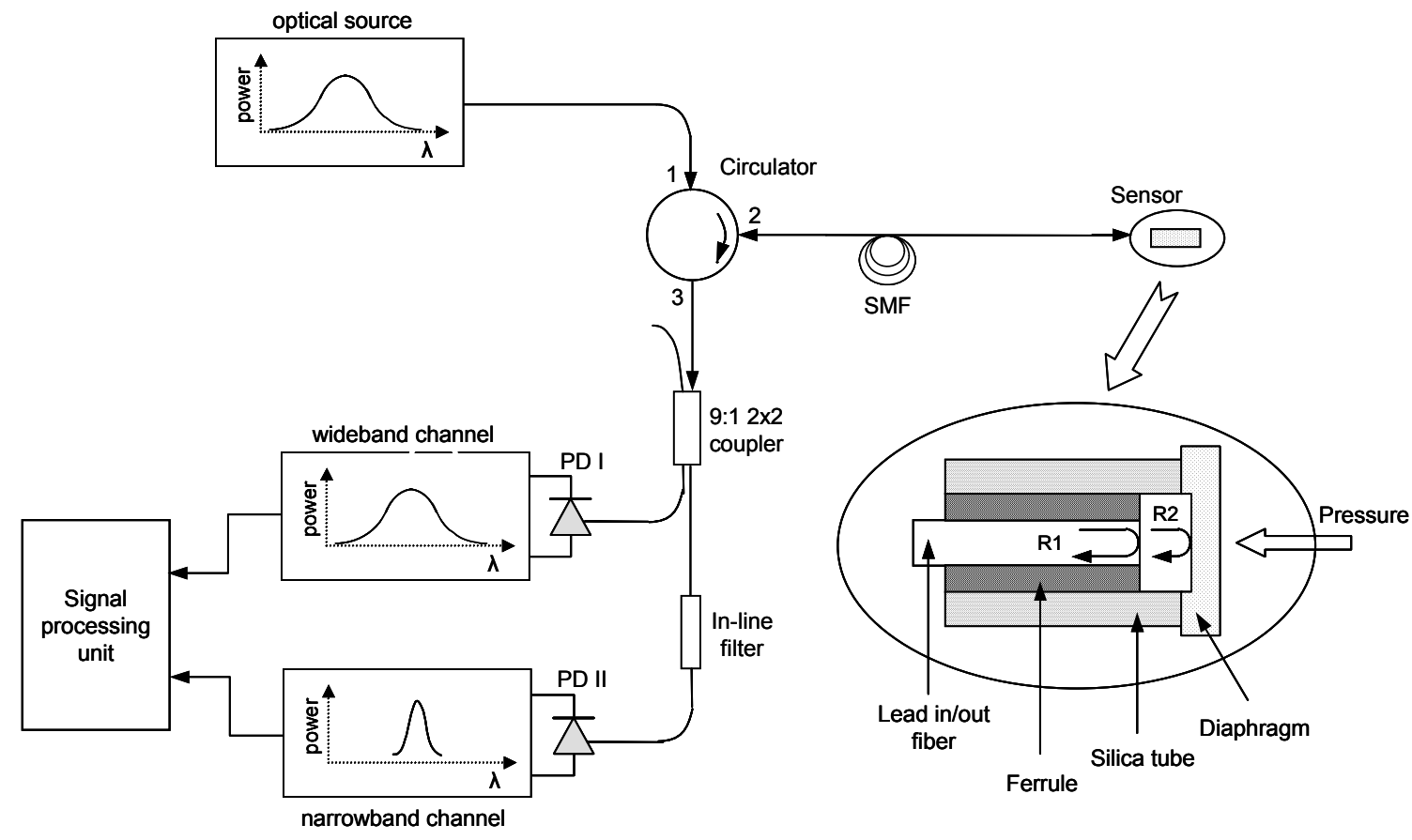

Figure 3.1. Schematic of basic diaphragm-based SCIIB sensors. 
and coated, into a capillary tube so that a Fabry-Perot cavity is formed between the fiber endface and a gold coated fused silica diaphragm. Light from the broadband source is launched into Port 1 of a circulator, outputs from Port 2 and propagates along the optical fiber to the sensor head. Similar to the system shown in Figure 2.1, the two reflections then travel back along the same fiber, to Port 3 of the circulator. The sensor output is then split into a wide band and a narrow band channel that are detected by two photodiodes.

\subsection{Principle of Operation}

The coherence length $\mathrm{L}_{\mathrm{c}}$ of a light source is inversely proportional to the spectral width and can be estimated by

$$
L_{C} \propto \frac{\lambda_{c}^{2}}{\Delta \lambda}
$$

where $\lambda_{c}$ is the central wavelength of the channel and $\Delta \lambda$ is its spectral width. To obtain a highly interfering signal from the F-P cavity, the optical path difference (OPD) between different beams has to be much smaller than the coherence length of the light. If the OPD of the interferometer is larger than the coherence length of the light, the reflected beams will not effectively interfere with each other to generate fringes.

When a low-finesse Fabry-Perot (F-P) interferometer formed by low-reflection mirrors is illuminated by a light source, the response is a periodic function similar to a two-beam interferometer that can be expressed as:

$$
\begin{aligned}
& I=E_{1}^{2}+E_{2}^{2}+2 \cdot e^{\left[-2 L / L_{c}\right]} \cdot E_{1} \cdot E_{2} \cdot \cos \left(\frac{4 \pi L}{\lambda_{c}}+\pi\right) \\
& =R \cdot A \cdot I_{0} \cdot\left[1+\eta^{2}(1-R)^{2}-2 \eta(1-R) \cos \left(\frac{4 \pi}{\lambda_{c}} L\right)\right], \\
& \approx 2 R \cdot A \cdot I_{0} \cdot\left[1-\eta \cos \left(\frac{4 \pi}{\lambda_{c}} L\right)\right]
\end{aligned}
$$

where: $E_{1}$ and $E_{2}$ are the magnitudes of the electrical fields of the reflected light at two airfiber endfaces

$I_{0}$ is the optical power of the source

$R$ is the reflectance of the boundary of the air and fiber endfaces

$L$ is the cavity length

$A$ is the optical loss coefficient along the whole optical path

$\eta$ is the coupling coefficient of the Fabry-Perot cavity. It is a function of the cavity length $\mathrm{L}$, the lateral offset, and the angular offset. Because the cavity length change is very small, it remains relatively constant.

The reflectance can be calculated by 


$$
R=\left[\frac{\left(n-n^{\prime}\right)}{\left(n+n^{\prime}\right)}\right]^{2},
$$

where $\mathrm{n}$ is the refractive index of the fiber core and diaphragm, and $n^{\prime}$ is that of the medium separating the fiber and the diaphragm, which in our case is air. The reflectance at the surfaces is thus about $4 \%$ of the total incident optical power.

Figure 3.2 illustrates the output interference fringes typically obtained from the diaphragmbased F-P sensors. The pressure signal generated by a turbine engine, for example, causes the deformation of the diaphragm, which modulates the sealed air gap length. The sensor therefore gives an output signal that corresponds to the pressure modulation of the engine.

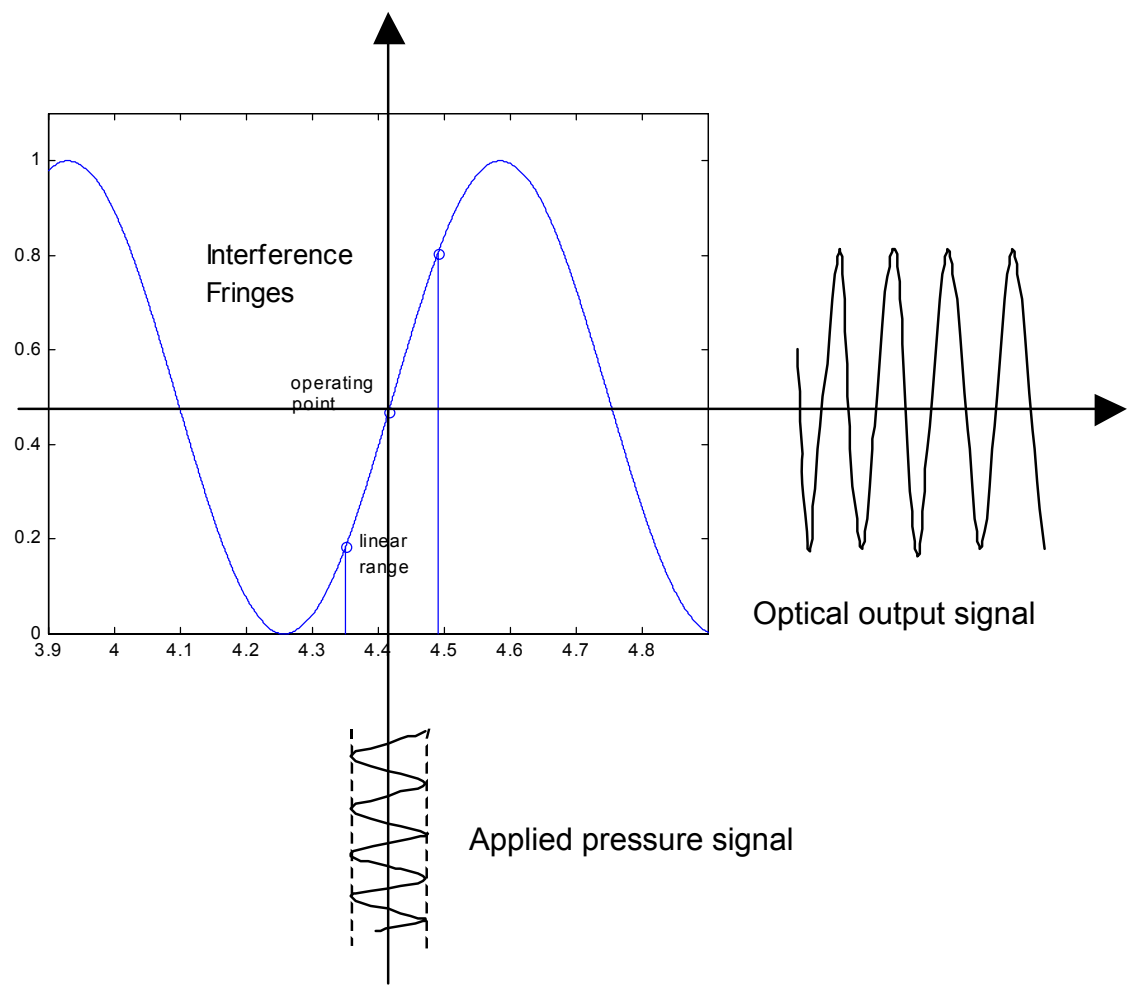

Figure 3.2. Illustration of the linear operating range of the sensor response curve.

The quality of the optical interference is described by the parameter fringe visibility $\gamma$, defined by:

$$
\gamma=\frac{I_{\max }-I_{\min }}{I_{\max }+I_{\min }},
$$

where $I_{\min }$ and $I_{\max }$ are the minimum and maximum intensities of the optical interference, respectively. Obviously, $\gamma$ depends on $\eta$ and $R$. 
The sensitivity of an interferometer $\left(I_{\text {sens }}\right)$ can be defined as the intensity change produced in response to unit air gap change as given by:

$$
I_{\text {sens }}=a b s\left(\frac{d I}{d L}\right)=a b s\left(\beta \cdot \frac{4 \pi}{\lambda_{c}} \sin \left(\frac{4 \pi}{\lambda_{c}} L\right)\right),
$$

where $\beta=2 R A I_{0} \eta$. The maximum sensitivity ( $I_{\text {sens }}(\max )$ ) obtained at the quadrature point of the interference fringes is:

$$
I_{\text {sens }}(\max )=\beta \frac{4 \pi}{\lambda_{c}} .
$$

Like regular interferometers, the measurement will have ultra-high sensitivity. One period of fringe variation corresponds to an air gap change of one-half of the optical wavelength. However, from Eq. (3-5), the measurement would suffer from the disadvantages of sensitivity reduction and fringe direction ambiguity when the sensor reaches peaks or valleys of the fringes. Sensitivity is reduced at the peak or valley of a fringe since at that point the change in optical intensity is nearly zero for a small change in the air gap. Fringe direction ambiguity refers to the difficulty in determining whether the air gap is increasing or decreasing by detecting the optical intensity. If a measurement starts with an air gap corresponding to the peak of a fringe, the optical intensity will decrease, regardless of whether the gap increases or decreases. Therefore, we define the operating range of the sensor $\left(I_{\text {operating }}\right.$ in $\left.\mu \mathrm{m}\right)$ as its sensitivity remaining within the $60 \%$ of the maximum sensitivity.

$$
I_{\text {operating }}=\text { Solution }\left\{I_{\text {sens }} \leq \beta \frac{4 \pi}{\lambda} \bullet 60 \%\right\} .
$$

Within this range, a pressure induced airgap change can be realized linearly by the sensor. To obtain the highest pressure sensitivity and resolution possible, it is very important to ensure the sensor work within its operating range.

An airgap change of a half of a wavelength can cause the relative movement of one whole fringe length. This would allow for ambiguity in the interpretation of the pressure signal. For example, if the pressure response was such that the diaphragm was deflected a distance equivalent to multiple wavelengths, the output signal could be from any of the sinusoidal repeat fringes; it would first be necessary to know which fringe one was on. Once the fringe was determined, the pressure measurement then would be absolute. It is not apparent however, how to determine which fringe you might be on. In principle, continuous tracking of phase changes in the interference fringes can yield information about the airgap changes in the sensor element. However, this is difficult to realize and suffers many disadvantages.

\subsubsection{Diaphragm Frequency Response}

A structural model for a diaphragm-based sensor is shown in Figure 3.3. For a free vibrating circular plate clamped at the edge, its natural frequency $f_{m n}$ is given by: 


$$
f_{m n}=\frac{\alpha_{m n}}{2 \pi r^{2}} \times \sqrt{\frac{D g}{h w}},
$$

where $\alpha_{\mathrm{mn}}$ is a constant related to the vibrating modes of the diaphragm, listed in Table 3.1; $r$ is the radius of the free area of the diaphragm (in our design, $r$ is the radius of a etched pit on a diaphragm); $h$ is the thickness of the diaphragm; $g$ is the gravitational constant; $w$ is the density of the diaphragm material; $D$ is the flexural rigidity of the diaphragm defined by:

$$
D=\frac{E h^{3}}{12\left(1-\mu^{2}\right)},
$$

where $\mu$ is the Poisson's ratio; E is the Young's modulus of the fused silica glass material. From the properties of fused silica shown in Table 3.2, the frequency response of the sensor can thus be calculated by combining Eq. (3-8) and (3-9):

$$
f_{m n}=2.749 \times 10^{9} \times \frac{\alpha_{m n}}{\alpha_{00}} \frac{h}{r^{2}}
$$

where $\mathrm{h}$ and $\mathrm{r}$ are specified in microns.

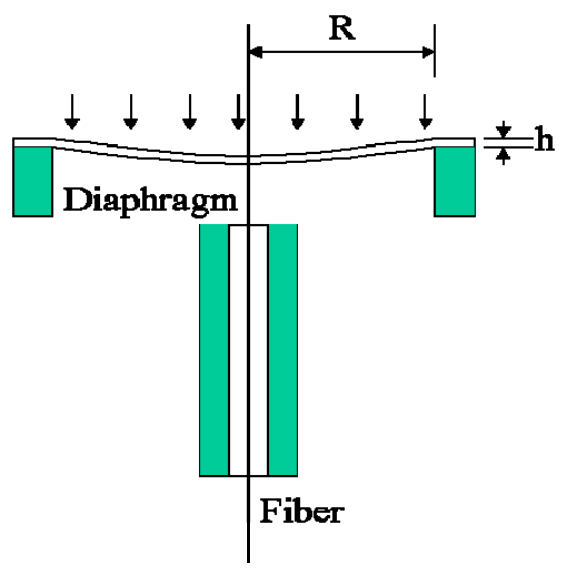

Figure 3.3. Structural model for diaphragm-based sensor.

As indicated by Equation (3-10), the sensor's frequency response is proportional to the thickness of the diaphragm and inversely proportional to the square of the effective diaphragm radius. In order to obtain a flat frequency response from DC to $150 \mathrm{kHz}$, let 800 $\mathrm{kHz} \geq f_{00} \geq 500 \mathrm{kHz}$. The relationships between frequency response and $\mathrm{r}$ and $\mathrm{h}$ are given in Figure 3.4. 
Table 3.1. Values of $\alpha_{\mathrm{mn}}$ (constant related to the vibrating modes of the diaphragm).

\begin{tabular}{rrrc}
\multicolumn{1}{c}{$\alpha_{\mathrm{mn}}$} & $\mathrm{n}=0$ & $\mathrm{n}=1$ & $\mathrm{n}=2$ \\
\hline $\mathrm{m}=0$ & 10.21 & 21.22 & 34.84 \\
\hline 1 & 39.78 & 60.82 & 84.58 \\
\hline 2 & 88.90 & 120.12 & 153.76 \\
\hline
\end{tabular}

Table 3.2. Properties of fused silica $\left(@ 25^{\circ} \mathrm{C}\right)$.

\begin{tabular}{lccc}
\hline Property & Symbol & Value & Unit \\
\hline Density & $w(\rho)$ & $2.202 \times 10^{3}$ & $\mathrm{~kg} / \mathrm{m}^{3}$ \\
\hline Young's Modulus & $\mathrm{E}$ & $73.73 \times 10^{9}$ & $\mathrm{~Pa}$ \\
\hline Poisson's Ratio & $\mu$ & 0.17 & - \\
\hline Max. tensile stress & $\rho_{\mathrm{m}}$ & $\sim 1.5 \times 10^{9}$ & $\mathrm{~Pa}$ \\
\hline Coefficient of thermal expansion & $\alpha_{\mathrm{T}}$ & $\sim 5.5 \sim 7.5 \times 10^{-7}$ & $/{ }^{\circ} \mathrm{C}$ \\
\hline
\end{tabular}

*Some of the properties of fused silica will be dependent not only on the composition of the glass but also on the thermal history of the glass.

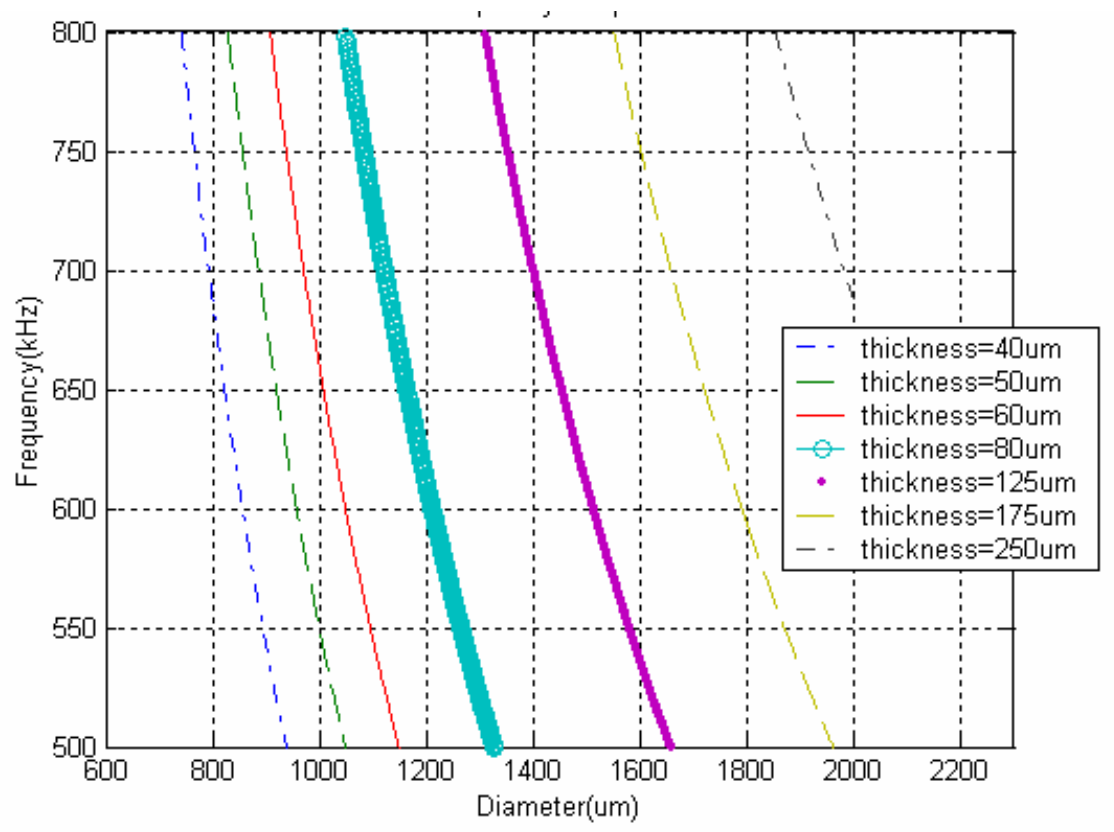

Figure 3.4. Predicted frequency response of the sensor versus pit diameter. 


\subsubsection{Sensitivity}

The diaphragm will be deflected when there is a differential pressure $\mathrm{p}$ between the inside and outside of the sealed cavity. The out-of-plane deflection of the diaphragm $y$ is a function of the pressure difference at any radium position a, as defined by:

$$
y=\frac{3\left(1-\mu^{2}\right) p}{16 E h^{3}} \times\left(r^{2}-a^{2}\right)
$$

Usually, we define the ratio between the deflection and the pressure difference as the diaphragm sensitivity $\left(\delta_{\text {diap }}\right)$ with respect to the applied pressure. When the fiber is positioned at the center of the diaphragm, only the center deflection $y_{0}$ is of interest, so $\delta_{\text {diap }}$ (for fused silica material) is given by

$$
\delta_{\text {diap }}=\frac{y_{0}}{p}=\frac{3\left(1-\mu^{2}\right)}{16 E h^{3}} \times r^{4}=1.71 \times 10^{-8} \frac{r^{4}}{h^{3}}
$$

where $y_{0}$ is in microns, and $p$ is in pound per square inch (psi), so $\delta_{\text {diap }}$ is in $\mu \mathrm{m} / \mathrm{psi}$. The dependence of the sensitivity on diaphragm thickness and diameter is shown in Figure 3.5.

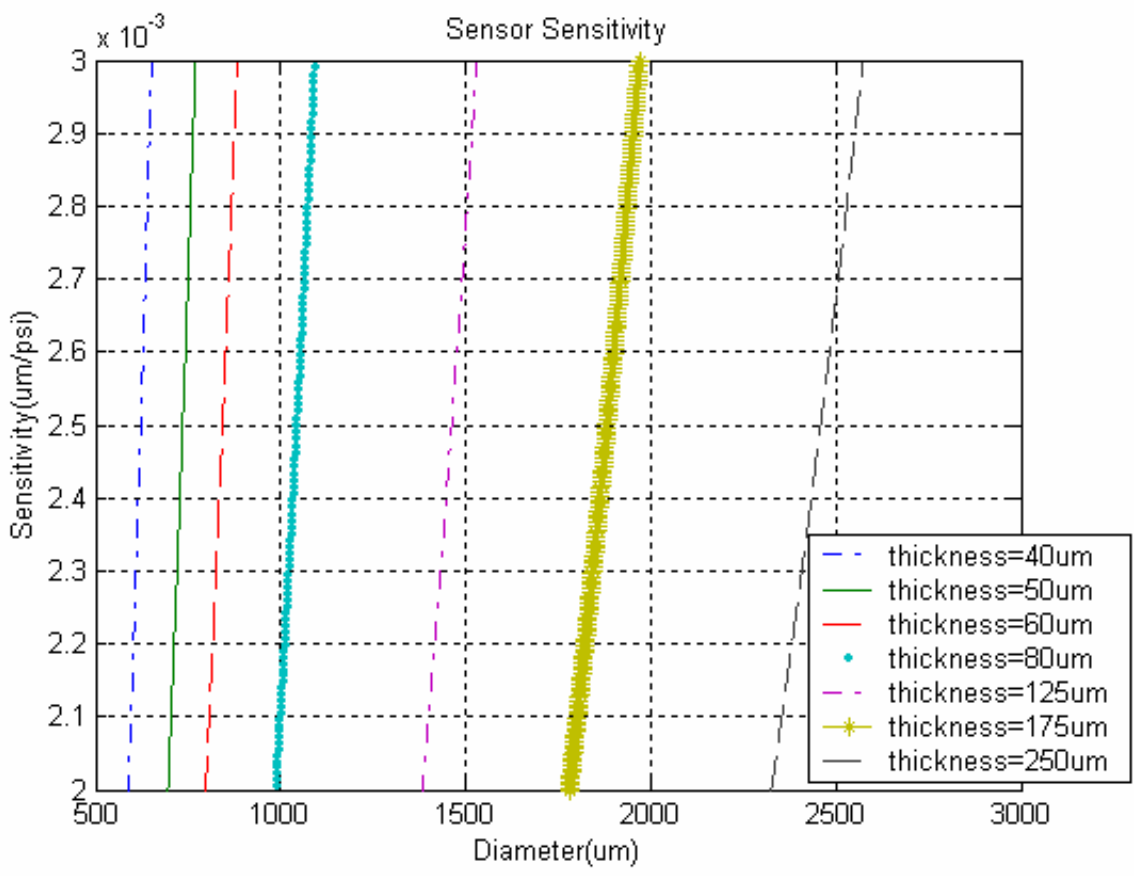

Figure 3.5. Predicted diaphragm sensitivity versus pit diameter. 


\subsubsection{Self-Calibration of the SCIIB Sensor Technology}

The self-calibrated interferometric/intensity based (SCIIB) sensor technology is adopted from the well-developed extrinsic Fabry-Perot interferometric (F-P) optical fiber sensors. SCIIB fiber sensors successfully combine the advantages of both interferometric and intensity-based fiber sensors in a single system. The underlying principle of the SCIIB system is to have a second channel as reference in addition to the signal channel. This makes it possible to compensate for the source power fluctuation and the fiber loss changes and therefore provide absolute measurement of various parameters.

As shown in Eq. (3-1), the coherence length of a light source is inversely proportional to the spectral width. If there are two channels, one with a wide spectrum and short coherence length while the other has a much narrower spectrum and longer coherence length, then by setting the OPD of the F-P cavity between the two, the narrowband channel will exhibit good fringes and can act as the signal while the wideband channel will have much weaker or no interference and thus can be used as a reference channel.

As shown in Figure 3.1, the reflected light from the sensor head is split into two channels by a coupler. The light in Channel 1 remains its original spectral width (wideband spectrum) while the light in Channel 2 has a much narrower spectrum after passing through an optical in-line bandpass filter. Now let us study the behavior of these two signals.

For convenience, assume that the optical spectrum of the LED can be approximated as a Gaussian profile with a FWHM (full-width-at-half-maximum) of $\Delta \lambda_{\text {FWHM, }}$

$$
I_{0}(\lambda)=I_{0} \exp \left(-\frac{\left(\lambda-\lambda_{C}\right)^{2}}{2 \Delta \lambda^{2}}\right)
$$

where $\Delta \lambda=\frac{\Delta \lambda_{\text {FWHM }}}{\sqrt{2 \ln 2}}$.

To calculate the interference of the reflected light, it should be noticed that this is a multibeam interference problem, especially when there is a high-reflectance coating on the surfaces. The first surface is the fiber end face and the second is the surface of the diaphragm. It should also be emphasized that the light from the fiber, after being reflected by the diaphragm, cannot be completely coupled back into the fiber. This loss is primarily due to the divergence of the beam as it propagates from the fiber endface to the diaphragm and back to the fiber endface. Another source of loss of the light signal is because the two surfaces are not perfectly parallel and the angle between the two will introduce a misalignment loss. We assume that after a round-trip reflection (fiber->diaphragm->fiber) the portion of the light coupled back into the fiber is a constant $\eta$, no matter how many times the beam has been reflected before this round-trip. Then for a specific wavelength $\lambda$, the total reflected signal coupled back into the fiber could be expressed as 


$$
\mathrm{I}_{\mathrm{r}}(\lambda)=\mathrm{I}_{0}(\lambda)\left(1-\mathrm{A}_{1}-\frac{\left(1-\mathrm{A}_{1}-\mathrm{r}_{1}\right)\left(1-\eta\left(1-\mathrm{A}_{1}\right) \mathrm{r}_{2}\right)}{1+\eta \mathrm{r}_{1} \mathrm{r}_{2}-2 \sqrt{\eta \mathrm{r}_{1} \mathrm{r}_{2}} \cos \frac{4 \pi \mathrm{L}}{\lambda}}\right)
$$

where $A_{1}$ is the absorption by the fiber end face, $r_{1}$ and $r_{2}$ are reflectance at the fiber end face and the diaphragm surface, $\eta$ is the back-coupling efficiency and $L$ is the length of the airgap.

For a channel centered at $\lambda_{\mathrm{c}}$ with a bandwidth of BW, the total light intensity at the detector is the integral of $I_{r}(\lambda)$ over its passband

$$
\begin{aligned}
\mathrm{I} & =\int_{\lambda_{\mathrm{c}}-\frac{\mathrm{BW}}{2}}^{\lambda_{\mathrm{c}}+\frac{\mathrm{BW}}{2}} \mathrm{I}_{\mathrm{r}}(\lambda) \mathrm{d} \lambda \\
& =\mathrm{I}_{0} \int_{\lambda_{\mathrm{c}}-\frac{\mathrm{BW}}{2}}^{\lambda_{\mathrm{c}}+\frac{\mathrm{BW}}{2}} \exp \left(-\frac{\left(\lambda-\lambda_{\mathrm{C}}\right)^{2}}{2 \Delta \lambda^{2}}\right)\left(1-\mathrm{A}_{1}-\frac{\left(1-\mathrm{A}_{1}-\mathrm{r}_{1}\right)\left(1-\eta\left(1-\mathrm{A}_{1}\right) \mathrm{r}_{2}\right)}{\left.1+\eta \mathrm{r}_{1} \mathrm{r}_{2}-2 \sqrt{\eta \mathrm{r}_{1} \mathrm{r}_{2}} \cos \frac{4 \pi \mathrm{L}}{\lambda}\right) \mathrm{d} \lambda}\right.
\end{aligned}
$$

For the wideband channel, the integral is over all wavelengths, i.e. $\mathrm{BW}=\infty$. From (3-15), it can be seen that if the ratio of the two channels is taken, a common factor of $\mathrm{I}_{0}$ will cancel out. This means the change of $\mathrm{I}_{0}$ due to light source fluctuation or fiber bending loss will not affect the ratio. This is one of the major advantages of the SCIIB system. Figure 3.6 (a), (b) and (c) are the simulation results of the wideband and narrowband channel and their ratio. Figure 2.7 (a) and (b) show that the coherent ability of the wideband channel is much less than that of narrowband channel. Thus if the airgap is chosen to be around $20 \mu \mathrm{m}$, the wideband signal will remain stable while the narrowband will still see strong interference.

The parameters in equation (3-14) need to be determined. $A_{1}$ has been measured by experimentation and appears to be around 15\%. $\eta$ can be estimated from the experimental data as has also been examined by theoretical calculation and is about $40 \%$ for an airgap of $20 \mu \mathrm{m} . \mathrm{r}_{1}$ and $\mathrm{r}_{2}$ should be determined such that the narrowband signal has both high average power and high visibility in order to increase the sensitivity of the system.

\subsection{Cleanroom Construction}

The fabrication of diaphragm-based Fabry-Perot sensors generally involves the following processes: diaphragm and fiber cleaning, photolithography, diaphragm etching, thin film deposition, diaphragm surface quality measurements, thin film characterization, and waferto-wafer or wafer-to-ferrule bonding. Fused silica wafers and optical fibers for pressure sensors must be washed and stored in, and the remaining manufacturing processes performed in a clean environment for the following reasons.

- The wet process station requires low particle concentration environment to keep the solutions clean and maintain wafer or fiber cleanness during transfers between different tanks.

- Particles on the wafers during the photolithography process may cause under-etch in the sensing area of the diaphragm or unwanted etch in the bonding area, resulting in a rough reflective or bonding surface, which is catastrophic for direct bonding. 


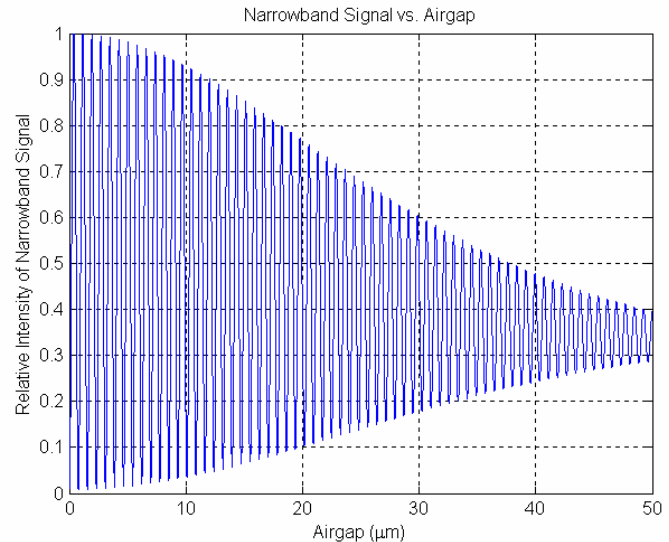

(a)

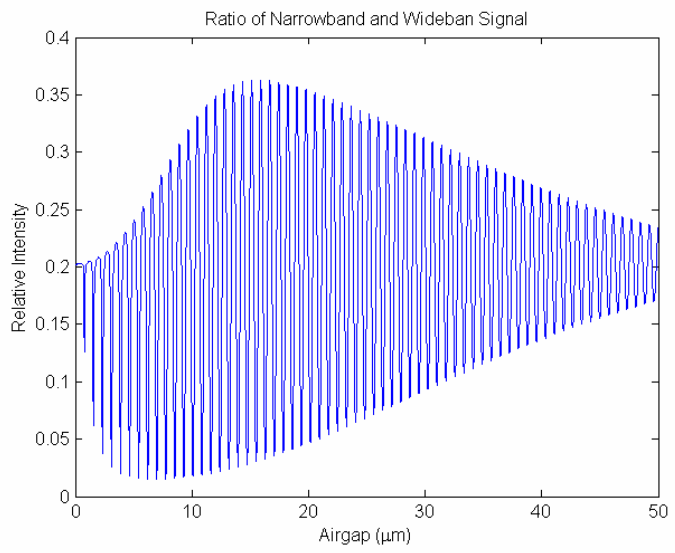

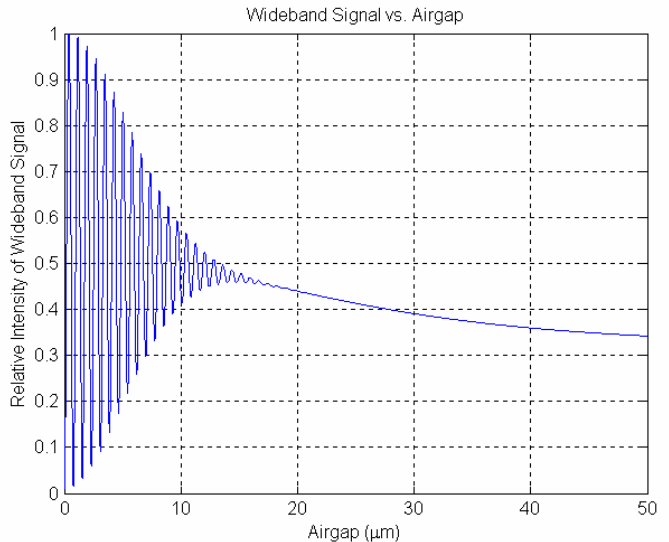

(b)

Figure 3.6. SCIIB (a) wideband signal; (b) narrowband signal; (c) ratio of the two.

(c)

- The profilometer, which is necessary for diaphragm roughness measurement and etched pit characterization, is very sensitive to particle contamination. The vertical accuracy of the profiler is $1.0 \mathrm{~nm}$. Any particles on the samples or on the stylus of the instrument larger than $1.0 \mathrm{~nm}$ may lower the measuring accuracy significantly.

- The ellipsometer exhibits better performance characteristics for thin film thickness and refractive index measurements in a cleanroom.

- Reflective coatings on both fibers and diaphragms are essential and crucial parts in the pressure sensors. Contaminated diaphragms and fibers may cause rough, cracked or even peeling coatings, and therefore degrade the performance of the sensors.

- Glass to glass bonding, especially the direct bonding process requires extremely clean and smooth surfaces. Any particles or contaminations may cause a failure of the bonding process.

- A clean environment ensures that no contamination occurs during transportation among the above processes. 
To fill this need, a clean facility was designed and installed at the Center for Photonics Technology. Office environments typically have a cleanness poorer than Class 100,000, which means the $0.5 \mu \mathrm{m}$ particle concentration is higher than $100,000 / \mathrm{ft}^{3}$, and definitely are not suitable for diaphragm-based fiber-optic Fabry-Perot sensor fabrication. In a laboratory, particles in the air generally come from several main sources, including personnel, machinery, and supplies that are brought into the lab. For example, people typically emit 510 million particles per minute.

The first step in reducing particles is to minimize these sources. Lab personnel wear "bunny suits" which cover their bodies and clothing and which block particle emission from these sources. The room was painted with cleanroom epoxy and floor sealant, and sticky mats at the entrance to the cleanroom are used to minimize particle generation. Since the source of particles can never be completely eliminated, constant filtration is used to remove particles as they are generated. This is accomplished by recirculating the lab air through High Efficiency Particle Air (HEPA) filters. The HEPA filters are $99.97 \%$ efficient at removing particles from the air. With careful attention to leaks and sealing around the filter edges, the exit air is typically better than Class 1 . Particles can also be introduced during chemical cleaning steps. Filtration of these chemicals is the normal method used to minimize this source of contamination. A special case is the water which is used in wafer rinsing, a common step in wafer cleaning procedures. A Barnstead NANOpure DIamond ultrapure water system fed by a DIamond RO reverse osmosis system is used to supply water containing $<0.005 \mathrm{Eu} / \mathrm{ml}$ of pyrogens, TOC values $<3 \mathrm{ppb}$, and resistivities up to $18.2 \mathrm{M} \Omega-\mathrm{cm}$.

Table 3.3. Detailed cleanroom specifications.

Cleanroom size: 16'x12'

Class: Class 1000 or better

Cleanroom structure:

- Hardwall modular clean room construction;

- 2'x4' Filter/Fan Units(115VAC/60Hz) and Ceiling Panels;

- T-bar fixtures

- 99.99\% efficient HEPA filter @ .3micron

- Acrylic panels;

- Control panel included;

- A sliding door is required;
Accessories:

- Fluorescent lights: No less than 27,360 foot candles;

- Two 110VAC 50/60Hz outlets;

- Two Nitrogen/Compressed air connections;

- Temperature and humidity sensors and display;

- Sticky mat: 24"x36";

- Sticky mat frame: 30"x42";

- 60"x30" cleanroom work bench;

- 48"x30" cleanroom work bench;

- Cleanroom chair;

- Wall-mount glove box holder;

- Wall-mount cabinet;

- Glove box for photolithography;

- Garment cabinet;

- Storage cabinet;

- Cleanroom vacuum cleaner;

- Cleanroom waste receptacle;

- Water \& gas pipes. 


\subsubsection{Design Specifications}

The layout and detailed specifications of the cleanroom design selected to satisfy the sensor fabrication requirements are shown in Figure 3.7 and Table 3.3. This design was implemented at CPT using the Terra Universal hardwall modular cleanroom shown in Figure 3.8. This modular cleanroom has eight HEPA filter units. A particle counter Model 229 from Pacific Scientific Instruments is used to detect the particle concentrations in the room.

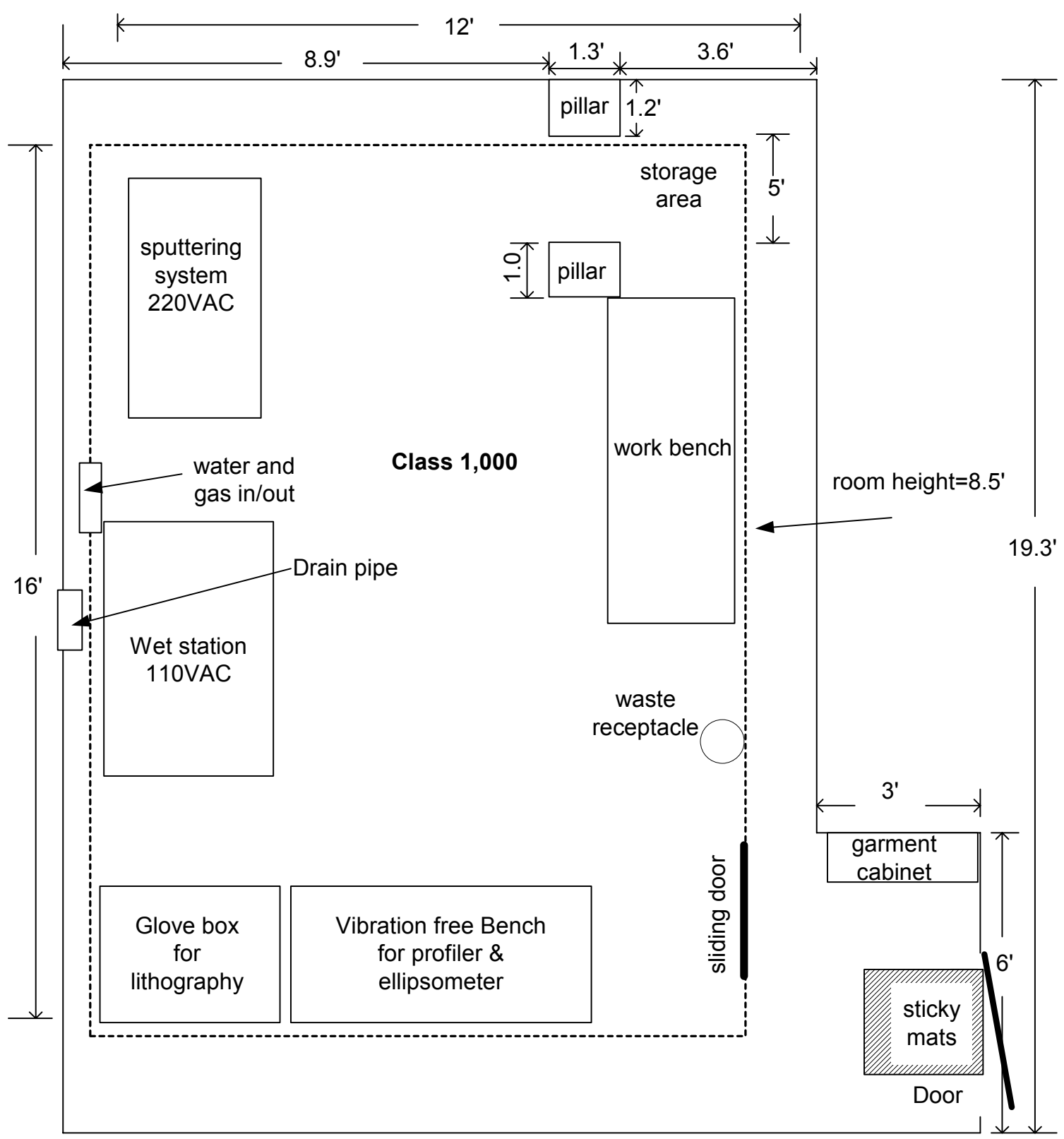

Figure 3.7. Cleanroom layout. 


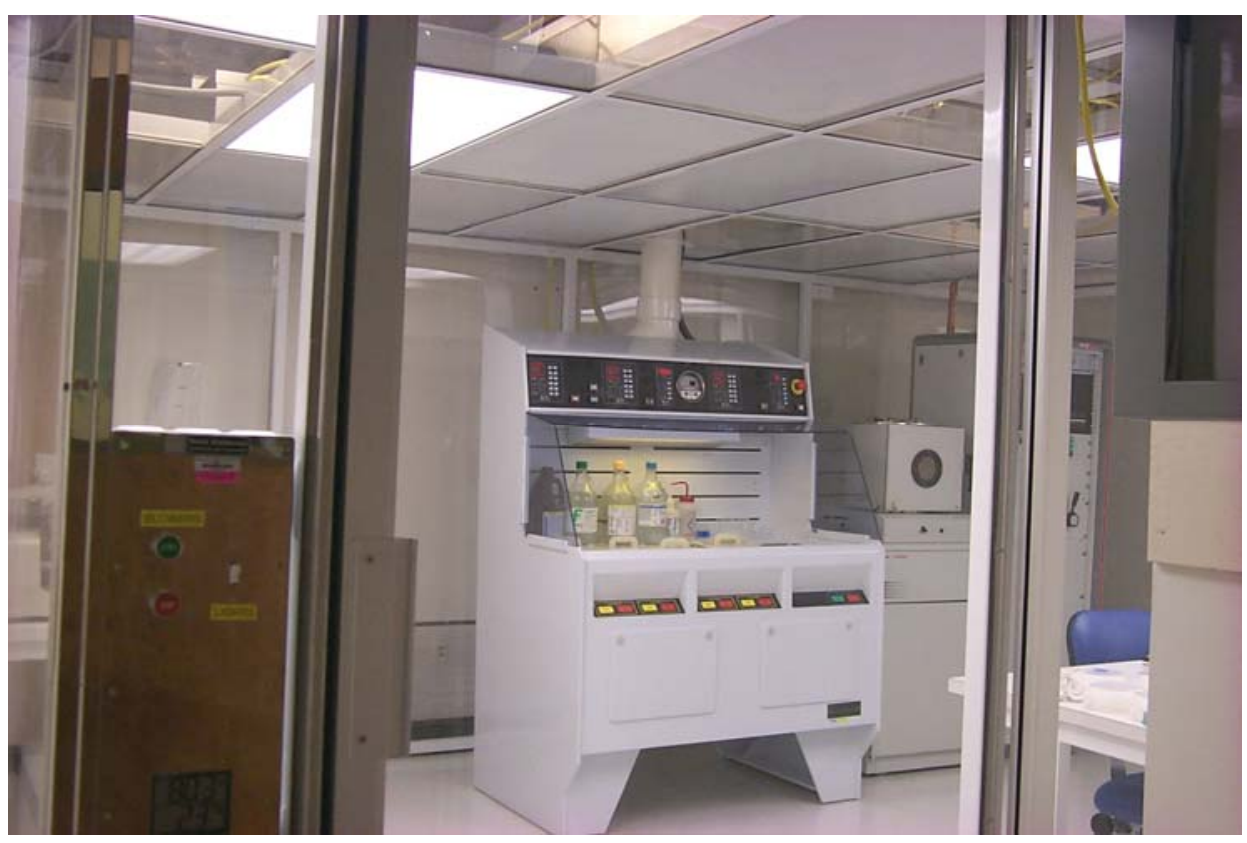

Figure 3.8. A view of the CPT cleanroom from the main door.

\subsubsection{Cleanroom Instrumentation}

A complete suite of cleanroom instrumentation has been set up to permit diaphragm and fiber cleaning, diaphragm etching, thin film deposition, diaphragm surface quality measurements, and thin film characterization. An AUTO 500 RF Sputtering System manufactured by BOC Edwards has been installed for metallic and dielectric thin film deposition (Figure 3.9). The AUTO 500 is a versatile front-loading system with a large box chamber measuring $500 \mathrm{~mm}$ deep x 500mm wide. It is designed for metal films, such as $\mathrm{Au}, \mathrm{Cr}$ and $\mathrm{Al}$ deposition, but can also be modified for dielectric films, such as $\mathrm{ZrO}_{2}, \mathrm{TiO}_{2}$, and $\mathrm{Al}_{2} \mathrm{O}_{3}$, permitting the deposition of reflective thin films in the lab.

A wet processing station made by Bold Technology (Figure 3.9) makes it very efficient to clean, etch and dry wafers in this cleanroom. Tanks are designed for RCA cleaning, HF etching, $\mathrm{KOH}$ etching, DI water rinsing and nitrogen drying with low sample volumes. Automatic temperature control, draining, timing and alarming are the other benefits of the station. 


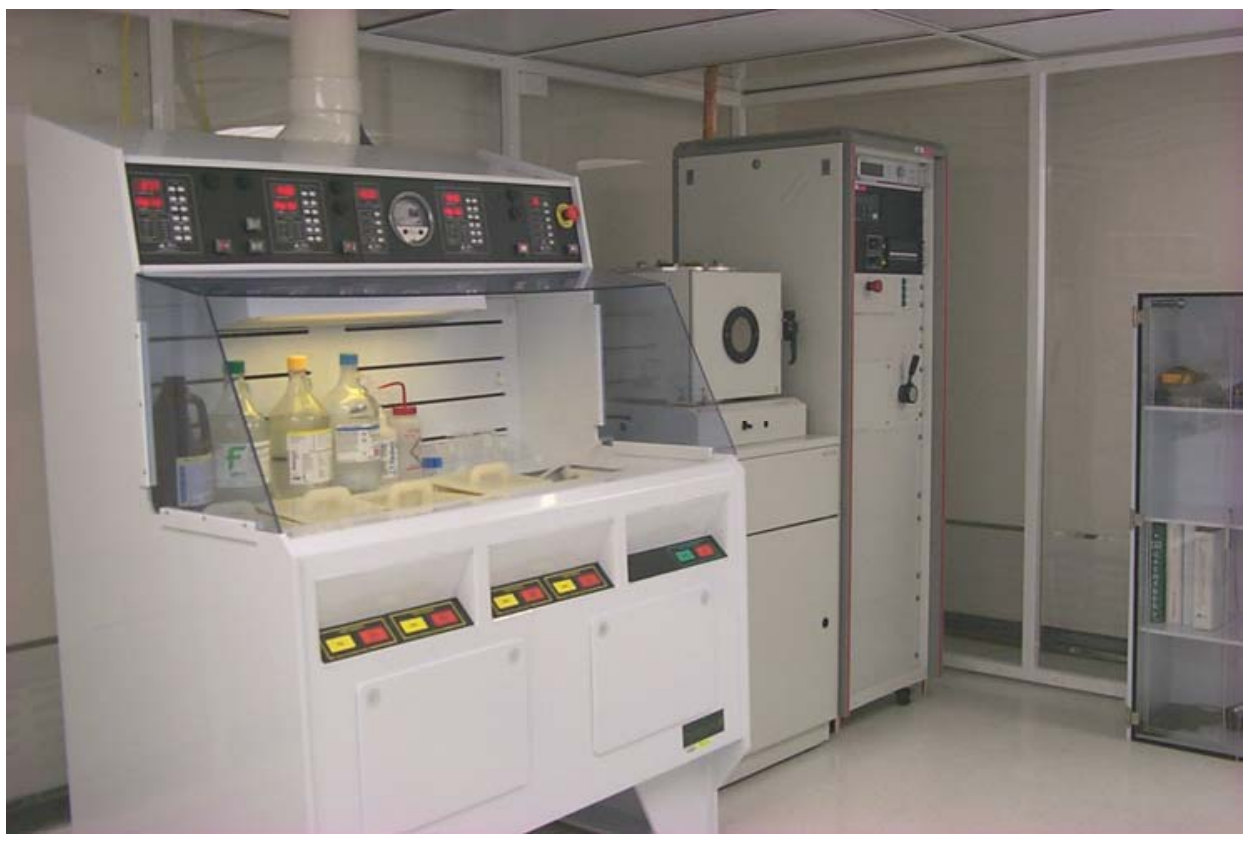

Figure 3.9. Bold Technology wet processing station (left) and Edwards AUTO 500 Sputtering System (right).

The DEKTAK 3 surface profiler from Veeco Metrology Group (Figure 3.10) is a very high precision instrument capable of measuring minute physical surface variations and is very sensitive to environment in which it is operated. It must be operated in an area free from excessive dust. Vibration levels must be low enough that fingertips cannot detect them, and therefore a vibration free bench should be used. The scan head should be covered with the environmental shield to eliminate drafts. Ambient temperatures should be between $18^{\circ} \mathrm{C}$ and $24^{\circ} \mathrm{C}\left(64^{\circ} \mathrm{F}\right.$ to $\left.75^{\circ} \mathrm{F}\right)$. The key technical specifications of the DEKTAK 3 profiler are listed in Table 3.4.

Table 3.4. Detailed specifications of DEKTAK3 profilometer.

Vertical Range: $100 \AA$ to $655 \mathrm{~K} \AA$

Vertical Resolution: $10 \AA$

Scan Length Range: 50 microns to $30 \mathrm{~mm}$

Scan Speed Range: Low, Medium, High

Scan Time Range: 3 seconds to 50 seconds

Software Leveling: Two-point programmable or cursor leveling

Stage Leveling: Manual
Stylus: Diamond, 12.5-micron radius

Stylus Tracking Force: $10 \mathrm{mg}$ to $50 \mathrm{mg}$, adjustable

Maximum Sample Thickness: $20 \mathrm{~mm}$

Sample Stage Diameter: $127 \mathrm{~mm}$

Sample Stage Rotation: $360^{\circ}$ continuous

Zoom Magnification: 90x

Camera: Solid state monochrome video camera 
The Gaertner Scientific LSE Stokes Ellipsometer (Figure 3.10) uses a compact StokesMeter to replace the typical ellipsometer rotating analyzer assembly and waveplate. Any point on the surface of transparent or absorbing samples up to $300 \mathrm{~mm}$ wide can be measured using the $6328 \AA$ source at a $70^{\circ}$ incidence angle. The manual tilt and height adjustment can be set using an alignment screen in the included Windows 2000/NT/Me/XP/9x LGEMP software. Film thickness and refractive index of a single layer on a substrate or the top layer on 1,2, or 3 known bottom layers can be calculated. Some key features are listed in Table 3.5.

Table 3.5. Detailed specifications of Gaertner ellipsometer.
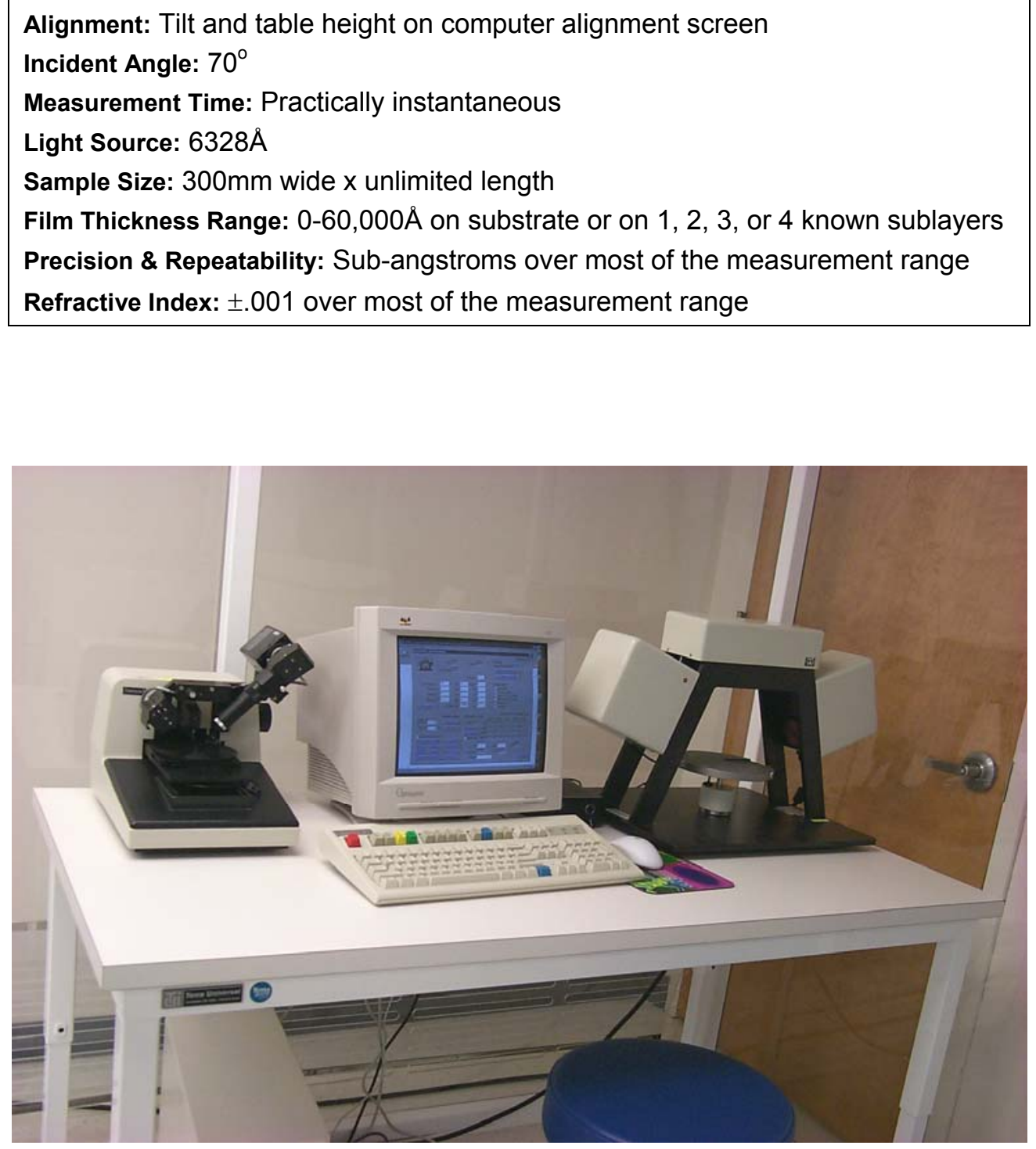

Figure 3.10. DEKTAK3 Profiler (left) and Gaertner Ellipsometer (right) in the cleanroom. 


\subsection{Ring-Based Sensor Fabrication}

Assembly of the sensor head can be achieved in a number of ways, including insertion of a ring between the ferrule and the diaphragm, etching of the diaphragm, and etching of the ferrule. In all three cases, bonding is the key element in sensor fabrication. The goal is to achieve the necessary pressure response with extremely low temperature dependence.

Because the diaphragm based pressure sensor is going to operate under harsh environmental conditions such as high temperature and high pressure, using materials with closely matched CTE (coefficient of thermal expansion) as that of optical fiber is very important to reduce thermally induced airgap changes, and thermal shock failures. Ordinary single mode optical fibers are made of a fused silica cladding material surrounding a germanium doped fused silica central core region. The sensor designs utilize fused silica for all of the components used in the sensor head including the tubing, the ferrule and the diaphragm, because fused silica has a very high softening point $(\sim 1585 \mathrm{oC})$, low CTE and high elastic modulus. Utilizing all fused silica components in the design, while increasing the maximum operating temperature and decreasing the thermal dependence of the sensor signal, also introduces some challenges such as difficulty in polishing, and difficulty in bonding. Therefore, some special techniques have been investigated and developed to fabricate sensors with fused silica wafers and tubing.

A substantial portion of the program was invested in investigating and optimizing diaphragm-based sensor fabrication. This section and the following two sections describe these efforts on ring-based sensors, etched diaphragm sensors, and laser-bonded sensors.

\subsubsection{Ring-Based Sensor Structure}

A major task in sensor fabrication is bonding the fused silica ferrule to the diaphragm. A number of different sensor designs have been considered to facilitate this bonding. In a ringbased sensor, a ring is inserted between the ferrule and diaphragm and melted under high temperature, forming a cavity without requiring pit etching. In addition, the diaphragm response is determined by the inner diameter of the ring, which can be closely controlled using this method. Figure 3.11 shows the structure of such a sensor. The fiber is bonded to the ferrule by solgel and a gold coating is sputtered onto both the fiber endface and diaphragm to increase the signal strength. Since gold films have the potential to survive under temperatures up to $800-900^{\circ} \mathrm{C}$, the ring material is chosen to be borosilicate, which has a softening point around $840^{\circ} \mathrm{C}$ in order to avoid damaging the gold. The length of the airgap can be adjusted before the fiber is bonded to the ferrule. 


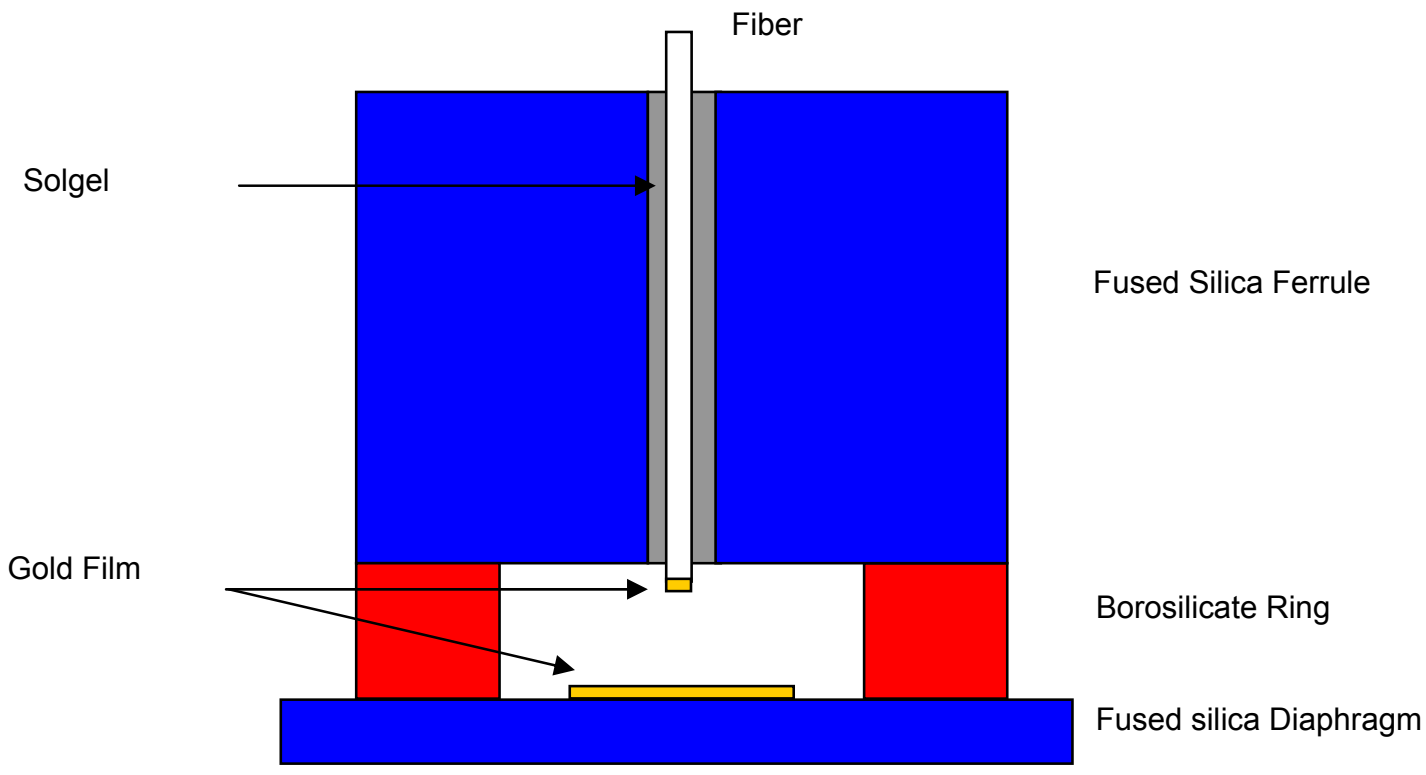

Figure 3.11. Structure of ring-based sensor.

\subsubsection{Ring Fabrication Procedure}

A long borosilicate tube, with a $1 \mathrm{~mm}$ I.D. and a $2 \mathrm{~mm}$ O.D matching with that of the ferrule, is first cut into small pieces by precision saw. Then these small segments are polished to reduce the thickness to about 200 microns. Diluted hydrofluoric acid (HF) is used to further thin the ring down to 30-50 microns, controlling the thickness through the etching rate. Ring thickness is monitored throughout the process.

\section{Thickness Measurement}

Ring thickness measurements are critical in sensor preparation. Thinner rings are preferred to reduce the temperature coefficient. However, if the ring is too thin, it will be difficult to handle and the surface quality of the ring will deteriorate, resulting in poor bonding with many voids. In addition, etching rate calculations require accurate thickness measurements. Figure 3.12 shows the measuring setup. A surface profiler is used to scan along the microscope slide, which is raised at one end by the ring. If the stylus scans a distance of $d$ and the height change is $\mathrm{h}$, the ring thickness $\mathrm{H}$ can be calculated simply as

$$
H=\frac{h}{d} L
$$

given the length of the slide L. Table 3.6 compares the thickness results obtained using this method and using a caliper for a borosilicate ring, a fused silica wafer and a cover glass. Careful measurements produce an error of about $\pm 1 \mu \mathrm{m}$. Typically, the ring is not very uniform and requires multiple measurements at different positions to determine an average thickness. 


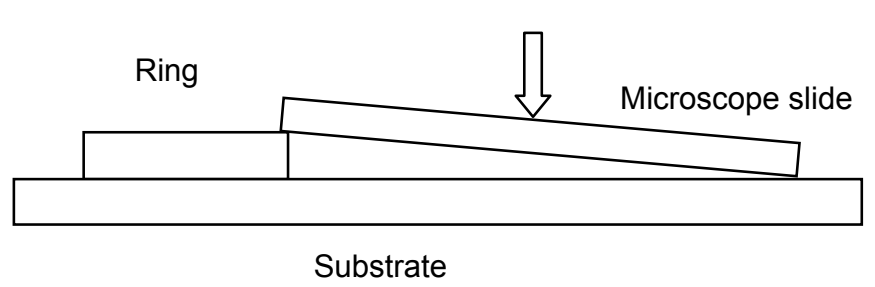

Figure 3.12. Borosilicate ring thickness experimental setup.

Table 3.6. Comparison of thickness measurement using the profile method and calipers.

\begin{tabular}{lcccc}
\hline & \multicolumn{1}{c}{ Ring } & Fused silica wafer & Cover glass & Error \\
\hline Profiler & $221.5 \mu \mathrm{m}$ & $200.7 \mu \mathrm{m}$ & $159.5 \mu \mathrm{m}$ & $\sim 1 \mu \mathrm{m}$ \\
\hline Caliper & $220 \mu \mathrm{m}$ & $210 \mu \mathrm{m}$ & $150 \mu \mathrm{m}$ & $\sim 10 \mu \mathrm{m}$ \\
\hline
\end{tabular}

\section{Etching}

Polishing the ring below $200 \mu \mathrm{m}$ is difficult; wet etching is used to further reduce the thickness. Higher concentrations of HF (50\%) will etch the ring faster at a rate about 8 $\mu \mathrm{m} / \mathrm{min}$. But the surface appears rough with a large number of pits, which could potentially be etched through to form a hole when the ring thickness becomes small. This problem could be alleviated by using a dilute HF solution (10\%), producing a slower etching rate. Table 3.7 lists the results obtained by etching a $175 \mu \mathrm{m}$-thick ring to a thickness of about 50 $\mu \mathrm{m}$ using a $10 \% \mathrm{HF}$ solution. The time of each etching was recorded and the etching rate determined by the thickness measurement. Figure 3.13 also shows the microscope pictures of this ring before and after the etching.

Table 3.7. Ring etching results.

\begin{tabular}{ccccc}
\hline Ring \# & $\begin{array}{c}\text { Avg. Thickness } \\
\text { Before Etching } \\
(\boldsymbol{\mu m})\end{array}$ & $\begin{array}{c}\text { Avg. Thickness } \\
\text { After Etching } \\
(\boldsymbol{\mu m})\end{array}$ & $\begin{array}{c}\text { Etching Time } \\
(\mathbf{m i n})\end{array}$ & $\begin{array}{c}\text { Etching Rate } \\
(\boldsymbol{\mu m} / \mathbf{m i n})\end{array}$ \\
\hline $\mathbf{1}$ & 175.0 & 150.1 & 60 & 0.207 \\
\hline $\mathbf{2}$ & 150.1 & 84.2 & 155 & 0.213 \\
\hline $\mathbf{3}$ & 84.2 & 50.5 & 90 & 0.187 \\
\hline
\end{tabular}




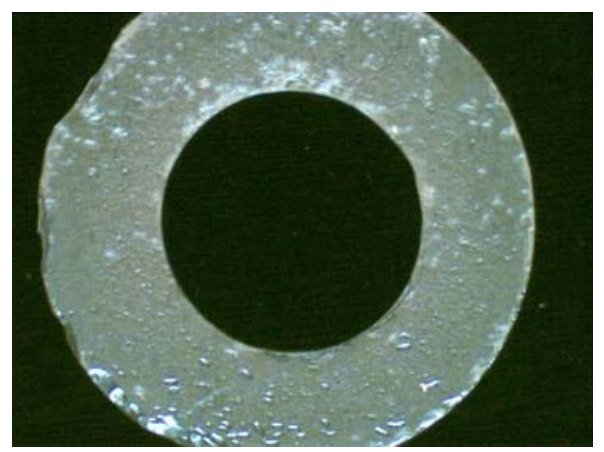

Before

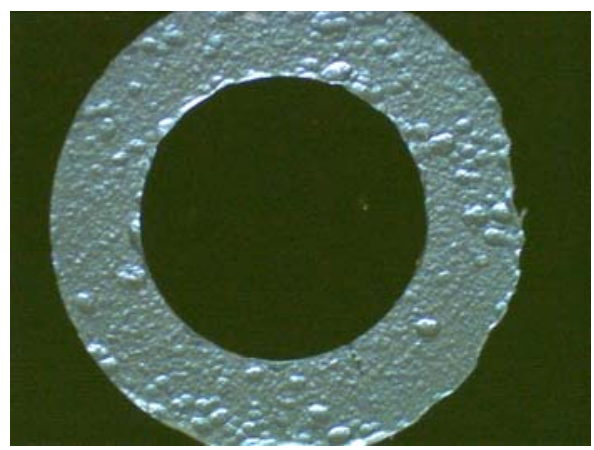

After

Figure 3.13. Surface inspection of ring before and after etching.

\subsubsection{Bonding}

To bond the ferrule with diaphragm, a ring is placed between the two and the entire assembly is heated in a furnace to about $850^{\circ} \mathrm{C}$, melting the ring. The cooling process needs to be slow to prevent the ring from cracking, which will result in leakage. Glass powder can be used around the sensor head to seal any bonding leakage. A sensor head bonded in this way is shown in Figure 3.14. At the center, the small hole in the ferrule is for fiber insertion. Fiber to ferrule bonding is achieved using sol-gel. After the fiber is inserted to the ferrule and the airgap is adjusted to proper value, a heat gun or $\mathrm{CO}_{2}$ laser is used to cure the sol-gel, forming the bond. Controlling the initial airgap is a key element in this process.

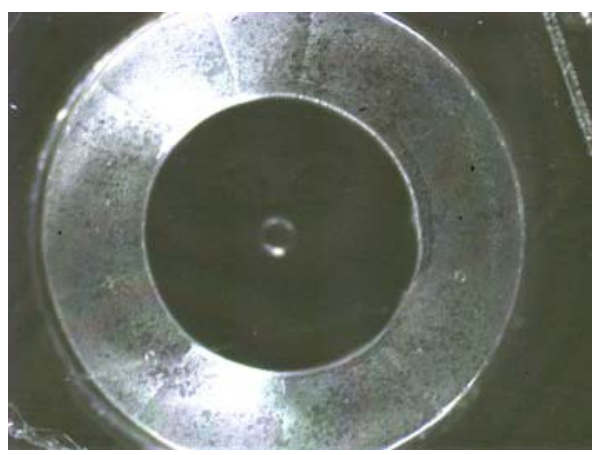

Figure 3.14. Ferrule and diaphragm bonded with ring.

\subsubsection{Sensor testing}

After fabrication, the sensor is tested for its sensitivity, repeatability, stability and temperature dependence. A diagram of the experimental setup is shown in Figure 3.15. The sensor is sealed in a tube placed in a temperature chamber, which can control the 
temperature. A gas system applies pressure by supplying compressed nitrogen to the tube. The pressure can vary from atmospheric pressure to about 200 psi. The reflected signal data is collected by a computer.

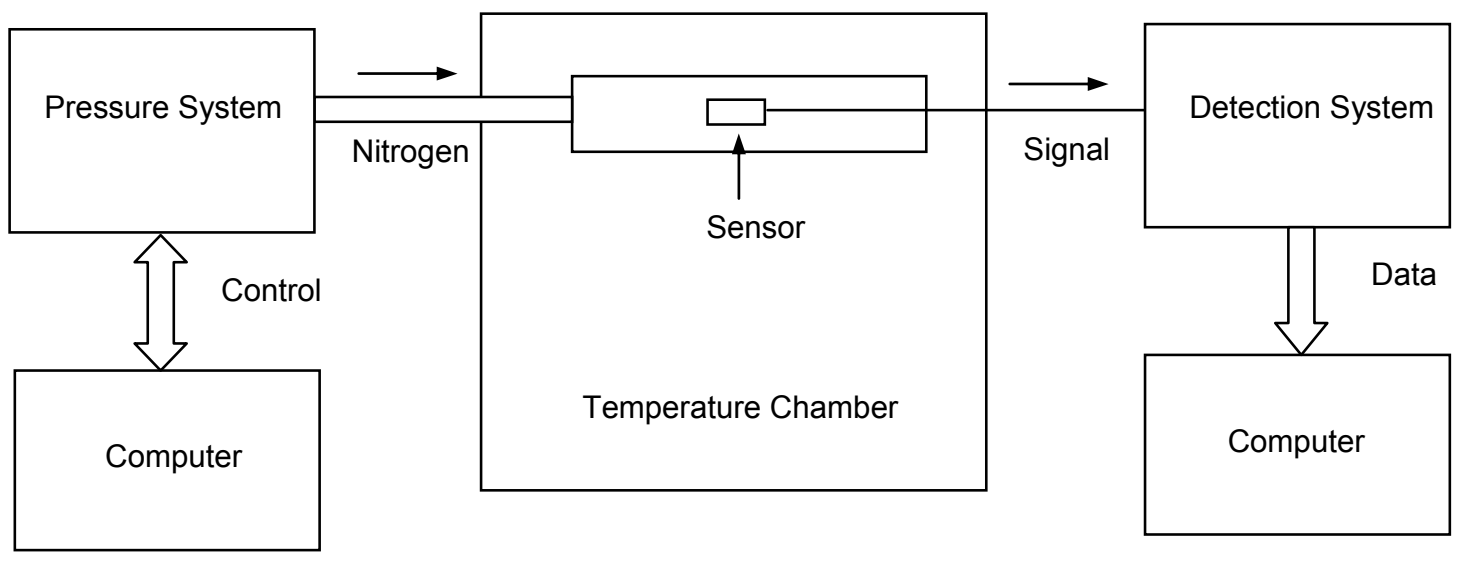

Figure 3.15. Pressure sensor testing setup.

Currently, the gold film shown in Figure 3.11 has not been used to increase the signal strength. Therefore the reflectance at both the fiber end and the diaphragm is relatively low $(\sim 4 \%)$, resulting in two-beam interference, producing a sinusoidal curve when the pressure varies, as shown in Figure 3.16.

Suppose the reflected light has an intensity of $I_{1}$ at the fiber endface and $I_{2}$ at the diaphragm. The complex amplitude of the total reflected light is

$$
\mathrm{A}=\sqrt{\mathrm{I}_{1}}+\sqrt{\mathrm{I}_{2}} \mathrm{e}^{-\Delta \phi}
$$

The reflected intensity is therefore

$$
\mathrm{I}_{\mathrm{r}}=\left|\mathrm{A}^{2}\right|=\mathrm{I}_{1}+\mathrm{I}_{2}+2 \sqrt{\mathrm{I}_{1} \mathrm{I}_{2}} \cos (\Delta \phi)
$$

The phase shift $\Delta \phi$ relates to the length of the airgap L, which is in turn determined by the initial airgap, diaphragm deflection due to pressure changes and the airgap change due to thermal expansion, i.e.,

$$
\Delta \phi=\frac{4 \pi}{\lambda} \mathrm{L}=\frac{4 \pi}{\lambda}\left(\mathrm{L}_{0}-\mathrm{S} * \mathrm{p}+\mathrm{C}_{\mathrm{T}} * \mathrm{~T}\right)=-\frac{4 \pi}{\lambda} \mathrm{S}\left(\mathrm{p}-\frac{\mathrm{C}_{\mathrm{T}} * \mathrm{~T}+\mathrm{L}_{0}}{\mathrm{~S}}\right)
$$


where $\lambda$ is the central wavelength of the narrowband, $L_{0}$ is the initial airgap, $S$ is the sensitivity of the sensor and $\mathrm{C}_{\mathrm{T}}$ is the temperature coefficient. Therefore the reflected intensity becomes

$$
\mathrm{I}_{\mathrm{r}}=\mathrm{I}_{1}+\mathrm{I}_{2}+2 \sqrt{\mathrm{I}_{1} \mathrm{I}_{2}} \cos \left(\frac{4 \pi}{\lambda} \mathrm{S}\left(\mathrm{p}-\frac{\mathrm{C}_{\mathrm{T}} * \mathrm{~T}+\mathrm{L}_{0}}{\mathrm{~S}}\right)\right)
$$

Each time a sensor is tested, the signal data are fit to a cosine function as follows.

$$
\mathrm{a}+\mathrm{b} * \cos (\mathrm{c} *(\mathrm{p}-\mathrm{d}))
$$

where $p$ is the pressure. Comparing Eq. 3-20 and 3-21 we can see the parameters are related by

$$
\mathrm{a}=\mathrm{I}_{1}+\mathrm{I}_{2} \quad \mathrm{~b}=2 \sqrt{\mathrm{I}_{1} \mathrm{I}_{2}} \quad \mathrm{c}=\frac{4 \pi}{\lambda} \mathrm{S} \quad \mathrm{d}=\left(\mathrm{C}_{\mathrm{T}} * \mathrm{~T}+\mathrm{L}_{0}\right) / \mathrm{S}
$$

Parameter $a$ and $b$ relate to the fringe visibility which is defined as $(a-b) /(a+b)$. Parameters $c$ and $\mathrm{d}$, are of most interest. $\mathrm{c}$ has to do with sensitivity $\mathrm{S}$ of the sensor, i.e., how much the diaphragm will deflect and the signal will change for a given pressure change. Parameter $\mathrm{d}$ determines the temperature coefficient, $\mathrm{C}_{\mathrm{T}}$.

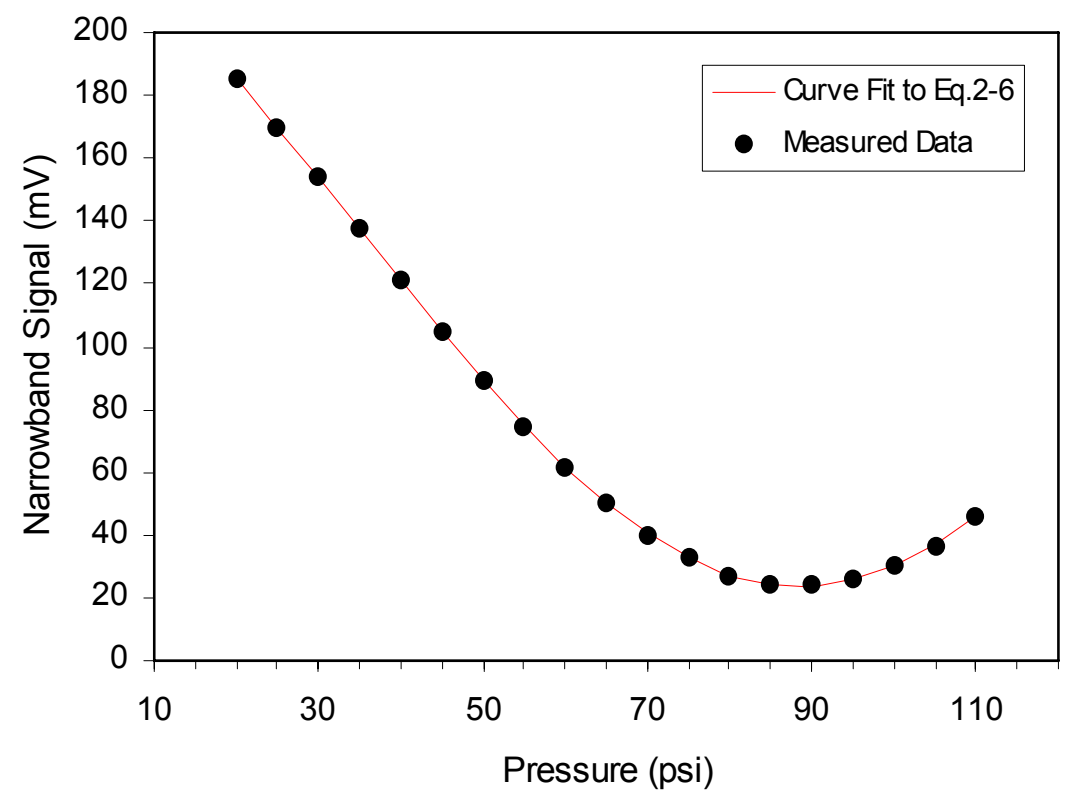

Figure 3.16. Experimental narrowband signal data and Eq. 3-22 curve fit.

\section{Sensitivity}

For the sensor signal shown in Figure 3.16, the sensitivity analysis was performed as follows. The data was fit to expression 3-22, resulting in 


$$
\mathrm{a}=132.6 \quad \mathrm{~b}=109.0 \quad \mathrm{c}=0.03023 \quad \mathrm{~d}=192.5 .
$$

Both the fit data and the original data are shown in Figure 3.16 and they match very well. The sensitivity can therefore be derived as

$$
\mathrm{S}=\mathrm{c} \frac{\lambda}{4 \pi}=0.03 * \frac{1550}{4 \pi}=3.7 \mathrm{~nm} / \mathrm{psi}
$$

From atmospheric pressure ( 14 psi) to 100 psi, the diaphragm will deflect about $(100-14) * S$ $=318.2 \mathrm{~nm}$. This is far beyond the linear range of the sensor $(\sim 200 \mathrm{~nm})$. This sensitivity may be reduced by increasing the diaphragm thickness or decreasing the response area, i.e. the ID of the borosilicate ring. Furthermore, though the airgap varies linearly with the pressure, the signal does not. The figure shows clearly that at a working pressure about 100 psi, the slope is much smaller and the actual signal is not very sensitive. This needs to be adjusted by carefully selecting the initial airgap. Controlling the response curve of the sensor to within the linear range remains a very important future task.

\section{Repeatability}

Repeatability indicates whether the sensor can repeat its performance under same conditions. The narrowband and wideband pressure response of the sensor was evaluated in the temperature chamber at $24^{\circ} \mathrm{C}$. The pressure was increased from atmospheric pressure to 110 psi and then decreased to atmospheric pressure. The two channels were recorded by the digital system. Figure 3.17 shows the narrowband signal of the first test round (14psi to $110 \mathrm{psi}$ to $14 \mathrm{psi}$ ). The increasing pressure and decreasing pressure curves are slightly different. The average relative error (relative difference between the two values when pressure is increased and decreased) is about $2 \%$. However, in the following tests the sensor was very repeatable. Figure 3.18 shows the narrowband signals of the 2 nd, 3rd and 4 th trials. These curves are very close to each other. The relative errors are $0.41 \%, 0.15 \%$ and $0.20 \%$. One possible reason for the difference between the first round and the rest is that the stress in the bond may change when the pressure is initially applied. Following this redistribution, the sensor output appears to be repeatable.

\section{Temperature Coefficient}

When the temperature of the sensor varies, the thermal expansion of its parts (ferrule, ring and fiber) will introduce an additional change in the length of the airgap, over and above the pressure-induced change. Since it is very difficult to compensate for this change, the goal is to make it as small as possible and so small that within the working temperature range (room temperature to about $800^{\circ} \mathrm{C}$ ) it can be neglected. Therefore, measuring the temperature coefficient $\mathrm{C}_{\mathrm{T}}$, i.e. the airgap change per ${ }^{\circ} \mathrm{C}$, is important. This is done by testing the sensor under different temperatures and extracting the c parameter in Eq. 3-22.

The same sensor used for the sensitivity and repeatability test was evaluated at $24^{\circ} \mathrm{C}, 14^{\circ} \mathrm{C}$ and $4^{\circ} \mathrm{C}$. The output signals are plotted in Figure 3.19. All the three curves were fit to equation 2-6 and the results listed in Table 3.8. 


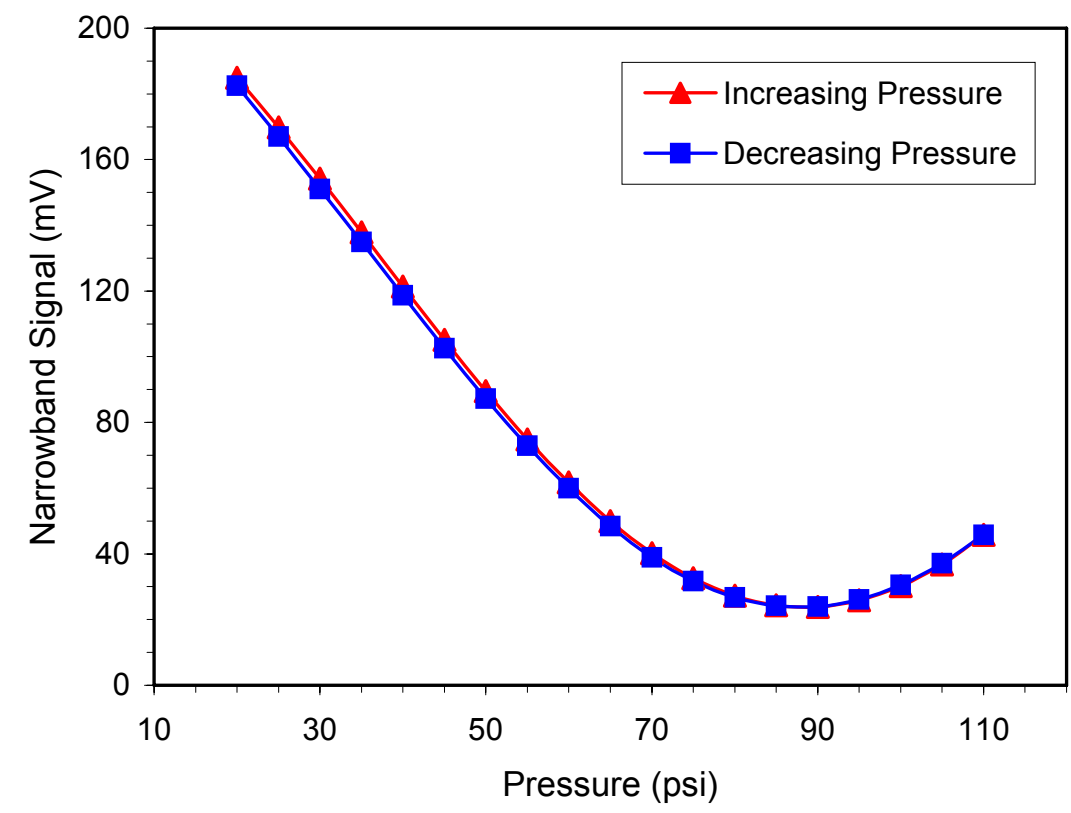

Figure 3.17. Narrowband signal in the $1^{\text {st }}$ test round.

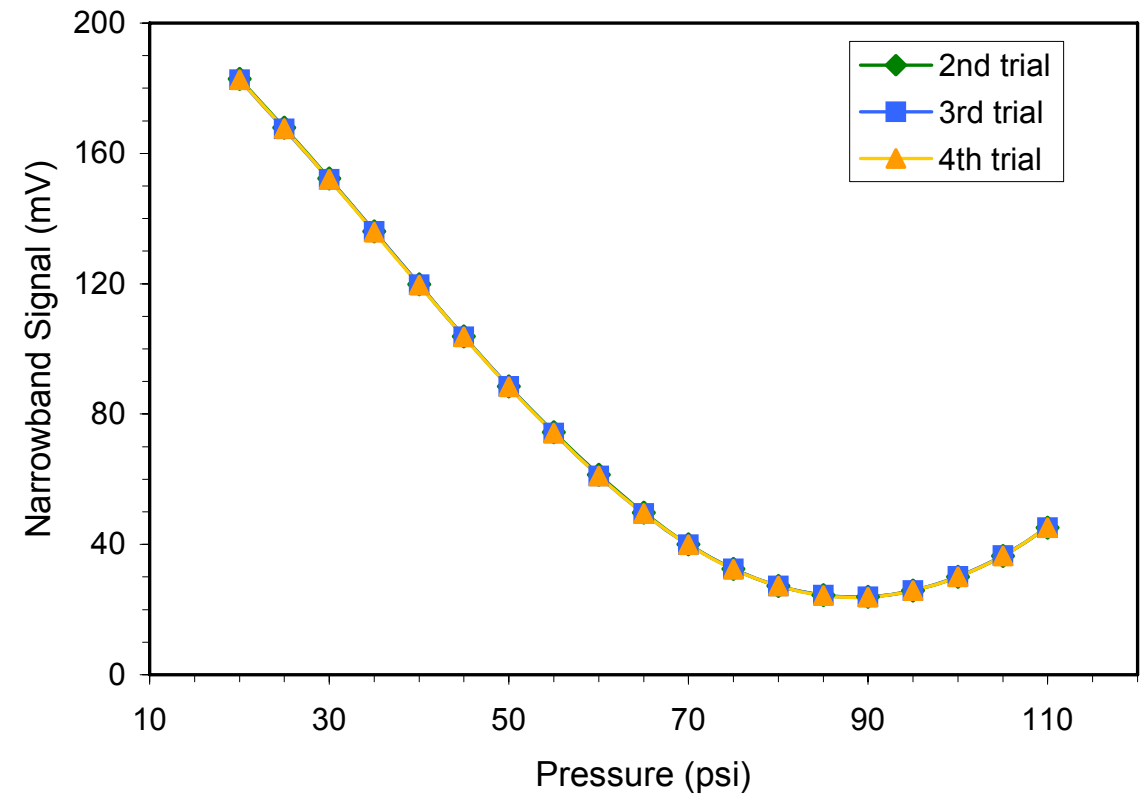

Figure 3.18. Narrowband signal in the $2^{\text {nd }}, 3^{\text {rd }}$ and $4^{\text {th }}$ trials. 


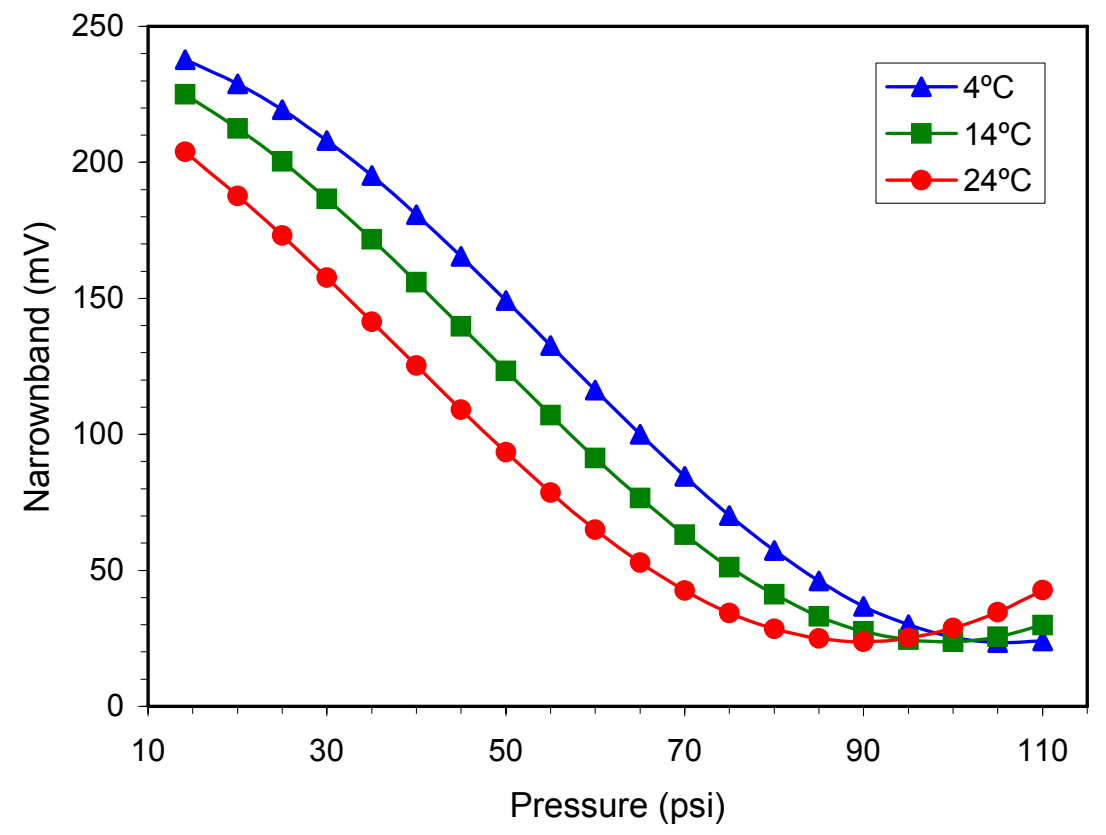

Figure 3.19. Narrowband signals at three operating temperatures.

Table 3.8. Fitting parameters from Eq. 3-22 for three operating temperatures.

\begin{tabular}{ccccc}
\hline $\begin{array}{c}\text { Temperature } \\
\left({ }^{\circ} \mathbf{C}\right)\end{array}$ & a & b & c & d \\
\hline $\mathbf{4}$ & 534.3 & 441.3 & 0.03014 & 158.9 \\
\hline $\mathbf{1 4}$ & 532.5 & 437.5 & 0.03021 & 151.0 \\
\hline $\mathbf{2 4}$ & 531.4 & 436.7 & 0.03003 & 142.2 \\
\hline
\end{tabular}

It is clear that $\mathrm{a}, \mathrm{b}$ and $\mathrm{c}$ are very close at different temperature while parameter $\mathrm{d}$ changes a great deal, indicating a large temperature coefficient.

Since $\mathrm{d}=\left(\mathrm{C}_{\mathrm{T}} * \mathrm{~T}+\mathrm{L}_{0}\right) / \mathrm{S}$ and $\mathrm{S}=\mathrm{c} \frac{\lambda}{4 \pi}$, then $\Delta \mathrm{d}=\mathrm{C}_{\mathrm{T}} \frac{\Delta \mathrm{T}}{\mathrm{S}}$ and so $\mathrm{C}_{\mathrm{T}}=\mathrm{S} \frac{\Delta \mathrm{d}}{\Delta \mathrm{T}}=\mathrm{c} \frac{\Delta \mathrm{d}}{\Delta \mathrm{T}} \frac{\lambda}{4 \pi}$. For a $10^{\circ} \mathrm{C}$ change from $4{ }^{\circ} \mathrm{C}$ to $14^{\circ} \mathrm{C}, \mathrm{C}_{\mathrm{T}}=-(158.9-151) / 10^{*} 0.03 * 1550 / 4 / \mathrm{pi}=-2.9 \mathrm{~nm} /{ }^{\circ} \mathrm{C}$.

For a $10^{\circ} \mathrm{C}$ change from $14^{\circ} \mathrm{C}$ to $24^{\circ} \mathrm{C}, \mathrm{C}_{\mathrm{T}}=-(151-142.2) / 10^{*} 0.03^{*} 1550 / 4 / \mathrm{pi}=-3.3 \mathrm{~nm} /{ }^{\circ} \mathrm{C}$.

The average value for $\mathrm{C}_{\mathrm{T}}$ is about $-3.1 \mathrm{~nm} /{ }^{\circ} \mathrm{C}$. This is very large. The negative value is unexpected since borosilicate has a larger coefficient of thermal expansion (CTE) than fused silica and thus the airgap should become larger when temperature increases. However, the 
observed negative coefficient indicates that the airgap actually decreases. The reason for this is possibly due to the sol-gel bonding between the fiber and ferrule and requires additional study.

\section{Long-Term Stability}

Long-term stability depends not only on the sensor, but on the entire system. The stability test lasted several days; however the total useful testing period was about 68 hours because the data collection program crashed from time to time. Figure 3.20 shows the narrowband signal, wideband signal and their ratio during the testing period. A discontinuity indicates that a program crash at that time. The figure clearly shows that taking the ratio of narrowband and wideband signal can reduce the fluctuations due to the light source or other components in the system. While short-term and long-term performance are both improved by taking ratio, the long-term stability is far from being satisfying (Relative error for longterm: Narrowband $-6 \%$ Wideband $-2.6 \%$ Ratio $-4.4 \%$ ). A number of sources could contribute to the large long-term shift. The sensor's temperature coefficient $\left(-3 \mathrm{~nm} /{ }^{\circ} \mathrm{C}\right)$ could account for the long-term signal shift because of the $\pm 0.5^{\circ} \mathrm{C}$ control precision within the temperature chamber.

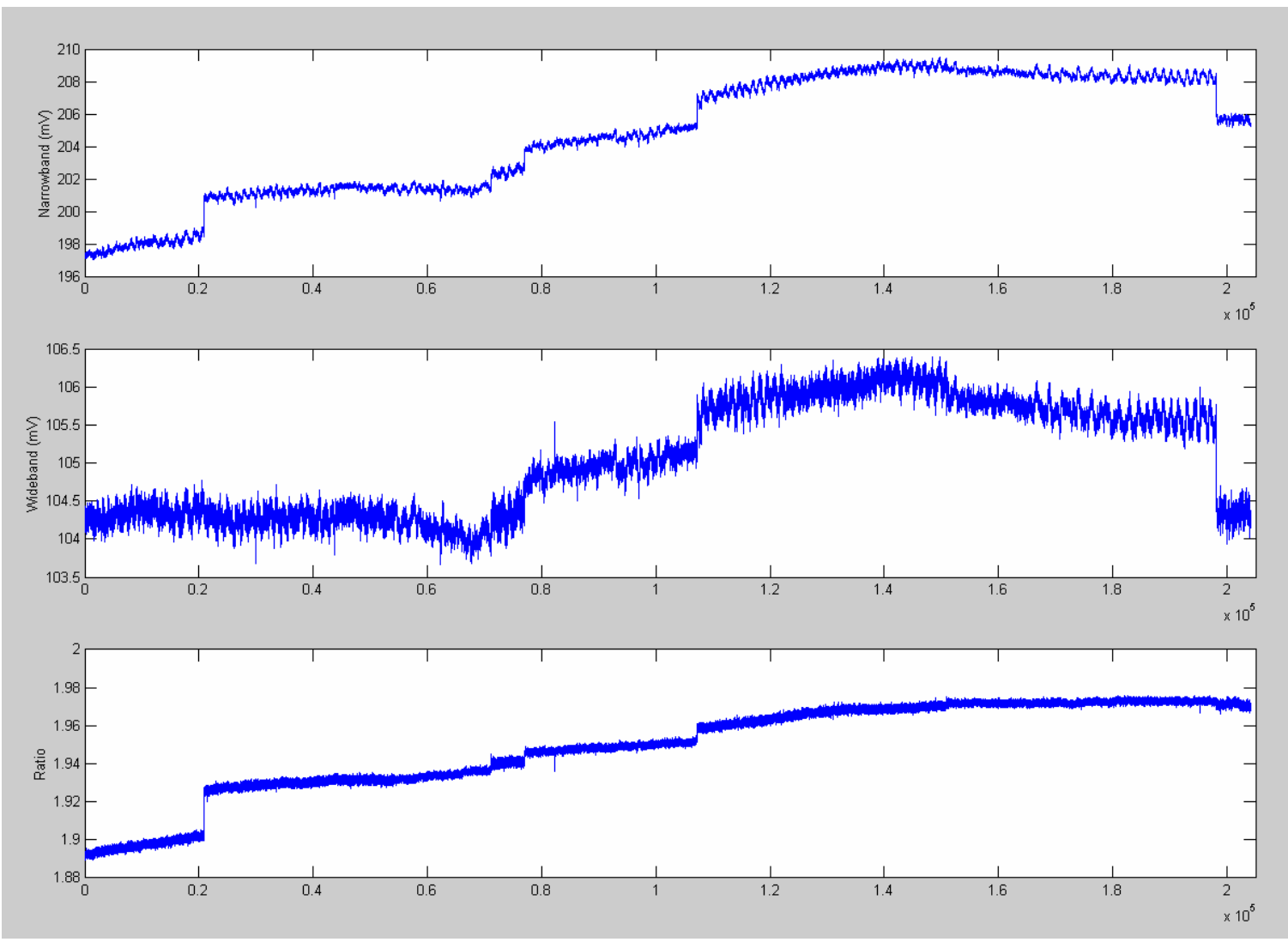

Figure 3.20. Stability of narrowband signal, wideband signal and their ratio during a several day test; total useful testing period was about 68 hours due to data collection program downtime. 
To further investigate the long-term shift, the light source was also tested for a possible spectrum shift that could lead to changes of narrowband signal and wideband signal. Figure 3.21 shows the test result for about 2 days. The three plots are: total power within the spectrum range which can be taken as the wideband signal, central power with a $16 \mathrm{~nm}$ range around the center wavelength which can be taken as the narrowband signal, and the ratio of the two. The relative errors are $0.73 \%, 0.67 \%$ and $0.31 \%$. Again, taking the ratio could reduce the fluctuation. A $0.31 \%$ error in 2 days is reasonable and far below the ratio of $4.4 \%$ in the previous section. This demonstrates that the LED source is not the primary source of the long-term shift. Reducing the sensor temperature coefficient is the most likely focus to achieve long-term stability.

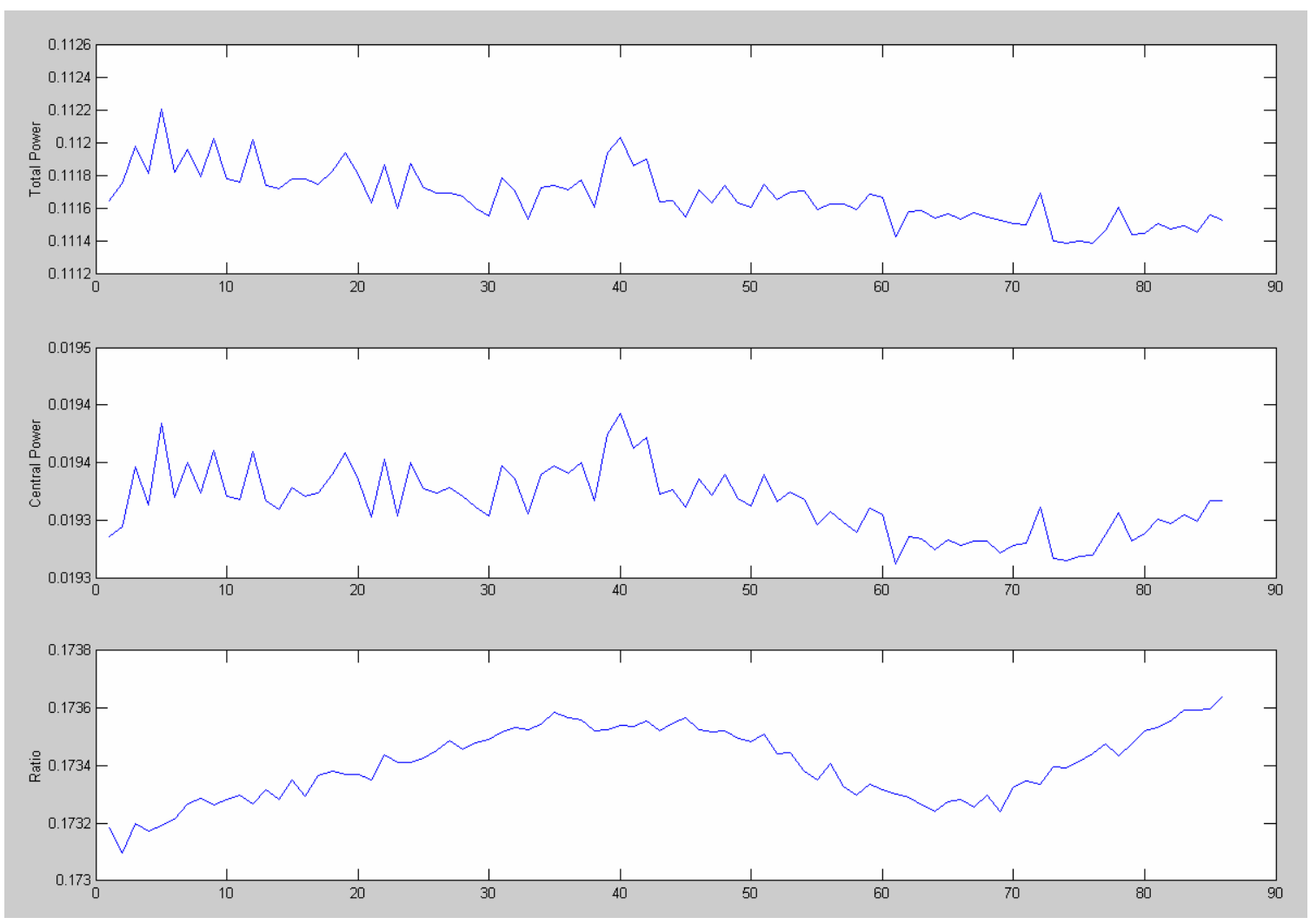

Figure 3.21. Stability of the LED source over two days. 


\subsection{Etched Diaphragm-Based Pressure Sensors}

A large portion of the second year of the program was spent developing pressure sensors based on the photolithographic fabrication of etched diaphragms (Figure 3.22). This method offers the advantage of batch processing capability. Several fabrication steps are required: diaphragm etching, bonding, and sensor evaluation. Each has been investigated and is described in this section.

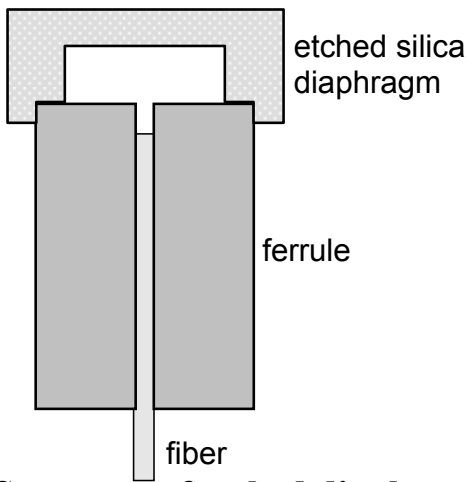

Figure 3.22. Structure of etched diaphragm-based sensor.

\subsubsection{Photolithography}

Deep wet etch process

Using a standard photolithography process, we can achieve very good quality etched pits in fused silica, as shown in Figure 3.23. However, the etch depth is limited to approximately 5 microns because photoresist cannot withstand buffered hydrofluoric acid (BHF) longer than 1 hour. An etch depth of at least 20 microns is required for SCIIB pressure sensor fabrication in order to reduce the gage length and limit the temperature effects. This can be accomplished by depositing additional masking layers between the photoresist layer and the wafer in order to further protect the wafer surface from HF attack. Chromium was selected as the additional masking layer for the following reasons.

- A sputtering system is available at CPT for chromium coating deposition.

- Chromium targets for the sputtering system are relatively inexpensive.

- A chromium layer can be easily removed using commercially available chromium mask etchant

- A chromium layer can improve the photoresist adhesion and prevent HF acid attack on the diaphragm.

In order to deep etch the fused silica diaphragm, several additional steps involving chromium deposition and removal must be added to the standard photolithography process. The entire process is illustrated in Figure 3.24. 


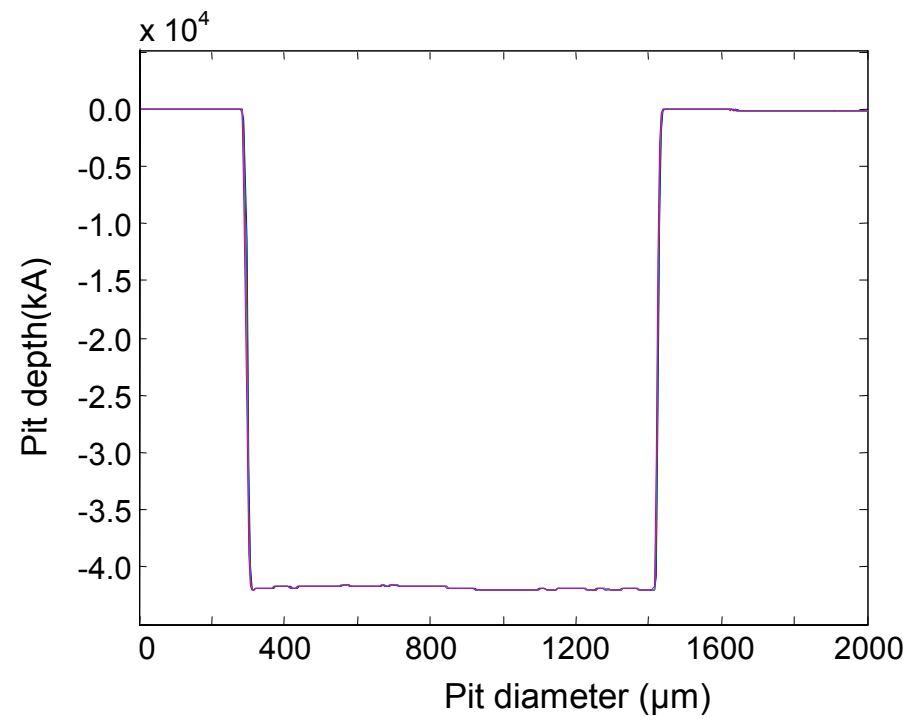

Figure 3.23. Wet etched pit depth in a fused silica diaphragm.

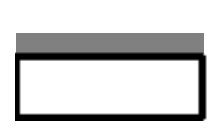

(a)

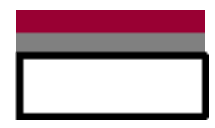

(b)

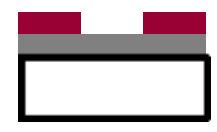

(c)

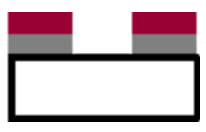

(d)

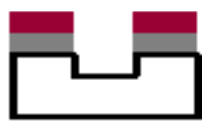

(e)

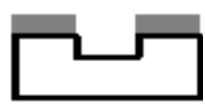

(f)

(g)

photoresist

chromium

\section{fused silica}

diaphragm

(a) Deposit chromium onto the diaphragm.

(b) Spin coat photoresist.

(c) UV expose and develop.

(d) Remove partial (exposed) chromium layer

(e) Wet etch by BHF.

(f) Remove photoresist.

(g) Remove chromium layer.

Figure 3.24. Deep wet etch process.

The chromium layer is deposited using the BOC Edwards RF sputtering system described in Section 3.2.2. Sputtering is a technology in which the material is released from the source at much lower temperature than in evaporation. The substrate is placed in a vacuum chamber with the source target and an inert gas (such as argon) is introduced at low pressure. A gas 
plasma is struck using an RF power source, causing the gas to become ionized. The ions are accelerated towards the surface of the target, causing atoms of the source material to break off in vapor form and condense on all surfaces including the substrate. This basic principle holds for all sputtering technologies. Differences between sputtering methods typically involve the manor in which the ion bombardment of the target is realized. A schematic diagram of a typical RF sputtering system is shown in Figure 3.25 below.

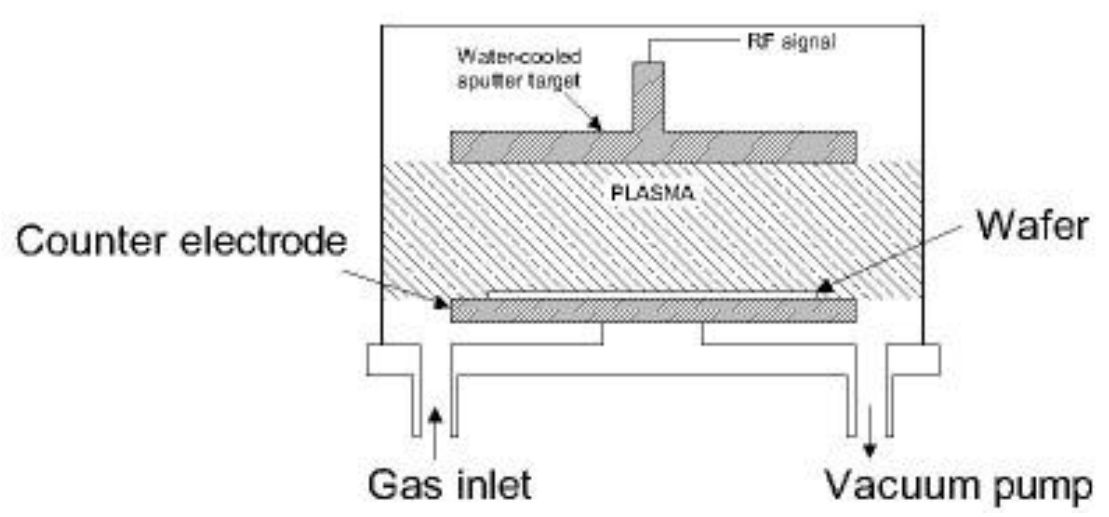

Figure 3.25. Typical RF sputtering system.

The thickness of chromium deposited depends on the RF power, process pressure and deposition time. Table 3.9 lists the results of chromium coating under different conditions. The profile of a diaphragm etched in BHF for 5 hours is shown in Figure 3.26. The pit depth is approximately 25 microns and the bottom surface is still very flat. Sensors made using such pits can exhibit visibility as high as $98 \%$. Even deeper pits can be formed using this method. A magnified image of a representative pit is shown in

Table 3.9. Chromium coating thickness resulting from various processing parameters.

\begin{tabular}{cccc}
\hline $\begin{array}{c}\text { Power } \\
(\mathbf{W})\end{array}$ & $\begin{array}{c}\text { Time } \\
(\mathbf{s e c})\end{array}$ & $\begin{array}{c}\text { Pressure } \\
(\text { Torr })\end{array}$ & $\begin{array}{c}\text { Thickness } \\
(\mathbf{n m})\end{array}$ \\
\hline 100 & 60 & $3.5 \mathrm{e}-3$ & 70 \\
\hline 100 & 30 & $3.6 \mathrm{e}-3$ & 55 \\
\hline 100 & 15 & $3.6 \mathrm{e}-3$ & 10 \\
\hline 100 & 30 & $4.4 \mathrm{e}-3$ & 40 \\
\hline 300 & 60 & $3.4 \mathrm{e}-3$ & 180 \\
\hline
\end{tabular}




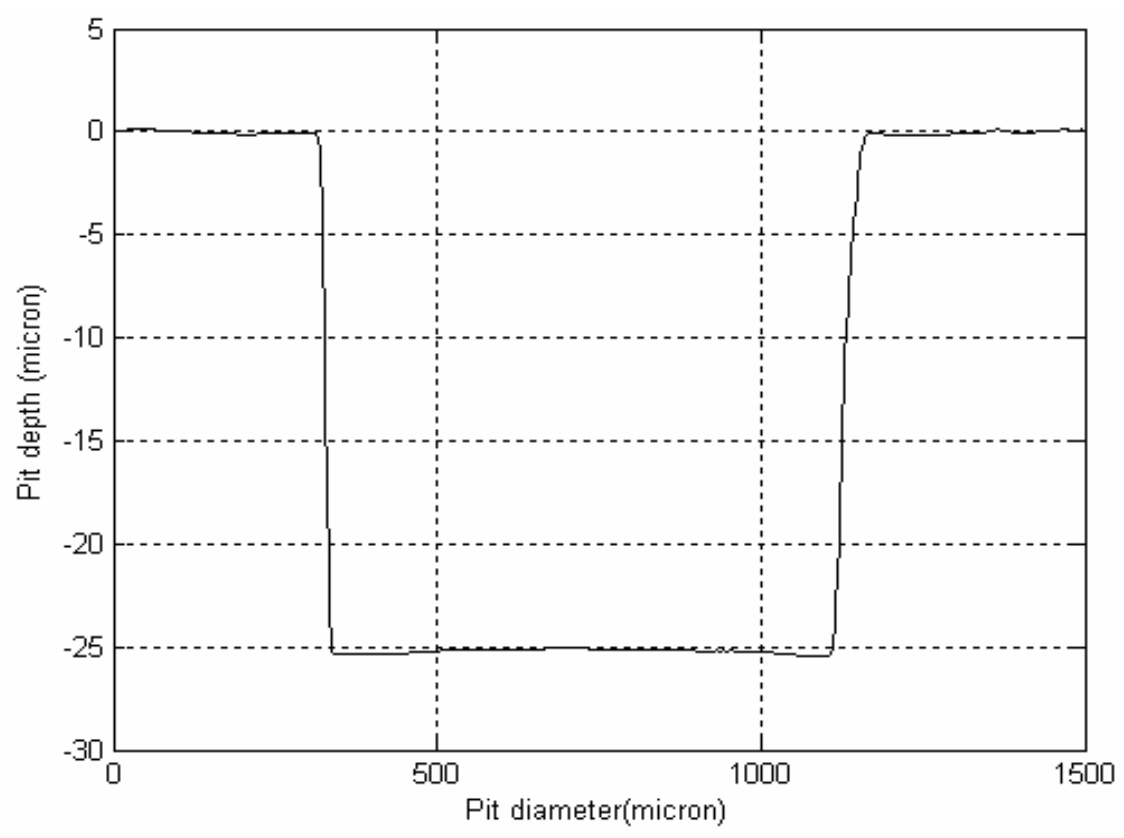

Figure 3.26. Profile of a fused silica diaphragm etched in BHF for 5 hours using the deep wet etching process illustrated in Figure 3.24.

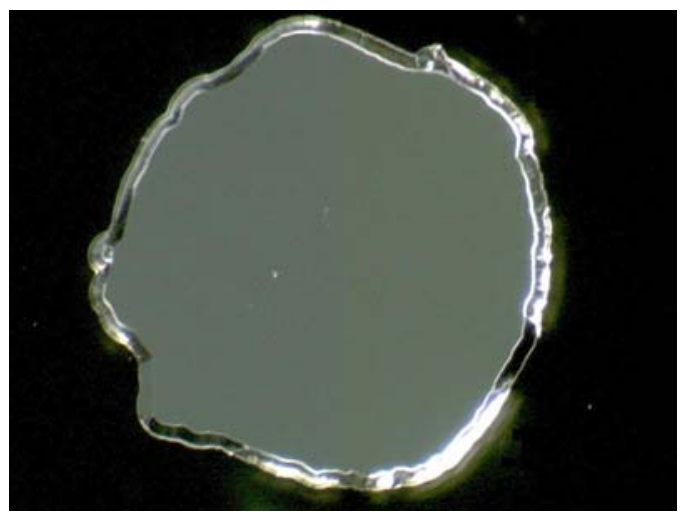

Figure 3.27. Representative etched pit in silica diaphragm.

\section{Two-step wet etching}

The center is the most critical part of the diaphragm pit. As shown in Figure 3.22, the central axis of the ferrule and the center of the pit should be aligned in order to obtain reach the maximum pressure sensitivity upon diaphragm deflection (Figure 3.28). A two-step etching structure is used to make the alignment easier using an outer pit diameter equal to the OD of the ferrule, and a deeper inner pit. Figure 3.29 illustrates the advantage of this two-step structure. 

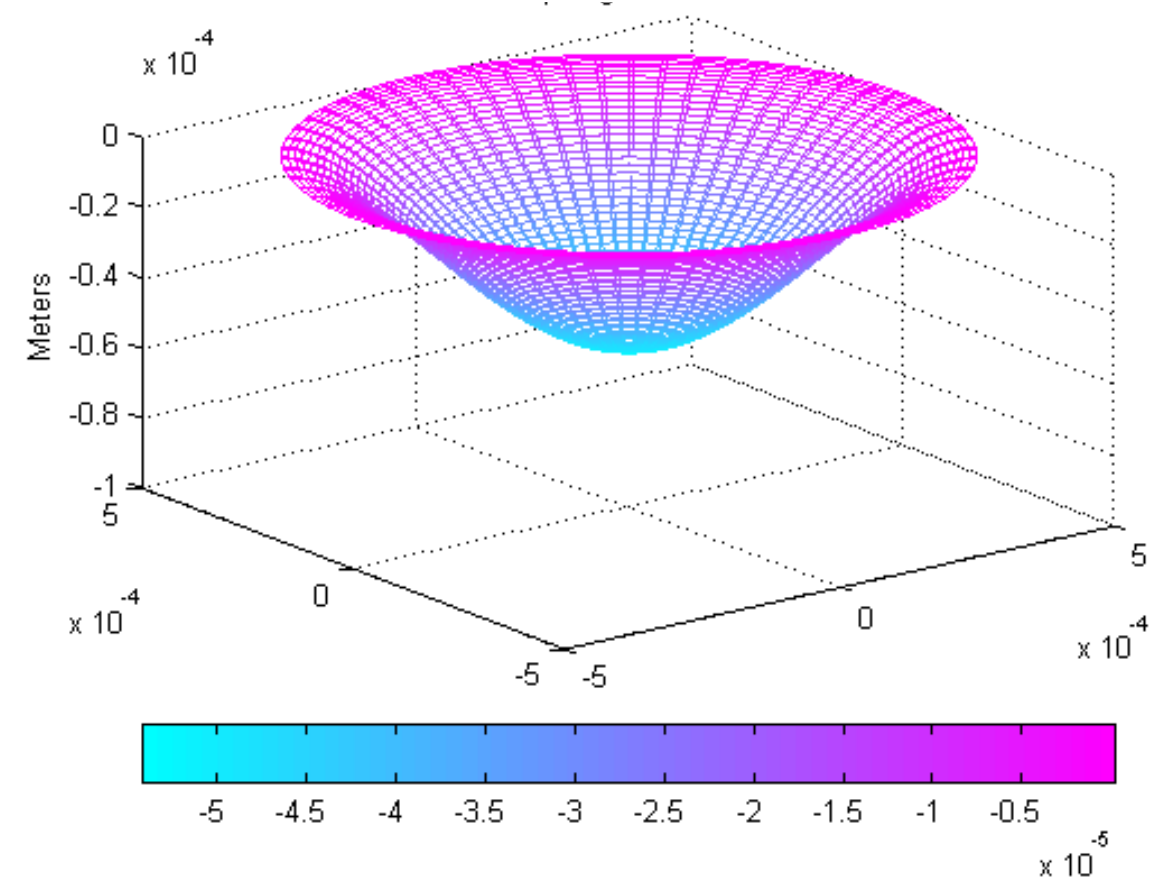

Figure 3.28. Computer simulation of fused silica diaphragm deflection.

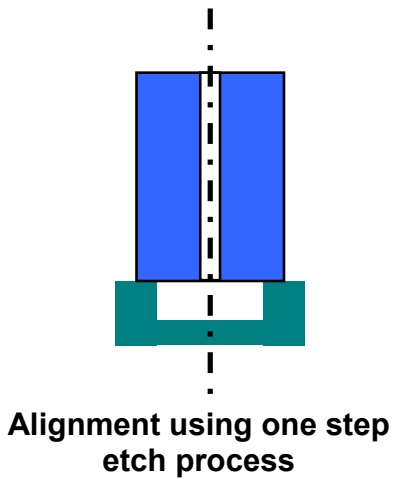

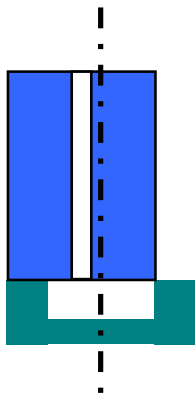

Misalignment using one step etch process

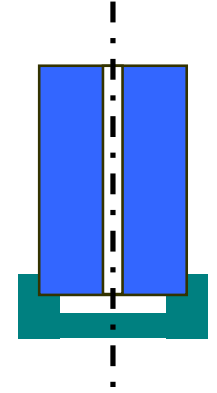

Simplified alignment using two step etch process

Figure 3.29. Two step etching process used to assist in diaphragm-ferrule alignment.

Figure 3.30 illustrates the additional processing steps necessary to achieve the two step etch structure. A 700nm chromium layer was deposited on the fused silica diaphragm. This layer improves the photoresist adhesion and results in a deeper etch depth. In steps (b) to (f) and (g) to (l) in Figure 3.30, the normal photolithography process has been used twice with two different size photo-masks. Figure 3.31 shows the profile of a representative two-step wet etched pit. The bonding of the diaphragm and the ferrule should occur on the bottom surface of the larger (outer) pit. 

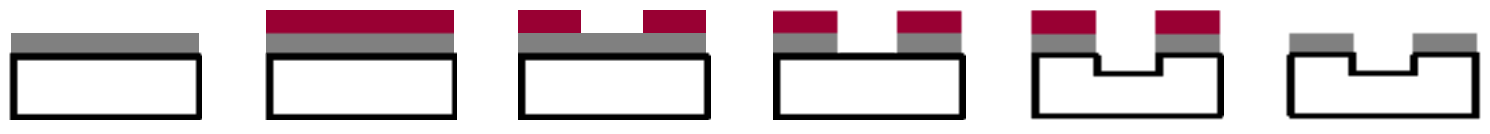

(a)

(b)

(c)

(d)

(e)

(f)
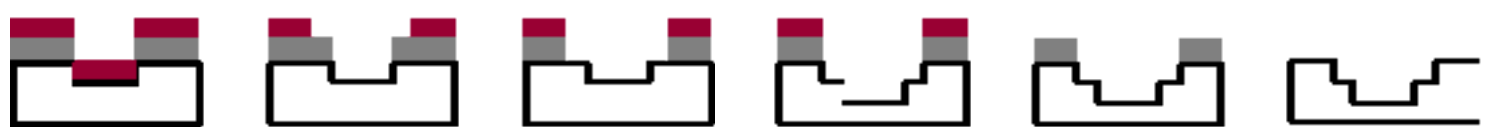

(g)

(h)

(i)

(j)

(k)

(l)

photoresist

chromium

(a) Deposit chromium onto the diaphragm.

(b) Spin coat photoresist.

(c) UV expose and develop.

(d) Remove partial (exposed) chromium layer.

(e) Wet etch by BHF.

(f) Remove photoresist. (g) Deposit second photoresist layer.

(h) UV expose and develop.

(i) Remove partial (exposed) chromium layer.

(j) Wet etch by BHF.

(k) Remove photoresist.

(l) Remove chromium layer.

Figure 3.30. Two step wet etch process.

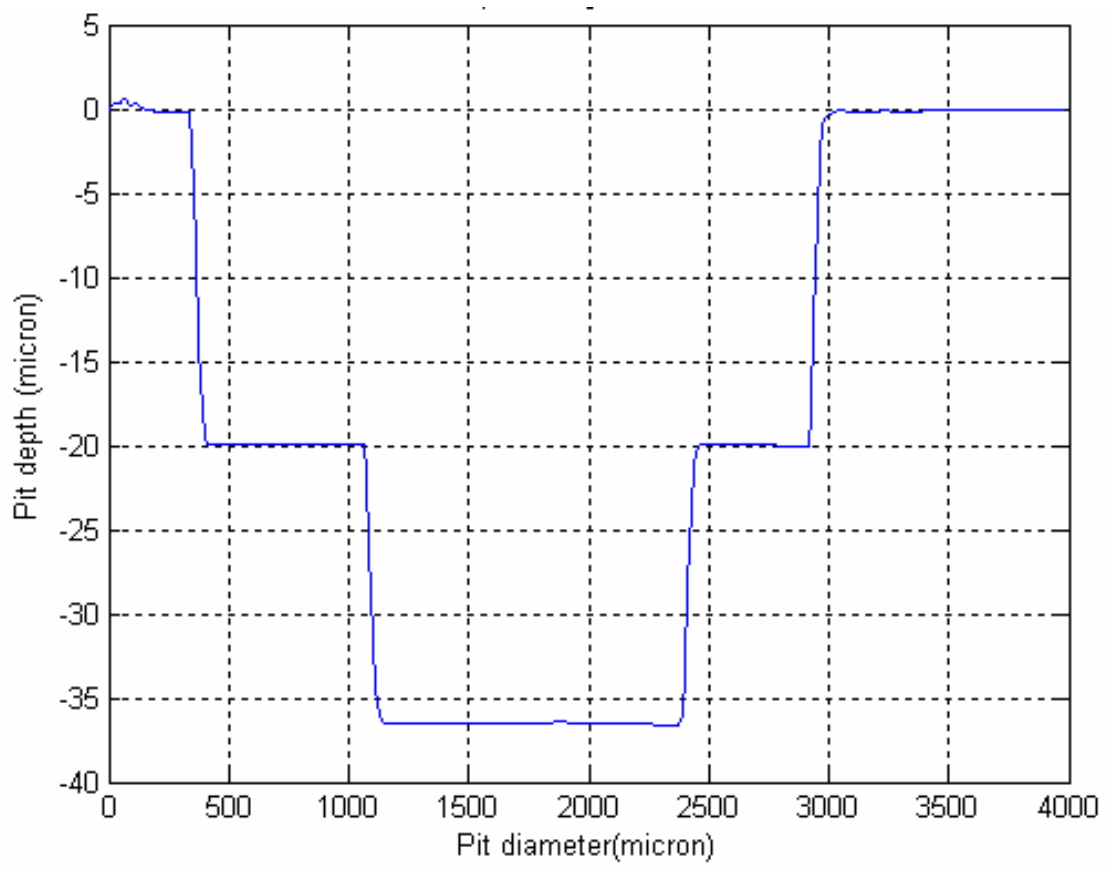

Figure 3.31. Depth profile results of two step wet etch in fused silica (DEKTAK3 profilometer). 


\section{Analysis of wet etch procedures}

Etch Rate: Wet etch rates can vary for reasons that are typically divided into three groups: the etch setup, the material being etched, and the layout and structure on the substrate. The most significant effects on wet-etch rate are as follows.

- Temperature

- Impurities in or on the material being etched

- Contamination

For fused silica, the etch rate in 5:1 BHF at room temperature is about $46.42 \mathrm{~nm}$ per minute (Figure 3.32). Using commercially available BHF etchant results in an etch rate of $83.3 \mathrm{~nm}$ per minute at room temperature (Figure 3.33). It is evident that the total etch rate curves are not linear; the etch rate decreases after an extended period of time.

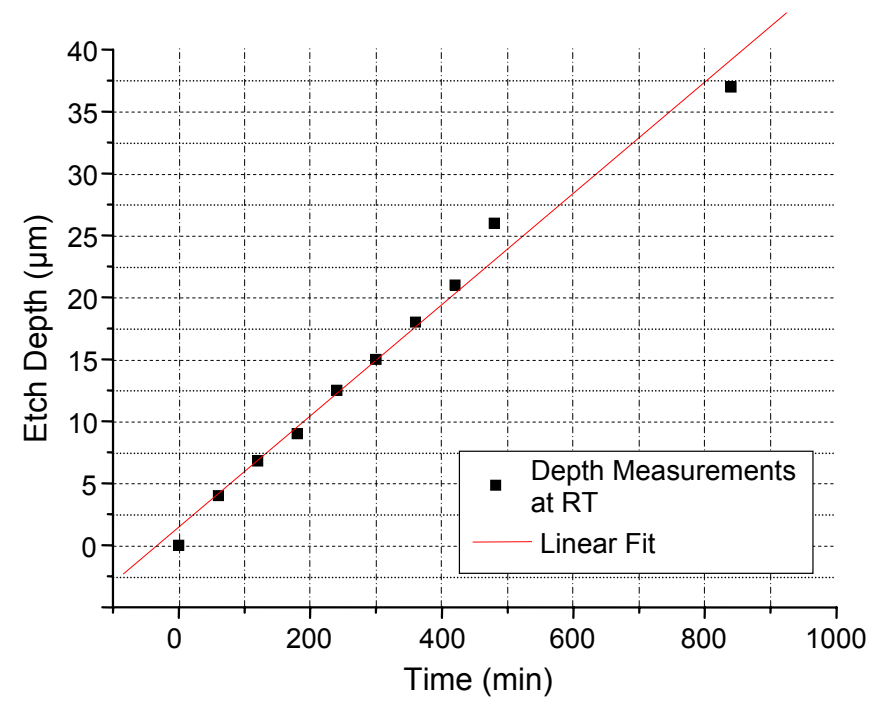

Figure 3.32. Etch depth results using 5:1 BHF; etch rate $=46.42 \mathrm{~nm} / \mathrm{min}$.

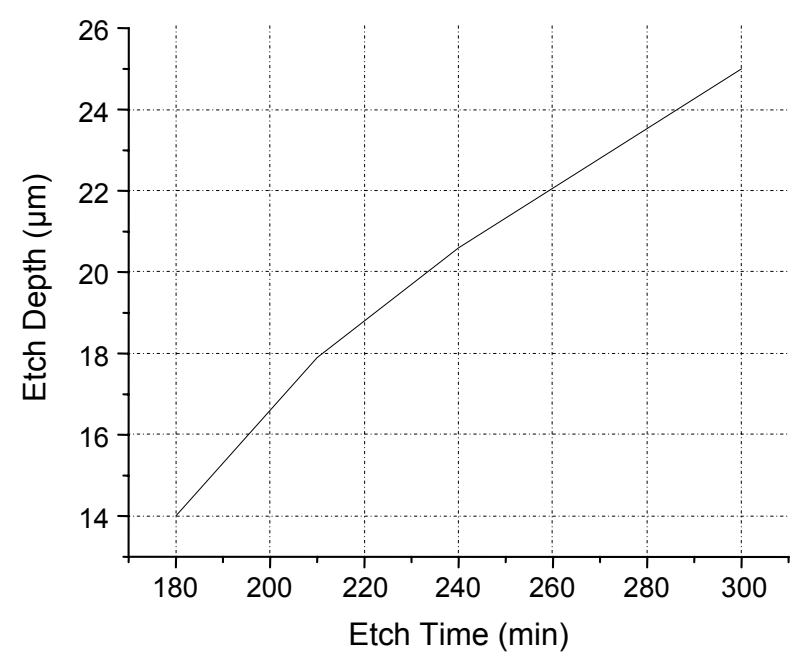

Figure 3.33. Etch depth results using commercial $\mathrm{BHF}$; etch rate $=83.3 \mathrm{~nm} / \mathrm{min}$. 
Surface roughness: The amount of buffer in the HF solution was the most important factor in terms of the resulting surface roughness. Pinholes appeared in the etched diaphragm if sufficient $\mathrm{NH}_{4} \mathrm{~F}$ was not added to the solution because the diaphragm was immersed in $\mathrm{HF}$ for too long. A smooth surface can be obtained by improving the BHF composition. This is illustrated by the two plots in Figure 3.34; the surface quality in the plot on the right was improved by increasing buffer concentration. In addition, reducing the concentration of HF produced better surface quality results.
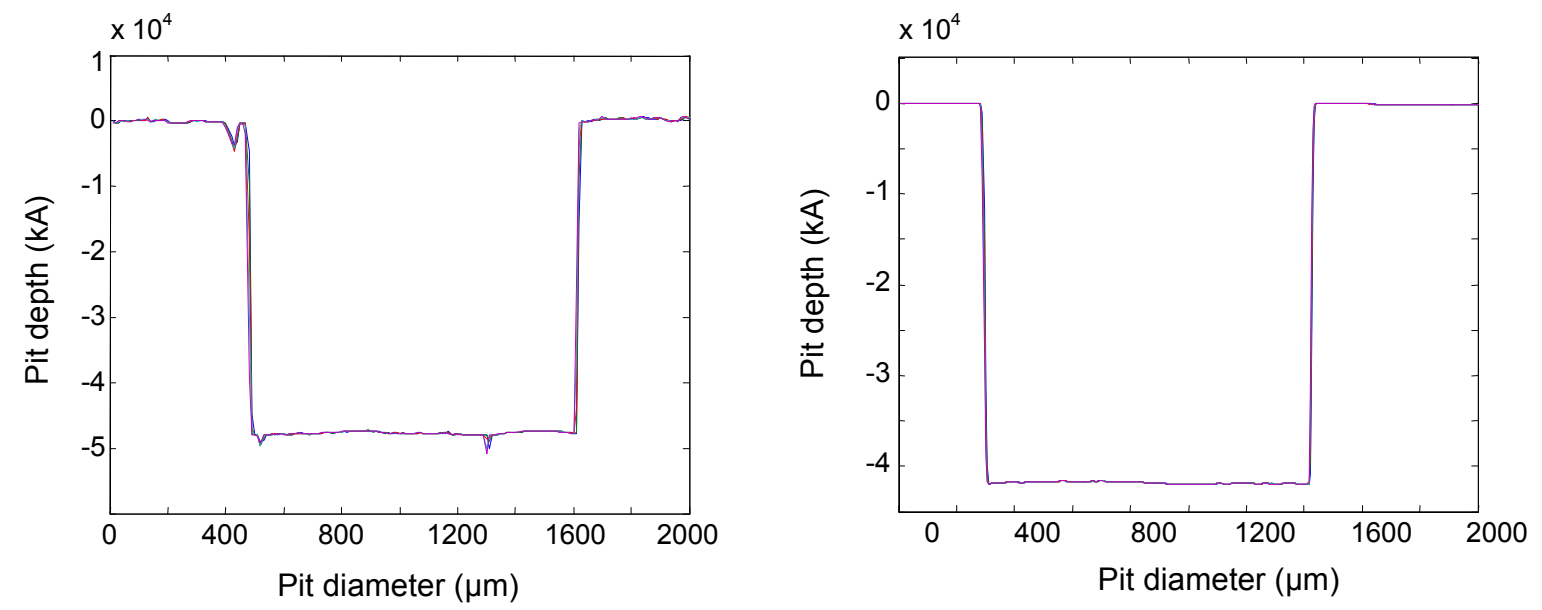

Figure 3.34. Improvements in pit surface quality through changes in BHF composition.

There is a trade-off between surface quality and etch rate. For our sensor fabrication, we selected commercially available BHF with higher concentration to etch the small pit. The etch rate is high and we can achieve pit depths as deep as $20 \mu \mathrm{m}$ in 4 hours. But some roughness developed after the four hours (Figure 3.34, 2nd plot). A reduced concentration of BHF (10:1) was then used to etch the larger pits. The depth of the larger pits is not critical and using a low HF concentration can mitigate the roughness of the small pits induced by the first etch process. The reflectance of the resulting etched pits can be as high as $4 \%$.

Diameter Deviation: The main factor affecting the diameter of the etched pit is the exposure process, including the UV light/substrate separation distance and the exposure time. The exposure must be uniform in energy and time across the substrate surface. If the UV light was too close to the substrate (Figure 3.35, left), some areas were overexposed and some other areas were underexposed because of the narrow UV beam. This caused the pit diameter to vary quite a bit. If the UV light was placed further away from the substrate, the exposure was uniform, but a longer exposure time was required (Figure 3.35, right). A separation distance of $8 \mathrm{~cm}$ and exposure time of 4 minutes were selected. 

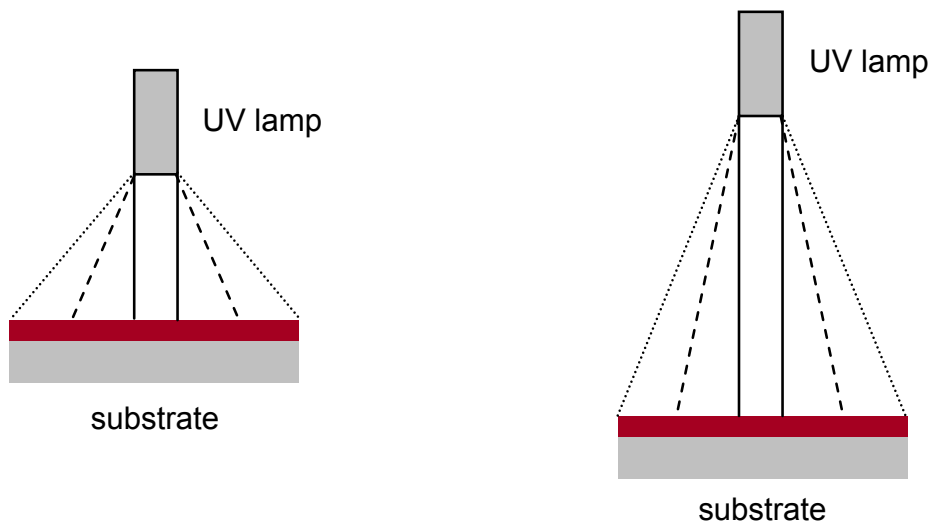

Figure 3.35. Impact of UV light/substrate separation distance on exposure uniformity.

A second factor that impacts the pit diameter is the etching parameters discussed above. The plot on the left in Figure 3.36 shows the profiles of some early etched pits. The diameter deviation was $73 \%$. The second plot illustrates the improvement in etched pit diameters achieved in recently fabricated pits. The sidewalls are very straight and smooth and the diameter deviation is only about $9.6 \%$. Controlling the pit diameter deviation is critical in determining the sensitivity of the resulting sensor.
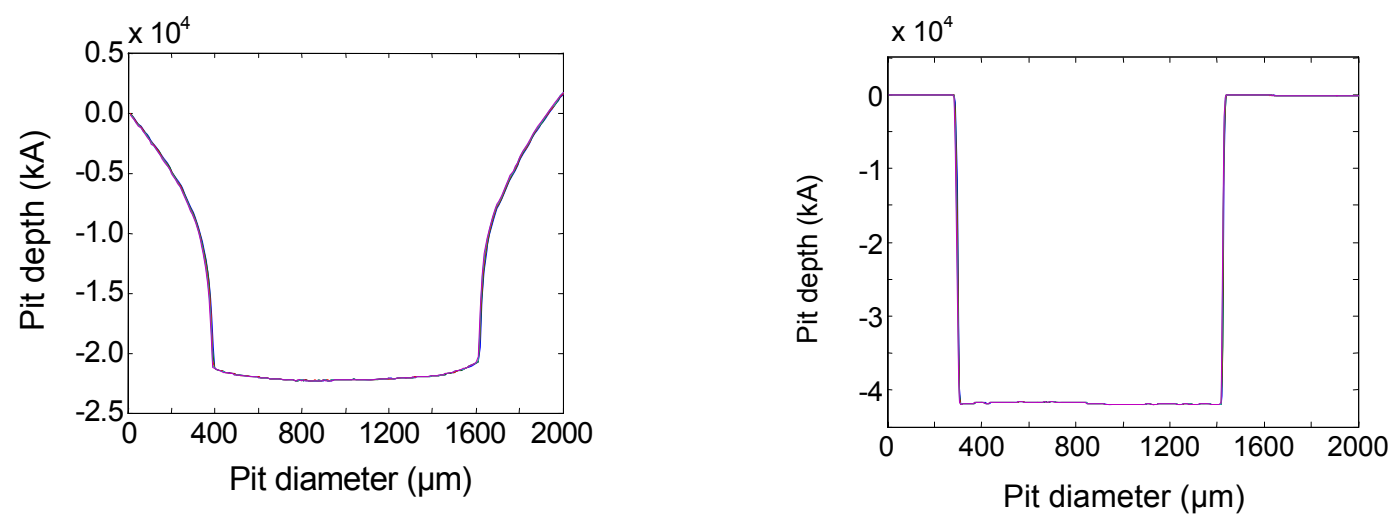

Figure 3.36. Improvements in diameter deviation resulting from process improvements. Pits on the left exhibit a diameter deviation of $73 \%$; those on the right only $9.6 \%$.

Etch depth uniformity: Figure 3.37 shows the depth profile of two etched pits from two different diaphragms. Both were etched in commercial BHF for 5 hours. The etch depth was controlled by BHF concentration, temperature and the time. Since HF is extremely dangerous we normally use it at room temperature. Commercial BHF was used in order to maintain BHF concentration. The pit depth uniformity was measured as $\pm 0.7 \%$. Figure 3.38 
shows the depth profile of six pits etched in the same diaphragm, resulting in a depth deviation of $\pm 04 \%$.

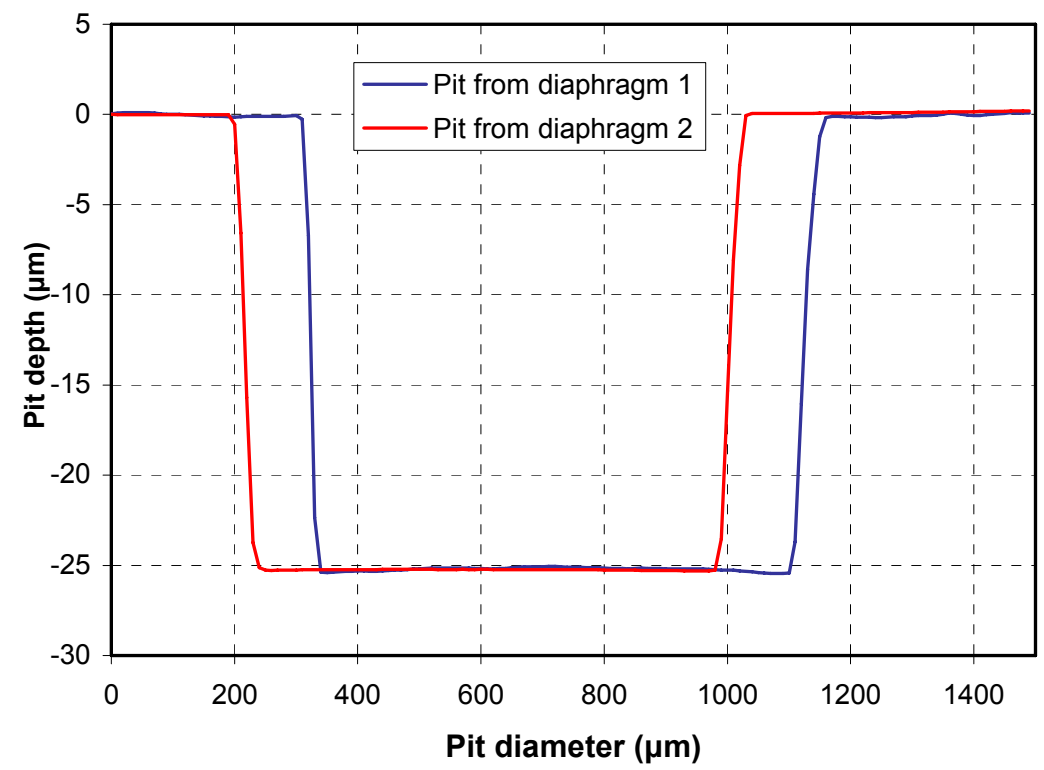

Figure 3.37. Depth profile results of etched pits from two different fused silica diaphragms (Veeco DEKTAK3 profilometer).

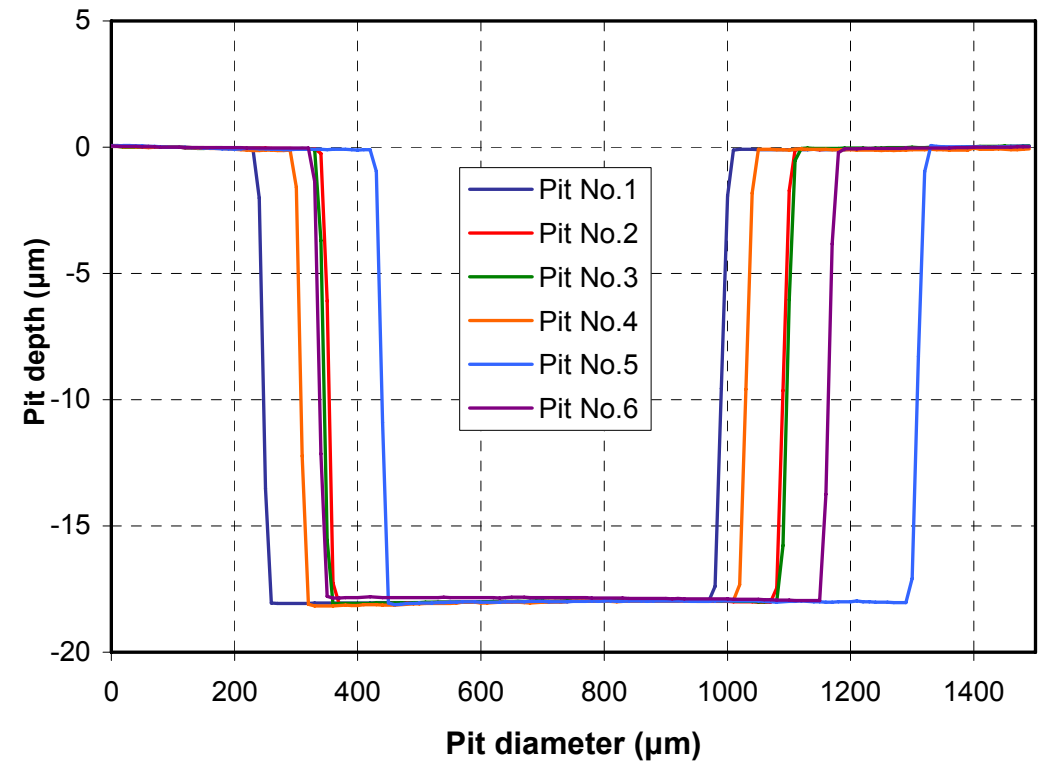

Figure 3.38. Depth profile results of six etched pits from the same fused silica diaphragm (Veeco DEKTAK3 profilometer). 


\subsubsection{Bonding}

Successful pressure sensor fabrication depends primarily on how well the diaphragm can be bonded with the ferrules. Several bonding mechanisms were investigated simultaneously in order to determine the most feasible method of bonding.

\section{Direct Bonding}

The direct-bond method relies on forces that naturally attract surfaces together when they are very smooth and flat. A range of mechanisms has been proposed to explain this initial contact attractive force. It is well known that smooth metal surfaces, if atomically clean, will bond together. This process is often referred to as "cold welding" and is typically achieved by cleaning and bringing into contact the metal surfaces in a vacuum to maintain cleanliness. This bond usually relies on plastic deformation of the metal to bring the atoms in close contact. Most direct bonding demonstrated for microstructures requires some surface treatment (e.g., hydration, oxygen plasma exposure) prior to contact to promote the surface attraction and bonding process. This is sometimes assisted by modest pressure to expel air from between the wafers and to initiate the contact. Bonding is usually followed by a thermal cycle, which increases the strength of the bond.

Batch processing capability makes the fabrication process more economical in terms of both cost and time. Direct bonding may be a route to batch bonding of diaphragms with ferrules. For that purpose, a chuck capable of holding many ferrules has been designed (Figure 3.39). The ferrules protrude out of the chuck a little in order to be able to polish all the ferrules at the same time without affecting the chuck and also to keep the ferrules at the same level after polishing, which will help in the final bonding. The glass substrate containing the etched pits are precisely placed on top of the polished ferrules and pressure applied for direct bonding. This is done under a microscope. Because gold deposited in the etch pits cannot withstand very high temperature, and the ferrules are attached to the chuck with an low temperature adhesive, pressure-assisted low temperature bonding process appears to be the best solution.

To begin using this bonding process for bonding diaphragms with ferrules, initial experiments involved bonding two fused silica glass wafers.

Wafer Requirements: Direct wafer bonding depends enormously on the chemical surface state of the wafers to be bonded, since intermolecular or interatomic force play a major role in obtaining good bond strength. Therefore, wafer surfaces must be sufficiently clean, flat and smooth. The literature survey indicates that wafers should have a surface roughness of no greater than about $10 \AA$ and a bow of less than $5 \mu \mathrm{m}$ (on a 4 " wafer). Also, protrusions from the surface (resulting from previous processing) of greater than $10 \AA$ can produce problems in the bonding. We therefore ordered wafers of the following specifications:

- $\quad$ Fused Silica Corning UV Grade $79805 \mathrm{G}$

- Optically polished

- 10/5 scratch/dig

- Dimension: 1 " X 1" X 0.005" $\pm 0.0004 "$ 


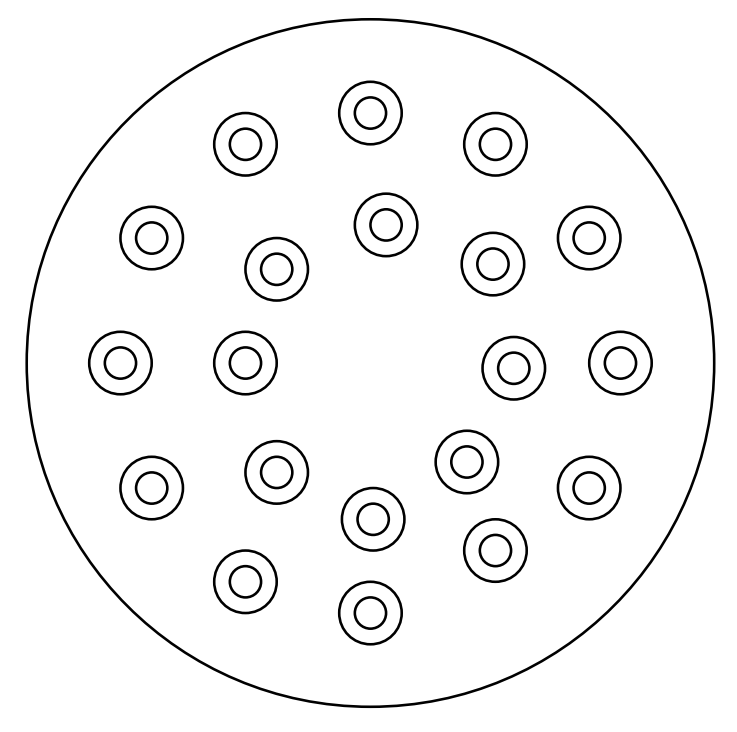

(a)

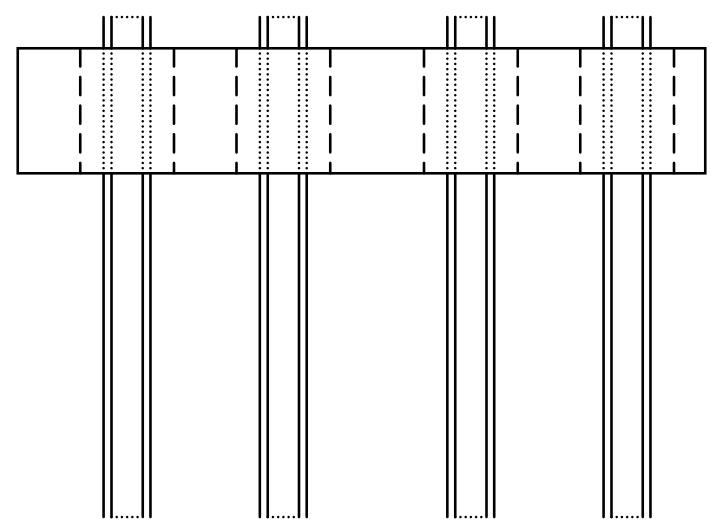

(b)

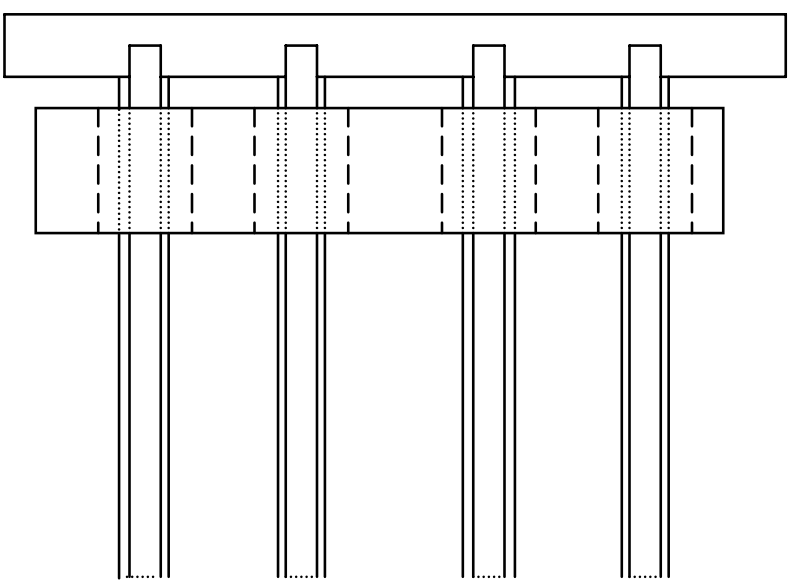

(c)

Figure 3.39. A special chuck has been designed for batch fabrication using direct bonding. (a) Top view of the chuck with the ferrules inserted in the holes and fixed by adhesive (b) side view (c) side view with the etched diaphragms bonded. 
Wafer Cleaning: First, the substrates are cleaned in an organic solvent to remove particles, dust and organic contamination. Then a sulphuric acid peroxide mixture $\left(5: 1 \mathrm{H}_{2} \mathrm{SO}_{4}: \mathrm{H}_{2} \mathrm{O}_{2}=\right)$ is used at room temperature for $20 \mathrm{~min}$ to increase the hydrophilicity of the glass substrates. Afterwards, due to the high viscosity of the sulphuric acid, wafers are intensively rinsed in deionized water and dried.

Immediately after cleaning, the two substrates are brought into contact and a variable external pressure is applied to the entire surface of the substrate pair (Figure 3.40). This pressure presents two advantages: First, it keeps the two substrates sufficiently close to allow the intermolecular force to be effective. Second, it produces a small deformation of the substrate surfaces, enabling the elimination of voids in the between the two substrates, originating from surface imperfections and roughness or trapped air. While continuously maintaining the applied pressure, the substrate stack is annealed to a specific temperature.

The chemical mechanism behind the bonding process is governed by the polymerization of the $\mathrm{Si}-\mathrm{OH}$ groups on the pre-bonded substrates into strong equivalent $\mathrm{Si}-\mathrm{O}-\mathrm{Si}$ bonds on the release of $\mathrm{H}_{2} \mathrm{O}[1]$. The effect of the pressure on the bonding strength is merely an enhancement of the contact surface between the two substrates, thereby changing the effective bonding area.

The entire wafer preparation and bonding process must be performed in very clean environment. Initial attempts in ordinary laboratory space did not yield any positive results. There will be a large number of particles moving around in an ordinary room and if the surface is contaminated by any of these particles at any time during the process, it will preclude the formation of a good bond. For instance, a $1 \mu \mathrm{m}$ particle can cause a void as large as $1 \mathrm{~cm}$ in diameter when direct bonding 8 in wafers[2]. Thus it is not only essential to have very clean wafers but also to have a very clean environment. The entire process was shifted to a Class 1000 clean room that has been newly added to our laboratory. The details of the set up of cleanroom, its specification and the instruments in that room are described in Section 3.2 .

The pressure applied to the wafer is $5 \mathrm{MPa}$. The arrangement for applying pressure is similar to that shown in Figure 3.40. The temperature of the furnace is maintained at $200^{\circ} \mathrm{C}$. The whole arrangement is maintained at that pressure and temperature for 15 hours. Inspection revealed that the wafers were bonded. Figure 3.41a shows the interface of the bonded wafers. In Figure 3.41b, we show the interface of the wafers that are not bonded. Many "Newton Rings" are evident, signifying that the wafers are not bonded at that location due to a void.

The bonding strength was then measured using the arrangement shown in Figure 3.42. One surface of the bonded wafer was epoxied to a string and fixed to a support. The other side was also epoxied to a string and a weight hung from the string. We measured the weight required to break the bond between the wafers. This weight multiplied by the bonding area gives the bonding strength. We found a bonding strength of $1 \mathrm{MPa}$, which is quite sufficient for our work. 


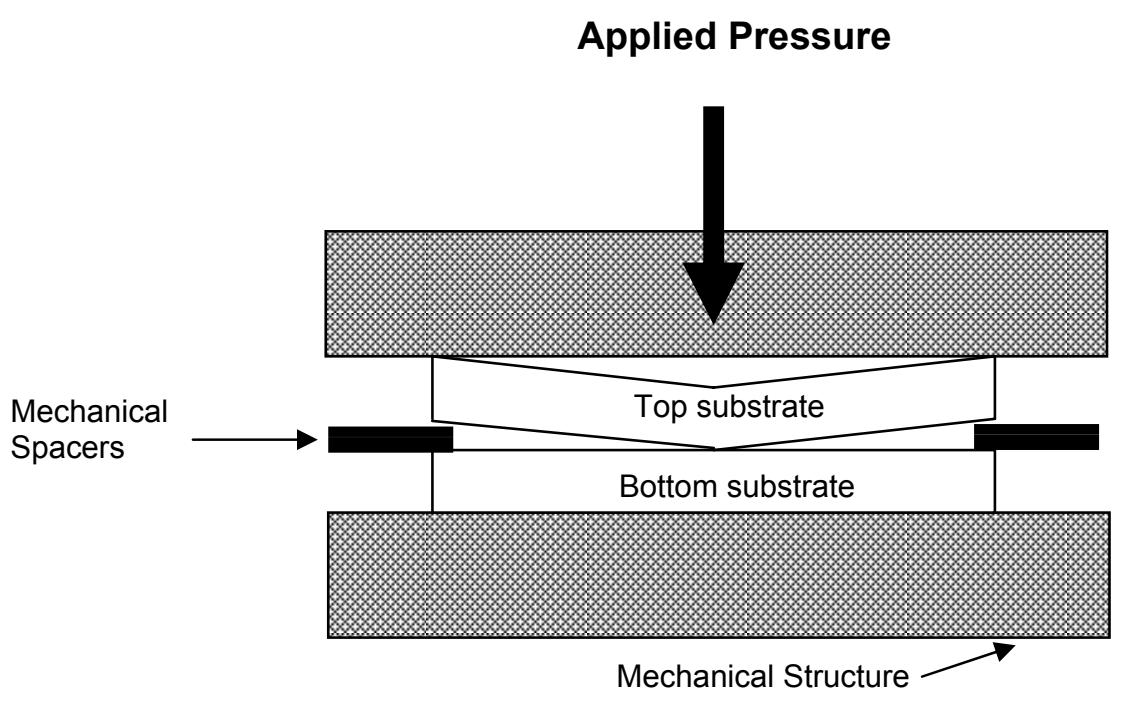

(a)

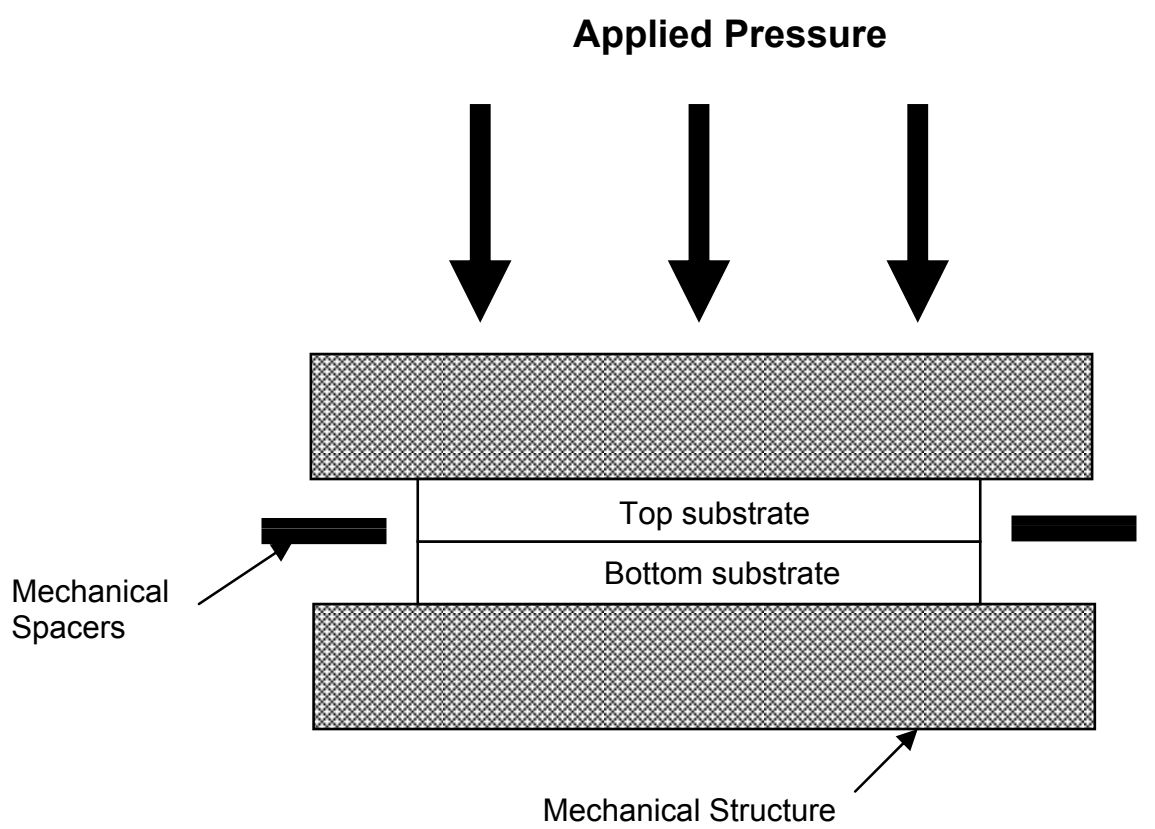

(b)

Figure 3.40. (a) Initially pressure is applied at the middle of one wafer to create a single point of contact between the pieces (b) Retracting the mechanical spacers creates a bonding wave that propagates from the middle of the sample toward the periphery. Surface cleanliness and roughness have a strong influence on the quality of the bond. 

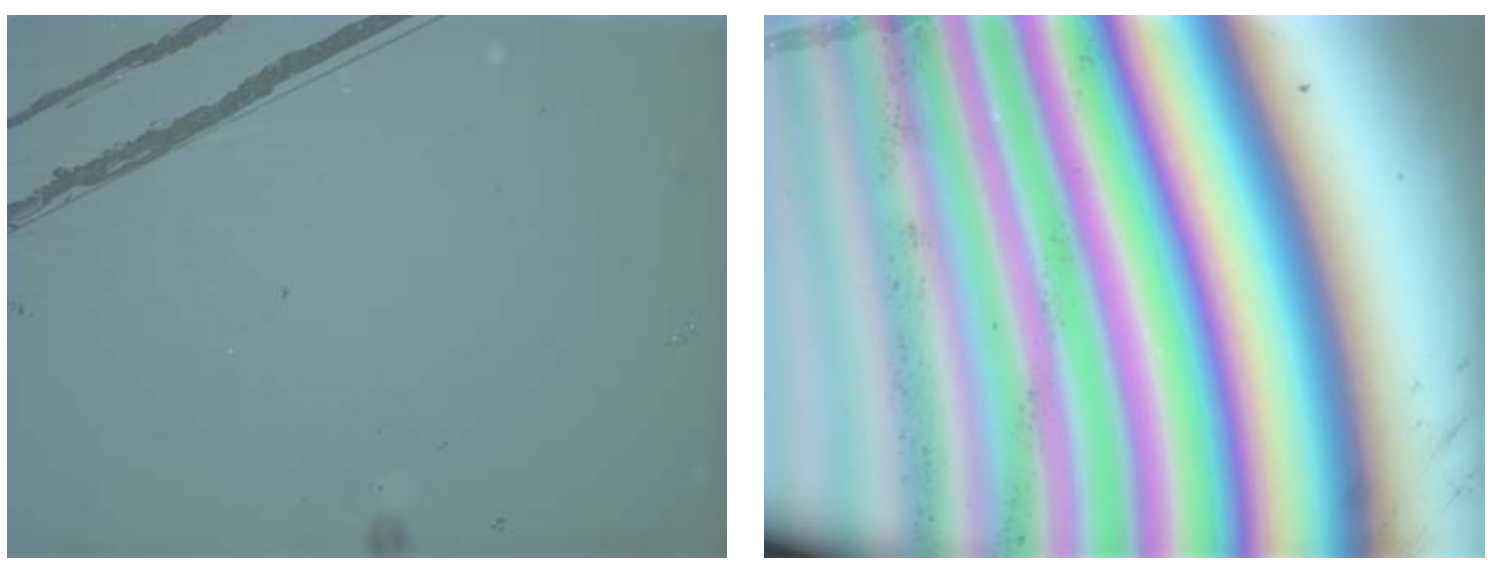

Figure 3.41. Portions of the interface of two bonded wafer (a) completely bonded (b) not bonded due to presence of void.

$\underline{\text { Room temperature hydroxide catalysis bonding }}$

We have investigated another low temperature bonding using the technique of hydroxidecatalysis bonding of fused silica. This is a room temperature process. The wafers need to be as flat and smooth as possible but it does not necessarily require a clean room environment. The entire process is represented pictorially in Figure 3.43.

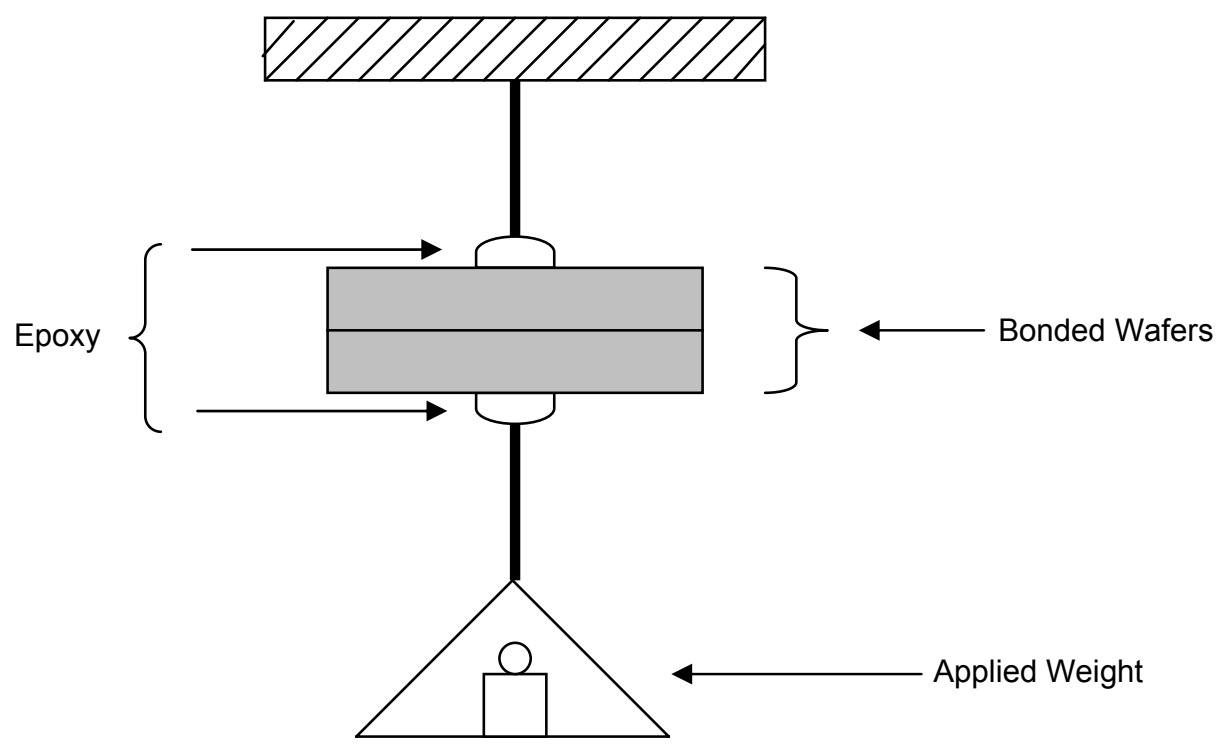

Figure 3.42. Bonding strength measurement setup. 

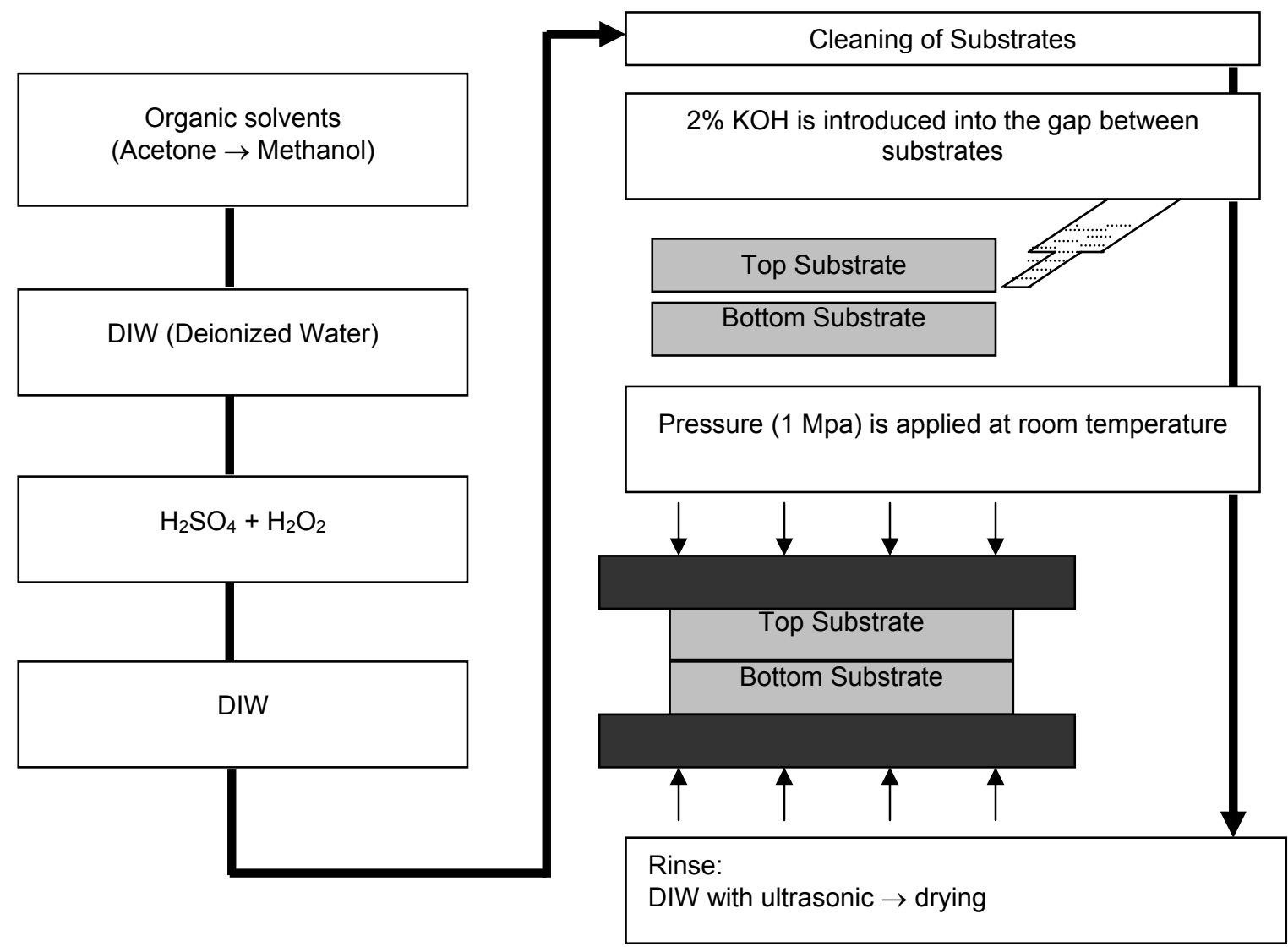

Figure 3.43. Hydroxide-catalysis room temperature fused silica bonding.

The chemical bonding process is initiated through hydroxide-catalysed surface hydration and dehydration at room temperature. The hydration activates the bonding surfaces by generating two surface silanol groups $(\mathrm{Si}-\mathrm{OH})$ per exposed siloxane bridge ( $\mathrm{Si}-\mathrm{O}-\mathrm{Si})$. In other words, the hydration helps to create in situ relatively loose near-surface siloxane networks, which serve as the basic filling material needed for bonding. The dehydration links two sterically accessible silanol groups to form a siloxane bridge, resulting in bonding between one substrate and the loose siloxane network generated in situ, and eventually between the two substrates[3]. Readjustments of the pieces being joined are possible within approximately 3040 minutes of the start of the joining process. The maximum bond strength is reached after several weeks, with the exact time depending on the concentration of the $\mathrm{KOH}$ solution and any mismatch in the surface features of the bonded pieces. However after only a few hours the bond has sufficient strength to allow the fused silica pieces to be handled in a regular environment. Figure 3.44 shows the bonded interface of a ferrule with the fused silica. As the ferrule surface is not very smooth, there are some portions that are not bonded properly. 


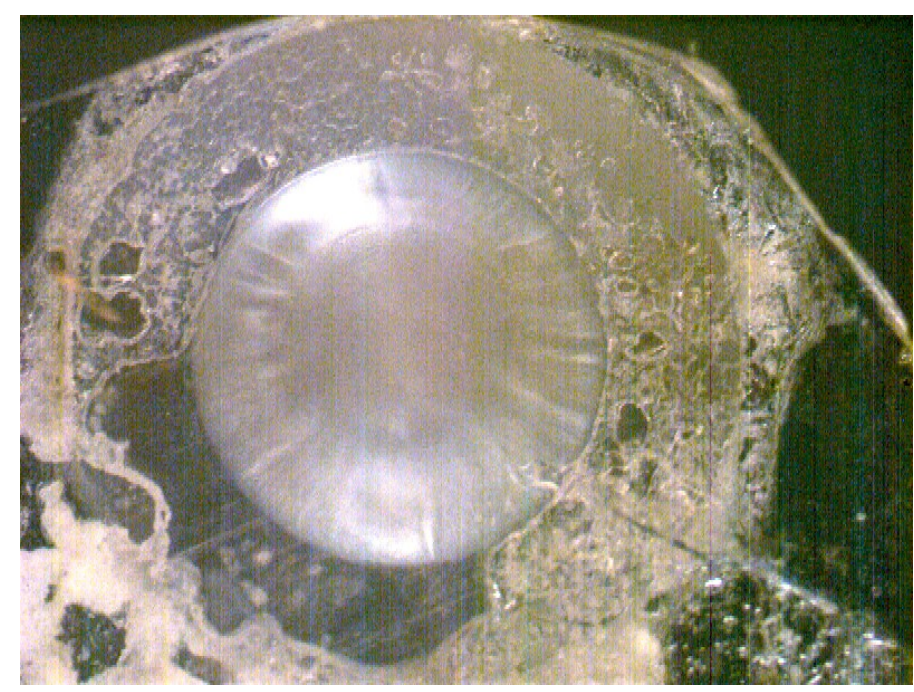

Figure 3.44. Interface of a wafer bonded with a ferrule bonded using the mechanism of hydroxide-catalysis bonding.

\section{Sol-Gel Bonding}

Direct bonding is the most promising way to reduce the temperature dependence of SCIIB pressure sensors. However, directly bonding places extremely high requirements on the surface quality and operating environment. Although we have successfully bonded two fused silica wafers together tightly, patterned diaphragm/diaphragm and patterned diaphragm/ferrule direct bonding are still under investigation. Intermediate layer bonding is currently the most feasible bonding method for our experiments. Sol-gel was selected as the intermediate layer for several reasons.

- Sol-gel is a low temperature process.

- Thin films can be formed by sol-gel.

- The final form of the sol-gel process "gel" can be high purity silica, this will reduce the thermal expansion of the intermediate layer;

- Sol-gel is available in our lab and it is inexpensive

Sol-gel was used for both diaphragm-ferrule bonding and ferrule-fiber bonding.

The term sol-gel was first coined in the late 1800s. It generally refers to a low-temperature method using chemical precursors that can produce ceramics and glasses with better purity and homogeneity than conventional high temperature processes. In general, the sol-gel process involves the transition of a system from a liquid "sol" (mostly colloidal) into a solid "gel" phase (usually silica). It is possible to fabricate ceramic or glass materials in a wide variety of forms: ultra-fine or spherical shaped powders, thin film coatings, ceramic fibers, microporous inorganic membranes, monolithic ceramics and glasses, or extremely porous aerogel materials.

Thin films can be produced on a piece of substrate by spin-coating or dip-coating. When the "sol" is cast into a mold, a wet "gel" will form. With further drying and heat-treatment, the 
"gel" is converted into dense ceramic or glass articles. The basic sol-gel reaction, shown in Figure 3.45, consists of two chemical reactions that produce a thin film when the sol is applied to a substrate. The first is a hydrolysis reaction, which produces the sol, and the second is a condensation reaction, which forms a macroscopic gel on the substrate, producing a thin film.

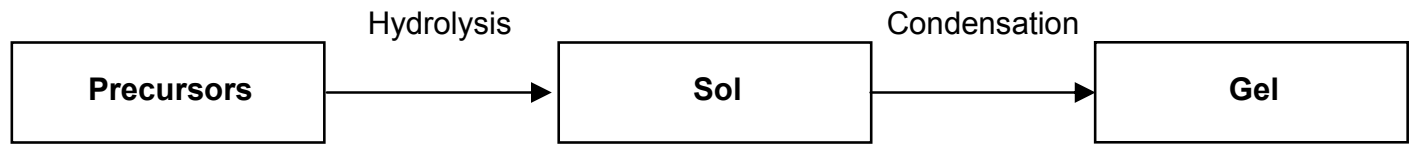

Figure 3.45. The basic sol-gel reaction,

This basic sol-gel reaction starts when the metal alkoxide $\mathrm{Si}\left(\mathrm{OCH}_{2} \mathrm{CH}_{3}\right)_{4}$ (tetraethoxysilane, or TEOS) is mixed with $\mathrm{H}_{2} \mathrm{O}$ and a hydrolysis reaction occurs. TEOS is used because it, along with other metal alkoxides, reacts readily with water. Ethanol is used as a solvent because water and alkoxides are immiscible. Providing there is sufficient water and a catalyst present, the hydrolysis reaction will be completed when all of the $\left(\mathrm{OCH}_{2} \mathrm{CH}_{3}\right)$ groups are replaced by $(\mathrm{OH})$ :

$$
\mathrm{Si}\left(\mathrm{OCH}_{2} \mathrm{CH}_{3}\right)_{4}+4 \mathrm{H}_{2} \mathrm{O} \rightarrow \mathrm{i}(\mathrm{OH})_{4}+4 \mathrm{CH}_{3} \mathrm{CH}_{2} \mathrm{OH} \text {, }
$$

producing a sol consisting of $\mathrm{Si}(\mathrm{OH})_{4}$ (silicic acid) and $\mathrm{CH}_{3} \mathrm{CH}_{2} \mathrm{OH}$ (ethanol).

Next, two partially hydrolyzed molecules can link together in a condensation reaction that liberates water:

$$
2 \mathrm{Si}(\mathrm{OH})_{4} \rightarrow(\mathrm{OH}) 3 \mathrm{Si}-\mathrm{O}-\mathrm{Si}(\mathrm{OH})_{3}+\mathrm{H}_{2} \mathrm{O} .
$$

As the reaction proceeds, the number of $\mathrm{Si}-\mathrm{O}-\mathrm{Si}$ bonds increases in a process called polymerization, which produces a macroscopic gel. When producing thin films this gel is formed by rapid evaporation of the solvent.

Diaphragm-ferrule bonding: When sol-gel is liquid, it expands, flows and it is very hard to control. There are two possible ways to apply sol-gel between the diaphragm and the ferrule. One is spin coating the sol-gel onto the diaphragm. The other way is apply sol-gel onto the ferrule end and keep the diaphragm clean. We finally chose the second method because a spin coated sol-gel layer will also cover the etched pit. Although the sol-gel layer can be as thin as several microns and cannot fill up the etched pit, the layer is not uniform and affects the light reflection. The entire diaphragm/ferrule bonding process is shown in Figure 3.46. 


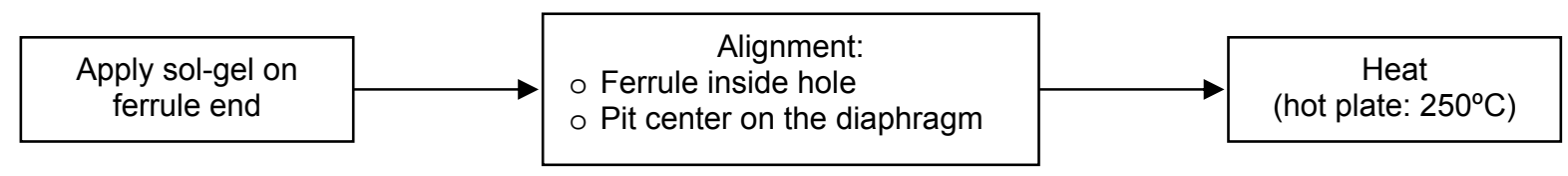

Figure 3.46. Sol-gel bonding process

As we can see from Figure 3.47, the bonding area is the area with sol-gel. The sol-gel area can even be a little bit larger than the etched pit as long as the inside hole of the ferrule is not blocked. A three dimensional micropositioner with a monitoring system is used to do the alignment. Figure 3.48 shows a picture of the bonding under the microscope.

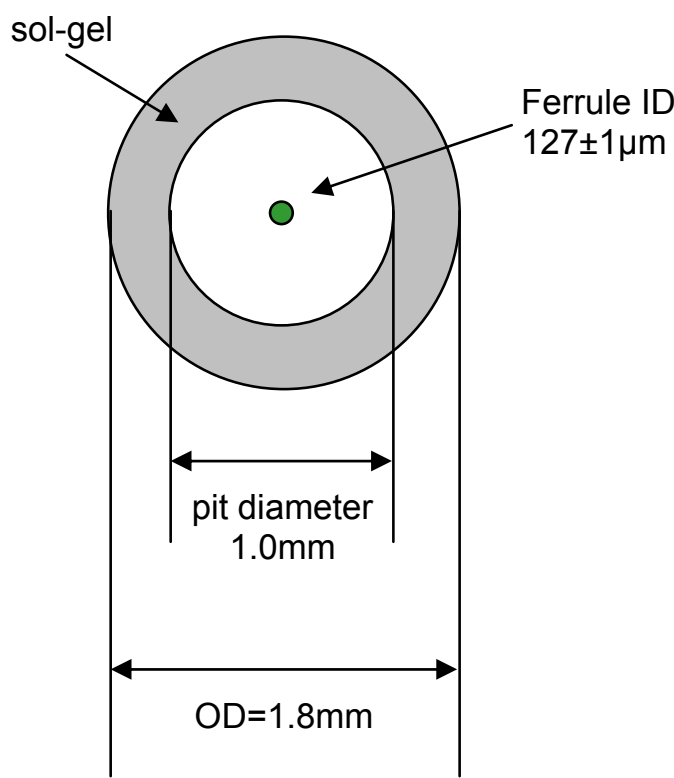

Figure 3.47. Application of sol-gel to the ferrule.

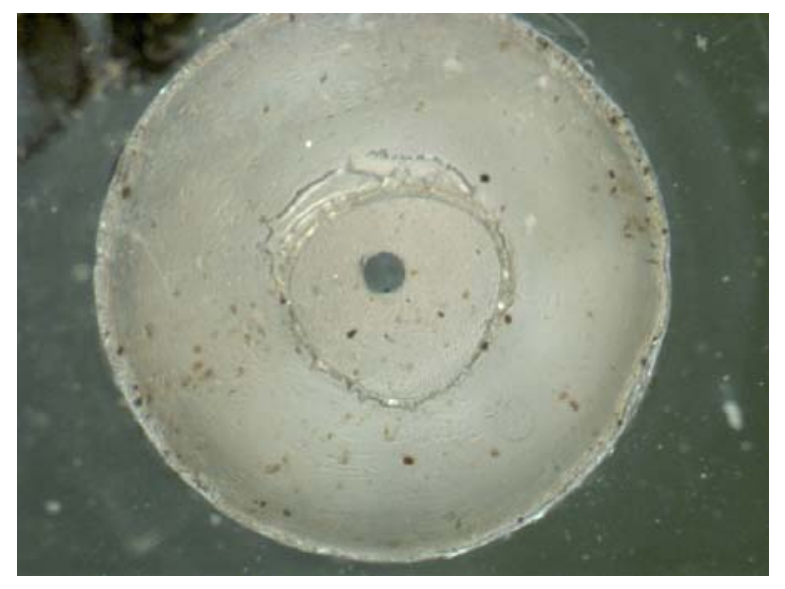

Figure 3.48. Sol-gel bonded diaphragm and ferrule.

Ferrule-fiber bonding: The final step of the sensor fabrication is to insert the fiber into the ferrule, adjust the air-gap, and bond them together. In this step, sol-gel is dip-coated around the surface of the fiber, and heated by using $\mathrm{CO}_{2}$ laser at very low power for several seconds. Any heating methods, (heat gun, hot plate, furnace or laser) can work, but after the air-gap adjustment, the position of both the fiber and the ferrule should not be moved on the positioning system. So the $\mathrm{CO}_{2}$ laser mounted on the positioning system is the best choice. 


\subsubsection{Sensor testing}

The etched diaphragm sensors were fabricated using the white light interferometry system, but tested using SCIIB system. Figure 3.49 shows the interferometric signal, air-gap and visibility obtained for a representative single mode fiber sensor. The visibility is very high, above $90 \%$.

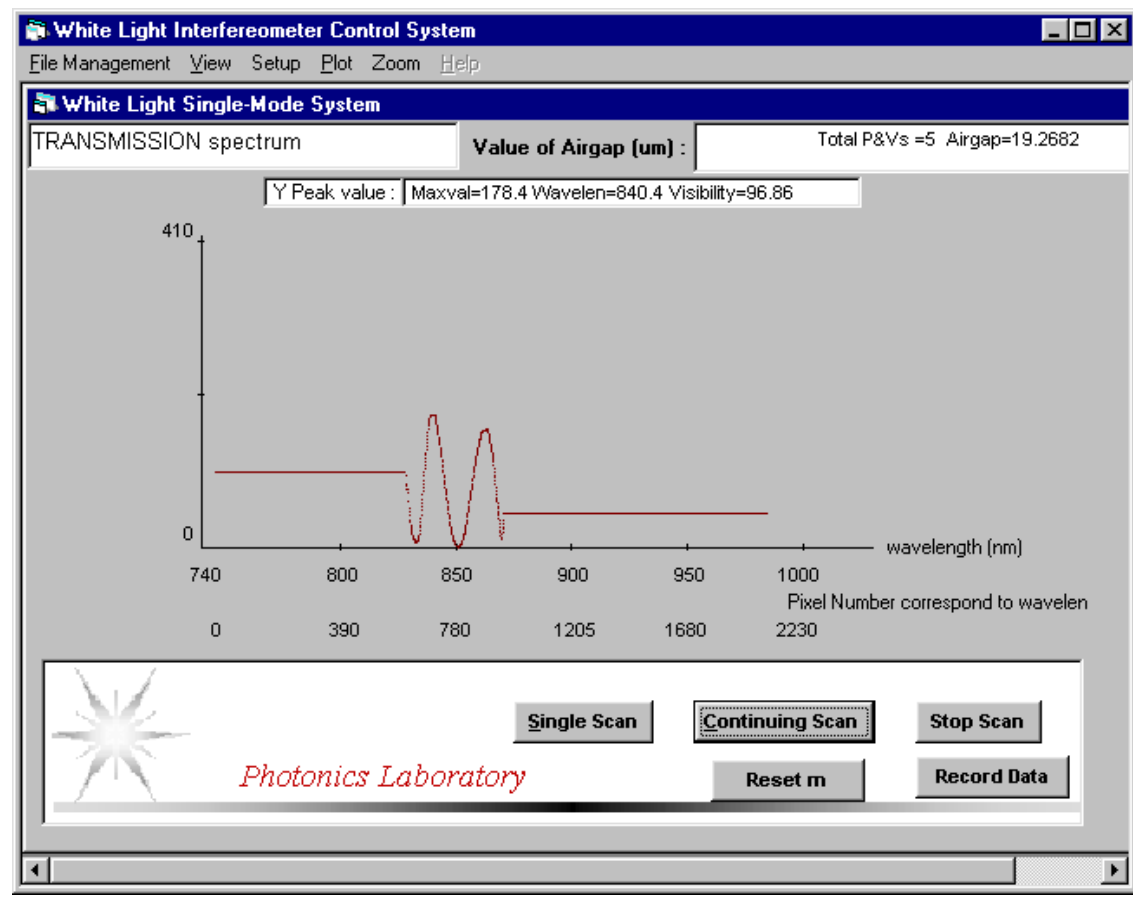

Figure 3.49. Interference fringes of a representative diaphragm-based pressure sensor.

Sensor performance was evaluated using a PSI (Pressure System, Inc) pressure controller and the single mode SCIIB system. The full-scale range of the PSI system is $200 \mathrm{psi}$. The parameters of the sensor tested are: as follows.

- Diaphragm thickness $=125 \mu \mathrm{m}$; Pit etch depth $=25 \mathrm{~mm}$, so the sensing thickness is $100 \mu \mathrm{m}$;

- $\quad$ Pit diameter $=1 \mathrm{~mm}$

The pressure test results between 13 psi to 200 psi are shown in Figure 3.50. The SCIIB system output was almost the same for increasing and decreasing pressure. The output of the SCIIB system is voltage; air gap changes cannot be read directly from the system without some data processing. Figure 3.51 shows the nonlinear fit to the test result, which can be used to obtain information on the air-gap change after we have obtained one cycle of the curve. For this sensor, 200 psi pressure results in an air gap change of $476.9 \mathrm{~nm}$.

Figure 3.52 presents a comparison of the results from the PSI system and the SCIIB system. The peak-peak value of ripples on the top of the PSI system output waveform was less than 1 psi. 


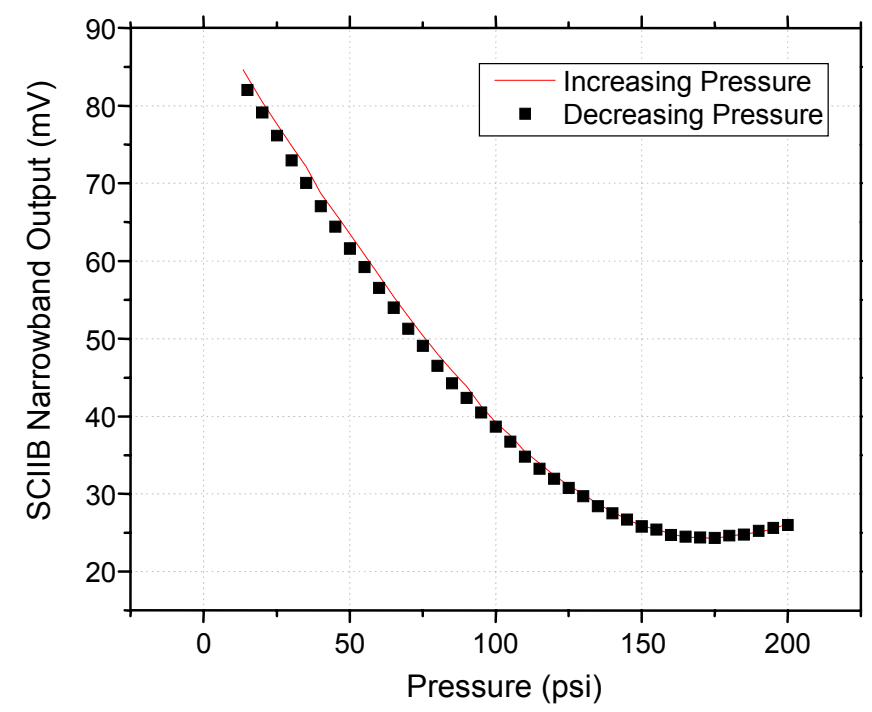

Figure 3.50. SCIIB pressure sensor test results for 13-200 psi and 200-13 psi.

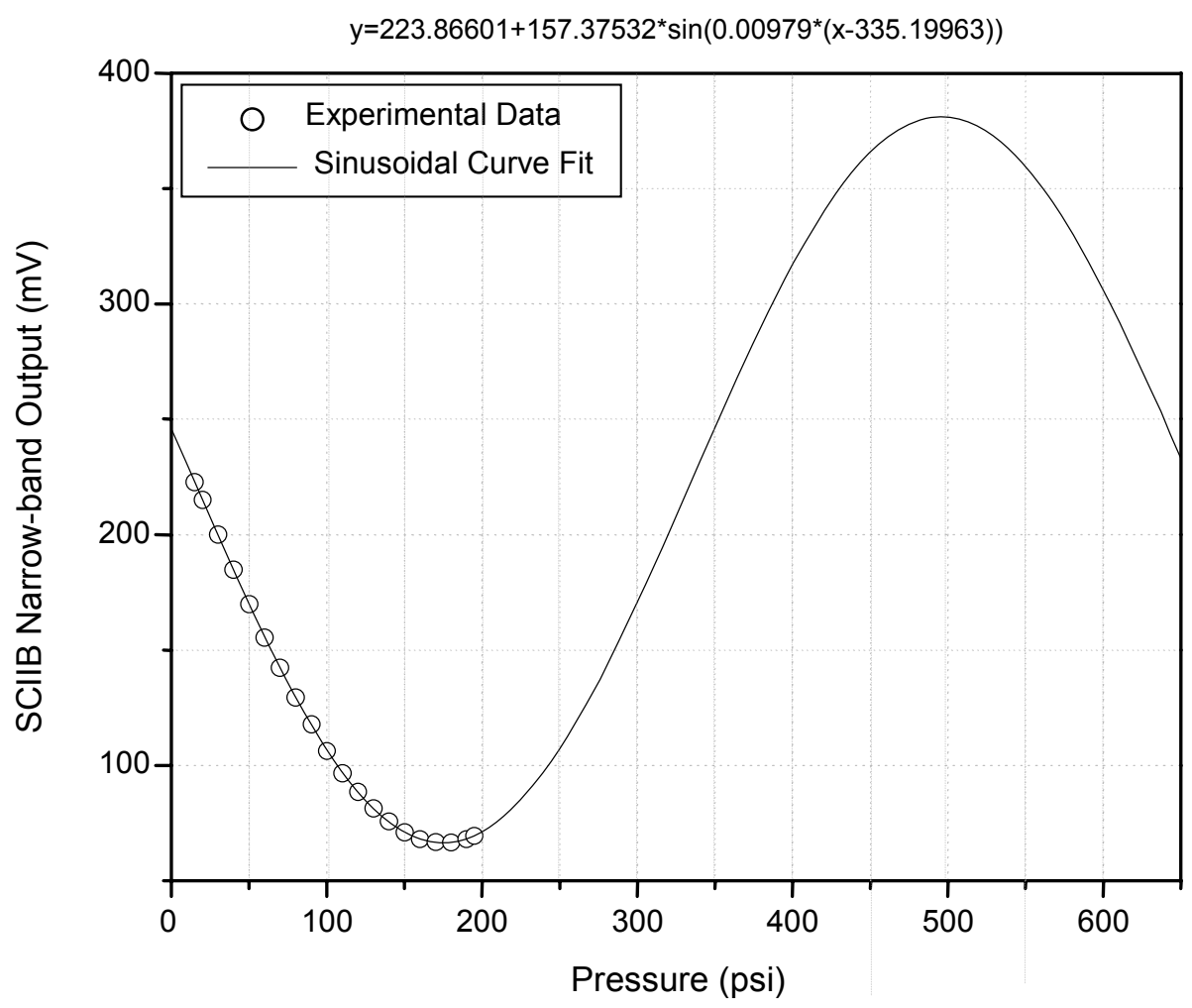

Figure 3.51. Nonlinear curve fit of test results. 

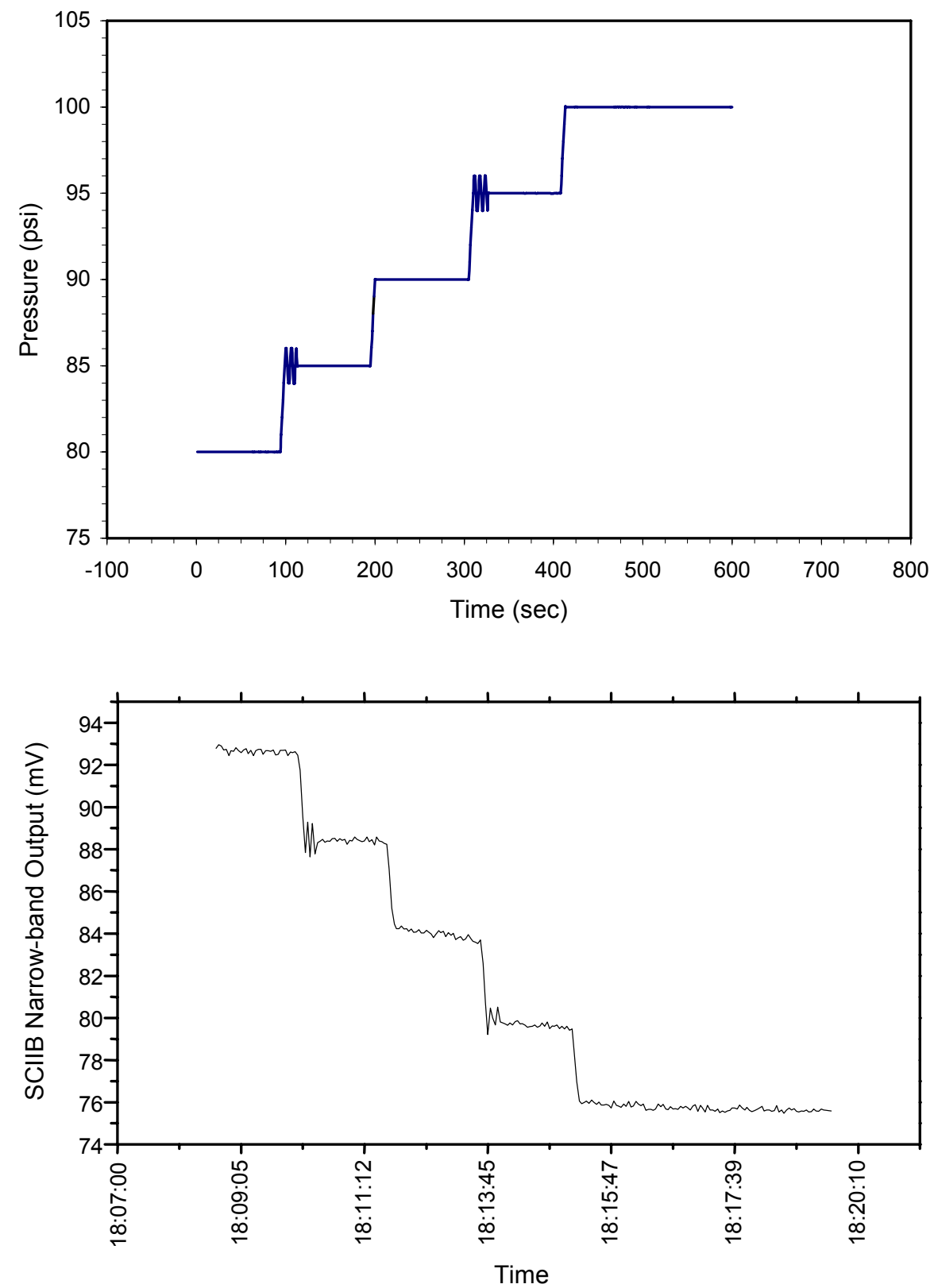

Figure 3.52. Comparison of PSI output (top) to SCIIB test results (bottom). 


\subsection{Laser Bonding-Based Pressure Sensors}

The sensor structure resulting from laser bonding of a fiber, ferrule, and diaphragm is shown in Figure 3.53. Rather than using a pit etched in the diaphragm, a pit is drilled in the ferrule to allow the diaphragm to deflect in response to pressure variations.

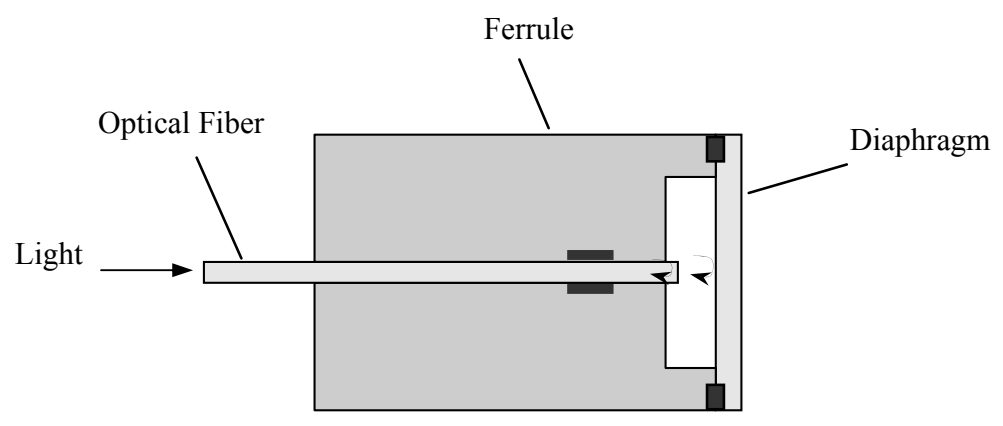

Figure 3.53. Diaphragm based fiber optic pressure sensor.

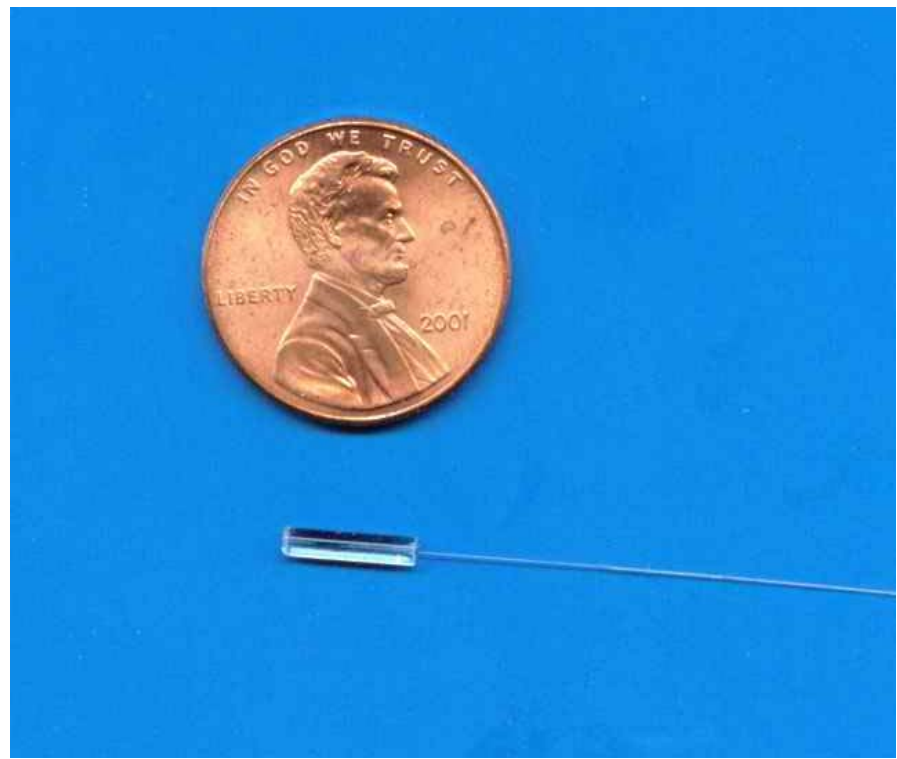

Figure 3.54. CPT's engine sensor; the sensor head is $8 \mathrm{~mm}$ long and $1.8 \mathrm{~mm}$ in diameter.

\subsection{1 $\mathrm{CO}_{2}$ Laser Sensor Fabrication System}

As shown in Figure 3.55 and Figure 3.56, the sensor fabrication system includes three subsystems, the carbon dioxide $\left(\mathrm{CO}_{2}\right)$ laser heating subsystem, the white light fiber optic interferometric airgap monitoring subsystem, and the step motor controlled rotary holder subsystem. 
A high-energy $\mathrm{CO}_{2}$ laser controlled by a computer is used as the heating source. It generates high-energy optical pulses at the wavelength of $10.6 \mu \mathrm{m}$. When silica glass is exposed to the $\mathrm{CO}_{2}$ laser output, it absorbs the optical energy and converts it to thermal energy, which allows silica glass materials to be locally heated to very high temperatures. The white light fiber optic interferometric system is used in the system to allow accurate monitoring of the sensor airgap. A holder for the ferrule is mounted on the step motor that is also controlled by the computer. This rotary holder allows the ferrule to be turned at the required speed corresponding to a specific laser bonding time. The computer controls the $\mathrm{CO}_{2}$ laser and the step motor simultaneously so the laser bonding process can be realized automatically.

\subsubsection{Diaphragm / Ferrule Bonding}

Bonding of the diaphragm and ferrule is shown in Figure 3.57 and Figure 3.58. The fused quartz diaphragm is bonded on the top of the ferrule, which is also made of fused quartz, by the $\mathrm{CO}_{2}$ laser. The pit in the ferrule, which can be mechanically machined or chemically etched, will allow the diaphragm to deflect in response to pressure variations outside.

A micrograph of a laser bonded diaphragm and ferrule is shown in Figure 3.59. The diameter of the bonding circle can be set as $0.5 \mathrm{~mm}$ or larger. The bonding edge is not round because the diaphragm was not perfectly diced before laser bonding and some eccentricity exists. However, this does not affect the sensor's performance.

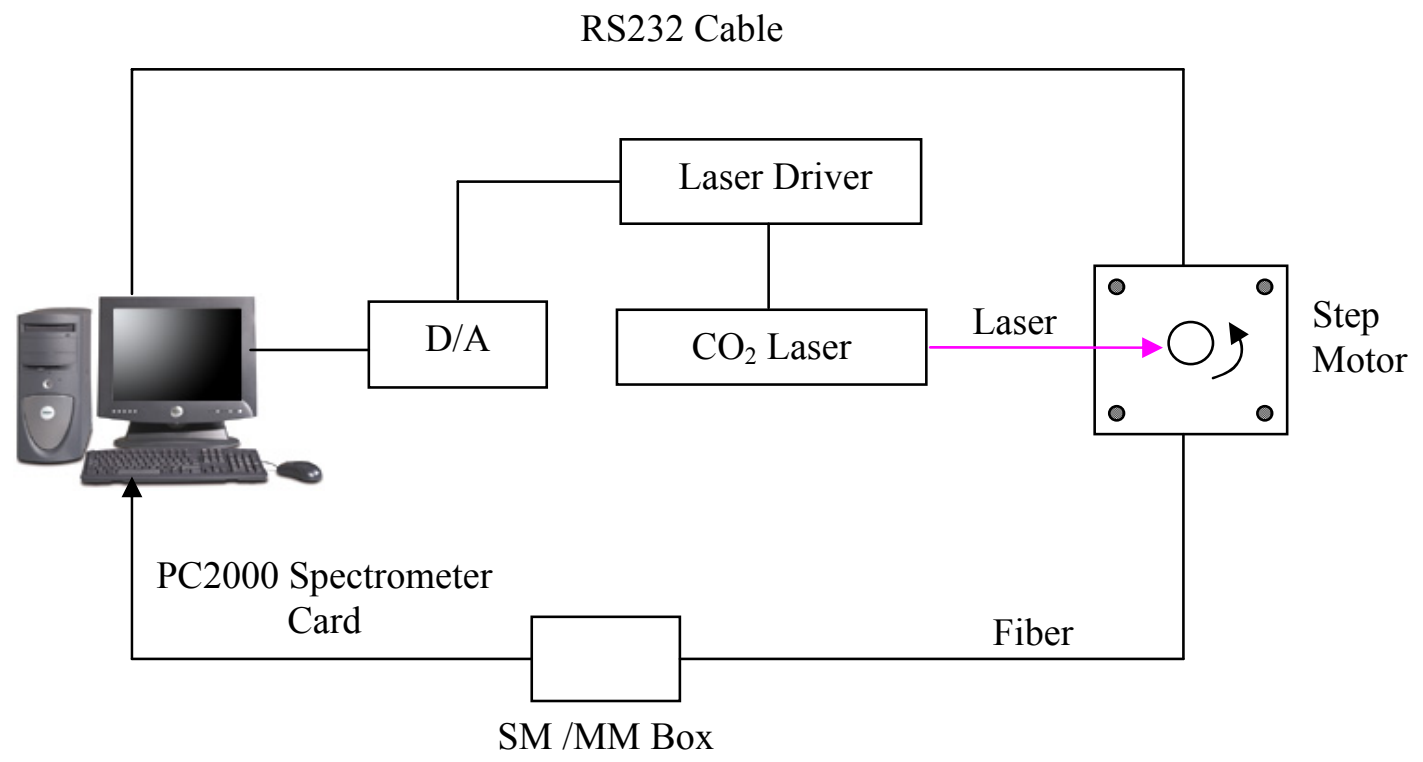

Figure 3.55. $\mathrm{CO}_{2}$ laser automatic sensor fabrication system. 


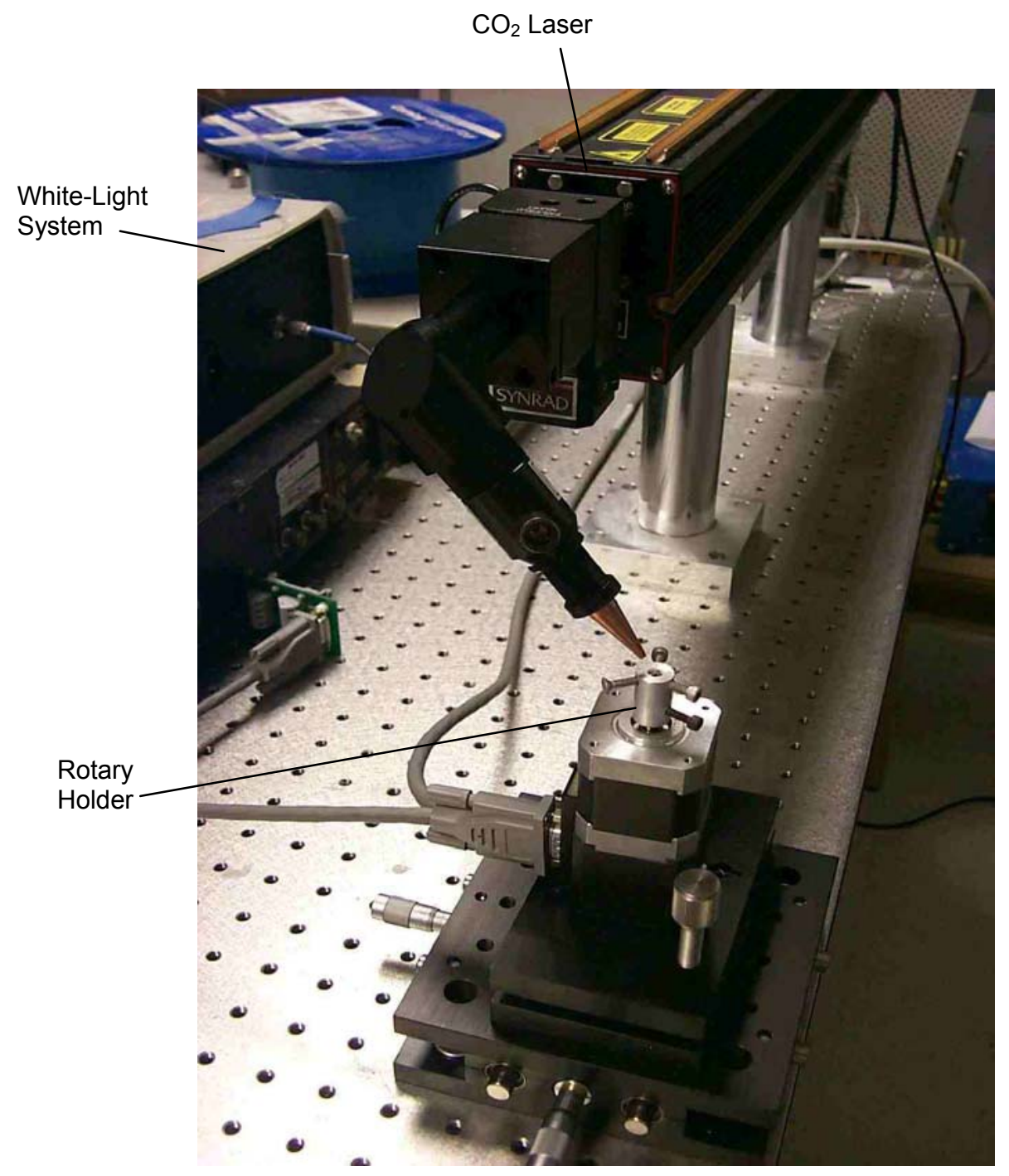

Figure 3.56. $\mathrm{CO}_{2}$ laser sensor fabrication system 


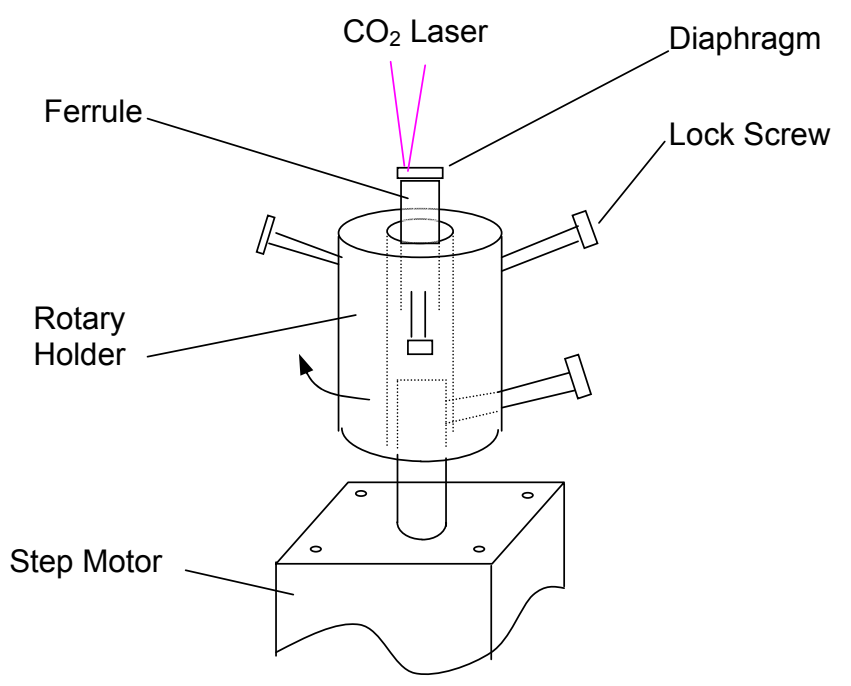

Figure 3.57. Rotary device and laser bonding process.
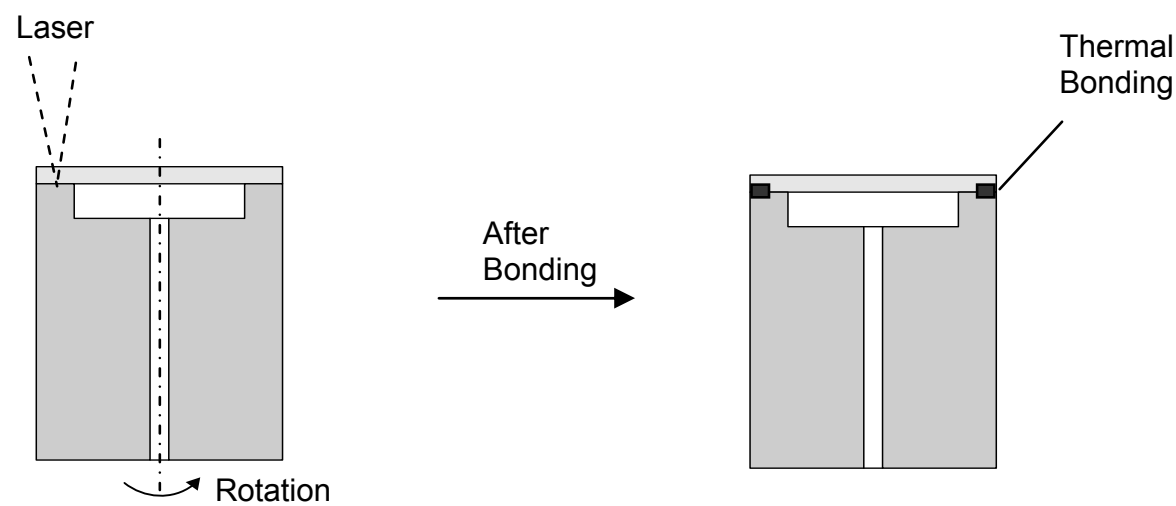

Figure 3.58. Diaphragm and ferrule thermal bonding.

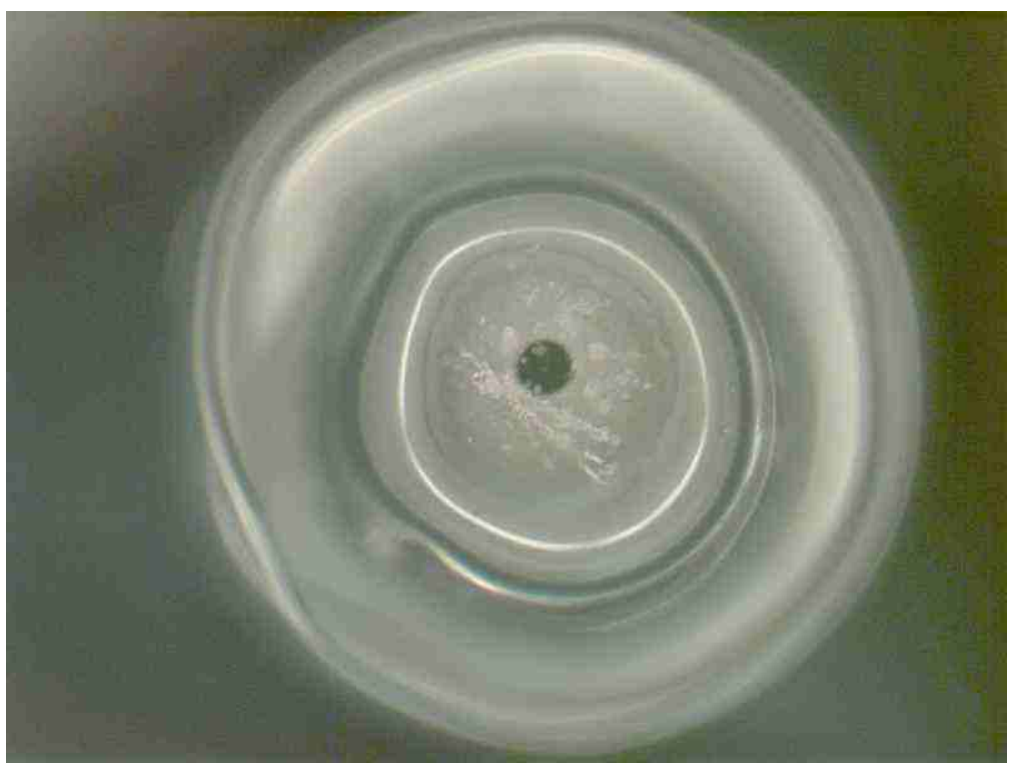

Figure 3.59. Micrograph of diaphragm and ferrule laser bonding (top view). 


\subsubsection{Ferrule / Fiber Bonding}

After the diaphragm and ferrule are bonded, a well cleaved fiber with its buffer removed is inserted in the ferrule. The airgap between the fiber endface and the inside surface of the diaphragm is adjusted by using the white-light interferometry measurement system. The same rotary device is then used to realize ferrule and fiber thermal bonding. The process is shown in Figure 3.60 and a micrograph of a laser-bonded ferrule and fiber is shown in Figure 3.61. The size of the sensor head is $5 \mathrm{~mm}$ long and $1.8 \mathrm{~mm}$ in diameter.

An alternative way to bond the fiber inside the ferrule is to use a high temperature flame to heat the ferrule and fiber to realize a hermetic seal, as shown in Figure 3.62 and Figure 3.63.
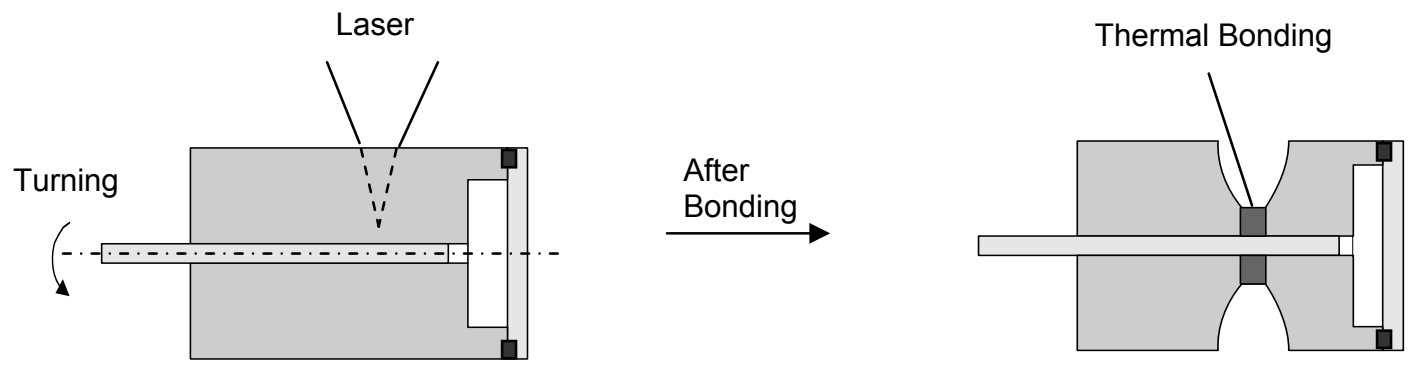

Figure 3.60. Fiber and ferrule thermal bonding.

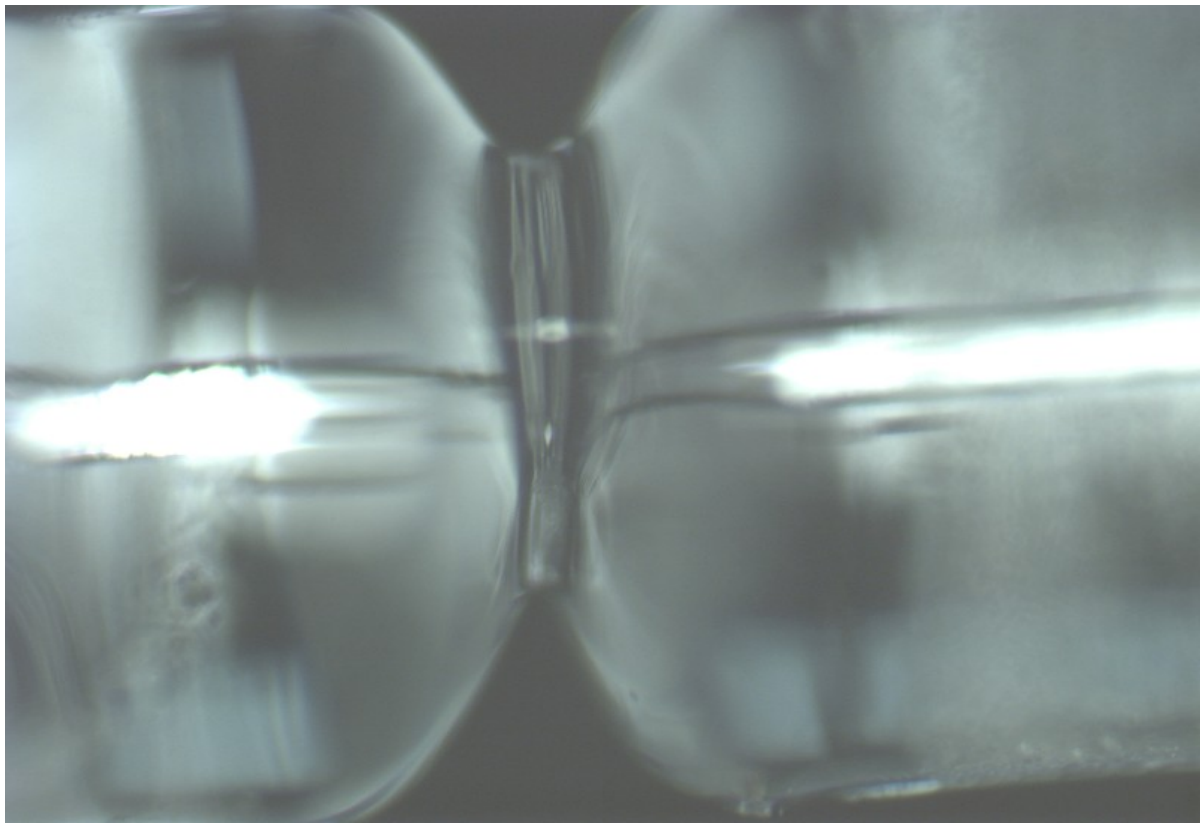

Figure 3.61. Micrograph of the ferrule and fiber laser bonding (side view). 


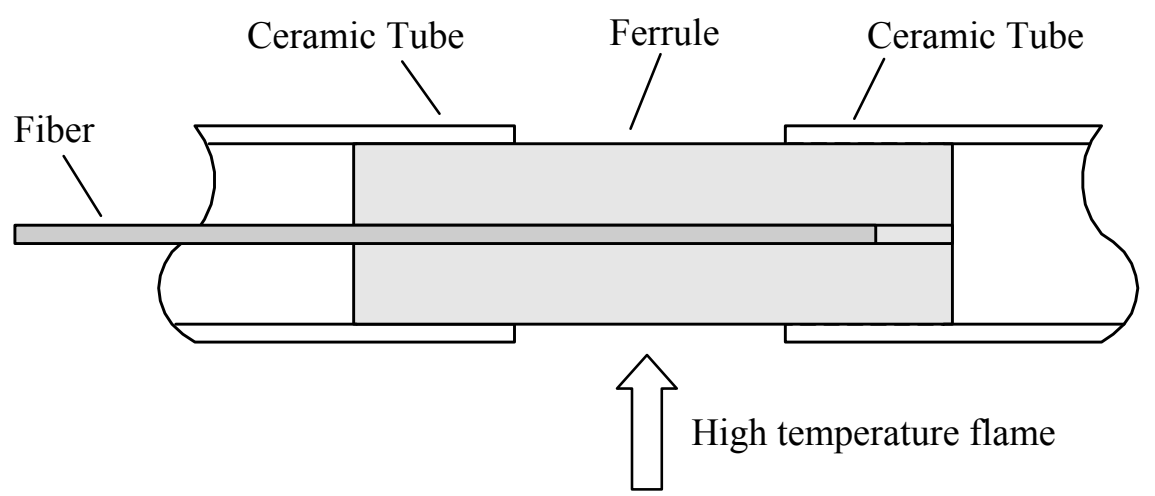

Figure 3.62. Thermal bonding between an optical fiber and a ferrule.

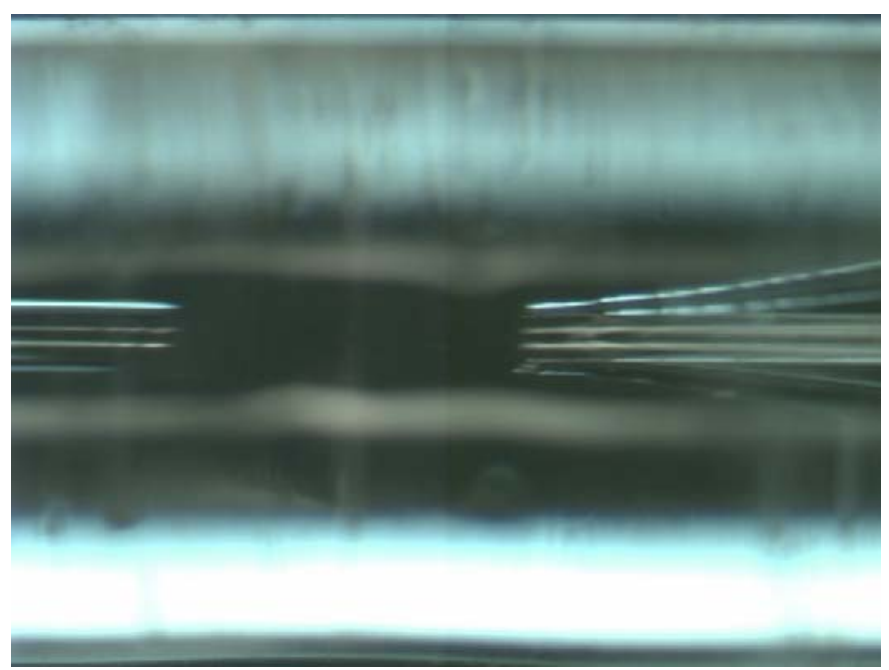

Figure 3.63. Photo of the fused bonding between an optical fiber and a ferrule.

\subsubsection{Sensor Testing}

The basic structure of the spectrometer-based white light system used to monitor the air gap during testing is shown in Figure 3.64. The data processing required to calculate the airgap from the fringes was realized in Visual Basic. The measurement window is shown in Figure 3.65 .

A multimode pressure sensor was placed in a furnace at atmospheric pressure. The temperature was increased from room temperature to $600^{\circ} \mathrm{C}$. The resulting sensor output is shown in Figure 3.66.

Two multimode pressure sensors were tested at room temperature, the results are shown in Figure 3.67 and Figure 3.68. The nonlinearity is caused by gauge measurement errors in the pressure system when the pressure is relative low (0-500psi). 


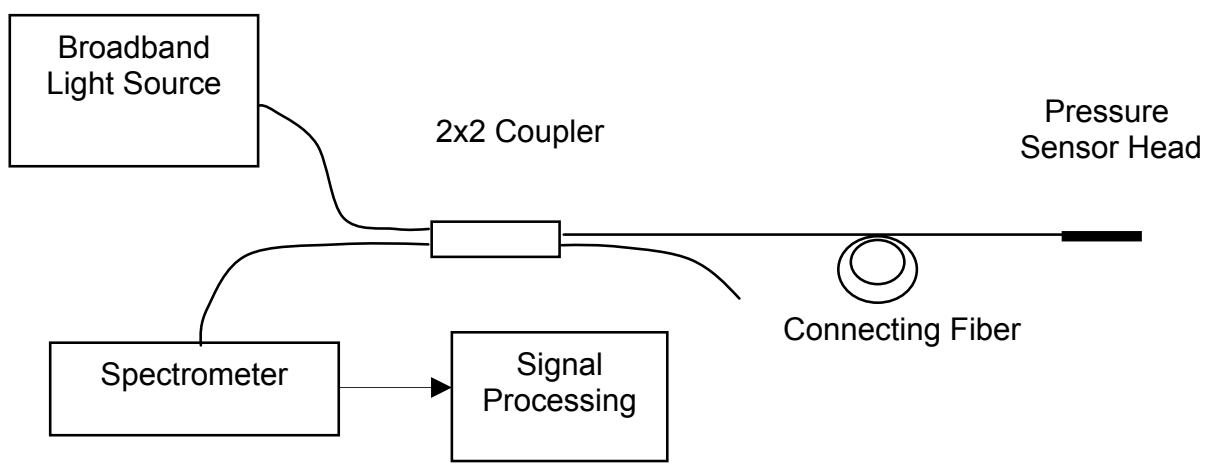

Figure 3.64. The basic structure of interferometer-based white light system.

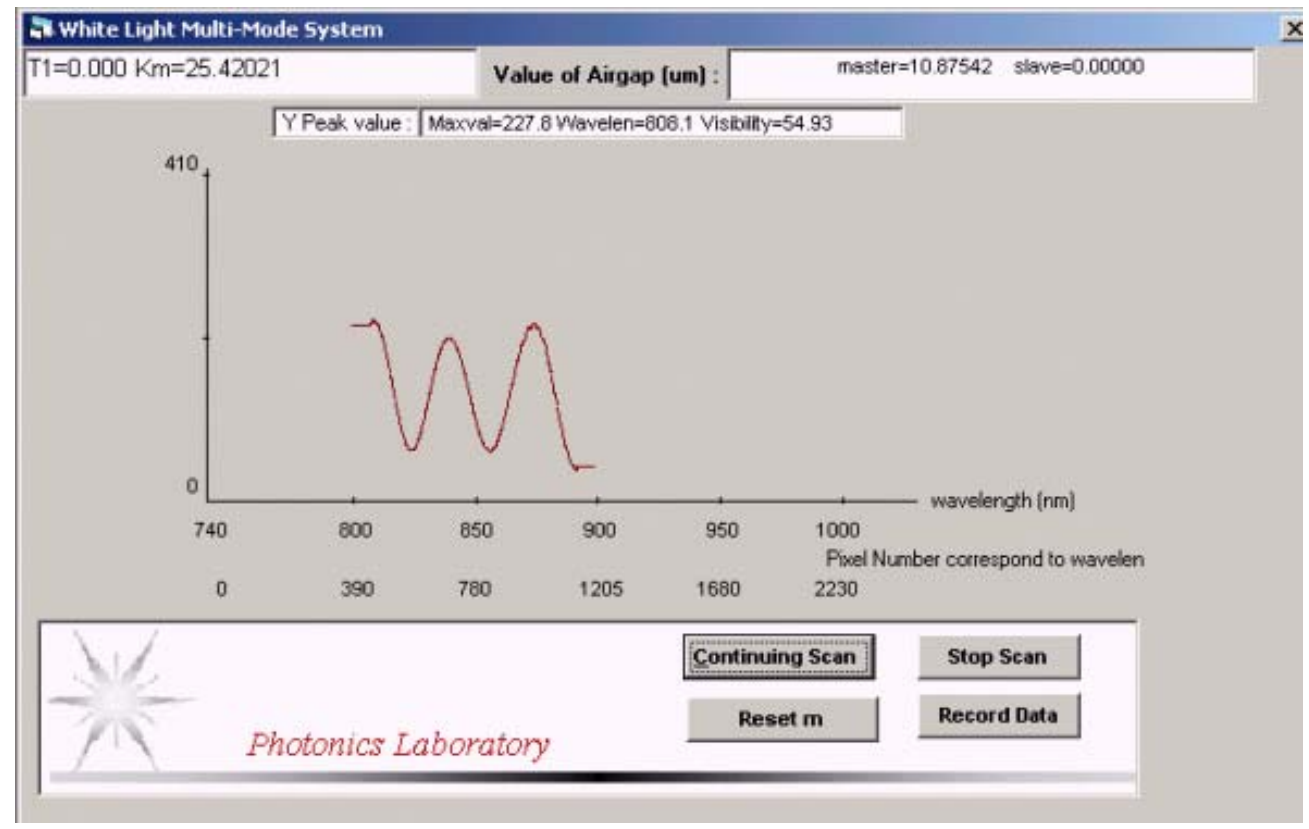

Figure 3.65. White-light airgap measurement window. 


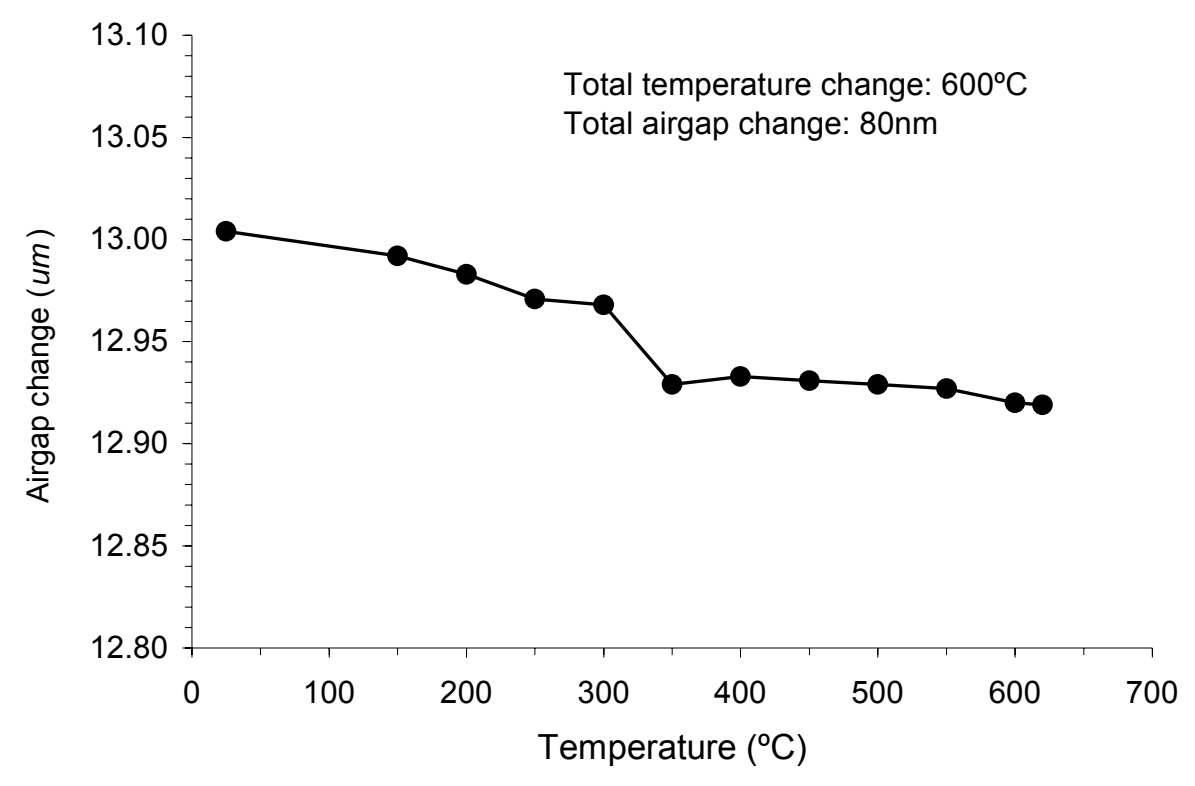

Figure 3.66. MMF engine pressure sensor temperature dependence test.

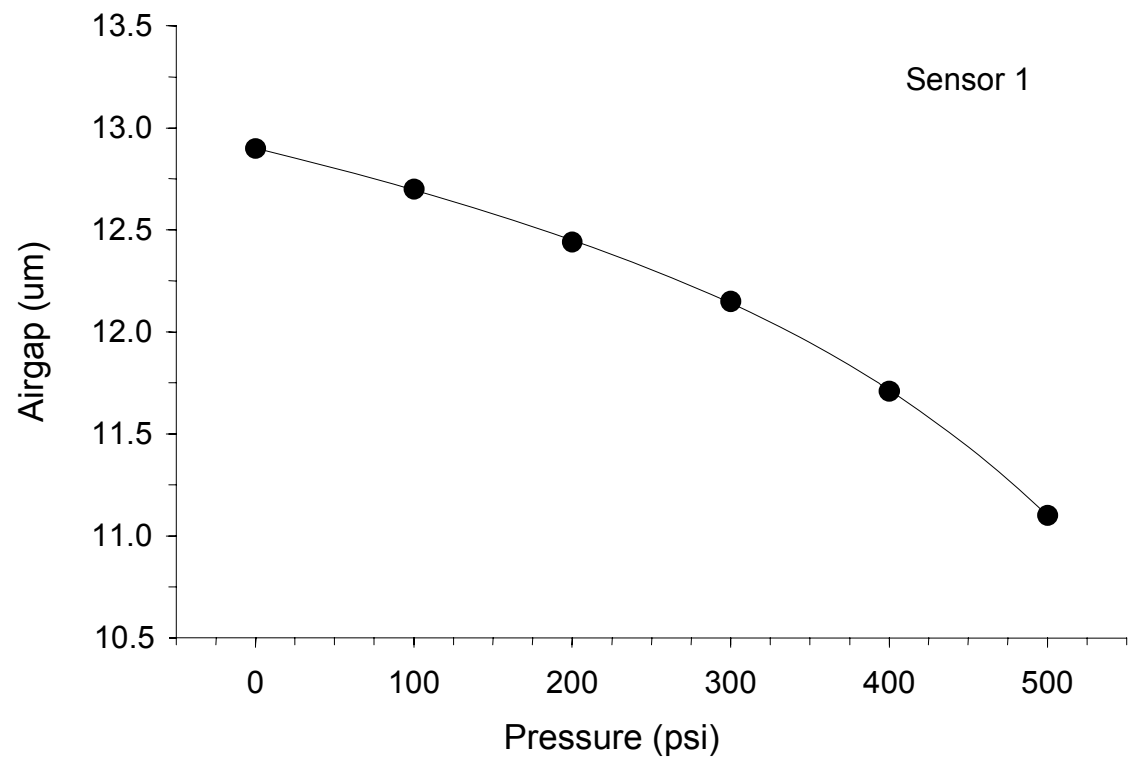

Figure 3.67. MMF engine pressure sensor pressure sensitivity test. 


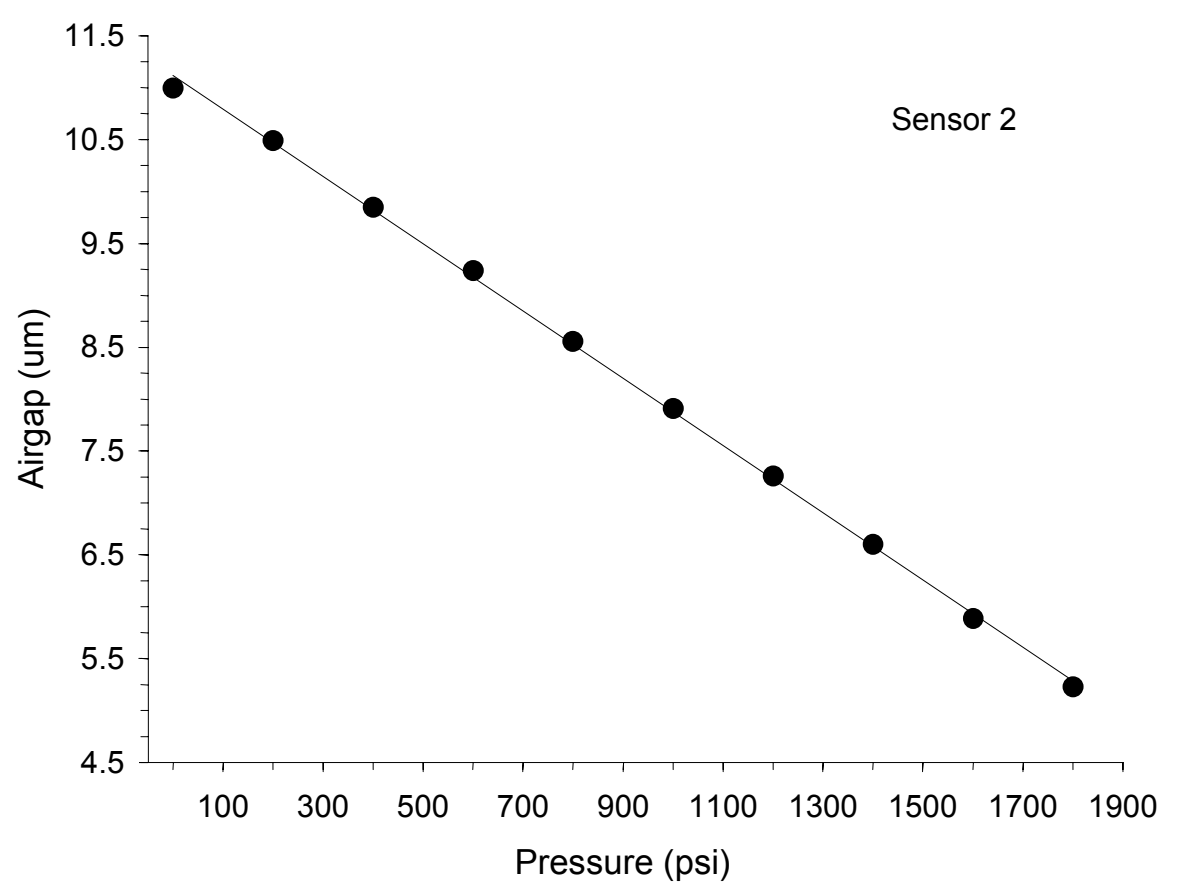

Figure 3.68. MMF engine pressure sensor pressure sensitivity test. 


\subsection{Pressure Sensor Testing}

\subsection{Sensor Testing System}

Figure 4.1 shows the schematic of the dynamic pressure test system. The dynamic pressure (ultrasonic wave) is generated by an ultrasonic air transducer purchased from APC Products, Inc. The transducer is sealed in an iron housing and faces the open end of a mullite tube connected to the house. The other end of the mullite tube connects to the pressure control system and the fiber optic sensor system through a T-fitting. All the connections to the housing and the ceramic tube are hermetically sealed by epoxy. The sensor is put into the central part of tube which is heated by a furnace.

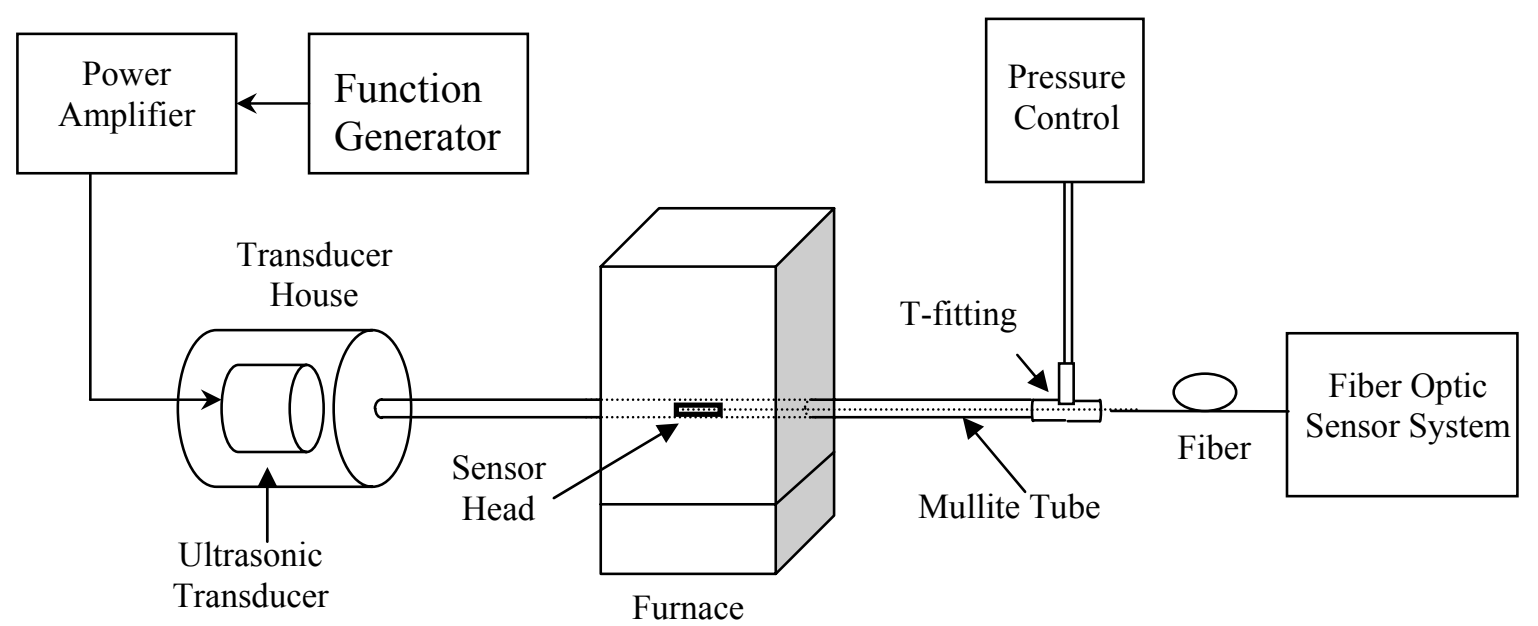

Figure 4.1. Schematic of the high temperature dynamic pressure test system.

The signal from a function generator is amplified by a high voltage power amplifier (Model 7602, Krohn-Hite Corporation) before inputting to the air transducer. From the manufacturer's test report, at ambient pressure, the transducer can generate a sound pressure level of $140 \mathrm{~dB}$ (corresponding to a peak-to-peak pressure change $0.058 \mathrm{psi}$ ) at a one meter distance when a $47 \mathrm{KHz}$ sinusoidal wave with 200 volts peak-to-peak is applied. The sound pressure increases as the background pressure is increased due to higher ultrasonic wave to air coupling efficiency at higher background pressure. The real sound pressure levels at the location of sensor and at different background pressures need to be calibrated.

The static background pressure inside the ceramic tube is controlled by the pressure control system (Model 9035, Pressure Systems) which is connected to a compressed nitrogen gas tank. The background pressure can be adjusted accurately from ambient pressure to 200 psi.

The mullite tube is approximately 1.5 meter long and only the central part where the fiber optic sensor is placed is heated. The temperature in the heated area can be adjusted from 
room temperature to $1200^{\circ} \mathrm{C}$ by the furnace. The heated length is about $40 \mathrm{~cm}$. All other units of the test system and the seals are contained in a safe low-temperature environment.

The specifications of our test system are listed here:

- Test temperature: up to $1200^{\circ} \mathrm{C}$;

- Test static background pressure: up to $200 \mathrm{psi}$;

- Test dynamic pressure: sound pressure more than $140 \mathrm{~dB}$ with a frequency of $47 \mathrm{kHz}$.

\subsection{Pressure Sensitivity Test}

A structure model for a diaphragm is shown in Figure 4.2.

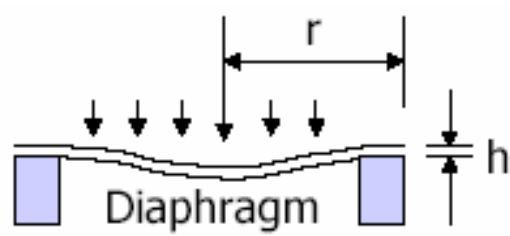

Figure 4.2. Diaphragm deflection model.

The equation for diaphragm deflection:

$$
y=\frac{3\left(1-\mu^{2}\right) P}{16 E h^{3}}\left(a^{2}-r^{2}\right)^{2}
$$

where $\mathrm{y}=$ deflection

$\mathrm{P}=$ pressure

$\mathrm{h}=$ thickness

$\mathrm{a}=$ radius of diaphragm

$\mathrm{E}=$ modulus of elasticity

$\mathrm{r}=$ radial distance

$\mu=$ Poisson's ratio

The sensor diaphragm thickness is $125 \mu \mathrm{m}$ and the diaphragm diameter is about $1.4 \mathrm{~mm}$. The calculated sensitivity of the sensor is about $2.1 \mathrm{~nm} / \mathrm{psi}$. The single mode fiber optic engine pressure sensor was tested at elevated temperatures and the results are shown in Figure 4.3, Figure 4.4 and Figure 4.5. The sensitivity of the sensor is about $2 \mathrm{~nm} / \mathrm{psi}$, which is close to the calculated value. To increase the sensitivity of the sensor, a thinner diaphragm or larger pit can be chosen.At temperatures above $600^{\circ} \mathrm{C}$, glass creep was observed which results in shifts of the curves in Figure 4.5. 


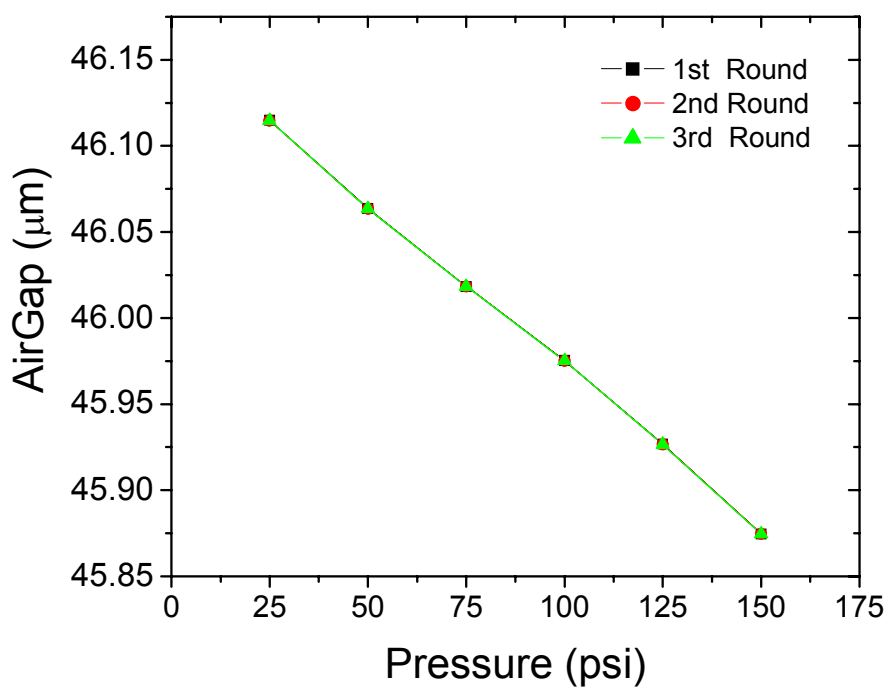

Figure 4.3. Pressure Response at $400^{\circ} \mathrm{C}$.

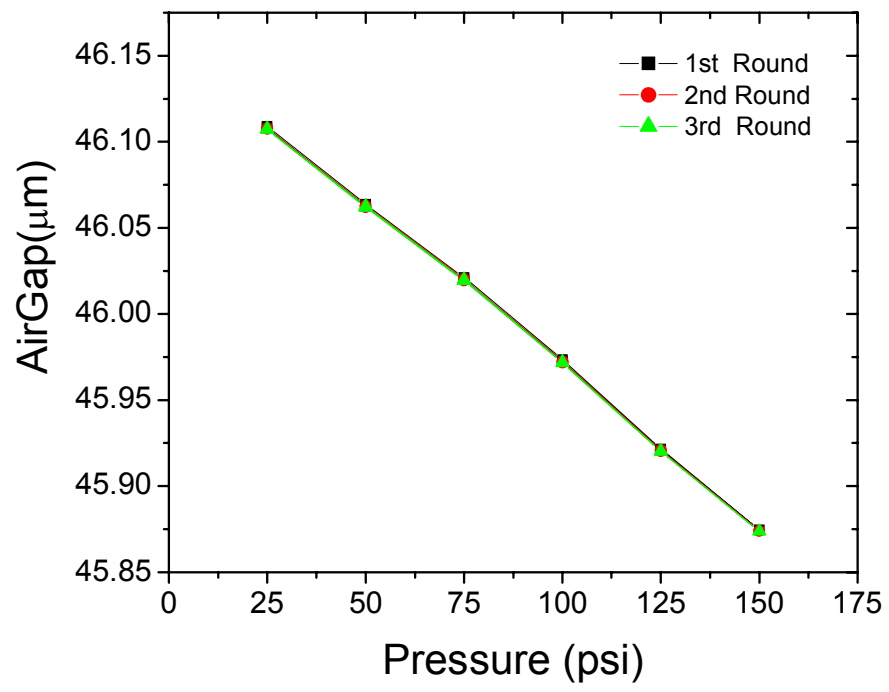

Figure 4.4. Pressure Response at $500^{\circ} \mathrm{C}$. 


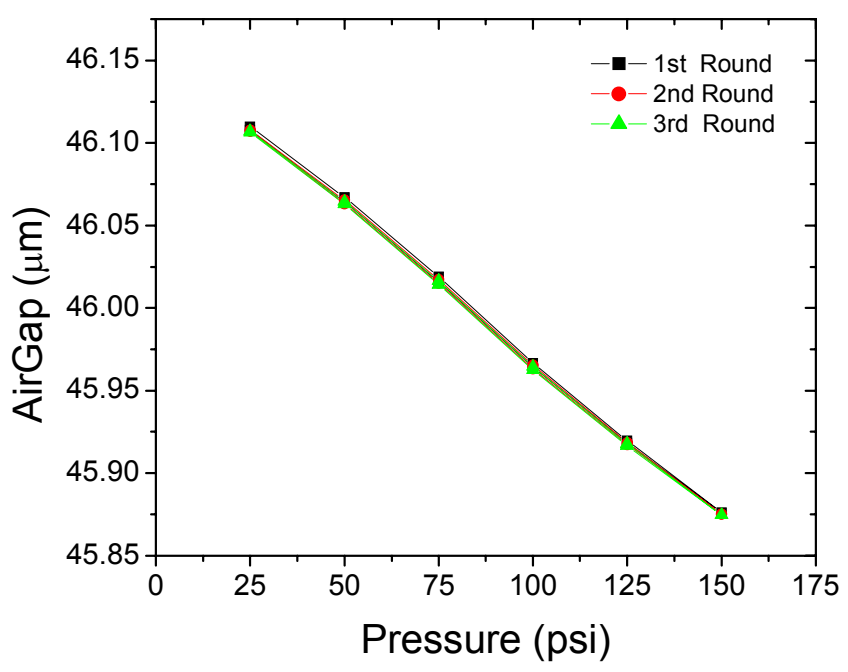

Figure 4.5. Pressure response at $600^{\circ} \mathrm{C}$.

\subsection{Dynamic Pressure Tests}

The test results at room temperature and atmospheric pressure are shown in Figure 4.6 and Figure 4.7. As shown in Figure 4.6, for the time domain, the sensor response is about 5mv peak to peak with its FFT having a $17 \mathrm{~dB}$ signal noise ratio. For Q-point tuning response in Figure 4.7, the maximum and minimum outputs are $3.4 \mathrm{v}$ and $1 \mathrm{v}$ respectively, so the sensor's visibility is $54.5 \%$ by the following equation:

$$
\text { Visibility }=\frac{\max -\min }{\max +\min } \times 100 \%
$$

\subsection{Temperature Dependence Test}

A multimode pressure sensor was placed in a furnace at atmospheric pressure. The temperature was increased from room temperature to $600^{\circ} \mathrm{C}$. The result is shown in Figure 4.8. Since the temperature induced pressure error is only $0.0169 \mathrm{psi} /{ }^{\circ} \mathrm{C}$ and the temperature will not change at high frequency, this temperature dependence will not affect the dynamic pressure measurement. 
3日-Apr-B4

MEASURE

$16: 42: 54$

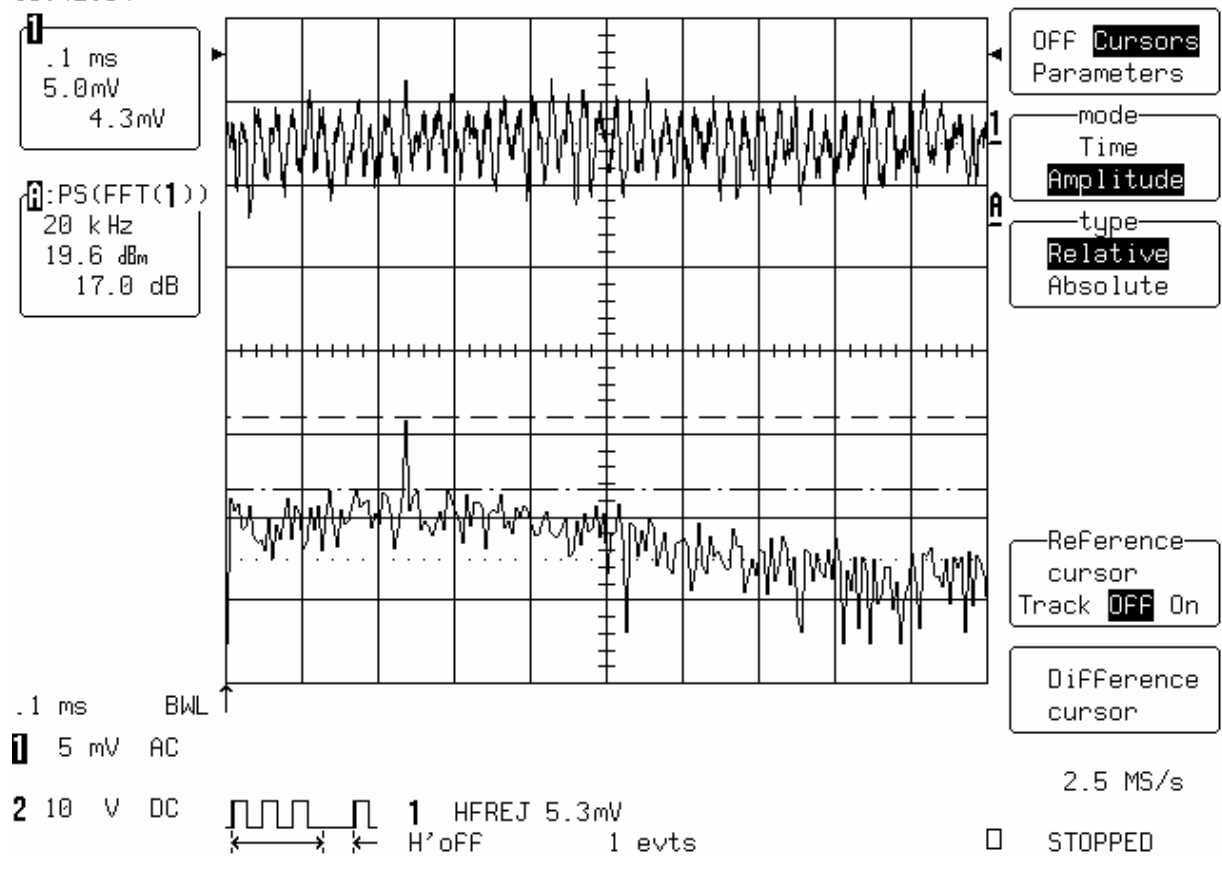

Figure 4.6. Engine sensor dynamic pressure response.

30-Apr-94

$16: 52: 00$

1 5 s

$1.09 \mathrm{~V}$

$0.87 \mathrm{~V}$

Ai: PS (FFT(1)

$.5 \mathrm{~Hz}$

$19.6 \mathrm{dBm}$

$17.0 \mathrm{~dB}$
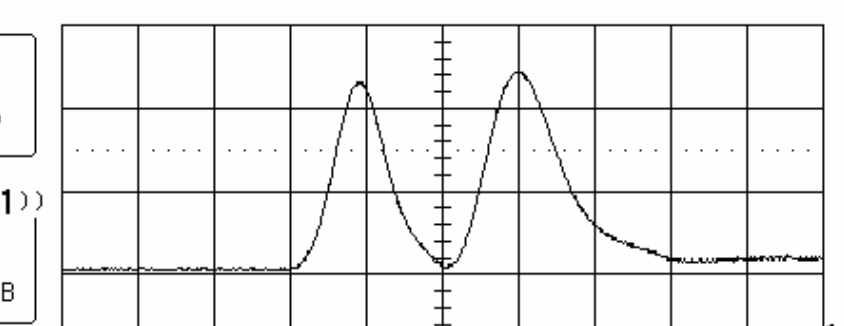

CHANNEL 1

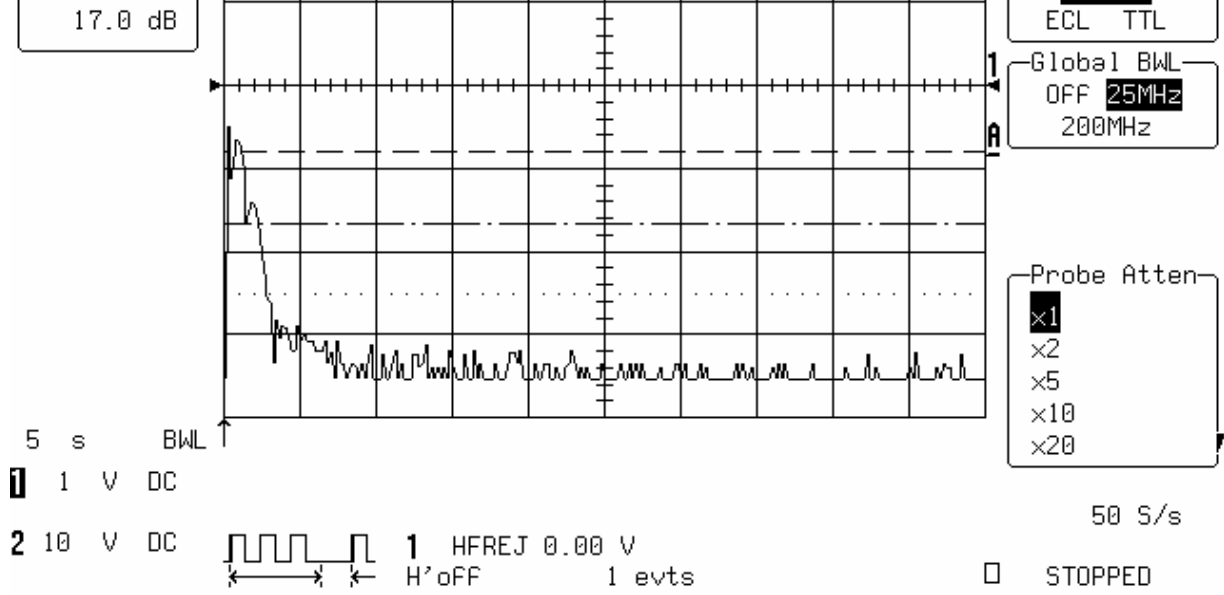

Figure 4.7. Engine sensor response using Q-point tuning. 


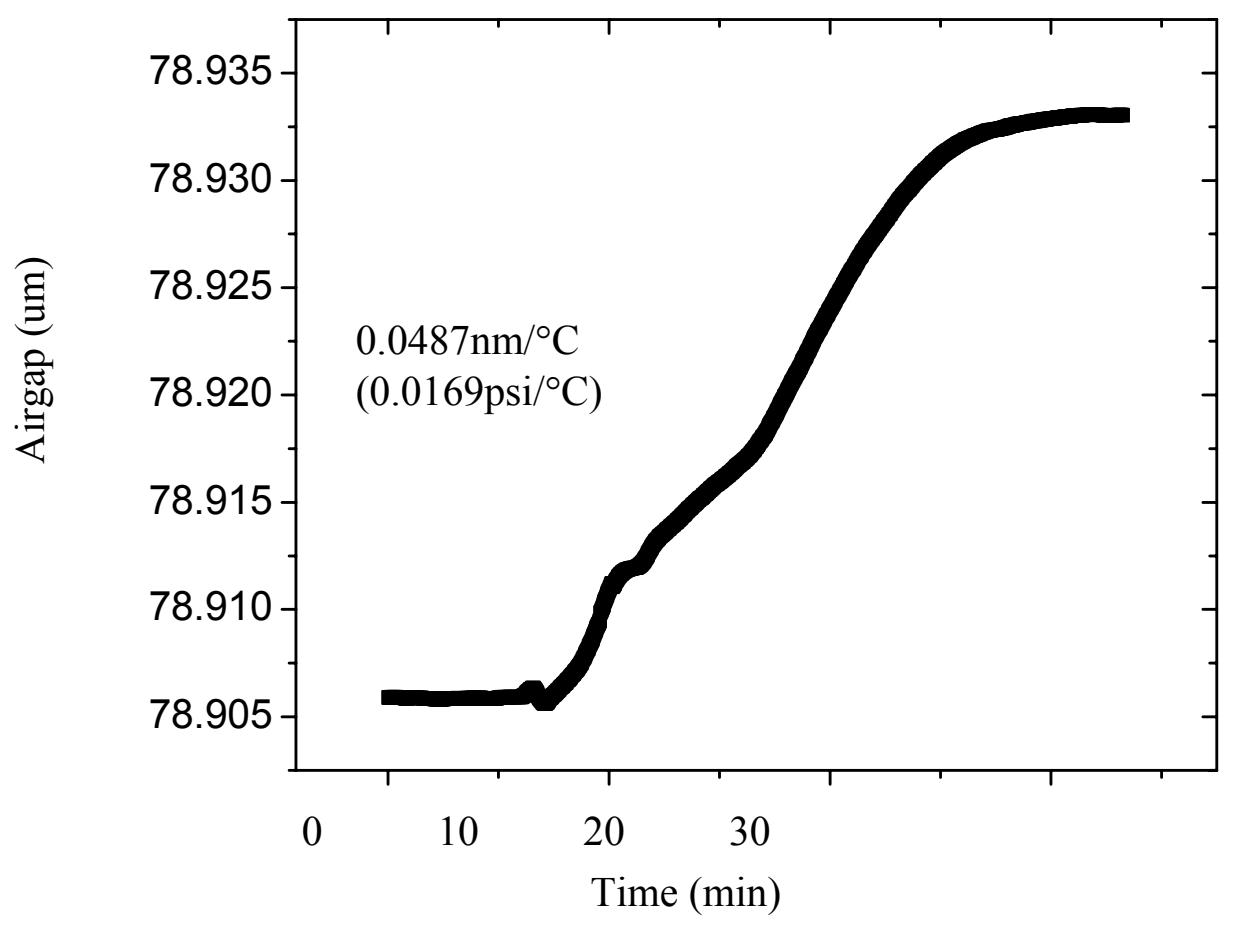

Figure 4.8. Engine sensor temperature dependence test $\left(25^{\circ} \mathrm{C}\right.$ to $\left.600^{\circ} \mathrm{C}\right)$ 


\subsection{Additional EFPI Sensor Development}

\subsection{Miniature Diaphragm-Based Sensors}

The miniature fiber-tip pressure sensor is a diaphragm-based extrinsic Fabry-Perot interferometric (EFPI) sensor, fabricated through a series of processes involving fusionsplicing, cleaving and etching. This section introduces its operating principles and discusses essential sensor fabrication procedures and signal detection and processing.

\subsubsection{Principles of Diaphragm-based EFPI Pressure Sensors}

The traditional structure of a diaphragm-based pressure sensor [4, 5] is shown in Figure 5.1. A hermetic Fabry-Perot cavity is formed between the fiber and the diaphragm. Two beams of reflected light, one from the fiber end and the other from the diaphragm, interfere with each other to modulate the returned optical spectrum.

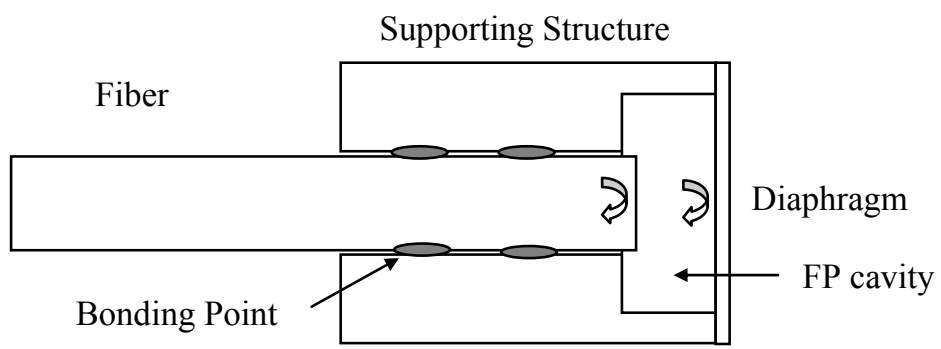

Figure 5.1. A traditional diaphragm-based EFPI pressure sensor structure.

The intensity of the reflected light can be expressed as

$$
\mathrm{I}_{\mathrm{r}}=\mathrm{I}_{1}+\mathrm{I}_{2}+2 \sqrt{\mathrm{I}_{1} \mathrm{I}_{2}} \cos \left(\phi_{0}+\frac{4 \pi \Delta \mathrm{L}}{\lambda}\right)
$$

where $I_{1}$ and $I_{2}$ are the light intensities reflected at the fiber end and the diaphragm respectively, $\varphi_{0}$ is the phase constant related to initial airgap and $\Delta \mathrm{L}$ is the airgap change. When the applied pressure changes, the diaphragm will deform accordingly, resulting in an air-gap change:

$$
\Delta \mathrm{L}=\frac{3\left(1-\mu^{2}\right) \mathrm{R}^{4}}{16 \mathrm{Eh}^{3}} \Delta \mathrm{P}
$$

where $\Delta \mathrm{P}$ is the pressure variation, $\mathrm{R}$ is the radius of the diaphragm, $\mathrm{E}$ is the Young's modulus for the material, $\mu$ is Poisson's ratio and $h$ is the thickness of the diaphragm. 
Therefore the airgap change is linearly dependent on the applied pressure. Resolving the airgap change from the spectral shift can thus provide information about pressure.

\subsubsection{Fiber Etching}

Fiber etching is a critical technique, making it possible to fabricate a similar sensor directly on a fiber tip, eliminating the use of a ferrule, and reducing the sensor size by an order of magnitude. Optical fibers are drawn from fused silica and chemically doped to slightly change the refractive index of either the core or cladding. Most fibers can be grouped into two categories based on dopant: fluorine-doped and germanium-doped. The former has a fluorine-doped cladding with a smaller refractive index than the core, while the latter is usually doped with germanium to increase the refractive index of the core, and is the one of interest in our sensor fabrication.

When immersed into hydrofluoric (HF) acid, the core and cladding region of Ge-doped fiber will etch differently due to their differing compositions. The relative rate of each can be controlled by the component ratios of buffered hydrofluoric acid (BHF) and the Ge dopant concentration. Figure 5.2 shows a $62.5 \mu \mathrm{m}$-core and a $50 \mu \mathrm{m}$-core graded index fiber etched in $50 \% \mathrm{HF}$ solution for 5 minutes. The cores of both fibers are clearly etched off to form a 'hollow tube' in the tip but claddings are nearly intact. The different etching rates indicate different dopant concentrations in the two fibers. Therefore it is possible to generate a small cavity on the fiber tip by etching the core without much damage to the cladding.
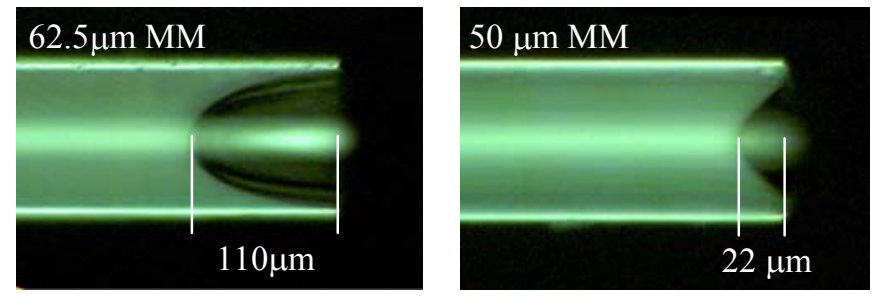

Figure 5.2. $62.5 \mu \mathrm{m}$-core fiber and a $50 \mu \mathrm{m}$-core fiber etched in $50 \%$ HF solution for 5 minutes. The cores of both fibers are clearly etched off to form a 'hollow tube' in the tip but claddings are nearly intact.

\subsubsection{Sensor Fabrication}

The basic structure of a fiber-tip sensor is shown in Figure 5.3, consisting of a single-mode (SM) fiber, an etch barrier layer, a Fabry-Perot cavity and a diaphragm. The structure is similar to a traditional sensor, but miniaturized at the fiber tip

A $10 \sim 20 \mu \mathrm{m}$ etch barrier fabricated from a special fiber (Figure 5.4) is used to protect the SM fiber from HF exposure, which may roughen the SM fiber surface, reducing the reflected signal strength significantly. Since the etching rate is very low, this layer can retain a very flat surface during cavity etching. This step can be omitted where signal to noise ratio is of less concern. 


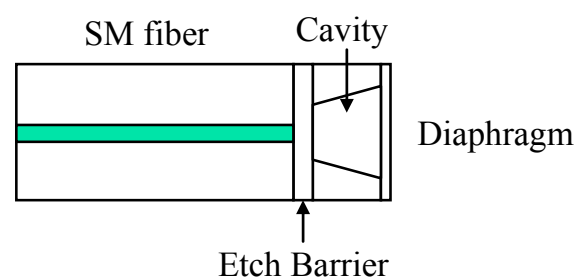

Figure 5.3. Basic structure of a miniature fiber tip pressure sensor. Slightly different interferometric structures are possible; only the simplest is presented here.
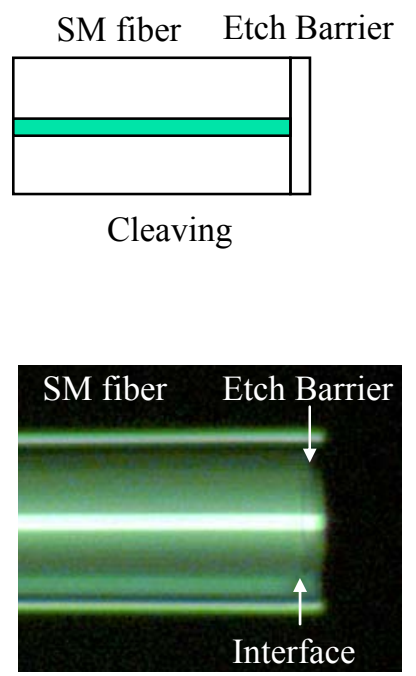

Figure 5.4. Fiber tip sensor etch barrier layer.

As discussed above, a cavity is formed in Ge-doped multimode (MM) fibers by immersion in $\mathrm{HF}$ acid. After the etch barrier is added, the fiber is spliced to a $62.5 \mu \mathrm{m}$-core MM fiber and then cleaved. The length of the MM fiber left is controlled to be approximately equal to that of the cavity. The fiber is immersed in HF for a time period determined by the cavity length and the core etching rate. A slight over-etch is required to ensure the etch reaches the barrier layer and the central portion of the cavity floor is flat. The fiber is now ready for diaphragm bonding. 


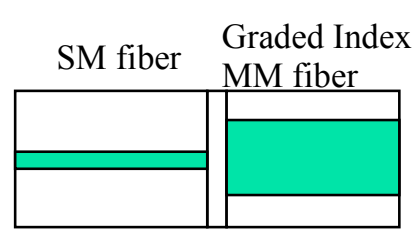

Fusion Splicing
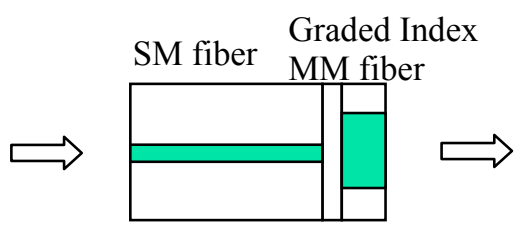

Cleaving
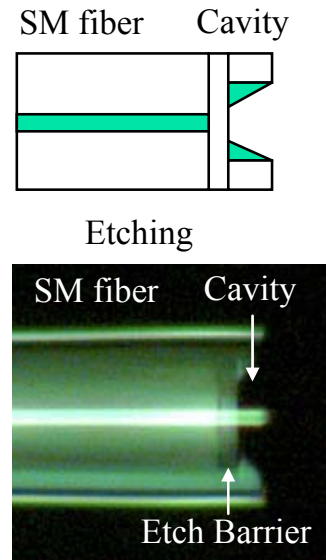

Figure 5.5. Fiber tip sensor cavity formation process.

As shown in Figure 5.6, a pure fused silica fiber is spliced with the cavity and then cleaved, leaving a 'diaphragm' on top of the cavity. To achieve the sensitivity required for physiologic pressure measurements, the diaphragm must be thinned to well below $10 \mu \mathrm{m}$. The splice in this step differs from previous ones. Standard splicing procedures are not applicable here due to their high arc power and long arc duration, which will overheat and collapse the wall of the cavity. Instead, low power arc is utilized for shorter duration, and the prefusion power and time is carefully chosen to minimize the amount of air trapped inside the cavity after the splice. This is essential for temperature cross-sensitivity reduction.

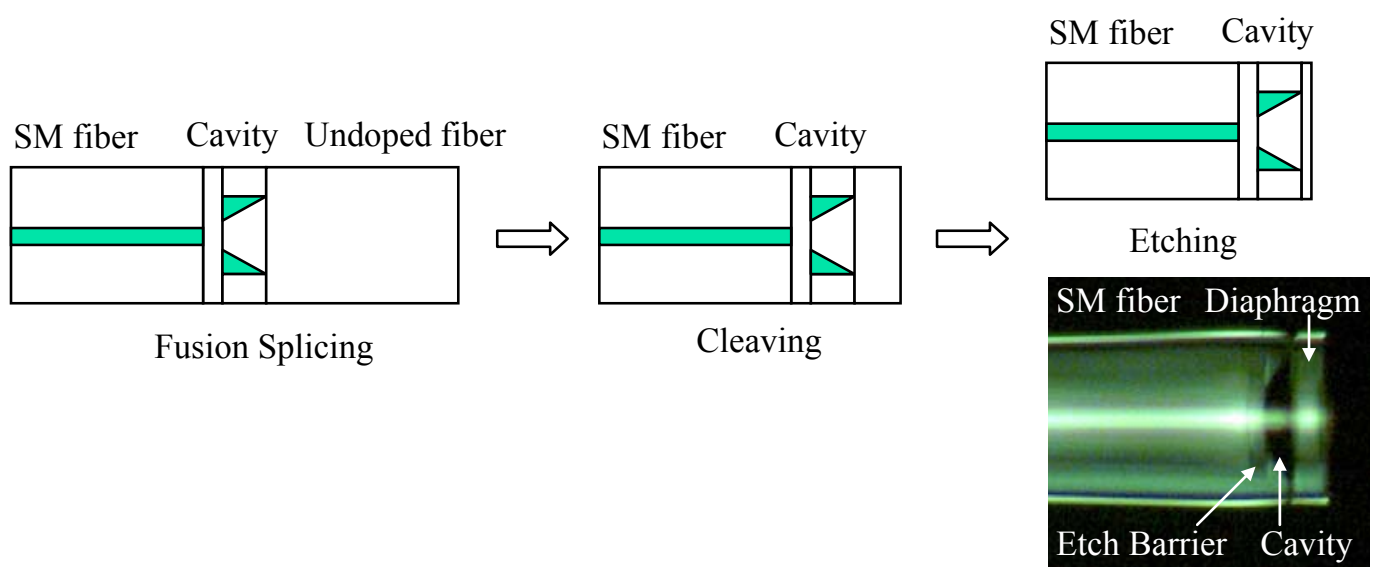

Figure 5.6. Fiber tip sensor diaphragm fabrication process. 
Three reflecting surfaces are evident: the etch barrier and the two diaphragm surfaces. Each will send a beam of light back into the fiber, resulting in a three-beam interference pattern, which is not the case discussed in section 5.1.1 for traditional EFPI sensors. In general, this will only cause minor signal processing difficulties because of the large difference between cavity length and diaphragm thickness.

\subsubsection{Sensor Testing}

The fiber tip sensor is fabricated with fused silica fibers only, eliminating the problem of thermal expansion mismatch between different materials. Therefore it is expected to work at high temperature. To explore its high temperature capability, sensor was tested using a setup shown in Figure 5.7. The sensor was sealed in a tube pressurized by a pressure calibrating system (Pressure System, Inc.) which controls the amount of compressed nitrogen flowing into the tube to change the pressure inside. The end of the tube is placed in a furnace, controlling the sensor's temperature. Sensor output was detected using Micron Optics Component Testing System (CTS), which is essentially a spectrometer with build-in tunable laser source and detector. The cavity length was determined by monitoring the interference pattern shift.

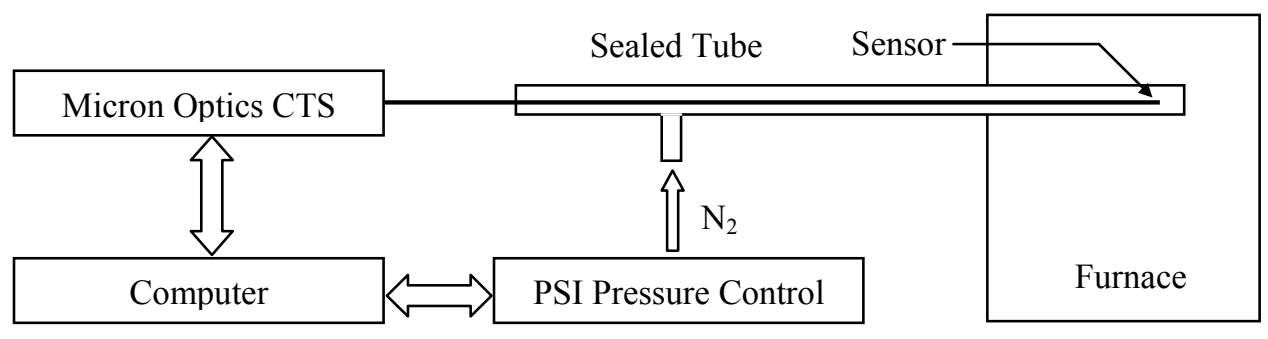

Figure 5.7. Fiber tip sensor test setup.

It was observed that the first time the sensor was heated up to high temperature the airgap changed drastically. However, after being kept at high temperature for a few hours, the sensor tended to reach a stable state. Figure 5.8 shows such a heating process. The sensor was heated from room temperature (RT) to $600^{\circ} \mathrm{C}$ and kept for a few hours (several hours of data was missing after about 50 mins due to program error). Then it was cooled down to $500^{\circ} \mathrm{C}$ and it can be seen the airgap does not change much. The reason may be because the stress at the splicing point began to be released slowly at elevated temperature. Therefore the first heating cycle will probably serve as an annealing process.

Figure 5.9 shows the sensor's pressure response at $500^{\circ} \mathrm{C}$ after 'annealing'. The performance is not as good as room temperature with a resolution about $2 \%$. Creep has been observed when sensor is under high pressure. This is because the viscosity of fused silica will decrease at higher temperature and cause the materials to deform very slowly when it is under stress. Details of the sensor's high temperature capability are currently under investigation. 


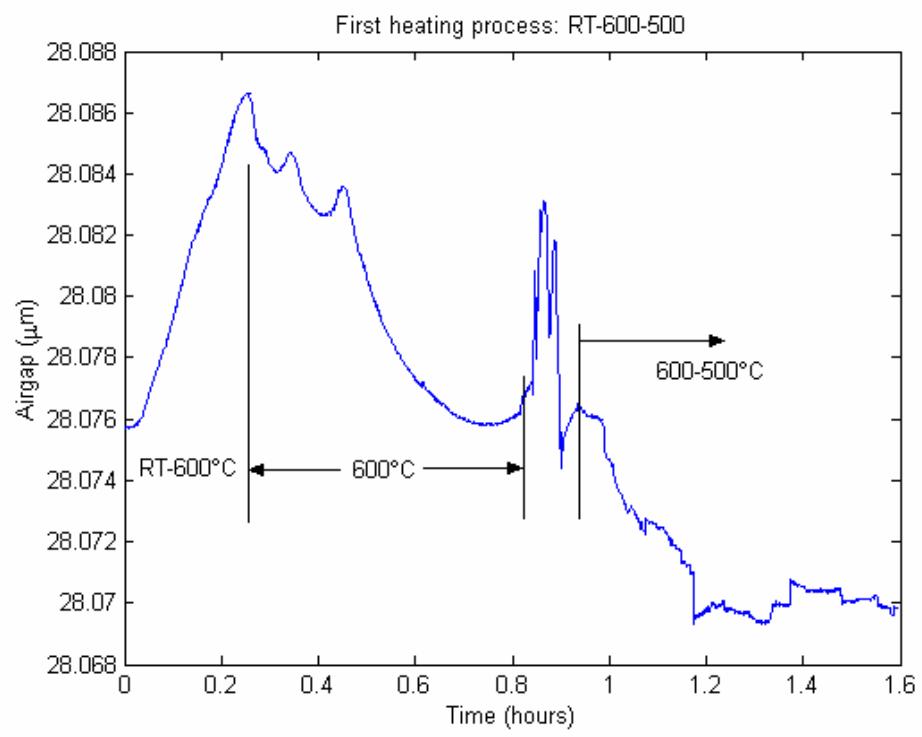

Figure 5.8. Airgap change in $1^{\text {st }}$ heating cycle.

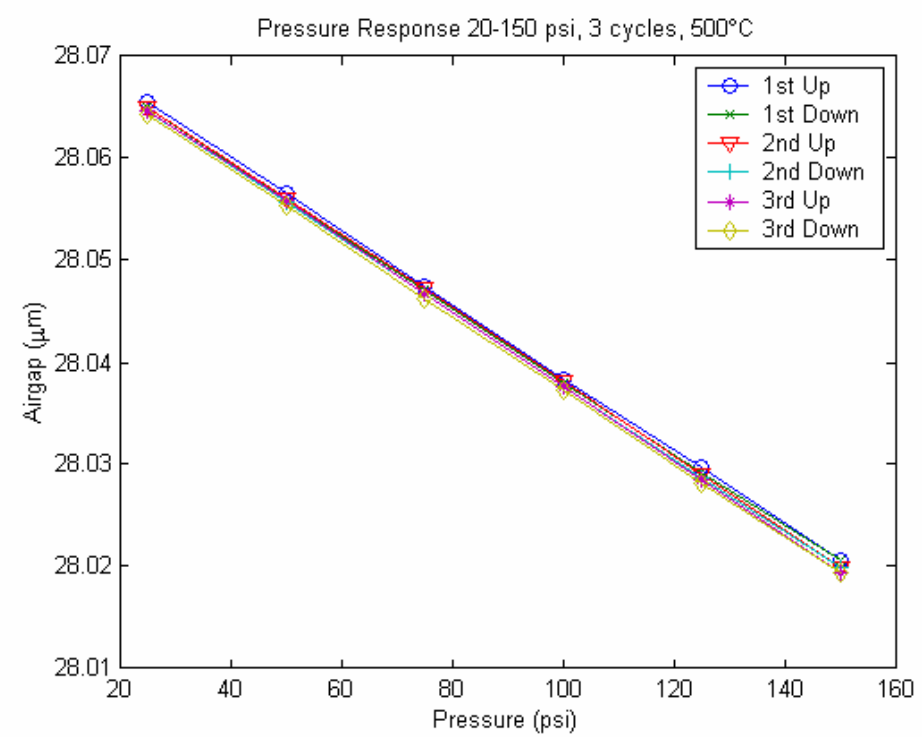

Figure 5.9. Sensor response at $500^{\circ} \mathrm{C}$.

\subsection{Hollow Fiber Based Miniature Sensor}

Rather than using a chemical etching process during the fabrication, this miniature sensor ( $125 \mu \mathrm{m}$ in diameter) can be fabricated by using a piece of hollow fiber. Eliminating the use of a chemical or hazardous material like HF, the whole fabrication process is safe, simple and inexpensive. The basic sensor structure is shown in Figure 5.10. The essential element is a piece of hollow fiber, which connects a fiber end to a diaphragm to form a Fabry-Pérot cavity. Cavity fabrication is realized by cleaving and splicing, completely eliminating any chemical etching process. This not only ensures that the whole process is clean, safe, and 
easy for anyone including those with no chemical material process training to operate, but also greatly simplifies laboratory maintenance without any need for hazardous waste disposal or fume hood cleaning. Also, this technique can be conveniently utilized by any laboratory including those without chemical processing facilities. Last but not least, the simpler fabrication process and fewer experimental material and tools undoubtedly lower the total cost.

\subsubsection{Fabrication Process}

The fabrication process is described as follows:

1. Splice a piece of hollow fiber at the cleaved end of a standard fiber.

2. Cleave the hollow fiber near the junction according to the cavity length requirement. The cavity length can be cleaved down to the order of micrometers under the inspection of a microscope or CCD.

3. Connect the diaphragm to the other end of the hollow fiber. The method can be splicing, agglutinating, heating, etc.

4. Control the diaphragm's thickness to the order of sub-microns by cleaving, polishing or dipping the fiber head into HF acid of suitable concentration. This last step is optional according to the diaphragm requirement.

The diaphragm can be a slice of optical fiber, sapphire fiber, ceramic plate, sapphire plate, rod, or combined layers. The whole process is monitored by a power measurement device (for example, a white light interferometry system). The interior diameter of the hollow fiber can be selected according to different requirements.

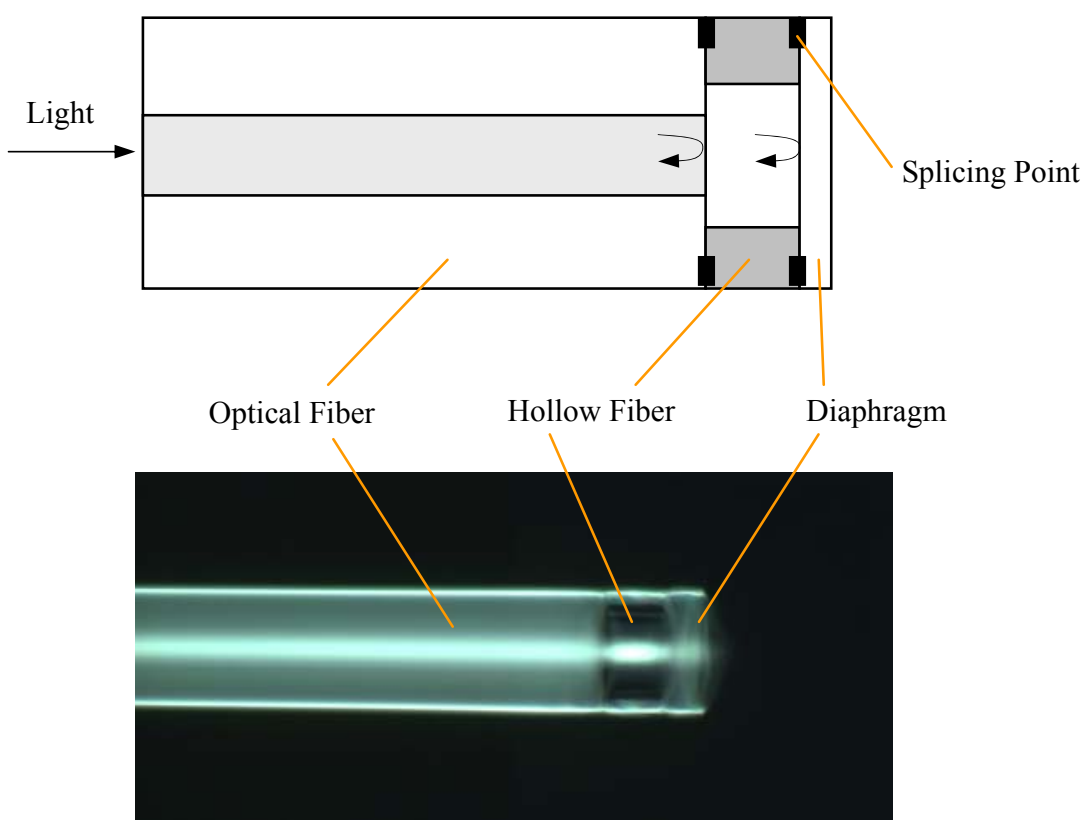

Figure 5.10. Schematic (top) and photograph (bottom) of the hollow fiber-based miniature sensor. 


\subsubsection{Novel Features}

This hollow fiber based miniature sensor has the following advantages:

- Miniature size: The whole structure is the size of the fiber, generally $125 \mu \mathrm{m}$ in diameter.

- Simple and efficient fabrication: The process mainly includes cleaving and bonding steps, and takes less than one hour in general.

- No chemical process involved: The entire process can be performed in a general photonics laboratory with a cleaver, a splicer and a microscope. The complete setup for the fabrication is shown in Figure 5.11. Since no hazardous material, such as HF, is involved, no fume hood or poisonous waste procedure is needed.

- Safe fabrication: The lack of chemicals ensures the method is safe and reliable. No chemical or hazard safeguard is needed. Anyone, including those without hazard protection training, can operate the tools

- Low cost: The materials for the structure, namely, optical fiber and hollow fiber, are commercially available products. Also, the tools for the fabrication, the cleaver and splicer, are common tools in a photonics laboratory. In addition, without any waste prpdcuts or hood maintenance fee, the cost is further reduced.

- High temperature capability: When the fiber, hollow fiber and diaphragm are sapphire, the structure can work at more than $2000^{\circ} \mathrm{C}$.

- High signal strength and visibility: The cavity surfaces are smooth, flat and parallel, which guarantees the quality of the signal.

- EMI immunity and electric passivity: Such an optical structure is immune to the electromagnetic interference.

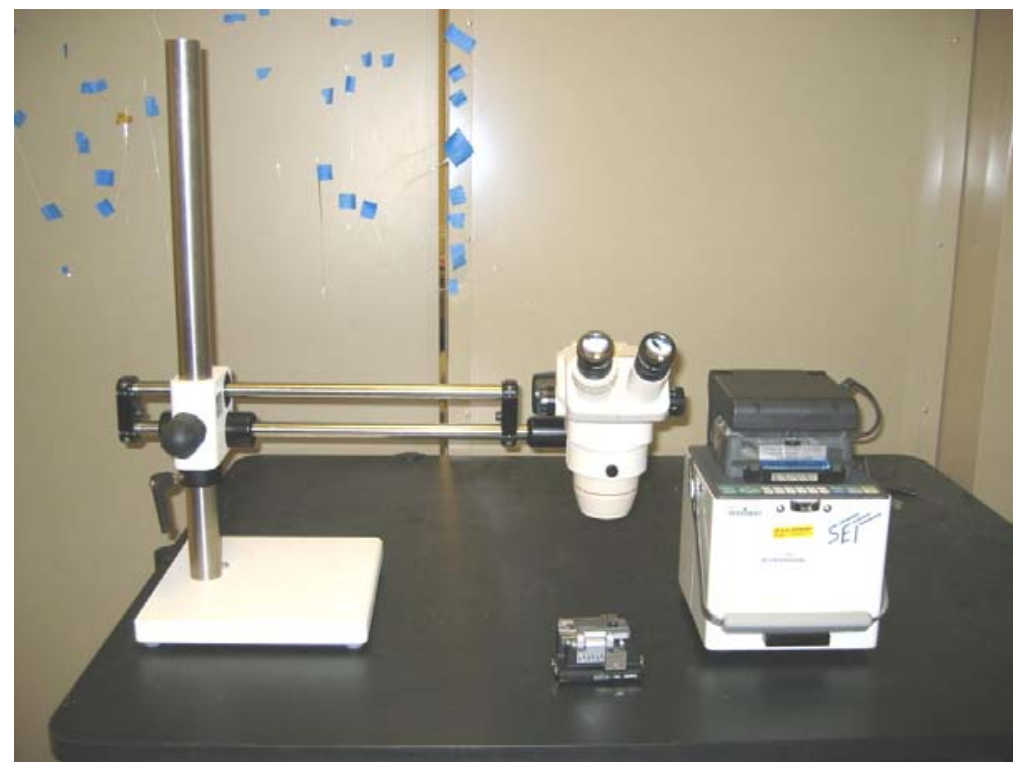

Figure 5.11. Complete setup for miniature sensor fabrication: cleaver, splicer and microscope. 


\subsubsection{Testing Results}

During fabrication and testing the hollow fiber-based miniature sensors were monitored by the CTS (High Resolution Swept Laser Interrogator) shown in Figure 5.12. It contains a very fast, high signal-to-noise ratio swept laser, high dynamic range detectors and amplifiers, high speed, simultaneously sampling data acquisition, and a real time operating system to tie all the components together and display data on an LCD. The laser is a mode-locked Er-doped fiber ring whose maximum average power is $0 \mathrm{dBm}$, and the peak pulse energy is $0.1 \mathrm{~nJ}$. It continuously sweeps 5 times per second in the wavelength range between $1520 \mathrm{~nm}$ and $1570 \mathrm{~nm}$.

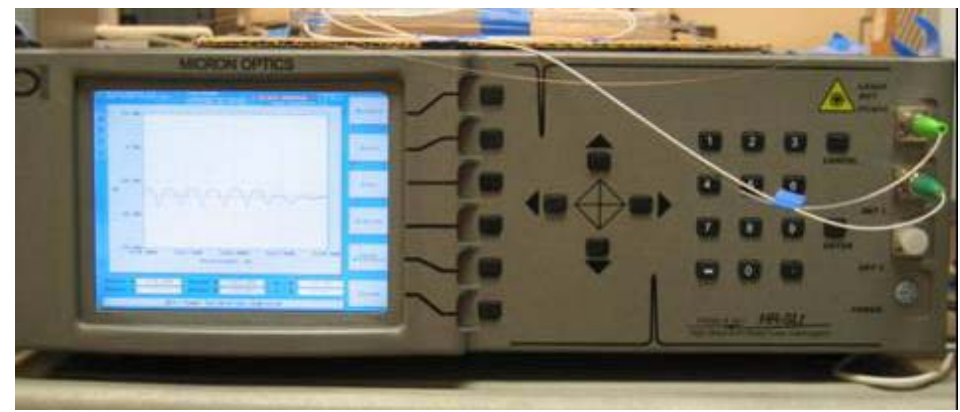

Figure 5.12. Micron Optics Component Test System (CTS), a high resolution swept laser interrogator utilized for sensor testing.

The testing setup is shown in Figure 5.13. The sensor is sealed in a tube where the pressure is supplied by a gas system and controlled by a PSI (Pressure System, Inc) pressure controller. The pressure can vary from atmospheric pressure to about 200 psi. The reflected signal data is collected by a computer.

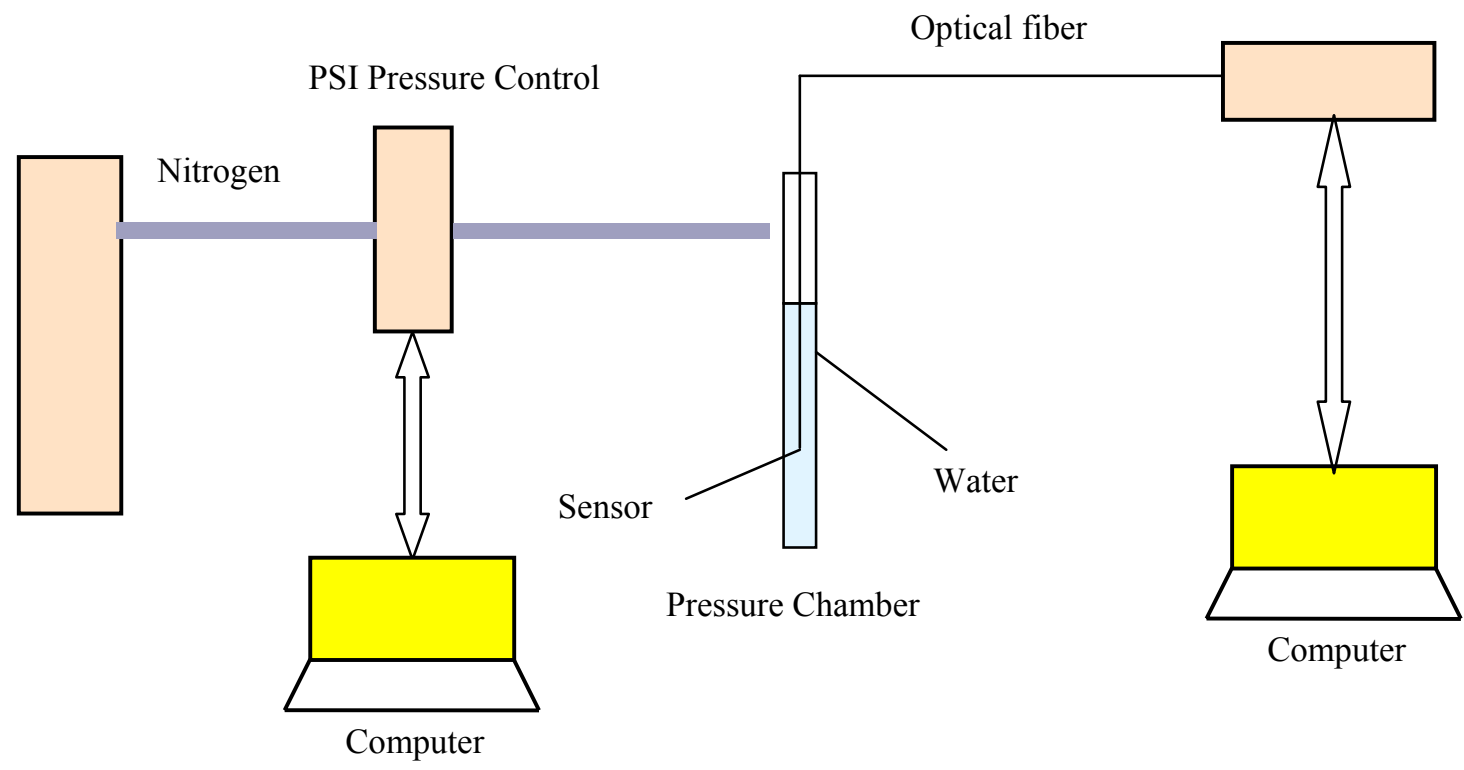

Figure 5.13. Pressure testing setup. 
After fabrication, the hollow fiber based miniature sensors were tested. Their interferometric signal, sensitivity, repeatability and temperature dependence are discussed in the following paragraphs.

\section{Interferometric Signal}

The reflectance at both the fiber end and the inner surface of the diaphragm are low (about $3.5 \%$ ), the response is periodic function similar to a two-beam interferometer, which can be described by

$$
I=E_{1}^{2}+E_{2}^{2}+2 E_{1} E_{2} \cos \left(\frac{4 \pi n d}{\lambda}\right)
$$

$E_{1}$ and $E_{2}$ : the magnitudes of the electrical fields of the reflected light at two fiber ends

$d:$ The length of the air gap of the Fabry-Pérot cavity

$\lambda$ : The wavelength

$n$ : The refractive index of the medium.

When $E_{1} \approx E_{2}$, and $I_{1}=E_{1}^{2}$, Eq. 3-3 can be simplified as:

$$
\begin{aligned}
& I=2 E_{1}^{2}\left[1+\cos \left(\frac{4 \pi n d}{\lambda}\right)\right] \\
& =2 I_{1}\left[1+\cos \left(\frac{4 \pi n d}{\lambda}\right)\right]
\end{aligned}
$$

As a consequence, the interference signal is a function of the cavity length. The pressure induced diaphragm deflection changes the sealed air gap length and modulates the signal. By monitoring the sensor output, the applied pressure can be measured. Figure 5.14 shows the interferometric signal obtained for a representative hollow fiber based miniature sensor. The signal is as strong as $33 \mathrm{~dB}$.

The pressure test results between 15psi and 135psi, in 30psi steps, are shown in Figure 5.15. We first increase the pressure, and then decrease it with the same step. After 3 rounds, the relationship between the airgap change and the applied pressure was obtained and is shown in Figure 5.16. 


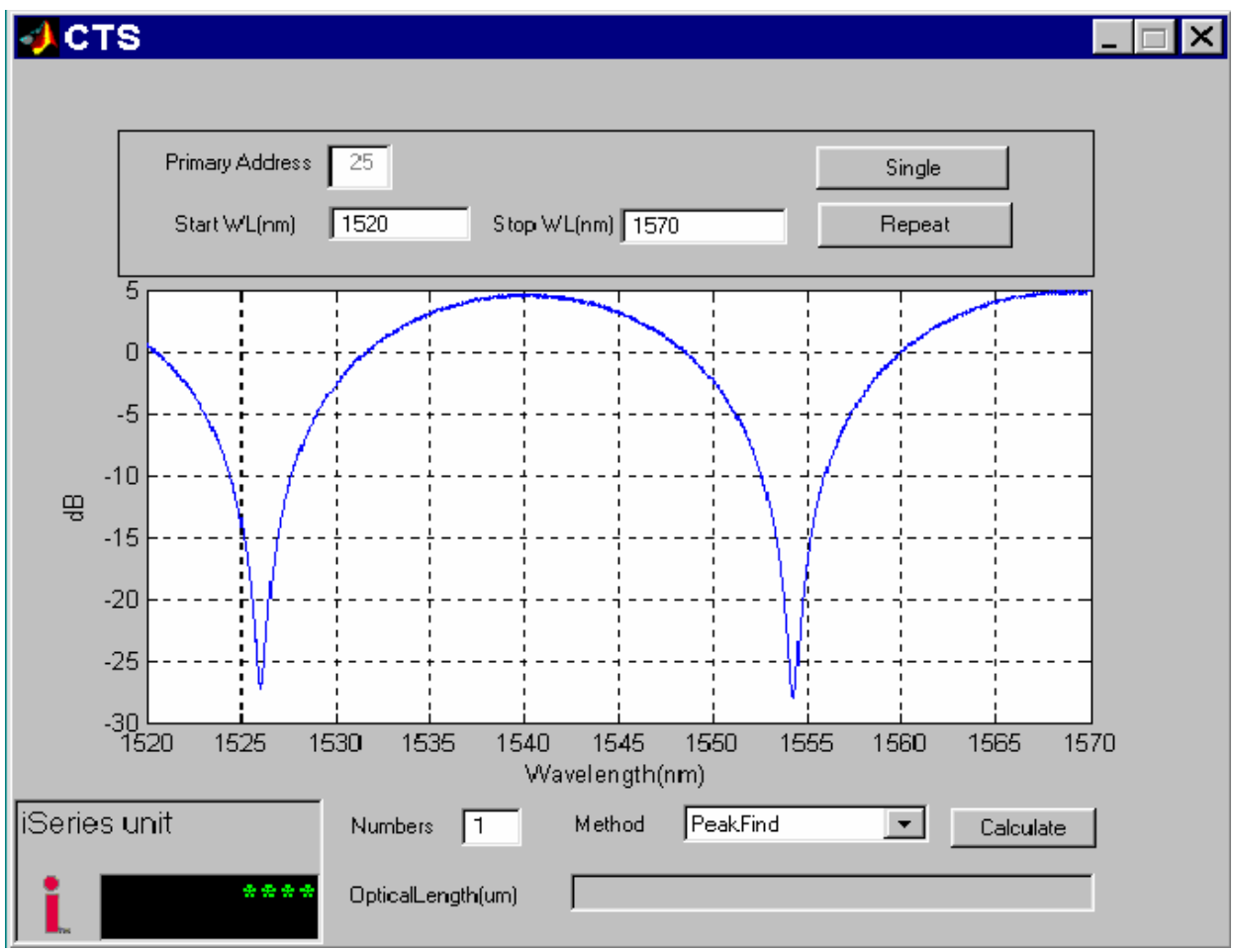

Figure 5.14. Interference fringes of a hollow fiber based pressure sensor.

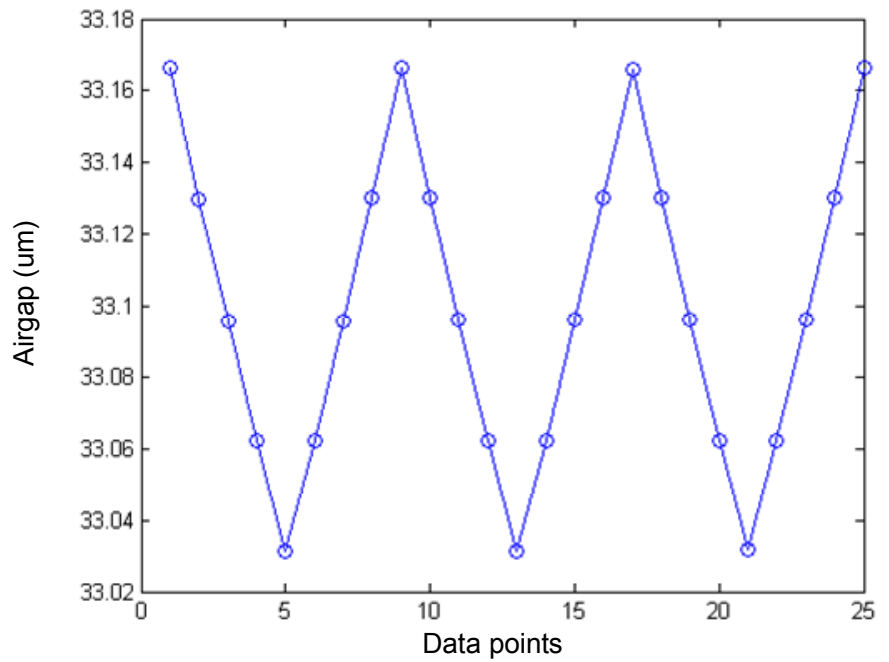

Figure 5.15. Airgap change versus pressure change for a hollow fiber-based pressure sensor. 


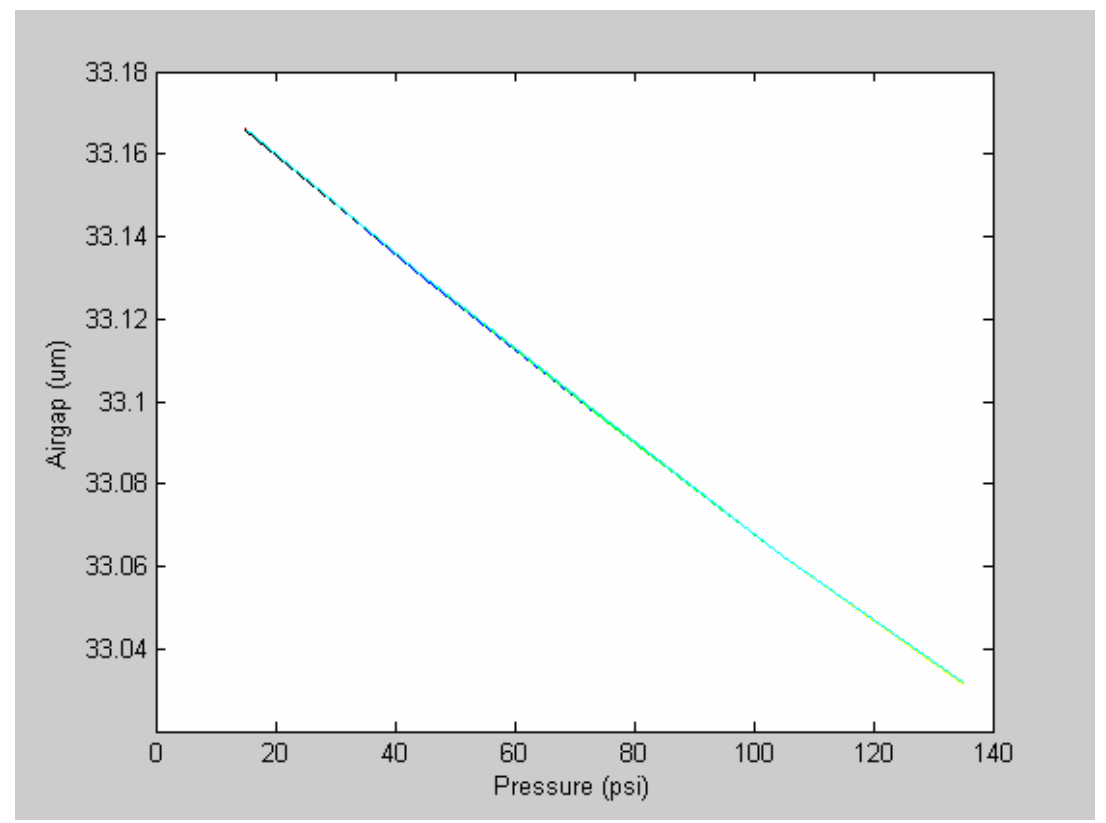

Figure 5.16. Repeatability of airgap change, six curves plotted together.

\section{Sensitivity}

Sensitivity, one of the most important performance indices of a pressure sensor, is defined by the ratio of the deflection to the pressure applied. For this sensor, shown in Figure 5.15 and Figure 5.16, 120psi pressure results in an air gap change of $140 \mathrm{~nm}$. So the sensitivity is about $1.17 \mathrm{~nm} / \mathrm{psi}$. The sensitivity can be further improved by reducing the thickness of the diaphragm.

\section{Repeatability}

Repeatability indicates whether the sensor can repeat its performance under the same conditions. The three cycle test producing six curves are plotted together in Figure 5.16. There is no apparent hysteresis. The repeatability and linearity are very good.

Figure 5.17 shows the enlarged picture of Figure 5.16 where the error is maximal. The maximum relative error (the ratio of the maximum difference to the full measurement scale) is about $0.0004 \%$. One possible reason for the error is that the stress in the bond changes when the pressure is applied.

\section{Temperature Coefficient}

When the temperature of the sensor varies, the thermal expansion of its parts (single mode fiber, hollow fiber and the diaphragm) will introduce an additional change in the length of the airgap, other than the pressure-induced change. In order to minimize this cross sensitivity effect on the signal acquisition, information of temperature coefficient $\mathrm{C}_{\mathrm{T}}$, i.e. the airgap change per ${ }^{\circ} \mathrm{C}$, is important. This can be obtained by testing the sensor under different temperatures. 


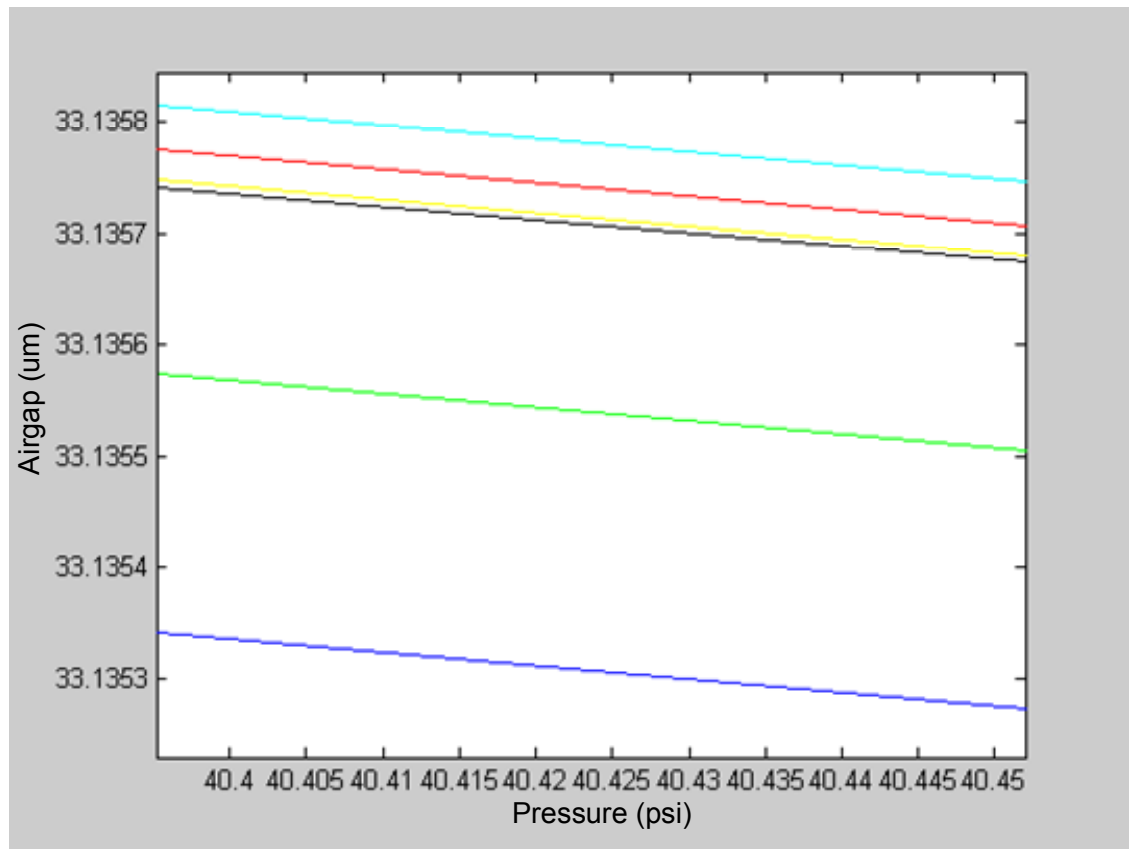

Figure 5.17. Enlarged plot of Figure 5.16.

The same sensor used for the sensitivity and repeatability test was evaluated at $100^{\circ} \mathrm{C}, 200^{\circ} \mathrm{C}$, $300^{\circ} \mathrm{C}, 400^{\circ} \mathrm{C}, 500^{\circ} \mathrm{C}$ and $600^{\circ} \mathrm{C}$. The output signals according to the temperature increase and decrease are plotted in Figure 5.18. The temperature coefficient is about $0.05 \mathrm{~nm} /{ }^{\circ} \mathrm{C}$. The airgap is larger when the temperature is decreasing than that when the temperature is increasing. The reason may lie in the insufficient time duration for the sensor to stabilize after heating. In our experiment, the time for the sensor to stabilize is only about 10 minutes.

\section{Dynamic Test Results}

To test the sensor's dynamic response, a $45 \mathrm{KHz}$ air transducer was used. The sensor head was placed close to the transducer with the distance about $0.5 \mathrm{~cm}$, as shown in Figure 5.19 . The sensor response under atmosphere pressure was tested by the SCIIB system and shown in Figure 5.20. The yellow curve indicates the sensor's time domain response from the narrow band of SCIIB system, and the red curve shows its FFT result. A sharp pulse (about $20 \mathrm{dBm}$ ) is observed at $45 \mathrm{KHz}$, which is the transducer's operating frequency. This signal can be demodulated to obtain the dynamic pressure information. 


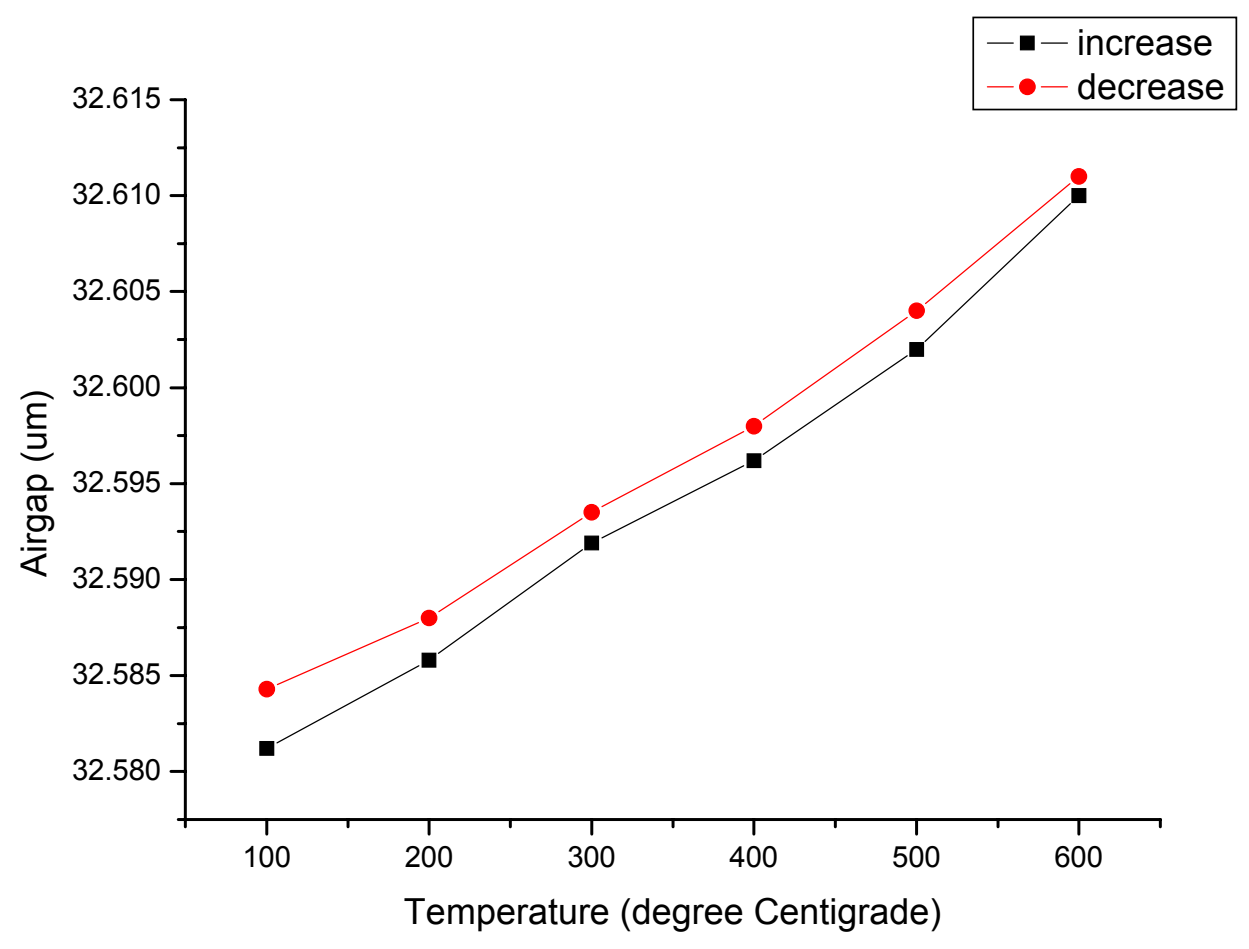

Figure 5.18. Sensor's temperature dependence.

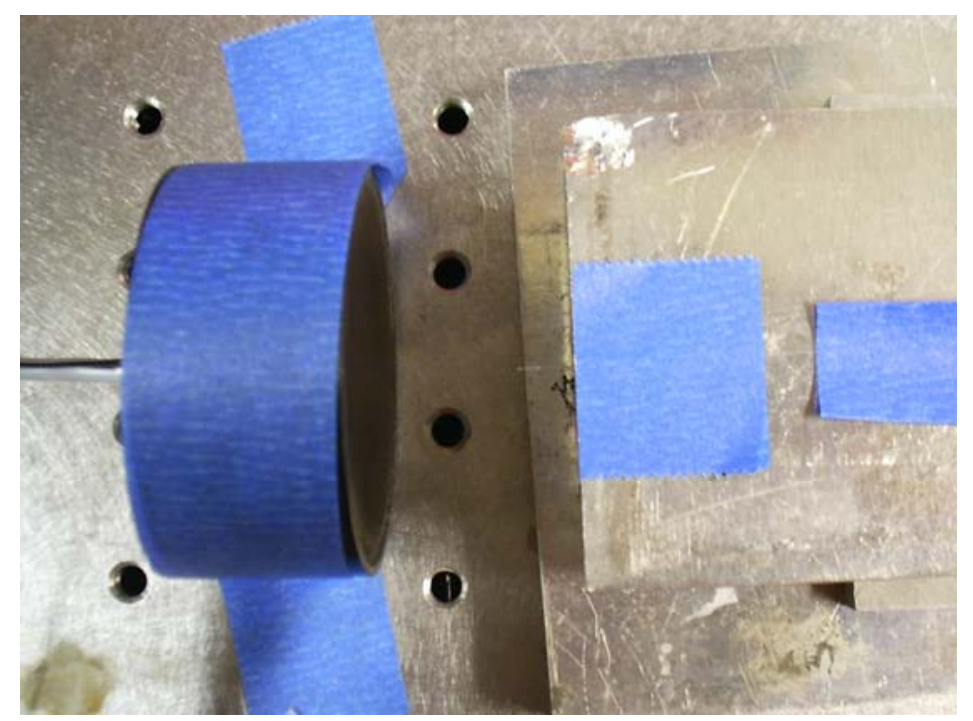

Figure 5.19. Relative position between the transducer and the sensor for dynamic testing. 


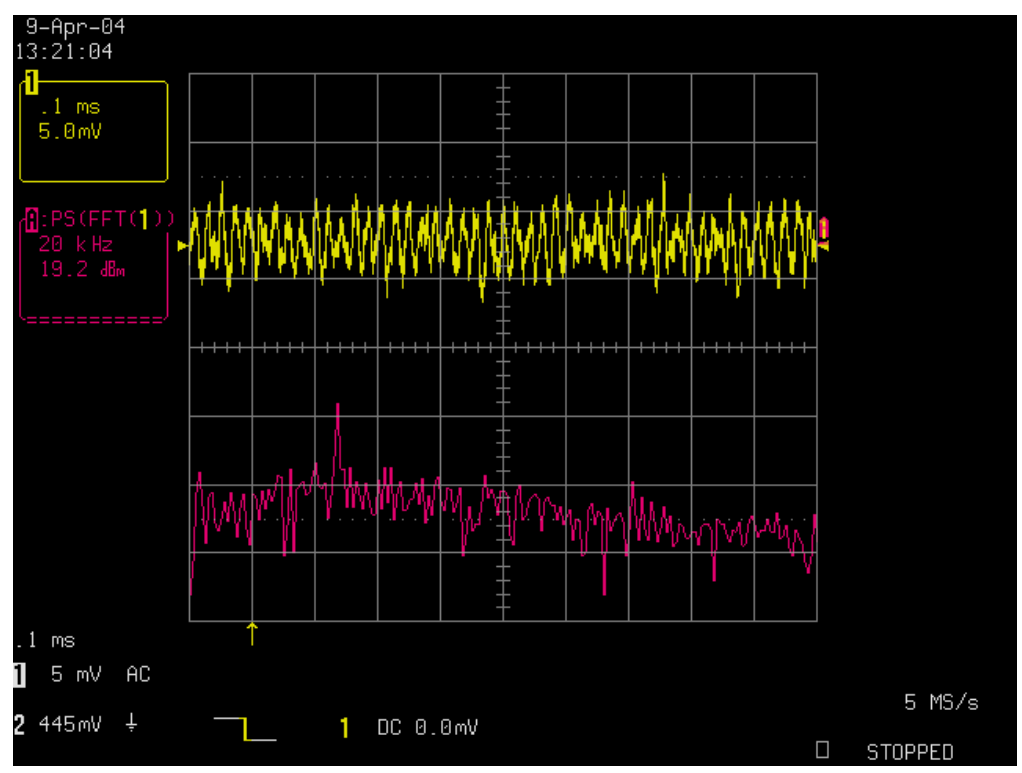

Figure 5.20. Dynamic test result of the sensor.

\subsection{Diaphragm-Based High Temperature Sensor}

This fiber optic temperature sensor is based on the interference generated by the two surfaces of a diaphragm. Figure 5.21 illustrates the sensing principle. The sensing element is the diaphragm, acting as an interferometer. Reflections at both sides of the diaphragm will interfere with each other, producing a modulated spectrum, whose pattern is determined by the optical path difference (OPD) between the two reflections. Thermal dependence of both the diaphragm thickness and the refractive index will make the OPD and thus the spectrum pattern sensitive to temperature variation. Therefore the information about temperature can be demodulated from the reflected signal. Mathematically, OPD can be expressed as

$$
\mathrm{OPD}=2 \mathrm{nd}
$$

where $\mathrm{n}$ is the refractive index of the material, $\mathrm{d}$ the thickness. Let $\alpha$ be the coefficient of thermal expansion (CTE) of the material and $\beta$ the thermal coefficient of refractive index, we have

$$
\mathrm{OPD}=2 \mathrm{~d}_{0}(1+\alpha \Delta \mathrm{T}) \cdot \mathrm{n}_{0}(1+\beta \Delta \mathrm{T})=2 \mathrm{n}_{0} \mathrm{~d}_{0}\left(1+(\alpha+\beta) \Delta \mathrm{T}+\alpha \beta \Delta \mathrm{T}^{2}\right)
$$

The temperature dependence of the OPD is clearly seen. 


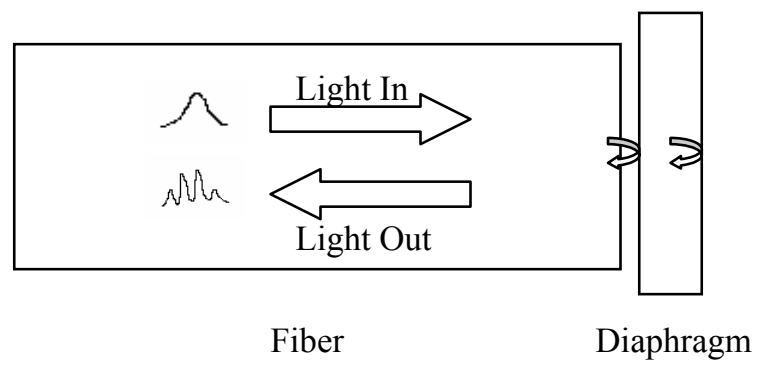

Figure 5.21. Sensor principle

\subsubsection{Sensor Fabrication}

The most advantageous feature of this scheme is that the fiber could be of any type, not only conventional telecommunication singlemode/multimode fibers. Even the highly multimoded sapphire fiber, in which it is usually very difficult to generate interference, can be used this way to get fringes. If the whole sensing head, including fiber, diaphragm and supporting structure, are made from sapphire or alumina, the sensor can be used as high temperature sensor capable of measuring temperature up to $1600^{\circ} \mathrm{C}$.

\subsubsection{Light Source and Detector:}

There are multiple possible combinations of light source and detector. Two possible choices are: whitelight source + spectrometer or laser + photodiode. For the first one, temperature is resolved from the modulated spectrum. For the second one, the light intensity at the photodiode will change accordingly.

\subsubsection{Preliminary Results}

Some preliminary experiments have been conducted. Three types of fibers have been tested using a white light system, as shown in Figure 5.22. Two type of wafers were used, $12 \mu \mathrm{m}-$ thick and $57 \mu \mathrm{m}$-thick fused silica wafer. The interference signals are shown in Figure 5.23.

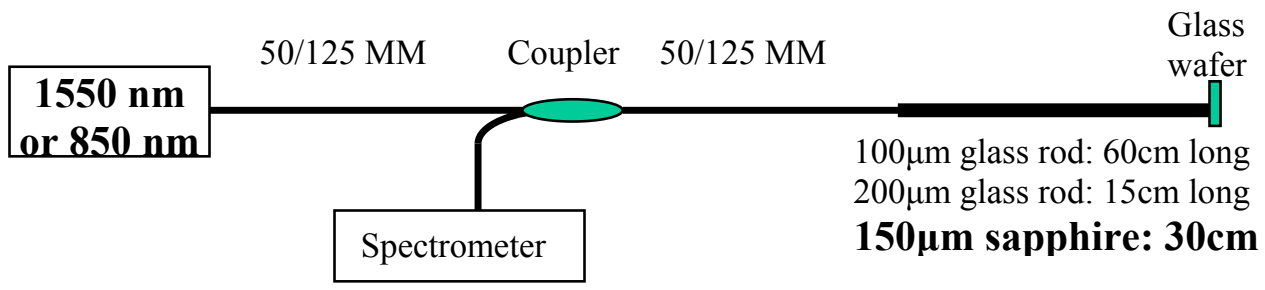

Figure 5.22. System setup. 


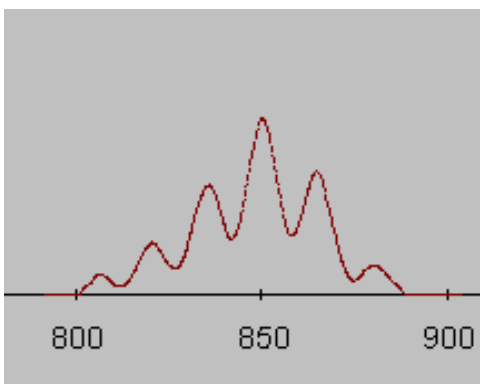

Fiber: $100 \mu m$ Silica Rod, Wafer: $12 \mu \mathrm{m}$ thick

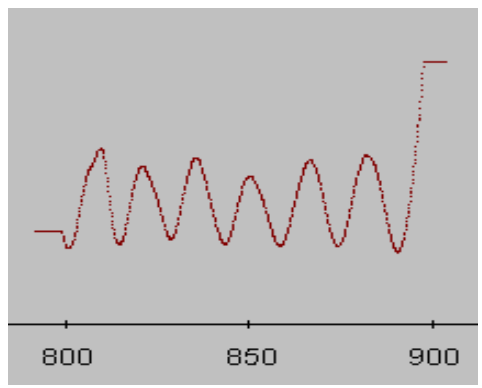

Fiber: $200 \mu m$ Silica Rod, Wafer: $12 \mu \mathrm{m}$ thick (Normalized spectrum)

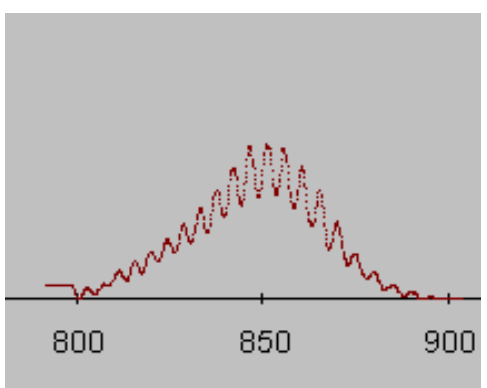

Fiber: $100 \mu \mathrm{m}$ Silica Rod, Wafer: $57 \mu \mathrm{m}$ thick

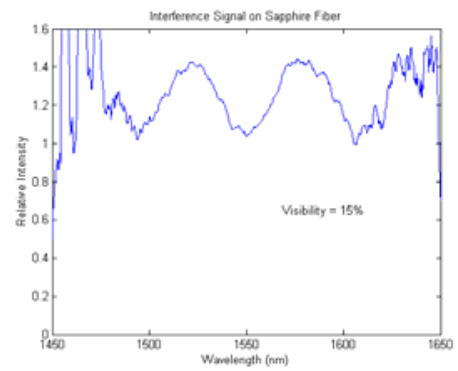

Fiber: $150 \mu \mathrm{m}$ Sapphire fiber, Wafer: $12 \mu \mathrm{m}$ thick (Normalized spectrum)

Figure 5.23. Interference signal generated by various types of fibers and wafers.

\subsection{Laser Ablation of Sapphire}

Sapphire has been widely used in microelectronics and sensor optics due to its unique properties. Sapphire has thermal stability up to $1600^{\circ} \mathrm{C}$ and very high stiffness (modulus). It is highly inert and resistant to attack in most process environments including hydrofluoric acid and the fluorine plasma applications commonly found in semiconductor wafer processing. Sapphire also has a wide transmission window ranges from below 0.2 microns, to over 4 microns. In turn, these properties make it difficult to process precisely by any micromachining techniques and therefore limit the wide applications of sapphire.

Despite this, several methods have been reported to structure sapphire, such as reactive ion etching (RIE), ion beam implantation ${ }^{[6]}$, laser induced backside wet etch (LIBWE)[7] and laser processing with excimer laser ${ }^{[6]}$, quasi-cw laser[8] and picosecond and subpicosecond laser[9]. Among these methods, RIE can provide the best surface quality, but etching depth is limited by the materials of the mask. The LIBWE method and other direct laser ablation give very poor surface quality though with high etch rate. Ion beam implantation followed by wet chemical etching results in better quality than laser ablation, but this method can only structure the sapphire with a depth around $1 \mu \mathrm{m}$. RIE and ion beam implantation are costly processes while laser ablation is cheaper. 
We have developed a novel method, laser ablation on a hot substrate, to etch sapphire by using an excimer laser. The principle of this method is shown in Figure 5.24. A laser beam is incident from the front side of the sapphire wafer while the backside (but not limited to the backside) of the sapphire wafer is heated to a certain temperature. Compared with direct laser ablation methods, backside heating laser ablation can provide extremely high surface quality (as shown in Figure 5.25), high etch rate $(83 \mathrm{~nm} / \mathrm{min}$ or even higher), great structure depth $(>50 \mu \mathrm{m})$, but requires low energy fluence $\left(<=1 \mathrm{~J} / \mathrm{cm}^{2}\right)$. In Figure 5.25 , two structures were obtained under almost the same conditions, except for the left one, the sapphire wafer was attached to a small furnace with temperature of $600^{\circ} \mathrm{C}$.

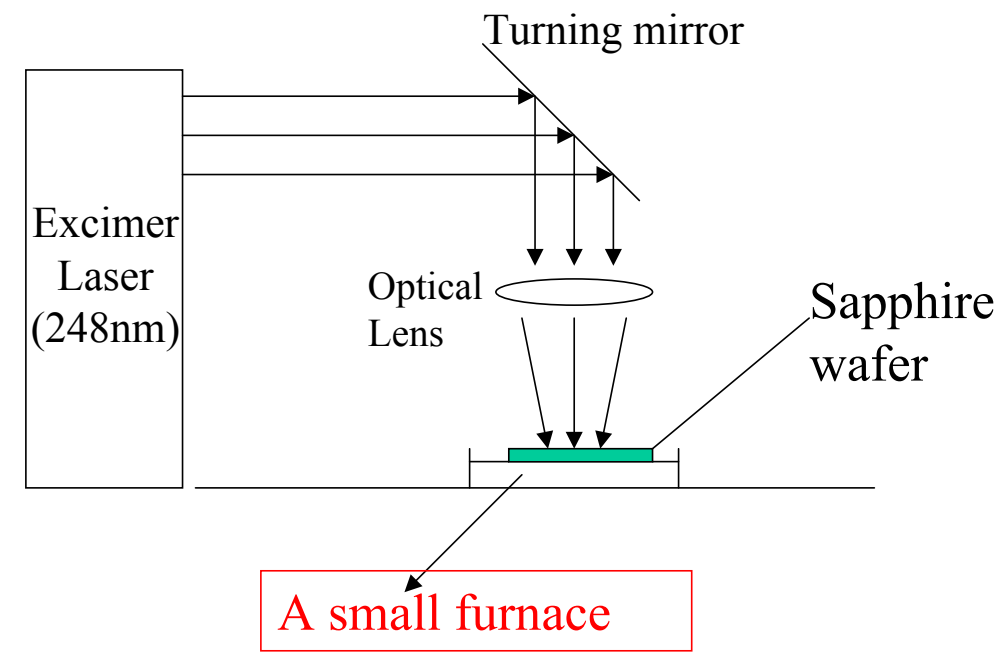

Figure 5.24. Principle of backside heating laser ablation
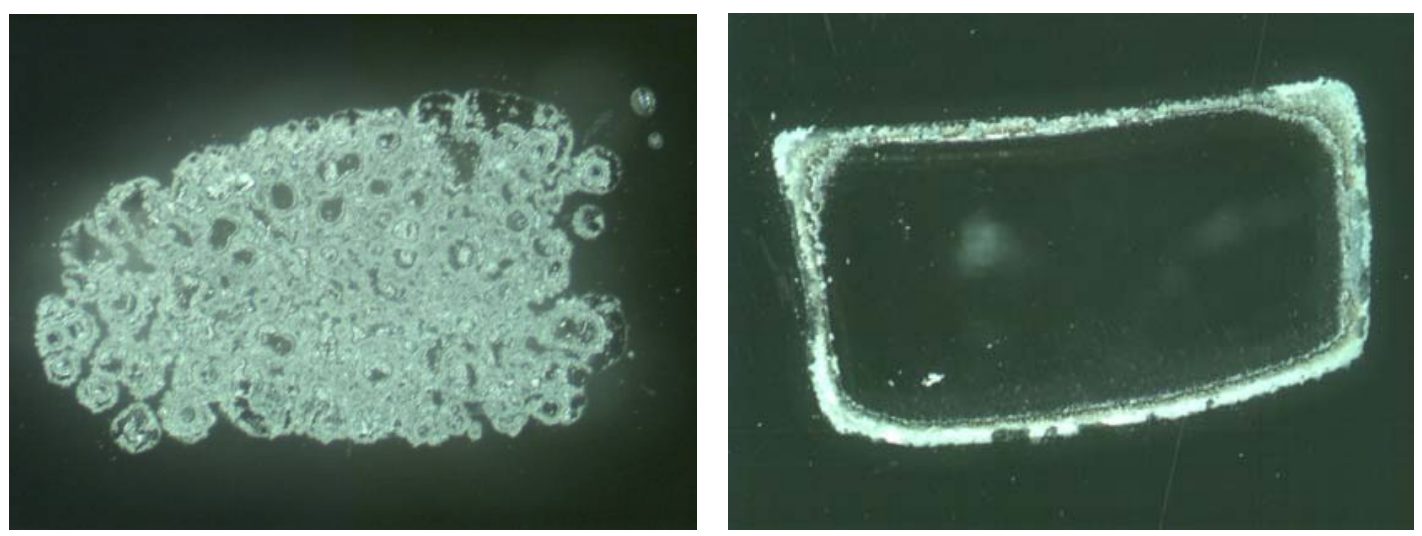

Figure 5.25. Surface quality produced with backside heating laser ablation (left) and direct laser ablation (right). 
The factors that influence the results are as follows:

- Laser beam shape;

- Heating temperature;

- Separation between last optic and the sample substrate;

- Energy density;

- Frequency;

- Number of pulses;

- Thickness of the sample

By choosing the correct value of these factors, and using more accurate optical components, the best result can be obtained. The re-deposition on the rims of the structure can be removed by wet etching. This method can also be used etch other materials, such as fused silica, quartz and zirconia. 


\subsection{A Novel Intrinsic Fabry-Perot Fiber Optic Sensor}

Among various optical fiber sensors, Fabry-Perot (FP) interferometric sensors are very promising because they are accurate and sensitive. Intrinsic Fabry-Perot sensors are "all-fiber sensors", which can reduce or eliminate the bonding problems experienced with extrinsic sensors. Intrinsic sensors are also more versatile for installation and are more robust. But intrinsic sensors are usually more difficult or require more expensive equipment to fabricate than extrinsic sensors. So from a commercial viewpoint, intrinsic sensors are significantly more expensive.

To fabricate in-line Fabry-Perot interferometers, the greatest focus has been on producing thin-film mirrors on the cleaved fiber end face through vacuum deposition, sputtering in a magnetron system or electron-beam evaporation. However, it is difficult to control the film thickness and flatness with precision. Moreover, the thin-film mirrors will be immediately damaged or deteriorated in quality by the splicing electric arc, and the reflectivity of the Fabry-Perot mirror cannot be controlled[10, 11].

A simple, novel process was developed to fabricate intrinsic Fabry-Perot interferometric sensors by wet chemical etching and fusion splicing. This process is not only a cost effective technology but also has potential for batch processing. Two structures can be generated by this process. Therefore, this sensor can provide multiple functions. It can be used to measure the temperature, strain, pressure, refractive index of chemicals and thin film thickness by itself. It can also be multiplexed with itself, with other type of Fabry-Perot sensors and with gratings to perform multiple point measurements or to function as temperature compensation element for other perturbation measurements.

\subsection{Sensor Fabrication}

Microfabrication techniques have been developed that allow differential etching of the core with respect to the cladding of the fiber. With suitable buffered acid solution, micro-pits can be obtained in the cleaved fiber end faces. These etched fibers can then be spliced with other unetched fibers to generate micro-air-gaps inside the fibers. The air-gap can be used as reflective mirrors to form Fabry-Perot interferometers with the cleaved fiber end faces or with other air-gaps, and function as sensing elements.

Usually, the core of the fiber is made out of germania doped silica, while the cladding is made out of pure silica. Using a suitable buffered acid solution $\left(\mathrm{NH}_{4} \mathrm{~F}: \mathrm{HF}: \mathrm{H}_{2} \mathrm{O}\right)$ for etching the core faster than the cladding results in a pit, whose base diameter corresponds to the single mode core of the fiber. There is a limit for the solution ratio between $\mathrm{NH}_{4} \mathrm{~F}$ and $\mathrm{HF}$ (volume ratio between $\mathrm{NH}_{4} \mathrm{~F}: \mathrm{HF}: \mathrm{H}_{2} \mathrm{O}$ is for this limit ratio 1.7:1:1) where the etching speed of the cladding equals that of the core. For values of the volume ratio of $\mathrm{NH}_{4} \mathrm{~F}$ to $\mathrm{HF}$ higher than 1.7:1, the core is etched more slowly than the cladding, resulting in an inversion of the 
etching speed. In this case, pure silica, i.e. the fiber cladding, is etched at a faster rate than doped silica, i.e. the core of the fiber, thus resulting in a corn on the fiber end face[12].

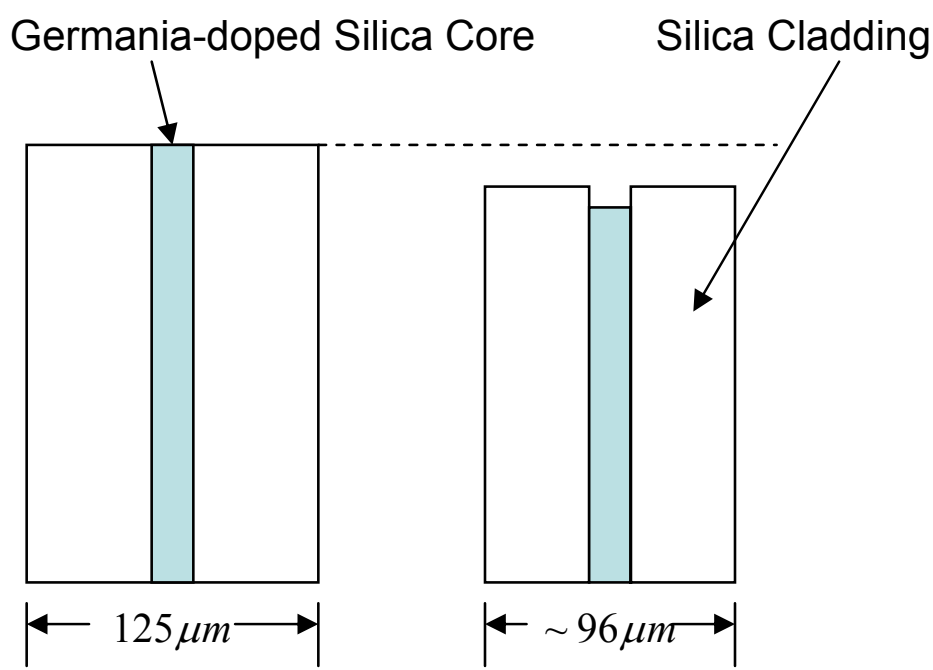

(a) (b)

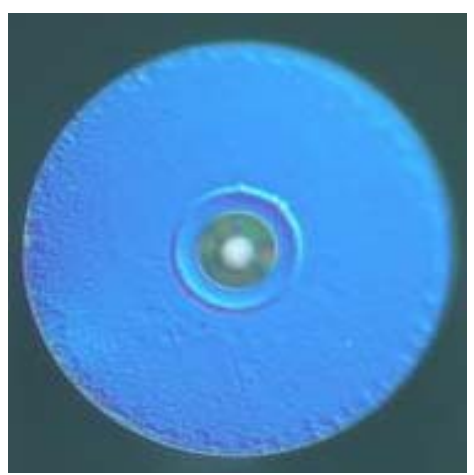

(c)

Figure 6.1. Differential wet chemical etching (a) Before etching; (b) After etching; (c) End face of etched fiber

The guiding fiber and sensing fiber used are Corning single-mode fiber (SMF-28). Before undertaking the fusion splicing process, one fiber is differentially etched with a suitable acid solution while the other fiber is well cleaved. Low arc currents and short splicing times are used to fusion splice them to create a micro-air-gap between etched fiber and unetched fiber. After this mirror is formed in the fiber, the bare fiber is cleaved to the desired Fabry-Perot cavity length. This structure is shown in Figure 6.2.

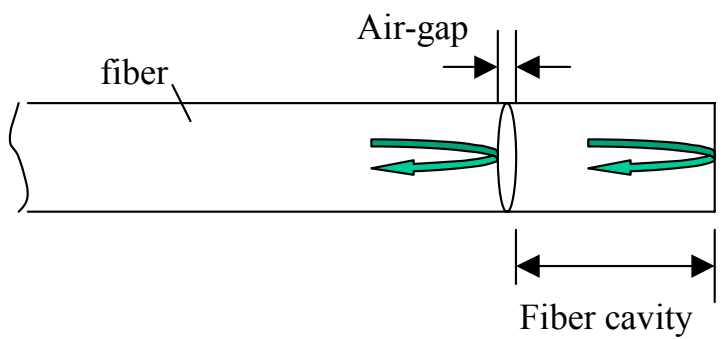

Figure 6.2. The $1^{\text {st }}$ structure of the sensor. 
The other possible structure of this sensor is created by adding to the above structure by splicing another etched fiber to the cleaved fiber end. Another identical micro-air-gap can be formed. These two micro-air-gaps function as two reflective mirrors of the interferometer. The length of the fiber cavity between the two micro-air-gaps is the Fabry-Perot cavity length. Compared with the first structure, the advantage of this structure is the capability of multiplexing with other sensors.

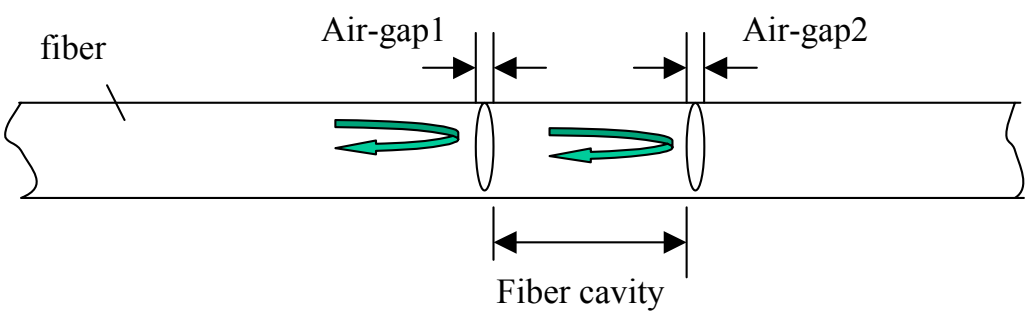

Figure 6.3. The $2^{\text {nd }}$ structure of the sensor.

\subsection{Theoretical Analysis}

When the light is guided into the sensor, the light reflected from the end surface of the airgap or from the cleaved fiber end face will generate constructive (i.e., reflections are in phase with each other) or destructive (i.e., reflections are out of phase with each other) interference. If the refractive index and/or length of fiber cavity are changed by perturbation, the reflective power will sweep through the maximum and minimum interference effects. These signals can be processed to determine the amount of perturbation.

Multiple cavities (including air-cavity and fiber-cavity) are formed in both structures of this sensor because of multiple reflective surfaces, especially in the $2^{\text {nd }}$ structure. For simplicity, we model the $1^{\text {st }}$ structure as the case of three reflective surfaces. Three reflective surfaces have its reflectance $\left(R_{1}, R_{2}, R_{3} R_{1}\right)$ and transmittance $\left(\eta_{1}, \eta_{2}, \eta_{3}\right)$. The cavity lengths are $\mathrm{L}_{1}$, $\mathrm{L}_{2}$ and $\mathrm{L}_{3}=\mathrm{L}_{1}+\mathrm{L}_{2}$. We assume that the energy of light from the source is 1 .

The phase shift can be obtained from

$$
\varphi=\frac{4 \pi n L \cos i}{\lambda},
$$

where $\mathrm{i}$ is the incident angle of the light. $\mathrm{n}$ is the refractive index of the fiber, and $\mathrm{L}$ is the length of the fiber cavity. 


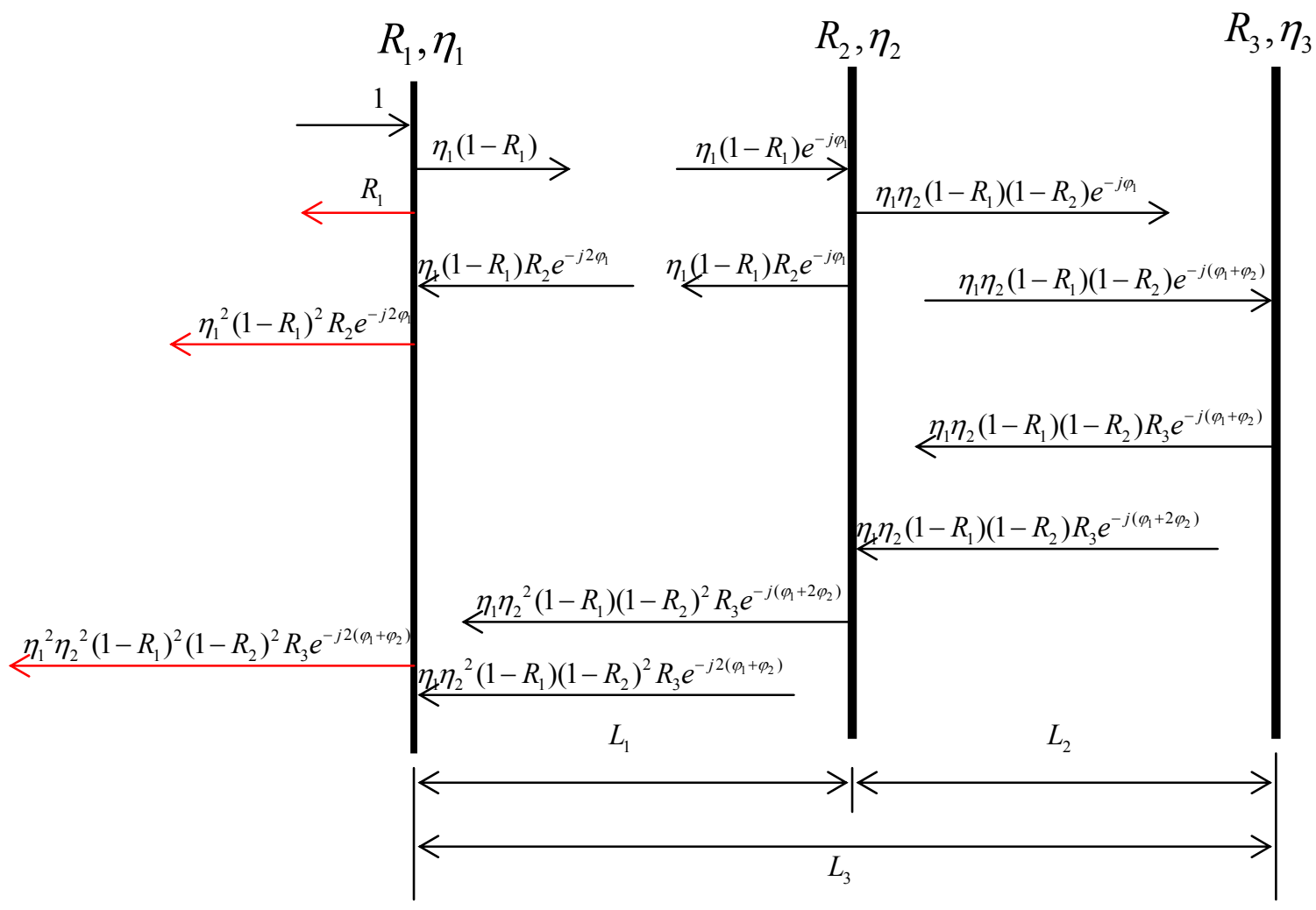

Figure 6.4. Theoretical model for multiple cavities

Three different rays are reflected back. They are:

Ray 1 with energy: $R_{1}$;

Ray 2 with energy: $\eta_{1}^{2}\left(1-R_{1}\right)^{2} R_{2} e^{-j 2 \varphi_{1}}$

Ray 3 with energy: $\eta_{1}{ }^{2} \eta_{2}^{2}\left(1-R_{1}\right)^{2}\left(1-R_{2}\right)^{2} R_{3} e^{-j 2\left(\varphi_{1}+\varphi_{2}\right)}$

We sum them together, the total energy received by the detector can be

$$
E=R_{1}+\eta_{1}^{2}\left(1-R_{1}\right)^{2} R_{2} e^{-j 2 \varphi_{1}}+\eta_{1}^{2} \eta_{2}^{2}\left(1-R_{1}\right)^{2}\left(1-R_{2}\right)^{2} R_{3} e^{-j 2\left(\varphi_{1}+\varphi_{2}\right)},
$$

and the intensity I is:

$$
I=E \times E^{*} .
$$

For this sensor, it is difficult to obtain the size of the air-gap. We did some simulation and compared the simulation spectrum with the spectrum captured from the CTS. We can approximately know that the size of air-gap is around $1.5 \mu \mathrm{m}$. As an example, we know that the fiber cavity length is $68.66 \mu \mathrm{m}$ from measurement. In simulation, we set $L_{2}=68.66 \mu \mathrm{m}$, and when $L_{1}=1.5 \mu \mathrm{m}$, the spectrum from the simulation (Figure 6.5) is close to the actual spectrum shown in Figure 6.6. 


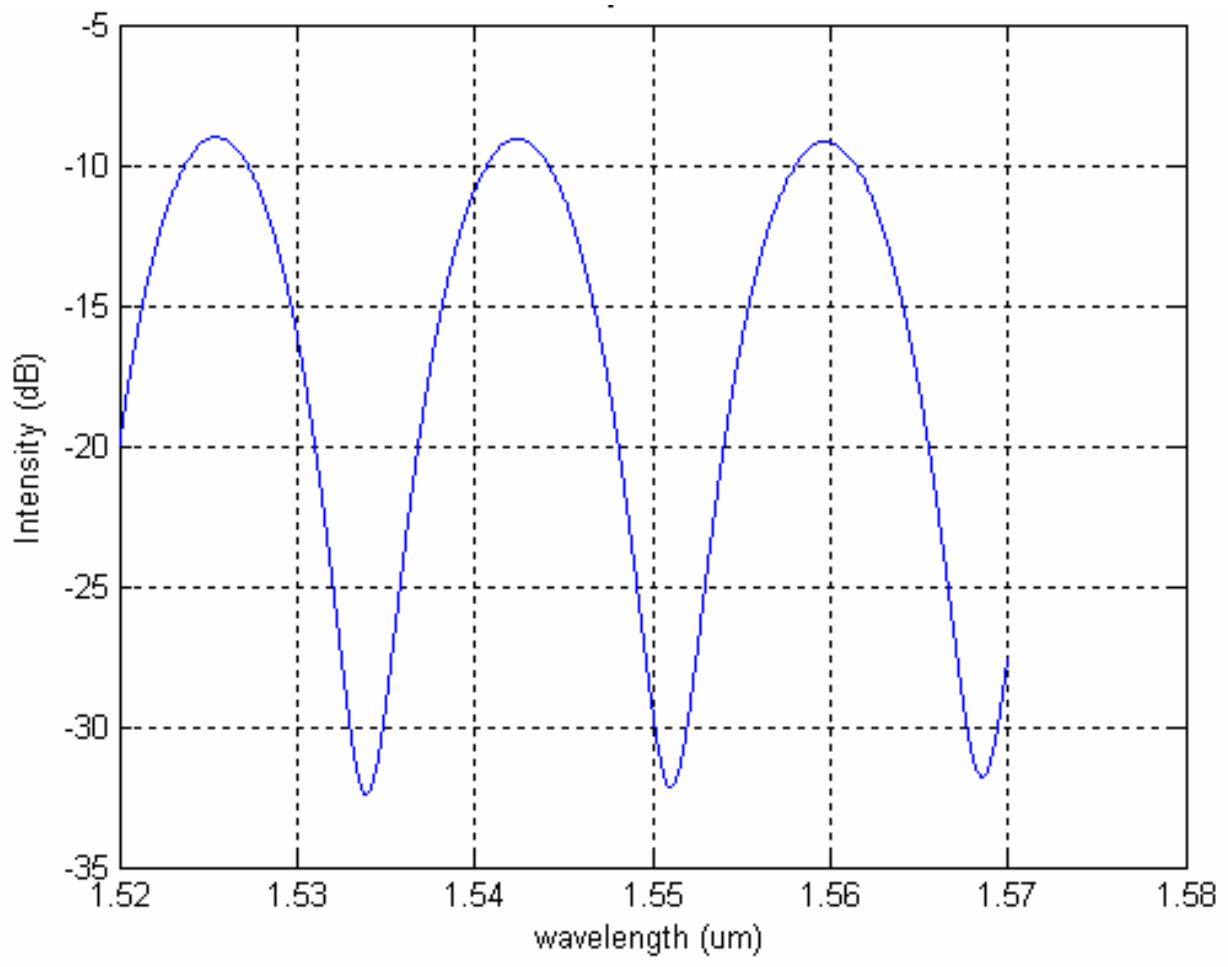

Figure 6.5. Simulation based on theoretical model

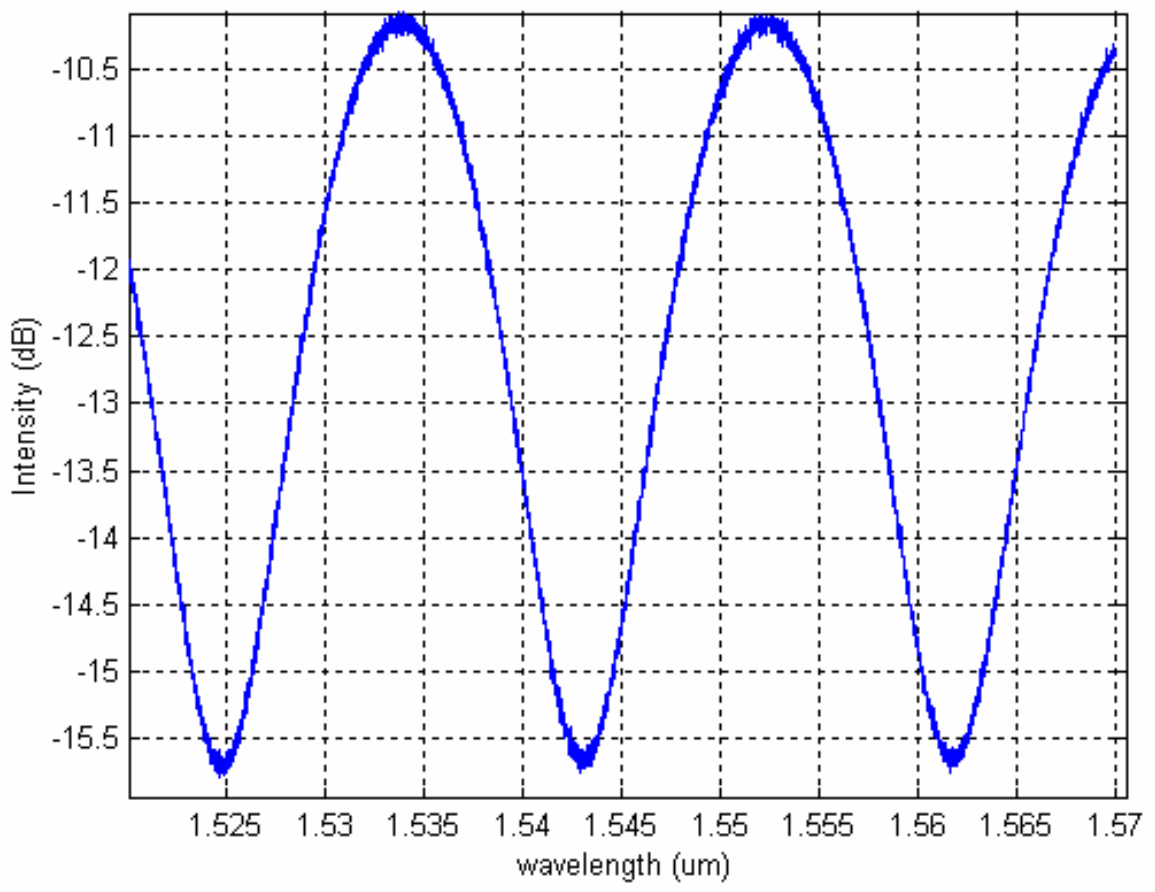

Figure 6.6. Interference fringes captured from testing system 


\subsection{System Setup and Measurement Results}

The Micron Optics CTS system was used to monitor the interference fringes. Signal processing provided the capacity to filter out the desired signal in the frequency domain. The principle of the CTS system is shown in Figure 6.7.

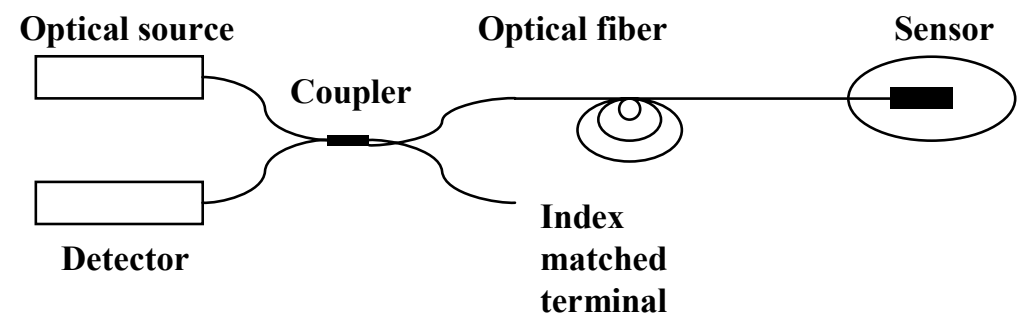

Figure 6.7. Principle diagram of the sensor system

\subsubsection{Temperature Measurement}

We used both structures of this sensor to measure temperature changes. Their performance as temperature sensors were demonstrated from room temperature to $700^{\circ} \mathrm{C}$.

The temperature measurement can be determined by

$$
\lambda=L\left(\frac{d n}{d T}+n \frac{d L}{d T}\right) \Delta T,
$$

where $\lambda$ is the wavelength of light source, $\mathrm{dn} / \mathrm{dT}$ is the thermooptic coefficient, and $\mathrm{dL} / \mathrm{dT}$ is the thermal expansion coefficient of the sensing fiber, $\Delta T$ is the temperature change per period of fringe. For Corning SMF-28, $\mathrm{n}=1.4488$ at wavelength of $1550 \mathrm{~nm}$, $d L / d T \approx 0.55 \times 10^{-6} /{ }^{\circ} \mathrm{C}, d n / d T \approx 9.2 \times 10^{-6} /{ }^{\circ} \mathrm{C}$.

We used a sensor with a fiber cavity length of $623.0656 \mu \mathrm{m}$ to measure the temperature. Our measurement showed that the optical length changed $4.41 \mathrm{~nm} /{ }^{\circ} \mathrm{C}$. In Figure 6.8 , temperature was increased four times and in Figure 6.9 , temperature decreased from $700^{\circ} \mathrm{C}$ to $50^{\circ} \mathrm{C}$ four times. These two figures showed that this sensor has high repeatability in temperature measurement. We show the hysteresis test of the sensor in Figure 6.10. The hysteresis in this measurement was caused by the speed difference of heating and cooling of the furnace which was used for testing. 


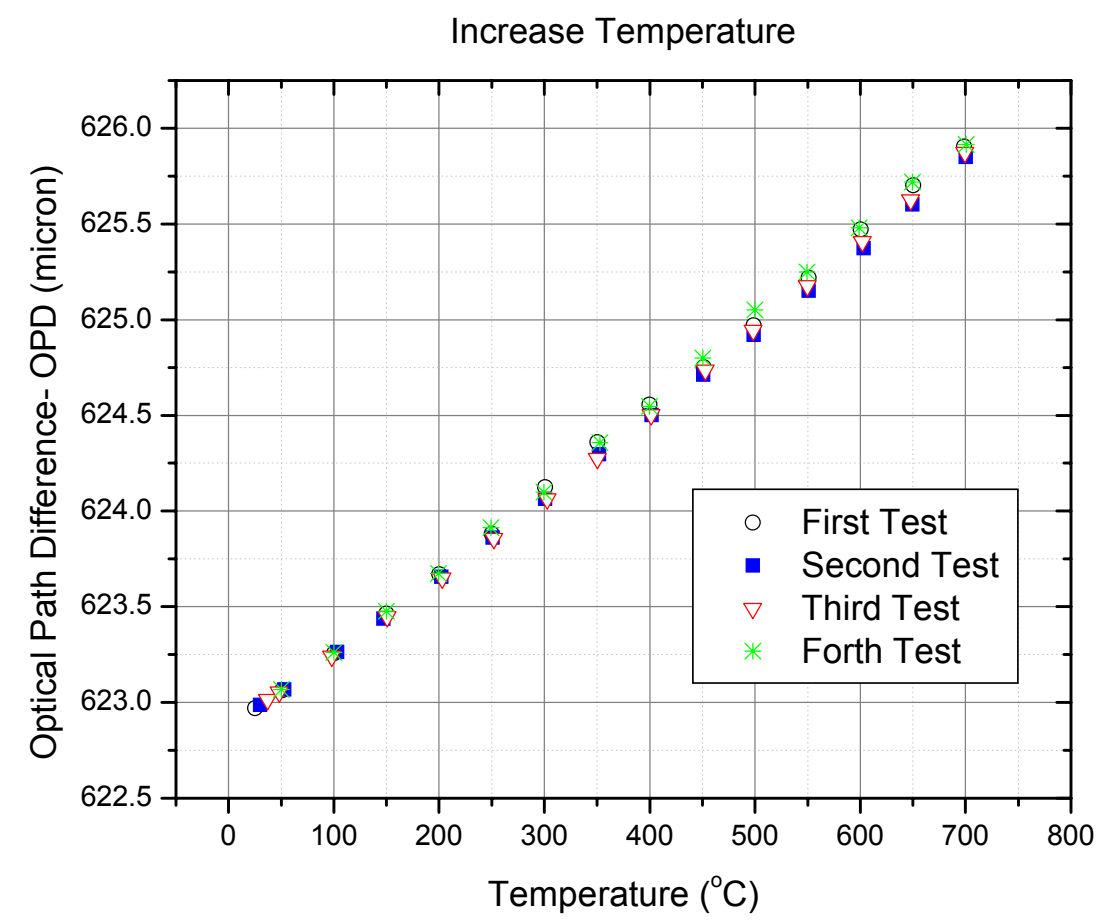

Figure 6.8. The output of OPD when the temperature was increased.

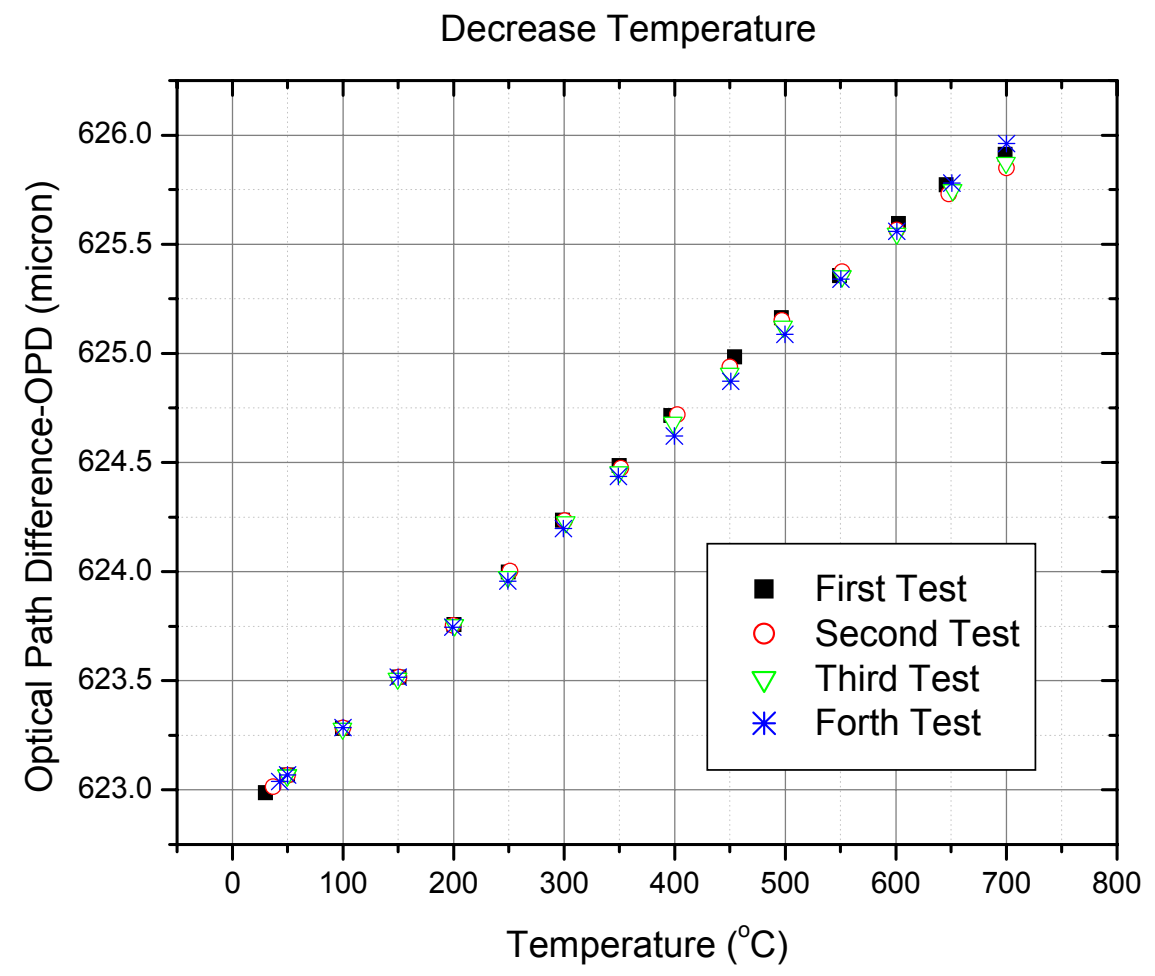

Figure 6.9. The output of OPD when the temperature was decreased. 


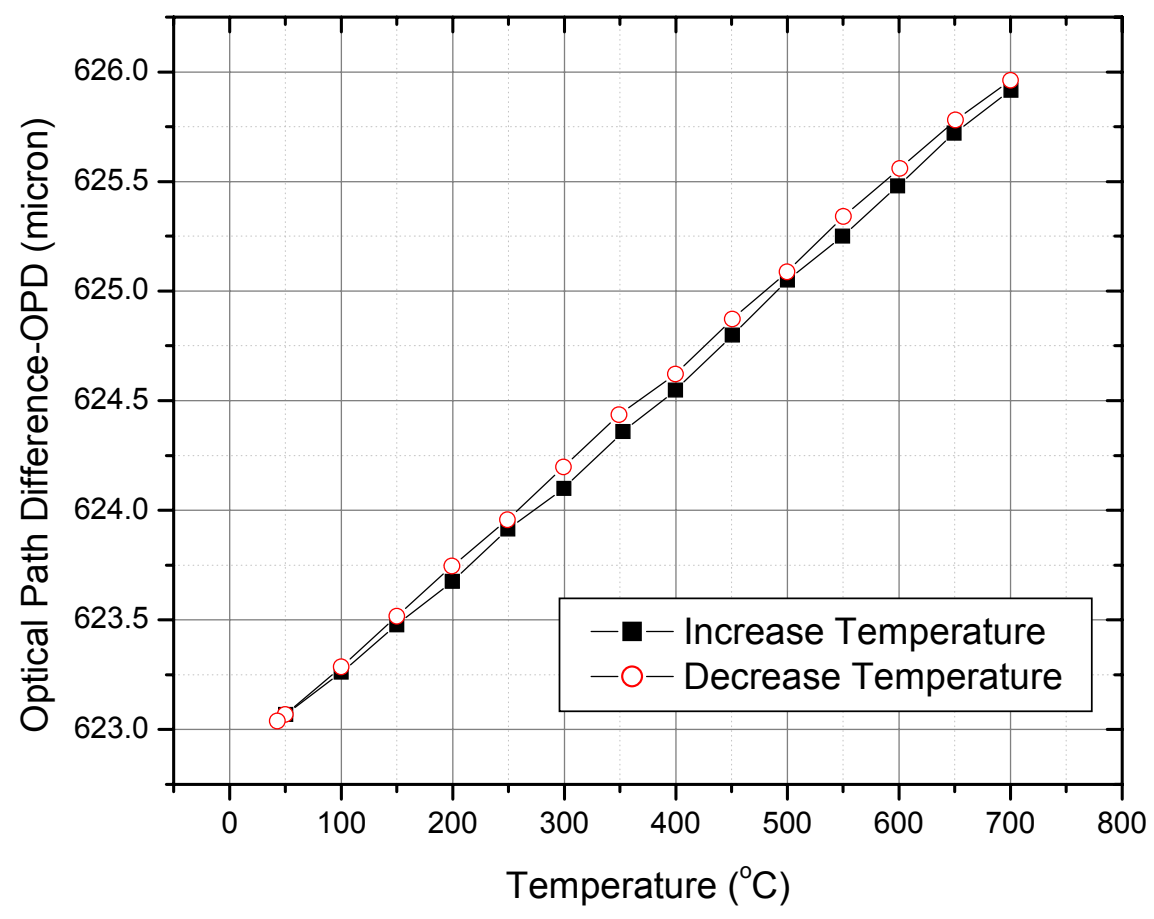

Figure 6.10. Hysteresis of temperature measurement.

\subsubsection{Strain Measurement}

For strain measurement, we use the $2^{\text {nd }}$ structure of the sensor to cascade two sensors to make multi-point strain measurements. We used a Foil Gauge as a reference to calibrate the optical sensors. The strain test setup is shown in Figure 6.11.

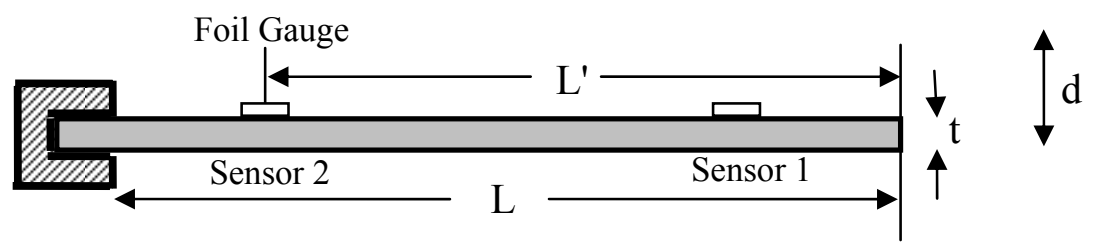

Figure 6.11. Setup for strain measurement.

Theoretically, the stain is

$$
\text { TheoryStrain }=\frac{3 L^{\prime} t d}{2 L^{3}},
$$

where $t$ is the thickness of the substrate to support the sensors and the $d$ is the height when one side of the substrate can be lifted up to cause the strain change. $L$ is the total length of the substrate and L' is the distance between the substrate end and where foil gauge was placed. 
If we measure the optical path changes to obtain the strain change information, we can use the following equations:

$$
\begin{aligned}
& \frac{\Delta O P D}{O P D_{0}}=\frac{\Delta n}{n_{0}}+\frac{\Delta l}{l_{0}}=0.737 \frac{\Delta l}{l_{0}} \\
& \text { OpticalStrain }=\frac{1}{0.737} \frac{\Delta O P D}{O P D_{0}}
\end{aligned}
$$

Figure 6.12 and Figure 6.13 show that the optical sensors' test results agreed with the results from foil strain test system. Figure 6.14 shows that the experimental results agree with the theoretical results.

\subsection{Temperature Compensation}

\subsubsection{Temperature Compensation for Long Period Gratings}

Long period gratings are very sensitive to temperature change. In order to compensate for temperature, we connected an intrinsic Fabry-Perot sensor with a LPG. The spectrum after multiplexing a FP sensor and a LPG is shown in Figure 6.15 and an FFT plot of it is shown in Figure 6.16.

\subsubsection{Temperature Compensation for Miniature Pressure Sensor}

Diaphragm-based miniature pressure sensors have the capability to work at high temperature. For the best performance, the effect of temperature also needs to be deleted. Multiplexing an intrinsic Fabry-Perot temperature sensor and extrinsic Fabry-Perot pressure sensor is a currently ongoing task. 


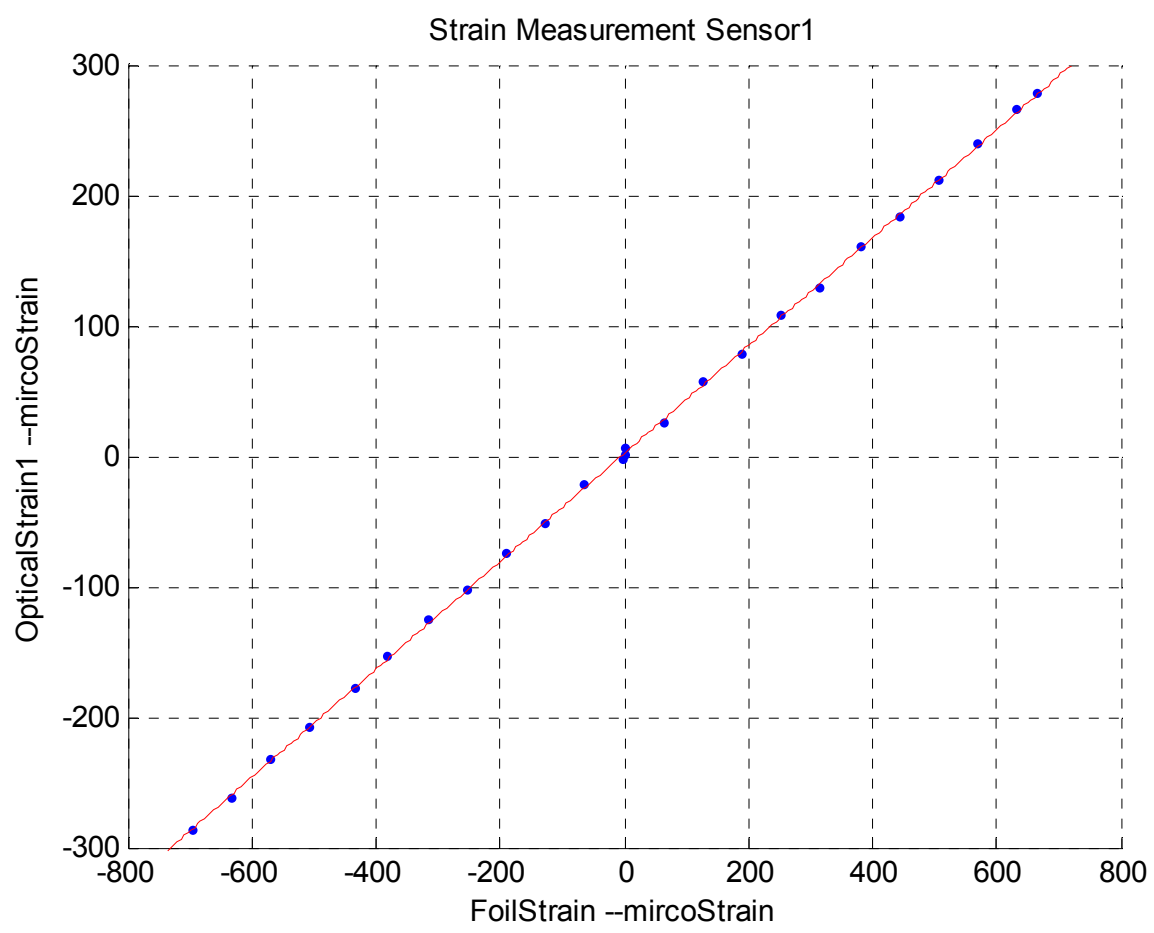

Figure 6.12. Strain test result of the $1^{\text {st }}$ sensor.

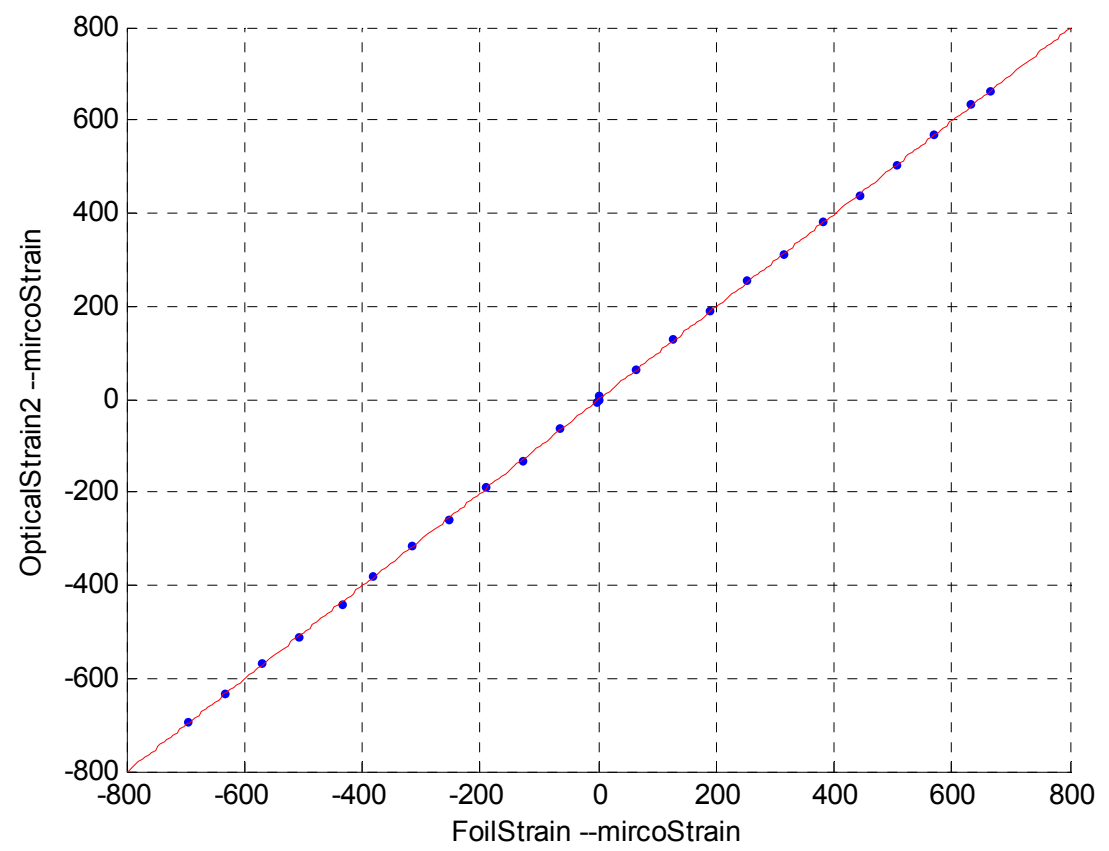

Figure 6.13. Strain test result of the 2 nd sensor. 


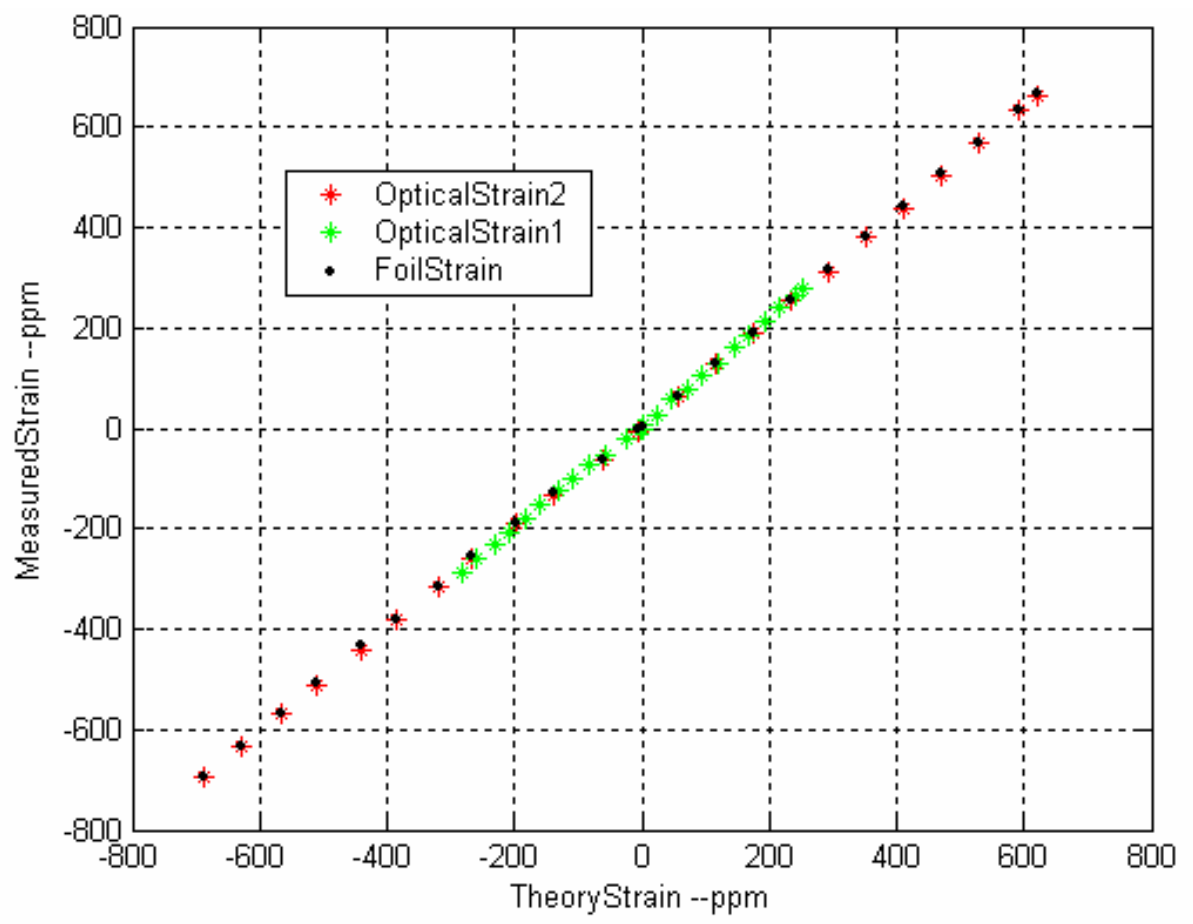

Figure 6.14. Comparison between the optical sensors and the foil strain gauge.

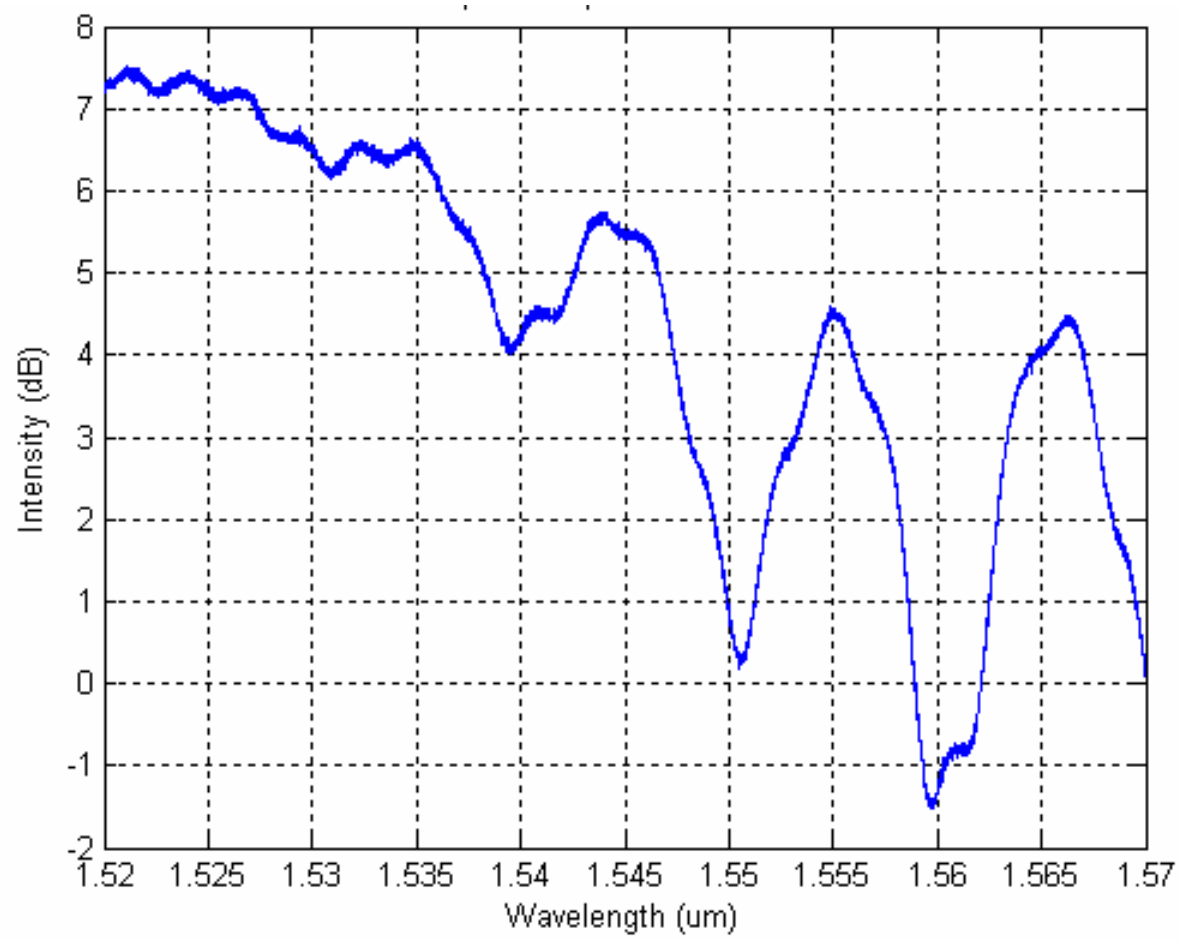

Figure 6.15. Spectrum of multiplexed FP sensor and LPG. 


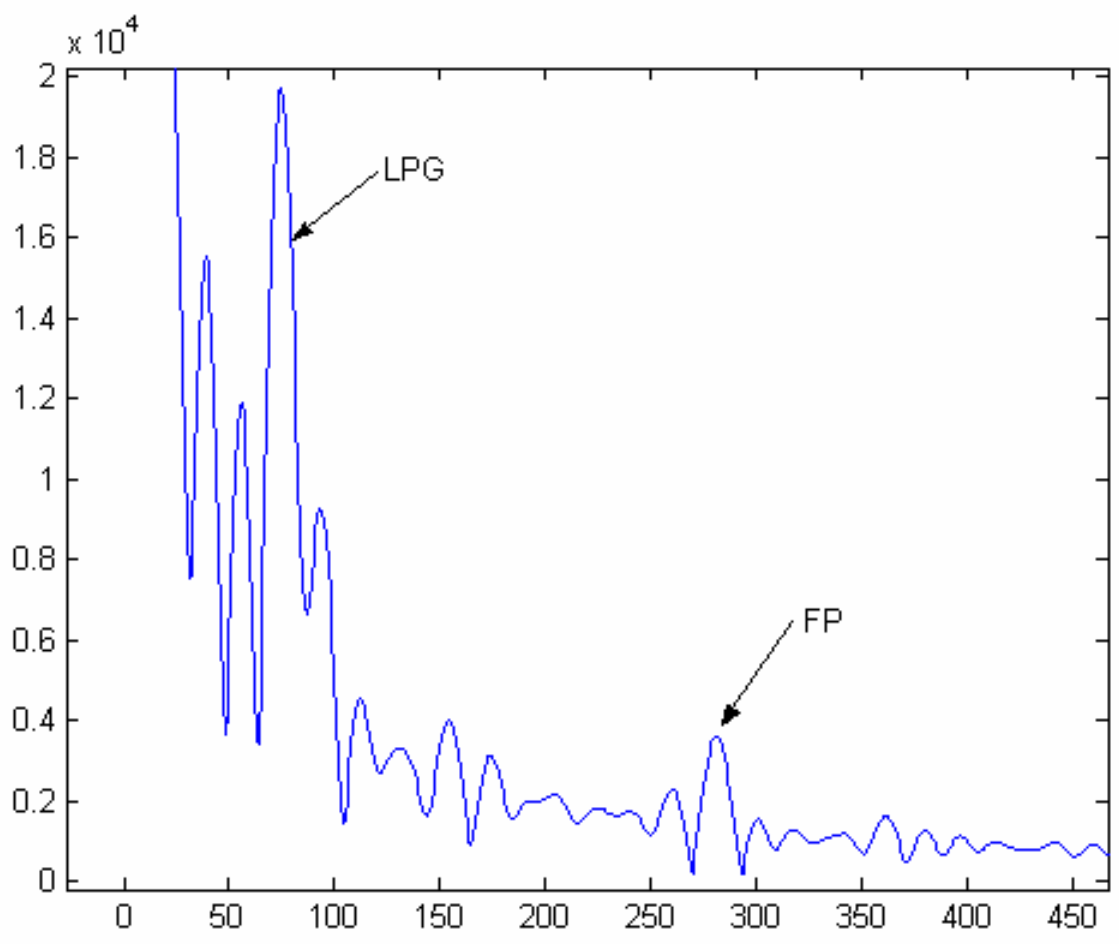

Figure 6.16. FFT of the spectrum of multiplexed FP and LPG. 


\subsection{Multiplexed Intrinsic Fabry-Perot Interferometric (IFPI) Sensors}

Fabry-Perot (FP) interferometric fiber sensors have attracted considerable interest for temperature, strain, pressure and many other physical parameter sensing in the past decade [13-20]. Lee and Taylor et al. demonstrated FP interferometers by building dielectric mirrors into the fiber[13-15]; Research groups at Virginia Tech developed extrinsic Fabry-Perot interferometer (EFPI) sensors by forming an air gap between the end faces of two uncoated fibers inserted into an alignment tube[16, 17]; Sirkis et al. introduced inline fiber etalons by fusion splicing two fibers with a section of hollow core fiber of the same outside diameter[18]. These FP interferometers had been utilized for various applications such as temperature, strain, pressure[17], electromagnetic field[21], and ultrasound sensing[5]. However, these extrinsic interferometers usually have relatively high reflectance and power loss and are therefore unable to be highly multiplexed.

In order to multiplex a very large number of sensors, intrinsic Fabry-Perot interferometers (IFPIs) with low reflectance are desirable. Recently the researchers in the Center for Photonics Technology (CPT) at Virginia Tech proposed and demonstrated several new methods to fabricate IFPI sensors inside a fiber[22], and also demonstrated a spatial frequency division multiplexing method that promises the potential of dense sensor multiplexing. Further, the latest experiment showed the capability of measurement of different physical parameters, including temperature, strain and pressure capable of operation at high temperatures above $600^{\circ} \mathrm{C}$.

\subsection{Principle of Operation of IFPI Sensor}

Large refractive index changes on the order of $10^{-2}$ can be achieved in both hydrogen loaded and commercially available Ge or B doped fiber by means of ultraviolet irradiation. When a segment of photosensitive fiber is exposed to UV, the refractive index of the fiber is changed, so a local Fresnel reflector is created that behaves as a partial mirror. A pair of such reflectors can form an intrinsic Fabry-Perot interferometric sensor that can be used to measure physical parameters such as temperature, strain, pressure, vibration etc.

Figure 7.1 shows the structure and point-by-point fabrication method of IFPI sensors. A narrow slit formed by two metallic plates is placed in front of the fiber to act as a shadow mask. The photosensitive fiber is irradiated on the side by UV. The position and width of the slit can be adjusted through 3-D translation stages so that the width of writing point can be controlled and a section of the laser beam profile can be selected for given laser beam pattern. In general, this width is set to about 20 30 microns in the manufacturing procedure. 


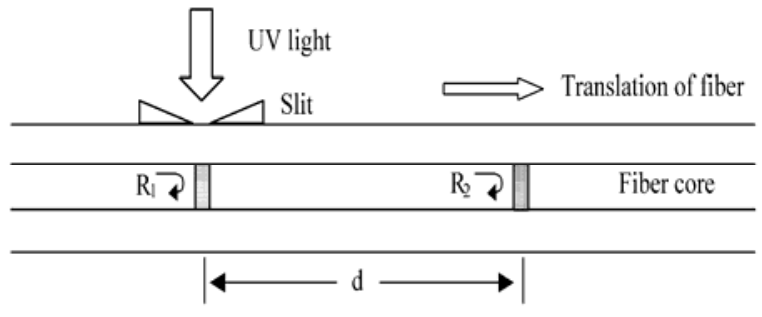

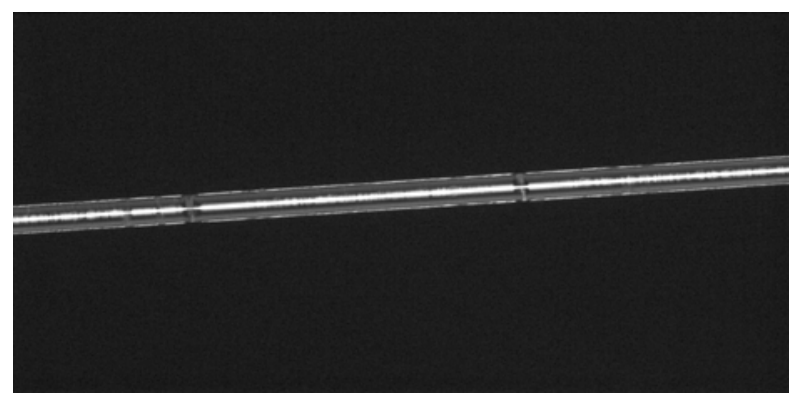

Figure 7.1. UV-induced IFPI sensor structure (left) and photograph (right).

The position of the fiber can also be adjusted through 3-D translation stages. After one point is written, the fiber is moved along the axial direction for a certain distance, then another point is written. These two points act as two partial mirrors and produce a Fabry-Perot cavity. Figure 7.1 shows a photograph of an IFPI sensor with FP cavity length of 1 millimeter. The optical path distance (OPD) between the two reflectors is

$$
L=2 n_{\text {eff }} d
$$

where $\mathrm{d}$ is the distance between the two reflectors and $\mathrm{n}_{\mathrm{eff}}$ is the effective refractive index of the fiber at a certain wavelength.

Due to the low reflectance at each reflector, the interference between the two reflections at wavelength $\lambda$ is approximately

$$
I=I_{0} \cdot\left[R_{1}+R_{2}+2 \sqrt{R_{1} R_{2}} \cos \left(\frac{2 \pi L}{\lambda}+\phi\right)\right]
$$

where

I Reflected light power measured at wavelength $\lambda$.

$\mathrm{I}_{0} \quad$ Power of the light source at wavelength $\lambda$.

$\mathrm{R}_{1}, \mathrm{R}_{2}$ Reflectivity of the reflectors at wavelength $\lambda$.

$\phi \quad$ Constant phase difference term due to the phase changes on reflection interface.

$\lambda \quad$ Wavelength of light source.

The normalized interference at wavelength $\lambda$ can be written as

$$
\frac{I}{I_{0}}=R_{1}+R_{2}+2 \sqrt{R_{1} R_{2}} \cos \left(\frac{2 \pi L}{\lambda}+\phi\right)
$$

The width of the exposed segment is controlled to be in orders of micrometers, a broadband Fresnel reflector can be achieved, the reflection coefficient $\mathrm{R}_{1}$ and $\mathrm{R}_{2}$ and phase term $\phi$ an be considered to be wavelength independent. From Eq. (5-3), it is known that the normalized interference for different wavelength is only related to the $L$ and $\lambda$. 
From Eq. (7-1)-(73), it can be seen that that the IFPI sensor can be used to measure any quantity that can change either the refractive index or fiber dimension or both. Some of the common parameters include temperature, strain and pressure etc. By measuring the interferogram of the interferometer in a certain wavelength range, the OPD $L$ can be estimated. Thus, the parameters that cause the OPD change can be determined.

\subsection{IFPI Sensor Fabrication}

An IFPI sensor fabrication system has been developed in Center for Photonics Technology at Virginia Tech. The system consists of an UV Excimer laser, optical components including UV-grade mirror and lens, metallic shadow mask, 3-D translation stages and a monitoring system. A KrF excimer laser (MPB Communication Inc, MSX-250) with 248nm wavelength is used as UV source. A UV-grade mirror and a UV-grade lens are used to focus the laser beam onto the side of the photosensitive fiber. A pair of metallic plates is used to form a narrow slit that just allow a small portion of laser beam to pass though. The two plates are mounted onto 3-D stages. The slit width can be controlled by change the positions of the two plates. The distance between the slit and the focus lens can also be adjusted so that the laser power density irradiated to the fiber can be controlled. The photosensitive fiber is placed behind the slit and contact with the metallic plates to reduce the influence of laser beam diffraction. The fiber is also mounted onto a 3-D translation stage so that the position of the laser writing point can be translated and the axis direction of the fiber can be adjusted to be perpendicular to the slit. An optical component test system (Micron Optics, SI720), which function as both a sweeping laser source and an optical spectrum analyzer, together with an optical circular, which couples the reflected light to optical detector, are used to monitor the sensor fabrication procedure. The operation principle of the monitoring and measuring system will be explained in Section 7.3 below. Finally, a microscope is used to observe the laser writing point so that the slit width can be roughly estimated. A diagram and photograph of the sensor fabrication system are shown in Figure 7.2.

We have fabricated IFPI Sensors with different cavity lengths from tens of micrometers to several centimeters to meet the sensitivity requirements of physical parameter measurement, and have demonstrated their potential for use in applications such as temperature, strain and pressure sensing, as discussed further in Section 7.4.

\subsection{Measuring System}

The reflection of the UV-induced IFPI sensor is pretty low. It is difficult to measure the interferogram by conventional methods that utilize a low-coherence light source and passive optical spectrum analyzer. In our measuring system, a Micron Optics Si720, which contains a sweeping laser source, detectors, intensity and wavelength referencing units and supporting electronic circuits, is used as an optical spectrum measuring equipment. The diagram of the measuring system for single or multiplexed IFPI sensors is shown in Figure 7.3. 

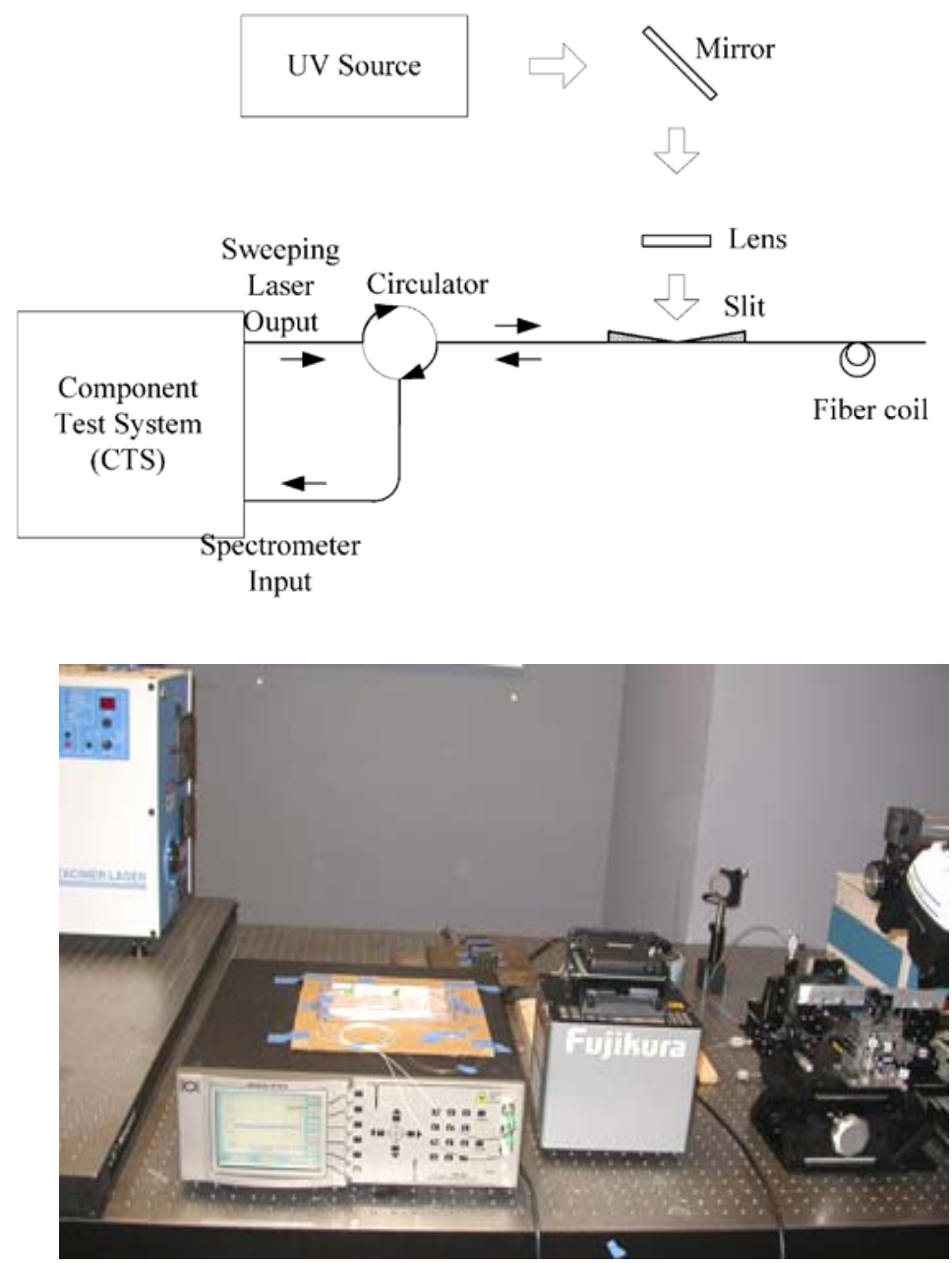

Figure 7.2. IFPI sensor fabrication system.

Optical Spectrum Analyzer

(Micron Optics Si720)

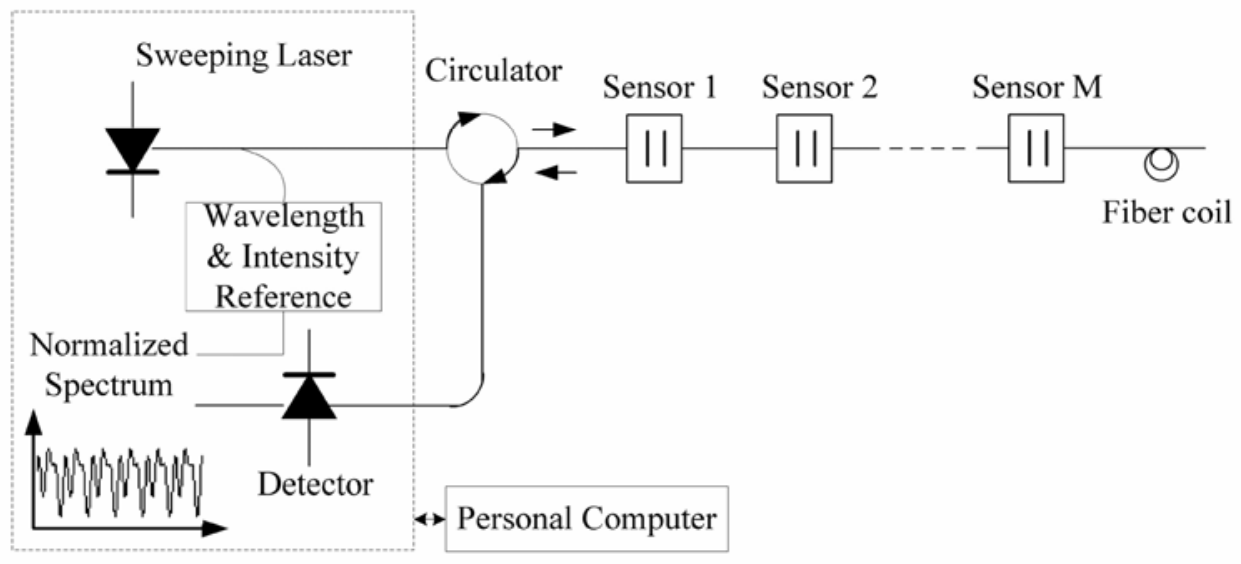

Figure 7.3. Diagram of IFPI measuring system. 
Narrowband light from the sweeping laser is launched into an optical circulator, which transmits the light to the IFPI sensors. At each sensor, part of the light is reflected back to the circulator. The circulator then couples the reflected light to an optical detector. The power of the reflected light is measured by the detector. A portion of the light is tapped from the light source inside the spectrum analyzer. The intensity and wavelength of the tapped light is measured as intensity and wavelength reference. Then the normalized interference at a certain wavelength can be measured by Eq. (5-3). The laser is operated to finish a sweep in 200 milliseconds. In total, 20,000 samples are measured in the $1520 \mathrm{~nm} \sim 1570 \mathrm{~nm}$ wavelength range with a wavelength step of 2.5 picometers during one sweep of the laser source. The measured interferogram is transferred to a personal computer via a GPIB interface. A Matlab program running on host computer is written to retrieve the interferogram data from the spectrum analyzer and to do the signal processing job to estimate the frequency and phase information of the interferogram.

We have developed an interferogram signal processing algorithm operating in wave number domain and spatial frequency domain. The measured interferogram in wave number domain may contain multiple spatial frequency components. FIR digital bandpass or lowpass filter is designed to select individual frequency component. Filtered signal in wave number domain will only contain single sinusoidal term. Then we use peak detection algorithm to find the positions of peaks and valleys of the sinusoid. Frequency and phase information of the sinusoid can be estimated by analyze positions of peaks and valleys. Therefore, the OPD of each IFPI sensor can be estimated.

\subsection{Measurement Results}

We have designed IFPI sensors to measure various physical parameters such as temperature strain and pressure etc.

\subsubsection{Temperature Sensors}

The optical path length (OPD) of the IFPI sensor will change due to the temperature dependence of the refractive index and thermal expansion. The change of the OPD is given by

$$
\delta L=2 *(\delta n * l+n * \delta l)=2 *\left(n_{T} * \Delta T * l+n * l * \alpha * \Delta T\right)
$$

where $\delta \mathrm{L}$ Change of OPD.

$\delta$ Change of refractive index.

$\delta 1$ Change of geometry length.

$\mathrm{n}$ Effective refractive index of fiber.

L Distance between two reflectors.

$\mathrm{n}_{\mathrm{T}}$ Thermal optic coefficient $\left(\sim 1.0^{*} 10^{-5} /{ }^{\circ} \mathrm{C}\right)$.

$\alpha$ Thermal expansion coefficient $\left(\sim 0.5^{*} 10^{-6} /{ }^{\circ} \mathrm{C}\right)$.

The potential of the IFPI for use as a temperature sensor has been experimentally confirmed. Figure 7.4 shows the temperature response of an initial IFPI temperature sensor with a $1000 \mu \mathrm{m}$ cavity length tested in the temperature range $25^{\circ} \mathrm{C}-650^{\circ} \mathrm{C}$. 


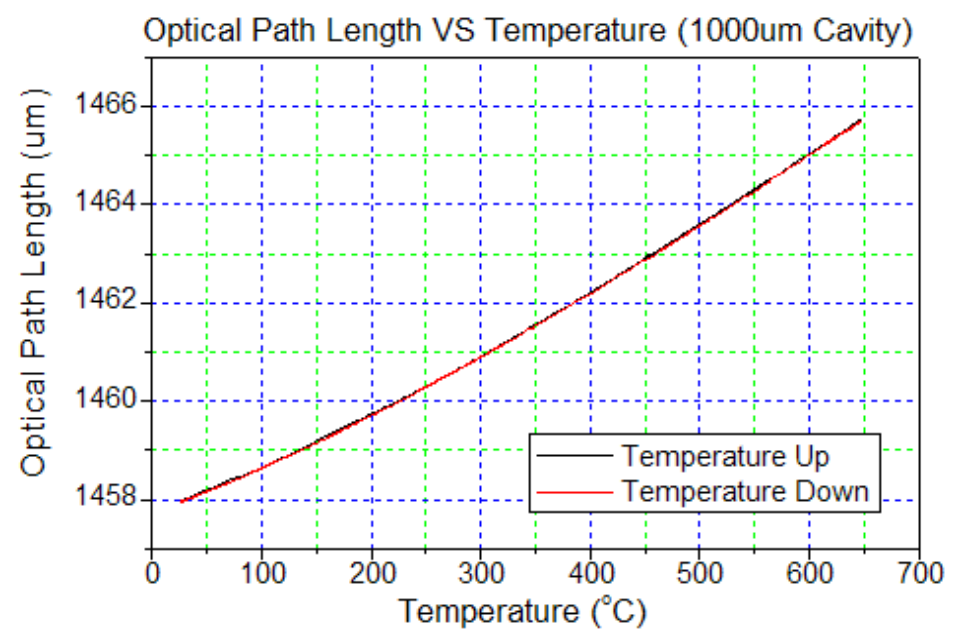

Figure 7.4. IFPI temperature sensor experimental results.

\subsubsection{Strain Sensors}

The IFPI sensor can be designed to measure the strain of the fiber caused by external tensile or shear stress. The optical path length (OPD) of the IFPI sensor will change due to the deformation of the cavity and the stress-optic effect of the optical fiber. The change of OPD is given by

$$
\delta L=2 *(\delta n * l+n * \delta l)=2 *\left(n_{c} * \varepsilon * l+n * \varepsilon * l\right)
$$

where $\delta \mathrm{L}$ Change of OPD.

$\delta$ Change of refractive index.

$\delta 1$ Change of geometry length.

$\mathrm{n}$ Effective refractive index of fiber.

1 Distance between two reflectors.

$\mathrm{n}_{\mathrm{c}}$ Stress-optic coefficient.

$\varepsilon \quad$ Strain of fiber defined as $\delta 1 / 1$.

Figure 7.5 shows test results of an IFPI strain sensor as a function of applied tensile stress.

\subsubsection{Sensors for Additional Parameters}

By applying a glass tube, the IFPI sensor can also be used to measure pressure, acoustic signals, and vibration. Figure 7.6 shows a schematic of the proposed structure of a mechanically packaged IFPI sensor head. The IFPI sensor (fabricated in the fiber) is inserted into a fused silica tube whose inside diameter (132um) matches the outer diameter of the bare fiber (125um). The tube and the fiber are welded together at both ends by using a $\mathrm{CO}_{2}$ laser. Figure 7.7 shows the test results of an IFPI pressure sensor. 


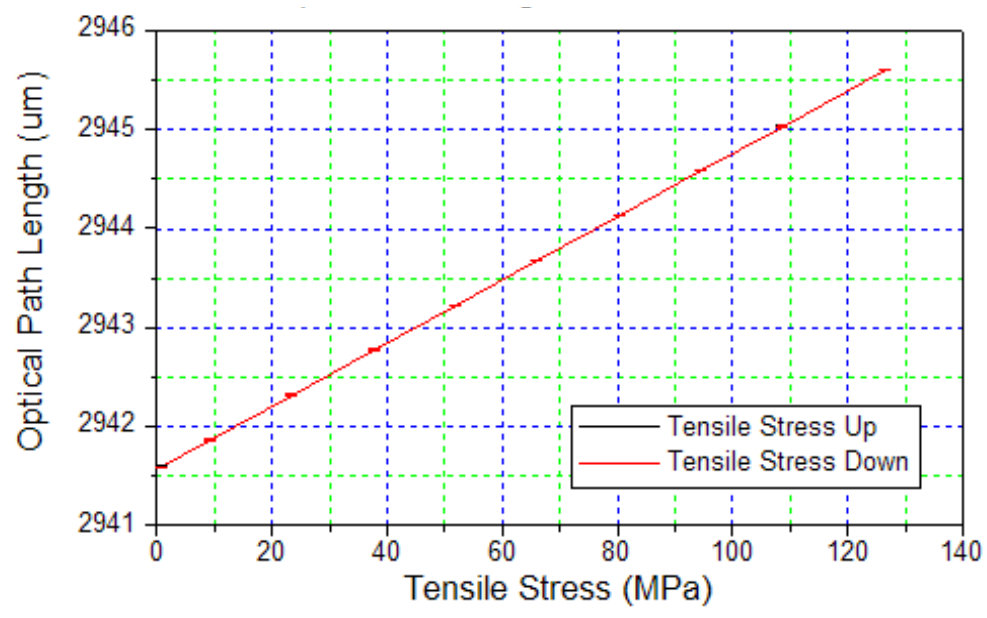

Figure 7.5. IFPI strain sensor experimental results.

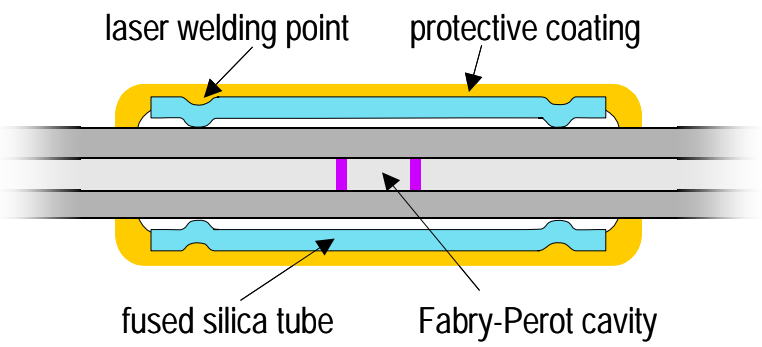

Figure 7.6. Schematic representation of the proposed IFPI pressure sensor.

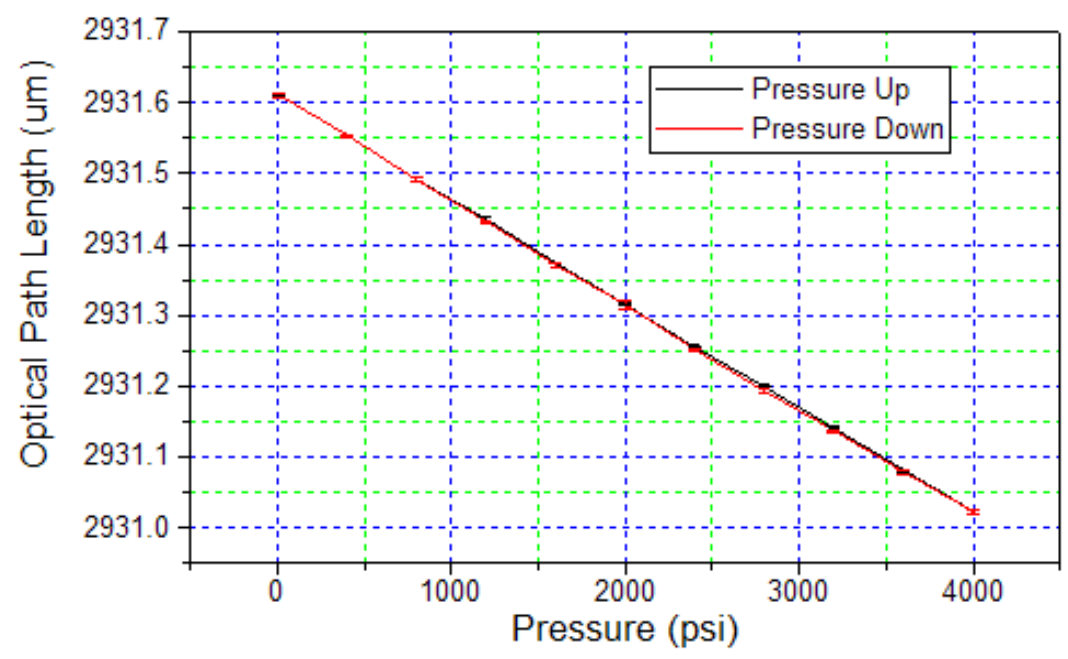

Figure 7.7. IFPI pressure sensor experimental results. 


\subsection{Multiplexing of IFPI Sensors}

The typical reflection of a UV-induced IFPI sensor is in the range of $-60 \mathrm{~dB}$ to $-50 \mathrm{~dB}$, depending on the refractive index profile achieved at the laser writing point. This results in minimal loss in transmitted light power. Typical IFPI sensors we have made using commercially available photosensitive fiber have an insertion loss less than $0.1 \mathrm{~dB}$. The power loss can be reduced to a very low level if the multiple splicing points between traditional fiber and photosensitive fiber can be eliminated. This type of low reflectance intrinsic FP sensor is a very good candidate for multiplexed sensor array and sensor networks.

Many multiplexing techniques for interferometric sensors have been investigated[23], including time[24, 25], frequency[26], wavelength[27], code[28], spatial[29], and coherence[30, 31] division multiplexing. Hybrid techniques that combine different multiplexing schemes have also been proposed[32]. We have demonstrated both time division multiplexing (TDM) and spatial frequency division multiplexing (SDM) schemes in laboratory tests using the UV-induced IFPI sensors.

\subsubsection{SDM Scheme}

In the SDM scheme, the sensors are sequentially arranged as shown in Figure 7.3. After intensity normalization and wavelength calibration, the interferogram of the IFPI sensors can be determined. If the cavity length of each sensor is different, the interferometric fringe pattern of each sensor will have different spatial frequency. By separating each frequency component and measuring its frequency and phase information, the optical path length of each IFPI sensor can be determined, so that the physical parameters surrounding the IFPI sensors can be measured.

The sensor frequency is dependent on the optical path distance (OPD) of the IFPI cavity. By designing sensors with different cavity lengths, we can locate these frequency components in the spatial frequency domain. When the source wavelength is swept from $1520 \mathrm{~nm}$ to $1570 \mathrm{~nm}$, a normalized interferogram from sensors can be measured and transferred to a computer for signal processing. The captured interferogram may contain multiple frequency components if multiple sensors are interrogated. A FFT based algorithm was developed to estimated the frequency and phase of these components. Figure 7.8 shows a representative interferogram and its FFT when 11 sensors are multiplexed.

The maximum frequency of these frequency components is limited by the coherence length of the light source and the number of sampling points of the data acquisition system. First, the maximum OPD of an IFPI sensor should not exceed the coherence length of the light source, otherwise the interference will not be detectable for this sensor. Second, the maximum frequency should be less than half of the sampling frequency according to the Nyquist sampling theorem. Because we are using a sweeping laser with narrow line width and a large number of data sampling points, a large frequency range is available; in our current SDM system, a spatial frequency bandwidth of $10 \mathrm{kHz}$ is available. If each sensor is designed to have a bandwidth of $5 \mathrm{~Hz}$, then the multiplexing capacity is approximately $10 \mathrm{k} / 5=2,000$. In an ideal situation, 2,000 sensors can be multiplexed if the power loss of each sensor is very low, cross-talk between sensors can be neglected, signal noise ratio (SNR) of 
each sensor is sufficient and signal processing speed is high enough. Practically, the multiplexing number of sensors can not reach the bandwidth limit due to degradation factors such as power loss, cross-talk, SNR etc. In a preliminary laboratory experiment, 20-30 sensors have been multiplexed on a single fiber for temperature sensing using the SDM technique with the OPD difference of each sensor of $150 \mathrm{um}$, and reasonable reflected light power and signal processing speed. If the influence of these degradation factors can be reduced, this number has the potential to be greatly increased.
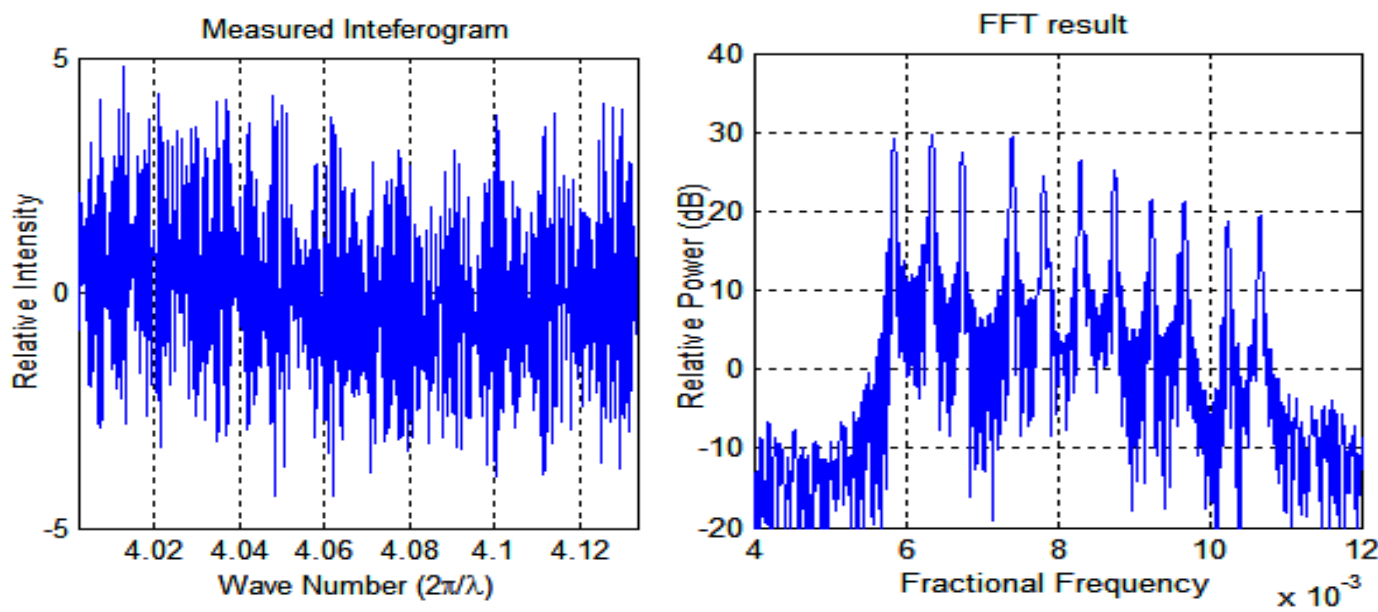

Figure 7.8. Interferogram and FFT result of signals from 11 sensors.

\subsubsection{TDM scheme}

In the TDM scheme, the sensors are also serially arranged and signals from different sensors are multiplexed in the time domain. An optical time domain reflectometer (OTDR) is often used to detect sensor signals of TDM scheme. A pulse of laser light is transmitted down to the sensor array. Individual sensors in the multiplexed sensor array are addressed by the time of flight of the optical pulses. The reflected pulses are amplitude modulated by the physical parameter to be measured. By measuring the intensity response of each sensor, the physical parameters of the environment surrounding the IFPI sensors can be determined. The diagram of TDM scheme is shown in Figure 7.9.

\subsubsection{Sensor Network With Star Topology}

Potentially, a large number of sensors can be multiplexed along a single fiber. Yet the number is limited due to technical and reliability reasons. To increase the multiplexing capability, a large number along single fiber is preferred. To decrease the fault probability of the sensor array, the number along single fiber should be limited. To obtain a good balance between these requirements, a sensor network with star topology can be utilized. Sensor arrays will be cyclically scanned by optical switching. The multiplexing scheme for each sensor array can be either TDM or SDM as discussed above. A sensor network with this kind of star topology has the potential to integrate a large number of sensors into one network with 
higher flexibility, reliability and reasonable response speed. The diagram of sensor network with star topology is shown in Figure 7.10.

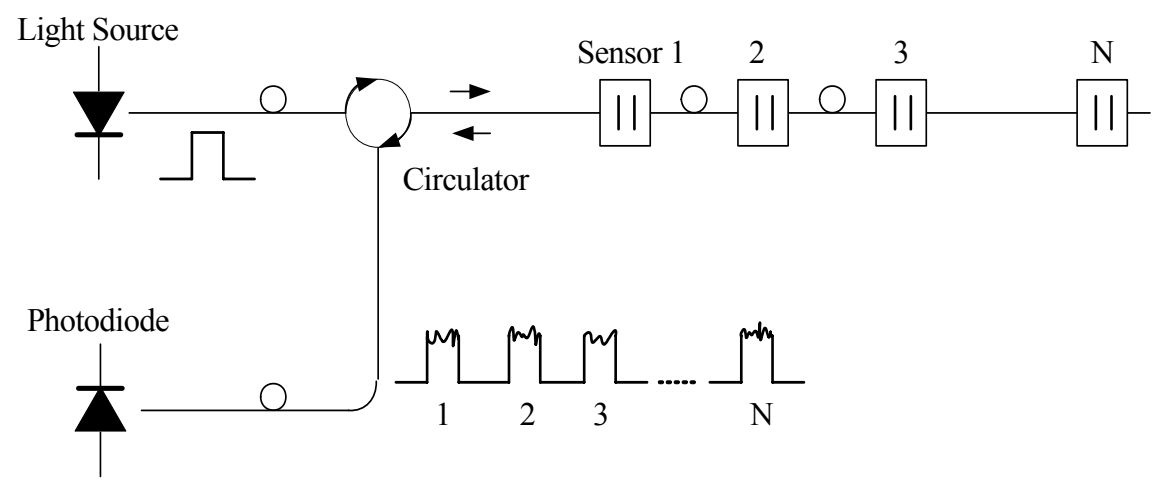

Figure 7.9. TDM multiplexing scheme.

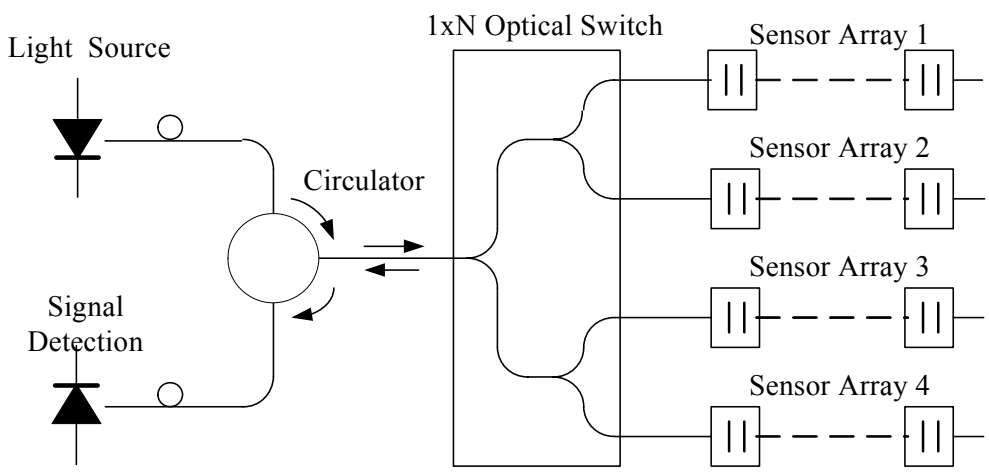

Figure 7.10. Optical switch based star topology structure. 


\subsection{Testing in Simulated Harsh Environments}

Single crystal sapphire and zirconia were exposed in glass melts in order to measure the corrosion rates at different temperatures and for different lengths of time. The thickness of the samples was measured before and after corrosion experiments and their difference represents the amount of corrosion in a glass medium.

\subsection{Experiment Procedures and Sample Preparation}

Single crystal sapphire and zirconia were cut using a diamond saw into strips (about $0.8 \mathrm{~mm}$ thick). One of each was placed in a $5 \mathrm{~mL}$ alumina crucible with crushed soda lime glass and heated at $1200^{\circ} \mathrm{C}, 1300^{\circ} \mathrm{C}$ and $1400^{\circ} \mathrm{C}$ for four different time periods: 1 day, 2 days, 3 days and 4 days. Upon cooling, slices were cut from the crucibles that included both sapphire and zirconia samples as in Figure 8.1.

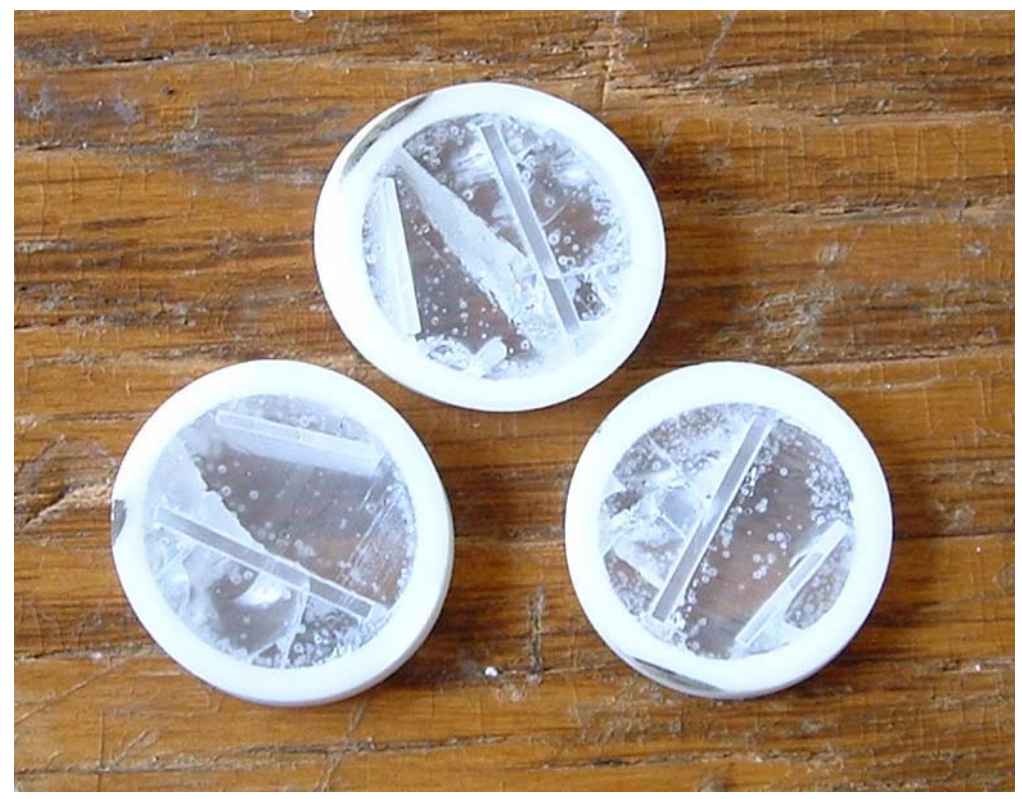

Figure 8.1. Slices of crucibles with sapphire and zirconia in soda lime glass.

\subsection{Thickness Measurements}

The initial thickness was measured with a vernier caliper. It was not possible to obtain a reliable thickness measurement after reaction in this way due to the fact that the sapphire and 
zirconia samples were encased in glass. Two methods were employed to measure the final thickness, dissolution by hydrofluoric acid and SEM analysis.

\subsubsection{HF Method}

The glass was first dissolved with hydrofluoric acid and then the sample thickness was measured with a vernier caliper. This method was very slow because soda lime glass takes a long time to dissolve in HF due to calcium fluoride residue formation. Another problem was that when the glass was dissolved there was nothing to hold cracked sample pieces together. This was especially important for the zirconia samples and in some cases, the zirconia samples fell into small pieces. This made thickness measurements difficult.

\subsubsection{Scanning Electron Microscopy (SEM)}

Micrographs of samples that included both interfaces were taken by SEM. The sample thickness was measured and multiplied by the magnification to obtain the final thickness of sapphire and zirconia. Since the interfaces of all samples were clear in general, this method worked well. Figure 8.2 shows a cross section of a sapphire sample in soda lime glass. The interface is clear, but it can be seen from this picture that the glass cracked around the samples. This cracking probably took place during the cooling process.

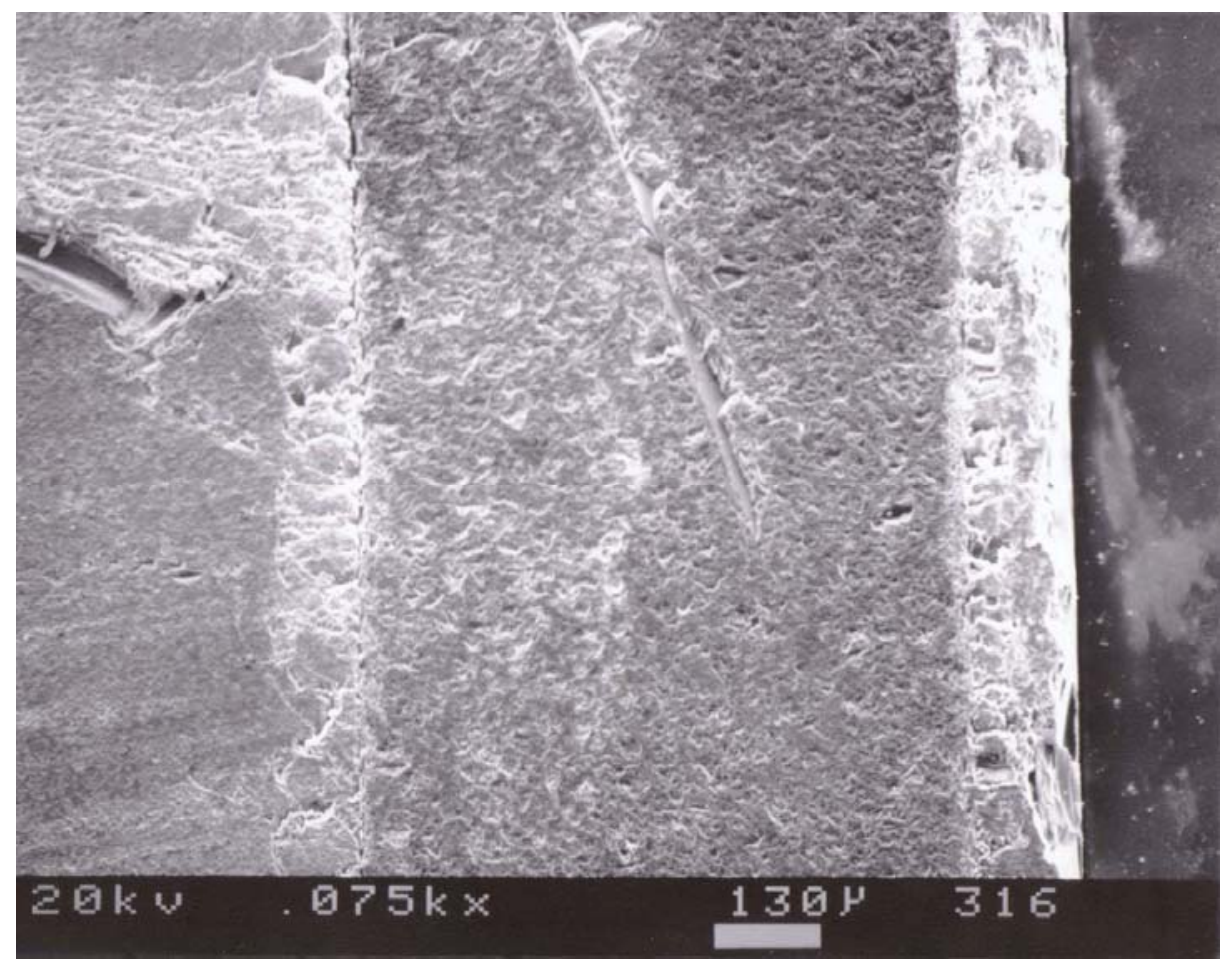

Figure 8.2. Cross section of sapphire in soda lime glass. 


\subsubsection{Results}

The results obtained to date are listed in Table 8.1 for both methods of thickness measurement. The goal was to obtain the change in thickness. Data obtained using the SEM method needs to be corrected for the angle of tilt of the specimen. The angle at which some samples are tilted has not been measured yet.

Table 8.1. Experimental thickness measurement data employing both methods.

\begin{tabular}{|c|c|c|c|c|c|c|c|c|c|c|c|c|c|c|c|c|c|c|c|}
\hline 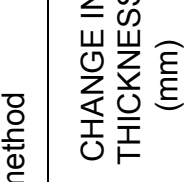 & & $\begin{array}{l}0 \\
0 \\
0 \\
0\end{array}$ & & & & $\stackrel{8}{\circ}$ & & & $\stackrel{\varphi}{0}$ & & & $\begin{array}{l}\text { ஜ } \\
\text { \& } \\
0 \\
0 \\
0\end{array}$ & & & & ஜ & & $\begin{array}{l}0 \\
0 \\
\infty \\
0 \\
0\end{array}$ & \\
\hline 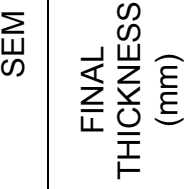 & & $\begin{array}{l}\frac{1}{L} \\
\stackrel{5}{0} \\
0\end{array}$ & & & & 咅 & & & : & & & $\begin{array}{l}\text { L } \\
\text { L } \\
\infty \\
0 \\
0\end{array}$ & & & & $\begin{array}{l}\hat{\theta} \\
0\end{array}$ & & $\begin{array}{l}\frac{\hat{\sigma}}{\pi} \\
\stackrel{1}{0}\end{array}$ & \\
\hline 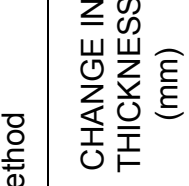 & & & $\begin{array}{l}\text { 守 } \\
\text { o } \\
\text { i }\end{array}$ & 0 & & $\begin{array}{l}\text { స̃. } \\
\text { ర. } \\
0\end{array}$ & & & & & & & $\begin{array}{l}0 \\
0 \\
0 \\
0 .\end{array}$ & & & 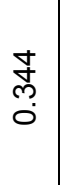 & & & \\
\hline 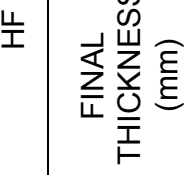 & & & $\begin{array}{l}\text { f } \\
\text { o } \\
0 \\
0\end{array}$ & $\stackrel{\infty}{\circ}$ & & $\begin{array}{l}\frac{\infty}{N} \\
0 \\
0\end{array}$ & & & & & & & $\begin{array}{l}\underset{\pi}{6} \\
0\end{array}$ & & 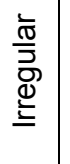 & $\begin{array}{l}\stackrel{0}{\sigma} \\
\stackrel{ \pm}{0}\end{array}$ & & & \\
\hline 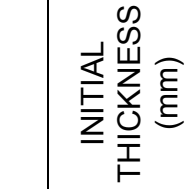 & $\begin{array}{l}\infty \\
0 \\
0\end{array}$ & $\stackrel{\infty}{0}$ & $\stackrel{\infty}{0}$ & $\stackrel{\infty}{0}$ & $\stackrel{\infty}{0}$ & $\begin{array}{l}\infty \\
0\end{array}$ & $\begin{array}{l}\infty \\
\stackrel{\infty}{0}\end{array}$ & $\stackrel{\infty}{0}$ & $\stackrel{\infty}{\circ}$ & $\stackrel{\infty}{0}$ & 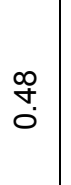 & 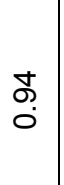 & $\begin{array}{l}0 \\
\stackrel{0}{0}\end{array}$ & $\begin{array}{l}\stackrel{R}{\stackrel{0}{0}} \\
\stackrel{0}{*}\end{array}$ & 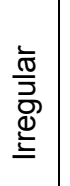 & $\begin{array}{l}\stackrel{0}{\circ} \\
0\end{array}$ & $\begin{array}{l}\frac{\bar{\pi}}{\bar{J}} \\
\stackrel{\Phi}{\underline{\underline{D}}}\end{array}$ & $\stackrel{\infty}{0}$ & $\begin{array}{l}\frac{\bar{\omega}}{\bar{\sigma}} \\
\bar{\sigma} \\
\underline{\underline{\omega}}\end{array}$ \\
\hline 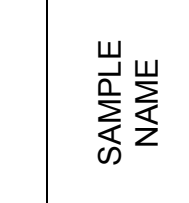 & 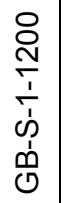 & 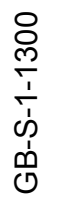 & 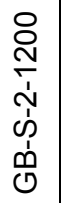 & 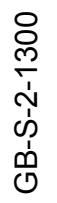 & 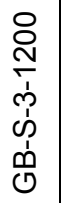 & 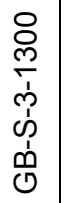 & 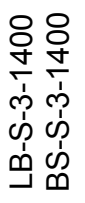 & 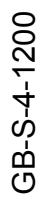 & 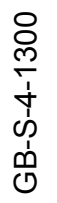 & 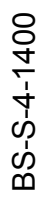 & 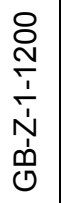 & 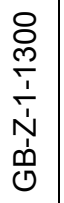 & 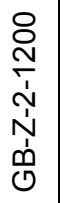 & 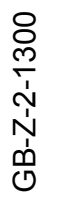 & 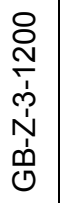 & 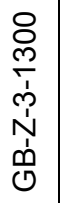 & 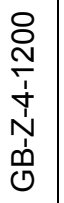 & 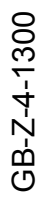 & 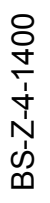 \\
\hline 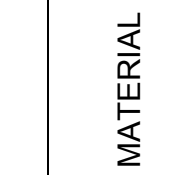 & \multicolumn{10}{|c|}{ 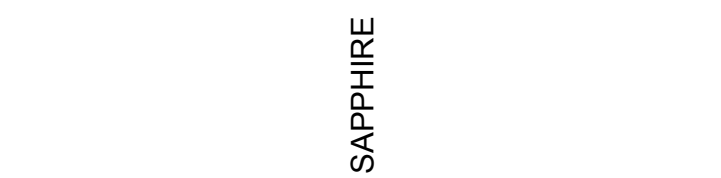 } & \multicolumn{9}{|c|}{ 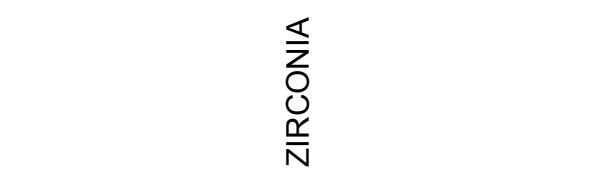 } \\
\hline 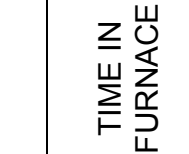 & \multicolumn{2}{|c|}{ 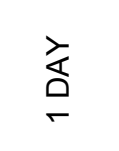 } & \multicolumn{2}{|c|}{ 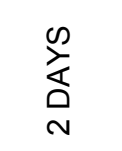 } & \multicolumn{3}{|c|}{ 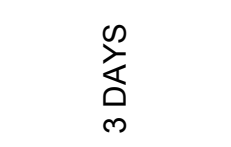 } & \multicolumn{3}{|c|}{$\begin{array}{l}\infty \\
\frac{1}{\Delta} \\
\dot{\sigma}\end{array}$} & \multicolumn{2}{|c|}{ 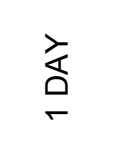 } & \multicolumn{2}{|c|}{$\begin{array}{l}\infty \\
\stackrel{\infty}{\Delta} \\
\sim\end{array}$} & \multicolumn{2}{|c|}{ 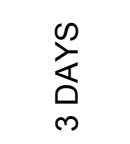 } & \multicolumn{3}{|c|}{ 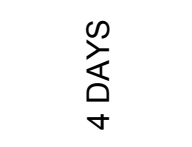 } \\
\hline$\sum_{\Perp}^{0} \underset{0}{0}$ & 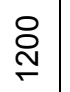 & ষ্লি & 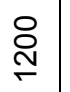 & 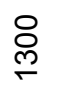 & $\underset{ָ}{\stackrel{ }{~}}$ & $\begin{array}{l}\text { ঃ্ল } \\
\end{array}$ & 守 & ્ֻญ & ষ্লি & ষ্ণে & 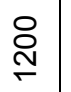 & $\begin{array}{l}\text { ঃ্লি } \\
-\end{array}$ & $\underset{ָ}{\stackrel{ }{\sim}}$ & 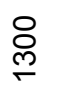 & $\underset{ָ}{\stackrel{ }{\leftarrow}}$ & 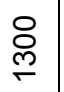 & $\underset{ָ}{\stackrel{ }{\leftarrow}}$ & 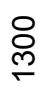 & $\stackrel{8}{\stackrel{+}{+}}$ \\
\hline
\end{tabular}




\subsection{Energy Dispersive X- ray Analysis (EDX)}

In order to observe the interface between sapphire and zirconia samples and glass, EDX measurements were performed. The elements selected for study were the major components of soda lime glass (silicon, sodium and calcium), and major components of the samples aluminum for sapphire and zirconium for zirconia.

Figure 8.3 represents the EDX analysis of the interface between a zirconia sample and soda lime glass heated at $1300^{\circ} \mathrm{C}$ for 3 days. Glass is on the left of the interface and the zirconia sample on the right. Four elements were observed: silicon (top left), sodium (top right), zirconium (bottom left), and calcium (bottom right). The interface is clear. The spots of silicon in zirconia could be due to smudging during the cross sectional cut, or filling of cracks in zirconia while the glass was still molten.

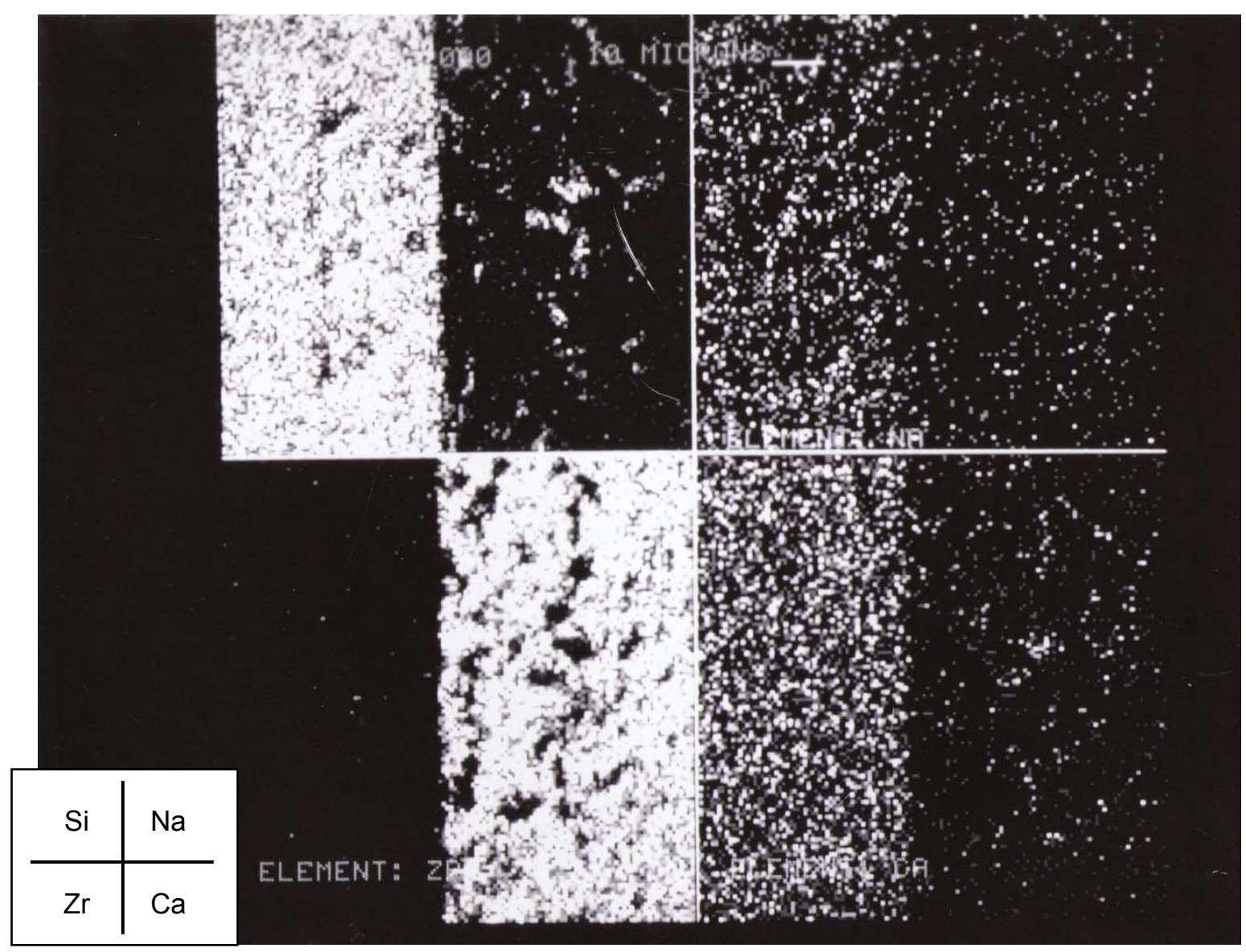

Figure 8.3. EDX of zirconia-glass interface for silicon (top left), sodium (top right), zirconium (bottom left) and calcium (bottom right).

Spot scans were performed on both sides of the interface to check for penetration of glass into sapphire and zirconia. The scans show that even close to the interface there is little mixing- the spot in the zirconia side of the interface shows mostly zirconia and the spot on the glass side shows mostly the elements of soda lime glass. 


\subsection{Instrumentation Development}

\subsection{System Structure}

The diaphragm-based SCIIB engine sensor system consists of the following sub-systems (Figure 9.1):

- A diaphragm based pressure sensor

- A high power light source system

- Optical components

- Two photodiodes and preamplifiers

- Voltage amplifiers and a analog divider

- $\mathrm{A} / \mathrm{D}$ and $\mathrm{D} / \mathrm{A}$ converters

- Digital signal processing board

- Power supply sub-system

Because of the large driving current required for the TEC, a second power supply is used to reduce the load of the power supply I. It is anticipated that this will help to reduce the noise that might be induced by large current fluctuations. Other subsystems will be discussed in detail below.

\subsubsection{Light Source}

The high power light source is further divided into three small systems, including a high power LED, a LED driver, and a TEC controller.

\section{High power LED}

To meet the requirement of high resolution and high SNR, a high power source is of great importance. The light source used in the system is a $1550 \mathrm{~nm}$ edge-emitting LED (ELED) which can launch a maximum power of $50 \sim 60 \mu \mathrm{W}$ into its single-mode fiber pigtail. Other optical components are thus required to work at $1550 \mathrm{~nm}$. The FWHM is about $83 \mathrm{~nm}$. Figure 9.21(a) is the I-P characteristic and Figure 9.21 (b) is the spectrum of the LED, both at $25^{\circ} \mathrm{C}$.

The most important parameters of the LED are its output power and the central wavelength (CWL). The output power is essential to the system resolution and should be as high as possible. The CWL will, when the light signal passes through the in-line filter (1551 nm CWDM filter), determine the narrowband signal intensity. Small variations of the CWL due to factors like temperature change will cause changes of the final signal and lead to system error and poor resolution. When the CWL matches the passband $(1551 \mathrm{~nm})$ of the in-line filter, the same variation will introduce smaller error than when there is a mismatch (see the filter section). Therefore the CWL should be around $1551 \mathrm{~nm}$. These two parameters will change with temperature and driving current. Figure 9.3(a) shows that when temperature 
increases the output power decreases and meanwhile the CWL shifts to longer wavelength (Figure 9.3 (b)), both are tested under a driving current of $\mathrm{I}=80 \mathrm{~mA}$.

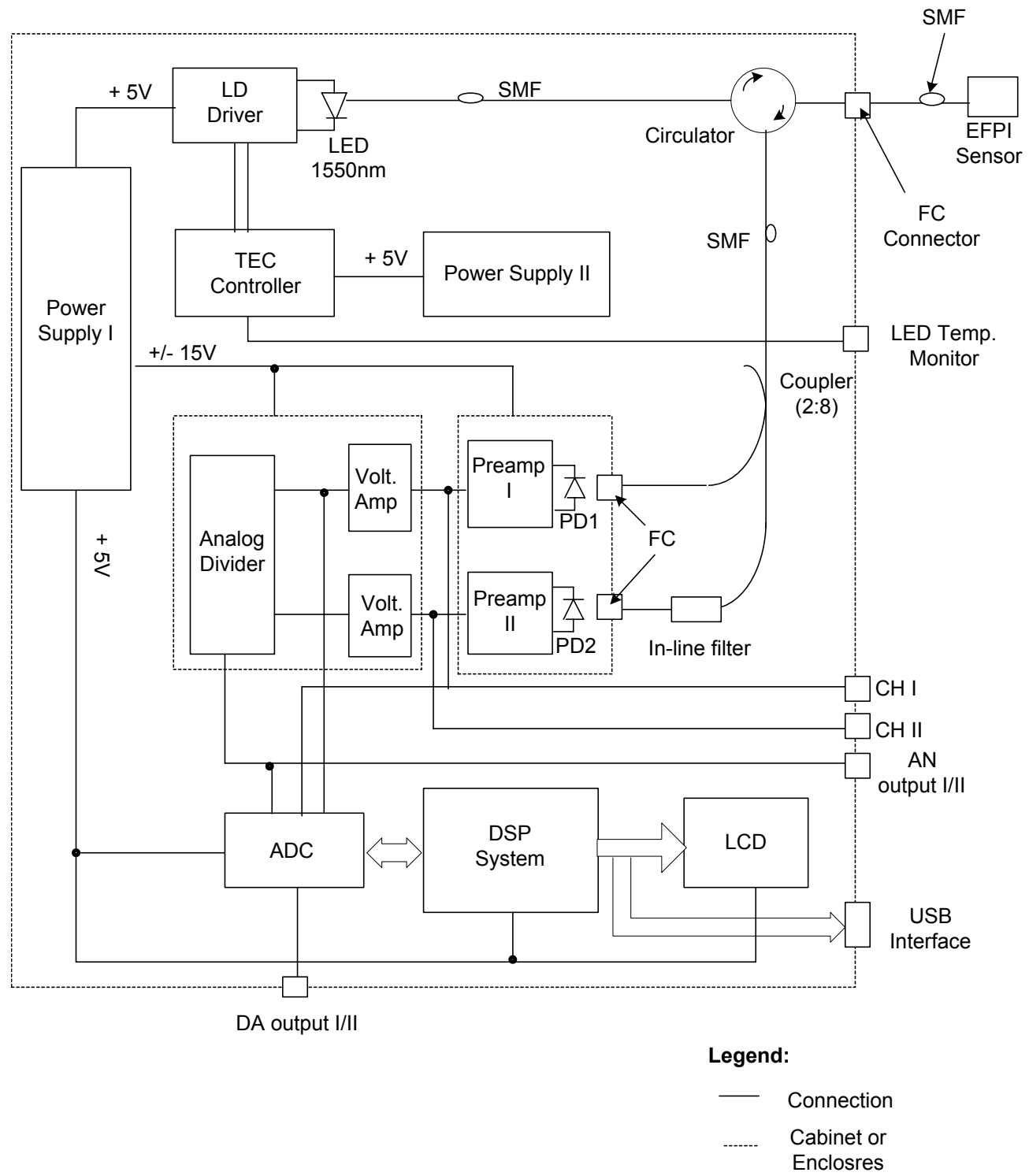

Figure 9.1. Schematic of the diaphragm based engine sensor system. 


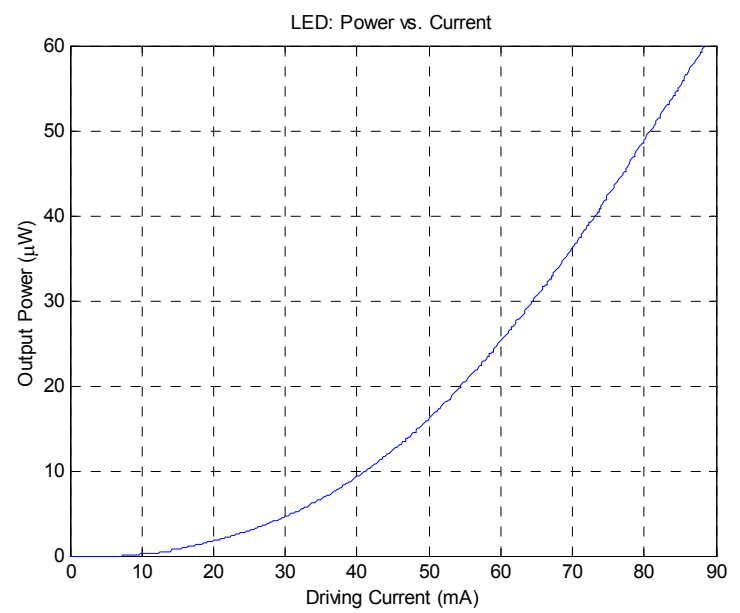

(a) I-P characteristic $\left(25^{\circ} \mathrm{C}\right)$

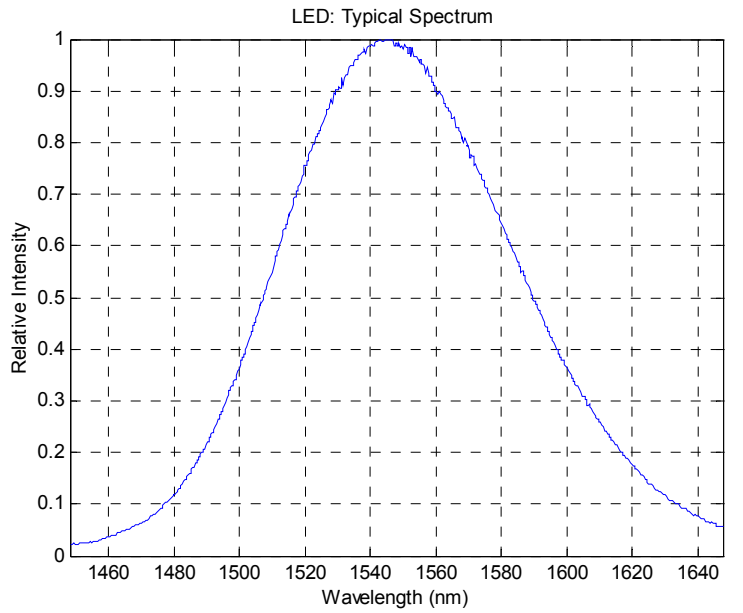

(b) Typical spectrum $\left(25^{\circ} \mathrm{C}\right)$

Figure 9.2. Characteristics of the high power LED.
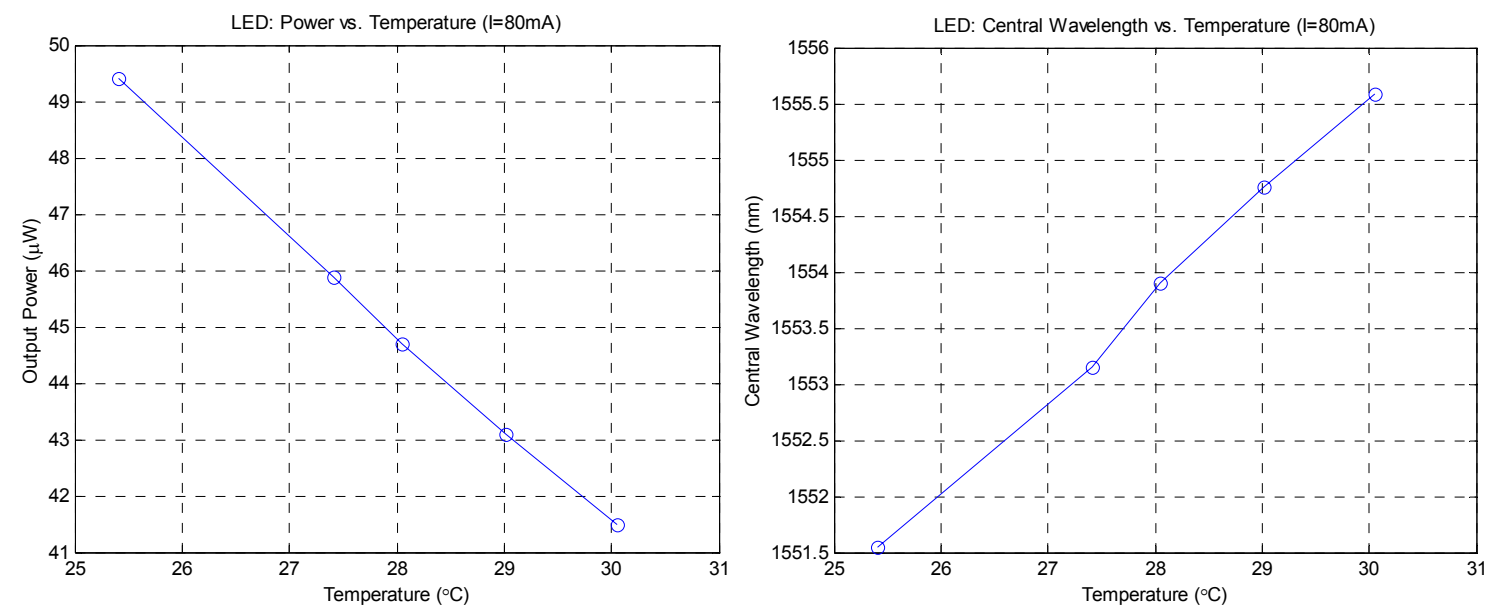

Figure 9.3. Temperature dependence of the optical power and CWL.

Figure 9.4(a) and (b) are P-I curves and CWL-I curves at different temperatures $20^{\circ} \mathrm{C}$, $25^{\circ} \mathrm{C}$ and $30^{\circ} \mathrm{C}$. From (a), the driving current at which the LED has a CWL of $1551 \mathrm{~nm}$ can be determined for different temperatures. In (b), optimal working points are labeled by open circles and the corresponding output power can thus be found. It can be seen that in order to have the highest power and the best CWL the LED has to work under high temperature and large driving current. However, both of conditions may shorten the operating life of the LED. Currently, the working conditions are chosen to be $84 \mathrm{~mA}$ and $25^{\circ} \mathrm{C}$, with an output power of $52 \mu \mathrm{W}$. 


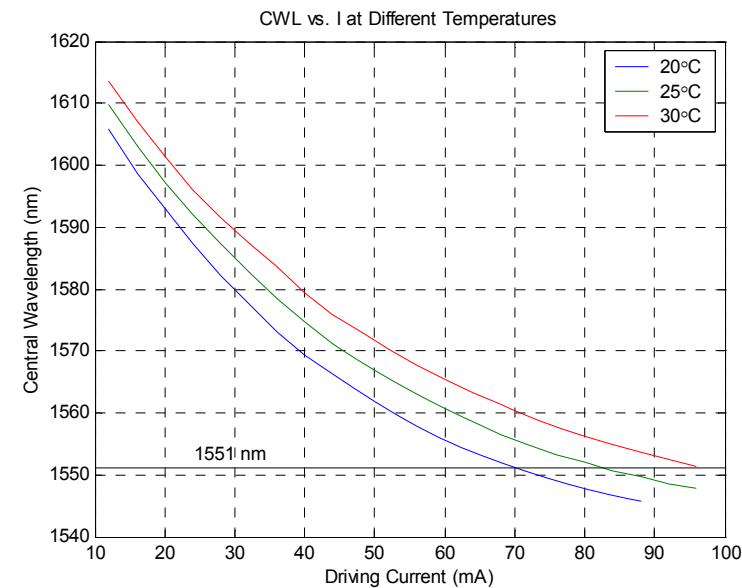

(a) CWL vs. I at $\mathrm{T}=20^{\circ} \mathrm{C}, 25^{\circ} \mathrm{C}$ and $30^{\circ} \mathrm{C}$.

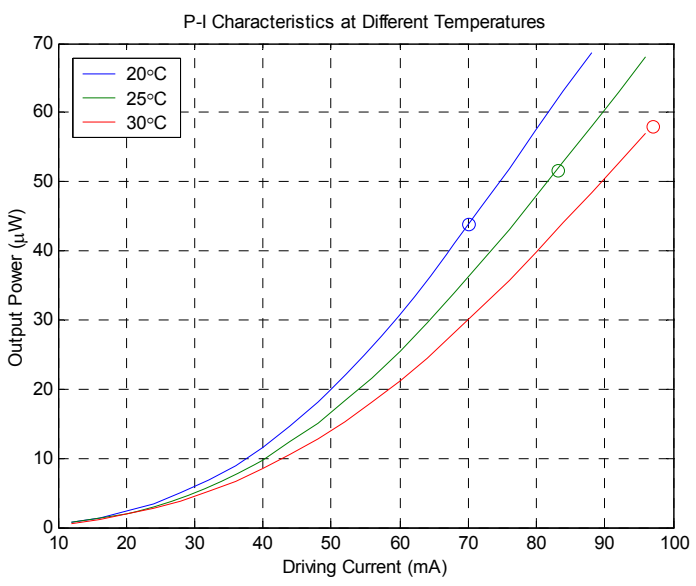

(b) $\mathrm{P}$ vs. I at $\mathrm{T}=20^{\circ} \mathrm{C}, 25^{\circ} \mathrm{C}$ and $30^{\circ} \mathrm{C}$.

Figure 9.4. Driving current dependence of the optical power and CWL at different temperatures.

\section{LED driver}

An LDD-200-1P laser diode driver from Wavelength Electronics was selected as the constant current source for the LED. When it operates in constant current mode, the LDD-200-1P can provide a $200 \mathrm{~mA}$ maximal driving current with $<50 \mathrm{ppm}$ current stability ( 24 hours) and noise $(\mathrm{rms})<5 \mu \mathrm{A}$. This low noise feature is very important to fulfill our sensors' resolution of $0.01 \%$. The output current can be precisely controlled with the on-board Output Current Adjust trim pot and modulated with a DC to $2 \mathrm{MHz}$ input signal.

\section{$\underline{\text { TEC controller }}$}

Since the LED's output power and spectrum are temperature dependent, temperature control is very necessary to reduce the temperature effects. Also because of the high output put of the LED, cooling is very important to protect the LED from being damaged.

The LED comes with a thermistor and an imbedded TEC. The temperature dependence of the resistance of the thermistor is given in Figure 9.24. When the temperature of the LED increases, the resistance of the thermistor decreases accordingly. The absolute rating for the TEC current at $25^{\circ} \mathrm{C}$ is $0.8 \mathrm{~A}$.

Thermoelectric coolers (TECs) are used in a variety of applications that require extremely stable temperature control. Because of their solid-state construction, these small devices are very reliable and relatively easy to use. However, before attempting to build high performance systems, a designer must have a good understanding of thermal management, techniques and carefully select the controller. The HTC-3000 Hybrid Temperature Controller from Wavelength Electronics is a linear controller designed for stable, low noise operation. The advanced and reliable circuitry of the HTC-3000 easily achieves $0.001{ }^{\circ} \mathrm{C}$ temperature stability even at ambient, and its independently adjustable proportional gain and integrator 
time constant can be modified to optimize temperature overshoot and stability. A schematic of the HTC-3000 is shown in Figure 5.5.

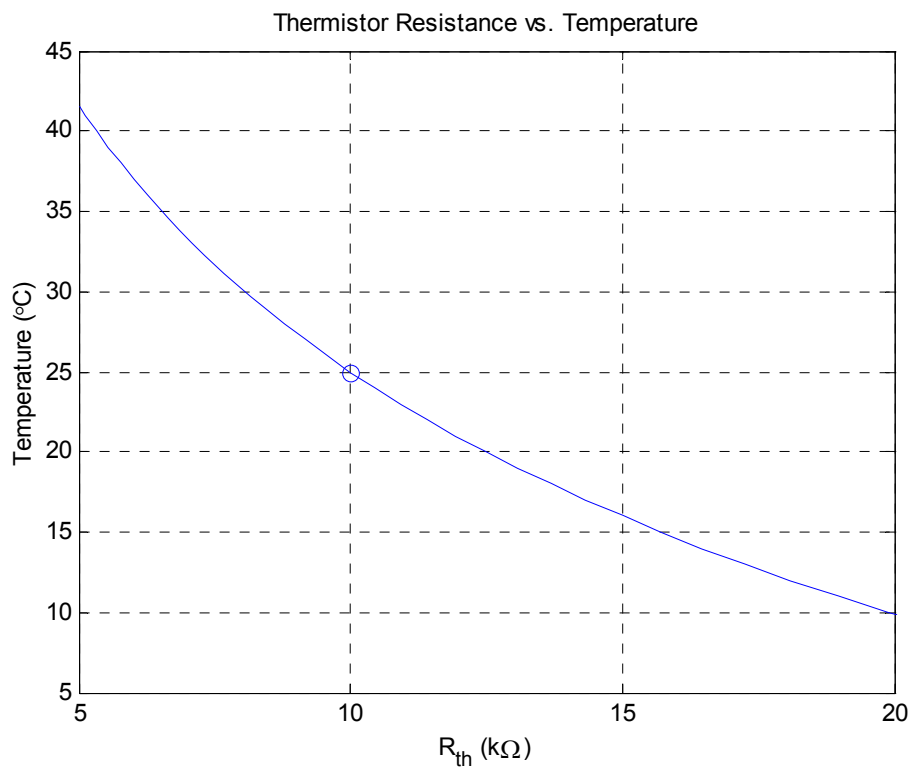

Figure 9.5. Temperature v.s. $\mathbf{R}_{\text {th }}$.

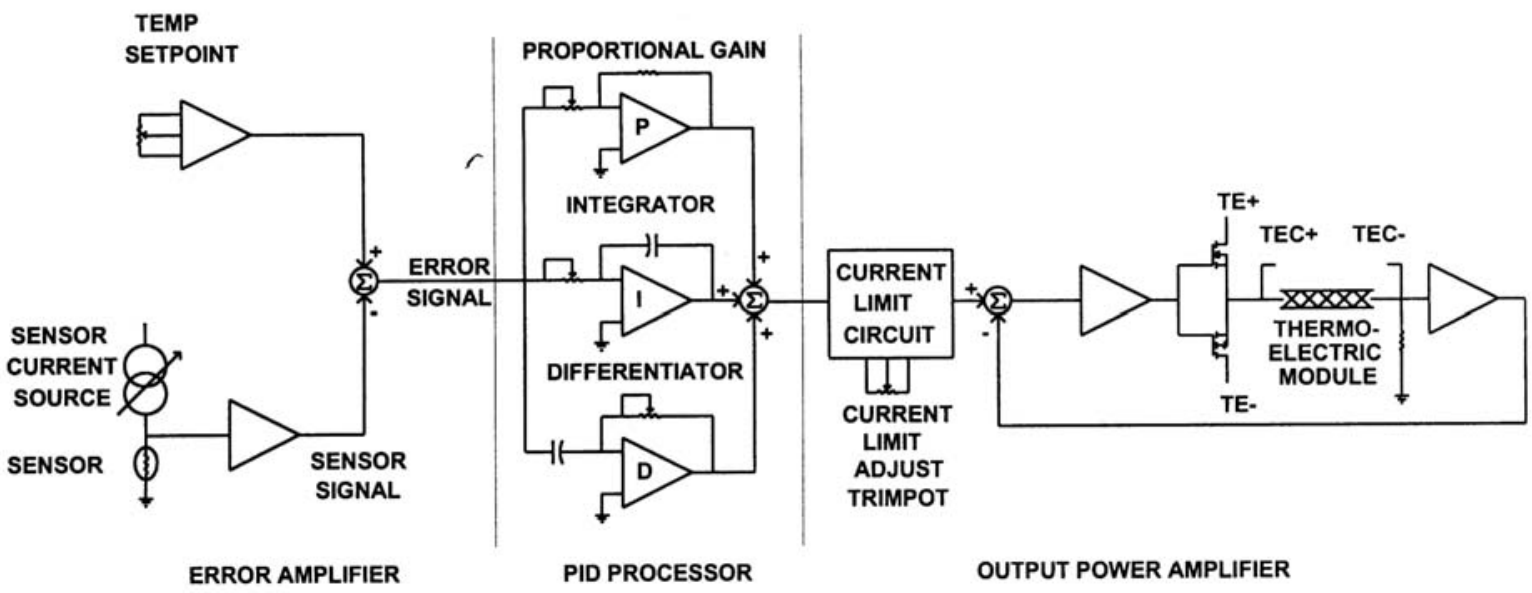

Figure 9.6. Schematic of the HTC-3000 Hybrid Temperature Controller (Wavelength Electronics). 


\subsubsection{Optical Components}

\section{Circulator}

As mentioned above, high resolution requires high signal power. Therefore, instead of a coupler that is commonly used but will cause a $6 \mathrm{~dB}$ loss of signal, a $1550 \mathrm{~nm}$ polarization insensitive optical circulator is used to make full use of the light power.

\section{Coupler}

After the light is reflected by the sensor head, it passes through the circulator and then is split into a broadband channel and a narrowband channel by a coupler. The broadband channel acts as a reference signal and the ratio of the two channels is taken to get rid of instability factors such as source power drift. Therefore, unlike the LED and the circulator, the coupler should work in a very stable manner and be able to maintain a constant splitting ratio. What is used here is a single-mode wideband coupler with operating wavelength of $1550 \mathrm{~nm} \pm 50$ $\mathrm{nm}$ and a splitting ratio of 90:10. The $90 \%$ channel is then filtered by a CWDM optical filter to form the narrowband signal, resulting in a similar level of power with the $10 \%$ channel which is directly taken as the wideband signal. The similar power level of the two channels is preferred because two 'identical' detecting circuits can be used to reduce the system error.

The temperature dependence of the $90 \%$ and $10 \%$ channels is tested and shown in Figure 9.7(a). In a temperature range of $15{ }^{\circ} \mathrm{C}\left(31 \sim 46{ }^{\circ} \mathrm{C}\right)$, the two channels remain quite stable with a relative error around $0.05 \%$ that is probably due to errors from the measuring instruments. Therefore, for room temperature operation, the splitting ratio of the coupler can be taken as a constant. Figure 9.7(b) shows the change of CWL with temperature. The variation is smaller than $0.2 \mathrm{~nm}$ and in a random manner, which is also within the precision of the measuring instruments.

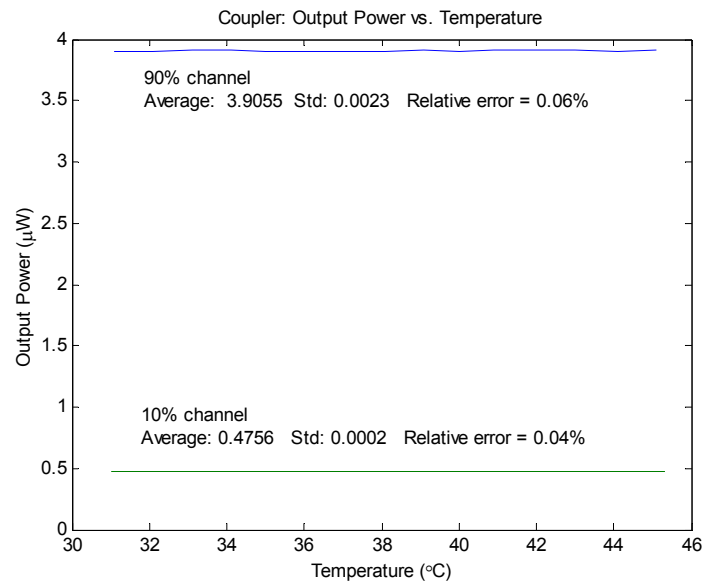

(a) Splitting ratio stability.

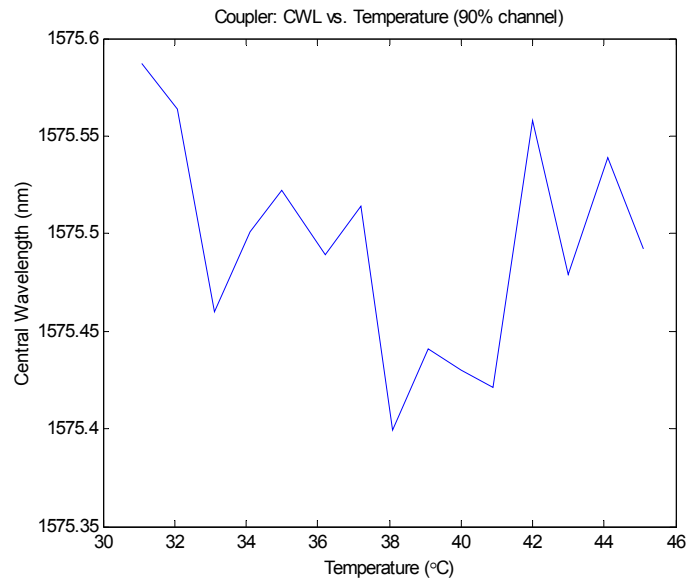

(b) CWL stability.

Figure 9.7. Coupler's temperature dependence. 


\section{In-line CWDM filter}

To reduce the loss of power, an in-line filter is used to filter the $90 \%$ channel of the coupler into a narrowband signal. The filter is a Coarse WDM (CWDM) filter working at $1551 \mathrm{~nm}$ with a center wavelength of about $1550.9 \mathrm{~nm}$. Figure 9.8 shows the passband spectrum of the filter. The bandwidth is about $16.9 \mathrm{~nm}$ at $0.5 \mathrm{~dB}$ and $18 \mathrm{~nm}$ at $3 \mathrm{~dB}$. Ideally, this filter can be well modeled as a rectangular passband centered at $1550.9 \mathrm{~nm}$ and a bandwidth of about $18 \mathrm{~nm}$.

In the LED section it was mentioned that the mismatch of the center wavelengths of LED and filter would lead to larger changes of the narrowband signal when the environment temperature varies. This result is shown in Figure 9.9, where the center wavelength of the filter is taken as $1551 \mathrm{~nm}$ and three values of LED center wavelength, $1551 \mathrm{~nm}$ (no CWL mismatch), $1552 \mathrm{~nm}$ (1 nm mismatch) and $1553 \mathrm{~nm}$ (2 nm mismatch), are used to calculate and compare the relative change of the narrowband signal. For each of the three LED CWLs, a drift of $\pm 0.5 \mathrm{~nm}$ is assumed. The figure shows that, for the same amount of drift, the narrowband signal will change much less when there is no mismatch (blue curve) than when there is mismatch (green and red curve). In the case of no mismatch, the relative change is within $0.01 \%$ which meets the requirement very well.

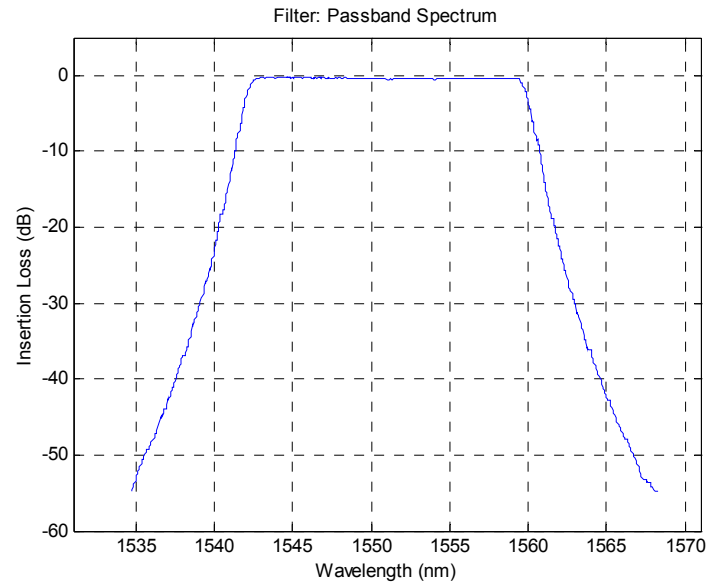

Figure 9.8. Spectrum of the in-line filter.

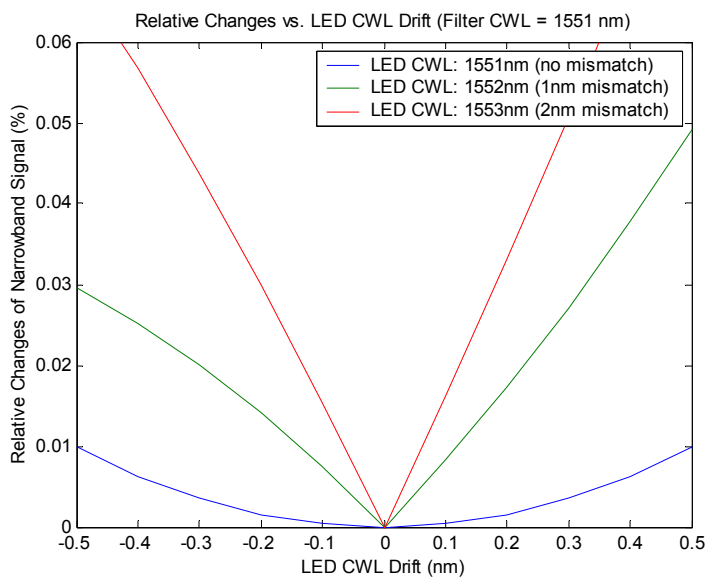

Figure 9.9. Temperature effects.

\subsubsection{Analog Circuitry}

As indicated in Figure 9.1, the analog circuitry in the system consisted of three main parts: two transimpedance preamplifiers, two voltage amplifiers, and a divider.

\section{$\underline{\text { Photodiodes }}$}

The function of a photodiode (PD) is to convert the optical power from the back-reflection of a SCIIB sensor into an electrical current signal. Since the PD is the current source of all the following electronic circuits, its performance is very important to the signal to noise ratio (SNR) of the whole system. The parameters that affect PD performance include: 
- Capacitance, which is a deterministic factor of the frequency response of the preamplifier;

- Dark current;

- Active area, which affects the coupling efficiency between a fiber and the detector, dark current, and capacitance;

- Responsivity;

- Package.

To satisfy the DC to $150 \mathrm{KHz}$ frequency bandwidth and $0.01 \%$ accuracy of the engine pressure measurement, the two photodiodes must have small active areas, low dark currents and high efficiencies. What's more important is that the two photodiodes have close performances to guarantee identical performances of the two channels. Two InGaAs PINs' (FD80FC) from Fermionics Opto-Technology have been chosen from four samples. They have same size $(80 \mu \mathrm{m})$, dark current $(\mathrm{ID}=0.04 \mathrm{nA})$, capacitance $(\mathrm{C}=0.68 \mathrm{pF} @ \mathrm{VR}=5 \mathrm{~V})$, and very close responsivity $(\mathrm{R} 1=0.84 \mathrm{~A} / \mathrm{W}, \mathrm{R} 2=0.83 \mathrm{~A} / \mathrm{W})$. Their $\mathrm{FC}$ receptacle packages make it easy to be integrated into a preamplifier case.

\section{Preamplifiers}

Because preamplifiers are positioned after the photodiodes and the current signal from the PDs are usually on the order of $100 \mathrm{nW}$ to $1 \mu \mathrm{W}$, preamplifiers are very critical to the SNR of the whole system. Ultra-low noise, wide-bandwidth, and high gain preamplifiers are required to achieve the $0.01 \%$ accuracy for pressure measurement. Figure 9.10 shows one of the two preamplifiers we have designed for diaphragm-based SCIIB sensors. It includes a currentvoltage converter, a gain control tripot, a low-pass filter and a matched output.

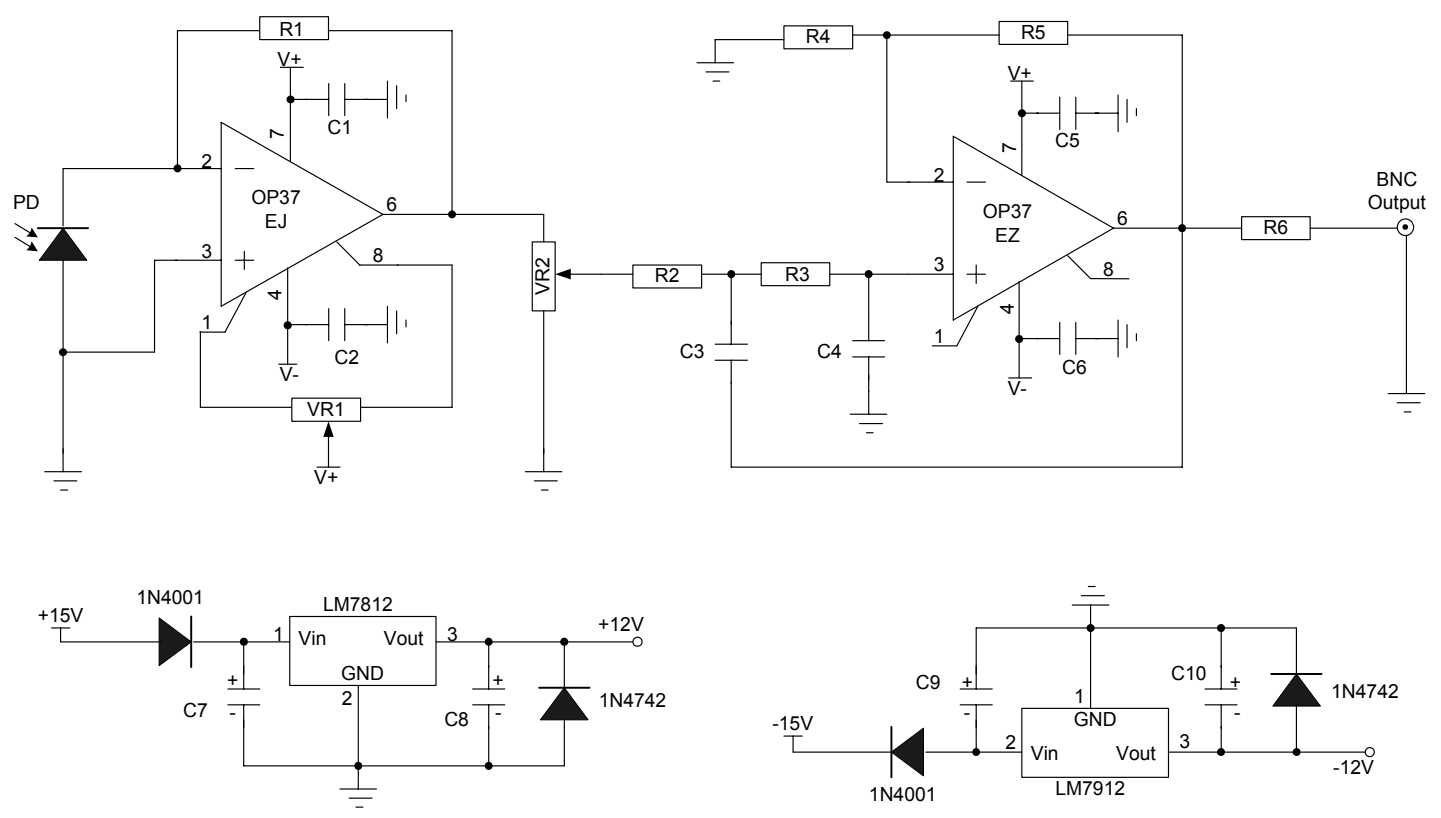

Figure 9.10. Schematics of optoelectronic circuits. 
A transimpedance amplifier is a component used to convert the PD signal from current to voltage for further process. It provides a good compromise between the low-noise characteristics of a high-impedance front-end and the wideband nature of a low-impedance voltage amplifier front-end. As shown in Figure 10, the amplifier is termed transimpedance because it utilizes shunt feedback around an inverting amplifier. The feedback stabilizes the transimpedance. The gain of the I-V converter is determined by the resistance of the feedback resister R1. Because ultra-high gain is necessary for the weak signal, a $1 \mathrm{M} \Omega$ low noise metal film resistor is used.

A second-order low-pass filter with a rolloff factor of $40 \mathrm{~dB} / \mathrm{dec}$ ade follows the transimpedance amplifier. The filter introduces very high attenuation to out of band noises, while provides a gain of R5 / R4 to the signal. The bandwidth is totally decided by the lowpass filter because the bandwidth of the transimpedance amplifier is still much higher than that of the filter. Therefore, the total bandwidth is given by:

$$
f_{0}=1 / 2 \pi \sqrt{R_{2} C_{3} R_{3} C_{4}}
$$

The gain of the preamplifier is continuously tunable with a tripot so that a large optical input range can be obtained. The total gain of the preamplifier is given by

$$
G=R \times R_{1} \times g \times\left(R_{5} / R_{4}\right)
$$

where $\mathrm{R}$ is the responsivity of the photodiode, and $\mathrm{g}$ is the attenuation factor of the tripot.

In addition to the careful design of the transimpedance amplifier and low-pass filter, some other techniques are also employed to reduce the noise power, thus improve the SNR and resolution of the system.

First of all, a short wire between a PD and the following current-voltage converter can avoid the EM noises that may be picked up by a long wire. The best way to reduce the distance is to mount the PD on the PCB. Therefore, a PD with a FC receptacle is selected so that the PD can be readily installed into an aluminum case.

Second, in order to reduce the noise from the power supply, voltage regulators and decoupling capacitors are used.

Good shielding is also helpful to reduce the electrostatic coupling, RF interference. Shield material and grounding determine the shield effectiveness. Making the shield with a material of high electrical conductivity ensures that the coupled currents produce little voltage drop across the shield. In our design, a cast aluminum case is used.

Finally, a $50 \Omega$ matching is preferred to a $50 \Omega$ input impedance $\mathrm{A} / \mathrm{D}$ converter to reduce the reflection.

Gain linearity and symmetry: The gain ranges of the two channels are G1 $=0 \sim 1.8 \times 10^{6}$ and $\mathrm{G} 2=0 \sim 1.8 \times 10^{6}$, respectively. Figure 9.11 shows the relationship between the input optical power and the corresponding preamplifier output for channel 1 . The curve is very linear with 
a correlation coefficient $\mathrm{R}=0.99998$. Figure 9.12 is the output voltage of channel 1 versus that of channel 2 for the same optical inputs and the correlation coefficient is $R=0.99999$.

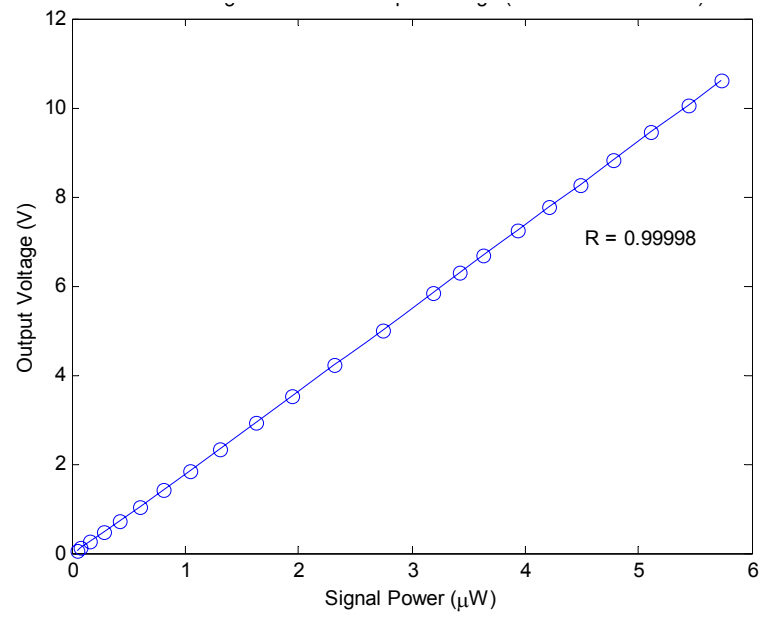

Figure 9.11. Gain linearity.

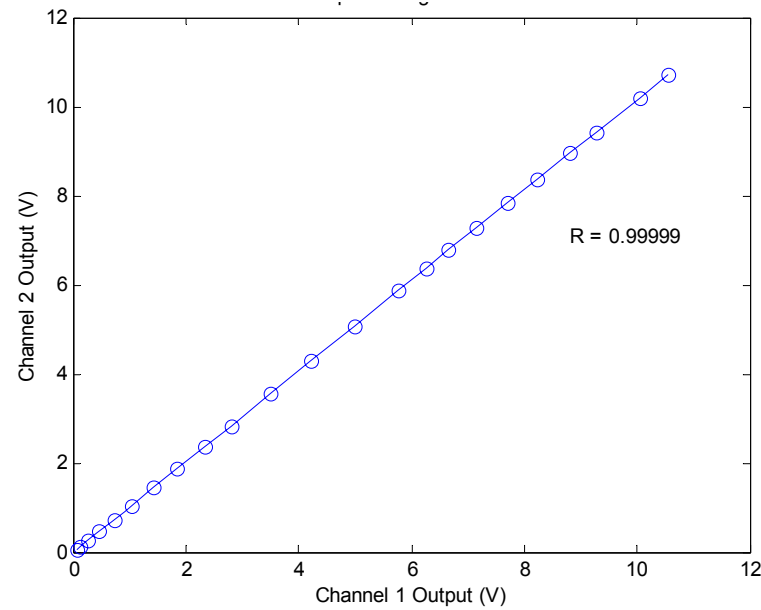

Figure 9.12. Gain symmetry.

Bandwidth: The frequency response of the preamplifier is shown in Figure 9.13. The two channels have very close frequency characteristics. The $3 \mathrm{~dB}$ bandwidth is $\mathrm{DC}$ to $230 \mathrm{kHz}$, and the gain flatness in the bandwidth $\mathrm{DC}-150 \mathrm{KHz}$ is less than $0.7 \mathrm{~dB}$. A gain peak around $580 \mathrm{kHz}$ might be due to the second-order filter.

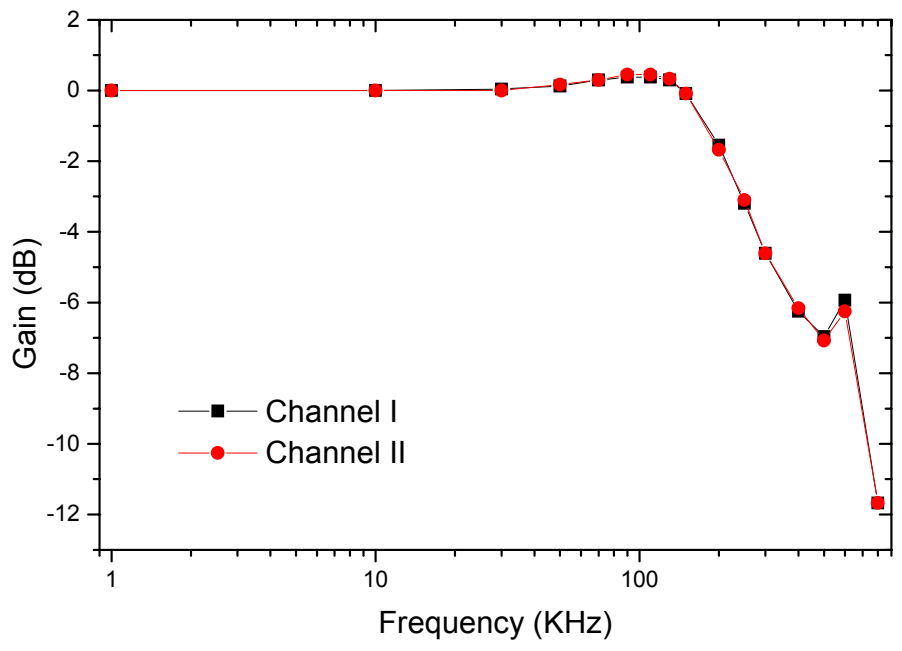

Figure 9.13. Frequency response of the two preamplifiers. 
Table 9.1. Noise performance.

\begin{tabular}{|c|c|c|}
\hline \multicolumn{3}{|c|}{ a. Noise caused by dark current and electronics $\left(T=19^{\circ} \mathrm{C}\right.$, Full gain) } \\
\hline \multicolumn{3}{|c|}{$\begin{array}{l}\text { Channel I: 6.3mV@1M } @ 1 \mathrm{M}, 2.9 \mathrm{mV} @ 50 \Omega \\
\text { Channel II: 6.0mV@, } 1 \mathrm{M} \Omega, 2.7 \mathrm{mV} @ 50 \Omega\end{array}$} \\
\hline \multicolumn{3}{|c|}{ b. DC optical input (Full gain, @ $1 M \Omega$, input directly from LED pigtail) } \\
\hline LED Driving Current (mA) & Channel I (mV) & Channel II (mV) \\
\hline 4 & 6.3 & 6.0 \\
\hline 8 & 6.3 & 6.0 \\
\hline 16 & 8.0 & 7.5 \\
\hline 24 & 11.0 & 10.7 \\
\hline
\end{tabular}

c. Crosstalk (Full gain, Mod. Index=0.1, 500nW input to Channel I)

\begin{tabular}{cccc} 
Frequency & Channel I $(\mathrm{mV})$ & & Channel II $(\mathrm{mV})$ \\
\cline { 1 - 1 } & 7.0 & 7.0 & 6.0 \\
$10 \mathrm{kHz}$ & 7.0 & 6.0 \\
$50 \mathrm{kHz}$ & 7.0 & 6.0 \\
$100 \mathrm{kHz}$ & 7.1 & 6.0 \\
$150 \mathrm{kHz}$ & & 6.1 \\
\hline
\end{tabular}

d. Offset adjustable range:

Channel I: $+77.9 \mathrm{mV} \sim-83.4 \mathrm{mV}$

Channel II: $+83.0 \mathrm{mV} \sim-101.2 \mathrm{mV}$

e. Temperature drift

No measurable temperature drift has been observed when the temperature of the preamplifiers is increased from $23^{\circ} \mathrm{C}$ to $45^{\circ} \mathrm{C}$.

\section{Analog Divider}

The divider function is implemented through the use of AD734BD and AD538 dividers.

The AD734BD has such favorable features as high accuracy ( $0.1 \%$ typical error), high speed (10 MHz full-power bandwidth), low distortion (-80dBc from any input), low noise (94 dB $\mathrm{SNR}, 10 \mathrm{~Hz}$ to $20 \mathrm{KHz}$ and $70 \mathrm{~dB} \mathrm{SNR}, 10 \mathrm{~Hz}$ to $10 \mathrm{MHz}$ ), and direct division mode (allowing the ratio of two channels to be obtained directly).

The AD538 is a monolithic real-time computational circuit that provides precision analog multiplication, division and exponentiation. No external components are required for onequadrant (positive input) division. It has wide input dynamic range, low error $(0.25 \%$ of reading possible), wide bandwidth $(400 \mathrm{kHz})$.

The accuracy and bandwidth requirement for this project is $150 \mathrm{kHz}$, with a resolution of $0.01 \%(80 \mathrm{~dB})$. The bandwidth of both dividers is more than sufficient to meet the 
requirements of the project, but the noise performance will require significant improvement in order to meet the $0.01 \%$ resolution.

$A D 734 B D$ divider: The connection diagram of AD734BD is shown in Figure 9.14. The uncommitted (open-loop) transfer function of the AD734 is:

$$
W=A_{o}\left[\frac{\left(X_{1}-X_{2}\right)\left(Y_{1}-Y_{2}\right)}{\left(U_{1}-U_{2}\right)}-\left(Z_{1}-Z_{2}\right)\right]
$$

where $A_{o}$ is the open-loop gain of the output op-amp, typically $72 \mathrm{~dB}$. When a negative feedback path is provided, the circuit will force the quantity inside the brackets essentially to zero, resulting in the equation:

$$
\frac{\left(X_{1}-X_{2}\right)\left(Y_{1}-Y_{2}\right)}{\left(U_{1}-U_{2}\right)}=\left(Z_{1}-Z_{2}\right)
$$

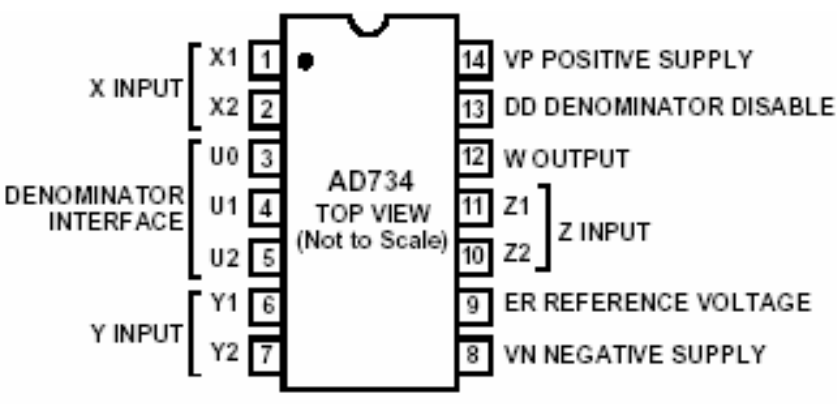

Figure 9.14. Connection Diagram of AD734.

In our application, we use the Direct Denominator Control model, which enables us to directly vary the denominator voltage. In addition to providing much higher accuracy and bandwidth, this mode also provides greater flexibility, because all inputs remain available. $Z_{1}$ was connected to output $W$ and $Z_{2}$ is connected to the signal ground. The $Y$ input is set to a fixed voltage ( $8 \mathrm{~V}-10 \mathrm{~V}$ adjustable), which can be regarded as the gain of the divider. Thus, the transfer function becomes:

$$
W=\frac{Y\left(X_{1}-X_{2}\right)}{U_{1}-U_{2}}
$$

To ensure negative feedback, the denominator $U=U_{1}-U_{2}$ must be positive. To avoid clipping at the positive and negative extremities of the $\mathrm{X}$-input, the ratio $X / U$ cannot exceed 1.25 (for $Y=10 \mathrm{~V}$ ). The schematic of the divider is shown as Figure 9.15. 


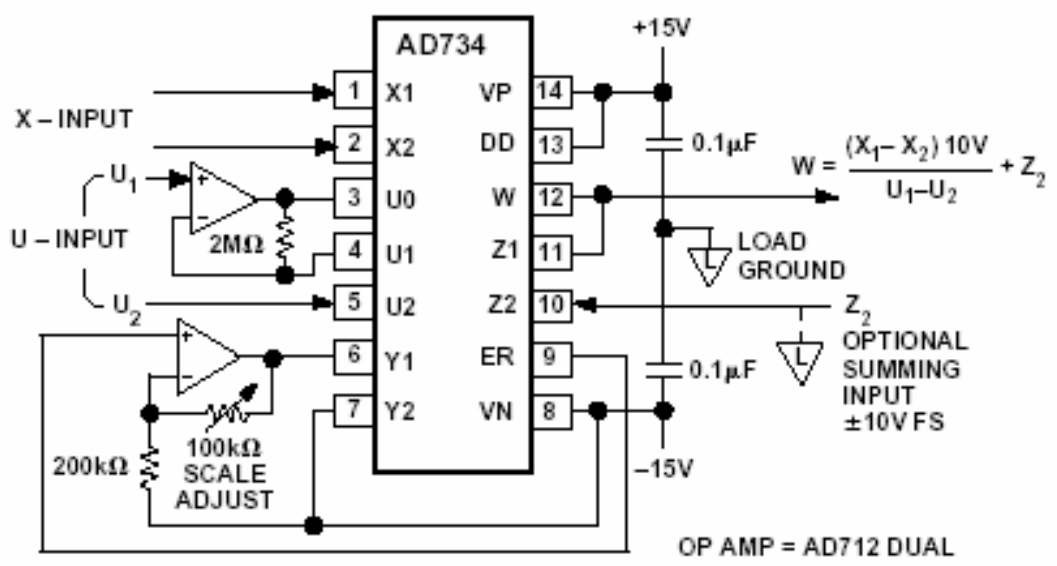

Figure 9.15. Two-Quadrant Divider with Fixed Voltage Scaling.

We call this a two-quadrant divider because the denominator $U=U_{1}-U_{2}$ must be positive as previously noted. The reference voltage output appearing between Pin 9 (ER) and Pin 8 (VN) is amplified and buffered by the second op amp, to impose $10 \mathrm{~V}$ across the $Y_{1} / Y_{2}$ input. Adjusting the potentiometer can change the scale. The $2 M \Omega$ resistor is included to improve the accuracy of the gain for small denominator voltages. This divider may also be viewed as a variable-gain element, whose output, in response to a signal at the $X$ input, is controllable by both the $Y$ input (for attenuation, using $Y$ less than $U$ ) and the $U$ input (for amplification, using $U$ less than $Y$ ). Its gain bandwidth product is $250 \mathrm{MHz}$. Usually the magnitude of $U$ input signal from preamplifier is around $100 \mathrm{mV}$, so the gain is $40 \mathrm{~dB}$ and the bandwidth is $2 \mathrm{MHz}$.

There are two voltage amplifiers prior to the divider corresponding to denominator and numerator input channel. The gain is adjustable with a maximum value of 5.6. The $3 \mathrm{~dB}$ bandwidth is

$$
f_{3 d B}=\frac{1}{2 \pi R C}=\frac{1}{2 \pi \times 56 \times 10^{3} \times 12 \times 10^{-12}}=236.8 \mathrm{KHz}
$$

Two jumpers follow the outputs of the two amplifiers so that we can choose to connect them directly with $\mathrm{A} / \mathrm{D}$ converter bypassing the divider.

AD538 divider: The functional diagram of AD538 is shown in Figure 9.16. The AD538's overall transfer function is $\mathrm{V}_{\mathrm{O}}=\mathrm{V}_{\mathrm{Y}}\left(\mathrm{V}_{\mathrm{Z}} / \mathrm{V}_{\mathrm{X}}\right)^{\mathrm{m}}$. Programming a particular function is via pin strapping. No external components are required for one-quadrant (positive input) multiplication and division. Exponentiation with an $m$ value from 0.2 to 5 can be implemented with the addition of one or two external resistors.

Figure 9.17 shows how the AD538 may be easily configured as a precision one-quadrant multiplier/divider. The transfer function $\mathrm{V}_{\mathrm{OUT}}=\mathrm{V}_{\mathrm{Y}}\left(\mathrm{V}_{\mathrm{Z}} / \mathrm{V}_{\mathrm{X}}\right)$ allows three independent input variables, a calculation not available with a conventional multiplier. In addition, the 1000:1 (i.e., $10 \mathrm{mV}$ to $10 \mathrm{~V}$ ) input dynamic range of the AD538 greatly exceeds that of analog multipliers computing one-quadrant multiplication and division. 


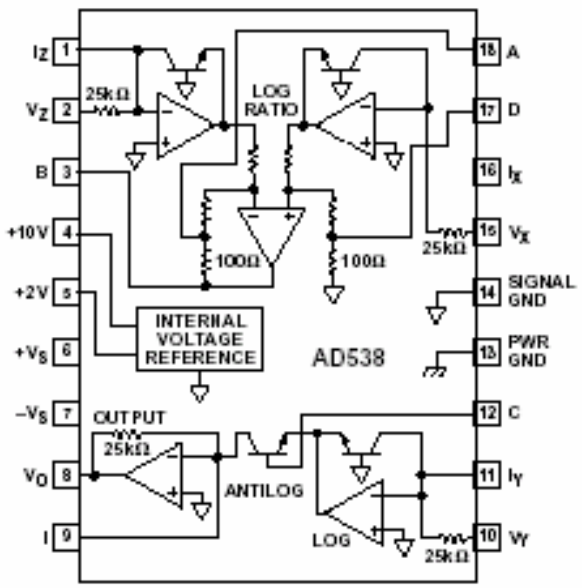

Figure 9.16. Functional diagram of AD538.

By using the $+10 \mathrm{~V}$ reference as the VY input, the circuit of Figure 9.16. is configured as a one-quadrant divider with a fixed scale factor. As with the one-quadrant multiplier, the inputs accept only single (positive) polarity signals. The output of the one-quadrant divider with a $+10 \mathrm{~V}$ scale factor is:

$$
V_{o}=10 \mathrm{~V}\left(\frac{V_{Z}}{V_{x}}\right)
$$

The typical bandwidth of this circuit is $370 \mathrm{kHz}$ with $1 \mathrm{~V}$ to $10 \mathrm{~V}$ denominator input levels. At lower amplitudes, the band-width gradually decreases to approximately $200 \mathrm{kHz}$ at the 2 $\mathrm{mV}$ input level.

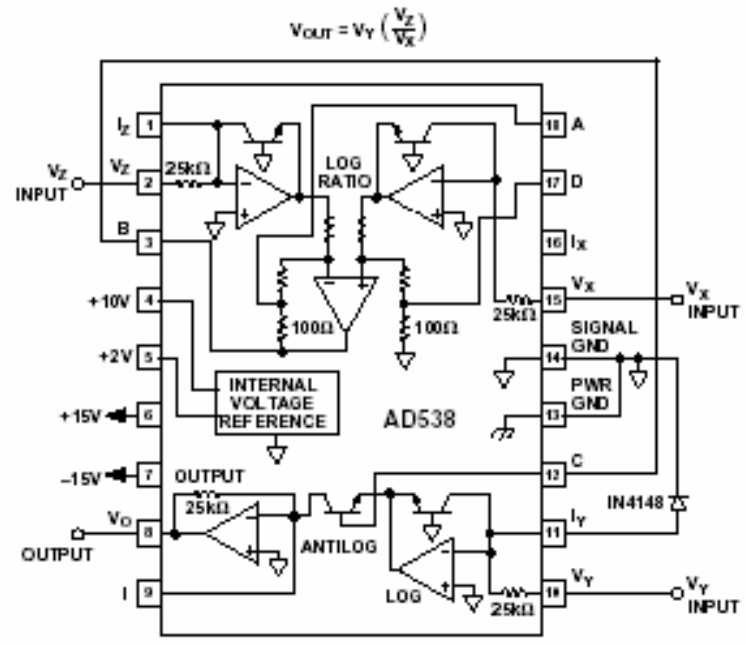

Figure 9.17. One-quadrant divider. 
We tested the frequency response, linear and noise performance of AD734BD and the frequency response and noise performance of AD538. These characteristics are discussed below.

Frequency Response: The two inputs are identical sinusoidal signals and their frequencies vary from $0 \mathrm{~Hz}$ to $150 \mathrm{kHz}$. The function of output versus frequency is shown in Figure 9.18.

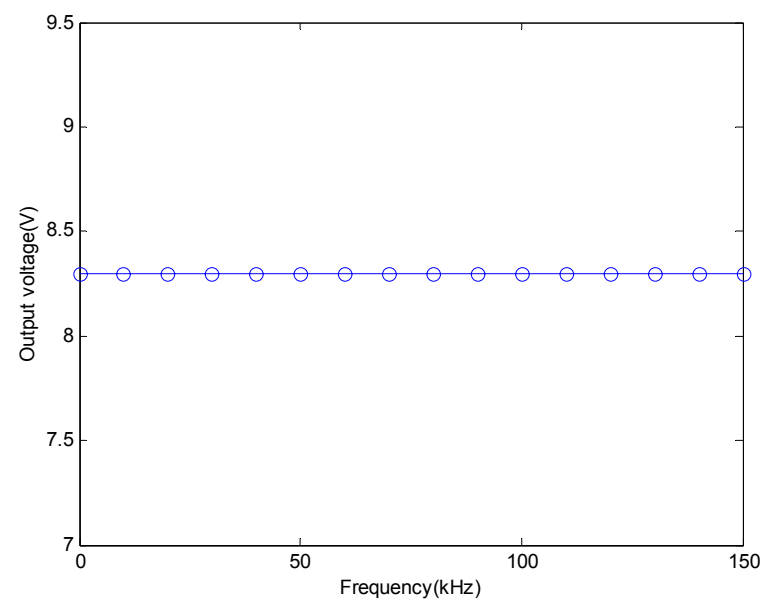

(a) AD734BD divider.

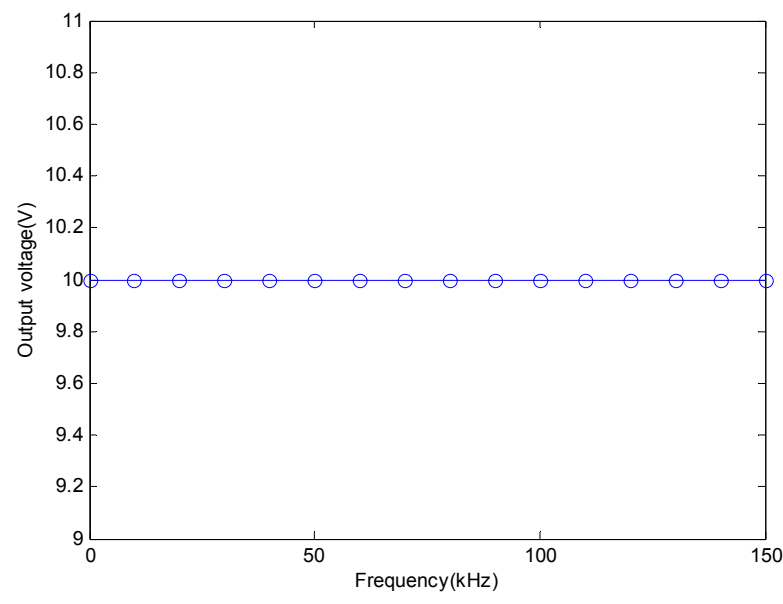

(b) AD538 divider.

Figure 9.18. Frequency Response.

Linear Performance of $A D 734 B D$ divider: The two inputs are still identical sinusoidal signals, but we can adjust the gain of each voltage amplifier such that the numerator is more or less than the denominator. Then we observed the output amplitude. The function of the output versus $V_{n} / V_{d}$ is shown in Figure 9.19. When $V_{n} / V_{d}$ reaches 1.4 , the output has been saturated. This result corresponds to the theoretical calculation. The output is $8.3 \mathrm{~V}$ as $V_{n} / V_{d}$ is 1 , thus the $Y$ input is set to $8.3 \mathrm{~V}$. Since the voltage supply is $12 \mathrm{~V}$, so

$$
\left(V_{n} / V_{d}\right)_{\max }=\frac{12}{8.3}=1.44 .
$$

Noise Performance: The signal to noise ratio is good when one input is a DC signal and the other is a sinusoidal signal. The noise on top of the DC signal is higher than we expected when the two inputs are identical sinusoidal signals. For the AD734BD divider, the noise increased with an increase in frequency and the noise has the same frequency as the input sinusoidal signal, not only white noise. For the AD538, the noise frequency didn't change much with the increase of the input signal frequency. The noise voltage is about $50 \mathrm{mV}$ on the top of a $10 \mathrm{~V}$ DC signal when the frequency is about $100 \mathrm{kHz}$. 


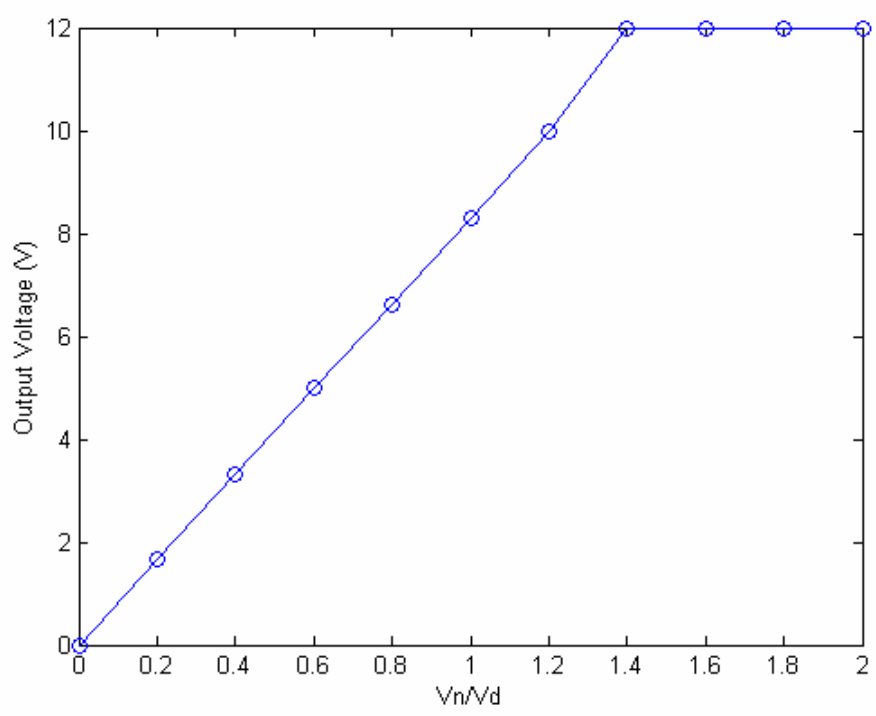

Figure 9.19. Linear performance of AD734B divider.

The high noise of both dividers may be due to the following factors.

- Radio frequency interference;

- Function generator for input;

- Ground;

- Power supply

\subsection{Grating-Assisted Operating-Point Tuning}

Fiber-optic Fabry-Perot interferometric (FFPI) sensors have been used for the detection and measurement of a large variety of physical parameters, such as temperature, vibration, pressure, acoustic wave, and strain, etc. Many of the FFPI sensors operate over the linear range of an interference fringe. Compared to FFPI sensors using fringe counting, sensors working in the linear region have the advantages of a linear transfer function, no fringe direction ambiguity, simple signal processing and high resolution, and are therefore suitable for detection of small gap changes and wideband applications. However, confining the operation to the linear region places difficult manufacturing constraints on the sensor head by requiring the initial cavity length to be positioned at the quadrature point (Q-point) or sometimes one end of a linear region, of the transfer function. Linear region sensors also suffer from signal fading caused by environment perturbations other than the measurand, which may drive the sensors out of the linear regions. This is extremely critical for FFPI sensors with medium or high finesse F-P cavities in order to obtain high sensitivity.

Current operating point stabilizing techniques include using a servo system, using a tunable light source, quadrature phase-shifted demodulation or dual wavelength interrogation, and 
direct spectrum detection. The servo system method is straightforward and useful for high frequency signal measurements, but the reference voltage may not be constant due to temperature drift, static bias change or source power fluctuation. Adjusting the operating point by changing the bias current of a laser diode may cause optical power fluctuation and is sensitive to backreflections. The quadrature phase-shifted demodulation or dual wavelength interrogation was originally developed by K. Murphy et al. to solve the nonlinear transfer function and directional ambiguity problems in extrinsic Fabry-Perot interferometric (EFPI) sensors, but it may also be used for operating point stabilizing for sensors working in the linear region as well. However, it is possible that neither of the two quadrature channels will operate at the optimal Q-point at a given time, and maintaining a $90^{\circ}$ phase shift is as hard as controlling the operating-point itself.

Strictly speaking, the spectrum detection method should not be categorized as an operatingpoint stabilizing method, although a linear response may be achieved. By using a diffraction grating or a Fizeau interferometer, the modulated broadband spectrum is detected by a CCD array and analyzed by a signal-processing unit. This method does not need to control the operating point of an FFPI sensor, provides the absolute and accurate value of the optical path difference (OPD) in a sensing interferometer, and is insensitive to power and spectral fluctuations of the light source. Its major disadvantage is that it is not suitable for real time detection of broadband signals, such as acoustic waves and high frequency pressure, because a large amount of time is required to process the huge amount of data from the CCD array. The achievable frequency response is about $1 \mathrm{kHz}$. Another disadvantage of spectrum detection is the high cost, especially for sensors operating at NIR wavelengths.

In this section, we describe an alternative demodulation technique with operating point tuning, called grating-assisted operating point tuning (GA-OPT), by using a diffraction grating with feedback control. The GA-OPT features dynamic operating-point control, high frequency response, insensitivity to light source fluctuation, and real time operation.

\subsubsection{GA-OPT Operating Principle}

The operating principle of an FFPI sensor system based on GA-OPT is shown in Figure 9.20. Operating point tuning is realized using two collimators, a diffraction grating, a short piece of multimode fiber (MMF), a signal processor, a motorized rotary stage and its drive. The light from a broadband source is launched into a single-mode fiber (SMF), propagates through a 3$\mathrm{dB}$ coupler, and arrives at a FFPI sensor, where it is modulated by the perturbation measurand. The wavelength-modulated signal propagates back through the same 3-dB coupler and reaches the first collimator. The collimated light beam illuminates a diffraction grating that can be rotated by a motorized rotary stage with extremely high resolution $(\sim 0.2$ $\mathrm{mrad})$. The diffraction collected by a second collimator focuses onto a 100/140 $\mu \mathrm{m}$ MMF, and only part of the total spectrum $(\lambda \pm \Delta \lambda)$ can be collected by the MMF. Instead of a SMF or a detector, a short MMF is used to increase the reception area and to facilitate spectrum monitoring with an optical spectrum analyzer (OSA). It may be replaced by the photodetector at the cost of reduced flexibility. A signal processor is used to separate the detected signal into a dc component and an ac component. The ac output directly represents the absolute value of the ac information of the perturbation signal, and its frequency response is limited only by the sensor head and the bandwidth of the electronic circuits. The angle of the grating, 
which is obtained from the number of the driving pulses or the amplitude of analog voltage to the step motor, contains the dc information of the perturbation signal. The dc output can be used to analyze the drift of the operating point $(\Delta V)$ from the set point $\left(V_{0}\right)$, and a control signal can be generated to adjust the angle of the diffraction stage, and thereby the center wavelength of the received spectrum. Since the operating point of an interference fringe depends on the center wavelength of the interrogation light, dynamic tuning of the operatingpoint can be realized by rotating the grating.

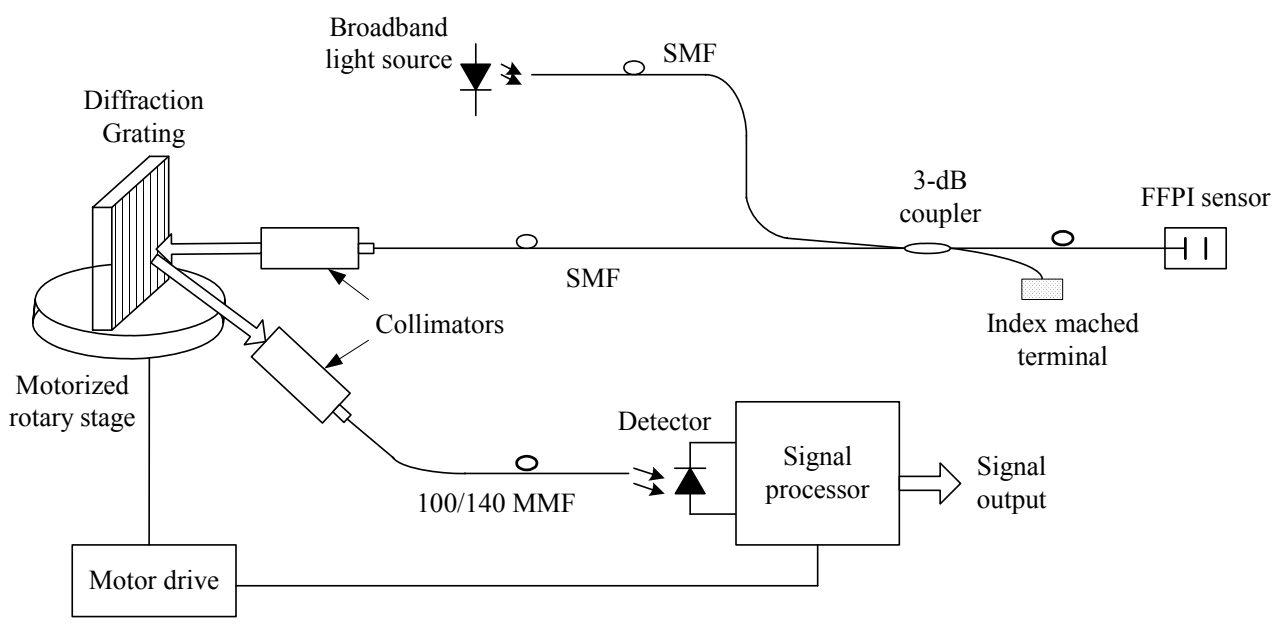

Figure 9.20. Illustration of the principle of an FFPI sensor system based on GA-OPT.

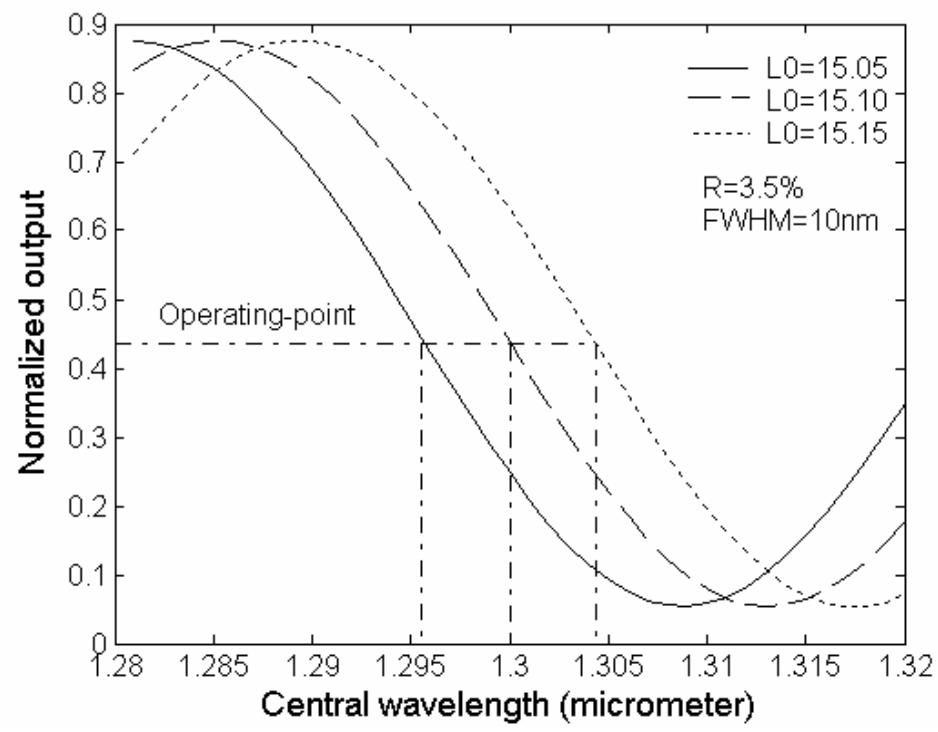

Figure 9.21. A theoretical calculation of the wavelength dependence of the operating point, where $2 \Delta \lambda=10 \mathrm{~nm}, R=3.5 \%$, and $F-P$ cavity length $L 0$ is in $\mu \mathrm{m}$. 
Figure 9.21 shows the theoretical wavelength dependence of the interference fringe on different F-P cavity lengths for a $1300 \mathrm{~nm}$ light source of a $40 \mathrm{~nm}$ spectrum width. If the F-P cavity length drifts from $L_{0}=15.05 \mu \mathrm{m}$ to $15.15 \mu \mathrm{m}$, the operating-point can be tracked by tuning the central wavelength from $1.296 \mu \mathrm{m}$ to $1.303 \mu \mathrm{m}$. Absolute measurement may be realized if $V_{0}$ can be calculated from a fringe measured during a reset scan without the presence of any perturbation signal. The tuning range is determined by the source spectrum width and the dc accuracy is limited by the resolution and repeatability of the rotary stage.

According to the grating equation, the relationship between the incident beam and $-1^{\text {st }}$ diffracted beam can be expressed as

$$
\sin (A)+\sin (B)=\lambda / d,
$$

where $A$ : the incident angle of the light beam from the input collimator with respect to the normal of the grating;

$B$ : the angle of -1 st diffraction with respect to the normal of the grating;

$\lambda$ : the wavelength of the light in the air;

$\mathrm{d}$ : the groove spacing of the grating.

Assuming a lens of focal length $f$ is used in the receiving collimator, we can calculate $B$ by

$$
\cos (B)=\frac{f(d \lambda / d x)}{d}
$$

and

$$
\frac{d \lambda}{d x}=\frac{\Delta \lambda}{D}
$$

where $\Delta \lambda$ - the spectral resolution;

$\mathrm{D}$ : the diameter of the core of the receiving MMF or the active area of the detector.

Assume $\lambda=1300 \mathrm{~nm}, d=106 / 750 \mathrm{~nm}, f=6.5 \mathrm{~mm}, \Delta \lambda=10 \mathrm{~nm}, D=0.1 \mathrm{~mm}$ for a $100 / 140 \mu \mathrm{m}$ MMF, and $\Delta \lambda / D=100 \mathrm{~nm} / \mathrm{mm}$. From Eq.(9-1) and (9-2), $B$ and $A$ can be obtained as $B=60.82^{\circ}$ and $A=5.85^{\circ}$. Obviously, the resolution $\Delta \lambda$ is variable by changing the fiber size, the focal length $f$ the incident angle and receive angle are fixed.

Figure 9.22 shows the calculated angular tuning range for a wavelength range of $1280-1320$ $\mathrm{nm}$ and the resulting receiving bandwidth change. An angular change of $1.2^{\circ}$ is sufficient to scan a wavelength range of $40 \mathrm{~nm}$, resulting in a bandwidth error of about $3.5 \%$. This error can be compensated during the calibration of the non-flat spectrum of the broadband source. 


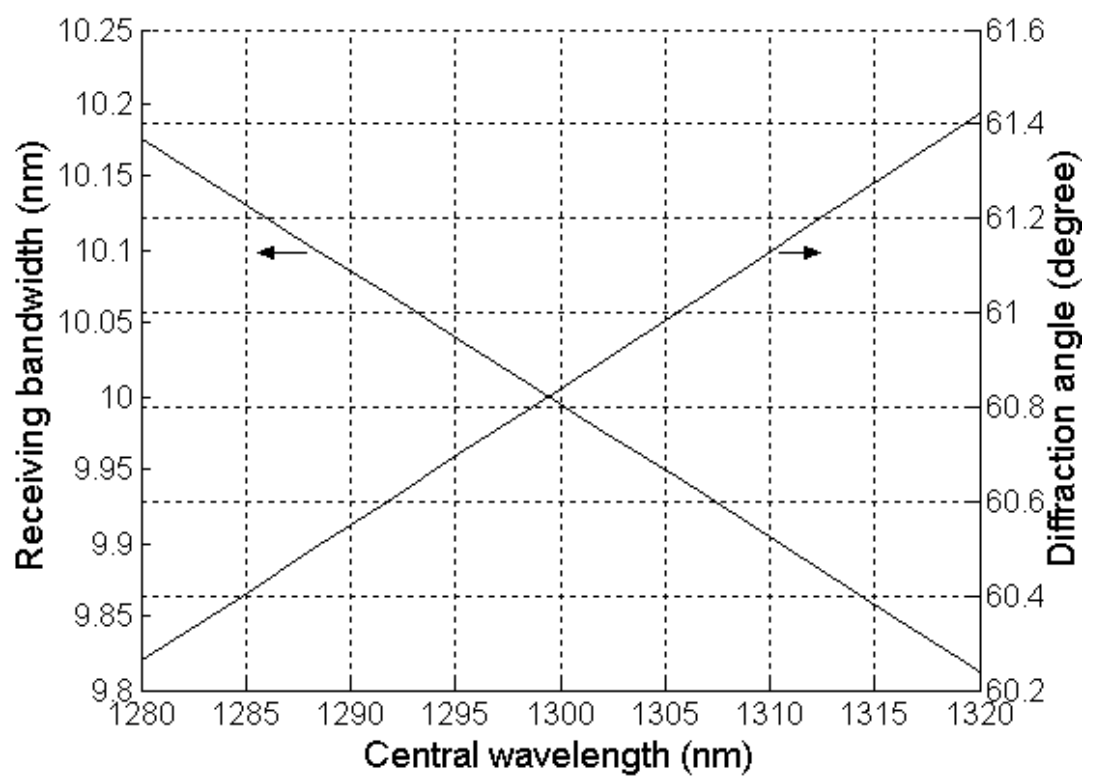

Figure 9.22. Angular range and receiving bandwidth $(2 \Delta \lambda)$ v.s. wavelength tuning range.

\subsubsection{Initial Experimental Results}

Figure 9.23 shows the spectral distributions received by the detector at different grating positions $\left(311^{\circ} 00^{\prime}, 310^{\circ} 42^{\prime}, 310^{\circ} 30^{\prime}, 310^{\circ} 12^{\prime}, 310^{\circ} 00^{\prime}, 309^{\circ} 42^{\prime}\right)$ and the original spectral distribution of the SLED. A $1300 \mathrm{~nm}$ SLED with a spectral width of $35 \mathrm{~nm}$ was used as the broadband source. By scanning the grating through an angle of $1^{\circ} 18^{\prime}$, a center wavelength change of $40 \mathrm{~nm}$ was achieved. This agrees well with the theoretical calculation given in Figure 9.22. The resulting spectral bandwidth (FWHM) is $\sim 5 \mathrm{~nm}$, only half of the theoretical value. This can be attributed to the misalignment between the $6.5 \mathrm{~mm}$ lens used in the experiment and the $100 / 140 \mu \mathrm{m} \mathrm{MMF}$ and the small aperture of $6.5 \mathrm{~mm}$ lens. This can be easily corrected through the use of a commercial collimator with a larger aperture. The insertion loss is about $15 \mathrm{~dB}$. The ripples on each spectrum curve are caused by the etalon formed by the end-face of the MMF and the input surface in the connector of the OSA. A tradeoff must be made between large spectral bandwidth for high optical power and small bandwidth for high fringe visibility and high operating point tuning resolution. 


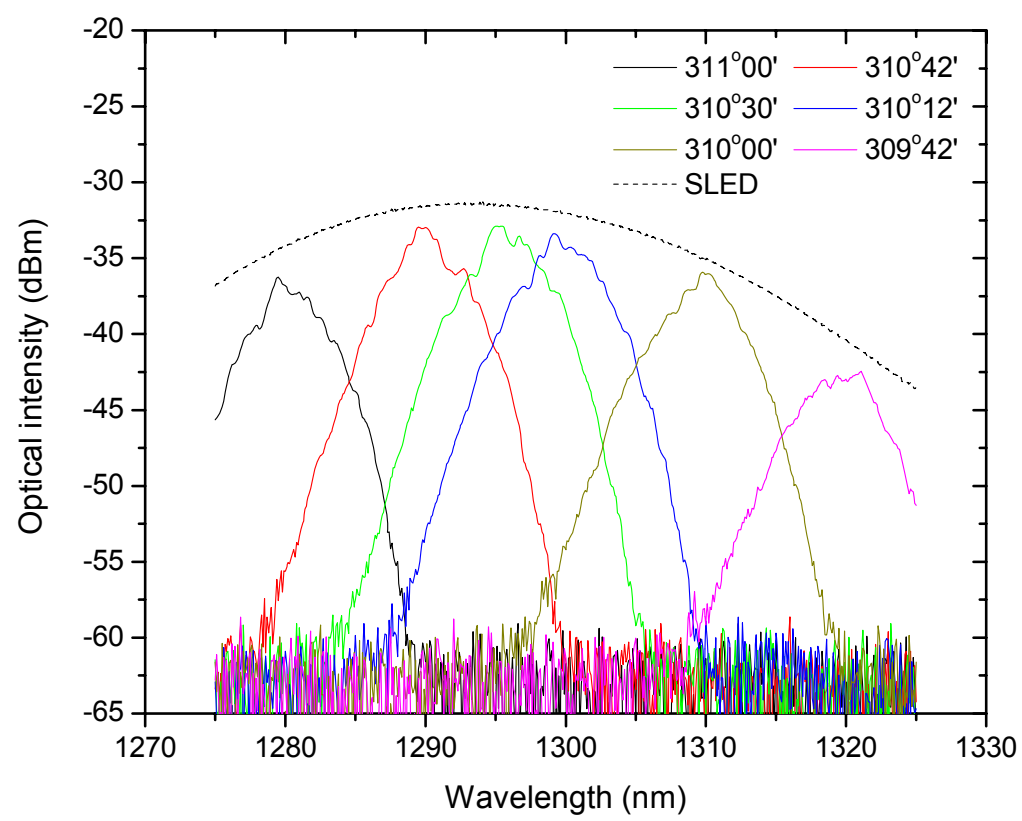

Figure 9.23. Spectral distributions received by the detector at different grating positions and the original spectral distribution of the SLED.

Figure 9.24(a) and (b) show the detected signal and its FFT from an experiment on acoustic wave detection, using a diaphragm-based FFPI sensor without operating point tuning. Obviously, the sensor was not in its linear region and the acoustic waveform is distorted. However, when GA-OPT was employed, the operating point was tuned close to its Q-point, and the sensor faithfully recovered the original waveform, as shown in Figure 9.24(c), with lower harmonic frequency as shown in Figure 9.24(d). The frequency response achieved is about $250 \mathrm{kHz}$, which is limited by the bandwidth of the electronic circuits.

\subsection{Digital Signal Processing (DSP) System}

The wideband and narrowband signals from the pre-amplifiers are processed by a DSP system. The main functions of the DSP system are:

- Data acquisition

- Calculation

- $\quad$ Signal filtering

- Linearization

- LCD screen display

- Communication with personal computer

A diagram of the DSP system is shown in Figure 9.25. The DSP system contains a standalone SBC6X DSP board, an A4D1 A/D module, a signal conditioning terminal board 
and an LCD display screen. The core of the DSP system is a $200 \mathrm{MHz}$ TMS320C6201 fixedpoint DSP processor. The wideband and narrow signals are converted to digital signals by the A4D1 A/D module. The ratios of the narrowband and the wideband signals are calculated then filtered by a low pass FIR filter so that high frequency noise can be removed. Then the filtered signals are linearized according to the real pressure values. All of these functions are implemented by the high performance DSP-based SBC6X controller. Real time pressure values and history curves can be displayed on the LCD display screen. Real time pressure values and buffered history curves can be communicated to a personal computer via a USB communication interface.

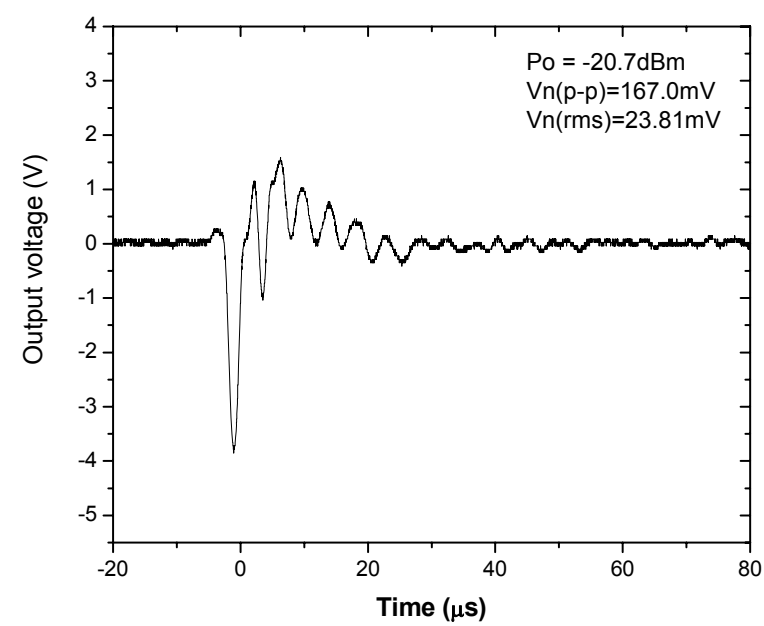

(a)

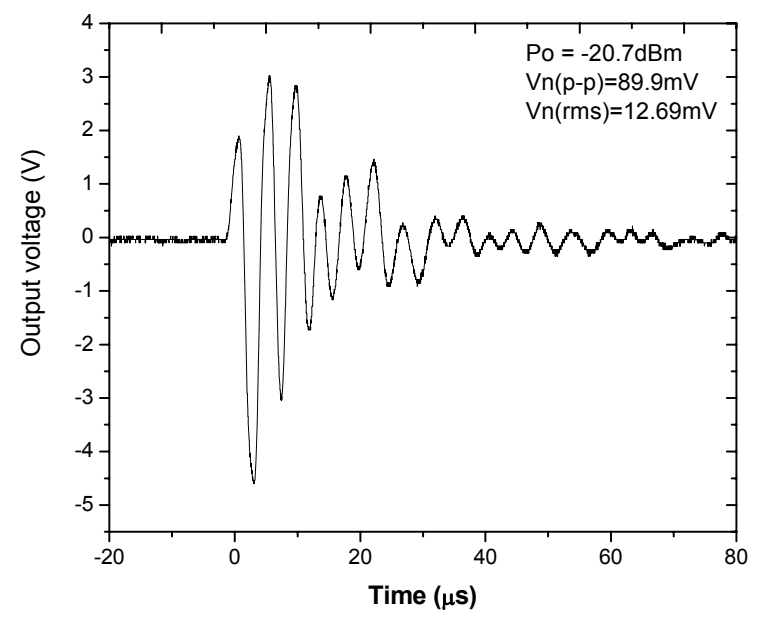

(c)

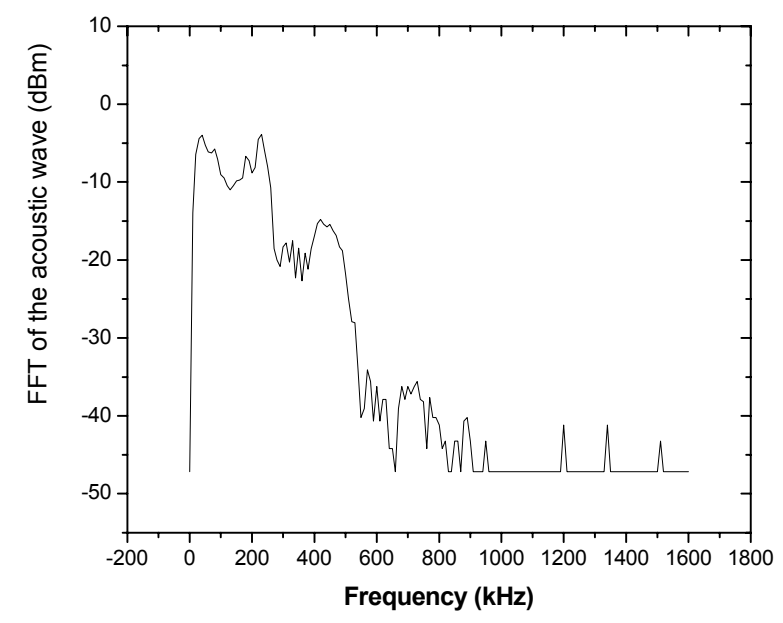

(b)

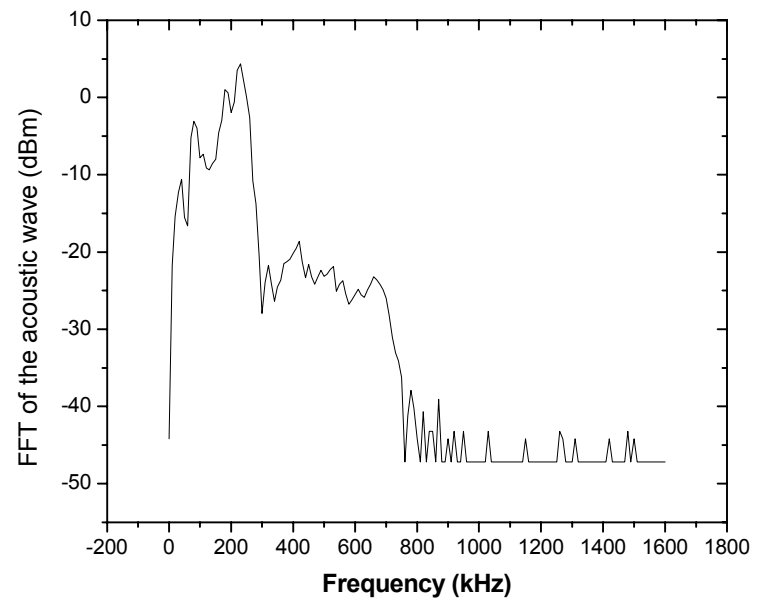

(d)

Figure 9.24. Acoustic wave and its spectrum detected by the fiber sensor without GA-OPT [(a) and (b)] and with GA-OPT [(c) and (d)]. 


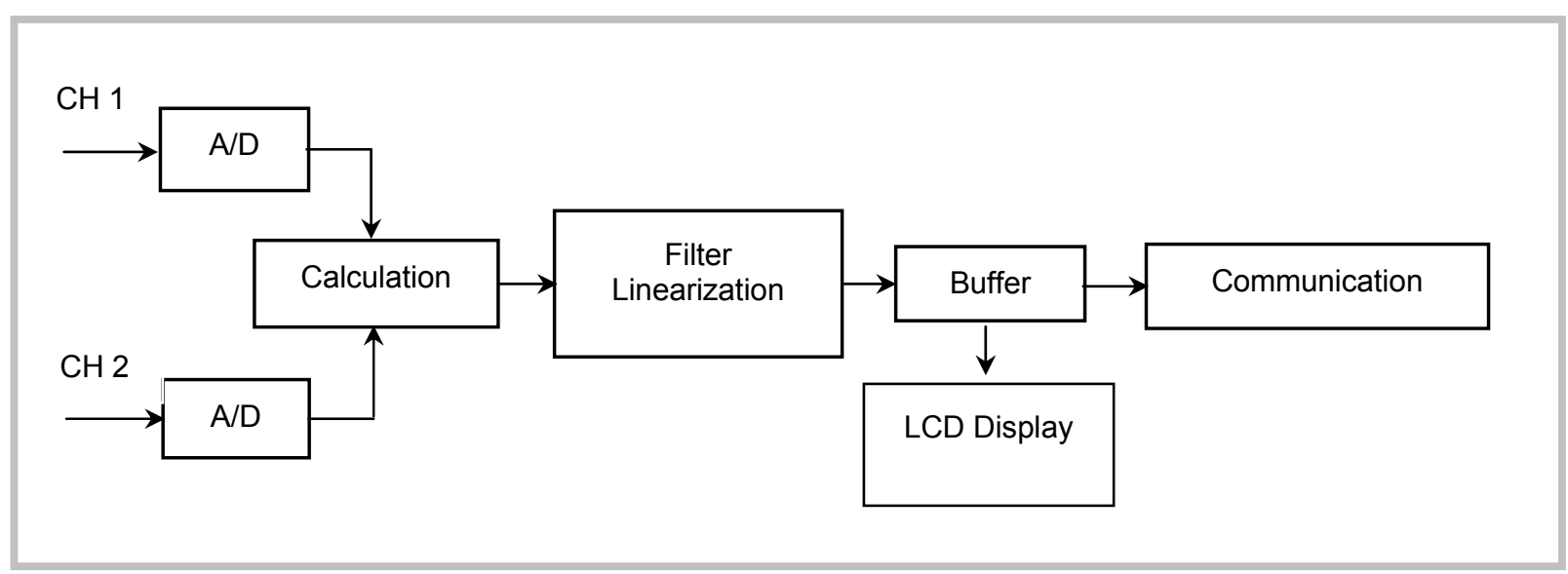

Figure 9.25. Signal flow diagram for the digital system.

\subsubsection{Functions of Digital System}

Data Acquisition

The A4D1 Analog I/O Module is a high-speed analog I/O module from Innovative Integration. Its main features are listed below.

A/D converters: 4 channels
Update rate: $10 \mathrm{MHZ}$ max
Resolution: $14-\mathrm{Bit}$
Analog input range: $+/-2 \mathrm{~V}$
S/N ratio: $75 \mathrm{~dB}$
64K FIFO

Two channels (channel 0 and channel 1) are used to sample the wideband and narrowband signals of pre-amplifiers. The sampling rate is programmable according to the signal bandwidth needed. It must be greater than twice that of the signal frequency. In this system, sampling rate is selected to be 8 times that of the signal frequency band. The desired signal bandwidth can be set to $50 \mathrm{kHZ}, 100 \mathrm{kHZ}$ or $150 \mathrm{kHZ}$, so the sampling rate can be set to 400 $\mathrm{kHz}, 800 \mathrm{kHz}$ and $1200 \mathrm{kHz}$ respectively. There is a 64K FIFO buffer in the A/D module. When half of the buffer is filled, the A/D module will generate an interrupt to the DSP processor and it will retrieve a block of data from the FIFO. The data processing speed benefits a great deal from this kind of block processing.

\section{Pressure Signal Calculation}

The narrowband digital signal is divided by the wideband digital signal producing the compensated pressure signals. 


\section{Signal Filtering}

The pressure signal is filtered by a low pass FIR digital filter to eliminate high frequency noise. The FIR filter is designed using a least squares method. The desired signal bandwidth can be set to $50 \mathrm{kHZ}, 100 \mathrm{kHZ}$ or $150 \mathrm{kHZ}$. The designed gain of the stop band is $-60 \mathrm{~dB}$ compared with $0 \mathrm{~dB}$ for pass band. The performance of the digital filter has been tested. The results show that it is fast enough to filter a signal with $150 \mathrm{kHz}$ bandwidth. Noise above the desired signal bandwidth will be remarkably well filtered.

\section{Linearization}

The relationship between the detected pressure signals and the real pressure values is not linear. It will be linearized by the digital signal processing unit. This will be implemented after the tested sensor performances are satisfactory.

\section{LCD Display}

The LCD display module is a GM24642 $240 * 64$ dot matrix LCD screen. The real time pressure data and history curves of pressure signals as well as raw narrowband and wideband signals can be displayed.

\section{Communication Interface}

The DSP target applications are downloaded and debugged via the RS232 interface on the SBC6X board. The maximum baud rate is $115 \mathrm{~kb} / \mathrm{s}$. The USB interface on the SBC6X board is used to communicate with a monitor application running on a host personal computer. The host program uses this interface to configure operating parameters of the target DSP application, and to retrieve pressure data from the DSP board. The maximum packet transfer rate is $12 \mathrm{Mbps}$.

\subsubsection{Software Development}

\section{Development Environment}

The software development environment includes following main parts:

- Code Composer Studio V2.0 from Texas Instruments

- Code Hammer PCI JTAG Emulator from Innovative Integration

- Zuma ToolSet from Innovative Integration

Code Composer Studio V2.0 is one of the most powerful integrated programming, debugging, and profiling development environment of the TMS320C6XXX series DSP applications from Texas Instruments. The main programming languages are $\mathrm{C} / \mathrm{C}++$ and assembly language. Code Hammer PCI JTAG Emulator is a PCI board plugged into a computer together with a JTAG head to simulate and debug real time DSP applications. Zuma ToolSet is a development package providing many useful library functions and development tools to develop applications for a SBC6X DSP board.

\section{Target Software}

Target software refers to the program running on the SBC6X DSP board. It is written using C and assembly language. The main functions of target program are: 
- System initialization

- Data acquisition through A4D1 A/D module

- Pressure signal calculation

- Low pass filtering

- Linearization of the pressure signals

- Real time pressure value display on LCD

- Communication with personal computer

The main program first initializes the DSP board and A/D, D/A and other I/O devices. Then it goes into a loop to process the sampled data, display it on the LCD screen and to handle the communication requests that the host computer has sent.

The data sampled by the A4D1 will go into a FIFO buffer on the A/D module. When half of the buffer is filled, an interrupt will be requested to the TMS320C6201 processor. In the interrupt handling routine, the sampled data in the FIFO will be read and calculated, then sent into the data buffer in the main system memory, and the data ready flag will be set to notify the main program that there are new data that need to be processed.

The main loop of the target program will check if there is new data need to be processed. If so, the sampled data will be filtered by a FIR low pass filter to eliminate high frequency noise. The filtering speed has been optimized with some routines written in assembly language. The DSP filter can process a 512 short integer data block within 260us. After the pressure signals are processed and linearized, the real time value of the pressure will be displayed on the LCD screen, and recent history values will be stored in a history data buffer. The display will be refreshed every $500 \mathrm{~ms}$. The recent history curve can be displayed on the LCD screen.

The target program will listen on the USB communication port. When there is a communication request, the target program will transfer relevant requested data to the host personal computer according to the different communication commands received.

\section{Host Software}

Host software refers to the program running on a personal computer to communicate with the DSP board. The platform of the host program is Microsoft Windows $9 \mathrm{X} / 2 \mathrm{~K}$. It is written using Microsoft Visual C++ V6.0. The main functions of the host program are:

- Parameter configuration of the target program

- Monitoring the DSP application through a high speed USB link

- Data logging

The operating parameters of the target DSP application can be set by the host application. These parameters include sampling rate, filter parameters, display mode etc. Both real time pressure data and pressure history can be transferred from the DSP target application to the host application. The host application can thus monitor the pressure signals through this communication link and the transferred pressure signal can be recorded through the datalogging module of the host application. 
The host program is a multithreaded application. Different threads are used to communicate with the DSP board, to display real time and history pressure signals and to log the data so that a high performance host program can be achieved.

\subsubsection{Prototype System Performance}

A prototype of the digital signal processing system has been implemented. The signal to noise ratio (SNR) of the DSP system itself is about $70 \mathrm{~dB}$. The noise performance of the entire system is limited to the SNR of the wideband and narrowband signals from the preamplifiers. The FIR digital filter can eliminate high frequency noise remarkably well. The signal processing speed is fast enough to process a signal which has a $150 \mathrm{kHz}$ bandwidth or even higher.

\subsection{Thermally Tunable Extrinsic Fabry-Perot Filter}

Tunable optical filters are a key component in the interrogation of optical sensors[33], in spectroscopy [34], as well as in video distribution networks [35]. A variety of bandpass tunable filters have been demonstrated in the past decades. Among these, acousto-optic tunable filters (AOTFs) [36], fiber Fabry-Perot tunable filters (FFP-TFs) [37], and micromachined tunable filters [38] have attracted the most interest. A significant amount of these efforts have been focused on meeting the requirements of WDM networks, in which a tuning range covering the erbium doped fiber amplifier spectral range $(\sim 40 \mathrm{~nm})$, a bandwidth smaller than $1.0 \mathrm{~nm}$ for high channel capacity, and high tuning speed on the order of microsecond or even faster are generally required [39]. However, in sensing systems, spectrometers and video networks, tunable filters of extremely low cost with a tuning range above $80 \mathrm{~nm}$, a passband from $0.5 \mathrm{~nm}$ to $10 \mathrm{~nm}$, an insertion loss less than $3.0 \mathrm{~dB}$, good repeatability and long term stability have obvious advantages. On the other hand, a tuning speed up to sub-second is still acceptable in some applications. Tunable filters addressing these application needs are desirable.

The thermally tunable filter demonstrated during the program has a tuning range of $90.8 \mathrm{~nm}$ with a temperature sensitivity of $1.63 \mathrm{~nm} /{ }^{\circ} \mathrm{C}$ and a long-term drift of less than $5.0 \mathrm{pm}$ in the $1550 \mathrm{~nm}$ band. The filter features polarization independence, high repeatability, and inherently low drift and low insertion loss.

\subsubsection{Principle of Operation}

The core of the device described here is basically a high-finesse EFPI that is formed by fusion bonding of two mirror-coated single-mode optical fibers to a short piece of borosilicate capillary tubing using fusion bonding, shown in Figure 9.26. For a Fabry-Perot interferometer, the resonant or transmission peaks occur when the optical phase difference $\delta=2 \pi \mathrm{nL}_{\mathrm{a}} / \lambda$ is equal to multiples of $2 \pi$, where $\mathrm{L}_{\mathrm{a}}$ is the distance between the two mirrors, and $\mathrm{n}=1.0$ is the refractive index of the air in the cavity. For a given $\mathrm{L}_{\mathrm{a}}$, multiple wavelengths satisfy the resonant conditions, resulting in multiple transmission bands. Changing $\mathrm{L}_{\mathrm{a}}$ results in the change of the resonant condition, and thus the shift of the transmission band. 


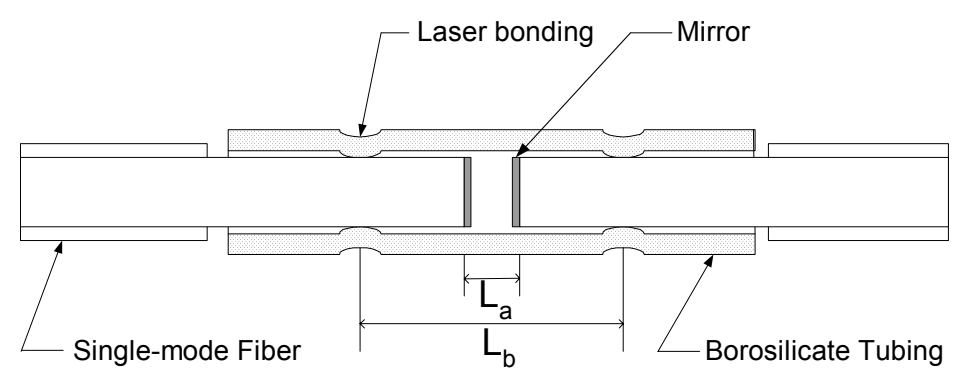

Figure 9.26. Schematic of the thermally tunable EFPI filter.

Assuming that the central wavelength of the filter passband is $\lambda_{0}$ when the airgap is $L_{a}$, the shift of the central wavelength $\Delta \lambda$ caused by an airgap change $\Delta L$ can be expressed as

$$
\Delta \lambda=\left(\Delta L / L_{a}\right) \cdot \lambda_{0}
$$

The wavelength shift is proportional to the ratio $\Delta L / L_{a}$, which means a large tuning range may be obtainable with a small change of the airgap, if a short cavity is used. Taking advantage of the differential thermal expansions of the borosilicate tubing and the silica fiber, continuous tuning of the filter passband can be achieved by locally cooling or heating the EFPI. Assuming that a temperature change from $T$ to $T+\Delta T$ is applied to an EFPI filter of gauge length $L_{b}$, corresponding to the distance between the two bonding points, resulting an airgap change from $L_{a}$ to $L_{a}+\Delta L, \Delta L$ is given by

$$
\Delta L=\left[\alpha_{b} L_{b}-\alpha_{f}\left(L_{b}-L_{a}\right)\right] \cdot \Delta T,
$$

where $\alpha_{b}$ and $\alpha_{f}$ are the coefficients of thermal expansion (CTE) of the borosilicate tubing and the fused silica fiber, respectively. $\alpha_{b}$ and $\alpha_{f}$ are constants from 0 to $300^{\circ} \mathrm{C}$. Substituting Eq. (9-5) into Eq. (9-4) and assuming that $L_{a}<<L_{b}$,

$$
\Delta \lambda=\left(L_{b} / L_{a}\right) \cdot\left(\alpha_{b}-\alpha_{f}\right) \cdot \lambda_{0} \cdot \Delta T .
$$

Eq. (9-6) indicates that the wavelength tuning is proportional to the temperature change. Since the borosilicate tubing has a CTE approximately 5 10 times higher than that of the silica fiber, a tuning range of above $100 \mathrm{~nm}$ can easily be achieved within a temperature range of less than $80^{\circ} \mathrm{C}$ (the normal operation range of a Peltier device).

As implied by Figure 9.26, the alignment between the two mirrors is automatically achieved because of the tight match between the inside diameter (I.D.) of the tubing and the diameter of the single-mode bare fiber. This self-alignment capability significantly reduces the coupling loss and eliminates the costly aligning process encountered by other tunable filter techniques. Furthermore high reliability is expected, since there are no moving parts and therefore no mechanical wear is involved. The long time stability of the filter is determined by the temperature stability of the Peltier device and its controller, which can be as low as $0.001^{\circ} \mathrm{C}$. 


\subsubsection{Filter Preparation}

A prototype tunable EFPI filter was constructed that operates in the $1550 \mathrm{~nm}$ band using SMF-28 single-mode fibers with a CTE of $5.6 \times 10^{-7} /{ }^{\circ} \mathrm{C}$, and Pyrex tubing with a CTE of $32.5 \times 10^{-7} /{ }^{\circ} \mathrm{C}$, an I.D of $127+1.5 \mu \mathrm{m}$ and an O.D. of $290 \mu \mathrm{m}$. The two multilayer dielectric mirrors were deposited on the cleaved ends of the fibers by Evaporated Coating, Inc.. The mirrors have a peak reflectance of $R=87.5 \%$ at $1565 \mathrm{~nm}$, reduced to $87.0 \%$ at $1529 \mathrm{~nm}$ and $1602 \mathrm{~nm}$. The fibers were fusion bonded to the Pyrex tubing using a pulsed $\mathrm{CO} 2$ laser at relatively high power, forming an airgap of $10.589 \mu \mathrm{m}$ at $T=25.45^{\circ} \mathrm{C}$ and a gauge length $\mathrm{L}_{\mathrm{b}} \approx$ $4.1 \mathrm{~mm}$. During the bonding process, an LED with a central wavelength of $1557.6 \mathrm{~nm}$ and a bandwidth $\Delta \lambda_{\mathrm{FWHM}}=61.7 \mathrm{~nm}$, and an AQ-6315A optical spectrum analyzer (OSA) were used to monitor the airgap length. Fine adjustment of the airgap can be made with low power $\mathrm{CO}_{2}$ laser pulses. Figure 9.27 shows an optical micrograph of the airgap.

The EFPI filter was then assembled into a 14-pin butterfly package with a thermoelectric cooling (TEC) module and a thermistor, shown in Figure 9.28. To achieve fast thermal response, the EFPI filter was brought in direct contact with the cold plate of the TEC and thermal compound was used to enhance thermal coupling between the TEC and the EFPI filter.

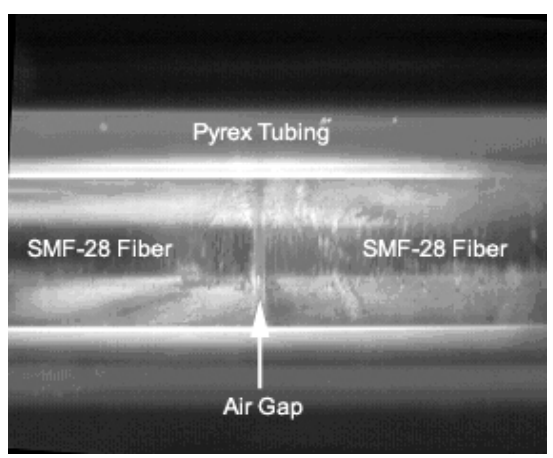

Figure 9.27. Optical micrograph of an EFPI filter.

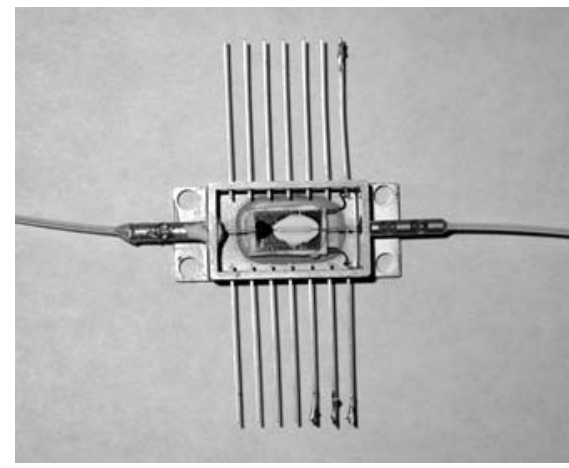

Figure 9.28. Photo of a packaged thermally tunable EFPI filter. 


\subsubsection{Filter Characterization}

To characterize the prototype filter, a TEC controller was constructed with a 24-hour temperature stability of $0.003^{\circ} \mathrm{C}$, whose input is set by a computer through a high resolution DAC card. The same light source and OSA for the fabrication was used for the performance evaluation. Figure 9.29 gives the output spectrum of the filter normalized to that of the source at a set voltage $\mathrm{V}_{\mathrm{SET}}=1.7 \mathrm{~V}$, corresponding to $\mathrm{T}=13.55^{\circ} \mathrm{C}$.

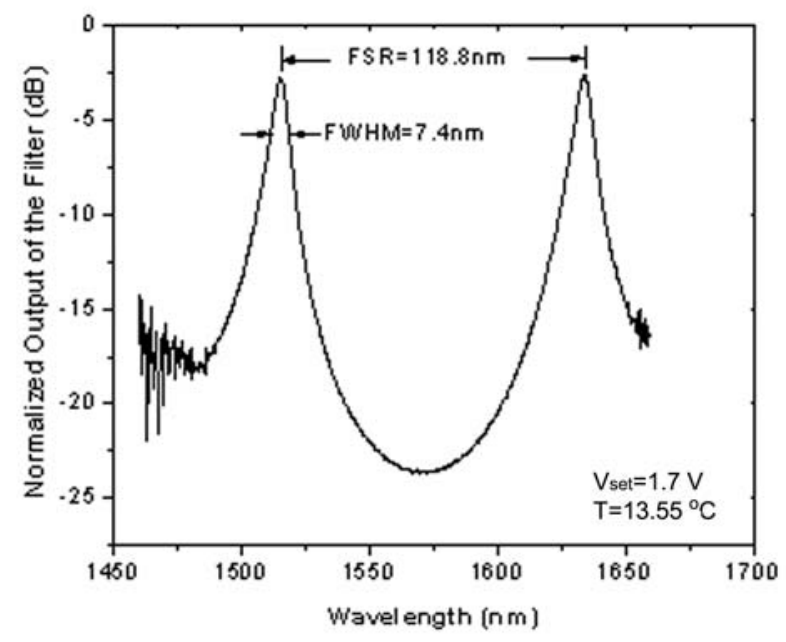

Figure 9.29. Normalized spectrum at $\mathrm{T}=13.55^{\circ} \mathrm{C}$.

A $3 \mathrm{~dB}$ bandwidth of $7.4 \mathrm{~nm}$ on the first passband, a free spectral range (FSR) of $118.8 \mathrm{~nm}$, and an insertion loss of $2.7 \mathrm{~dB}$ were measured at this temperature. From the positions of the two peaks, the airgap can be readily calculated as $10.425 \mu \mathrm{m}$. The FSR may be doubled by reducing the airgap to a few micrometers. A narrower passband as low as $1.0 \mathrm{~nm}$ can be obtained by using either mirrors of higher reflectance or longer cavity length. The major sources of the insertion loss include misalignments between the two fibers, absorption and asymmetry of the two mirrors. The misalignments includes the end separation, which is small in this case, and the tangential misalignment caused by the mismatch between the diameter of the fiber and the I.D. of the tubing. Insertion loss close to $1.0 \mathrm{~dB}$ can be expected by using tubing of smaller I.D. and higher quality thin films on polished fiber ends.

Continuous tuning of the passband has been achieved by scanning the output voltage of the DAC. Figure 9.30 shows the dependence of the central wavelength of the filter on the control voltage with a $3^{\text {rd }}$ order exponential curve fit. A tuning range of $90.8 \mathrm{~nm}$ was obtained with a voltage change of $1.7 \sim 0.2 \mathrm{~V}$, or a temperature change from $13.55^{\circ} \mathrm{C}$ to $69.28^{\circ} \mathrm{C}$. This tuning range agrees well with the predicted value of $89.8 \mathrm{~nm}$ by Eq. (9-6). The $3^{\text {rd }}$ order exponential dependence of the central wavelength on the control voltage can be explained by the similar dependence of the resistance of the thermistor on temperature. When converting the voltage to corresponding temperature, very good linear dependence of the wavelength on temperature resulted, as shown in Figure 9.31. A temperature sensitivity of $1.63 \mathrm{~nm} /{ }^{\circ} \mathrm{C}$ has been measured from the linear fit. The long time drift of the filter passband 
has been determined by the temperature stability, which is less than $5.0 \mathrm{pm}$ in the laboratory environment.

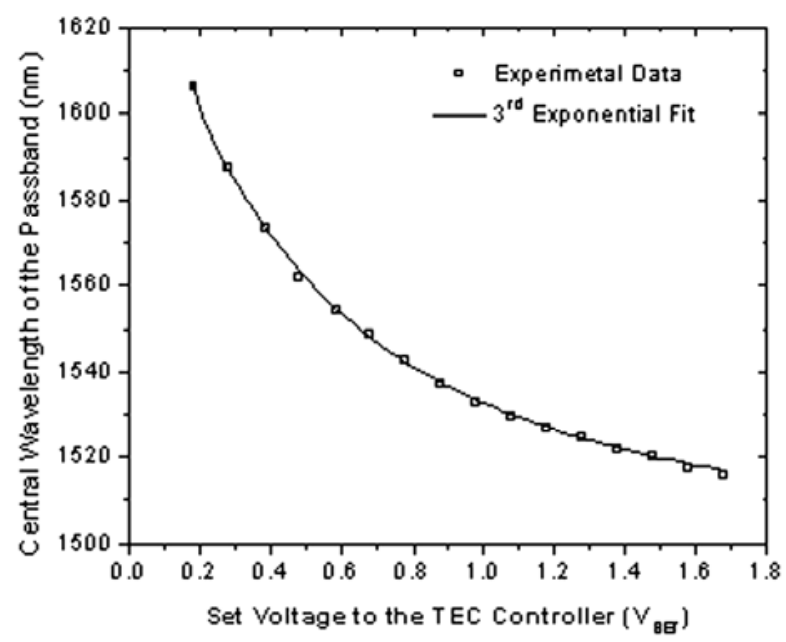

Figure 9.30. Tuning curves of the thermally tunable EFPI filter.

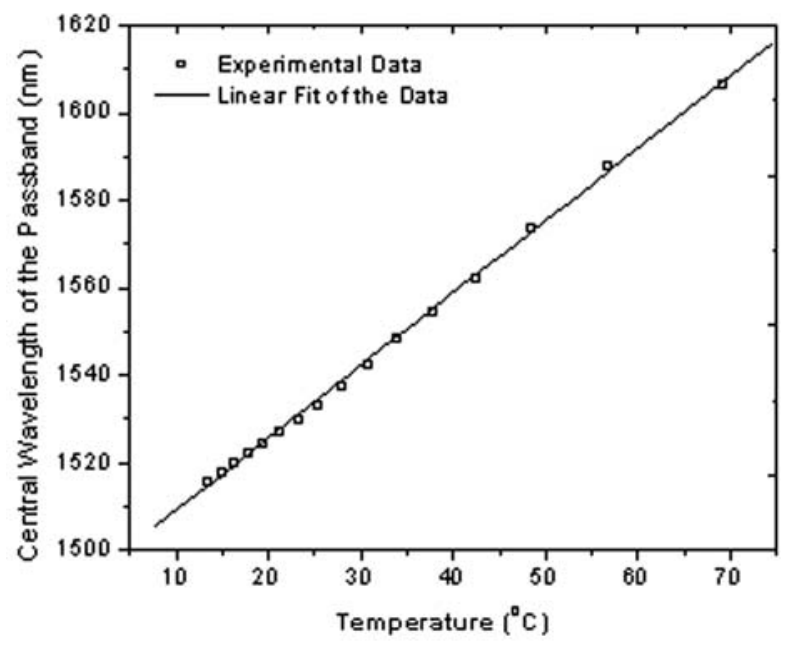

Figure 9.31. Filter's temperature sensitivity and linearity.

A bandwidth of $7.4 \mathrm{~nm}$ at $1515.6 \mathrm{~nm}, 6.58 \mathrm{~nm}$ around $1565 \mathrm{~nm}$ and $7.0 \mathrm{~nm}$ at $1606.4 \mathrm{~nm}$ was measured. This bandwidth change is a combined effect of the airgap change with temperature and the non-flat reflectance spectrum of the mirrors, which has a peak at $1565 \mathrm{~nm}$. The bandwidth can be described by $F W H M=(1-R) \cdot F S R /\left(\pi R^{1 / 2}\right)$ and $F S R$ is inversely proportional to the airgap $L_{a}$, and thus to $T$. On the other hand, the peak reflectivity makes the filter have a smaller FWHM around $1565 \mathrm{~nm}$ than at other wavelengths.

The time needed to scan the whole tuning range was less than 2 seconds. This relatively low speed can be attributed to the large package size and the associated thermal mass. Much 
higher scanning speeds can be expected by reducing the package size, or directly depositing an electrically resistive coating onto the capillary tubing of the filter.

\subsection{Data Processing System}

Fiber sensors researched and developed by CPT featuring small size, high accuracy and high reliability, have already been used in the measurement of temperature and pressure in harsh environment. However, the data acquisition and processing system that we are using for these tiny sensors, which includes a personal computer, and a CTS or OSA, is so bulky, expensive and fragile, has limited the broad application of those high quality sensors. Therefore, the research and development of a data acquisition and processing system with portable size, ease of use, cost effectiveness, high flexibility and reliability will become an indispensable part of our sensors, and will also propel the application of them in daily life.

\subsubsection{Objective}

1. To use the Oceanoptics spectrometer, 850nm white light source and a PC to set up a Fiber Sensor Spectrum Analysis System, which is just like the OSA we now have.

2. To set up an independent (without PC) Fiber Sensor Spectrum Analysis System that has features of portability, cost effectiveness, low power consumption, data storage, communication, and simple application to many kinds of fiber sensors.

3. To transfer CPT's specialized data processing software into it.

\subsubsection{Current Data Processing System}

The smallest data processing system that we have now includes a USB spectrometer and a PC. A block diagram is shown in Figure 9.32, and the components are listed in Table 9.2.

\subsubsection{Independent Fiber Sensor Data Processing System}

A block diagram of the designed data processing system is shown in Figure 9.33, and the components are listed in 


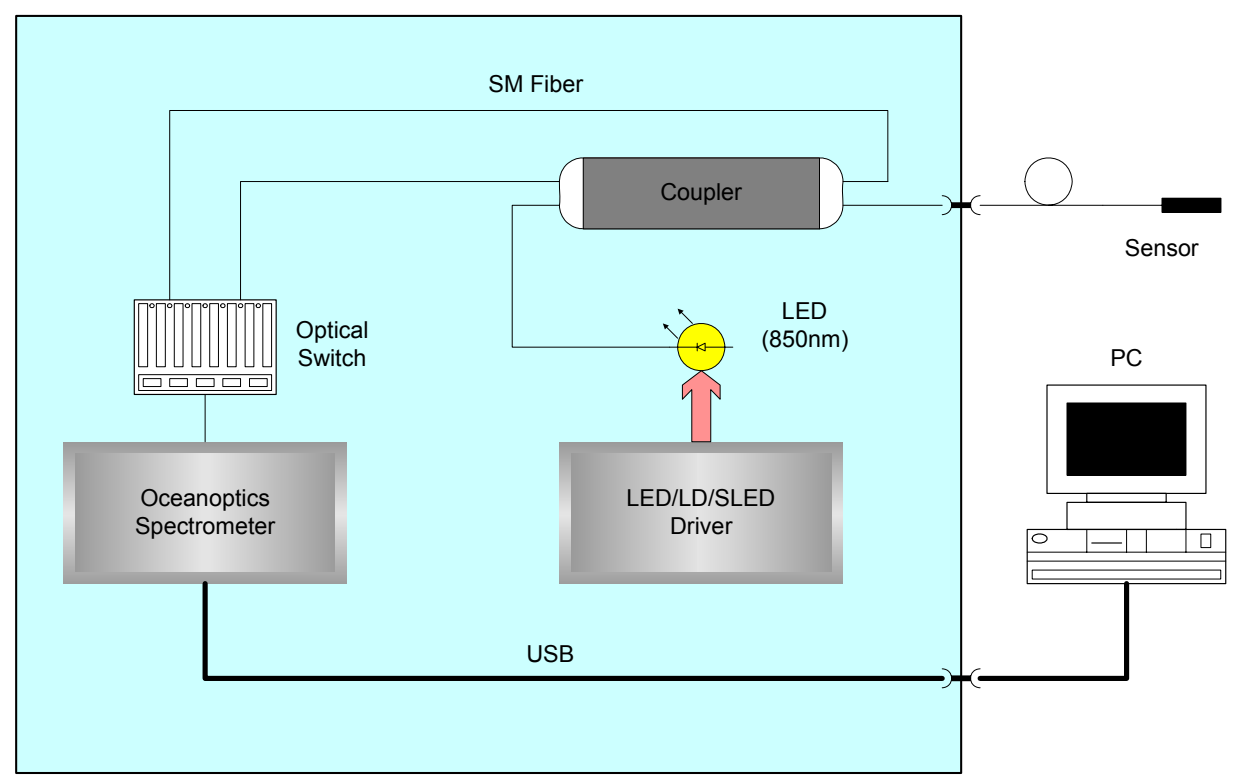

Figure 9.32. Block diagram of current data processing system.

Table 9.2. Component listing for current data processing system.

\begin{tabular}{|c|c|c|c|}
\hline Item & Description & Manufacturer & Price (USD) \\
\hline \multirow{2}{*}{ Spectrometer } & USB2000 & \multirow{2}{*}{ Oceanoptics } & \multirow{2}{*}{2,200} \\
\hline & $200 \sim 1100,0.3 \mathrm{~nm}$ & & \\
\hline LED & $850 \pm 30 \mathrm{~nm}, 410 \mathrm{uW}, 100 \mathrm{~mA}$ & Honeywell & 10 \\
\hline LED Driver & $5 \mathrm{~V}, 500 \mathrm{~mA}$ & Wavelength Eletr. & 330 \\
\hline Coupler & $2 * 2,50 \% / 50 \%$, SMF, $850 \mathrm{~nm}$ & ACPhotonics & 50 \\
\hline Optical Switch & $850 \pm 40 \mathrm{~nm}, 1 \times 2, \mathrm{MM}$ & ACPhotonics & $600^{*}$ \\
\hline $\mathrm{PC}$ & Dell Dimension 4600 & Dell & 510 \\
\hline & Total Cost & $\$ 3,10$ & \\
\hline & Total Volume & $0.7 \mathrm{~m}$ & \\
\hline
\end{tabular}

* The optical switch is multimode. A singlemode switch was unavailable and its price was prohibitive; therefore we do not use it in this system. However, if an optical switch was used, we could obtain the background spectrum at any time, then the measurement result would be very accurate, even if the background spectrum profile changed during detection. 


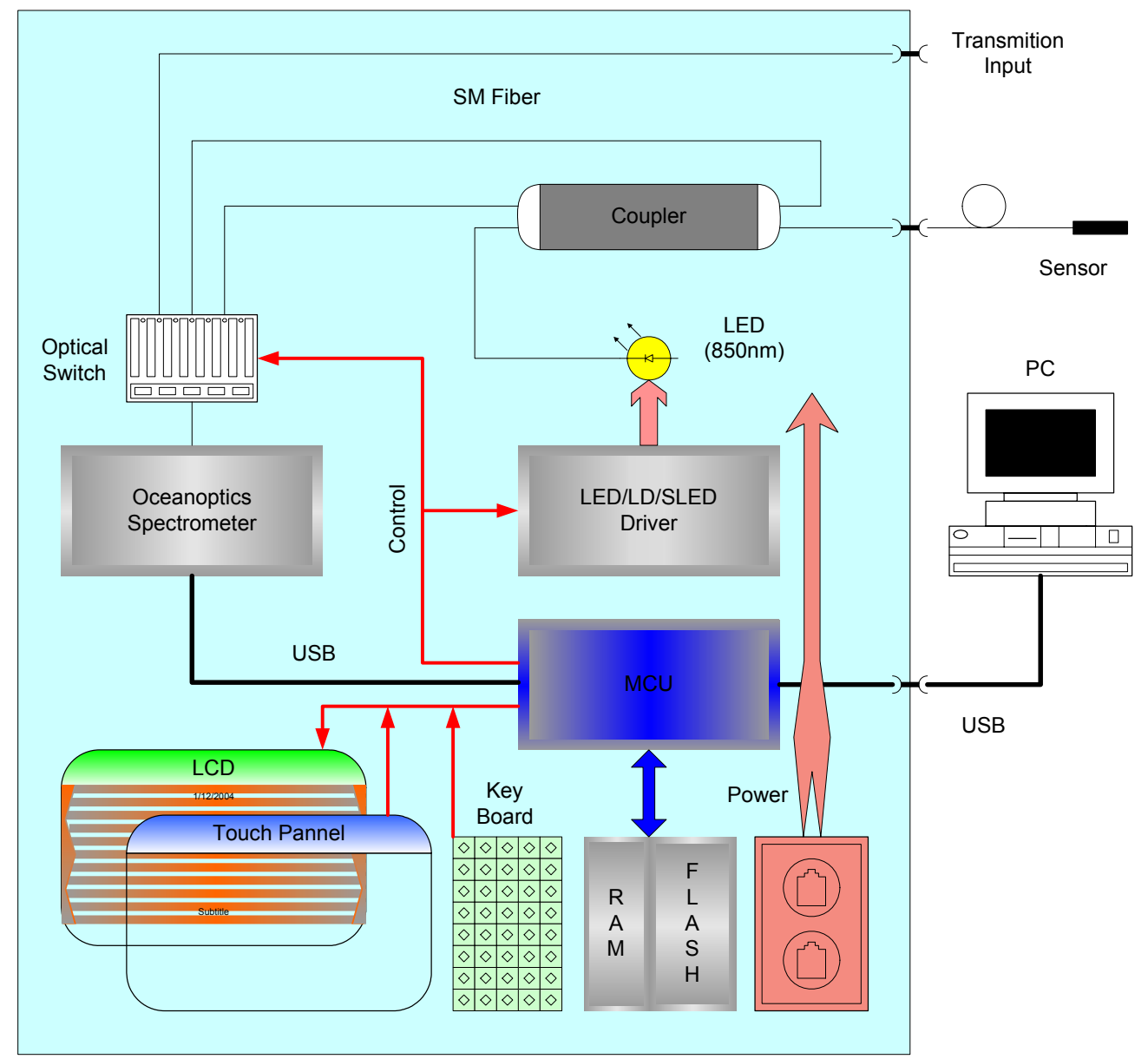

Figure 9.33. Block diagram of newly designed data processing system. 
Table 9.3. Component listing for new data processing system.

\begin{tabular}{|c|c|c|c|}
\hline Item & Description & Manufacturer & Price (USD) \\
\hline \multirow{2}{*}{ Spectrometer } & USB2000 & \multirow{2}{*}{ Oceanoptics } & \multirow{2}{*}{2,200} \\
\hline & $200 \sim 1100,0.3 \mathrm{~nm}$ & & \\
\hline LED & $850 \pm 30 \mathrm{~nm}, 410 \mathrm{uW}, 100 \mathrm{~mA}$ & Honeywell & 10 \\
\hline LED Driver & $5 \mathrm{~V}, 500 \mathrm{~mA}$ & Wavelength Eletr. & 330 \\
\hline \multicolumn{2}{|l|}{ Shell } & \multicolumn{2}{|r|}{50} \\
\hline Coupler & $2 * 2,50 \% / 50 \%$, SMF, $850 \mathrm{~nm}$ & ACPhotonics & 50 \\
\hline Optical Switch & $850 \pm 40 \mathrm{~nm}, 1 \times 2, \mathrm{MM}$ & \multirow[t]{2}{*}{ ACPhotonics } & $600^{*}$ \\
\hline $\mathrm{MCU}$ & Motorola i.XM1 & & 15 \\
\hline Flash & Am29LV640D,4M×16bits & AMD & 15 \\
\hline SDRAM & IS41LV16100,1M×16bits×2,3.3V & ISSI & 12 \\
\hline \multicolumn{2}{|l|}{ Power Module } & TI/National & 13 \\
\hline \multirow{2}{*}{$\begin{array}{l}\text { Color LCD } \\
\text { Module }\end{array}$} & $640 \times 4806.4 "$ & \multirow{2}{*}{ Sharp/LG Philips } & \multirow{2}{*}{150} \\
\hline & With Touch Panel & & \\
\hline \multirow{2}{*}{ Key Board } & Membrane Keypad & & \multirow{2}{*}{5} \\
\hline & Tact Switches & & \\
\hline PCB Board & 4 layer, $14 \mathrm{~cm} \times 14 \mathrm{~cm}$ & & 20 \\
\hline \multirow[t]{2}{*}{$\begin{array}{l}\text { Peripheral } \\
\text { Components }\end{array}$} & $\begin{array}{l}\text { Resistances, Capacitances, } \\
\text { Transformers, Inductors, etc. }\end{array}$ & & 20 \\
\hline & Total Cost & \multicolumn{2}{|l|}{2,890} \\
\hline \multicolumn{2}{|r|}{ Total Volume } & \multicolumn{2}{|c|}{$0.12 \mathrm{~m}^{3}$} \\
\hline
\end{tabular}

*Again, since an appropriate optical switch $(1 \times 3$ at $850 \mathrm{~nm})$ is not available, manual plugs will be used instead. 


\section{MCU Candidates}

The basic requirements for the micro controller unit (MCU) in this system are as follows.

\section{a) USB host or RS232 interface}

Since the spectrometer USB2000 made by Oceanoptics has USB and RS232 interfaces, the MCU should have one of these ports to control and acquire data. Because the spectrometer requires an extra special cable and power module to realize a RS232 port, a USB port will be utilized..

b) USB client interface

This port permits the data communication and control from a PC to our system.

c) LCD controller and touch panel interface

This system needs a LCD screen to display spectrum curves, command menu, calculation results and so on. If the MCU selected has an embedded LCD controller, much less work would have to be done to connect to an LCD. For use as experimental equipment, a color TFT LCD could be chosen, but for industrial use for fixed purpose, monochrome, or even a simple digits LCD is sufficient. If we use a touch panel for pointing input, a touch panel interface will facilitate our work.

d) Enough GPIO points for controlling use The MCU needs enough GPIO points, at least 5, to control the optical switch, LED driver, power module and so on.

e) High calculation speed The main tasks of the MCU are data acquisition, LCD refreshment and data transmission. The data analysis tasks, such as peak seeking, flitting, FFT, and averaging will not sacrifice much MCU capability, therefore any MCU with calculation speed greater than 10 MIPS will be qualified.

f) CCD interface A CCD will be used to detect the spectrum generated by a grating, so the MCU should have a CCD interface.

g) Easy connection to ADC

In the future, we may need to acquire data from a photo diode or non-standard CCD, so an easy connection to $\mathrm{ADC}$ is a good requirement.

There are many MCU choices for our system which satisfy all the requirements mentioned above. Three were selected for further consideration.

Motorola i.MX1 (\$15): The new MC9328MX1 features the advanced and power-efficient ARM920T ${ }^{\mathrm{TM}}$ core that operates at speeds up to $200 \mathrm{MHz}$. Integrated modules such as an LCD controller, static RAM, USB support, an A/D converter (with touch panel control), and an $\mathrm{MMC} / \mathrm{SD}$ host controller offer a suite of peripherals for volume data storage. It also has a multimedia accelerator, which has MAC for FIR and FFT operation. However, the i.MX1 only has a USB client port; we would need to use an extra USB chip to expand a host port. 


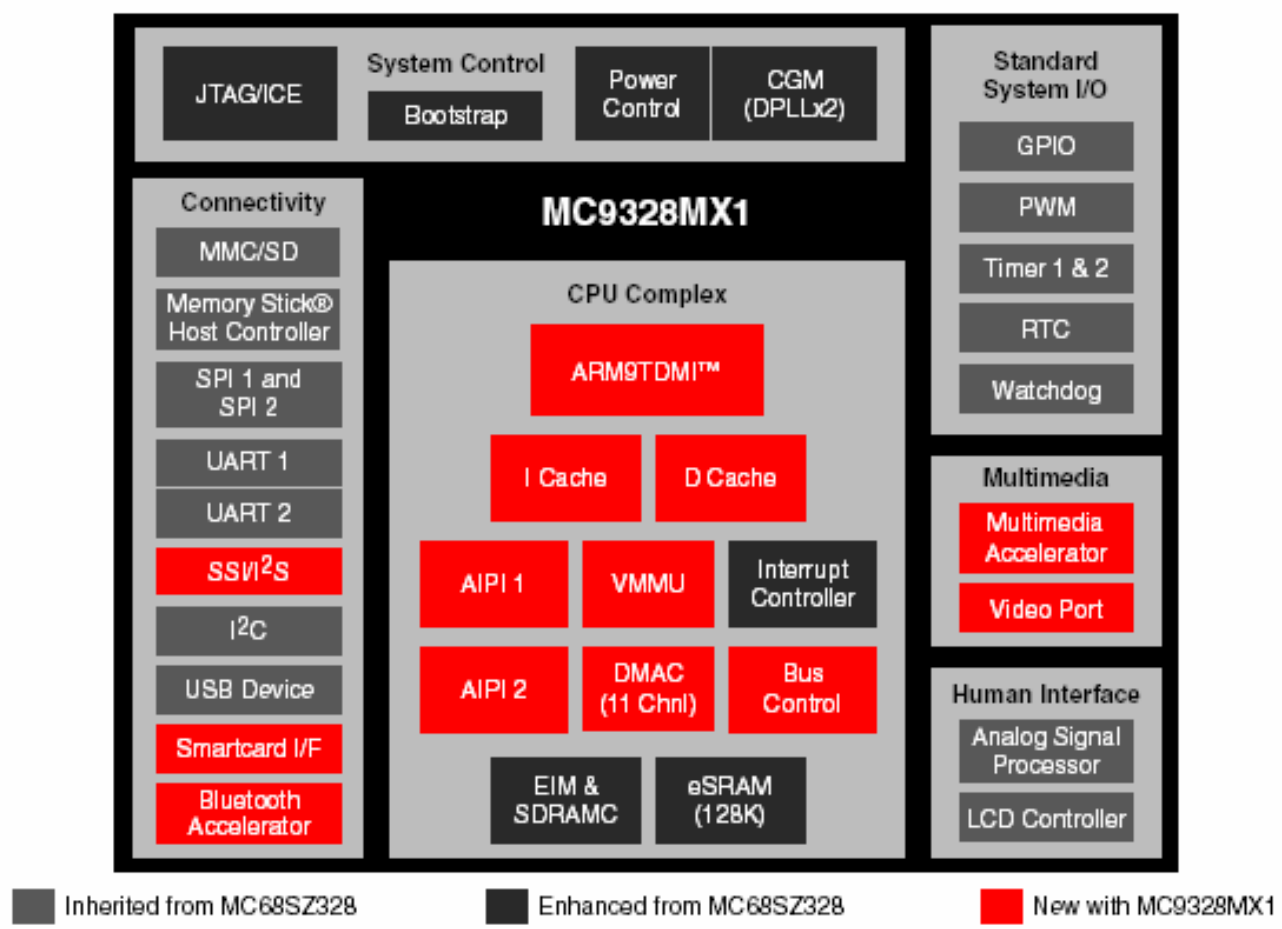

Figure 9.34. i.MX1 Functional Block Diagram.

Intel PXA255 Processor (\$18): The Intel PXA255 processor is a highly integrated, 32-bit RISC processor that combines the efficiency of Intel design with the ARM v.5TE instruction set architecture. Key features are as follows.

- Low power, high performance 32-bit Intel XScale® core-based CPU (200, 300 and $400 \mathrm{MHz}$ )

- ARM* Architecture v.5TE compliant

- Intel® Superpipelined RISC Technology utilizing advanced Intel $0.18 \mu$ process for high core speeds at low power

- Intel® Media Processing Technology including 40-bit accumulator and 16-bit SIMD to enhance audio/video decode performance

- Integrated Memory and PCMCIA/Compact Flash Controller with $100 \mathrm{MHz}$ Memory Bus, 16-bit or 32-bit ROM/Flash/SRAM (six banks), 16-bit or 32-bit SDRAM, SMROM (four banks), as well as PCMCIA and Compact Flash for added functionality and expandability

- System Control Module includes 17 dedicated general-purpose interruptible I/O ports, real-time clock, watchdog and interval timers, power management controller, interrupt controller, reset controller, and two on-chip oscillators

- Peripheral Control Module offers 16 channel configurable DMA controller, integrated LCD controller with unique DMA for fast color screen support, Bluetooth** I/F, serial ports including IrDA, I2C, I2S, AC97, three UARTs(1 Full $\mathrm{H} / \mathrm{W}$ flow control), SPI and enhanced SSP, USB end point interface, and $\mathrm{MMC} / \mathrm{SD}$ Card Support for expandable memory and I/O functionality 
However, the PXA255 also only has a USB client port, requiring the use of an extra USB chip to expand a host port. In addition, the PXA255 dose not have an ADC and CCD interface.

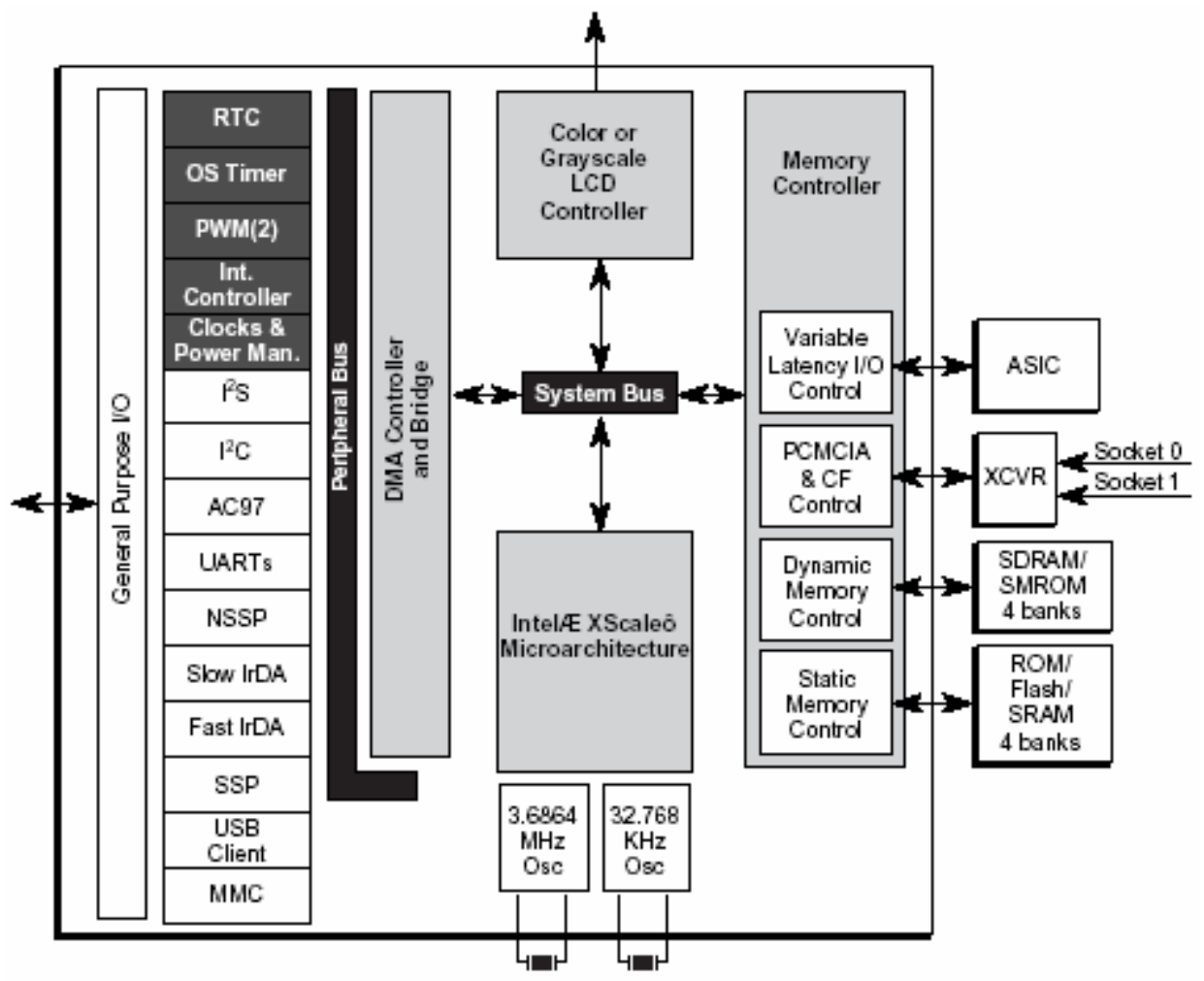

Figure 9.35. PXA255 Functional Block Diagram.

TI OMAP1510 (\$35): The OMAP1510 combines the TMS320C55x ${ }^{\mathrm{TM}}$ DSP core with a TIenhanced ARM925 processor, which provide the balance of high performance and low power consumption necessary for modern portable products, such as $2.5 \mathrm{G}$ and $3 \mathrm{G}$ wireless handsets and PDAs to run applications such as streaming video, audio, mobile commerce, location-based services and others.

TMS320C55x DSP Core:

- $\quad$ Up to $200 \mathrm{MHz}$ (maximum frequency)

Voltage: $1.5 \mathrm{v}$ nominal

- One/two instructions executed per cycle

- $32 \mathrm{~K} \times 16$-bit on-chip dual-access RAM (DARAM) (64 KB)

- $48 \mathrm{~K}$ x 16-bit on-chip single-access RAM (SARAM) (96 KB)

- $16 \mathrm{~KB}$ I-cache, $8 \mathrm{~KB}$ D-cache

- Video hardware accelerators for DCT, iDCT, pixel interpolation, and motion estimation for video compression 
TI925T ARM9TDMI Core:

- Up to $175 \mathrm{MHz}$ (maximum frequency)

Voltage: $1.5 \mathrm{v}$ nominal

- 16KB I-cache; 8KB D-cache

- 192-KB of shared internal SRAM - frame buffer

- Support for 32-bit and 16-bit (Thumb mode) instruction sets

- Data and program MMUs

- Two 64-entry translation look-aside buffers (TLBs) for MMUs

- 17-word write buffer

Application Subsystem:

- Supports Linux ${ }^{\circledR}$, Microsoft ${ }^{\circledR}$ Windows ${ }^{\circledR}$ CE, Palm OS ${ }^{\mathrm{TM}}$ and Symbian OS, Microsoft Smartphone 2002, Microsoft Pocket PC 2002 and Nokia Series 60

- Three 32-bit timers and watchdog timer

- 32-kHz timer

- Level1/Level2 interrupt handlers

- USB1.1 host interface with up to 3 ports

- USB1.1 function interface

- One integrated USB transceiver for either host or function

- Multichannel buffered serial port (McBSP)

- $\mathrm{I}^{2} \mathrm{C}$ master and slave interface

- Micro-wure serial interface

- $\mathrm{MMC} / \mathrm{SD}$ interface

- $\mathrm{HDQ} / 1$-wire interface

- Camera interface for CMOS sensors

- ETM9 trace module for TI925T debug

- Keyboard matrix interface $(6 \times 5$ or $8 \times 8)$

- Up to 10 MPU general-purpose I/Os

- Pulse-width tone (PWT) interface

- Pulse-width light (PWL) interface

- Two LED pulse generators

- Real-time clock

- LCD controller with dedicated system DMA channel

- Two multichannel buffered serial ports

- Two multichannel serial interfaces

- Three UARTs (one supporting SIR mode for IrDA)

- Four interprocessor mailboxes

- Up to 14 shared general-purpose I/Os

However, the OMAP1510 does not have an ADC, although it has a number of high performance ADC chips which can be easily connected to OMAP.

According to the analysis above, no MCU can totally satisfy our requirements. The OMAP1510 has the best performance, and its DSP portion will give us plenty of room for further calculations. But it has the highest price. Comparing the i.MX1 and PXA255, the i.MX1 has more of the ports we want, and both of them have the calculation capability we need. 


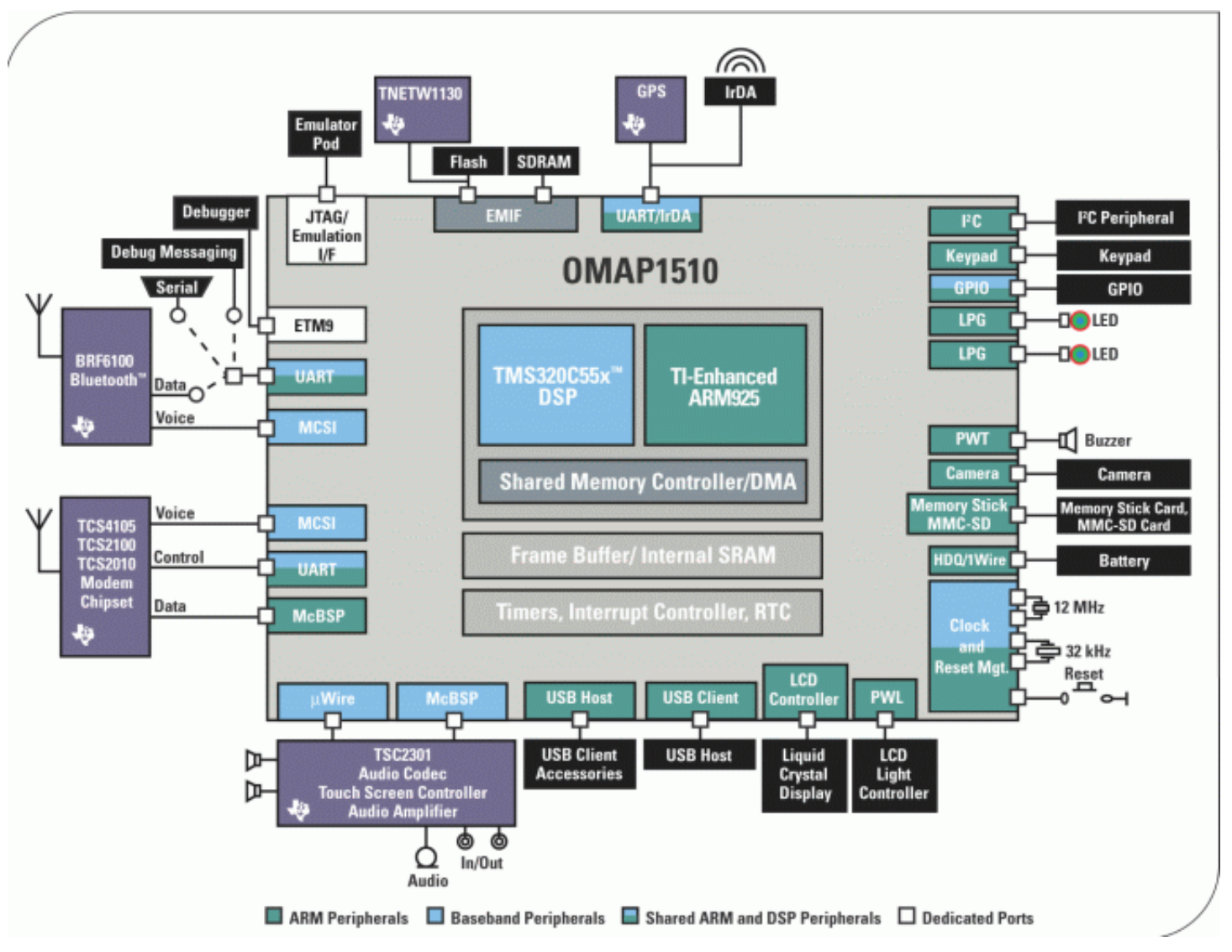

Figure 9.36. OMAP1510 Functional Block Diagram.

\subsubsection{System Assembly}

A Motorola i.MX1 ADS (Advanced Development System) has been purchased to facilitate the system development work. The ADS has provided the i.MX1 CPU, enough memory, a LCD, and some peripheral interfaces (see Figure 9.37). We still need to develop a USB host port which will be used to control the spectrometer.

\section{USB Host Card}

A Philips ISP1161A1 was selected to expand two USB host ports and one USB client port to the i.MX1. A USB host daughter card was designed, which can be easily plugged into the SoDIMM slot of the i.MX1 ADS. Figure 9.38 shows a schematic of the card and Figure 9.39 is the assembled PCB board. We are currently programming the USB host driver to achieve control and data acquisition of the spectrometer. Figure 9.40 shows all the parts of this new system.

After the USB driver programming, the system application program will be written. Then, a new smaller system schematic and PCB will be designed to take the place of the ADS and USB host card. In fact, the size of a finished system PCB board, with CPU, memory, USB ports, power and LCD circuits on it, will not be larger than the size of the LCD screen. 


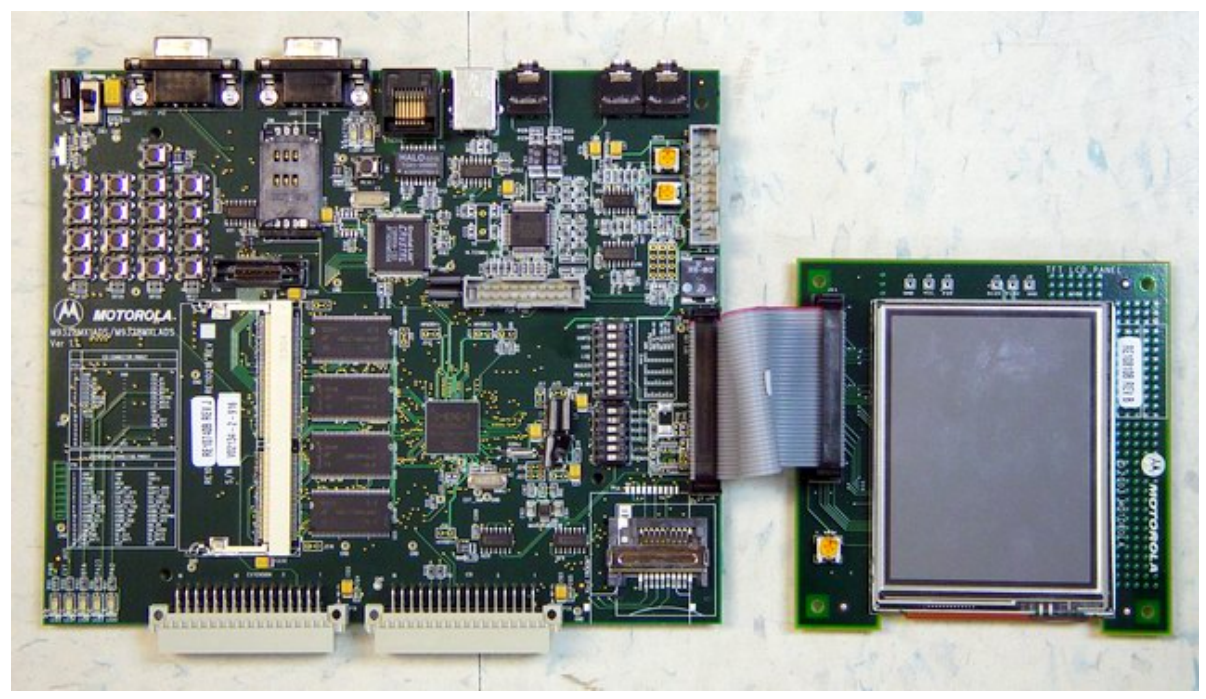

Figure 9.37. Motorola i.MX1 ADS.

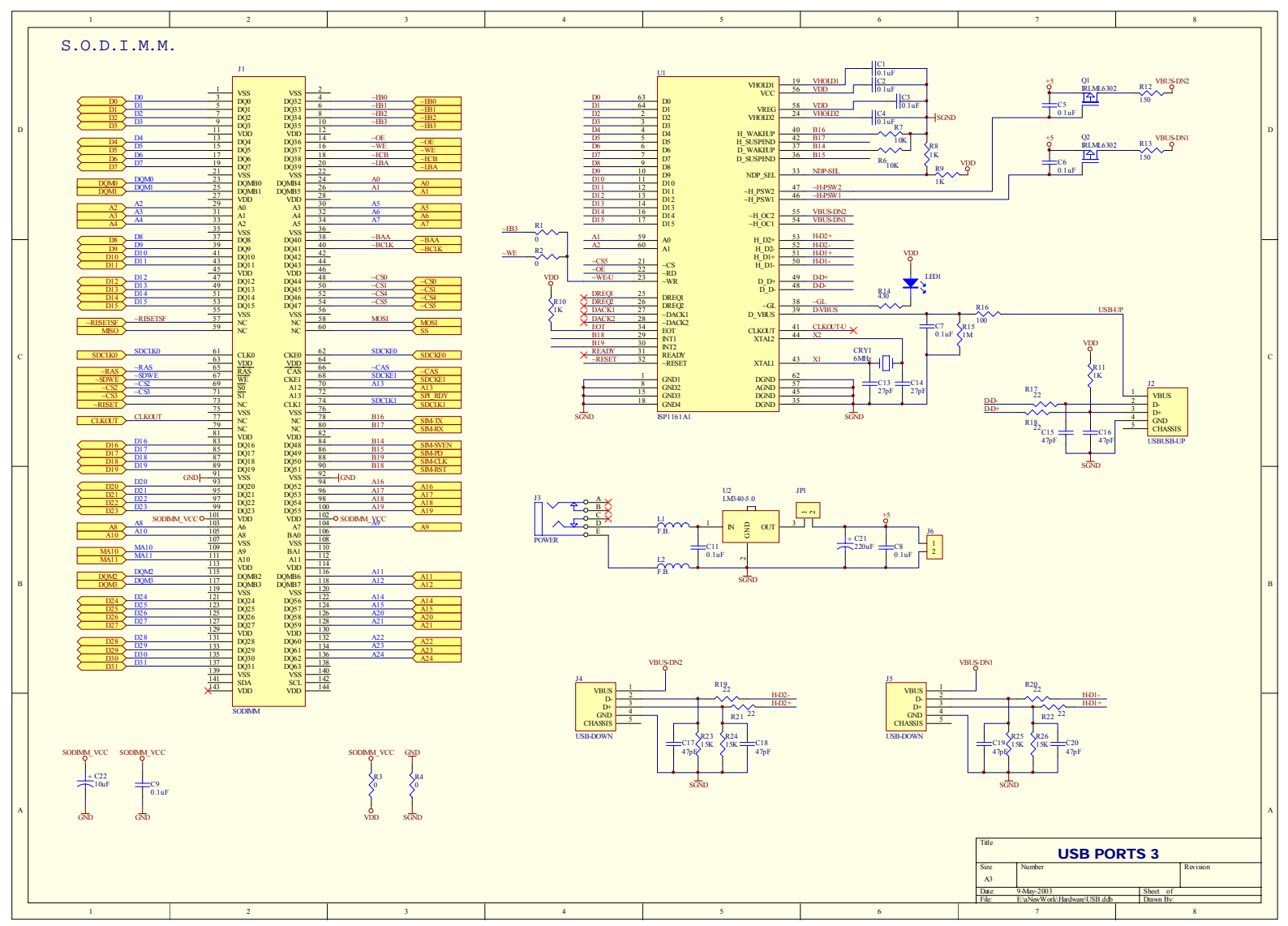

Figure 9.38. Schematic of USB host card. 


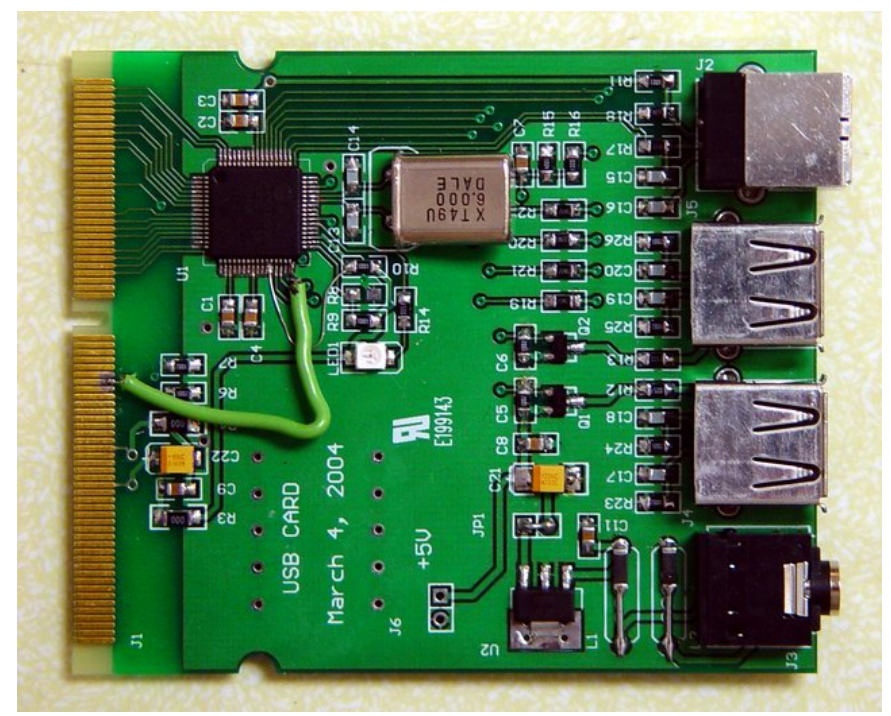

Figure 9.39. Assembled USB host card.

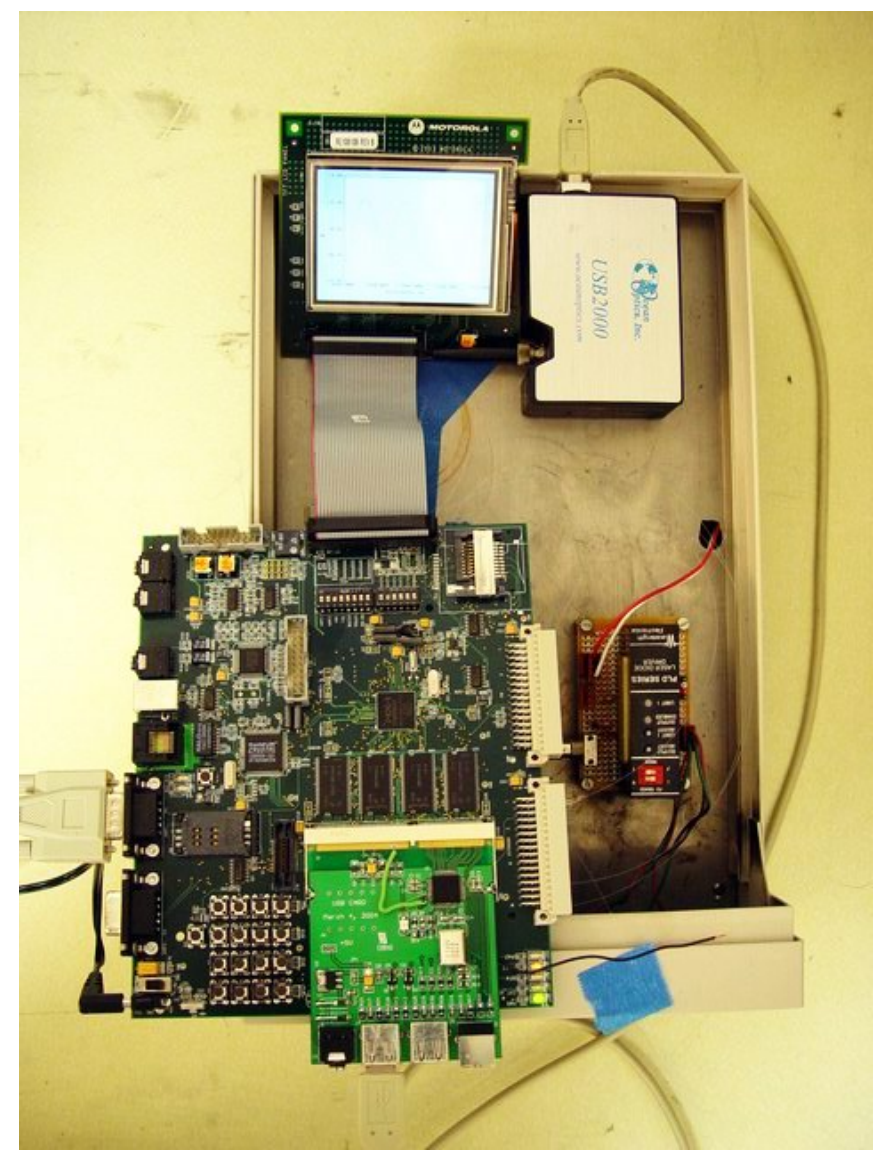

Figure 9.40. Preliminary system panoramas. 


\subsection{Fiber Optic Engine Pressure Sensor Field Test}

On June $24^{\text {th }}, 2004$, the Center for Photonics Technology at Virginia Tech successfully field tested three types of fiber optic pressure sensors near the fan of a jet engine: a diaphragmferrule based sensor and two miniature fiber sensors. This section describes the installation of the sensors in the engine and analyzes the performance of the optical sensors compared to that of a commercial Kulite sensor. Results from both sensors indicate that the acoustic pressure near the engine fan is about 0.89 psi. In addition, the phase delay between the optical sensor and the Kulite sensor is in good agreement with the value calculated based on the distance between these two sensors. The test demonstrated not only good sensor performance, but also that the sensor packaging and installation are suitable for engine operation.

\subsection{Sensor Packaging}

For protection and installation purposes, the engine sensor must be packaged in a metal housing. This sensor package protects both the sensor head and the bare fiber near the sensor head. The package design is shown in Figure 10.1 and the packaged sensors are shown in Figure 10.2

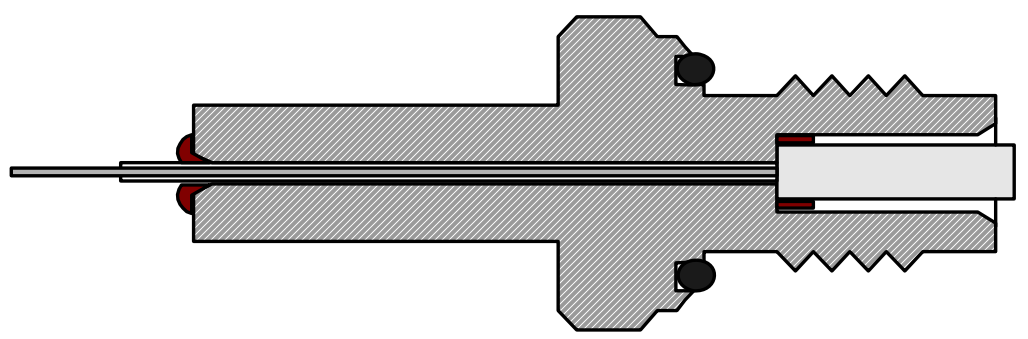

Figure 10.1. Engine sensor package design.
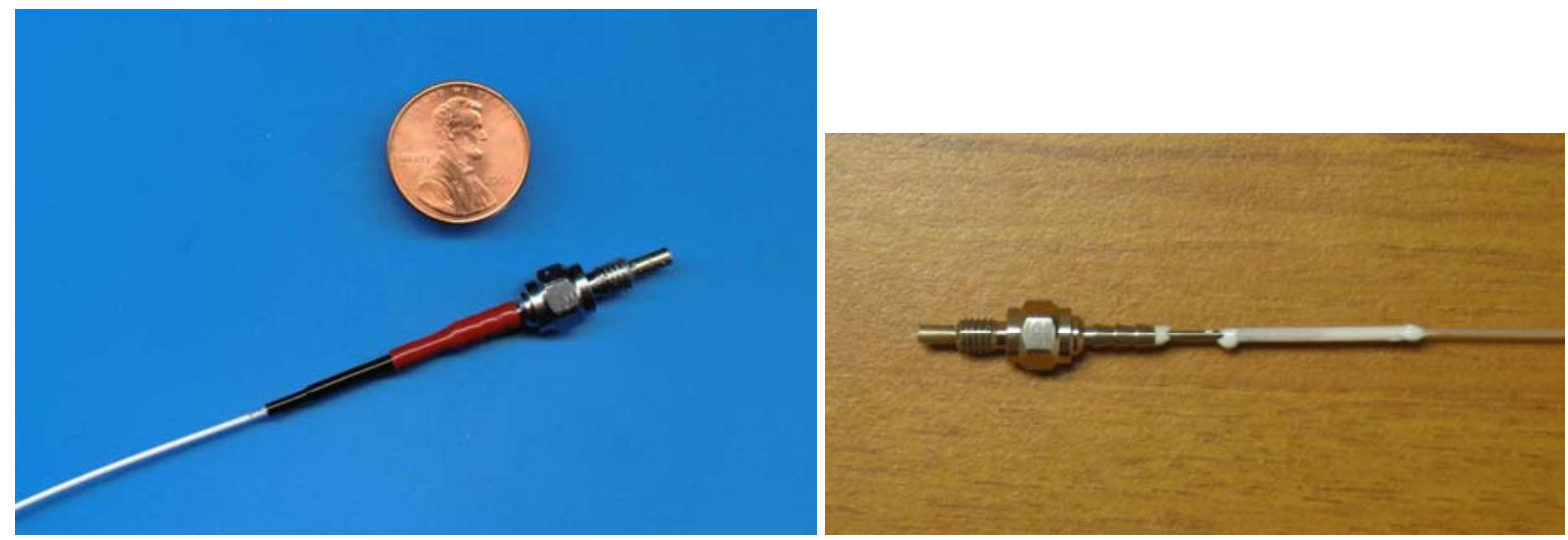

Figure 10.2. Representative packaged diaphragm-ferrule based fiber optic pressure sensor (left) and miniature sensor (right). 


\subsection{System Setup and Sensor Installation}

The F109 turbofan test engine is shown in Figure 10.3. Five holes were drilled in the aluminum ring at the engine inlet (Figure 10.4). The reference Kulite sensor was installed in the center hole and the fiber sensors in the remaining four holes. The miniature sensors, in the outer holes, were located $40 \mathrm{~mm}$ from the Kulite sensor. All five sensors were connected with long cables to the control room (Figure 10.5). The signals from the fiber and Kulite sensors were collected simultaneously and displayed on oscilloscope.

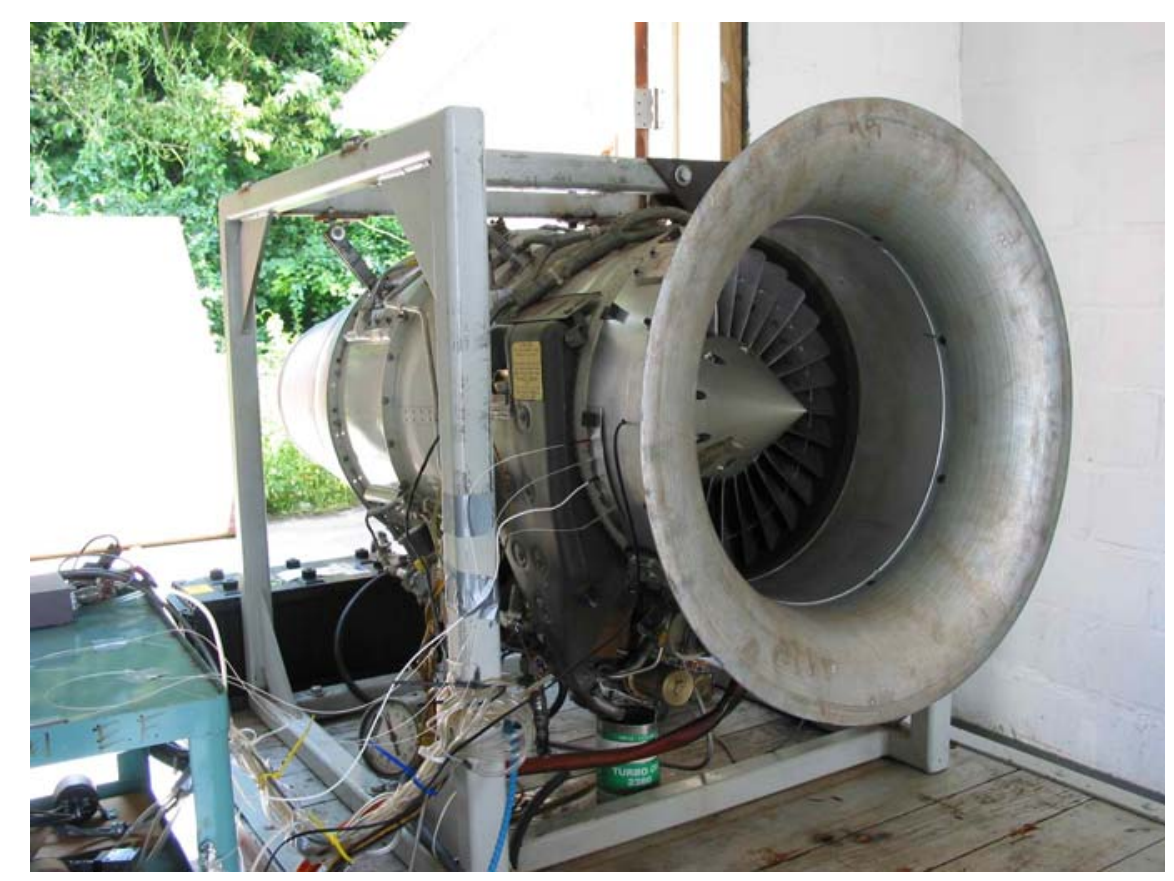

Figure 10.3. Photo of the turbofan engine.

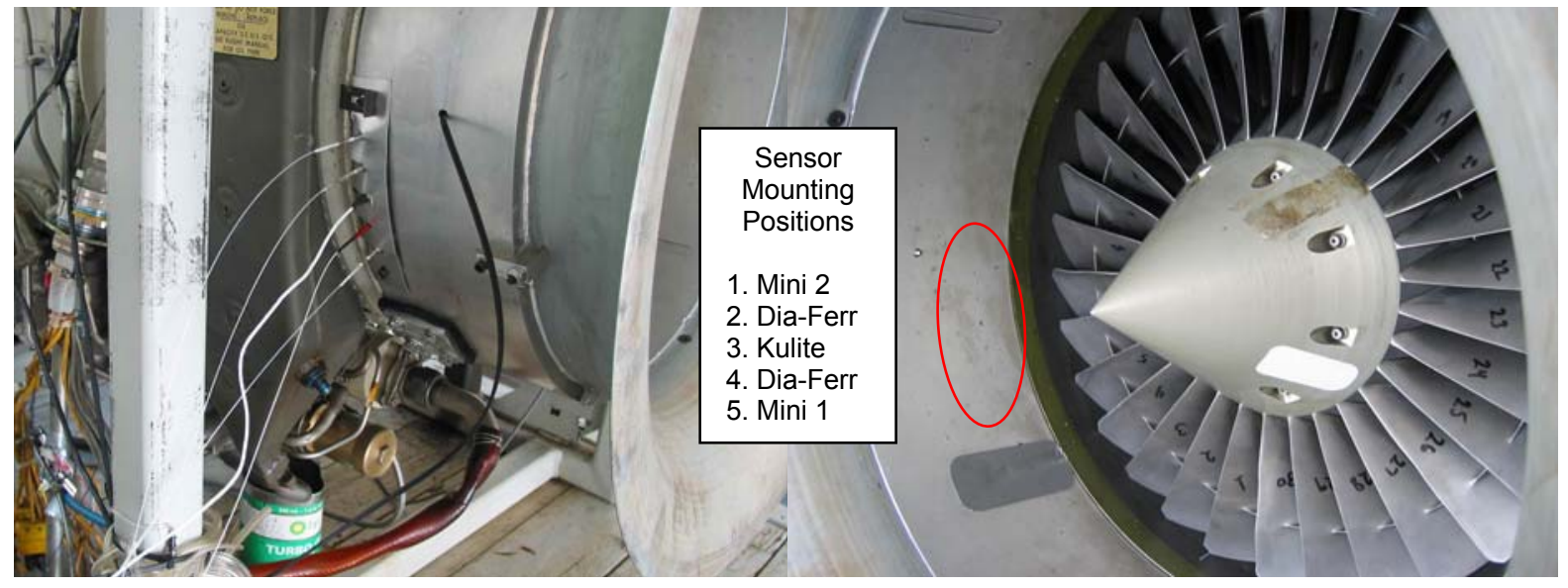

Figure 10.4. Kulite and fiber sensors installed on the engine. 


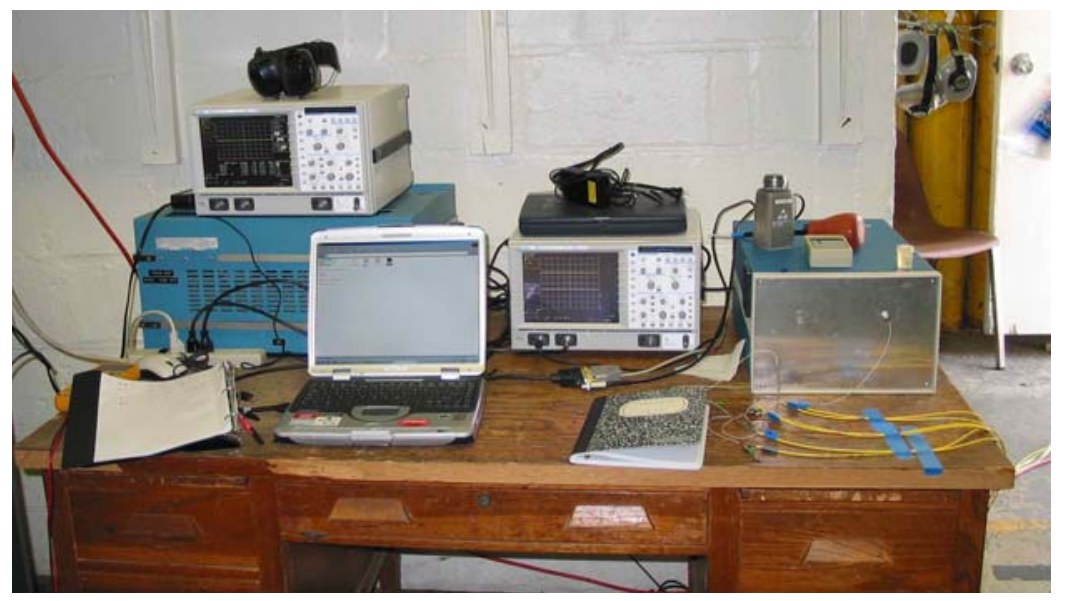

Figure 10.5. System instrumentation in control room.

\subsection{Test Results}

The following data was collected when engine was running at $90 \%$ of its full speed.

\subsubsection{Diaphragm-Ferrule Based Sensor}

The time domain and frequency domain signals for the diaphragm-ferrule based sensor are shown in Figure 10.6 and Figure 10.7, respectively. As discussed above, there is a phase difference (time delay) between the two signals due to the slightly different installation locations. According to the cross-correlation between the two signals, this delay is $0.058 \mathrm{~ms}$. The time domain signals were processed using the Matlab FFT function to obtain the frequency data listed in Table 10.1. The results of the Kulite and diaphragm-based sensors are very consistent.

The fundamental frequency at $5.93 \mathrm{kHz}$ is due to the effect of the blades passing since the rotation speed of the fan with 30 blades is set to about $12000 \mathrm{rpm}$. By precise calculation, the actual fan rotation speed is

$$
5.93 \times 1000 \mathrm{~Hz} \times 60 \mathrm{~s} / 30=11860(\mathrm{rpm}) .
$$

According to the Kulite calibration data, the output of the sensor is $33.3 \mathrm{mV} / \mathrm{psi}$. The peak to peak output at $5.93 \mathrm{kHz}$ was $22.7544 \mathrm{mV}$, resulting in a dynamic pressure of:

$$
P_{\text {kulite }}=\frac{22.7544 m V}{33.3 m V / p s i}=0.683 \text { psia } \text {. }
$$

Based on the diaphragm-ferrule fiber sensor calibration data, the sensor output is $87 \mathrm{mV} / \mathrm{psi}$. The peak to peak output at $5.93 \mathrm{kHz}$ was $60.1268 \mathrm{mV}$, resulting in a dynamic pressure of:

$$
P_{\text {fiber }}=\frac{60.1268 \mathrm{mV}}{87.0 \mathrm{mV} / \mathrm{psi}}=0.691 \mathrm{psia} .
$$

This result is consistent with that of the reference Kulite sensor. 

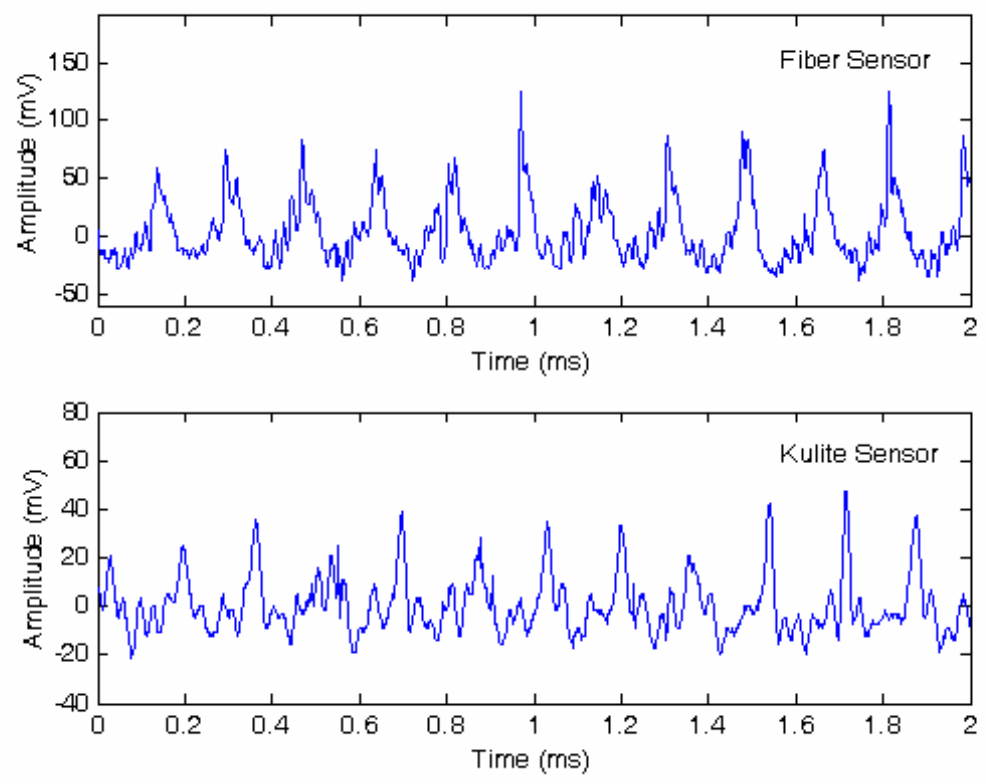

Figure 10.6. Diaphragm-ferrule based fiber sensor and Kulite sensor signals in time domain.
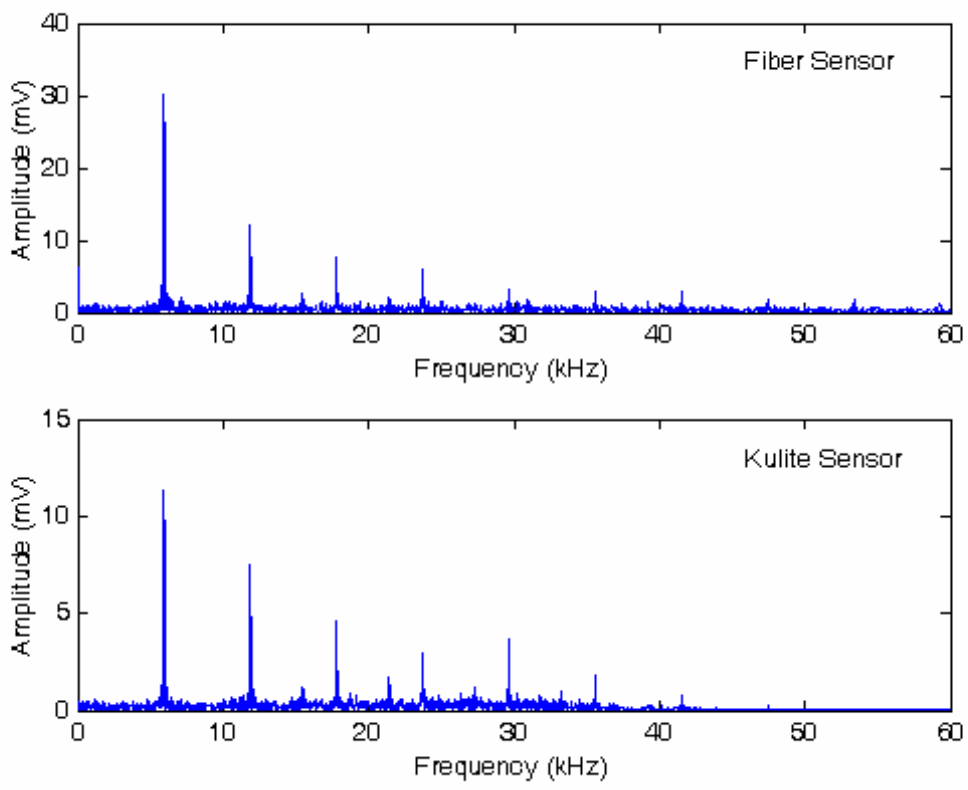

Figure 10.7. Diaphragm-ferrule based fiber sensor and Kulite sensor signals in frequency domain. 
Table 10.1. Main frequencies measured by Kulite and diaphragm-ferrule based sensors.

\begin{tabular}{|c|c|c|c|c|}
\hline \multirow{2}{*}{} & \multicolumn{2}{|c|}{ Frequency $(\mathrm{KHz})$} & \multicolumn{2}{c|}{ Amplitude (mV) } \\
\cline { 2 - 5 } & $\begin{array}{c}\text { Diaphragm- } \\
\text { Ferrule Sensor }\end{array}$ & Kulite Sensor & $\begin{array}{c}\text { Diaphragm- } \\
\text { Ferrule Sensor }\end{array}$ & Kulite Sensor \\
\hline 1 & 5.930 & 5.930 & 30.0634 & 11.3772 \\
\hline 2 & 11.860 & 11.865 & 12.1294 & 7.4924 \\
\hline 3 & 17.795 & 17.800 & 7.7381 & 4.5748 \\
\hline 4 & 23.725 & 23.720 & 6.0433 & 2.9771 \\
\hline 5 & 29.660 & 29.660 & 3.2610 & 3.7025 \\
\hline 6 & 35.590 & 35.595 & 2.9957 & 1.8222 \\
\hline
\end{tabular}

\subsubsection{Miniature Sensor Type 1}

The response of the first type of miniature sensor was calibrated as $15.7 \mathrm{mV} /$ psi. Figure 10.8 and Figure 10.9 show the time domain and frequency domain signals for the miniature sensor and the Kulite sensor. Both have a high response to the fundamental frequency and its multiple frequencies. Here the analysis and comparison of the responses are given up to the sixth frequency. Their frequencies and corresponding signal amplitude are listed in Table 10.2 .
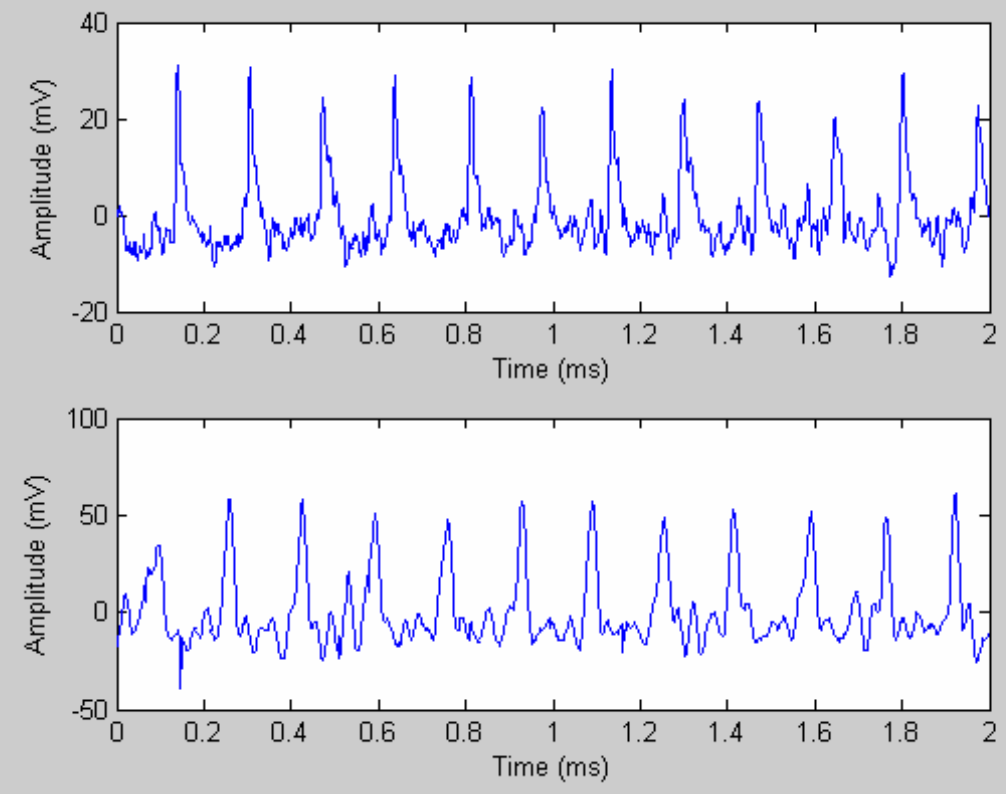

Figure 10.8. Time domain response of the optical sensor compared with that of the Kulite sensor. (The upper plot shows the response of the optical sensor; while the lower one shows the response of the Kulite sensor.). 

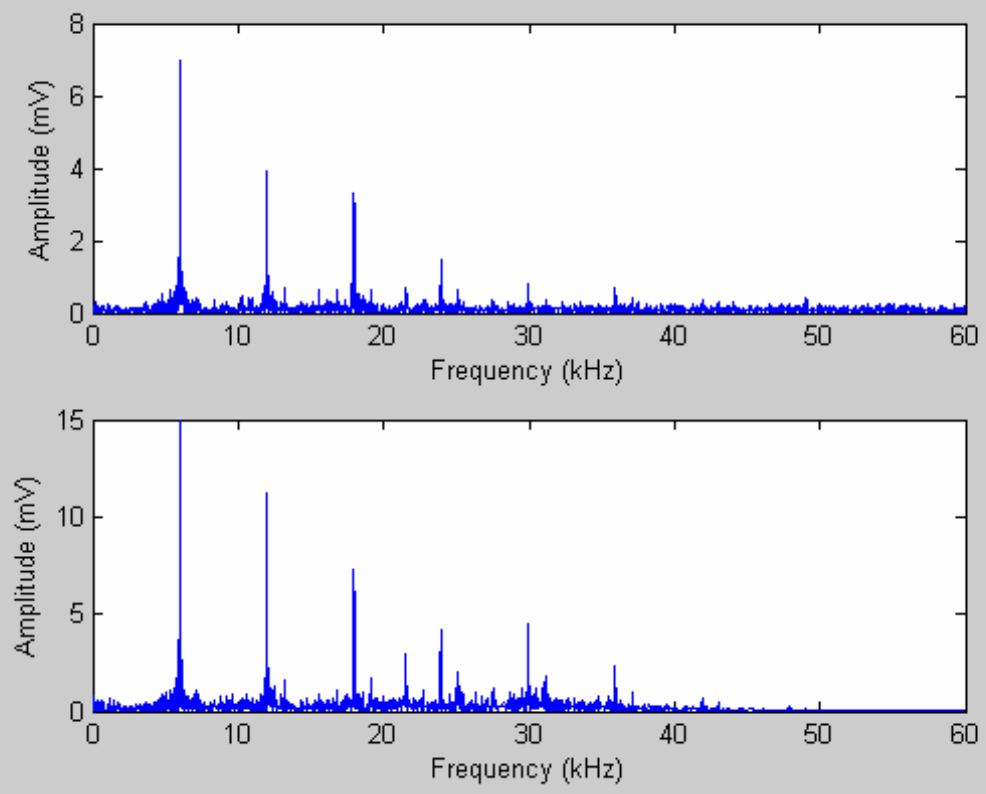

Figure 10.9. Frequency response of the optical sensor compared with that of the Kulite sensor. (The upper plot shows the response of the optical sensor; while the lower one shows the response of the Kulite sensor.).

Table 10.2. Comparison results between the first miniature fiber sensor and the Kulite sensor

\begin{tabular}{|c|c|c|c|c|}
\hline \multirow{2}{*}{} & \multicolumn{2}{|c|}{ Frequency $(\mathrm{kHz})$} & \multicolumn{2}{c|}{ Amplitude (mV) } \\
\cline { 2 - 5 } & Mini Sensor 1 & Kulite Sensor & Mini Sensor 1 & Kulite Sensor \\
\hline 1 & 5.985 & 5.985 & 7.0198 & 14.8556 \\
\hline 2 & 11.970 & 11.970 & 3.9559 & 11.1985 \\
\hline 3 & 17.960 & 17.955 & 3.3262 & 7.2848 \\
\hline 4 & 23.945 & 23.940 & 1.4419 & 4.1817 \\
\hline 5 & 29.940 & 29.930 & 0.8255 & 4.5464 \\
\hline 6 & 35.923 & 35.915 & 0.6702 & 2.3301 \\
\hline
\end{tabular}

The peak to peak output of the Kulite sensor at the fundamental frequency was $29.7112 \mathrm{mV}$, resulting in a dynamic pressure of $29.7112 / 33.3 \approx 0.8922 \mathrm{psi}$. At the same frequency, the miniature fiber sensor's peak to peak output was 14.0396, resulting in a dynamic pressure measurement of 14.0396/15.7 0.8942 psi. Except for a slight discrepancy which may be caused by calibration error, these results are quite consistent with each other. The obvious time delay between the miniature fiber sensor and the Kulite sensor is due to the distance between the two installation positions. An estimation of the resulting time delay can be calculated as follows. 
The distance between the Kulite sensor and the optical sensor is $40 \mathrm{~mm}$, the radius of the ring surrounding the fans is $254 \mathrm{~mm}$, and the rotation speed of the fan is about 200 revolutions per second. The time delay between the two sensors can therefore be estimated as

$$
\frac{40}{2 \pi \times 254} \times \frac{1}{200} \approx 0.13(m s) .
$$

Figure 10.8 shows that the time delay between the optical sensor and the Kulite sensor is about $0.12 \mathrm{~ms}$, which agrees well with the estimation. The error may be caused by the rough measurement of the distance of the position and the ring radius. Also, the rotation speed is not an ideal constant when the engine is operated at $90 \%$ of its full speed.

\subsubsection{Miniature Sensor Type 2}

Figure 10.10 and Figure 10.11 show the outputs from both the mini-sensor and the Kulite sensor. Output voltages recorded by oscilloscope are plotted in Figure 10.10, which clearly shows that pressure is periodic. As discussed above, the Kulite signal trailed the sensor signal because of their different mounting positions. By calculating the cross-correlation of the two signals, this delay was determined to be about $0.105 \mathrm{~ms}$. Figure 10.11 shows the FFT of the outputs. The frequency and amplitude of up to $6^{\text {th }}$ harmonic for both sensors are listed in Table 10.3. The frequencies for both sensors match very well.
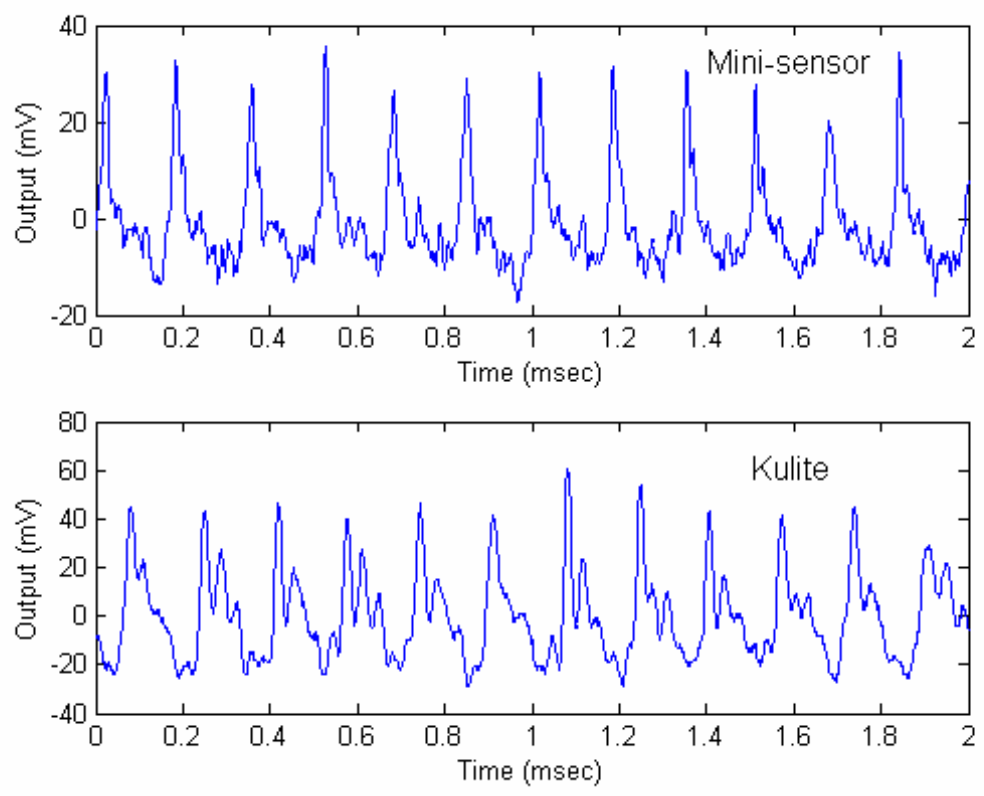

Figure 10.10. Mini-sensor output vs. Kulite sensor output, time domain signal . 

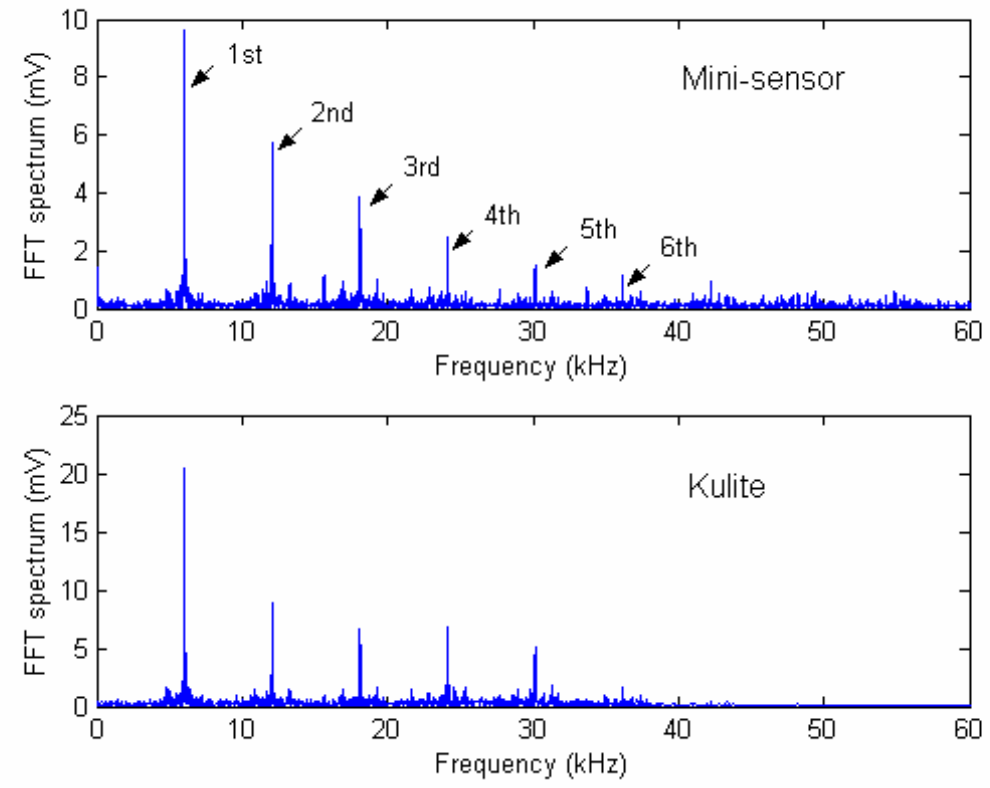

Figure 10.11. Mini-sensor output vs. Kulite sensor output, FFT spectrum.

Table 10.3. Comparison results between the second miniature fiber sensor and the Kulite sensor

\begin{tabular}{|c|c|c|c|c|}
\hline \multirow{2}{*}{ Peak \# } & \multicolumn{2}{|c|}{ Frequency $(\mathrm{kHz})$} & \multicolumn{2}{c|}{ Amplitude $(\mathrm{mV})$} \\
\cline { 2 - 5 } & Mini-sensor 2 & Kulite & Mini-sensor 2 & Kulite \\
\hline 1 & 6.030 & 6.030 & 9.59 & 20.44 \\
\hline 2 & 12.060 & 12.060 & 5.71 & 8.83 \\
\hline 3 & 18.085 & 18.090 & 3.88 & 6.69 \\
\hline 4 & 24.115 & 24.120 & 2.47 & 6.82 \\
\hline 5 & 30.145 & 30.145 & 1.51 & 5.03 \\
\hline 6 & 36.180 & 36.175 & 1.11 & 1.59 \\
\hline
\end{tabular}

The miniature sensor's dynamic response was calibrated to be about $18 \mathrm{mV} / \mathrm{psi}$. From Table 10.3 , the amplitude of the first harmonic measured by the sensor was about $9.59 \mathrm{mV}$. So the peak-to-peak pressure change is $2 * 9.59 \mathrm{mV} /(18 \mathrm{mV} / \mathrm{psi})=1.1 \mathrm{psi}$.

The Kulite sensor has a sensitivity of $33 \mathrm{mV} / \mathrm{psi}$. It measured a dynamic pressure change of $20.44 \mathrm{mV}$ in amplitude, which corresponds to a peak-to-peak pressure change of about

$2 * 20.44 \mathrm{mV} /(33 \mathrm{mV} / \mathrm{psi})=1.2 \mathrm{psi}$. 


\subsection{Conclusion}

The field test result confirms that the fiber optic sensor design can function safely and reliably near the engine fan. During a field test of about two hours, the optical sensors' package was robust enough for the engine operation. In addition, the optical sensor was able to measure the acoustic pressure near the engine fan. Its performance is consistent with that of the commercial Kulite sensor.

Furthermore, theoretical calculation and analysis indicate that the sensors can work well under higher temperature and pressure environments, where most current commercial sensors cannot survive. This was to be further demonstrated experimentally prior to the Energy Efficiency Science Initiative budget cuts. A second field test was scheduled at Pratt \& Whitney in Connecticut. 


\subsection{Product Development}

\subsection{Publications}

\subsubsection{Journal Articles}

1. B. Yu and A. Wang, "Grating-Assisted Demodulation of Interferometric Optical Sensors , Appl. Opt. 42(34), 6824-6829 (2003)

2. B. Yu and A. Wang, "Thermally tunable Extrinsic Fabry-Perot filter," IEEE Photonics Technol. Lett., 16(10) 2296-8 (2004).

3. J. Xu, X. Wang, K. L. Cooper, and A. Wang, "Miniature all-silica fiber optic pressure and acoustic sensors," Opt. Lett. 30(24), 3269-3271 (2005).

4. F. Shen and A. Wang, "Frequency-estimation-based signal-processing algorithm for white-light optical fiber Fabry-Perot interferometers," Appl. Opt. 44(25), 5206-5214 (2005).

5. M. Han, X. Wang, J. Xu, K. Cooper, and A. Wang, " Diaphragm-based extrinsic FabryPerot interferometric optical fiber sensor for acoustic wave detection under high background pressure e," Opt. Eng. 44, 060506 (2005).

6. F. Shen, J. Xu, A. Wang, "Measurement of the frequency response of a diaphragm-based pressure sensor by use of a pulsed excimer laser," Opt. Lett. 30(15), 1935-1937 (2005).

7. B. Yu, A. Wang, G. Pickrell, J. Xu, "Tunable-optical-filter-based white-light interferometry for sensing," Opt. Lett. 30(12), 1452-1454 (2005).

8. Y. Zhu and A. Wang, "Miniature fiber optic pressure sensor," IEEE Photon. Technol. Lett., 17(2), 447-449 (2005).

9. W. Peng, G.R. Pickrell, A. Wang, "High-temperature fiber optic cubic-zirconia pressure sensor", Opt. Engin. 44(12), 124402 (2005).

10. Y. Zhu, Z. Huang, F. Shen, A. Wang, "Sapphire-fiber-based white-light interferometric sensor for high-temperature measurements," Opt. Lett. 30(7), 711 (2005).

11. J. Xu, G. Pickrell, W. Peng, X. Wang, K. Cooper and A. Wang, "A novel temperatureinsensitive optical fiber pressure sensor for harsh environments," IEEE Photon. Technol. Lett., 17(4) 870 (2005).

12. Z. Huang, X. Chen, Y. Zhu, and A. Wang, "Wave-front splitting intrinsic Fabry-Perot fiber optic sensor," Opt. Eng. Lett., 44(7), 070501 (2005). 
13. F. Shen and A. Wang, "Fast-Fourier-transform based numerical integration method for the Rayleigh-Sommerfeld diffraction formula," Appl. Opt. 45(6), 1102-1110 (2006).

14. J. Xu, X. Wang, K. Cooper, A. Wang, G. Pickrell, "Miniature temperature-insensitive fiber optic pressure sensor," IEEE Photon. Tech. Lett., 18 (10), 1134-6 (2006).

15. X. Wang, J. Xu, Y. Zhu, K. L. Cooper, A. Wang, "An all fused silica miniature optical fiber tip pressure sensor," Opt. Lett. 31(7), 885-887 (2006).

16. Y. Zhu, K. L. Cooper, G. R. Pickrell, and A. Wang, "Fiber-tip pressure sensor for hightemperature applications," J. Lightwave Technol., 24(2), 861-9 (2006).

\subsubsection{Conference Proceedings}

1. A. Wang, "Optical fiber sensors for energy-production and energy-intensive industries.", Advanced Sensor Systems and Applications, Invited Paper, Proc. SPIE 4920, pp. 377381 (2002).

2. B. Yu, G. R. Pickrell, and A. Wang, "Grating-assisted operating-point tuning for fiber optic Fabry-Perot interferometric sensors," Industrial and Highway Sensors Technology,Proc. SPIE 5272, pp. 41-48, Providence, RI, (Mar 2004).

3. X. Chen, B. Yu, Y. Zhu, A. Wang, "Deep wet etching on fused silica material for fiber optic sensors" Micromachining and Microfabrication Process Technology IX, Proc. SPIE 5342, pp. 128-136 (2004).

4. J. Xu, G.R. Pickrell, B. Yu, M. Han, Y. Zhu, X. Wang, K.L. Cooper, A. Wang, "Epoxyfree high-temperature fiber optic pressure sensors for gas turbine engine applications" Sensors for Harsh Environments, Proc. SPIE 5590, pp. 1-10 (2004).

5. Y. Zhu, G.R. Pickrell, X. Wang, J. Xu, B.Yu, M. Han, K.L. Cooper, A. Wang, A. Ringshia, W. Ng "Miniature fiber optic pressure sensor for turbine engines" Sensors for Harsh Environments, Proc. SPIE 5590, pp. 11-18, Philadelphia (Oct 2004).

6. Y. Zhu, Z. Huang, M. Han, F. Shen, G. Pickrell, and A. Wang, "Fiber optic hightemperature thermometer using sapphire fiber," Sensors for Harsh Environments, Proc. SPIE 5590, pp. 19-26, Philadelphia (Oct 004).

7. F. Shen, W. Peng, K.L. Cooper, G. Pickrell, A. Wang, "UV-induced intrinsic Fabry-Perot interferometric fiber sensors" Sensors for Harsh Environments, Proc. SPIE 5590, pp. 4756 (2004).

8. X. Chen, F. Shen, A. Wang, Z. Wang, Y. Zhang, "Novel Fabry-Perot fiber optic sensor with multiple applications" Sensors for Harsh Environments, Proc. SPIE 5590, pp. 111121 (2004). 
9. X. Wang, J. Xu, Y. Zhu, B. Yu, M. Han, K. L. Cooper, G. R. Pickrell, A. Wang, A. Ringshia, and W. Ng, "Verifying an all fused silica miniature optical fiber tip pressure sensor performance with turbine engine field test," Sensors for Harsh Environments II, Proc. SPIE vol. 5998, 169-177, Boston (Oct 2005).

10. J. Xu, G. R. Pickrell, X. Wang, B. Yu, K. L. Cooper, and A. Wang, "Vacuum-sealed high temperature high bandwidth fiber optic pressure and acoustic sensors," Sensors for Harsh Environments II, Proc. SPIE 5998, 599809, Boston (Oct 2005).

11. F. Shen, Z. Huang, Y. Zhu, M. R. Coffey, G. R. Pickrell, and A. Wang, "Frequencydivision-multiplexed intrinsic Fabry-Perot interferometric fiber sensors for quasidistributed temperature monitoring in a selective catalytic reduction unit," Sensors for Harsh Environments II (invited paper), Proc. SPIE 5998, 59980H, Boston (Oct 2005).

12. J. Xu, G. R. Pickrell, K. L. Cooper, X. Wang, and A. Wang, "High-temperature thermometer with fiber optic readout," Sensors for Harsh Environments II, Proc. SPIE 5998, 59980B, Boston (Oct 2005).

13. J. Xu, X. Wang, K.L. Cooper, G.R. Pickrell, A. Wang, "Miniature fiber optic pressure and temperature sensors," Fiber Optic Sensor Technology and Applications IV, Proc. SPIE 6004, pp. 600403, Boston (Oct 2005).

14. F. Shen, Z. Wang, K.L. Cooper, G.R. Pickrell, A. Wang, "UV-induced intrinsic FabryPerot interferometric sensors and their multiplexing for temperature and strain sensing," Sensors and Smart Structures for Civil, Mechanical, and Aerospace Systems, Proc. SPIE 6174, pp. 111-120, San Diego, CA (Feb. 2006).

15. X. Wang, J. Xu, K.L. Cooper, A. Wang, "Miniature optical fiber sensor for pressure measurements," Photorefractive Fiber and Crystal Devices: Materials, Optical Properties, and Applications XII, Proc. SPIE 6314, pp. 63141K, San Diego, CA (Aug 2006).

\subsection{Web sites}

Portions of http://www.ee.vt.edu/ photonics

\subsection{Technologies}

The most significant technologies developed under this program are:

- A laser bonded silica high temperature fiber sensor with a high temperature capability up to $700^{\circ} \mathrm{C}$ and a frequency response up to $150 \mathrm{kHz}$

- The world's smallest fiber Fabry-Perot high temperature pressure sensor (125 x $20 \mu \mathrm{m})$ with $700^{\circ} \mathrm{C}$ capability

- UV-induced intrinsic Fabry-Perot interferometric sensors for distributed measurement. 
- A single crystal sapphire fiber-based sensor with a temperature capability up to $1600^{\circ} \mathrm{C}$.

These technologies have been well demonstrated and laboratory tested. Our work plan included conducting major field tests of these technologies at EPRI, Corning, Pratt \& Whitney, and Global Energy; field validation of the technology is critical to ensuring its usefulness to U.S. industries. However, due to budget cuts, DOE was unable to follow through with its funding commitment to support Energy Efficiency Science Initiative projects and this final phase was eliminated.

\subsection{Patents}

Virginia Tech Intellectual Properties, Inc. (VTIP), a non-profit corporation affiliated with the university is charged with the mission of protecting and utilizing IPs for the benefit of the university. All IPs assigned to the university flows to VTIP by assignment for operational management. Therefore, Virginia Tech patent disclosures are identified by a VTIP Disclosure Number.

\subsubsection{Patent Disclosures}

- VTIP No. 04.030 Method for Hermetic Bonding of Sapphire or Alumina Y. Zhu, G. Pickrell, A. Wang

- VTIP No. 04.028 Signal Processing Algorithm for White Light Fiber Fabry-Perot Interferometric Sensors M. Han, A. Wang

- VTIP No. 04.001 Miniature Fabry-Perot Structure $\mathrm{X}$. Wang, J. Xu, Y. Zhu, A. Wang

- VTIP No. 03.148 A Novel Optical Fiber and Ferrule Hermetic Thermal Bonding Method J. Xu, A. Wang

- VTIP No. 03.141 Laser Heated Sapphire-to-Sapphire Direct Bonding M. Han, A. Wang

- VTIP No. 03.132 A Novel Method of Micromachining Sapphire with Excimer Laser X. Chen, B. Dong, R. Chakraborty, A. Wang

- VTIP No. 03.126 Novel Mini-Size Optical Fiber Fabry-Perot Sensors J. Xu, A. Wang

- VTIP No. 03.103 Diaphragm-Based High Temperature Sensor Y. Zhu, Z. Huang, A. Wang

- VTIP No. 03.098 Thermally Tunable Fiber Fabry-Perot Filters B. Yu, A. Wang

- VTIP No. 03.089 Sapphire Fiber-Based Interferometric Sensors M. Han, A. Wang

- VTIP No. 03.081 Bi-directionally Tunable Optical Filters 
B. Yu, A. Wang

- VTIP No. 03.066 A Novel Method to Fabricate Intrinsic Fiber Optic Fabry-Perot Interferometer

X. Chen, A. Wang

- VTIP No. 03.055 Novel Split Wavefront Interferometer Z. Huang, A. Wang

- VTIP No. 03.054 Optical Crystal Based Fiber Optical Sensor for Ultrahigh Temperature W. Peng, A. Wang, G. Pickrell, Y. Zhang

- VTIP No. 02.127 Diaphragm-based Fabry-Perot Tunable Filter B. Yu, A. Wang

\subsubsection{Patent Applications}

- VTIP No. 03.012 Operating-Point Tuning Technique for Fiber-Optic Fabry-Perot Interferometric Sensors

B. Yu, A. Wang

U.S. Patent Application Serial No. 10/824,600

- VTIP No. 03.093 Miniature High Temperature Pressure Sensor Fabricated on Fiber Tip Y. Zhu, A. Wang

Patent Application Serial No. 10/911,635

$\mathrm{PCT} / \mathrm{US} 04 / 028535$

- VTIP No. 03.032 Optical Fiber Pressure Sensors for Ultrahigh Temperature Applications J. Xu, A. Wang

Patent Application Serial No. 10/791,842

- VTIP No. 02.062 Intrinsic Fabry-Perot Optical Fiber Sensors and their Multiplexing A. Wang Patent Application Serial No. 10/656,256

PCT/US2003/027933 


\subsection{Conclusions and Future Work}

Pressure and temperature sensors are vitally important for efficient energy use and low environmental emissions in many industrial processes, especially energy intensive industries such as power generation, metal casting, glass, and transportation sectors. However, many of these processes involve various harsh environmental conditions, such as high temperature, high pressure, chemical corrosion, and strong electromagnetic interference (EMI), which often makes conventional sensors and measurement devices difficult to apply. For example, the boilers or turbines in power generation plants operate at very high temperature and pressure for increased efficiency, and accurate pressure monitoring is required to provide the combustion process information needed to control the fuel-air mixture and residence time in the flame zone in order to attain desired emissions levels, and to provide seamless fuel transitions on duel-fuel turbines. In the transportation sector, the accurate monitoring and control of pressure and temperature in the combustion chamber of aircraft and locomotive engines can increase efficiency and reduce fuel consumption and pollutant emission.

For pressure measurements at elevated temperatures, industry primarily relies on semiconductor pressure sensors, which have several major drawbacks, including rather limited maximum operating temperature $\left(482^{\circ} \mathrm{C}\right.$ for the latest model $)$, poor reliability at high temperatures, extreme sensitivity to temperature, and susceptibility to electromagnetic interference (EMI). Kulite and other sensor manufacturers have been increasing the operating temperatures of silicon pressure sensors for some time, but they have reached the practical temperature limit at which silicon can operate. For temperatures of $500^{\circ} \mathrm{C}$ and higher, silicon deforms plastically. As with other electrical or electronic sensors, susceptibility to EMI remains a major concern.

For temperature measurements, the primary tool is the thermocouple. Again, susceptibility to EMI is a major concern. Moreover, commercially available thermocouples exhibit very short lifetimes and rather poor reliability in corrosive environments when the temperature is above $1500^{\circ} \mathrm{C}$, which is typical of many industrial environments.

Improved temperature and pressure sensors have been identified as a critical need in numerous industrial sectors, including power generation [40], transportation [41], and the targeted Industries of the Future (glass, steel, aluminum, and metal casting) [42], in order to improve energy efficiency and reduce pollutant emissions. Sensors that can reliably operate at the required temperatures up to $1600^{\circ} \mathrm{C}$ and pressures up to $450 \mathrm{psi}$ can result in savings of 17 to 45 trillion BTU in the glass industry, 26 trillion BTU in the steel industry, and 0.25 quadrillion BTU across the IOF industries [42].

In addition to energy savings, improved harsh environment sensing capabilities will provide for reductions in emissions such as nitrogen oxide, carbon monoxide, carbon dioxide, and unburned hydrocarbons through higher operating temperatures and leaner mixtures in turbine systems, significant reductions in greenhouse gas emissions in the glass industry, and minimization of waste through improved process control and the resulting reduction of scrap and rework. 
In this program, Virginia Tech's Center for Photonics Technology (CPT) has developed optical fiber-based sensing technologies and associated signal processing algorithms to provide self-calibrating and absolute measurements in the harsh environments of energy intensive industrial processes, combining the advantages of interferometric and intensitybased sensing.

CPT has demonstrated a number of sensing configurations using a range of materials and sensor sizes, suitable for the wide range of environmental conditions found in industrial processes. The most significant technologies developed under this program are:

- A laser bonded silica high temperature fiber sensor with a high temperature capability up to $700^{\circ} \mathrm{C}$ and a frequency response up to $150 \mathrm{kHz}$

- The world's smallest fiber Fabry-Perot high temperature pressure sensor $(125 \mathrm{x}$ $20 \mu \mathrm{m})$ with $700^{\circ} \mathrm{C}$ capability

- UV-induced intrinsic Fabry-Perot interferometric sensors for distributed measurement.

- A single crystal sapphire fiber-based sensor with a temperature capability up to $1600^{\circ} \mathrm{C}$.

The next step is to build packaged, field-ready systems based on this technology for deployment at industrial sites.

A variety of diaphragm-based Fabry-Perot interferometric (DFPI) pressure sensors were demonstrated with diameters ranging from a few millimeters (ferrule-based) [43-45] down to only 125 microns (fiber tip based) [46-48]. The ferrule-based sensors can operate at temperatures up to $700^{\circ} \mathrm{C}$ and with a sensitivity of $2.93 \mathrm{~nm} / \mathrm{psi}$, a resolution of $0.01 \mathrm{psi}(68.9$ $\mathrm{Pa}$ ) and temperature dependence $0.0076 \mathrm{psi} /{ }^{\circ} \mathrm{C}\left(52.4 \mathrm{~Pa} /{ }^{\circ} \mathrm{C}\right)$. Simple fabrication processes have been developed to produce hermetic seals with negligible CTE mismatches. The sensors exhibited a linear response in the available testing range from 0 to $200 \mathrm{psi}(1.38 \mathrm{MPa})$, and being composed entirely of fused silica, the structure is very reliable, corrosion resistant and immune to EMI. With the same structure, sensors with various sensitivities can be fabricated as needed by changing only the diaphragm thickness or the ferrule pit diameter on order to adapt to the needs of various industries.

As with the ferrule-based sensors, the fiber tip sensors are composed entirely of fused silica, resulting in a mechanically robust structure with a diameter of only 125 microns. We have demonstrated their operation up to $710{ }^{\circ} \mathrm{C}$ in our laboratory with repeatability better than $1 \%$. The maximum operating temperature is limited by the mechanical creep of the fused silica diaphragm. The system is able to resolve a pressure change about 0.0013 psi due to the high performance of both the algorithm and instrumentation.

For even higher temperatures (up to $1600^{\circ} \mathrm{C}$ with excellent repeatability), sapphire fiberbased sensors were developed. A key challenge with interferometric single-crystal sapphire sensors is the large number of propagating modes, which make it extremely difficult to generate high quality interference fringes. Angular offsets as small as $0.01^{\circ}$ can significantly reduce fringe visibility. To overcome this difficulty, a sensor was developed that uses a sapphire wafer as the extrinsic Fabry-Perot cavity to ensure high surface quality and 
excellent parallelism. To reduce the overall system cost, the sensor head is coupled to a silica fiber. A prototype sensor was tested from $230-1600^{\circ} \mathrm{C}$ with excellent reproducibility. The sensor is interrogated by digital signal processing, which removes the blackbody radiation and other sources of error. The measured accuracy of $\pm 0.2 \%$ full scale was limited by the accuracy of the B-type thermocouple used as a reference $( \pm 0.5 \%$ full scale over $800^{\circ} \mathrm{C}$ ). The sensor is expected to work close to sapphire's melting point, which is greater than $2000^{\circ} \mathrm{C}$.

The developed pressure sensors were successfully field tested near the fan of a F109 turbofan test engine. The peak to peak output of both the fiber sensors and the semiconductor reference sensor indicate that the acoustic pressure near the engine fan is about $0.89 \mathrm{psi}$. In addition, the phase delay between the optical sensor and the Kulite sensor is in good agreement with the value calculated based on the distance between these two sensors. The test demonstrated not only good sensor performance, but also that the sensor packaging and installation are suitable for engine operation.

These technologies have been well demonstrated and laboratory tested. Our work plan included conducting major field tests of these technologies at EPRI, Corning, Pratt \& Whitney, and Global Energy; field validation of the technology is critical to ensuring its usefulness to U.S. industries. However, due to budget cuts, DOE was unable to follow through with its funding commitment to support Energy Efficiency Science Initiative projects and this final phase was eliminated. 


\section{References}

[1] Q. Y. Tong, T. H. Lee, and U. Gosele, "The role of surface chemistry in bonding of standard silicon wafers," J. Electrochem. Soc, 144, 384-391, 1997.

[2] L. Ristic, Sensor Technology and Devices. Boston: Artech House, 1994.

[3] S. Rowan, S. M. Twyford, J. Hough, D. H. Gwo, and R. Route, "Mechanical loss associated with the technique of hydroxide-catalysis bonding of fused silica," Phys. Lett. A, 246, 471-478, 1998.

[4] J. Deng, H. Xiao, W. Huo, M. Luo, R. G. May, A. Wang, and Y. Liu, "Optical fiber sensor-based detection of partial discharges in power transformers," Optics and Laser Technology, 33 (5), 305-311, 2001.

[5] B. Yu, D. W. Kim, J. Deng, H. Xiao, and A. Wang, "Fiber Fabry-Perot Sensors for Partial Discharge Detection in Power Transformers," Applied Optics-OT, 42 (16), 3241-3250, 2003.

[6] A. Crunteanu, P. Hoffmann, M. Pollnau, and C. Buchal, "Comparative study on methods to structure sapphire," Applied Surface Science, 208-209, 322-326, 2003.

[7] M. K. Ram, P. Bertoncello, H. Ding, S. Paddeu, and C. Nicolini, "Cholesterol biosensors prepared by layer-by-layer technique," Biosens. Bioelec., 16, 849-856, 2001.

[8] S. I. Dolgaev, A. A. Lyalin, A. V. Simakin, and G. A. Shafeev, "Fast etching of sapphire by a visible range quasi-cw laser radiation," Applied Surface Science, 96-98, 491-495, 1996.

[9] D. Ashkenasi, A. Rosenfeld, H. Varel, M. Wahmer, and E. E. B. Campbell, "Laser processing of sapphire with picosecond and subpicosecond pulses," Applied Surface Science, 120, 65-80, 1997.

[10] C. E. Lee, W. N. Gibler, R. A. Atkins, J. J. Alcoz, and H. F. Taylor, "Metalembedded fiber optic Fabry-Perot sensors," Optical Society of America, 1991.

[11] J. Hodak, R. Etchenique, E. J. Calvo, K. Singhal, and B. P. N., "Layer-by-Layer SelfAssembly of Glucose Oxidase with a Poly(allylamine)ferrocene Redox mediator," langmuir, 13 (10), 2708-2716, 1997.

[12] A. Klini, E. B. David, S. Emonin, P. Papadopoulos, J. P. Goudonnet, and G. Kotrotsios, "Reproducible Optical Fiber Tips for Photon Scanning Tunneling Microscopy with Very Small (<5 ) Cone Angle," Journal of Lightwave Technology, 16 (7), 1998.

[13] C. E. Lee and H. F. Taylor, "Interferometric optical fiber sensors using internal mirrors," Electronics Letters, 24, 193, 1988.

[14] C. E. Lee, H. F. Taylor, A. M. Markus, and E. Udd, "Optical Fiber Fabry-Perot Embedded Sensor, Optical Letters, 14 1225-7, 1989," Optics Letters, 14, 1225-1227, 1989.

[15] C. E. Lee and H. F. Taylor, "In-line Fiber Fabry Perot Interferometer With High Reflectance Internal Mirrors," Journal of Lightwave Technology, 10, 1992.

[16] K. A. Murphy, "Extrinsic Fabry Perot Optical Fiber Sensor," Proc. OFS-8, Monterey, CA, 1992. 
[17] A. Wang, H. Xiao, J. Wang, Z. Wang, W. Zhao, and R. G. May, "Self-Calibrated Interferometric/Intensity-Based Optical Fiber Sensors," J. Lightwave Tech., 19 (10), 1495-1501, 2001.

[18] J. Sirkis, T. A. Berkoff, R. T. Jones, H. Singh, A. D. Kersey, E. J. Friebele, and M. A. Putnam, "In-line fiber etalon (ILFE) fiber-optic strain sensors," Journal of Lightwave Technology, 13 (7), 1256-1268, 1995.

[19] J. A. Greene, K. A. Murphy, A. J. Plante, V. Bhatia, and M. Sen, "Broadband photoinduced Fresnel reflectors," Lasers and Electro-Optics Society Annual Meeting, LEOS '94, vol. 2, 1994.

[20] J. A. Greene, T. A. Tran, K. A. Murphy, A. J. Plante, V. Bhatia, M. Sen, and R. O. Claus, "Photoinduced Fresnel Reflectors for Point-wise and Distributed Sensing Applications," Proc. SPIE, vol. 2444, 1995.

[21] K. D. Oh, J. Ranade, V. Arya, A. Wang, and R. O. Claus, "Optical fiber Fabry-Perot interferometric sensor for magnetic field measurement," Photonics Technology Letters, 9 (6), 797-799, 1997.

[22] A. Wang, Intrinsic Fabry-Perot optical fiber sensors and their multiplexing, U.S. Patent, patent pending.

[23] A. D. Kersey, "A Review of Recent Developments in Fiber Optic Sensor Technology," Optical Fiber Technology, 2 (3), 291-317, 1996.

[24] A. D. Kersey, A. Dandridge, and A. B. Tveten, "Time-division multiplexing of interferometric fiber sensors using passive phase generated carrier interrogation," Optics Letters, 12 (10), 775, 1987.

[25] S. C. Huang, W. W. Lin, and M. H. Chen, "Time-division multiplexing of polarization-insensitive fiber-optic Michelson interferometric sensors," Optics Letters, 20 (11), 1244, 1995.

[26] S. F. Collins, B. T. Meggit, A. W. Palmer, and K. T. V. Grattan, "A multiplexing scheme for optical fiber interferometetric sensors using an FMCW generated carrier," Proc. OFS-8, 1992.

[27] A. D. Kersey, "Demonstration of a hybrid time/wavelength division multiplexed interferometric fibre sensor array," Electronics Letters, 27 (7), 554-555, 1991.

[28] F. Kullander, "Code division multiplexing in interferometric optical fiber sensor networks," Optical Fiber Sensors Conference Technical Digest, 2002., vol. 1, 2002.

[29] Y. Hu and S. Chen, "Two-dimensional encoding scheme for digital spatial domain multiplexed large-scale optical fiber sensor arrays," IEEE Transactions on Instrumentation and Measurement, 46 (5), 1160, 1997.

[30] J. Brooks, R. Wentworth, R. Youngquist, M. Tur, B. Kim, and H. Shaw, "Coherence multiplexing of fiber-optic interferometric sensors," Journal of Lightwave Technology, 3 (5), 1062- 1072, 1985.

[31] W. J. Bock, "Coherence-Multiplexed Fiber-Optic Sensor Systems for Measurements of Dynamic Pressure and Temperature Changes," IEEE Instru. and Meas. Tech. Conf.,, Hungary, 2001.

[32] G. A. Cranch and P. J. Nash, "Large-scale multiplexing of interferometric fiber-optic sensors using TDM and DWDM," Journal of Lightwave Technology, 19 (5), 687, 2001. 
[33] M. G. Xu, H. Geiger, and J. P. Dakin, "Interrogation of fibre-optic interferometric sensors using acousto-optic tunable filter," Electronics Letters, 31 (17), 1487-88, 1995.

[34] G. Lammel, S. Schweizer, S. Schiesser, and P. Renaud, "Tunable optical filter of porous silicon as key component for a MEMS spectrometer," $J$. Microelectromechanical Systems, 11 (6), 815-827, 2002.

[35] H. Kobrinski and K.-W. Cheung, "Wavelength-Tunable Optical Filters: Applications and Technologies," IEEE Communications Magazine, 53-63, Oct 1989.

[36] D. A. Smith, J. E. Baran, J. J. Johnson, and K.-W. Cheung, "Integrated-Optic Acoustically-Tunable Filters for WDM Networks," IEEE J. on Selected Areas in Communications, 8 (6), 1151-1159, 1990.

[37] J. Stone and L. W. Stulz, "Pigtailed high-finesse tunable fibre Fabry-Perot interferometers with large, medium and small free spectral ranges,"" Electronics Letters, 23 (15), 781-782, 1987.

[38] T. Amano, "Design and Fabrication of GaAs-GaAlAs Micromachined Tunable Filter with Thermal Strain Control,"' Journal of Lightwave Technology, 21 (3), 596-601, 2003.

[39] D. Sadot and E. Boimovich, "Tunable Optical Filters for Dense WDM Networks,""' IEEE Communication Magazine, 50-55, Dec 1998.

[40] R. L. Anderson, D. N. Fry, and J. A. McEvers, "Advanced Turbine Systems Sensors and Controls Needs Assessment Study Final Report," Oak Ridge National Laboratory ORNL/TM-13335, 1997.

[41] W. F. Cullinane and R. R. Strange, "Gas Turbine Engine Validation Instrumentation: Measurements, Sensors and Needs," Proc. SPIE, vol. 3852, A. Wang, Ed., 1999.

[42] "Assessment of the Impact of Improved Harsh Environment Sensing on U.S. Industrial Energy Usage and Waste Production," Oak Ridge National Laboratory report prepared for U.S. Department of Energy - Office of Industrial Technology.

[43] M. Han, X. Wang, J. Xu, K. L. Cooper, and A. Wang, "A diaphragm-based extrinsic Fabry-Perot interferometric optical fiber sensor for acoustic wave detection under high background pressure," Opt. Eng., 44 (6), 060506, 2005.

[44] J. Xu, G. R. Pickrell, X. Wang, W. Peng, K. L. Cooper, and A. Wang, "A Novel Temperature-Insensitive Optical Fiber Pressure Sensor for Harsh Environments," Phot. Technol. Lett., 17 (4), 870-872, 2005.

[45] X. Wang, J. Xu, Y. Zhang, K. L. Cooper, A. Wang, and Z. Tu, "Label-free DNA Sequence Detection Using Oligonucleotide Functionalized Optical Fiber," Appl. Phys. Lett., in press.

[46] X. Wang, J. Xu, Y. Zhu, B. Yu, M. Han, K. L. Cooper, G. R. Pickrell, A. Wang, A. Ringshia, and W. Ng, "Verifying an all fused silica miniature optical fiber tip pressure sensor performance with turbine engine field test," Sensors for Harsh Environments II, vol. 5998, A. Wang, Ed., Boston, 2005.

[47] L. Song, K. L. Cooper, Z. Wang, A. Wang, and Y. Liu, "Position Location of Partial Discharges in Power Transformers Using Fiber Acoustic Sensor Arrays," Opt. Engin., in press.

[48] Y. Zhu and A. Wang, "Miniature Fiber-Optic Pressure Sensor," Phot. Technol. Lett., 17 (2), 447-449, 2005. 


\section{List of Acronyms and Abbreviations}

$\mathrm{A} / \mathrm{D}$, analog to digital

APP, Advanced Pressure Products, Inc.

BHF, buffered hydrofluoric acid

CCD, charge couple device

CPT, Center for Photonics Technology

CTE, coefficient of thermal expansion

EFPI, extrinsic Fabry-Perot interferometer

EMI, electromagnetic interference

FWHM, full width half maximum

GRIN, graded index

IOF, Industries of the Future

LED, light emitting diode

MCU, micro controller unit

MMF, multimode fiber

NA, numerical aperture

PC, personal computer

PZT, lead zirconium titanate

SCIIB, self-calibrated interferometric/intensity-based

SLED, superluminescent light emitting diode

SMF, single mode fiber

SNR, signal to noise ratio

VTPL, Virginia Tech Photonics Laboratory (now Center for Photonics Technology) 


\title{
Optical Fiber Sensors for Energy-Production and Energy Intensive Industries
}

\author{
Anbo Wang \\ Center for Photonics Technology \\ Bradley Department of Electrical \& Computer Engineering \\ Virginia Polytechnic Institute and State University, Blacksburg, VA 24061-0111, USA
}

\begin{abstract}
This paper presents a review of the sensor research at Virginia Tech Center for Photonics Technology. Some of these sensors are intended for energy-production and energy-intensive industries, such as power, oil, coal, transportation, metal casting, and glass manufacturing. The sensors of different types are presented. Some recent experimental results are briefly discussed.
\end{abstract}

\subsection{INTRODUCTION}

Optical fibers have been shown to be attractive in the fabrication of a variety of physical, chemical, and bio sensors because of a number of inherent such advantages as remote interrogation, potential of multiplexing, capability of operation in extreme physical conditions, non-electrically conducting, small size, and low weight. In the past three decades, many fiber sensor concepts and principles have been proposed, demonstrated and or developed into practical devices and systems. However, the real importance and potential of fiber sensors was not widely recognized and accepted in the energy-production and energy-intensive sectors until recent years. In these sectors, there are a wide range of extreme physical and chemical conditions which make conventional electrical and electronic sensors and measurement devices difficult to apply. Consequently, R\&D investment by industry is picking up in an observable pace, and it is believed that the pace will continue in the foreseeable future. There is no doubt that this trend will dramatically accelerate the industrialization of a range of fiber sensors as what has happened to fiber gyroscopes many years ago.

The optical sensor research at the Virginia Tech Center for Photonics Technology (VTCPT) has been mainly focused on the development of sensors for measurement of pressure, temperature, strain, acoustic waves, flow, electric partial discharges, surface mapping and 3-D temperature imaging. Most of these sensors are based on optical fibers, including silica glass fiber with various polymer and metallic coatings and single-crystal sapphire fiber waveguides. In terms of the optical parameters being modulated, these sensors could be approximately classified into interferometric, polarimetric, intensity-based, and wavelength-coded devices. This paper presents several examples of the sensors recently developed at Virginia Tech.

\subsection{Self-Calibrated Interferometric/Intensity-Based Sensors}

Many of the fiber sensors developed to date belong to the categories of interferometers in which optical phase is affected, and intensity-based devices in which optical intensity is modulated. In 
general, interferometric sensors have the advantages of ultra-high resolution, accuracy and configuration versatility. However, they have the disadvantages of relative measurement, costly signal processing and low frequency response. In contrast to fiber interferometers, the intensitybased devices have the advantages of simple signal demodulation, absolute measurement, and high frequency response. Recently, researchers at VTCPT developed a new sensor, - the self-calibrated interferometric/intensity-based (SCIIB) sensor, which combines the two types in one sensor system.

The basic principle of the SCIIB sensor is illustrated in Figure 1. The system involves a sensor probe, optoelectronic signal processing and an optical fiber linking the sensor head and signal processing unit. The light from an optical source is launched into a two-by-two fiber coupler and propagates along the optical fiber to the sensor head. As shown in the enlarged view of the sensor head, the lead-in fiber and a reflecting fiber are thermally fused to a hollow glass tube with a predetermined air-gap separation between the two fibers. The incident light is first partially reflected at the endface of the lead-in fiber. The remainder of the light propagates across the air gap to the reflecting fiber endface, where a second reflection is generated. The two reflections then travel back along the same lead-in fiber through the same fiber coupler to the photodetection end.

In order to overcome common problems associated with regular fiber interferometric sensors, such as relative measurement, low frequency response, and complexity in signal demodulation, a special optoelectronic signal demodulation method is employed that allows self-calibrating and absolute measurement with high frequency response. As a result, the new sensor possesses the best features of both fiber interferometry and intensity-based sensors. To our knowledge, this is the first design to realize successful combination of fiber interferometric and intensity-based sensors into a single sensor system. Because of these combined simultaneous advantages, the new sensor scheme is therefore extremely attractive for the fabrication of a variety of sensors for accurate and reliable measurement of various physical parameters that can be related to microdisplacement. Various sensors have been designed and fabricated based on the basic SCIIB sensor configuration.

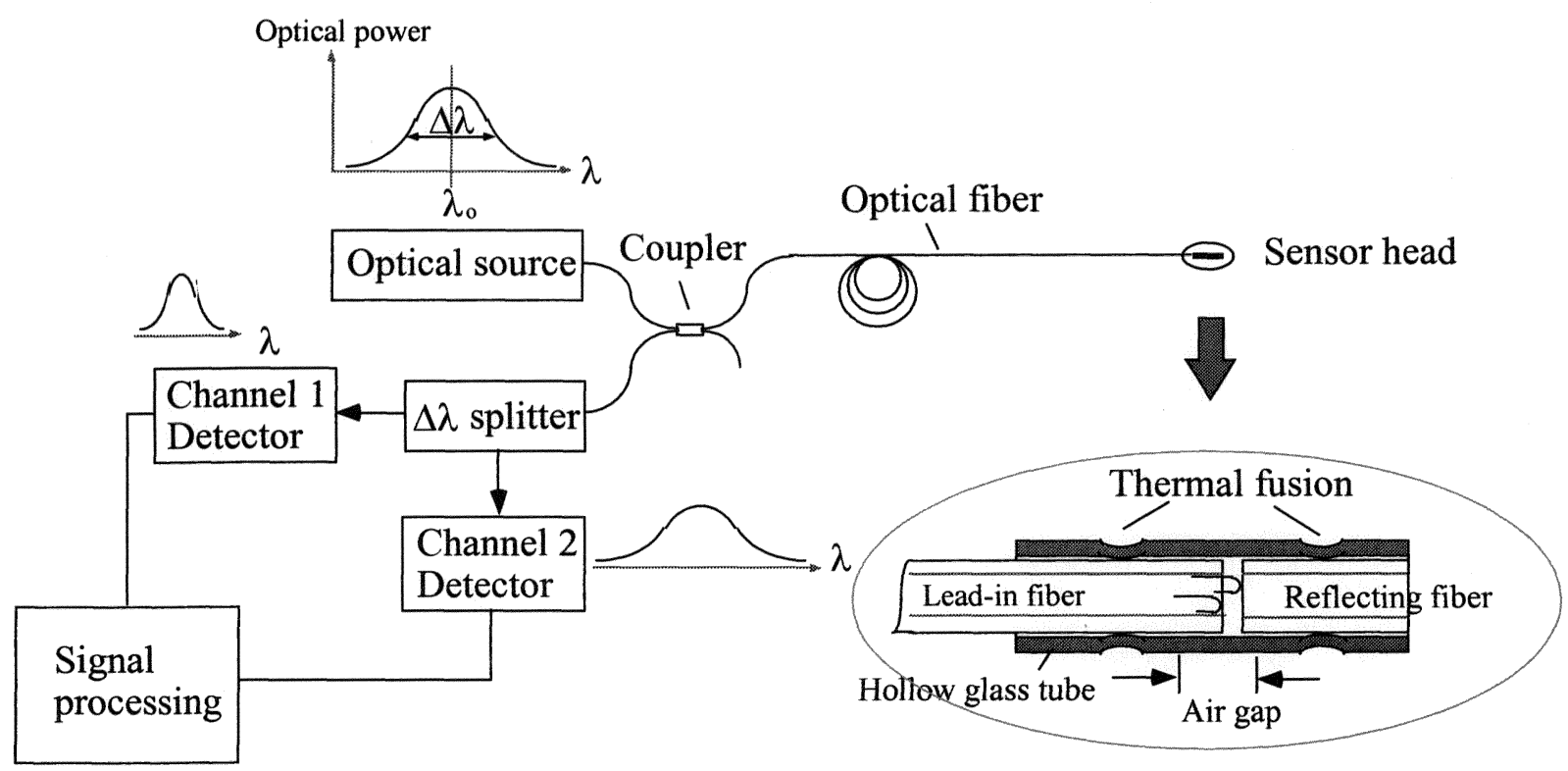

Figure 1. Illustration of the principle of the self-calibrated interferometric/intensity-based (SCIIB) fiber sensor. 


\section{Pressure Sensors}

For the sensor geometry shown in Figure 1, when a pressure is applied to the sensor head, due to the longitudinal and lateral compression of the glass alignment tube, the air gap separation in the sensor will change. The air gap variation can be expressed as

$$
\Delta=\frac{L}{E} \frac{p r_{0}^{2}}{r_{o}^{2}-r_{i}^{2}}(1-2 \mu)
$$

where $E$ is the Young's modulus of the tube material, $\mu$ is Poisson ratio, $L$ is the distance between the two thermal fusion points, and $r_{o}$ and $r_{i}$ are the outer and inner radii of the glass tube. Equation (1) indicates that the air gap change is directly proportional to the applied pressure. In addition, the equation also shows the involvement of several sensor parameters, including the inner and outer diameters of the hollow glass tube, the distance between the two fusion bonding points and the mechanical properties of the hollow glass material. The sensor thus offers excellent design flexibility for different dynamic measurement ranges. Moreover, since the glass tube can be made of the same material as the fiber, the thermal expansion of the tube is balanced by the thermal expansions of the lead-in and the reflecting fibers, so that ultra-low temperature dependence is therefore expected.

Based on Equation (1), various sensors were designed and fabricated for different dynamic measurement ranges. Some of the typical testing results are presented in Figures 2-4. Figure 5 shows the results of the temperature tests. As expected, the pressure sensor shows a very low level of temperature dependence.

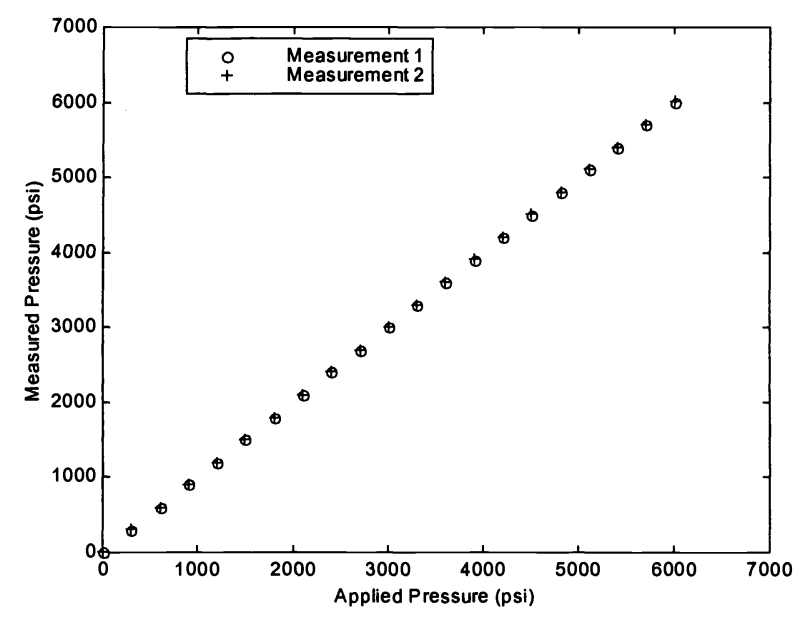

Figure 2. Results from a SCIIB pressure sensor with a $200 \mu \mathrm{m}$ gage length.

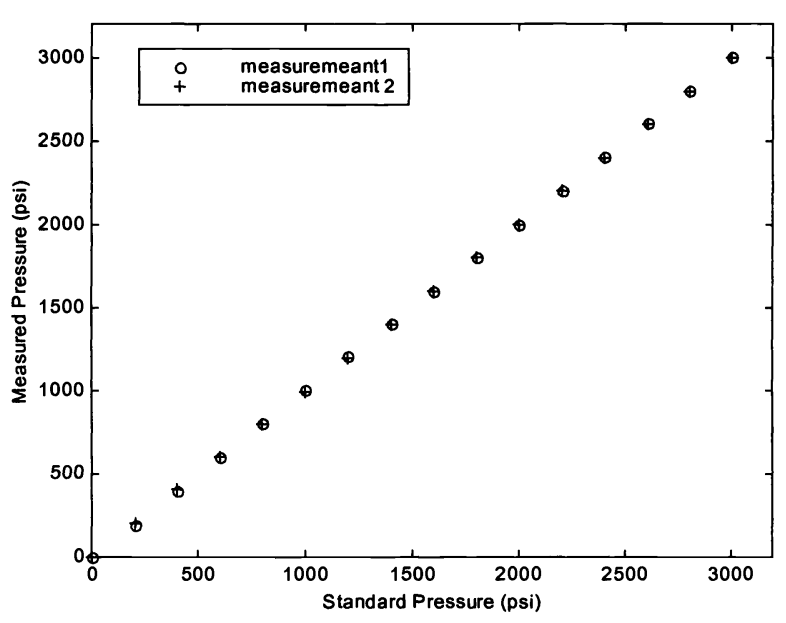

Figure 3. Results from a SCIIB pressure sensor with a $350 \mu \mathrm{m}$ gage length. 


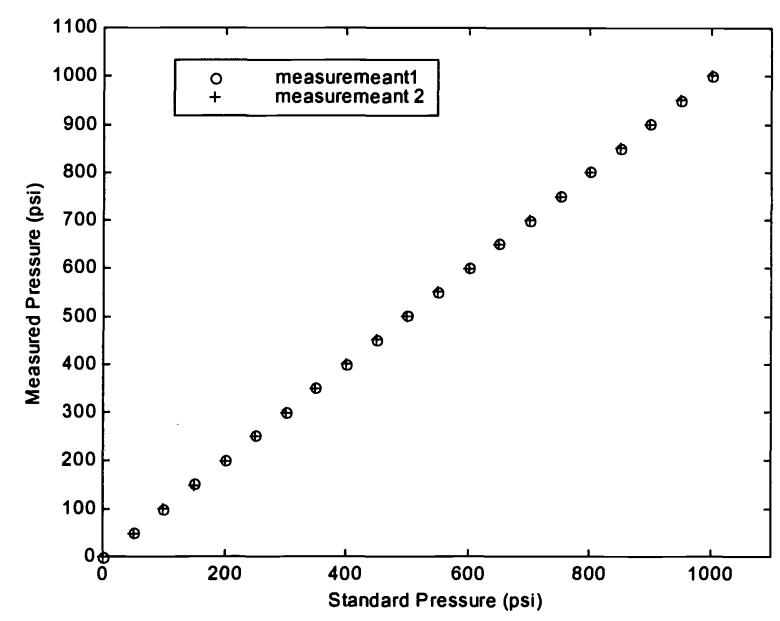

Figure 4. Results from a SCIIB pressure sensor with a $600 \mu \mathrm{m}$ gage length.

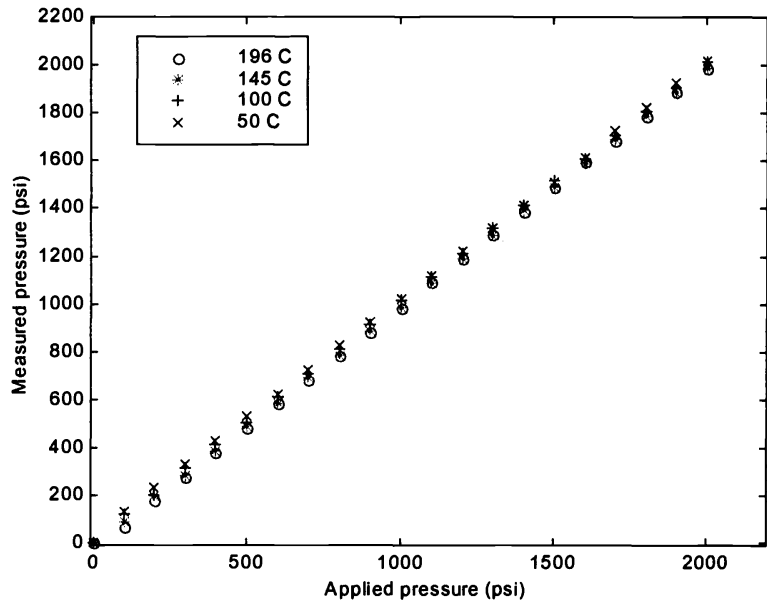

Figure 5. Testing results of temperature dependence of pressure sensor.

\section{$\underline{\text { Temperature Sensors }}$}

Contrary to the SCIIB-based pressure measurement, a temperature-induced air gap change is necessary for temperature measurement. One of the ways to produce a temperature-sensitive sensor is to use a sensor tube having a coefficient of thermal expansion different from that of the fibers, so that temperature changes produce a differential thermal expansion between the tube and the fibers. Because of the ultra-high sensitivity and accuracy in the measurement of small air gap changes, the SCIIB-based temperature sensors would allow excellent measurement resolution and accuracy. Again, the sensor could be designed to have varying measurement ranges by properly selecting such parameters as tube material and distance between the two thermal fusion points. The pressure dependence of the temperature measurement could be eliminated with the use of a pressure isolation glass tube.

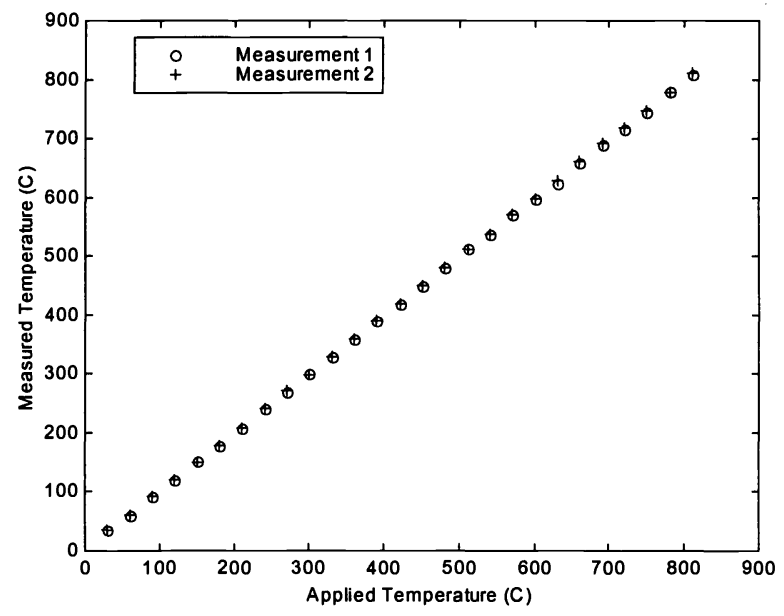

Figure 6. Measurement results obtained with the SCIIB temperature sensor over $800^{\circ} \mathrm{C}$.

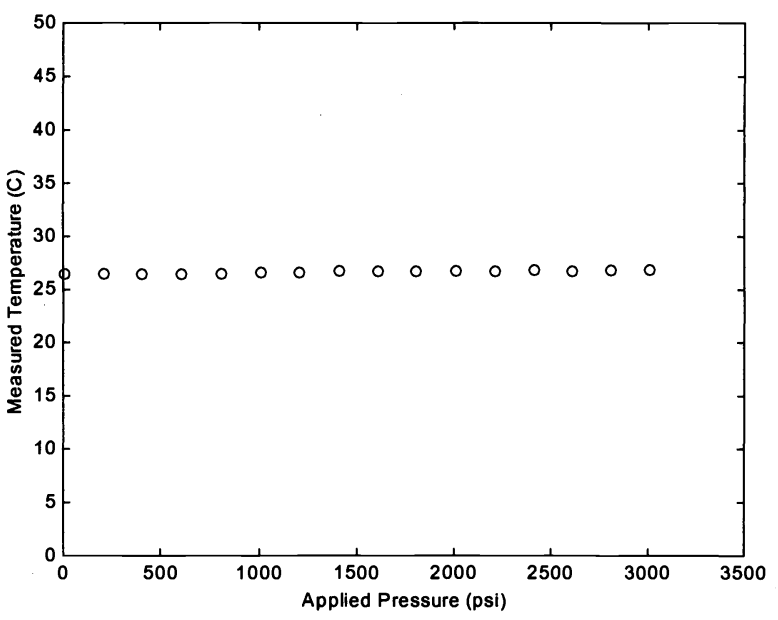

Figure 7. Results of pressure sensitivity of the CIIB temperature sensor. 
Figure 6 presents a typical response of a SCIIB temperature sensor. As expected, the sensor shows reliable measurement at temperatures above $800^{\circ} \mathrm{C}$. Figure 7 shows no observable cross-sensitivity between temperature and pressure.

Based on the principle described above, various different SCIIB sensors have been designed and fabricated for oil downhole measurement, gas turbine engine monitoring and aerospace materials evaluations.

\subsection{Sapphire-Based Polarimetric High Temperature Sensors}

Single-crystal sapphire is an attractive material for the fabrication of sensors intended for harsh environment applications because of its high melting temperature $(>2000 \mathrm{C})$, outstanding resistance to chemical corrosion, excellent optical transparency over a large spectral range, and commercial availability of different sizes and geometries. VTCPT has done extensive research concerning sapphire element-based or sapphire fiber-based sensors for measurement of temperature, strain and pressure. This section presents one example of the research in this area.

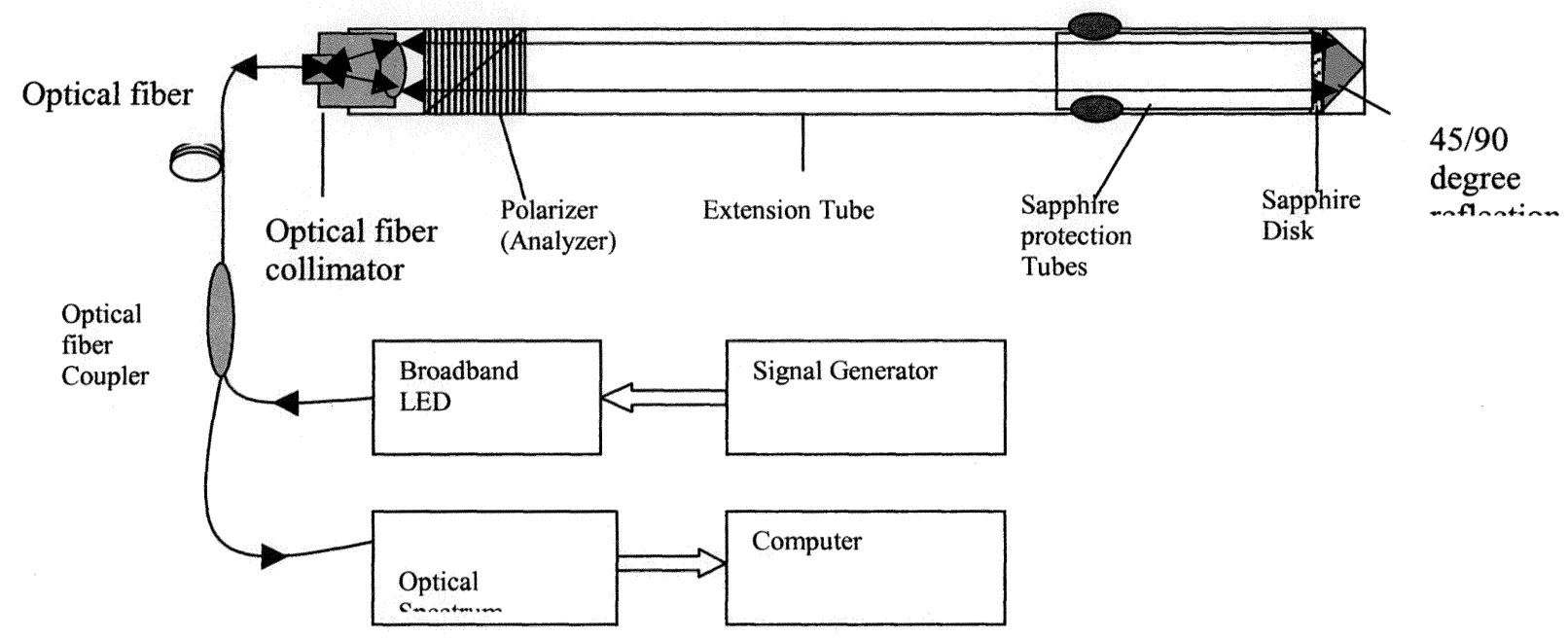

Figure 8. Schematic of the sapphire-based high temperature sensor.

Figure 8 shows the basic principle of a sapphire element-based polarimetric sensor for ultra-high temperature measurement. The light produced from a light emitting diode (LED) is launched into a multimode optical fiber and propagates through a $3 \mathrm{~dB} 2 \mathrm{X} 2$ fiber coupler to the sensor probe. In the probe, the light is first linearly polarized with its polarization direction aligned at 45 degrees with respect to the fast or slow axis of a single-crystal sapphire disk. Because of the birefringence of sapphire, the sapphire disk, which functions as the temperature sensing element, delays the two decomposed polarization components of the light along the fast and the slow axes differently. When the two co-propagating orthogonal polarization components are combined at a polarization analyzer, which is virtually the same as the input polarizer when the sensor is operated in a reflection mode, the light spectrum after the analyzer then carries the information about the birefringence of the sapphire disk, which is a function of temperature. The transmitted light spectrum can be expressed as: 


\title{
Grating-assisted demodulation of interferometric optical sensors
}

\author{
Bing Yu and Anbo Wang
}

\begin{abstract}
Accurate and dynamic control of the operating point of an interferometric optical sensor to produce the highest sensitivity is crucial in the demodulation of interferometric optical sensors to compensate for manufacturing errors and environmental perturbations. A grating-assisted operating-point tuning system has been designed that uses a diffraction grating and feedback control, functions as a tunablebandpass optical filter, and can be used as an effective demodulation subsystem in sensor systems based on optical interferometers that use broadband light sources. This demodulation method has no signaldetection bandwidth limit, a high tuning speed, a large tunable range, increased interference fringe contrast, and the potential for absolute optical-path-difference measurement. The achieved 40-nm tuning range, which is limited by the available source spectrum width, 400-nm/s tuning speed, and a step resolution of $0.4 \mathrm{~nm}$, is sufficient for most practical measurements. A significant improvement in signal-to-noise ratio in a fiber Fabry-Perot acoustic-wave sensor system proved that the expected fringe contrast and sensitivity increase. (C) 2003 Optical Society of America

OCIS codes: $\quad 060.2370,050.1950,070.6020$.
\end{abstract}

\section{Introduction}

Fiber-optic interferometers, especially fiber FabryPerot interferometers (FFPI), have found increased applications in the past decade for the detection and measurement of a large variety of physical parameters, such as temperature, ${ }^{1-4}$ vibration, ${ }^{1,5}$ pressure,,$^{3,4,6,7}$ acoustic waves, ${ }^{1,5,8-11}$ and strain. ${ }^{12,13}$ Many interferometric sensors operate over the linear region of an interference fringe. , $3,4,8,9,11_{\text {Compared }}$ with sensors that use fringe counting, $5,9,11$ sensors that operate in the linear region have the advantages of a linear transfer function, no ambiguity in fringe direction, simple signal processing, and highest sensitivity at the quadrature point ( $Q$ point). They are, therefore, suitable for detection of small optical path differences (OPDs) and wideband applications. However, confining the operation to the linear region places difficult manufacturing constraints on the sensor head by requiring that the initial cavity length be positioned at the $Q$ point or sometimes at one end of

The authors are with the Center for Photonics Technology, Virginia Polytechnic Institute and State University, 460 Turner Street, Blacksburg, Virginia 24061-0287. B. Yu's e-mail address is biyu@vt.edu.

Received 29 May 2003; revised manuscript received 8 August 2003.

0003-6935/03/346824-06\$15.00/0

(C) 2003 Optical Society of America a linear region of the transfer function. ${ }^{6}$ The sensors also suffer from signal fading caused by environmental perturbations, such as temperature changes, which may drive the sensors out of the linear region. The drifts are even more critical for Fabry-Perot (F-P) sensors; medium- or high-finesse F-P cavities are often necessary for higher measurement sensitivity ${ }^{1,11}$ because of their steeper fringe slopes. Therefore, poor control of the operation point has been a major factor that limits the practical application of interferometric optical sensors in the sensor industry.

Several techniques have been developed by various researchers to stabilize the $Q$ point, for example, intentionally applying an acoustic bias, ${ }^{1}$ tuning the static OPD in stages, ${ }^{14}$ and using a tunable light source, ${ }^{8,9}$ quadrature phase-shifted demodulation or dual-wavelength interrogation, $, 5,6,12,15$ or direct spectrum detection. ${ }^{6,13,16,17}$ In the acoustic bias method, the low-frequency component output of a FFPI acoustic-wave sensor was compared with a constant voltage corresponding to the setting operating point, and the difference was used to adjust the acoustic bias through a voice coil. This method does compensate for environmental drifts, but adjusting the operating point with a voice coil limits its use only to acoustic detection, and the coil may cause extra noise. A static OPD is tuned in a Mach-Zehnder interferometer by use of a fiber stretcher. The quadrature point nearest maximum visibility can be locked with 
a feedback-control servo system, and a temperature drift from $25{ }^{\circ} \mathrm{C}$ to $55^{\circ} \mathrm{C}$ has been successfully compensated for. However, tuning the OPD may cause excess power fluctuation in the fiber, and it is not suitable for use with sensors with F-P interferometers. Adjusting the operating point by changing the bias current of a tunable laser diode is another effective approach that has been used by many researchers. The disadvantages of using a tunable laser are its optical power fluctuation, high sensitivity to backreflection, laser mode hopping, and high cost. Quadrature phase-shifted demodulation, or dualwavelength interrogation, was originally developed by Murphy et al.12 to solve problems of a nonlinear transfer function and directional ambiguity in extrinsic F-P sensors, but it may also be used for the demodulation of sensors operating in the linear regions. However, it is possible that both channels operate away from their optimal $Q$ points at the same time, even if a $90^{\circ}$ phase shift can be maintained during the measurement, which is as hard as to control the operating point itself.

Strictly speaking, the spectrum detection method should not be categorized into a kind of operatingpoint stabilizing method, though linear response can be achieved. ${ }^{13}$ Direct spectrum detection uses a diffraction grating or a Fizeau interferometer, and the modulated broadband spectrum is detected by a CCD array and analyzed by a signal-processing unit. This procedure is also called white-light interferometry and actually does not need to control the operating point of an interferometric sensor. It provides an absolute and accurate value of the OPD in a sensing interferometer and is insensitive to power and spectral fluctuations of the light source. Its major disadvantage is that it is not suitable for real-time detection of broadband signals such as acoustic waves and high-frequency pressure because a large amount of time is required for processing the huge amounts of data from a CCD array. Another disadvantage of spectrum detection is its high cost, especially for sensors operating in the near-infrared region, where expensive detector arrays must be used.

In this paper we present an alternative demodulation technique with operation-point tuning by use of a diffraction grating with feedback control, called grating-assisted operating-point tuning (GA-OPT). This demodulation method has no ac bandwidth limit, a high tuning speed, a large tunable range, increased interference fringe contrast, and the potential for absolute optical-path-difference measurement. An experiment in which a fiber F-P acoustic-wave sensor system is used to prove the feasibility of the design is also presented.

\section{Wavelength Dependence of Operation Points}

Interferometric-intensity-based detection is a widely used demodulation technique in optical interferometric sensors such as fiber F-P interferometers, MachZehnder, and Sagnac sensors. When a monochromatic light of wavelength $\lambda$ is used to inter- rogate the sensors, the optical intensity of the twobeam interference between the sensing beam and the reference beam can be expressed as

$$
I=I_{1}+I_{2}+2 \sqrt{I_{1} I_{2}} \cos \phi,
$$

where $I_{1}$ and $I_{2}$ represent the optical intensities of the sensing beam and the reference beam, respectively, and

$$
\phi=2 \pi(n l) / \lambda
$$

is the phase difference caused by the OPD $n l$ between the two beams, where $n$ is the refractive index of the medium and $l$ is the physical path difference. The optical intensity arriving at the photodetector is a simple cosine function, called interference fringes, of $n l$, which is a function of perturbations in the measurant and the background. Obviously, sensors have zero sensitivity at the peaks or the valleys of the fringes, and the maximum sensitivity and the most nearly linear response at the $Q$ points, where $\phi=$ $\pi / 2+\mathrm{m} \pi, m=0,1,2, \ldots$ It is of absolute advantage to design a sensor operating at the $Q$ points for the highest sensitivity and the lowest signal distortion. However, any sensor fabrication tolerance, temperature-induced drift, or other environmental perturbation can easily drive a sensor away from the $Q$ points. Notice that the interference fringes and thereby the $Q$ points are wavelength dependent for all interferometers; one may dynamically control the operation points by tuning the wavelength of the interrogation lights to compensate for the phase drifts, that is,

$$
\Delta \phi=\Delta \phi_{(n l)}+\Delta \phi_{\lambda}=\frac{2 \pi}{\lambda} \Delta(n l)-\frac{2 \pi(n l)}{\lambda^{2}} \Delta \lambda=0,
$$

or

$$
\Delta \lambda=\frac{\lambda}{(n l)} \Delta(n l),
$$

where $\Delta \phi_{(n l)}$ and $\Delta \phi_{\lambda}$ are the phase changes caused by environmental disturbance $[\Delta(n l)]$ and wavelength tuning $[\Delta \lambda]$. Usually $n l$ is at least 1 order larger than $\lambda$, which means that a large drift can be compensated for by a relatively small change in the wavelength. The wavelength tuning can be achieved by use of a tunable laser, though this may cause some problems such as optical power fluctuation and sensitivity to backreflections.

For a broadband light source, the wavelength dependence of the interference fringe is much more complex than for a monochromatic source. The interference fringes are determined not only by the central wavelength but also by the spectrum width of the interrogation lights. The total optical intensity arriving at the photodetector has to be computed by integration over the whole spectrum of the light source. Figure 1 is a theoretical calculation of the wavelength dependence of the interference fringes at 


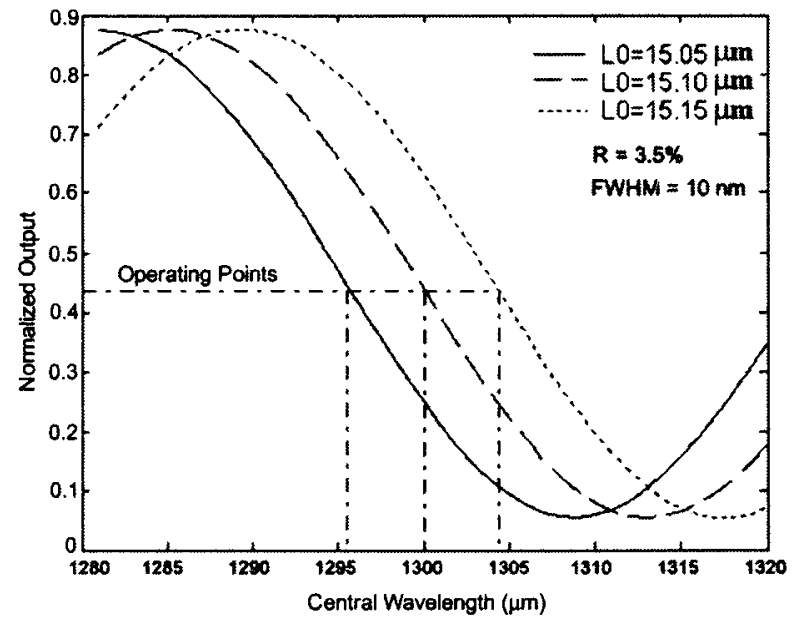

Fig. 1. Theoretical calculation of the wavelength dependence of operation points. $\mathrm{R}$, reflectivity.

three cavity lengths $(L 0)$ of a low-finesse FFPI sensor, ${ }^{11}$ where a 1300 -nm light source of a $35-\mathrm{nm}(3-\mathrm{dB})$ spectral width was used, the output was normalized to the source intensity distribution, and the bandwidth of the interrogation lights was limited to $2 \Delta \lambda=$ $10 \mathrm{~nm}$. For example, if the OPD, for any reason, changes from $L 0=15.05$ to $15.15 \mu \mathrm{m}$, one can track the operation point by tuning the central wavelength from 1.296 to $1.304 \mu \mathrm{m}$. The central wavelength of the interrogation light can be tuned by use of GAOPT, as is discussed in Section 3.

\section{Grating-Assisted Operation-Point Tuning}

A schematic illustration of a white-light interferometric sensor system based on GA-OPT is shown in Fig. 2. Operation-point tuning is achieved with two collimators, a diffraction grating, a short piece of multimode fiber (MMF), a photodetector, a preamplifier, a computer with a data-acquisition card, and a motorized rotary stage and its driver. The light from a broadband source is launched into a single-mode fiber (SMF), propagates through a 3-dB coupler, and arrives at an interferometric sensor, where it is modulated by the perturbation measurand. The phase-modulated light propagates back through the same 3-dB coupler and reaches the first collimator. The collimated light beam illuminates

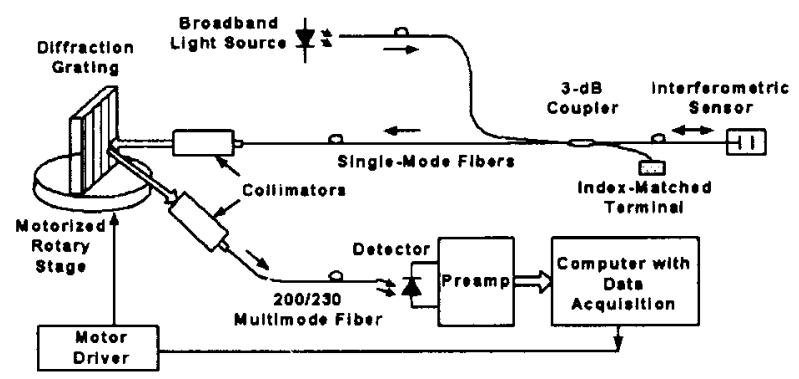

Fig. 2. Schematic diagram of a FFPI sensor system based on GA-OPT. a diffraction grating, which can be rotated by a motorized rotary stage with high resolution $(\sim 0.2$ mrad). The -1 st diffractions collected by a second collimator are focused into a $200-230-\mu \mathrm{m}$ MMF, and only part of the total spectrum $(\lambda \pm \Delta \lambda)$ can be collected by the MMF.

Instead of a SMF or a detector, a short MMF is used to increase the reception area and to facilitate the spectrum monitoring with an optical spectrum analyzer. The MMF can be removed and the detector can be placed on the focal point of the receiving collimator with a penalty of less flexibility. A transimpedance preamplifier converts the photocurrent into voltage, and a subsequent divider separates the signal into dc and ac components, which are amplified and filtered further. A computer with a dataacquisition card is used to record and to calibrate the $\mathrm{dc}$ and ac signals. The calibration reference, a representation of the original optical intensity distribution, is an output-position curve obtained by scanning of the whole wavelength range with a cleaved fiber, instead of a sensor, connected.

The calibrated ac signals directly represent the absolute value of the ac information of the measurand, and the frequency response is limited only by the sensor head and the bandwidth of the electronics. The calibrated dc signals represent the current operation point on the transfer function. Ideally, this dc voltage $(V)$ should to be equal to the setting point $\left(V_{0}\right)$, most likely the $Q$ point. However, any change in manufacturing tolerance and environment may cause an undesired change of the OPD of the sensor and thereby a drift of dc voltage $V$. The difference $\Delta V$ between the dc output and the setting point is the control signal that can be used to adjust the position of the grating, and therefore the central wavelength of the interrogation lights, through a motorized rotary stage. By this feedback control, the optimal operation point can be dynamically maintained, and the dc signal may also be recovered from the position of the grating if environmental effects can be determined. Obviously, the tuning range is limited only by the source spectrum, and the dc accuracy is limited by the resolution and the repeatability of the rotary stage.

According to the grating equation, the relationship between the incident beam and the -1 st diffracted beam can be expressed as

$$
\sin (A)+\sin (B)=\lambda / d,
$$

where $A$ is the incident angle of the light beam from the input collimator to the normal of the grating surface, $B$ is the angle of the -1 st diffraction to the normal of the grating surface, $\lambda$ is the wavelength of light in air, and $d$ is the groove spacing of the grating.

Assuming that a lens of focal length $f$ is used in the receiving collimator, we can calculate $B$ from

$$
\cos (B)=\frac{f(\Delta \lambda / D)}{d}
$$




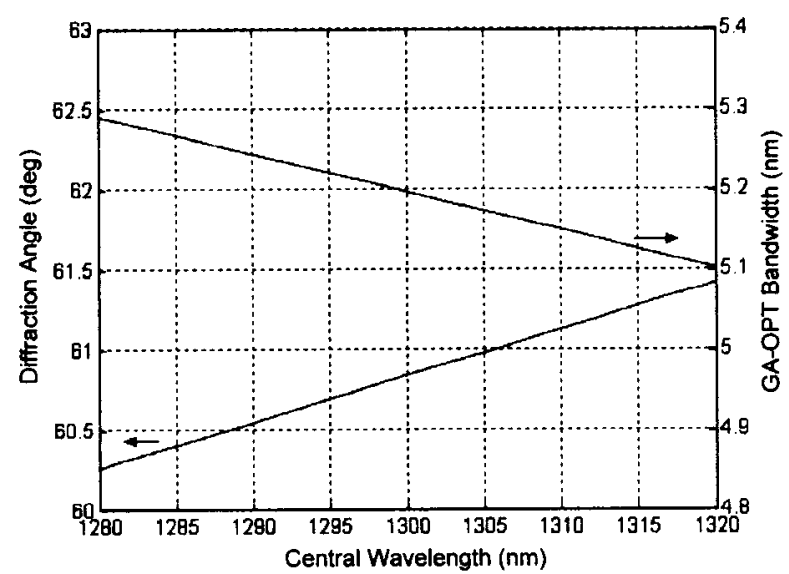

Fig. 3. Wavelength dependence of the diffraction angle and GAOPT bandwidth $(\Delta \lambda)$.

where $\Delta \lambda$ is the spectrum resolution and $D$ is the diameter of the core of the receiving MMF or the active area of the detector.

With $\lambda=1300 \mathrm{~nm}, d=1 / 750 \mathrm{~mm}, f=25.0 \mathrm{~mm}$, $D=0.2 \mathrm{~mm}$ for a $200-2300-\mu \mathrm{m} \mathrm{MMF}$, and $B-A=$ $55^{\circ}$, Fig. 3 gives the calculated angular tuning range for wavelength tuning from 1280 to $1320 \mathrm{~nm}$ and the resultant change in receiving bandwidth. An angular change of $1.2^{\circ}$ is enough for scanning a wavelength range of $40 \mathrm{~nm}$, and a bandwidth error of $\sim 3.5 \%$ may result. This bandwidth error may induce an error in the optical intensity distribution from the original light source distribution that one can easily compensated for by scanning the whole spectrum range and storing the new intensity distribution during the reset. Obviously, finer tuning or smaller $\Delta \lambda$ can be achieved, with a penalty of higher insertion loss, by use of smaller-diameter fiber $D$ or a longer focal length $f$ if the groove spacing, incident angle $A$, and diffraction angle $B$ have been determined.

\section{Experiments}

To verify the feasibility of operation-point tuning we designed and fabricated GA-OPT, as shown in Fig. 4. A holographic diffraction grating of 750 grooves $/ \mathrm{mm}$ was glued onto a motorized rotary stage. The motor has a step resolution of $0.2 \mathrm{mrad}$ and a rotation speed of $2 \mathrm{rpm}$, which means that a central wavelength resolution of $0.38 \mathrm{~nm}$ and a tuning speed of $400 \mathrm{~nm} / \mathrm{s}$ can be achieved. Collimator I is the input collimator, and collimator II is the output collimator, which has a focal length of $25 \mathrm{~mm}$. The input SMF is connected to an interferometric optical sensor, and the output 200-230- $\mu \mathrm{m}$ MMF is connected to an optical receiver. The control interface board interconnects the motor and the motor controller.

In Fig. 5 are shown the spectra received by the detector at various grating positions or central wavelengths $(1276.4,1288.0,1298.0,1307.9$, and 1317.9 $\mathrm{nm})$ and the original spectrum of the superluminescent light-emitting diode (SLED), where the sensor

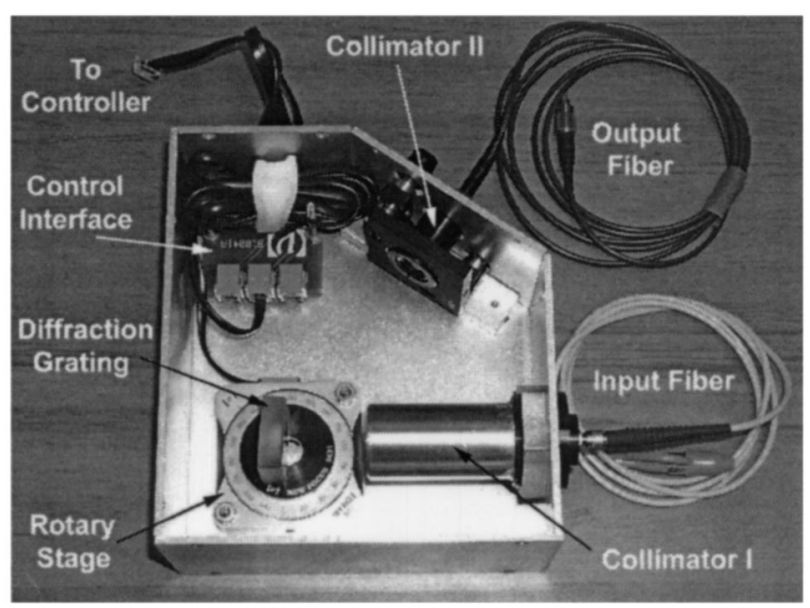

Fig. 4. Top view of GA-OPT.

was replaced with a cleaved SMF. A 1296-nm SLED with a spectral width of $35 \mathrm{~nm}$ was used as the broadband source. Scanning the grating at an angle of $1.2^{\circ}$ achieved a central wavelength change of $40 \mathrm{~nm}$. This agrees well with the theoretical calculation given in Fig. 3. The resultant spectrum bandwidth (FWHM) is $4.30-4.65 \mathrm{~nm}$, an average of $0.7 \mathrm{~nm}$ less than the theoretical results. This difference is believed to be caused by misalignment between the $25-\mathrm{mm}$ lens in collimator II and the 200-230- $\mu \mathrm{m}$ MMF as well as by the relatively small aperture of collimator II. The total insertion loss is $\sim 11 \mathrm{~dB}$. As mentioned in Section 3, a trade-off must be made between large bandwidth for high optical power and small bandwidth for high fringe visibility and high resolution of operation-point tuning.

The wavelength scan outputs of a FFPI sensor and the theoretical results in atmospheric pressure are shown in Fig. 6(a). The peak amplitudes are unequal because of the Gaussian spectrum distribution of the 1296-nm SLED source. The peak position differences between the experimental results and the

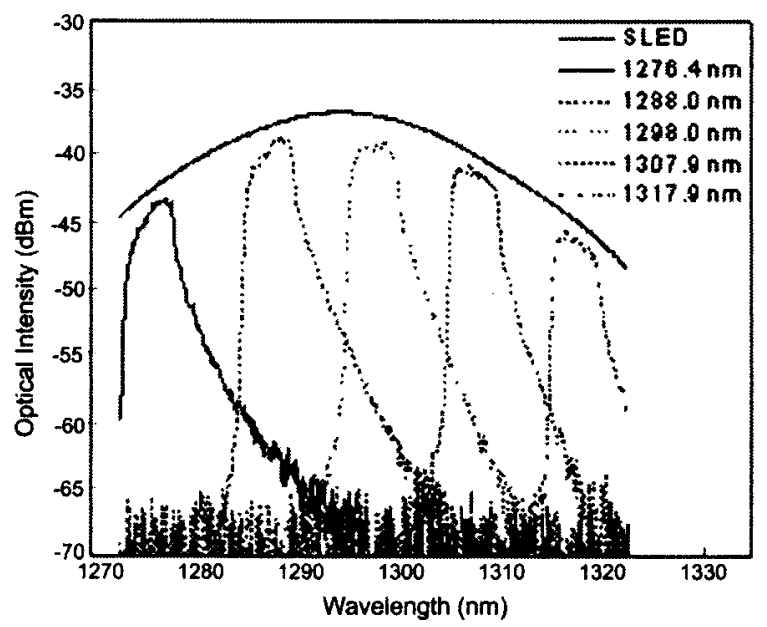

Fig. 5. Spectra received by the detector at several grating positions and the original spectrum of the SLED. 

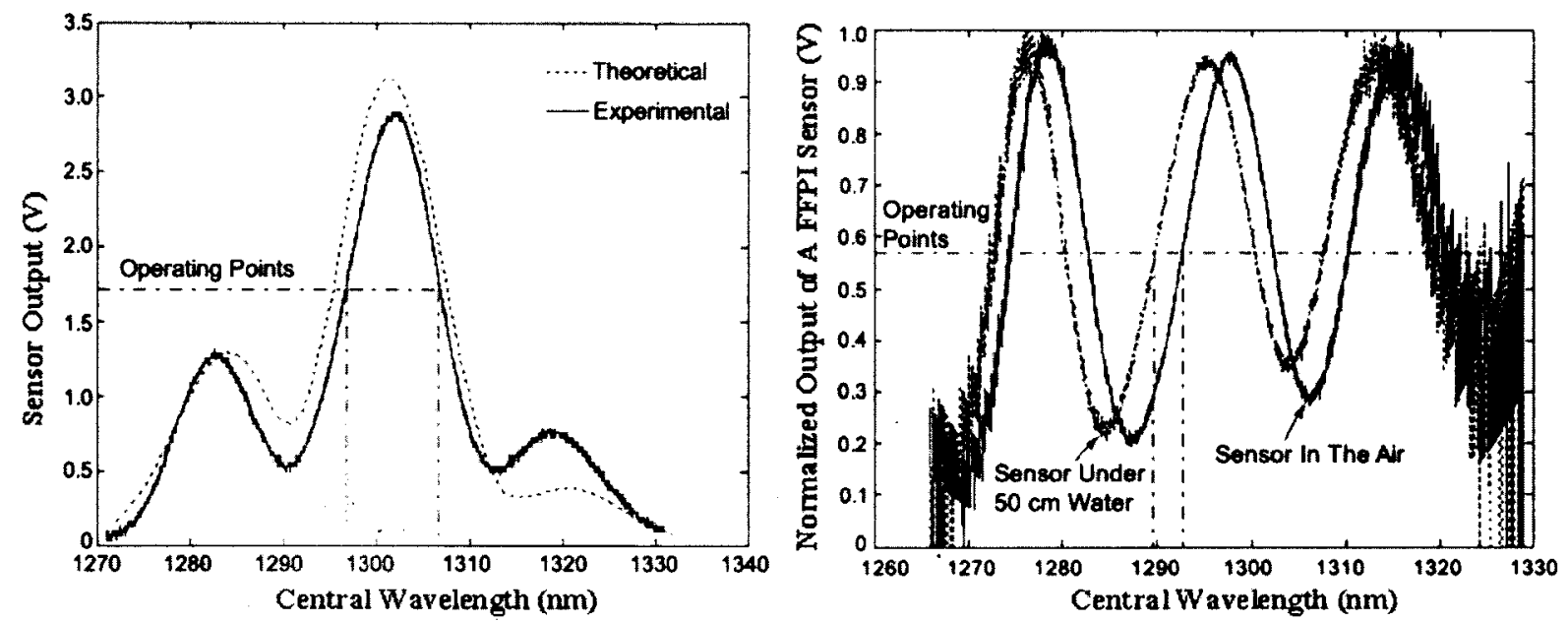

Fig. 6. Output of a FFPI sensor: (a) theoretical result and sensor output, (b) normalized sensor outputs at different static pressures.

theoretical calculation are caused by cavity-length measurement error, whereas the amplitude differences are believed to be caused by the offset of the spectrum distribution from the ideal Gaussian distribution used for the calculation and by misalignment of GA-OPT or of the sensor's F-P cavity. Figure 6(b) gives the scan outputs of a FFPI sensor in the atmospheric environment and under $50 \mathrm{~cm}$ of water normalized to the source spectrum. A $Q$-point drift of $\sim 3 \mathrm{~nm}$ resulted from the $50-\mathrm{cm}$ static water pressure. Obviously there is more than one $Q$ point on the fringes, but only those two $Q$ points on the highest fringe have the best signal-to-noise ratio and thereby are suitable as optimal operation points.

A diaphragm-based FFPI acoustic-wave sensor ${ }^{11}$ was chosen to test the performance of the developed GA-OPT in practical applications. This sensor was designed for partial-discharge detection in power transformers. Because this sensor was fabricated by use of thermal fusion bonding, which might cause large cavity-length error and may suffer from high static pressure in a transformer tank full of mineral oil, operation point control has been a major challenge, and very low sensitivity may result. A scan output of this sensor in an acoustic-wave test setup is given in Fig. 7(a). The acoustic-wave outputs at marked points A-E in Fig. 7(a) are shown in Fig. 7(b), along with that from a sensor system without GAOPT. Obviously, the original system has a low signal-to-noise ratio (SNR) because of the short coherence length of the SLED source compared with the sensor's cavity length, the unknown operation point, or both. When GA-OPT is used, the sensor performs differently at different operation points. At points $\mathrm{A}$ and $\mathrm{C}$ the $Q$ points of the interference fringes, the sensor has the best SNR, which is attributed to the highest sensitivity of the $Q$ point and the increased fringe contrast. An improvement of $\sim 15$ $\mathrm{dB}$ in SNR compared with that of the original sensor can easily be achieved, even if the original sensor was operating at its $Q$ point. The sensor's SNR has only moderate improvement at points $\mathrm{D}$ and $\mathrm{E}$, though they are $Q$ points too, because of the lower fringe slope at D or the low absolute optical intensity at E, both caused by the nonflat SLED spectrum. The

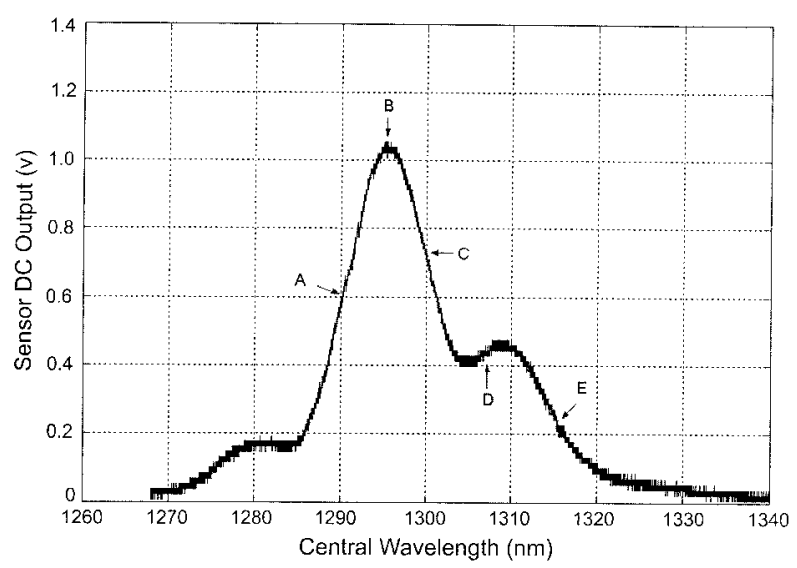

(a)

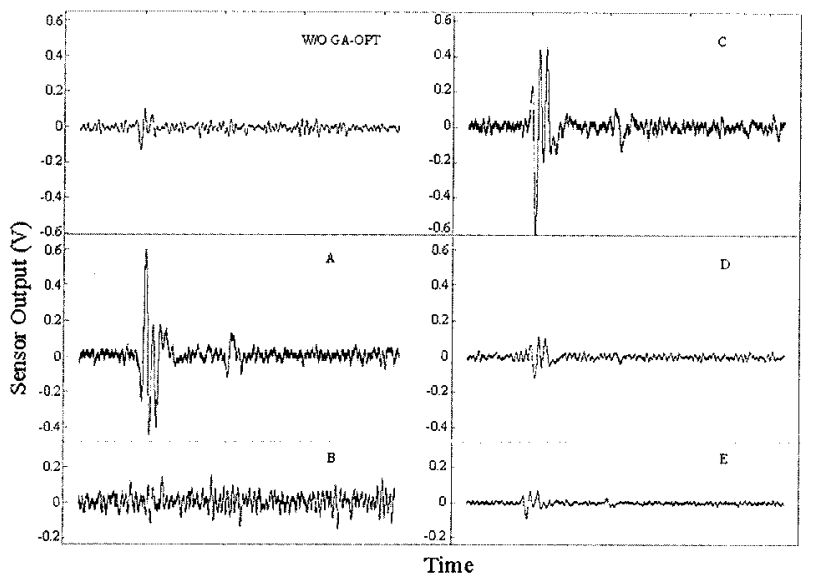

(b)

Fig. 7. Acoustic wave detection with a diaphragm-based FFPI sensor and GA-OPT: (a) scanning for the operation point, (b) acoustic-wave outputs without (WO) GA-OPT and with GA-OPT but at different operation points. 
sensor has the lowest SNR at B because of the zero sensitivity at the fringe peaks (or valleys). Therefore points $\mathrm{A}$ and $\mathrm{C}$ are the ideal operation points of this FFPI sensor. We also observed that the acoustic-wave outputs at these two points have different polarities and different dynamic ranges that may be sensitive for some measurements. When the operation point is determined, the last task is to maintain the sensor operation at the setting point. This can easily be achieved by the feedback-control system shown in the system diagram of Fig. 2. A signal-processing system that uses a computer and a data-acquisition card makes data recording and calibration easy and fast.

\section{Discussion}

In conclusion, an effective demodulation technique has been developed for dynamic compensation of manufacturing errors and environmentalperturbation-induced drifts in interferometric sensors. The total effect of all low-frequency drifts, including dc signals, in the sensor system can be measured by the position of the diffraction grating. The dc signals may be recoverable if other effects, such as temperature, are known or can be measured. If such is the case, the dc dynamic range of the sensor system can be greatly increased and is limited only by the source bandwidth. Obviously, the GA-OPT does not impose any limit on the ac signal, and the frequency response of the sensor system depends only on the sensor probe itself and the electronic bandwidth.

In addition to operation-point control, GA-OPT brings another benefit to the demodulation system, namely, improved sensitivity. The improvement comes from the reduction of the bandwidth of the interrogation lights and thereby from the increase of the interference fringe contrast. ${ }^{11}$ Although the fringe contrast is still smaller than that of a sensor system with a laser source, this demodulation approach retains all the advantages of a white-light system over a laser system.

The potential for absolute OPD measurements is another important feature of GA-OPT. By scanning the wavelength, one may calculate the absolute OPD between the sensing channel and the reference channel from the scanning output, as was done by Egorov et al. with a spectrometer and a CCD array. ${ }^{16}$ Compared with the method described in Ref. 16, the advantage of the GA-OPT is greatly reduced cost, especially for sensors operating in the near-infrared region.

This research was supported by the U.S. Department of Energy under contract DE-FC-01GO11050. The authors acknowledge useful discussions with Yibing Zhang of the Center for Photonics Technology, Virginia Polytechnic Institute and State University.

\section{References}

1. T. Yoshino, K. Kurosawa, K. Itoh, and T. Ose, "Fiber-optic Fabry-Perot interferometer and its sensor application," IEEE Trans. Microwave Theory Tech. MTT-30, 1612-1620 (1982).

2. C. E. Lee and H. F. Taylor, "Fiber-optic Fabry-Perot temperature sensor using a low-coherence light source," J. Lightwave Technol. 9, 129-134 (1991).

3. R. A. Wolthuis, G. L. Mitchell, E. Saaski, J. C. Hartl, and M. A. Afromowitz, "Development of medical pressure and temperature sensors employing optical spectrum modulation," IEEE Trans. Biomed. Eng. 38, 974-981 (1991).

4. A. Wang, H. Xiao, J. Wang, Z. Wang, W. Zhao, and R. G. May, "Self-calibrated interferometric-intensity-based optical fiber sensors," J. Lightwave Technol. 19, 1495-1501 (2001).

5. N. Furstenau, M. Schmidt, H. Horack, W. Goetze, and W. Schmidt, "Extrinsic Fabry-Perot interferometer vibration and acoustic sensor systems for airport ground traffic monitoring," IEE Proc. Optoelectron. 144, 134-144 (1997).

6. W. Pulliam, P. Russler, R. Mlcak, K. Murphy, and C. Kozikowski, "Micromachined, $\mathrm{SiC}$ fiber optic pressure sensors for high temperature aerospace applications," in Industrial Sensing Systems, A. Wang and E. Udd, eds., Proc. SPIE 4202, 21-30 (2000).

7. Y. Kim and D. P. Neikirk, "Micromachined Fabry-Perot cavity pressure transducer," IEEE Photon. Technol. Lett. 7, 14711473 (1995).

8. J. J. Alcoz, C. E. Lee, and H. F. Taylor, "Embedded fiber-optic Fabry-Perot ultrasound sensor," IEEE Trans. Ultrason. Ferroelectr. Freq. Control 37, 302-306 (1990).

9. J. F. Dorighi, S. Krishnaswamy, and J. Achenbach, "Stabilization of an embedded fiber optic Fabry-Perot sensor for ultrasound detection," IEEE Trans. Ultrason. Ferroelectr. Freq. Control 42, 820-824 (1995).

10. K. A. Murphy, M. F. Gunther, A. Wang, and R. O. Claus, "Detection of acoustic emission location using optical fiber sensors," in Smart Structures and Materials 1994: Smart Sensing, Processing, and Instrumentation, J. S. Sirkis, ed., Proc. SPIE 2191, 282-290 (1994).

11. B. Yu, D. W. Kim, J. Deng, H. Xiao, and A. Wang, "Fiber Fabry-Perot sensors for partial discharge detection in power transformers," Appl. Opt. 42, 3241-3250 (2003).

12. K. Murphy, M. F. Gunther, A. M. Vengsakar, and R. O. Claus, "Quadrature phase-shifted, extrinsic Fabry-Perot optical fiber sensors," Opt. Lett. 16, 273-275 (1991).

13. C. Belleville and G. Duplain, "White-light interferometric multimode fiber-optic strain sensor," Opt. Lett. 18, 78-80 (1993).

14. A. S. Gerges, F. Farahi, T. P. Newson, J. D. C. Jones, and D. A. Jackson, "Fibre-optic interferometric sensor utilising low coherence length sources: resolution enhencement," Electron. Lett. 24, 472-474 (1988).

15. M. Schmidt and N. Fürstenau, "Fiber-optic extrinsic FabryPerot interferometer sensors with three-wavelength digital phase demodulation," Opt. Lett. 24, 599-601 (1999).

16. S. A. Egorov, A. N. Mamaev, I. G. Likhachiev, Y. A. Ershov, A. S. Voloshin, and E. Nir, "Advanced signal processing method for interferometric fiber-optic sensors with straightforward spectral detection," in Sensors and Controls for Advanced Manufacturing, B. O. Nnaji and A. Wang, eds., Proc. SPIE 3201, 44-48 (1998).

17. B. Qi, G. Pickrell, J. C. Xu, P. Zhang, Y. H. Duan, W. Peng, Z. Y. Huang, W. Huo, H. Xiao, R. G. May, and A. Wang, "Novel date processing techniques for dispersive white light interferometer," Optical Engineering (to be published). 


\title{
Grating-assisted Operating-Point Tuning for Fiber-Optic Fabry-Perot Interferometric Sensors
}

\author{
Bing $\mathrm{Yu}^{1}$, Gary Pickrell, and Anbo Wang, Senior Member, IEEE \\ Center for Photonics Technology, Virginia Polytechnic Institute and State University \\ 460 Turner St., Blacksburg, VA 24061-0287
}

\begin{abstract}
A grating-assisted operating-point tuning system was designed to dynamically stabilize the operating-point of a fiber Fabry-Perot interferometric sensor to compensate for manufacturing errors and environment perturbation induced drifts. The system uses a diffraction grating and a feedback control, functioning as a tunable bandpass optical filter, and can be used as an effective demodulation subsystem in sensor systems based on optical interferometers using a broadband light source. This demodulation method features unlimited signal detection bandwidth, high tuning speed, large tunable range, increased interference fringe contrast, and a potential of absolute optical path difference measurement. Sensitivity improvements were demonstrated with a fiber Fabry-Perot acoustic wave sensor system.
\end{abstract}

Keywords: interferometric sensors, operating-point control, diffraction grating, feedback control.

\section{INTRODUCTION}

Fiber-optic interferometers, especially fiber Fabry-Perot interferometers (FFPI), have found increased applications in the last decade for the detection and measurement of a large variety of physical parameters, such as temperature, vibration, pressure, acoustic wave, and strain, etc. Many of the interferometric sensors operate over the linear region of an interference fringe or transfer function ${ }^{[1]-[6]}$. Sensors operating in the linear region have advantages over sensors using fringe counting ${ }^{[5]-[7]}$, such as linear transfer function, no fringe direction ambiguity, simple signal processing and highest sensitivity at the quadrature point (Q-point). They are, therefore, suitable for small optical path difference (OPD) change detection and wideband applications. However, confining the operation to the linear region places difficult manufacturing constraints on the sensor head by requiring the initial cavity length to be positioned at the Q-point or at one end of the linear region of the transfer function ${ }^{[6]}$. They also suffer from signal fading caused by environmental perturbations, such as temperature changes, which may drive the sensors out of the linear region. The drifts are even more critical for Fabry-Perot sensors with medium or high finesse F-P cavities that are necessary for higher measurement sensitivity ${ }^{[1][6]}$ because of their steeper fringe slopes. Therefore, poor control of the operating-point has been a major factor that limits the practical industrial application of interferometric optical sensors.

Currently, several techniques have been developed by different researchers to stabilize the Q-point, for example, by intentionally applying an acoustic bias ${ }^{[1]}$, tuning the static OPD using stages ${ }^{[8]}$, using a tunable light source ${ }^{[4]}[5]$, quadrature phase-shifted demodulation or dual wavelength interrogation ${ }^{[7][9][10][11]}$, or direct spectrum detection ${ }^{[9][12]}$ ${ }^{[13]}$. In the acoustic bias method, the low-frequency component output of an FFPI acoustic wave sensor was compared with a constant voltage corresponding to the operating set-point, and the difference was used to adjust the acoustic bias through a voice coil. This method does compensate for environmental drifts, but adjusting the operating-point with a voice coil limits it only to acoustic detection and may induce extra noise from the coil. The tuning of the static OPD is realized in a Mach-Zehnder interferometer by using a fiber stretcher. The quadrature point nearest to maximum visibility can be locked with a feedback control servo system and a temperature drift from $25^{\circ} \mathrm{C}$ up to $55^{\circ} \mathrm{C}$ has been successfully compensated. However, tuning the OPD may cause excess power fluctuation in the fiber, and it is not a suitable approach for sensors with F-P interferometers. Adjusting the operating-point by changing the bias current of a tunable laser diode is another effective approach that has been used by many researchers. The disadvantages of using a tunable laser are optical power fluctuation, high sensitivity to backreflections, laser mode hopping, and relatively high cost. The quadrature phase-shifted demodulation or dual wavelength interrogation was originally developed by K. Murphy, et.

${ }^{1}$ biyu@ vt.edu; phone 1540 231-7070; fax: 1540 231-2158 
al. ${ }^{[10]}$ to solve the nonlinear transfer function and directional ambiguity problems in extrinsic Fabry-Perot interferometric (EFPI) sensors, but it may also be used for the demodulation of sensors operating in the linear regions. However, it is possible that both channels are operating away from their optimal Q-points at the same time, even if a $90^{\circ}$ phase shift can be maintained during the measurement, which is difficult to control.

Strictly speaking, the spectrum detection method should not be categorized as an operating-point stabilizing method, though linear response can be achieved ${ }^{[12]}$. Direct spectrum detection uses a diffraction grating or a Fizeau interferometer, and the modulated broadband spectrum is detected by a CCD array and analyzed by a signal-processing unit. This approach is also called whitelight interferometry and actually does not need to control the operating-point of an interferometric sensor. It provides an absolute and accurate value of the OPD in a sensing interferometer, and is insensitive to the power and spectral fluctuations of the light source. Its major disadvantage is that it is not suitable for real time detection of a broadband signal, such as acoustic waves and high frequency pressure fluctuations because a large amount of time is required to process the huge data from a CCD array. Another disadvantage of the spectrum detection is the high cost, especially for sensors operating in the near infrared (NIR) region, where an expensive detector array must be used.

In this article, we present an alternate operating-point stabilization approach using a diffraction grating with a feedback control, referred to as grating-assisted operating point tuning (GA-OPT). This approach features unlimited bandwidth, high tuning speed, large tunable range, increased interference fringe contrast, and the potential for absolute optical path difference measurement. Experimental results of an FFPI acoustic wave sensor system used to prove the feasibility of the design is also presented.

\section{OPERATING-POINTS OF AN INTERFEROMETRIC SENSOR}

Interferometric-intensity-based detection is a widely used demodulation technique in optical interferometric sensors, such as FPI, Mach-Zehnder and Sagnac sensors. When monochromatic light of wavelength $\lambda$ is used to interrogate the sensors, the optical intensity of the two-beam interference between the sensing beam and the reference beam can be expressed as

$$
I=I_{1}+I_{2}+2 \sqrt{I_{1} I_{2}} \cos [2 \pi(n l) / \lambda]
$$

where $I_{l}$ and $I_{2}$ represent the optical intensities of the sensing beam and the reference beam, respectively, $(n l)$ is the OPD between the two beams, where $\mathrm{n}$ is the refractive index of the medium and $l$ is the physical path difference. The optical intensity arriving at the photodetector is a simple cosine function, named interference fringes, of $(n l)$, which is a function of both the measurant and background perturbations. Obviously, sensors have zero sensitivity at the peaks or the valleys of the fringes, and maximal sensitivity and linear response at the Q-points, where the phase difference

$$
\phi=2 \pi(n l) / \lambda=\pi / 2+m \pi
$$

where the interference order $m=0,1,2, \ldots$. Sensors operating around the Q-points will have the highest sensitivity and the lowest signal distortion. However, any sensor fabrication tolerance, temperature-induced drift, and other environment perturbations can easily drive a sensor away from its Q-points. Noticing that the interference fringes and therefore Qpoints are wavelength dependent for all interferometers, the operating-points may be dynamically controllable by tuning the wavelength of the interrogation signal to compensate for the phase drifts, that is

$$
\Delta \phi=\Delta \phi_{(n l)}+\Delta \phi_{\lambda}=\frac{2 \pi}{\lambda} \Delta(n l)-\frac{2 \pi(n l)}{\lambda^{2}} \Delta \lambda=0
$$

or

$$
\Delta \lambda=\frac{\lambda}{(n l)} \Delta(n l)
$$


where $\Delta \phi_{(n l)}$ and $\Delta \phi_{\lambda}$ are the phase changes caused by environment disturbance $\Delta(n l)$ and wavelength tuning $\Delta \lambda$, respectively. Usually $(n l)$ is at least one order larger than $\lambda$, which means a large drift can be compensated by a relatively small change of the wavelength. The wavelength tuning can be realized by using a tunable laser, though this may cause some problems, such as optical power fluctuation, sensitivity to back-reflections and high cost.

For a broadband light source, the wavelength dependence of the interference fringe is much more complex than for a monochromatic source. The interference fringes are determined by not only the central wavelength, but also the spectrum width of the interrogation lights as well as the physical path difference. The total optical intensity arriving at the photodetector has to be computed by integration over the entire spectrum of the light source. Fig. 1 is a theoretical calculation of the wavelength dependence of the interference fringes at different cavity lengths $\left(L_{0}\right)$ of a low-finesse FFPI sensor ${ }^{[6]}$, where a $1300 \mathrm{~nm}$ light source with a $40 \mathrm{~nm}$ (FWHM) spectral width was used. The output is normalized to the source intensity distribution and the bandwidth of the interrogation signal is limited to $2 \Delta \lambda=10 \mathrm{~nm}$. As an example, if the OPD, for any reason, changes from $L_{0}=15.05 \mu \mathrm{m}$ to $15.15 \mu \mathrm{m}$, the operating-point can be tracked by tuning the central wavelength from $1.296 \mu \mathrm{m}$ to $1.304 \mu \mathrm{m}$. The central wavelength of the interrogation light can be tuned by using a GAOPT that is discussed in the next section.

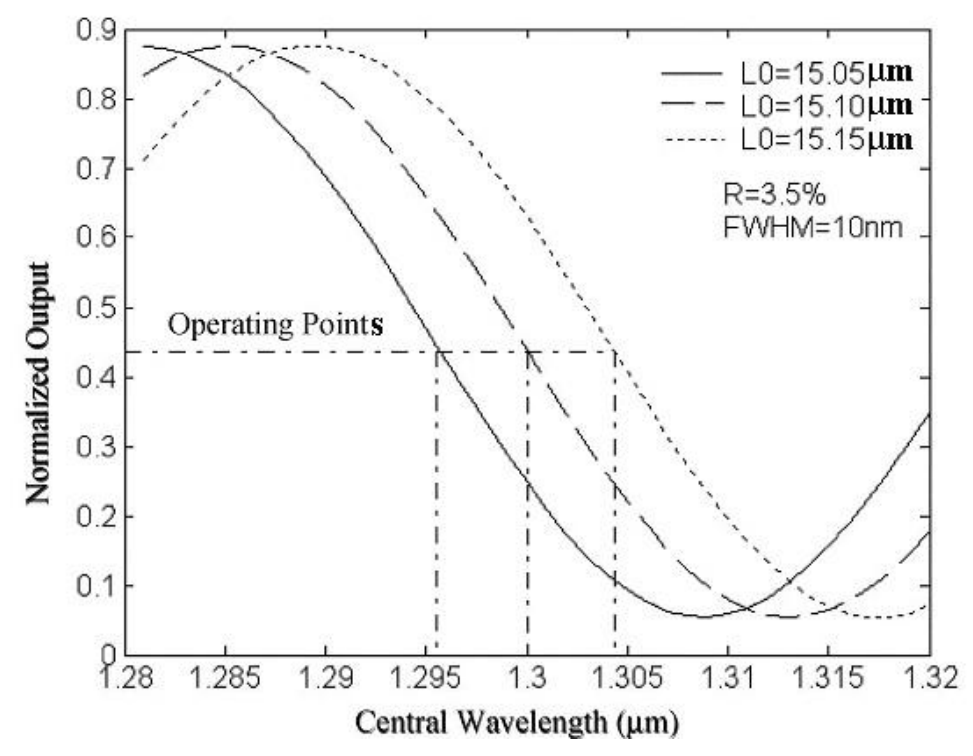

Fig. 1 Theoretical calculation of the wavelength dependence of operating-points of an FFPI sensor, where $2 \Delta \lambda=10 \mathrm{~nm}, \mathrm{R}=3.5 \%$.

\section{GRATING-ASSISTED OPERATING-POINT TUNING}

A schematic illustration of a whitelight interferometric sensor system based on a GA-OPT is shown in Fig.2. The operating-point tuning is realized with two collimators, a diffraction grating, a short piece of multimode fiber (MMF), a photodetector, a preamplifier, a computer with a data acquisition card, and a motorized rotary stage. The light from a broadband source is launched into a single-mode fiber (SMF), propagates through a 3-dB coupler, and arrives at an interferometric sensor, where it is modulated by the perturbation measurand. The phase-modulated light propagates back through the same 3-dB coupler and reaches collimator I of the GA-OPT, which is shown in detail in the inset. The collimated light beam illuminates a diffraction grating, which can be rotated by a motorized rotary stage with high resolution $(\sim 0.2 \mathrm{mrad})$. The $-1^{\text {st }}$ diffractions collected by collimator II are focused into a short 200/230 $\mu \mathrm{m}$ multimode fiber (MMF), and only part of the total spectrum $(\lambda \pm \Delta \lambda)$ can be collected by the MMF. 


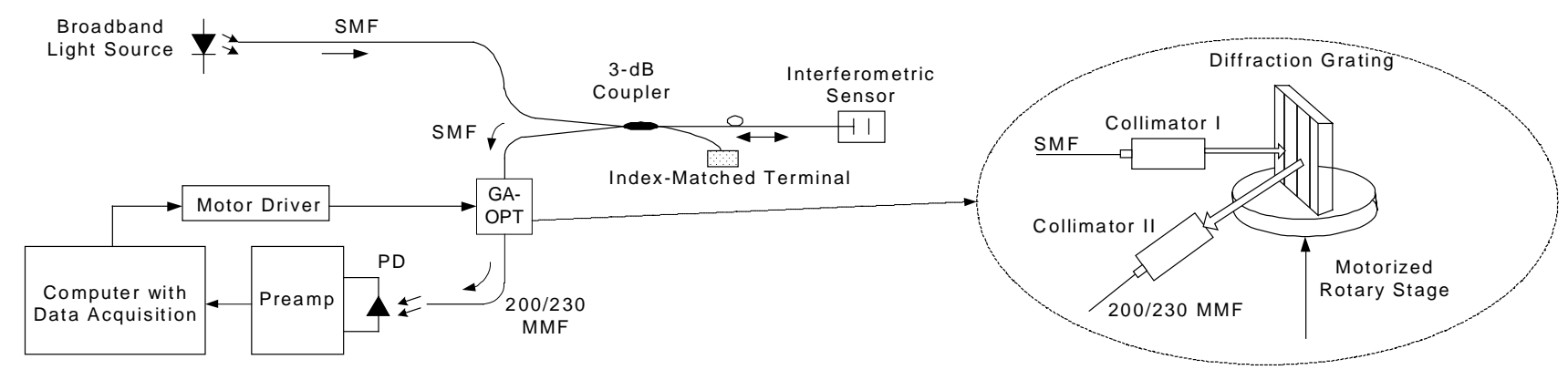

Fig. 2 Principle diagram of a whitelight interferometric sensor system based on GA-OPT.

Instead of a SMF or a detector, a short MMF is used to increase the reception area and to facilitate the spectrum monitoring with an optical spectrum analyzer (OSA). The MMF can be removed and the detector can be placed on the focal point of the receiving collimator. However, this will provide less flexibility. A transimpedance preamplifier converts the photocurrent into voltage, and a subsequent splitter separates the signal into two components that are further amplified and filtered into DC and AC components. A computer with a data acquisition card is used to record and calibrate the DC and AC signals. The calibration reference, a representation of the original optical intensity distribution, is an output-position curve obtained by scanning the whole wavelength range with a cleaved fiber, instead of a sensor, connected. The calibrated AC signals directly represent the absolute value of the AC information of the measurand, and the frequency response is limited only by the sensor-head and the bandwidth of the electronics. On the other hand, the calibrated DC signals represent the current operating-point on the transfer function. Ideally, this DC voltage (V) should to be equal to the set-point $\left(\mathrm{V}_{0}\right)$, which is most likely the Q-point. However, any manufacturing tolerance and environment change may cause an undesired change of the OPD of the sensor, thereby causing drift of the operatingpoint and the DC voltage $\mathrm{V}$. The difference $\Delta V$ between the DC output and the set-point is the control signal that can be used to adjust the position of the grating through a motorized rotary stage, and therefore the central wavelength of the interrogation signal. By this feedback control, the optimal operating-point can be dynamically maintained, and the DC signal may also be recovered from the position of the grating if environmental effects can be determined. Obviously, the tuning range is limited only by the source spectrum and the DC accuracy is limited by the resolution and repeatability of the rotary stage.

According to the grating equation, the relationship between the incident beam and the $-1^{\text {st }}$ diffracted beam can be expressed as

$$
\sin (A)+\sin (B)=\lambda / d
$$

where $A$ - the incident angle of the light beam from the input collimator respect to the normal of the grating surface;

$B$ - the angle of $-1^{\text {st }}$ diffraction with respect to the normal of the grating surface;

$\lambda$ - the wavelength of the light in air;

$d$ - the groove spacing of the grating.

Assuming a lens of focal length $f$ is used in the receiving collimator, we can calculate $B$ by

$$
\cos (B)=\frac{f(\Delta \lambda / D)}{d}
$$

where $\Delta \lambda$ - the spectrum resolution;

$D$ - the core diameter of the receiving MMF or the active area of the detector.

Fig. 3 gives the calculated angular tuning range for a wavelength tuning from 1280 to $1320 \mathrm{~nm}$ and the resulting receiving bandwidth change for $\lambda=1300 \mathrm{~nm}, d=1 / 750 \mathrm{~mm}, f=25.0 \mathrm{~mm}, D=0.2 \mathrm{~mm}$ for a $200 / 230 \mu \mathrm{m} \mathrm{MMF}$, and $B-A=55^{\circ}$. An angular change of $1.2^{\circ}$ is enough to scan a wavelength range of $40 \mathrm{~nm}$, which produces a bandwidth error of about 
$3.5 \%$. This bandwidth error may induce an optical intensity distribution error out of the original light source distribution, but can easily be compensated by scanning the whole spectral range and storing the new intensity distribution during the reset. Obviously, finer tuning or smaller $\Delta \lambda$ can be realized, with the penalty of higher insertion loss, by using smaller diameter fiber $D$ or longer focal length $f$ if the groove spacing, incident angle $A$ and diffraction angle $B$ have been determined.

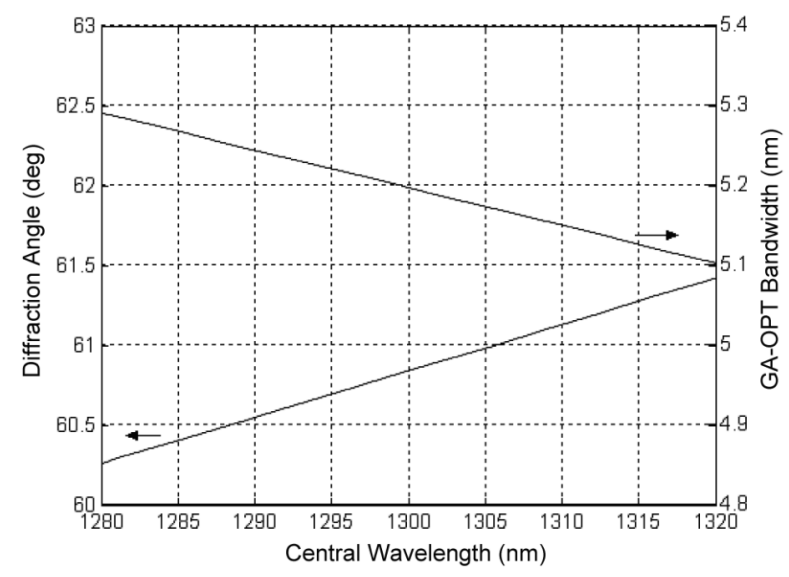

Fig. 3 Wavelength dependence of the diffraction angle and the GA-OPT bandwidth $(\Delta \lambda)$.

\section{EXPERIMENTS}

In order to verify the feasibility of the operating-point tuning, a GA-OPT was designed and fabricated, as shown in Fig.4. A holographic diffraction grating of 750 grooves per millimeter was glued onto a motorized rotary stage. The motor has a step resolution of $0.2-\mathrm{mrad}$ and a rotation speed of $2 \mathrm{RPM}$, which means a central wavelength resolution of $0.38 \mathrm{~nm}$ and a tuning speed of $400 \mathrm{~nm} / \mathrm{s}$ can be achieved. Collimator I is the input collimator, and collimator II is the output collimator which has a focal length of $25 \mathrm{~mm}$. The input single-mode fiber is connected to an interferometric optical sensor, while the output $200 / 230 \mu \mathrm{m}$ multimode fiber is connected to an optical receiver. The control interface board interconnects the motor and the motor controller.

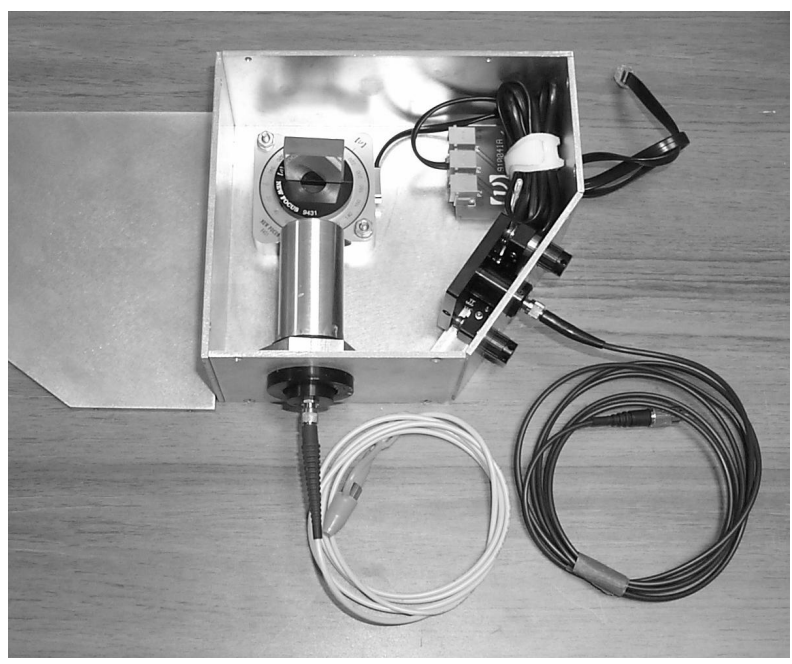

Fig. 4 Top view of the GA-OPT.

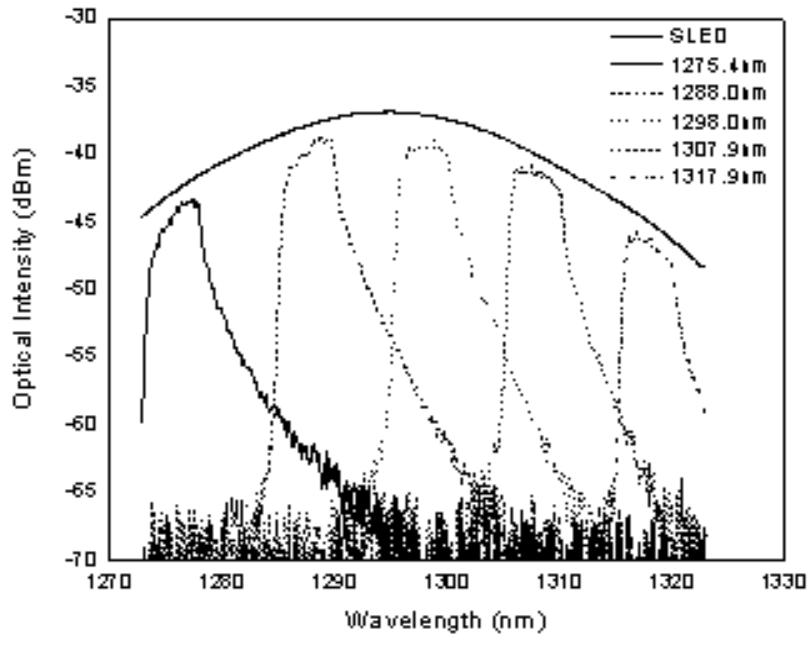

Fig. 5 Spectrum received by the detector at different grating positions and the original spectrum of the SLED. 
Fig. 5 shows the output spectrums received by the detector at different grating positions or central wavelengths $(1276.4 \mathrm{~nm}, 1288.0 \mathrm{~nm}, 1298.0 \mathrm{~nm}, 1307.9 \mathrm{~nm}$, and $1317.9 \mathrm{~nm})$ and the original spectrum of the SLED, where the sensor was replaced with a cleaved single-mode fiber. A $1298 \mathrm{~nm}$ SLED with a spectrum width of $35 \mathrm{~nm}$ was used as the broadband source. By scanning the grating by an angle of $1.2^{\circ}$, a central wavelength change of $40 \mathrm{~nm}$ was achieved. This agrees well with the theoretical calculation given in Fig.3. The resulted spectrum bandwidth (FWHM) is between 4.30 and $4.65 \mathrm{~nm}$, an average of $0.7 \mathrm{~nm}$ less than the theoretical results. This is believed to be caused by the misalignments between the $25 \mathrm{~mm}$ lens in Collimator II and the 200/230 $\mu \mathrm{m}$ MMF as well as the relatively small aperture of Collimator II. The total insertion loss is about $11 \mathrm{~dB}$. As mentioned in the previous section, a tradeoff must be made between large bandwidth for high optical power and small bandwidth for high fringe visibility and high resolution of operating-point tuning.

A diaphragm-based FFPI acoustic wave sensor ${ }^{[6]}$ was chosen to test the performance of the developed GA-OPT in practical applications. This sensor was designed for partial discharge detection in power transformers. Since this sensor was fabricated using thermal fusion bonding which might cause large cavity length error and may suffer from high static pressure in a transformer tank full of mineral oil, operation point control has been a major challenge. Fig. 6 gives the scan outputs of the FFPI sensor under normal atmospheric conditions and under $50 \mathrm{~cm}$ water normalized to the source spectrum. A Q-point drift of about $3 \mathrm{~nm}$ resulted due to the $50 \mathrm{~cm}$ static water-pressure. Obviously, there are more than one Q-point on the fringes, but only those two Q-points with the highest intensity have the best signal-to-noise ratio, and are optimal operating-points.

A scan output of this sensor in an acoustic wave test setup is given in Fig.7 with the acoustic wave outputs at the marked points A - C shown in Fig.8 with that from a sensor system without a GA-OPT. The original system has very low signal-to-noise ratio (SNR) because of the short coherence length of the SLED source compared to the sensor's cavity length and/or the unknown operating-point. When a GA-OPT is used, the sensor has different performances at different operating-points. At points A and C, the Q-points of the interference fringes, the sensor has the best SNR due to the highest sensitivity of the Q-points and the increased fringe contrast. A SNR improvement of about $15 \mathrm{~dB}$ can easily be achieved over the original sensor even if it was operating at its Q-point. Although D and E are Q-points too, they are generally not suitable for the operating-points because of the lower fringe slope at $\mathrm{D}$ or low absolute optical intensity at E caused by the non-flat SLED spectrum. The sensor has the lowest SNR at B because of the zero sensitivity at the fringe peaks (or valleys). Therefore, points A and C are the ideal operating-points of this FFPI sensor. We also observed that the acoustic wave outputs at these two points have different polarities and different dynamic ranges that may be sensitive for some measurements. When the operating-point is determined, the last task is to maintain the sensor in the operational range at the set-point. This can be easily realized by feedback control system shown in the system diagram of Fig.2. A signal processing system using a computer and a data acquisition card makes the data acquisition and calibration easier and faster.

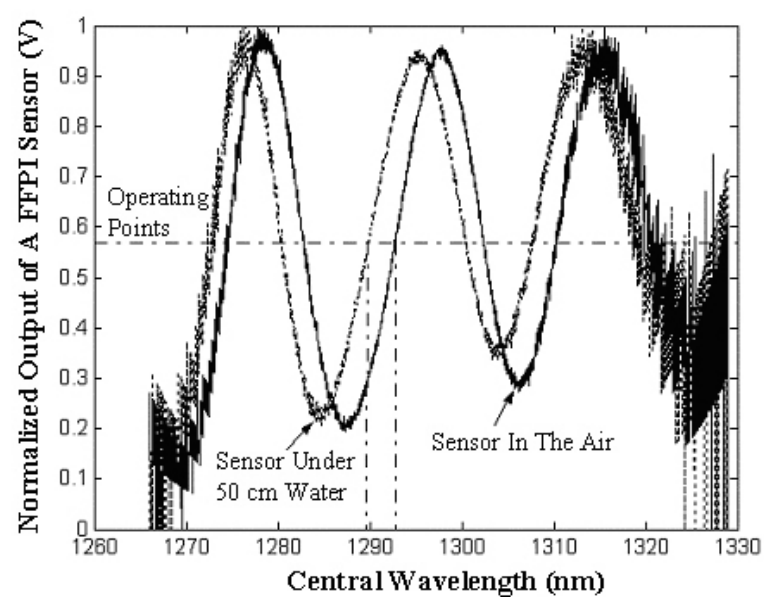

Fig.6 Normalized sensor output at different static pressures.

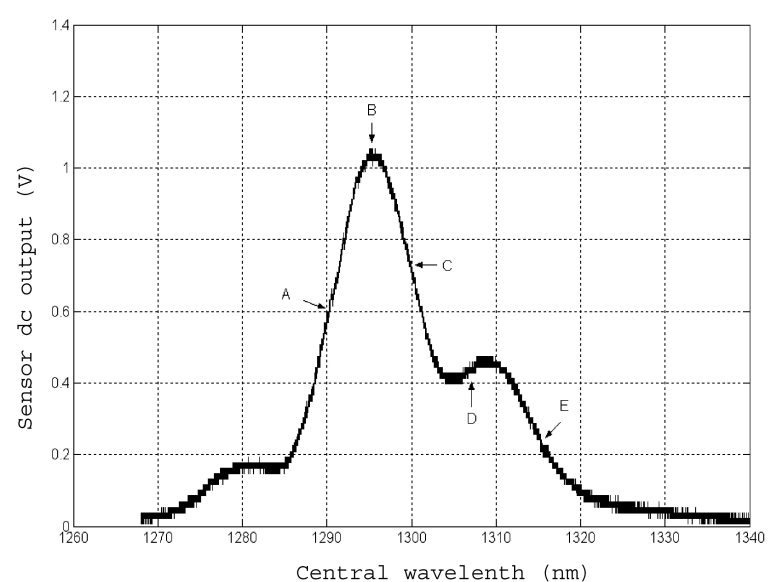

Fig.7 Scanning for operating-point. 


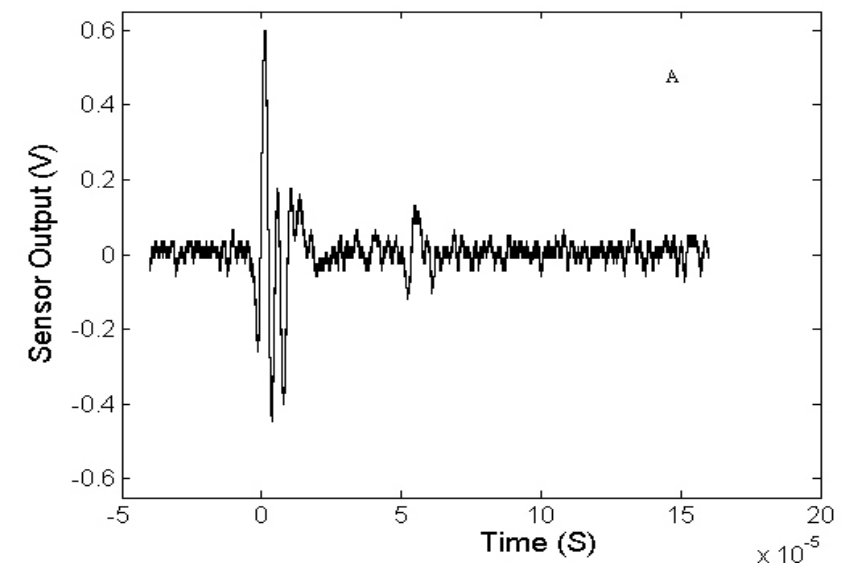

(a)

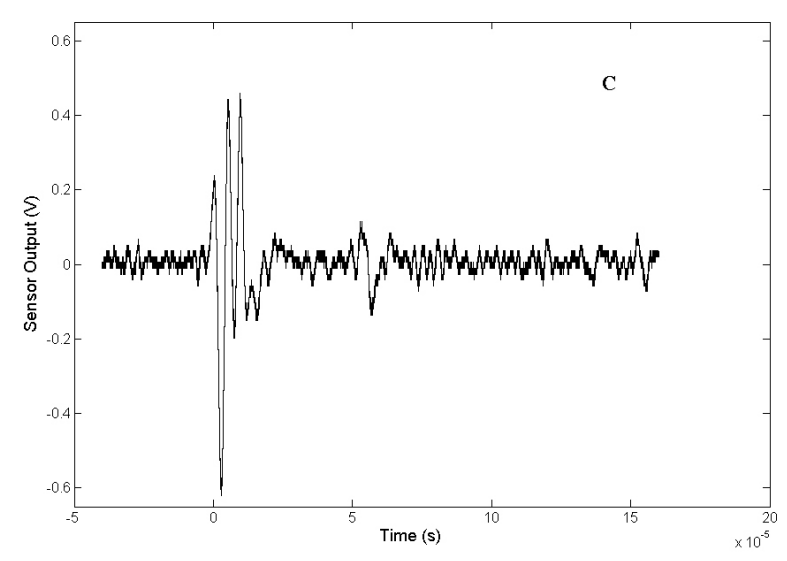

(c)

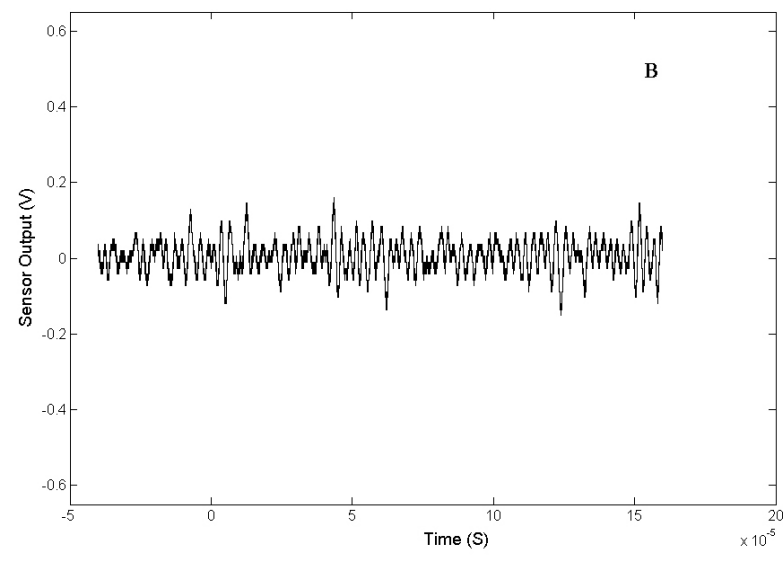

(b)

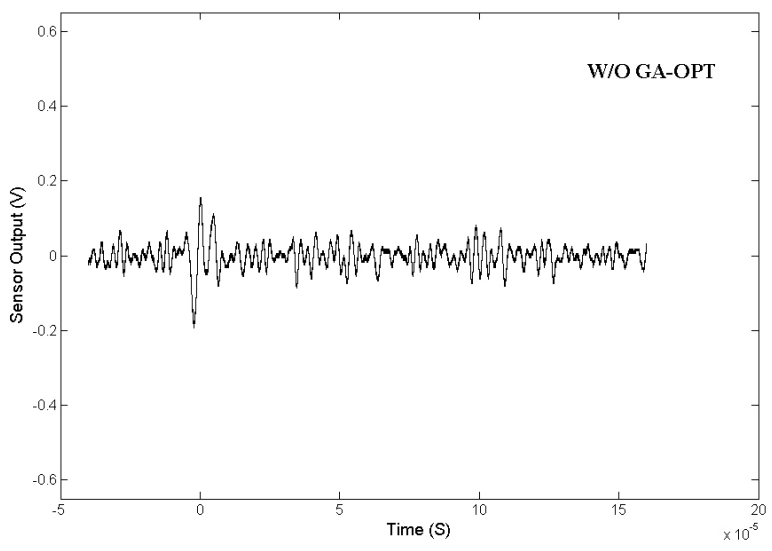

(d)

Fig.8 Acoustic wave detection using a diaphragm-based FFPI sensor and a GA-OPT at the operating-points (a) point A, (b) point $\mathrm{B}$, (c) point $\mathrm{C}$ and (d) without the GA-OPT.

\section{CONCLUSIONS}

In conclusion, an effective operating-point stabilization approach has been developed for dynamical compensation of manufacturing errors and environment perturbation induced drifts in interferometric sensors. The total effect of all low frequency drifts, including dc signals, in the sensor system can be measured by the position of the diffraction grating. The dc signals may be recoverable if other effects, such as temperature, are known or can be measured. If this is the case, the dc dynamic range of the sensor system can be greatly increased and is limited only by the source bandwidth. Obviously, the GA-OPT does not apply any limit on the ac signal, and the frequency response of the sensor system depends only on the sensor probe itself and the electronic bandwidth.

The achieved $40 \mathrm{~nm}$ tuning range which is limited by the available source spectrum width, $400 \mathrm{~nm} / \mathrm{s}$ tuning speed and a step resolution of $0.4 \mathrm{~nm}$ are sufficient for most practical sensor measurements.

In addition to the operating-point control, the GA-OPT brings another benefit into the demodulation system, which is improved sensitivity. The improvement comes from the reduction of the bandwidth of the interrogation signal, and 
thereby the increase of the interference fringe contrast ${ }^{[6]}$. Although the fringe contrast is still smaller than that of a sensor system with a laser source, this demodulation approach keeps all the advantages of a whitelight system in comparison to a laser system.

Potential for absolute OPD measurements is another important feature of the GA-OPT. By scanning the wavelength, the absolute OPD between the sensing channel and the reference channel may be calculated from the scanning output.

\section{ACKNOWLEDGEMENT}

This research was supported by the U.S. Department of Energy (DOE) under contract DE-FC-01GO11050.

\section{REFERENCES}

1. T. Yoshino, K. Kurosawa, K. Itoh, and T. Ose, "Fiber-Optic Fabry-Perot Interferometer and Its Sensor Application," IEEE Trans. on Microwave Theory and Techniques, vol. MTT-30, no. 10, pp. 1612-1620, Oct. 1982.

2. R. A. Wolthuis, G. L. Mitchell, E. Saaski, J. C. Hartl, and M. A. Afromowitz, "Development of Medical Pressure and Temperature Sensors Employing Optical Spectrum Modulation," IEEE Trans. on Biomedical Engineering, vol. 38, No. 10, pp.974-981, Oct. 1991.

3. Anbo Wang, Hai Xiao, J. Wang, Zhiyong Wang, W. Zhao, and R. G. May, "Self-calibrated interferometricintensity-based optical fiber sensors," J. Lightwave Tech., vol. 19, no. 10, 1495-1501, 2001.

4. J. J. Alcoz, C. E. Lee, and H. F. Taylor, "Embedded fiber-optic Fabry-Perot ultrasound sensor," IEEE Trans. on Ultrasonics, Ferroelectrics, and Frequency Control, vol. 37 no.4, 302-306, July 1990.

5. J. F. Dorighi, S. Krishnaswamy, and J. Achenbach, "Stabilization of an embedded fiber optic Fabry-Perot sensor for ultrasound detection," IEEE Trans. on Ultrasonics, Ferroelectrics, and Frequency Control, vol. 42 no. 5, 820-824, Sept. 1995.

6. Bing Yu, Dae Woong Kim, Jiangdong, Deng, Hai Xiao, and Anbo Wang, "Fiber Fabry-Perot Sensors for Partial Discharge Detection in Power Transformers", Applied Optics, Vol.42, No. 16, pp. 3241-50, 2003.

7. N.Furstenau, M.Schmidt, H.Horack, W. Goetze, and W. Schmidt, "Extrinsic Fabry-Perot interferometer vibration and acoustic sensor systems for airport ground traffic monitoring”, IEE Proc. Optoelectronics, Vol. 144, No.3, pp. 134-144 (1997).

8. A. S. Gerges, F. Farahi, T. P. Newson, J. D. C. Jones, D. A. Jackson, "Fibre-optic interferometric sensor utilising low coherence length sources: Resolution enhancement," Electron. Lett. 24, no. 8, 472-474, 1988.

9. W. Pulliam, P. Russler, R. Mlcak, K. Murphy, C. Kozikowski, "Micromachined, SiC fiber optic pressure sensors for high temperature aerospace applications," in SPIE Proc.: Industrial Sensing Systems, edited by Anbo Wang and Eric Udd, SPIE vol. 4202, pp. 21-30, 2000.

10. K. Murphy, M. F. Gunther, A. M. Vengsakar, and R. O. Claus, "Quadrature phase-shifted, extrinsic Fabry-Perot optical fiber sensors," Optics Letters, vol. 16, No. 4, pp. 273-275, Feb. 1991.

11. M. Schmidt and N. Fürstenau, "Fiber-optic extrinsic Fabry-Perot interferometer sensors with three-wavelength digital phase demodulation," Opt. Lett., vol. 24, No. 9, pp. 599-601, May 1999.

12. C. Belleville, G. Duplain, "White-light interferometric multimode fiber-optic strain sensor," Optics Letters, 18(1), pp. 78-80, 1993.

13. S. A. Egorov, A. N. Mamaev, I. G. Likhachiev, Y. A. Ershov, A. S. Voloshin, and E. Nir, "Advanced signal processing method for interferometric fiber-optic sensors with straightforward spectral detection," in Proc. Sensors and Controls for Advanced Manufacturing, edited by B. O. Nnaji and A. Wang, SPIE Proc., Vol. 3201, pp. 44-48, 1998. (Pittsburgh, PA, 14-15 Oct. 1997). 


\title{
Deep wet etching on fused silica material for fiber optic sensors
}

\author{
Xiaopei Chen*, Bing Yu, Yizheng Zhu, Anbo Wang \\ Center for Photonics Technology, Virginia Polytechnic Institute and State University \\ 460 Turner Street, Suite 303, Blacksburg, VA 24061
}

\begin{abstract}
In this paper, deep microstructures on fused silica material, which are useful for fabrication of the fiber optic sensors, were obtained by using a wet chemical etching process. The etching solutions and the masking materials used for developing deep structure are described in this paper. The etch rate of a fused silica diaphragm in room temperature ranged from $46 \mathrm{~nm}$ per minute to $83 \mathrm{~nm}$ per minute with different concentrations of Buffered Hydrogen Fluoride (BHF). The etch depth of one step etching was $25 \mu \mathrm{m}$ with the surface roughness less than $20 \mathrm{~nm}$ (peak-to-peak value). The optical reflectance from the deep etched surface was $4 \%$, which is the same as a well-cleaved fiber end face. This result made the visibility of interference fringes from the single mode fiber optic sensors to be as high as $96 \%$. Furthermore, two-step structures on the fused silica diaphragms with the total depth greater than $35 \mu \mathrm{m}$ are demonstrated. To the best knowledge of the authors, this is the deepest structure produced by wet etching process on fused silica material. Fiber optic pressure sensors based on deep etched diaphragms were fabricated and tested. Fabrication of microstructures on the fiber end faces by using this process is therefore possible.
\end{abstract}

Keywords: Deep wet etching, fused silica, optical fibers, fiber optic sensors

\section{INTRODUCTION}

Fiber optic sensors offer many advantages over conventional electrical or electromechanical sensors. First, optical fiber is a dielectric, so it is not subject to electromagnetic interference that might be present in the sensing environment. Secondly, fiber-optic sensors can function under harsh environment, such as high temperature, toxic or corrosive atmospheres where metals or other materials can be corroded. In addition, semiconductor based photo-detectors and laser diode sources are usually small and light weight, so fiber-optic sensors are useful as sensing devices for a wide range of physical and chemical phenomena including temperature, pressure, acoustic field, position, rotation, electrical current, liquid level, biochemical composition, and chemical concentration. Therefore, fiber optic pressure sensors, which can survive at high temperature, provide a promising field with clear advantages over conventional sensors.

Fused silica was chosen for high temperature pressure sensitive material because of its inertness and stability at high temperatures, and excellent optical transparency. In addition, fused silica offers an extremely low coefficient of thermal expansion and high Young's Modulus, which make it immune to stress effects from thermal shock or thermal gradients. It is widely available, hard and easy to fabricate into specialized components at modest cost. Thus it is a nearly ideal sensor material.

Many methods have been used for micro-fabrication of fused silica. Reactive Ion Etching (RIE) ${ }^{[1]}$ is the most conventional method. Laser ablation ${ }^{[2]}$, short-wavelength laser processing ${ }^{[3]}$, and ultra-short-pulse laser micromachining ${ }^{[4]}$ also have been intensely investigated. Laser Induced Back Side Etch (LIBWE) of fused silica is another method reported by researchers in Japan ${ }^{[5]}$ recently. However, deep wet chemical etching of fused silica material is a less investigated process though it is usually inexpensive and easy process.

In this paper, a process based on deep wet chemical etching of fused silica diaphragms for fabricating fiber optic pressure sensors is described. The sensor structure requires a deep pit on the fused silica diaphragm to form an extrinsic Fabry-Perot (FP) interferometric cavity with the optical fiber end-face. Therefore, etching solutions that provide cavities of the desired shape and surface quality, and masking layers that can withstand the etchant for several hours are needed. Both these issues have been overcome and finally, pressure test results of this sensor structure are presented. 


\section{DEEP WET ETCHING OF FUSED SILICA}

Wet etching is a simple technology, which could give good results with suitable combination of etchant and mask material. Since fused silica is a noncrystalline (glass) form of silicon dioxide (quartz, sand) ${ }^{[6]}$, acidic ammonium fluoride solution, called buffered hydrogen fluoride (BHF) was used because of its reactivity to silicon compounds ${ }^{[7]}$. But the etch depth can be only up to $5 \mu \mathrm{m}$. Currently no photoresist can withstand BHF for long time (more than 1 hour). For deeper structures, additional masking layers that can be deposited onto diaphragm have to be used. This mask layer should be deposited between the photoresist layer and the diaphragm in order to further protect the diaphragm surface from HF attack. Different mask materials, such as Plysilicon, amorphous silicon ${ }^{[8]}$, and Hexamethyldisilazane (HDMS $)^{[9]}$ have been reported for deep wet etching process. But these materials cannot be later removed easily. Chromium was chosen as the metal mask because chromium layers can be easily removed by a commercial chromium mask etchant.

Figure 1 shows the process for realization of a deep structure on fused silica material for pressure sensor fabrication. This process consists of deposition of a chromium thin film by a sputtering system (a), photolithographic imaging (b)-(d) and selective wet etching (e) and remove the masks (f), (g).

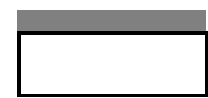

(a)

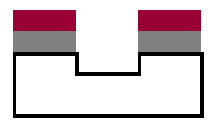

(e)

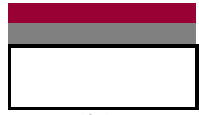

(b)

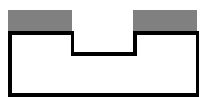

(f)

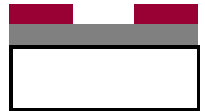

(c)

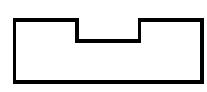

$(\mathrm{g})$

Photoresist

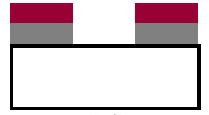

(d)

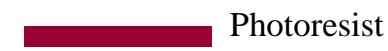

Chromium

Figure 1. Deep wet etch process

There is a large variety of different fused silica types. Some of them are not pure, which contain aluminium oxide. The impurity causes the etched pit to be filled with a white powder after etching, indicating aluminium oxide residuals ${ }^{[8]}$. We used fused silica diaphragms made of Valley Design, Inc. with thickness of $125 \mu \mathrm{m}$. This is an extremely pure fused silica material with high surface quality. Fused silica diaphragms were wet etched by BHF. The chemical composition of BHF is usually a mixture of $40 \% \mathrm{NH} 4 \mathrm{~F}$ and $49 \%$ or $50 \% \mathrm{HF}$ ranging in weight ratio of $\mathrm{NH}_{4} \mathrm{~F}: \mathrm{HF}_{\text {from }}$ 5:1 to 30:1. Buffering with $\mathrm{NH}_{4} \mathrm{~F}$ helps stabilize the etch rate. BHF was purchased from Sigma Aldric $\left(35.1 \% \mathrm{NH}_{4} \mathrm{~F}\right.$, $6.3 \% \mathrm{HF})$.

The chromium layer was coated by a sputtering system (100W, 3.5e-3 Torr process pressure for 15 to 60 second). The thickness of the chromium layer is around 10 to $70 \mathrm{~nm}$. This layer enhanced the adhesion of the photoresist (AZ TFP 650 ) to the substrate. In this way, the photoresist can withstand in the BHF solution without peeling off for at least 7 hours. Thus the etch depth can be as deep as $35 \mu \mathrm{m}$ after 7 hours' etching in the BHF solution at room temperature. The chromium layer can be easily lift off by the chromium mask etchant purchased from Transene Company, Inc.

A surface profiler was used to measure the etched substrate. Four aspects of the etch results, etch rate, surface roughness, diameter deviation and etch depth uniformity, were examined in deep etching fused silica material process. These four aspects were chosen because they all have effects on pressure sensor performance. Usually the surface roughness will affect the sensor visibility, the diameter deviation will affect the sensitivity of the sensor and the etch depth uniformity affects the repeatability of the sensor fabrication.

\subsection{Etch rate}


The wet etch rates can vary for reasons that are usefully divided into three groups: the etch setup, the material being etched, and the layout and structure on the substrate. The most significant effects on wet-etch rates are as follows:

- Temperature;

- Impurities in or on the material being etched;

- Contamination

Figure 2 shows the relationship between the etch depth and the etch time provided by commercially available BHF at room temperature. It is noticed that the etch rate curve is not linear. After certain amount of time, the etch rate decreased a little. The etch rate of silicon dioxide increases linearly with the concentrations of both $\mathrm{HF}^{-} \mathrm{and}_{2} \mathrm{HF}^{-} \mathrm{HF}$ molecules and $\mathrm{HF}_{2}^{-}$ions are consumed with time and make the $\mathrm{HF}$ concentration smaller than it was at the very beginning. If we do want to etch a sample in the BHF solution for long time, bigger container with more BHF solution is suggested.

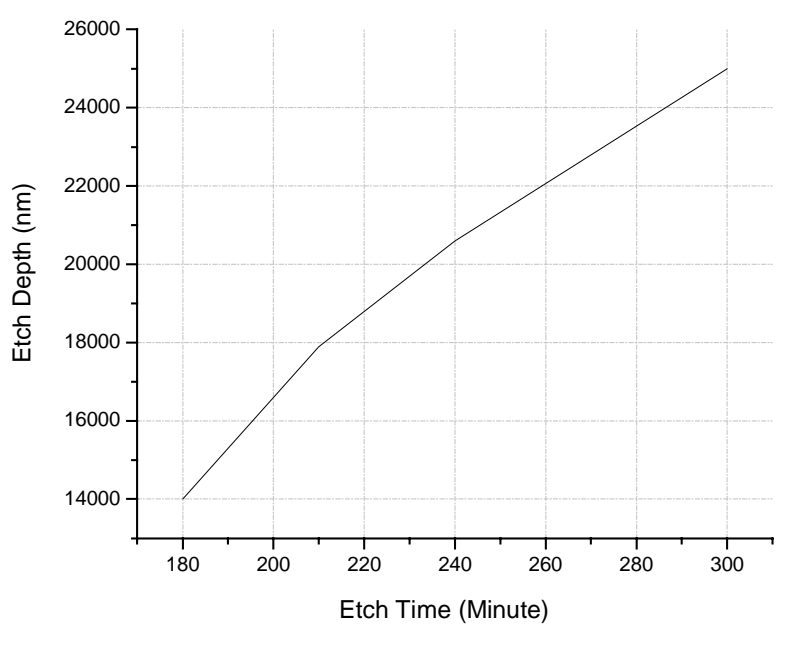

Figure 2. Etch rate of commercial BHF

\subsection{Surface roughness}

The amount of ammonium fluoride $\left(\mathrm{NH}_{4} \mathrm{~F}\right)$ in the BHF solution was the most important factor that affected the surface cleanness. Some pin holes appeared on the etched diaphragm if we didn't mix enough $\mathrm{NH}_{4} \mathrm{~F}$. The smoothness of the surface can be ensured through wet etching by the improvement of the compositions of the BHF solution. For the left plot in Figure 3-13, we mix $\mathrm{H}_{2} \mathrm{O}, \mathrm{HF}_{4} \mathrm{~F}$ and $\mathrm{HF}$ with the percentage of 58\%: 20\%:22\% and for the right plot in Figure 3, the concentration ratio changed to be: $\mathrm{H}_{2} \mathrm{O}: \mathrm{NH}_{4} \mathrm{~F}: \mathrm{HF}=58 \%: 36 \%: 6 \%$. We can see from the two plots in Figure 3 that the surface quality in the second plot is significantly improved by the changes of the BHF compositions.

Also, the lower concentration of the HF, the better surface quality we can get. So chemical etching is a possible means to mitigate the growth of surface damage on fused silica. There is a trade-off between the surface quality and the etch rate. For our sensor fabrication, we choose commercially available BHF with higher concentration to etch at the first (first etch process). The etch rate is high so we can get as deep as $20 \mu \mathrm{m}$ pits in 4 hours. But the etched surface became a little bit rough after long time etching. Then we changed to use 10:1 BHF (second etch process). Since we don't care much about the depth at the second etch process, lower concentration of HF was used to mitigate the surface roughness of the small pits caused by the first etch process. The reflectance of the etched pit on the fused silica diaphragm can be as high as $4 \%$ with normal light incidence. This is the same reflectance a with well-cleaved optical fiber end face.

\subsection{Diameter deviation}

The main factor that affects the diameter of the etched pit is the UV exposure process including the distance between the UV light source and the substrate and the exposure time. Ideally, different areas of the substrate should be exposed by the same energy and the same period. If we put the UV light too close to the substrate (Figure 4., left), some areas 
were over-exposed while some other areas were under-exposed because of the limited size of the UV light. This caused the diameter of the size to vary substantially.

If we place the UV light source far away from the substrate, the exposure would be more uniform as illustrated in Figure 4 (right plot). But longer exposure time is needed. In this experiment, the distance between the UV light and the substrate was $8 \mathrm{~cm}$ and the exposure time was 4 minutes.

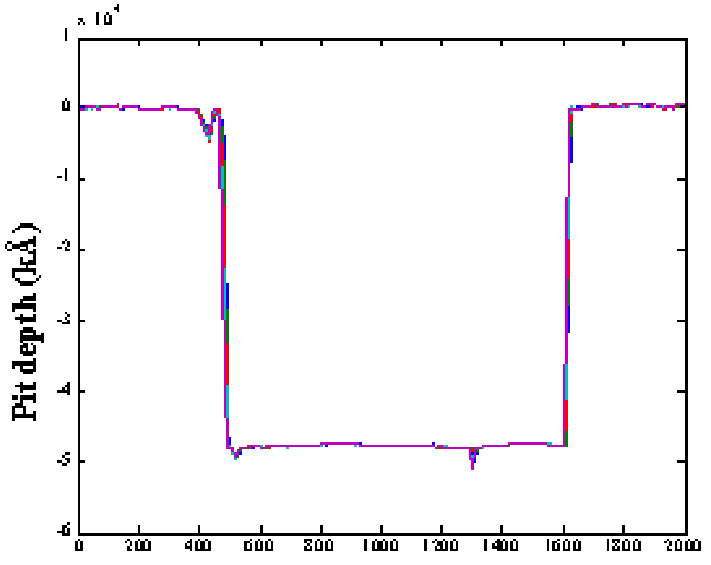

Pil diameter (mic ron)

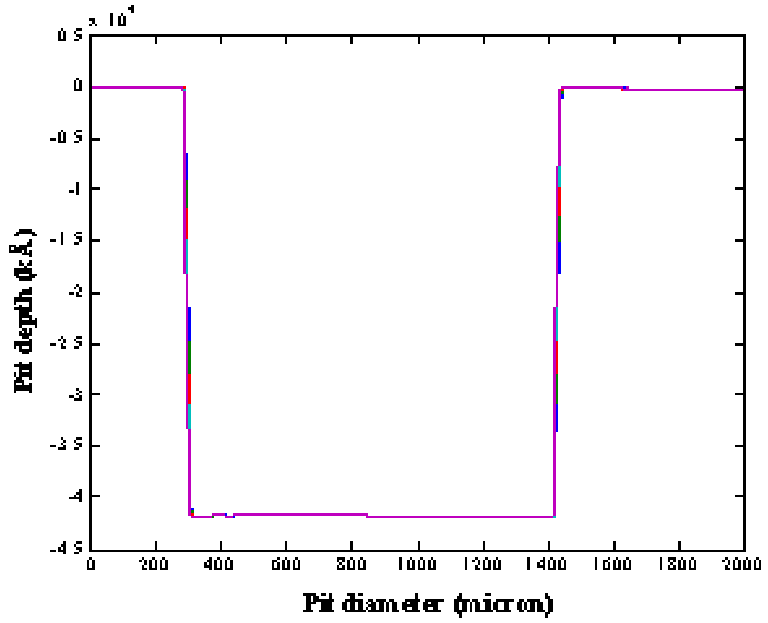

Figure 3. Surface quality improvement

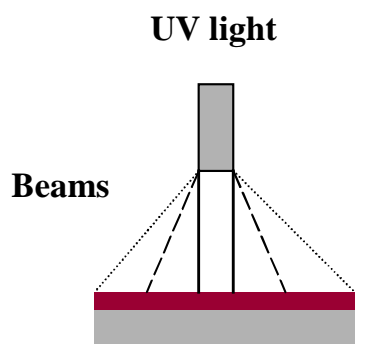

Substrate

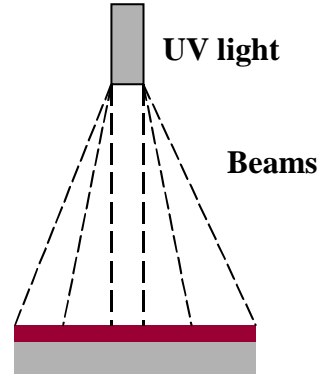

Substrate

Figure 4. UV light exposure

We can see from the plot in Figure 5 that the sidewalls of the 2 nd pit are very straight and smooth. Therefore, the deviations of the etched pit diameter are reduced. We can also see that in the second plot the diameter deviation is: $(1.14-1.04) / 1.04 * 100 \%=9.6 \%$, but in the first plot, the diameter deviation is: $(1.8-1.04) / 1.04 * 100 \%=73 \%$. The sensitivity of the sensor can be better controlled with the reduced deviation of the pit diameter per our calculation.

\subsection{Etch depth uniformity}

Etch depth uniformity was examined when the structures were etched under the same conditions on two different diaphragms or on the same diaphragm. Figure 6 shows two etched pits on two different diaphragms. These two diaphragms were both etched in the commercial BHF for 5 hours respectively. The etch depth was controlled by BHF 
concentration, the temperature and the time. Since HF is extremely dangerous, we normally only use it under the room temperature. And commercial BHF is used in order to keep the BHF concentration unchanged. Figure 7 shows six etched pits on the same diaphragm. We can see from both Figure 6 and Figure 7, that wet chemical etch can provide the same etch depth under the same conditions.
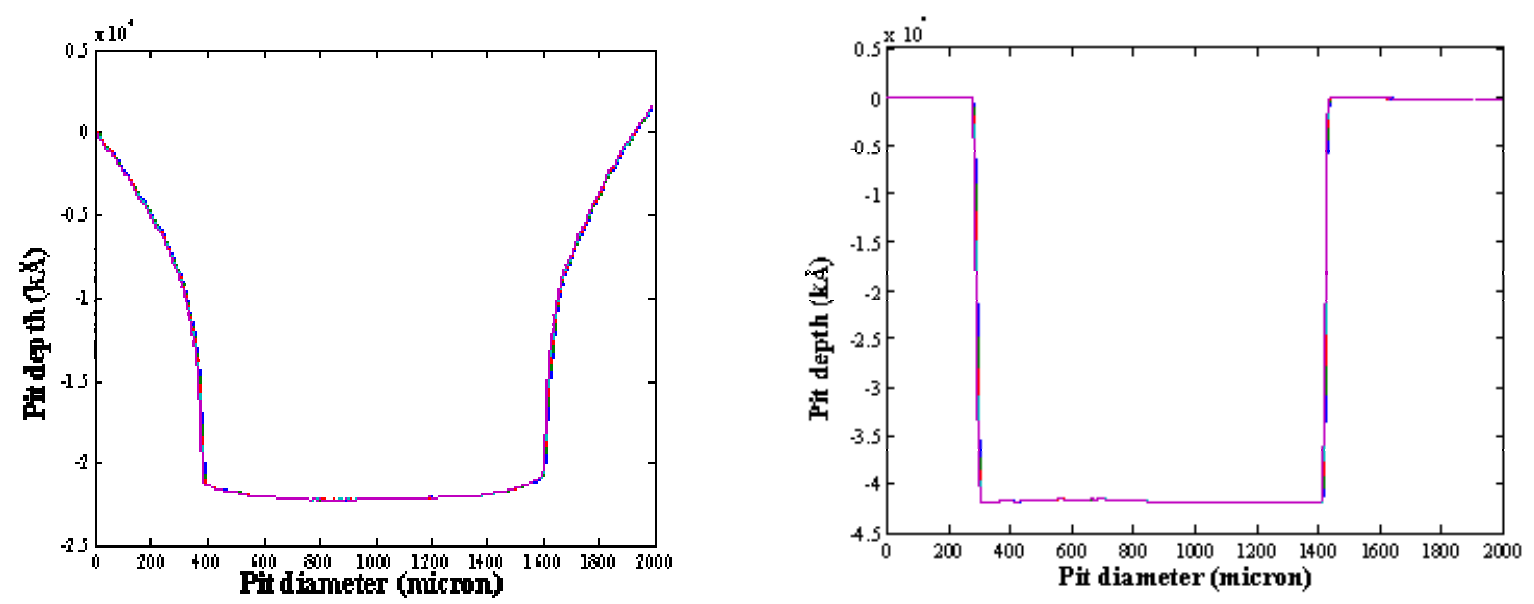

Figure 5. Diameter Deviation

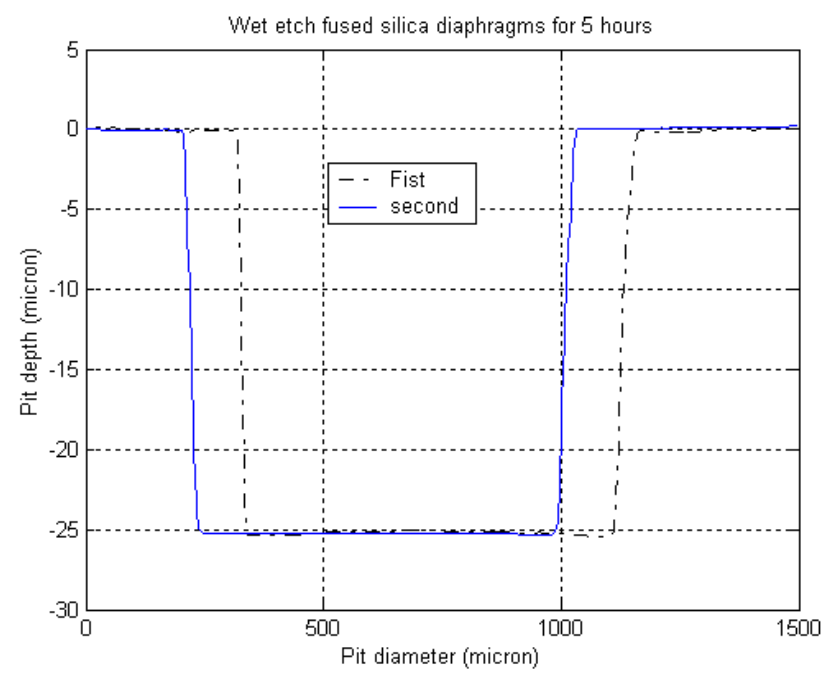

Figure 6. Etched pits on two different diaphragms 


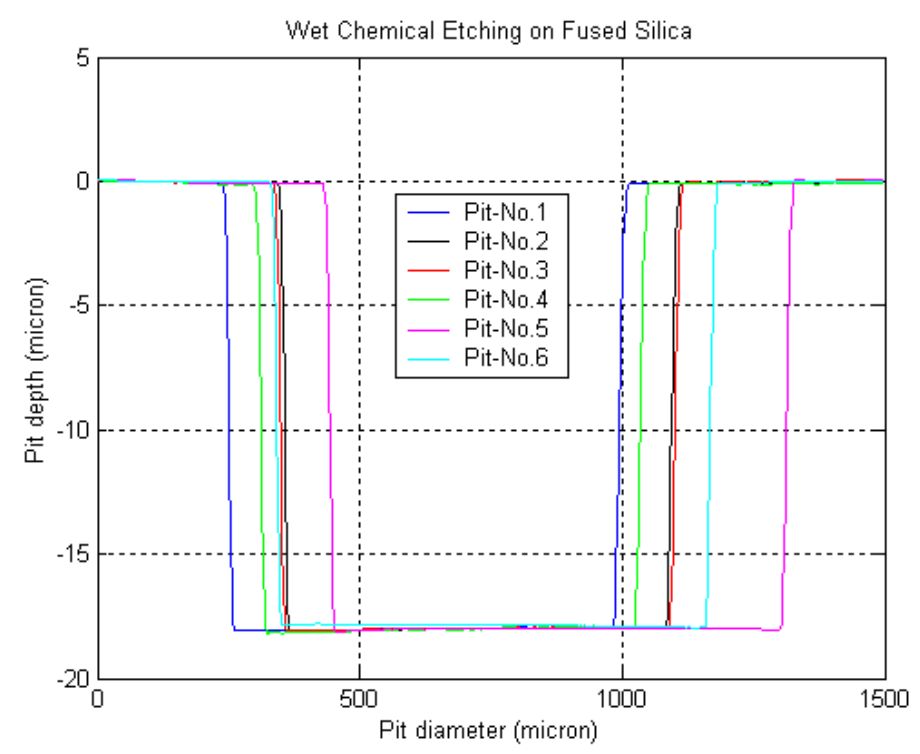

Figure 7. Etched pits on the same diaphragm

\section{FIBER OPTIC PRESSURE SENSOR}

The geometry of the diaphragm based pressure sensor can be illustrated using Figure 8. The interference cavity is formed by wet chemical etching of a round pit on the diaphragm. When static or dynamic pressures are applied, the diaphragm will deform, and as the consequence the cavity length will change.

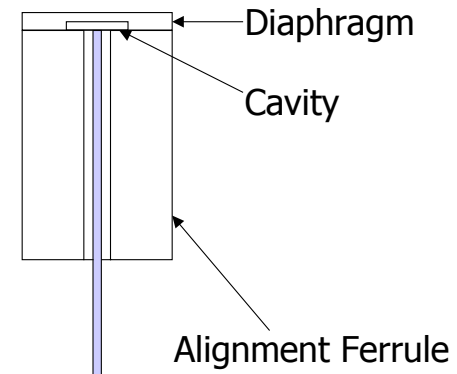

Fiber

Figure 8. Geometry of diaphragm based pressure sensor probe

Figure 9 shows the configuration of an Extrinsic Fabry-Perot Interferometric (EFPI) pressure sensor system. A single mode silica optical fiber transmits light from a $1550 \mathrm{~nm}$ laser diode to the sensor element through a $2 \mathrm{x} 2$ coupler. At the sensor head, the light signal is partially reflected and partially transmitted across the gap formed by the end of the input fiber and the fused silica diaphragm. The light from the first interface (the input fiber end) and the light reflected at the second interface (the pit on the diaphragm) travel back to a photo-detector where a sinusoidal interference signal can be monitored due to differential phase changes between the two light beams as a result of changes in the EFPI sensor cavity spacing. 


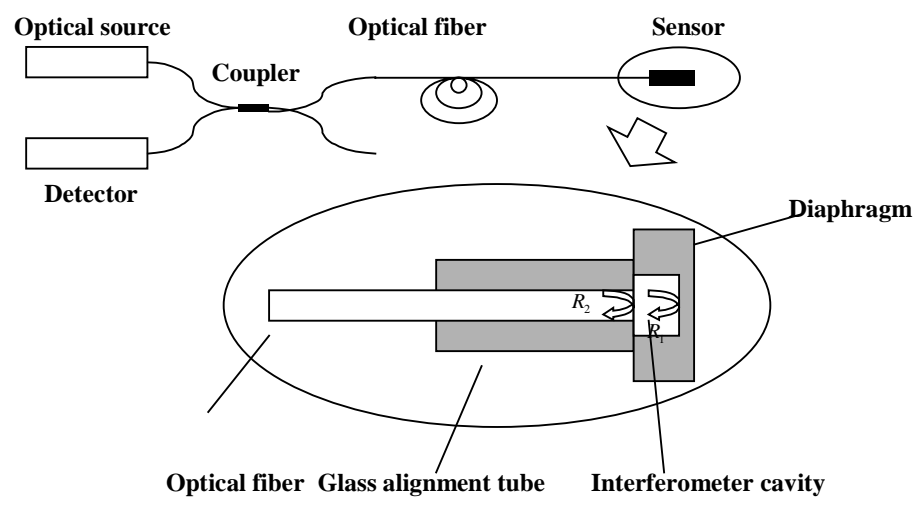

Figure 9. Configuration of the sensor system

For a single-mode fiber Fabry-Perot sensor, the visibility of the interference fringe which is a function of the reflectance of both two reflected surfaces, can reach as high as $95 \%$ to $99 \%$ as shown in Figure 10 . The etching surface quality of the fused silica diaphragm played an important role for this high visibility. These two plots were copied from the white light interferometric system. The left plot shows the interference fringes. The right plot shows the normalized interference fringes by the source spectrum. The cavity length (also called air-gap) and the interference fringe visibility can be read directly from the right plot.

The pressure sensor test results are as shown in Figure 11 and Figure 12. The sensor used in this test was a single-mode fiber sensor with $95 \mu \mathrm{m}$ thickness (after etching) diaphragm and $1 \mathrm{~mm}$ diameter etched pit. Due to the sinusoidal nature of the interference signal, the direct output is a nonlinear function of the applied pressure as shown in Figure 11. The sensitivity of this sensor to the pressure was controlled by the fused silica diaphragm etch depth and the diameter of the etched pit.
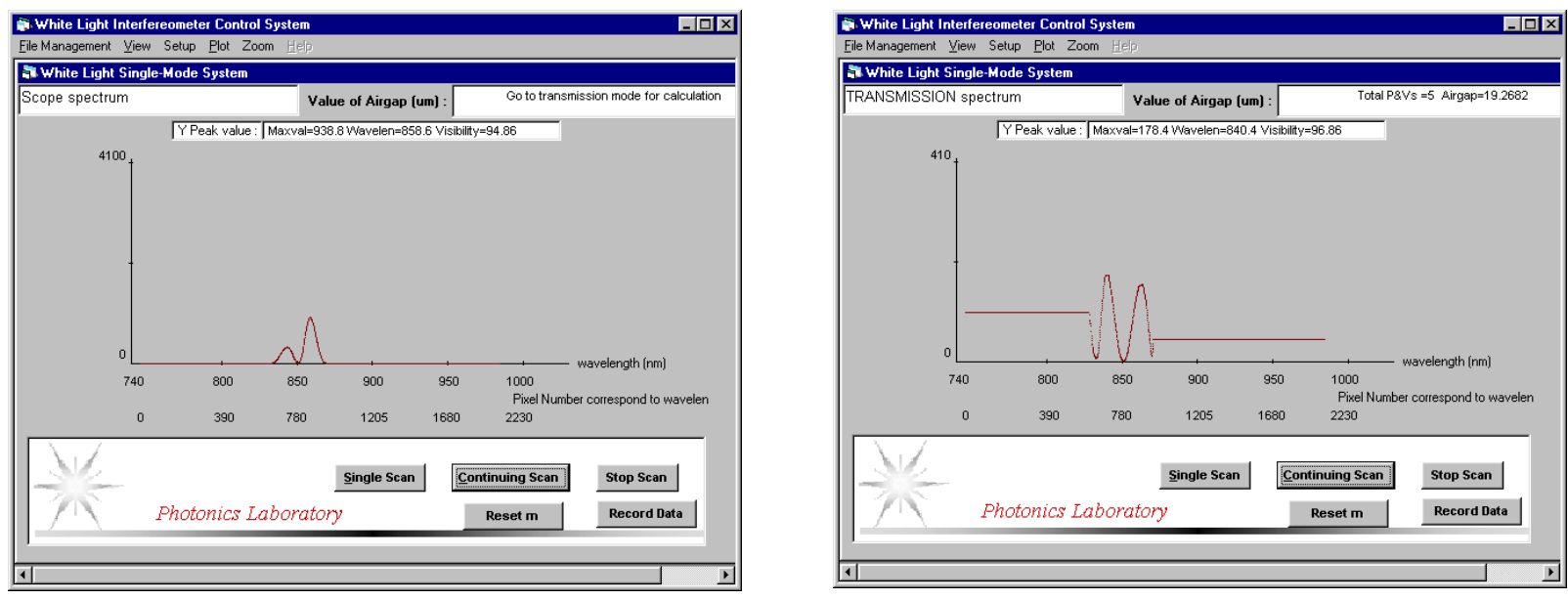

Figure 10. White-light interferometric signal 


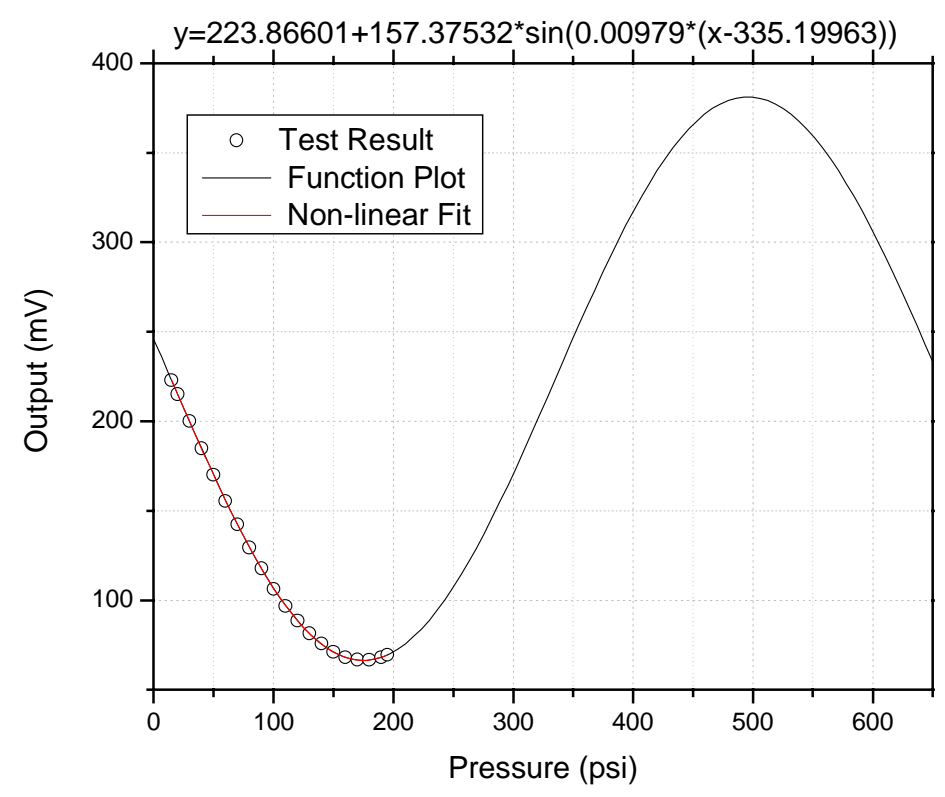

Figure 11. Static pressure measurement

The difference between outputs for a given pressure value under rising and falling directions is termed hysteresis. ${ }^{[10,11]}$ The sensor probe is made of fused silica material so the operating range of the sensor in terms of pressure induced strain is very small. Therefore, the hysteresis of the sensor is expected to be small. Hysteresis of the pressure sensor was measured by cycling the applied pressure between 14psi and 200psi. The measurement results are shown in Figure 12. The experimental results confirm our expectation. There was no noticeable hysteresis found within the operation range.

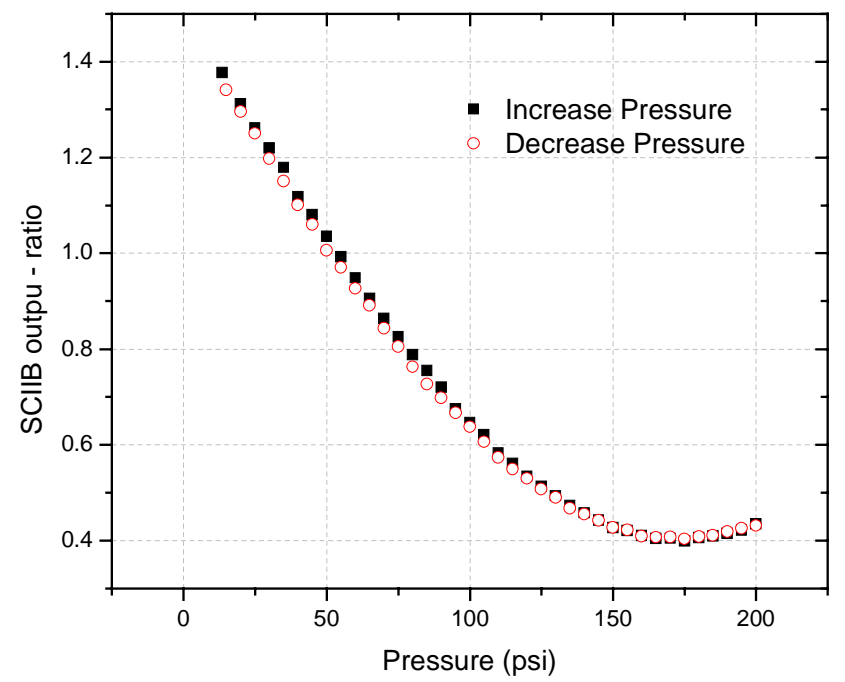

Figure 12. Hysteresis of the pressure sensor 


\section{CONCLUSION}

We have presented a deep wet chemical etching process for fabricating optical fiber pressure sensors. The wet chemical etching and thin film deposition methods were successfully combined and used in the sensor fabrication process. A very good adhesion of photoresist to the chromium mask was achieved and led to excellent etching results. Especially, these techniques were implemented on the fused silica diaphragms instead of silicon wafers. About twenty pits could be fabricated in the same process. The total time used in the photolithography process and wet etching on a diaphragm can be as short as one hour. The patterns transferred onto the fused silica diaphragms are uniform, with smooth surface and great depth that suffice the requirements of the pressure sensor application. It is revealed that wet etching process is a repeatable, cost effective and reliable technique.

\section{REFERENCES}

1. Savander, Pekka, “Microlens arrays etched into glass and silicon”, Optics \& Lasers in Engineering. v 20 n 21994. pp $97-107$

2. J. Ihlemann, B. Wolff-Rottke, “Excimer laser micro machining of inorganic dielectrics", Applied Surface Science, V106, (1996) pp 282-286

3. K. Sugioka, S. Wada, H. Tashiro, K. Toyada, A. Nakamura, "Novel ablation of fused quartz by preirradiation of vacuum-ultraviolet laser beams followed by fourth harmonics irradiation of Nd:YAG laser", Applied Physics Letters, 65, (1994) pp. 1510-1512

4. H. Varel, D. Ashkenasi, A. Rosenfeld, M. Wahmer, E.E.B. Campbell, "Micromachining of Quartz with ultrshort laser pulses”, Applied Physics, A 65, (1997), pp 367-373

5. J. Wang, H.Niino, A. Yabe,"One-step microfabrication of fused silica by laser ablation of an organic solution", Applied Physics, A 68, p111-113, 1999

6. http://www.accuraturs.com/fused.html;

7. Hirohisa Kikyuama, Nobuhiro Miki, Kiyonori Saka, Jun Takano, Ichiro Kawanabe, Masayuki Miyashita, and Tadahiro Ohmi, "Principle of Wet Chemical Processing in ULSI Microfabrication", IEEE Transactions on Semiconductor Manufacturing, Vol. 4, No. 1, 1991

8. A.Berthold, P.M. Sarro, M.J. Vellekoop,"Two-step Glass Wet-etching for Micro-fluidic Devices", Proceeding of the SeSens workshop, p613-616, 2000

9. Axel Gross, Matthias Grewe and Henning Fouckhardt, "Deep wet etching of fused silica glass for hollow capillary optical leaky waveguides in microfluidic devices", Journal of Micromechanics and Microengineering, Vol. 11, p 257262,2001

10. Hai Xiao, dissertation for Ph.D., "Self-Calibrated Intensity/Interferometric Based (SCIIB) Fiber Optic Pressure Sensor", 2000

11. Jiangdong Deng, preliminary exam for Ph.D., "High Sensitivity Optical Fiber Interferometric Acoustic Sensors for Detecting Acoustic Emission", March 2001

*xichen5@vt.edu; phone 1540 231-8359; fax 15402312158 


\title{
Thermally Tunable Extrinsic Fabry-Pérot Filter
}

\author{
Bing Yu, Gary Pickrell, and Anbo Wang, Senior Member, IEEE
}

\begin{abstract}
This letter describes a thermally tunable filter of extremely low cost, suitable for optical sensing, spectrometry, and video distribution. The filter utilizes a high-finesse extrinsic fiber Fabry-Pérot interferometer formed by fusion bonding of two mirror-coated single-mode fibers to a short piece of borosilicate tubing. Continuous wavelength tuning is achieved using a Peltier device. We demonstrate a prototype filter operating at $1550 \mathrm{~nm}$ with a tuning range of $90.8 \mathrm{~nm}$ and a temperature sensitivity of $1.63 \mathrm{~nm} /{ }^{\circ} \mathrm{C}$. The filter features polarization independence, high repeatability, and inherently low drift and low insertion loss.
\end{abstract}

Index Terms-Extrinsic Fabry-Pérot interferometer, laser bonding, tunable optical filter.

\section{INTRODUCTION}

$\mathbf{T}$ UNABLE optical filters are a key component in wavelength-division-multiplexing (WDM) networks for channel selection, wavelength add-drop, and circuit switching [1]. They are also important devices in the interrogation of optical sensors [2], in spectroscopy [3], as well as in video distribution networks [4]. A variety of bandpass tunable filters have been demonstrated in the past decades, among these, acoustooptic tunable filters [5], fiber Fabry-Pérot (FFP) tunable filters [6], and micromachined tunable filters [7] have attracted the most interest. A significant amount of these efforts have been focused on meeting the requirements of WDM networks, in which a tuning range covering the erbium-doped fiber amplifier spectral range $(\sim 40 \mathrm{~nm})$, a bandwidth smaller than $1.0 \mathrm{~nm}$ for high channel capacity, and high tuning speed on the order of microseconds, or even faster, are generally required [1]. However, in sensing systems, spectrometers, and video networks, tunable filters of extremely low cost with a tuning range above $80 \mathrm{~nm}$, a passband from 0.5 to $10 \mathrm{~nm}$, an insertion loss less than $3.0 \mathrm{~dB}$, good repeatability, and long-term stability have obvious advantages. On the other hand, a tuning speed up to subseconds is still acceptable in some applications. Tunable filters addressing these application needs are desirable.

The extrinsic FFP interferometer (EFPI) has found tremendous applications in fiber-optic sensors [8] and FFP tunable filters [6], because of its simple structure, compact size, and insensitivity to environment changes. In this letter, we present an inherently inexpensive thermally tunable filter based on an EFPI, suitable for optical sensing, spectrometer, and video distribution. The filter demonstrated has a tuning sensitivity of $1.63 \mathrm{~nm} /{ }^{\circ} \mathrm{C}$, and features polarization independence, high repeatability, and inherently low drift and low insertion loss.

\footnotetext{
Manuscript received April 16, 2004; revised June 3, 2004. This work was supported by DOE under Contract DE-FC36-01GO11050.

The authors are with the Center for Photonics Technology, Virginia Polytechnic Institute and State University, Blacksburg, VA 24061 USA (e-mail: biyu@vt.edu; pickrell@vt.edu; awing@ vt.edu).

Digital Object Identifier 10.1109/LPT.2004.833890
}

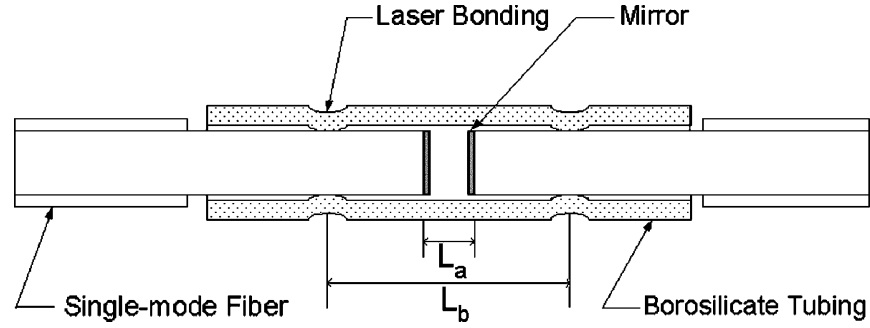

Fig. 1. Schematics of a thermally tunable EFPI filter.

\section{PRINCIPLE OF OPERATION}

The core of the device described here is basically a high-finesse EFPI that is formed by fusion bonding of two mirror-coated single-mode optical fibers to a short piece of borosilicate capillary tubing using fusion bonding, shown in Fig. 1. The resonant or transmission peaks occur when the optical phase difference $\delta=2 \pi L_{a} / \lambda$ is equal to multiples of $\pi$, where $L_{a}$ is the distance between the two mirrors and $\lambda$ is the optical wavelength in vacuum. Changing $L_{a}$ results in the change of the resonant condition, and thus, the shift of the transmission band.

Assuming that the central wavelength of the filter passband is $\lambda_{0}$ when the airgap is $L_{a}$, the shift of the central wavelength $\Delta \lambda$ caused by an airgap change $\Delta L$ can be expressed as

$$
\Delta \lambda=\left(\frac{\Delta L}{L_{a}}\right) \cdot \lambda_{0}
$$

The wavelength shift is proportional to the ratio $\Delta L / L_{a}$, which means a large tuning range may be obtainable with a small change of the airgap, if a short cavity is used. Taking advantage of the differential thermal expansions of the borosilicate tubing and the silica fiber, continuous tuning of the filter passband can be achieved by locally cooling or heating the EFPI. Assuming that a temperature change from $T$ to $T+\Delta T$ is applied to an EFPI filter of gauge length $L_{b}$, corresponding to the distance between the two bonding points, resulting in an airgap change from $L_{a}$ to $L_{a}+\Delta L, \Delta L$ is given by

$$
\Delta L=\left[\alpha_{b} L_{b}-\alpha_{f}\left(L_{b}-L_{a}\right)\right] \cdot \Delta T
$$

where $\alpha_{b}$ and $\alpha_{f}$ are the coefficients of thermal expansion (CTE) of the borosilicate tubing and the fused silica fiber, respectively. $\alpha_{b}$ and $\alpha_{f}$ are constants from $0{ }^{\circ} \mathrm{C}$ to $300{ }^{\circ} \mathrm{C}$. Substituting (2) into (1) and assuming that $L_{a} \ll L_{b}$

$$
\Delta \lambda=\left(\frac{L_{b}}{L_{a}}\right) \cdot\left(\alpha_{b}-\alpha_{f}\right) \cdot \lambda_{0} \cdot \Delta T .
$$

Equation (3) indicates that the wavelength tuning is proportional to the temperature change. Since the borosilicate tubing has a CTE approximately $5 \sim 10$ times higher than that 


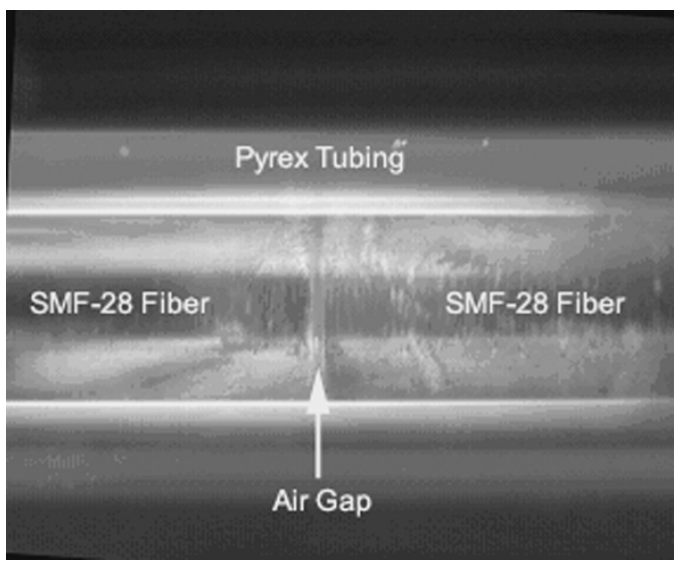

Fig. 2. Optical micrograph of an EFPI filter.

of the silica fiber, a tuning range of above $100 \mathrm{~nm}$ can easily be achieved within a temperature range of less than $80^{\circ} \mathrm{C}$ (the normal operation range of a Peltier device).

As implied by Fig. 1, the alignment between the two mirrors is automatically achieved because of the tight matching between the inside diameter (I.D.) of the tubing and the diameter of the single-mode bare fiber. This self-alignment capability significantly reduces the coupling loss and eliminates the costly aligning process encountered by other tunable filter techniques. Furthermore, high reliability is expected since there are no moving parts and, therefore, no mechanical wear is involved. The long time stability of the filter is determined by the temperature stability of the Peltier device and its controller, which can be as low as $0.001{ }^{\circ} \mathrm{C}$.

\section{FILTER PREPARATION}

A prototype tunable EFPI filter was constructed that operates in the 1550-nm band using SMF-28 single-mode fibers with a CTE of $5.6 \times 10^{-7} /{ }^{\circ} \mathrm{C}$, and Pyrex tubing with a CTE of $32.5 \times 10^{-7} /{ }^{\circ} \mathrm{C}$, an I.D. of $127+1.5 \mu \mathrm{m}$, and an outside diameter of $290 \mu \mathrm{m}$. The two multilayer dielectric mirrors were deposited on the cleaved ends of the fibers by Evaporated Coating, Inc.. The mirrors have a peak reflectance of $R=87.5 \%$ at $1565 \mathrm{~nm}$, but down to $87.0 \%$ at 1529 and $1602 \mathrm{~nm}$. The fibers were fusion bonded to the Pyrex tubing using a pulsed $\mathrm{CO}_{2}$ laser at relatively high power, forming an airgap of $10.589 \mu \mathrm{m}$ at $T=25.45{ }^{\circ} \mathrm{C}$ and a gauge length $L_{b} \approx 4.1 \mathrm{~mm}$. During the bonding process, a light-emitting diode with a central wavelength of $1557.6 \mathrm{~nm}$ and a bandwidth $\Delta \lambda_{\mathrm{FWHM}}=61.7 \mathrm{~nm}$, and an AQ-6315A optical spectrum analyzer (OSA) were used to monitor the airgap length. Fine adjustment of the airgap can be made with low power $\mathrm{CO}_{2}$ laser pulses. Fig. 2 shows an optical micrograph of the airgap.

The EFPI filter was then assembled into a $14 \mathrm{p}$-i-n butterfly package with a thermoelectric cooling (TEC) module and a thermistor. To achieve fast thermal response, the EFPI filter was brought in direct contact with the cold plate of the TEC and thermal compound was used to enhance thermal coupling between the TEC and the EFPI filter.

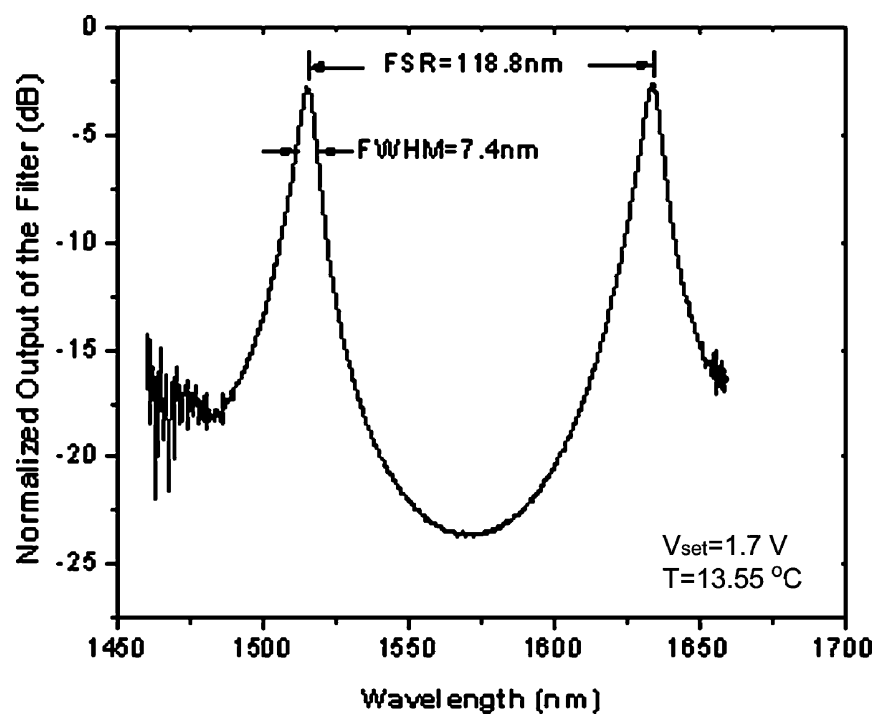

Fig. 3. Normalized spectrum at $T=13.55^{\circ} \mathrm{C}$.

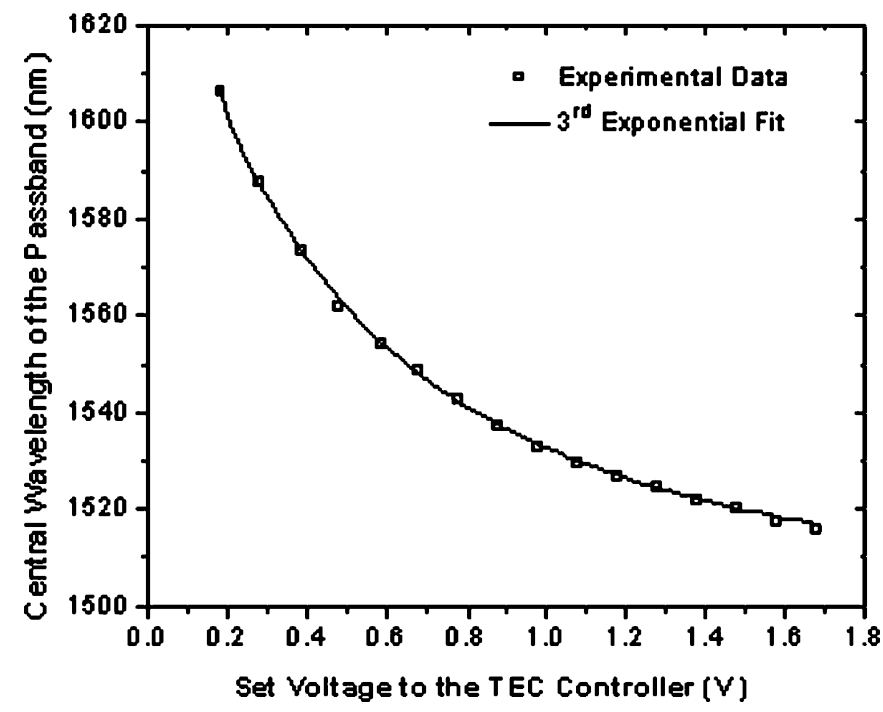

Fig. 4. Tuning curves of the thermally tunable EFPI filter.

\section{Filter CharacterizATION}

To characterize the prototype filter, a TEC controller was constructed with a 24-h temperature stability of $0.003{ }^{\circ} \mathrm{C}$, whose input is set by a computer through a high resolution data acquisition card (DAC) card. The same light source and OSA for the fabrication was used for the performance evaluation. Fig. 3 gives the output spectrum of the filter normalized to that of the source at a set voltage $V_{\mathrm{SET}}=1.7 \mathrm{~V}$, corresponding to $T=13.55^{\circ} \mathrm{C}$.

A 3-dB bandwidth of $7.4 \mathrm{~nm}$ on the first passband, a free spectral range (FSR) of $118.8 \mathrm{~nm}$, and an insertion loss of $2.7 \mathrm{~dB}$ were measured at this temperature. From the positions of the two peaks, the airgap can be readily calculated as $10.425 \mu \mathrm{m}$. A narrower passband as low as $1.0 \mathrm{~nm}$ can be obtained by using either mirrors of higher reflectance or longer cavity length. The major sources of the insertion loss include misalignments between the two fibers, absorption and asymmetry of the two mirrors. Insertion loss close to $1.0 \mathrm{~dB}$ can be expected by using tubing of smaller I.D. and higher quality thin films on polished fiber ends. 


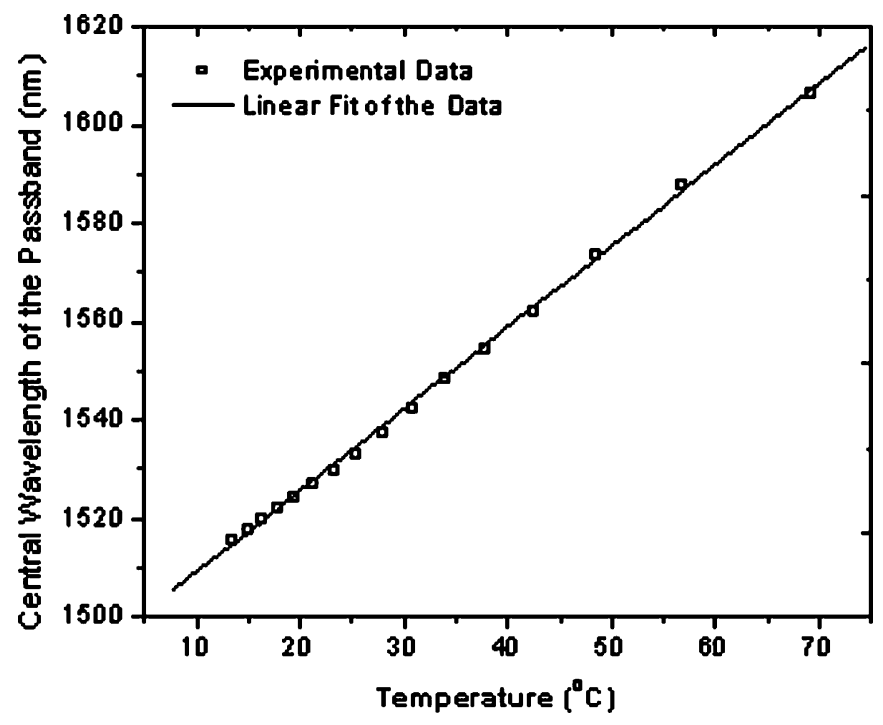

Fig. 5. Filter's temperature sensitivity and linearity.

Continuous tuning of the passband has been achieved by scanning the output voltage of the DAC. Fig. 4 shows the dependence of the central wavelength of the filter on the control voltage with a third-order exponential curve fit. A tuning range of $90.8 \mathrm{~nm}$ was obtained with a voltage change of $1.7 \sim 0.2 \mathrm{~V}$, or a temperature change from $13.55^{\circ} \mathrm{C}$ to $69.28^{\circ} \mathrm{C}$. This tuning range agrees well with the predicted value of $89.8 \mathrm{~nm}$ by (3). The third-order exponential dependence of the central wavelength on the control voltage can be explained by the similar dependence of the resistance of the thermistor on temperature. When converting the voltage to corresponding temperature, very good linear dependence of the wavelength on temperature resulted, as shown in Fig. 5. A temperature sensitivity of $1.63 \mathrm{~nm} /{ }^{\circ} \mathrm{C}$ has been measured from the linear fit. The long time drift of the filter passband has been determined by the temperature stability, which is less than $5.0 \mathrm{pm}$ in $24 \mathrm{~h}$ in the laboratory environment.

An FSR change from $118.8 \mathrm{~nm}$ at $13.55^{\circ} \mathrm{C}$ to $108.8 \mathrm{~nm}$ at $69.28{ }^{\circ} \mathrm{C}$ was observed which can be explained by FSR $=$ $\lambda^{2} / 2 L_{a}$. A bandwidth of $7.4 \mathrm{~nm}$ at $1515.6 \mathrm{~nm}, 6.58 \mathrm{~nm}$ around $1565 \mathrm{~nm}$, and $7.0 \mathrm{~nm}$ at $1606.4 \mathrm{~nm}$ was measured. This bandwidth change is a combined effect of the airgap change and the nonflat reflectance spectrum of the mirrors, which makes the filter have a smaller full-width at half-maximum (FWHM) around $1565 \mathrm{~nm}$ than at other wavelengths. The airgap dependence of the bandwidth can be described by FWHM $=$ FSR $/ F$, where FSR is inversely proportional to the airgap $L_{a}$, and thus, to $T$, and $F$ is the finesse determined by the mirror reflectance.

The time needed to scan the whole tuning range was less than $2 \mathrm{~s}$. This relatively low speed can be attributed to the large package size and the associated thermal mass. Much higher scanning speeds can be expected by reducing the package size, or directly depositing an electrically resistive coating onto the capillary tubing of the filter.

\section{SUMMARY}

We have demonstrated a thermally tunable EFPI filter that has a tuning range of $90.8 \mathrm{~nm}$ with a temperature sensitivity of $1.63 \mathrm{~nm} /{ }^{\circ} \mathrm{C}$ and a long-term drift of less than $5.0 \mathrm{pm}$ in the 1550-nm band. The filter features polarization independence, high repeatability, and inherently low drift and low insertion loss. This filter can be used in an optical sensing system as a demodulation component, and in a spectrometer for bio- and chemical-sensing.

\section{REFERENCES}

[1] D. Sadot and E. Boimovich, "Tunable optical filters for dense WDM networks," IEEE Commun. Mag., vol. 36, pp. 50-55, Dec. 1998.

[2] M. G. Xu, H. Geiger, and J. P. Dakin, "Interrogation of fiber-optic interferometric sensors using acousto-optic tunable filter," Electron. Lett., vol. 31, no. 17, pp. 1487-1488, 1995 .

[3] G. Lammel, S. Schweizer, S. Schiesser, and P. Renaud, "Tunable optical filter of porous silicon as key component for a MEMS spectrometer," $J$. Microelectromech. Syst., vol. 11, no. 6, pp. 815-827, 2002.

[4] H. Kobrinski and K.-W. Cheung, "Wavelength-tunable optical filters: applications and technologies," IEEE Commun. Mag., vol. 27, pp. 53-63, Oct. 1989.

[5] D. A. Smith, J. E. Baran, J. J. Johnson, and K.-W. Cheung, "Integratedoptic acoustically-tunable filters for WDM networks," IEEE J. Select. Areas Commun., vol. 8, pp. 1151-1159, Aug. 1990.

[6] J. Stone and L. W. Stulz, "Pigtailed high-finesse tunable fiber Fabry-Pérot interferometers with large, medium and small free spectral ranges," Electron. Lett., vol. 23, no. 15, pp. 781-782, 1987.

[7] T. Amano et al., "Design and fabrication of GaAs-GaAlAs micromachined tunable filter with thermal strain control," J. Lightwave Technol., vol. 21, pp. 596-601, Mar. 2003.

[8] K. A. Murphy, M. F. Gunther, A. Wang, R. O. Claus, and A. M. Vengsarkar, "Extrinsic Fabry-Pérot optical fiber sensor," in 8th Optical Fiber Sensors Conf., Jan. 29-31, 1992, pp. 193-196. 


\title{
Epoxy-free high temperature fiber optic pressure sensors for gas turbine engine applications
}

\author{
Juncheng $\mathrm{Xu}^{*}$, Gary Pickrell, Bing Yu, Ming Han, Yizheng Zhu, Xingwei Wang, \\ Kristie L Cooper, Anbo Wang \\ Center for Photonics Technology \\ Bradley Department of Electrical and Computer Engineering \\ Virginia Polytechnic Institute and State University, Blacksburg, VA 24061 USA
}

\begin{abstract}
Pressure measurements at various locations of a gas turbine engine are highly desirable to improve the operational performance and reliability. However, measurement of dynamic pressure (1psi $(6.9 \mathrm{kPa})$ variation superimposed on the static bias) in the operating environment of the engine, where temperatures might exceed $600^{\circ} \mathrm{C}$ and pressures might exceed 100psi $(690 \mathrm{kPa})$, is a great challenge to currently available sensors. To meet these requirements, a novel type of fiber optic engine pressure sensor has been developed. This pressure sensor functions as a diaphragm-based extrinsic Fabry-Perot interferometric (EFPI) sensor. The structure of the sensor head, composed entirely of fused silica, allows a much higher operating temperature to be achieved in conjunction with a low temperature dependence. The sensor head and the fiber tail have been packaged in a metal fitting connected to a piece of metal extension tubing, which improves the mechanical strength of the sensor and facilitates easy sensor installation. The sensor exhibited very good performance in an engine field test, demonstrating not only that the sensors' package is robust enough for engine operation, but also that its performance is consistent with that of a commercial Kulite sensor.
\end{abstract}

Keywords: Fiber optic sensor, pressure sensor, turbine engine

\section{INTRODUCTION}

Dynamic pressure measurements are highly desirable in aerodynamics applications, for example, in gas turbine engines. Pressure variations are of frequencies ranging from several hertz to on the order of $10 \mathrm{kHz}$ at different locations in the engine. Conventional semiconductor pressure transducers are widely used for these measurements. However, they are sensitive to temperature variations and electromagnetic interference (EMI) and are temperature limited to $500^{\circ} \mathrm{C}$. Fiber optic sensors offer many advantages as compared to other types of sensors, including small size, long life, high temperature capability, immunity to EMI, and extreme reliability.

Diaphragm-based extrinsic Fabry-Perot interferometer (EFPI) fiber optic sensors have been developed for many years ${ }^{[1]-}$ [7]. The most common approach to fabricating such sensors involves applying epoxy to bond both the diaphragm and the fiber to the holding ferrule or tube. However, epoxy will exhibit a time-dependent viscoelastic dimensional change and will decompose at high temperatures.

\footnotetext{
*juxu1@vt.edu; telephone 540-231-8359; fax 540-231-2158
} 
In this paper, we describe a silica diaphragm-based fiber optic pressure sensor that offers high sensitivity, high bandwidth and can be operated at temperatures up to $700^{\circ} \mathrm{C}$ with low temperature dependence. Some experimental results from laboratory measurements and dynamic pressure measurements in an engine field test will be presented.

\section{PRINCIPLE OF OPERATION}

The sensor head functions as a diaphragm-based extrinsic Fabry-Perot interferometric (EFPI) sensor, which offers high resolution and high frequency response. The basic configuration of the sensor system is shown in Fig. 1 and the sensor head structure is shown in Fig. 2. The fiber end face and diaphragm inside surface form a Fabry-Pérot (F-P) interferometer. The length of the F-P cavity (air-gap) will decrease with deflection of the diaphragm as a result of the applied pressure. The sensor lead-in fiber is connected to an operating-point tuning ${ }^{[8]}$ enhanced self-calibrated interferometric-intensity-based (SCIIB) ${ }^{[9]}$ signal interrogation system to monitor air-gap changes during pressure measurements. Light is injected into the optical fiber and partially reflected $(4 \%)$ by the end face of the fiber and the inside surface of the diaphragm. Then the two reflections propagate back through the same fiber and generate interference fringes, which are demodulated to determine the air-gap thickness. The intensity of the sensor output will change sinusoidally with the air-gap changes. In order to eliminate fringe direction ambiguity, the sensor is designed such that it is operated within a linear range of one fringe as shown in Fig. 3.

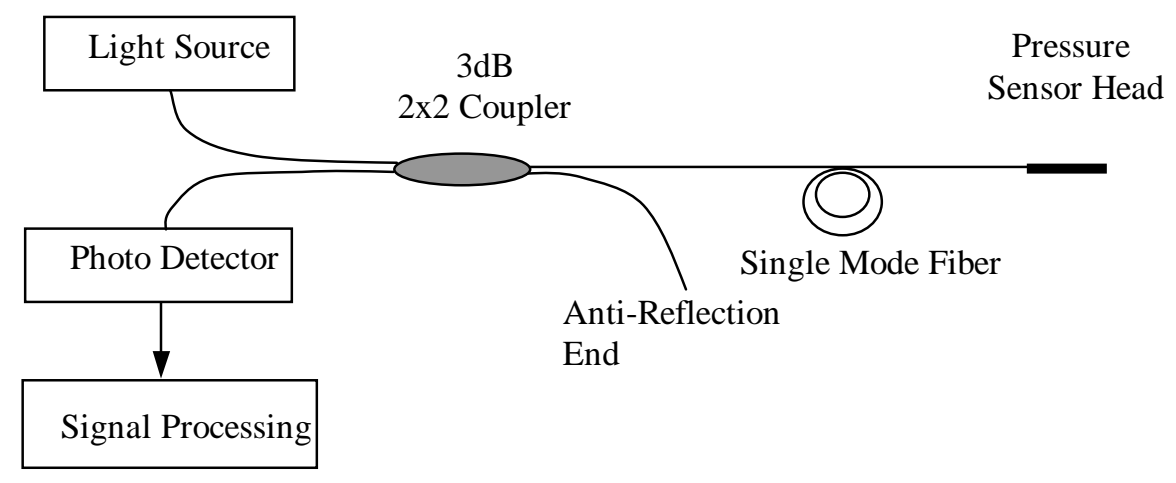

Fig. 1. Schematic of diaphragm-ferrule based fiber optic pressure sensor interrogation system.

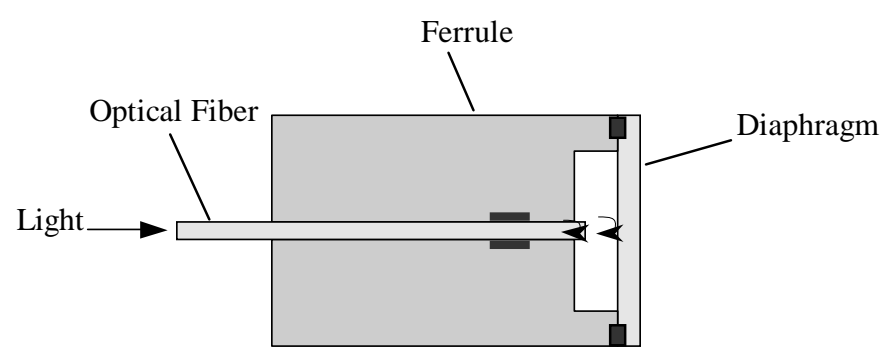

Fig. 2. Diaphragm-based fiber optic pressure sensor. 


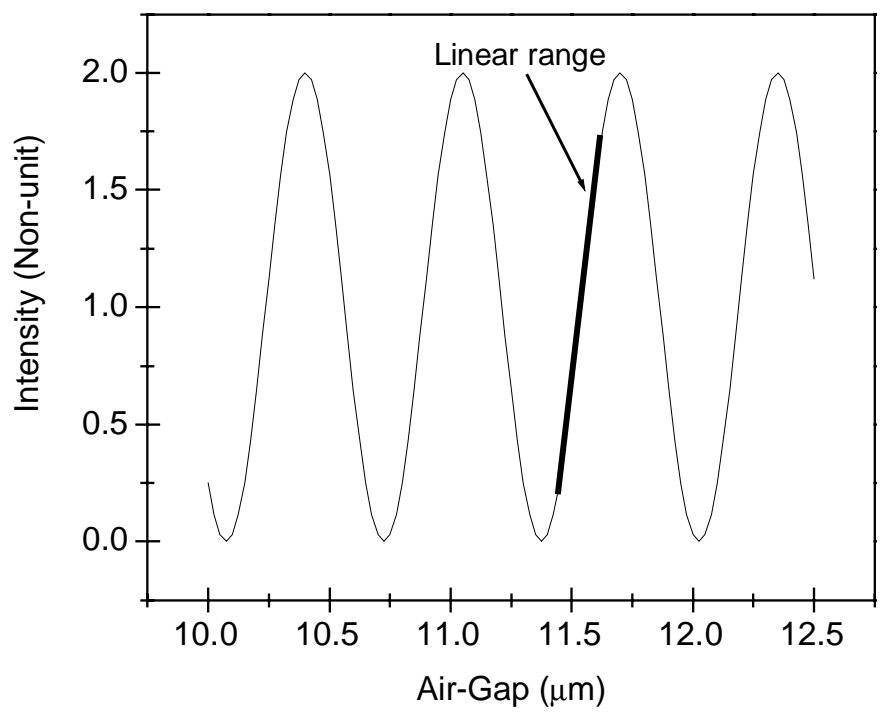

Fig. 3. Illustration of interference fringes versus sensor air-gap.

\section{SENSOR DESIGN}

\subsection{Diaphragm deflection under pressure}

A round diaphragm clamped rigidly at its edges is shown in Fig. 4. The diaphragm will be deflected under a uniform pressure P. The out-of-plane deflection of the diaphragm $y$ is a function of the pressure difference and the radial distance: ${ }^{[10]}$

$$
y=\frac{3\left(1-\mu^{2}\right) P}{16 E h^{3}}\left(r^{2}-d^{2}\right)^{2}
$$

$$
\text { where } \begin{aligned}
& y=\text { deflection } \\
& P=\text { pressure } \\
& h=\text { diaphragm thickness } \\
& r=\text { effective diaphragm radius } \\
& E=\text { Young's Modulus } \\
& d=\text { radial distance } \\
& \mu=\text { Poisson's Ratio }
\end{aligned}
$$

Usually, we define the ratio between the deflection and the pressure difference as the diaphragm pressure sensitivity (Y). When the optical fiber is positioned to face the center of the diaphragm, only the center deflection $\mathrm{y}_{\mathrm{c}}$ is of interest, and $\mathrm{Y}_{\mathrm{c}}$ (for fused silica material at $25^{\circ} \mathrm{C}$ ) is given by

$$
Y_{c}=1.71 \times 10^{-8} \frac{r^{4}}{h^{3}}(\mu \mathrm{m} / \mathrm{psi}),
$$

where $\mathrm{r}$ and $\mathrm{h}$ are in microns. 


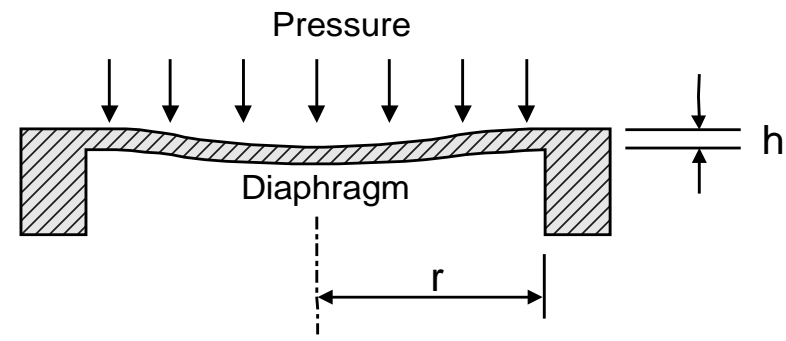

Fig. 4. Deflection of a rigidly clamped flat diaphragm under uniform pressure.

The relationships between pressure sensitivity and $\mathrm{r}$ and $\mathrm{h}$ are illustrated in Fig. 5. Since the central wavelength of the light source in the sensor system is about $1300 \mathrm{~nm}$, the linear range of the sensor falls within about $250 \mathrm{~nm}$ of air-gap variation. This linear range is related to a $0.6 \sim 1 \mathrm{~V}$ voltage output change, which is $0.8 \mathrm{~V}$ on average. The resolution of the sensor signal interrogation system is about $2 \mathrm{mV}$; to achieve a resolution of $0.1 \mathrm{psi}$ in pressure measurement, the diaphragm sensitivity should be higher than $6.25 \mathrm{~nm} / \mathrm{psi}$. If the effective diaphragm diameter is $1.5 \mathrm{~mm}$, the diaphragm thickness should be no more than $95 \mu \mathrm{m}$.

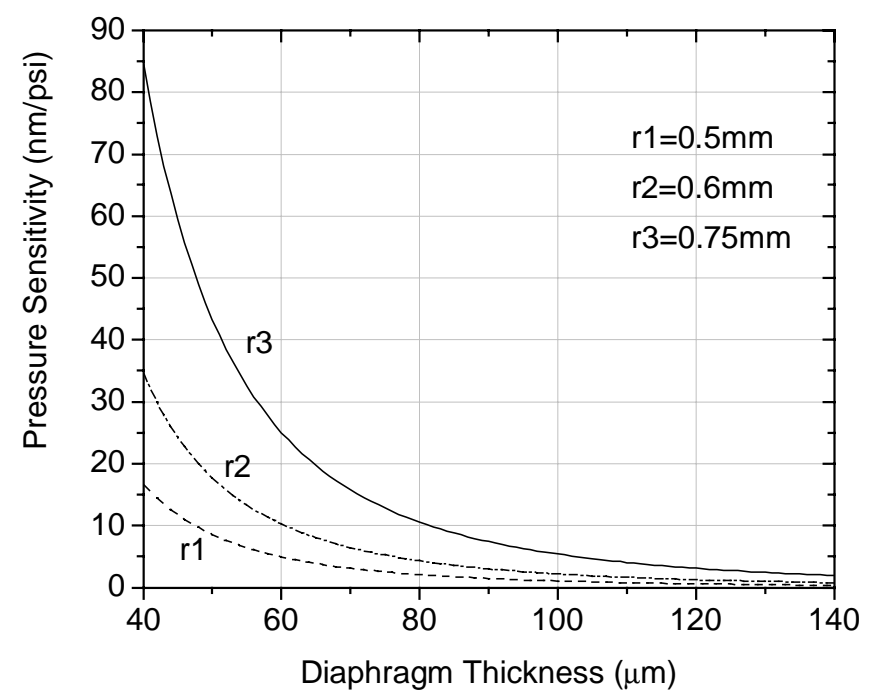

Fig. 5. Theoretical pressure sensitivities of the sensor for three different diaphragm radius values.

\subsection{Diaphragm frequency response}

In addition to the consideration for diaphragm sensitivity, the diaphragm frequency response is another important issue. We define the diaphragm as a free vibrating circular plate clamped rigidly at the edge. Its natural frequency $f_{m n}$ is expressed follows ${ }^{[10]}$ :

$$
f_{m n}=\frac{\alpha_{m n}}{4 \pi} \sqrt{\frac{E}{3 w\left(1-\mu^{2}\right)}}\left(\frac{h}{r^{2}}\right)
$$

where $\alpha_{m n}$ is a constant related to the vibrating modes of the diaphragm 
$h$ is the thickness of the diaphragm

$r$ is the effective diaphragm radius

$w$ is mass density of the diaphragm material

$\mu$ is the Poisson's ratio

$E$ is the Young's modulus of the diaphragm material

For the lowest natural frequency, $\alpha_{00}=10.21$, and based on the properties of fused silica, the frequency response of the diaphragm can thus be calculated as follows:

$$
f_{00}=2.742 \times 10^{9} \frac{h}{r^{2}}(\mathrm{~Hz})
$$

where $h$ and $r$ are in microns.

The relationships between frequency response and $r$ and $h$ are illustrated in Fig. 6. In the engine sensor field test described below, the blade passing frequency (BPF) is around $6 \mathrm{kHz}$, which is the fundamental frequency. The higher order harmonic frequencies might exceed $36 \mathrm{kHz}$. In order to faithfully respond to these dynamic pressures, the sensor natural resonant frequency should be at least three to five times as high as the highest applied frequency. To obtain a flat frequency response from $\mathrm{DC}$ to $50 \mathrm{KHz}$, let $f_{00}>250 \mathrm{kHz}$.

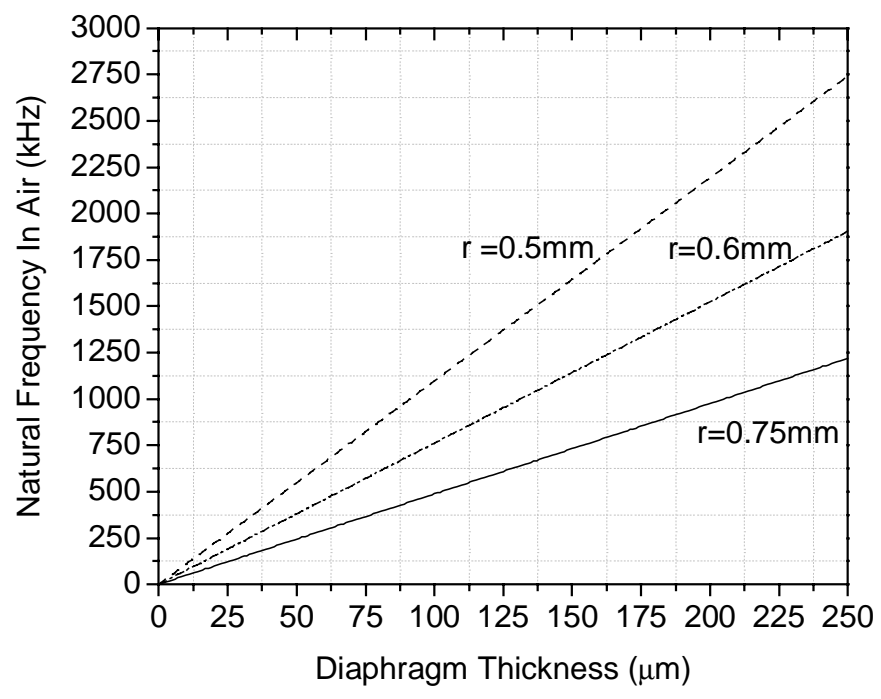

Fig. 6. Theoretical natural frequency of the sensor.

As indicated by Equation (3), the sensor's frequency response is proportional to the thickness of the diaphragm and inversely proportional to the square of the effective diaphragm radius, which is in contradiction with the diaphragm sensitivity. Therefore, some tradeoff must be made in sensor design. We chose the diaphragm thickness $h=60 \mu \mathrm{m}$ and its effective radius $r=0.75 \mathrm{~mm}$, so the calculated pressure sensitivity and natural resonant frequency is $292 \mathrm{kHz}$ and $25 \mathrm{~nm} / \mathrm{psi}$, which meets the requirements.

\section{SENSOR FABRICATION}

As shown in Fig. 7., a $60 \mu \mathrm{m}$ thick fused silica wafer was bonded to the top of the ferrule, which was also made of fused

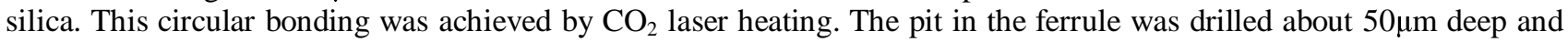
$1.5 \mathrm{~mm}$ in diameter, which will allow the diaphragm to deflect in response to pressure variations outside. To bond the fiber and the ferrule, the laser beam was defocused and directed to the side of the rotating ferrule and fiber. The ferrule and fiber were rotated clockwise and then counterclockwise alternately, to avoid twisting the fiber tail. 

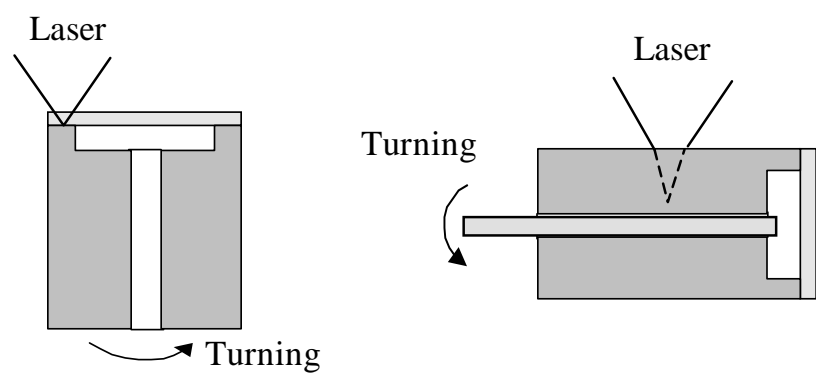

Fig. 7. Illustration of diaphragm to ferrule and fiber to ferrule thermal bonding.

The size of the sensor head is $8 \mathrm{~mm}$ long and $1.8 \mathrm{~mm}$ in diameter. Fig. 8 is a photo of the unpackaged sensor and the packaged one is shown in Fig. 9. In the sensor package, a stainless steel 316 fitting with 10-32 UNF-2A threads was used. The hex to tip length is about $11.3 \mathrm{~mm}$. The sensor fiber tail was also protected.

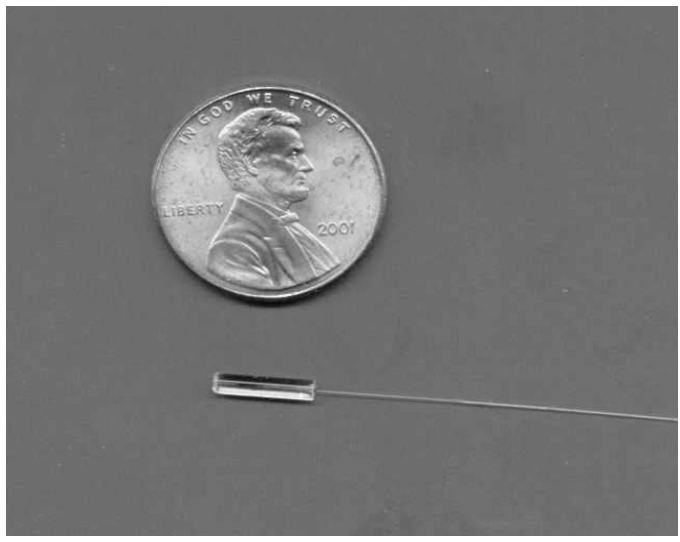

Fig. 8. Photo of the engine pressure sensor.

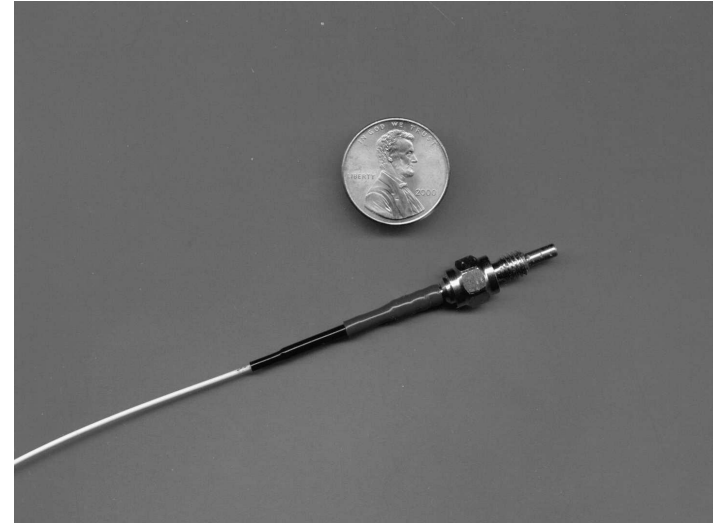

Fig. 9. Photo of the packaged engine pressure sensor.

\section{SENSOR CALIBRATION}

The schematic of the static pressure test system is shown in Fig. 10. The sensor head is sealed in a closed end ceramic tube with its fiber tail connected to the sensor interrogation system. The static pressure inside the ceramic tube is controlled by the pressure control system (Model 9035, Pressure Systems), which is connected to a compressed nitrogen gas tank.

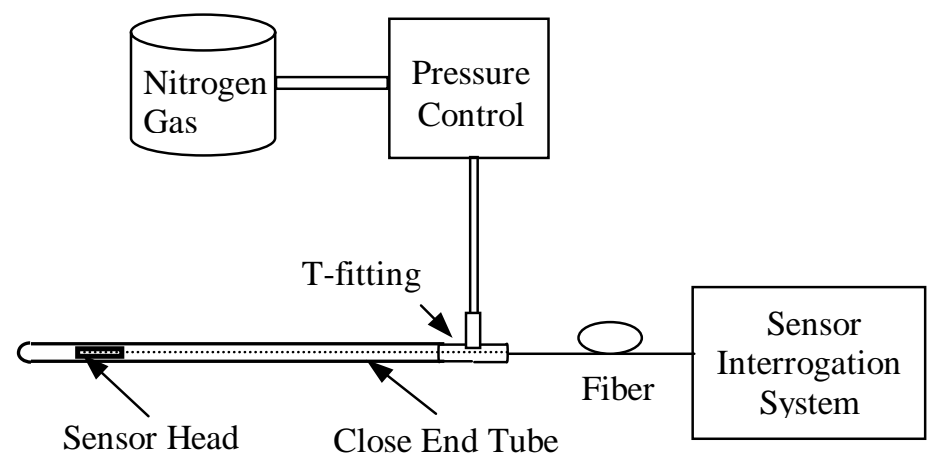

Fig. 10. Schematic of the controlled pressure testing system. 
The static pressure response of the pressure sensor is shown in Fig. 11. The sensor pressure sensitivity is about $87 \mathrm{mV} / \mathrm{psi}$. Therefore, the sensor system resolution is about $0.023 \mathrm{psi}$ (159Pa). To investigate the sensor response to dynamic pressure thoroughly, a dynamic pressure calibration will be needed, such as a shock tube testing. ${ }^{[1]}$

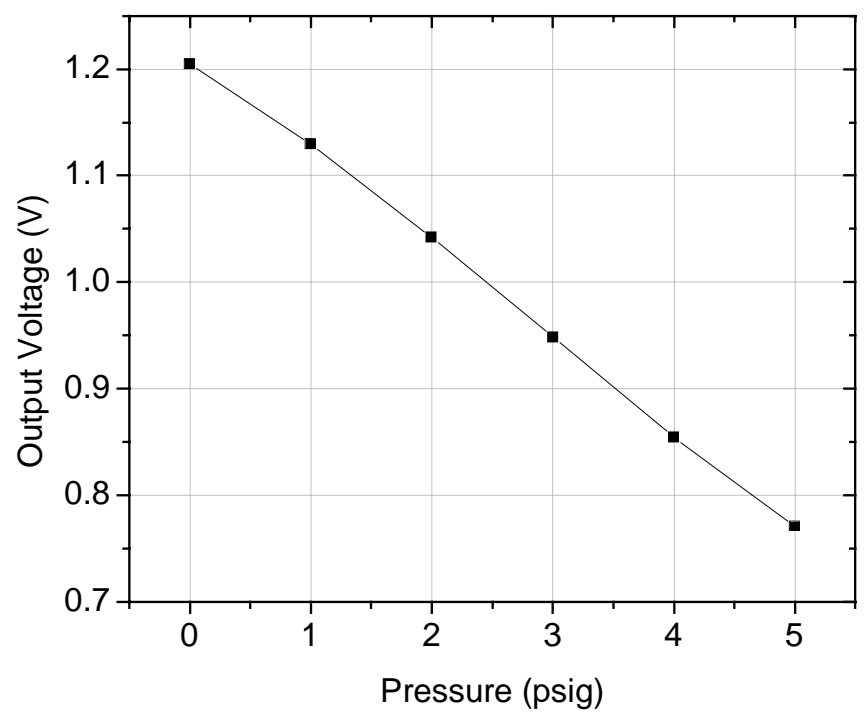

Fig. 11. Static pressure response of the diaphragm-ferrule based pressure sensor.

\section{ENGINE SENSOR FIELD TEST}

The turbine engine for the sensor field test in Blacksburg, Virginia, U.S., is a F-109 turbofan test engine manufactured by Allied Signal. The fan consists of 30 blades and the pressure sensors were flush mounted on the in-let aluminum ring near the blades (see Fig. 12. and Fig. 13).

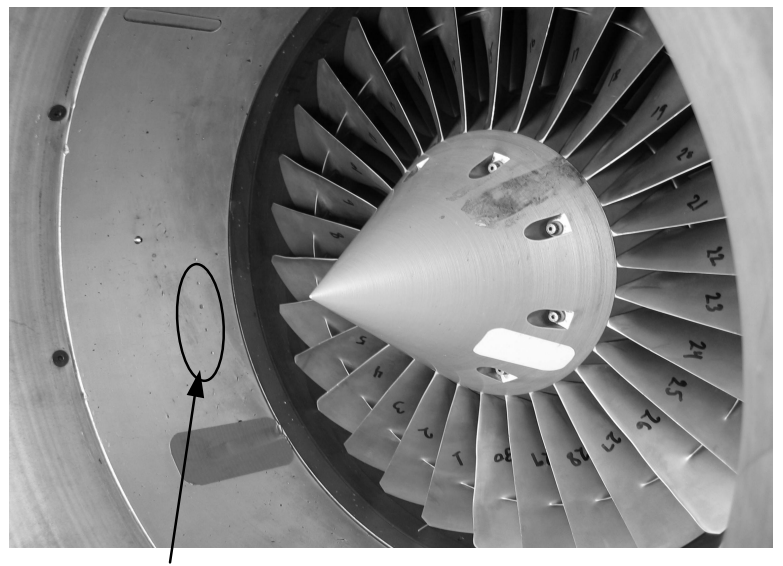

Flush Mount Holes

Fig. 12. Engine pressure sensor installation.

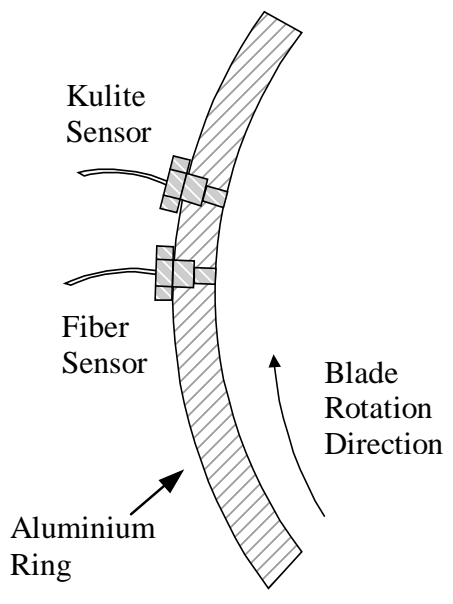

Fig. 13. Illustration of the sensor installation. 
The time domain and frequency domain signals for the diaphragm-ferrule based sensor are shown in Fig. 14 and 15, respectively. In the time domain signals, there is a phase difference (time delay) between the two signals due to the slightly different installation locations. The signal of the Kulite sensor had about $0.06 \mathrm{~ms}$ delay compared with that of the fiber sensor. This is because the two sensors were installed at different locations in the engine inlet ring. (see Fig. 13.) The time domain signals were processed using the Matlab FFT function to obtain the frequency data listed in Table 1. The results of the Kulite and diaphragm-based sensors are very consistent.
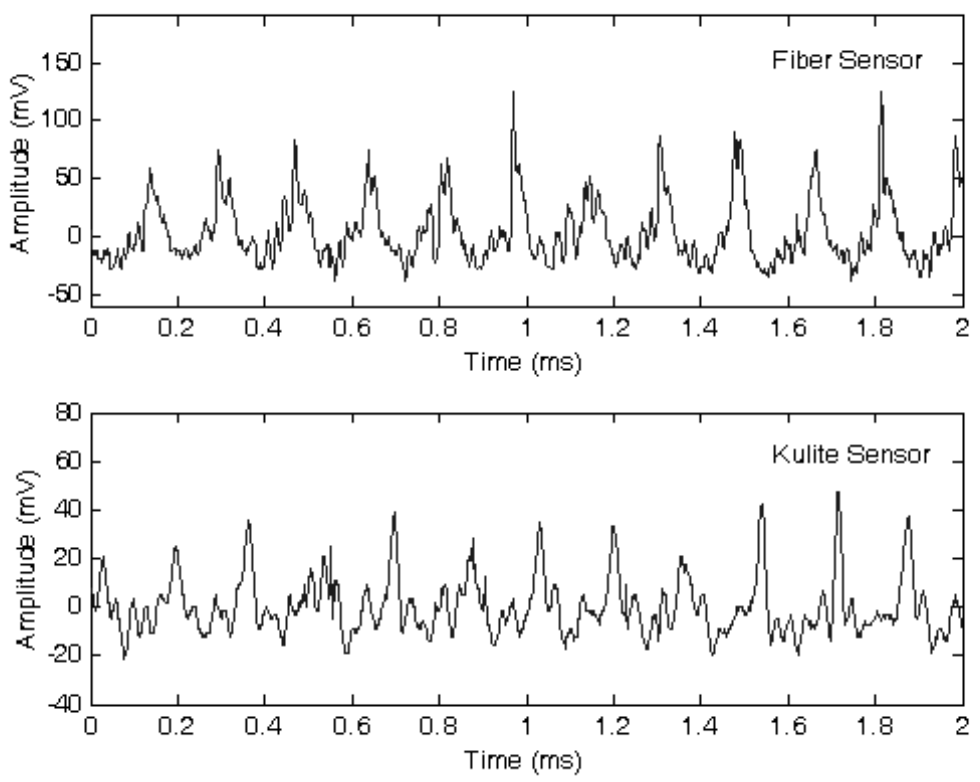

Fig. 14. Diaphragm-ferrule based fiber sensor and Kulite sensor signals in time domain
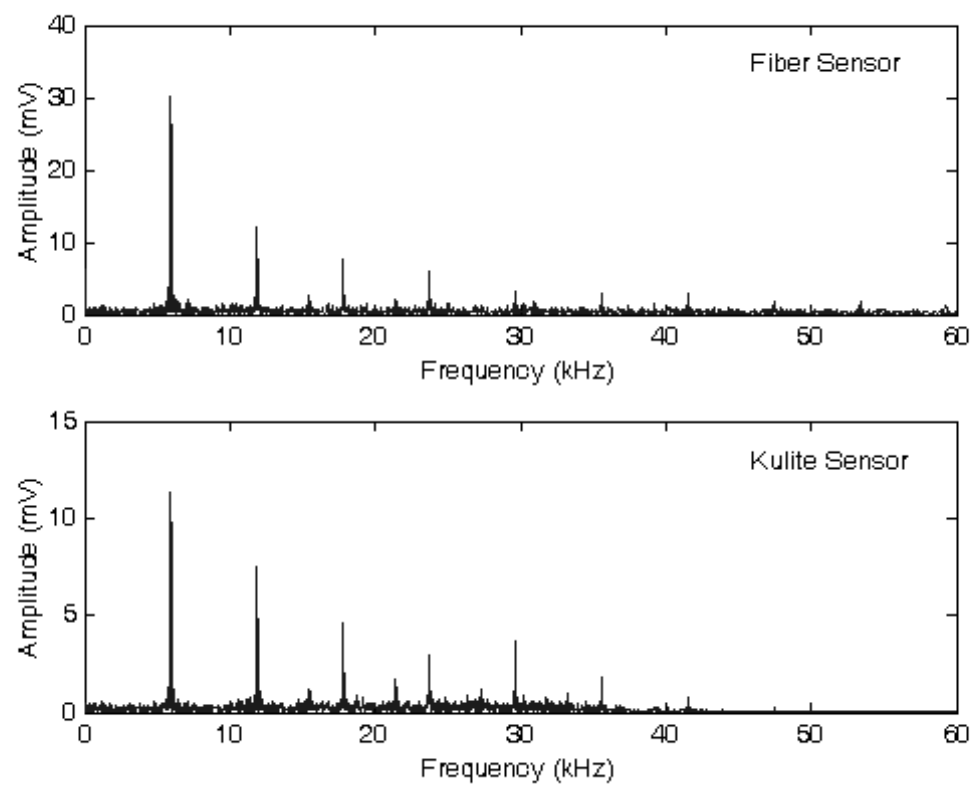

Fig. 15. Diaphragm-ferrule based fiber sensor and Kulite sensor signals in frequency domain. 
Table 1. Main frequencies measured by Kulite and diaphragm-ferrule based sensors.

\begin{tabular}{|c|c|c|c|c|}
\hline \multirow{2}{*}{} & \multicolumn{2}{|c|}{ Frequency (KHz) } & \multicolumn{2}{c|}{ Amplitude (mV) } \\
\cline { 2 - 5 } & $\begin{array}{c}\text { Diaphragm-Ferrule } \\
\text { Sensor }\end{array}$ & Kulite Sensor & $\begin{array}{c}\text { Diaphragm-Ferrule } \\
\text { Sensor }\end{array}$ & Kulite Sensor \\
\hline 1 & 5.930 & 5.930 & 30.0634 & 11.3772 \\
\hline 2 & 11.860 & 11.865 & 12.1294 & 7.4924 \\
\hline 3 & 17.795 & 17.800 & 7.7381 & 4.5748 \\
\hline 4 & 23.725 & 23.720 & 6.0433 & 2.9771 \\
\hline 5 & 29.660 & 29.660 & 3.2610 & 3.7025 \\
\hline 6 & 35.590 & 35.595 & 2.9957 & 1.8222 \\
\hline
\end{tabular}

The fundamental frequency at $5.93 \mathrm{kHz}$ is due to the effect of the blades passing since the rotation speed of the fan is set to about $12000 \mathrm{rpm}$. By precise calculation, the actual fan rotation speed is

$$
5.93 \times 1000 H z \times 60 s / 30=11860(r p m) .
$$

According to the Kulite pressure sensor calibration data, the output of the sensor is $33.3 \mathrm{mV} / \mathrm{psi}$. The peak to peak output at $5.93 \mathrm{kHz}$ was $22.7544 \mathrm{mV}$, resulting in a dynamic pressure of:

$$
P_{\text {kulite }}=\frac{22.7544 m V}{33.3 m V / p s i}=0.683(\text { psid }) .
$$

Based on the diaphragm-ferrule fiber sensor calibration data, the sensor output is $87 \mathrm{mV} / \mathrm{psi}$. The peak to peak output at $5.93 \mathrm{kHz}$ was $60.1268 \mathrm{mV}$, resulting in a dynamic pressure of:

$$
P_{\text {fiber }}=\frac{60.1268 m V}{87.0 m V / p s i}=0.691(\text { psid }) \text {. }
$$

This result is consistent with that of the reference Kulite sensor.

\section{CONCLUSIONS}

The all silica diaphragm sensor design offers high bandwidth $(>50 \mathrm{kHz})$. The engine pressure sensor exhibited a sensitivity of $87.0 \mathrm{mV} / \mathrm{psi}(12.6 \mathrm{mV} / \mathrm{kPa})$ and a resolution about $0.023 \mathrm{psi}(159 \mathrm{~Pa})$. The engine field test result confirms that the fiber optic sensor design can function safely and reliably near the engine fan. During a field test of about two hours, the optical sensors' package was robust enough for engine operation. In addition, the optical sensor was able to measure the acoustic pressure of $5.93 \mathrm{kHz}$ and $0.7 \mathrm{psi}(4.8 \mathrm{kPa})$ near the engine fan. Its performance is consistent with that of the commercial Kulite sensor. Further work will investigate the sensor pressure response at elevated temperatures and its temperature cross sensitivity. This fiber optic sensor was made entirely of silica glass, allowing operating temperatures well above $500^{\circ} \mathrm{C}$, which is higher than a conventional semiconductor based pressure sensor. 


\section{ACKNOWLEDGEMENTS}

This work was supported by US Department of Energy (DOE) under contract DE-FC36-01GO11050. The authors would like to thank Dr. Wing Ng, Aditya Ringshia and Hugh Hill of the Dept. of Mechanical Engineering of Virginia Tech. for providing the engine test facilities and technical support, and Dr. Bo Song for many helpful discussions.. The authors appreciate the technical assistance of Frank Caldwell.

\section{REFERENCES}

1. Rao, Y.J., Jackson, D.A., Jones, R. and Shannon, C. "Development of prototype fibre-based Fizeau pressure sensors with temperature compensation.” J. Lightwave Technol., Vol. 12, pp. 1685-1695, 1994.

2. Belovlov, M.I.; Bubnov, M.M.; Semjonov, S.L.; "High Sensitive Fiber Interferometric Pressure Sensor", Lasers and Electro-optics Europe, 1996. CLEO/Europe Conference, pp. 192-192. Sept. 1996.

3. O. Tohyama, M. Kohasi, M. Sugihara, H. Itoh, "A fiber-optic pressure microsensor for biomedical applications", Sensors and Actuators, A 66, pp. 150-154, 1998.

4. Jiangdong. Deng, Hai Xiao, Wei Huo, Ming Luo, Russell G. May, Anbo Wang and Yilu Liu, " Optical Fiber Sensor-based detection of Partial Discharges in Power Transformers ", Optics and Laser Technology, Vol. 33, No. 5, pp. 305-311, July 2001.

5. Bing Yu, Dae Woong Kim, Jiangdong Deng, Hai Xiao, and Anbo Wang, "Fiber Fabry-Perot Sensors for Detection of Partial Discharges in Power Transformers", Applied Optics-OT, Vol. 42 Issue 16, pp. 3241-3250, June 2003.

6. Gander, M.J., et al, "Embedded micromachined fiber-optic Fabry-Perot pressure sensors in aerodynamics applications", IEEE Sensors Journal, Vol. 3, pp. 102-107, Feb. 2003.

7. Toshima, K.; Watanabe, T.; Kaneko, Y.; Maesako, T.; Takahashi, K., "Diaphragm-Type Optical Fiber Pressure Sensor with a Sleeve for Fiber Insertion", Micromechatronics and Human Science, 2003. MHS 2003. Proceedings of 2003 International Symposium, pp. 171-174, 19-22 Oct. 2003.

8. Bing Yu, Gary R. Pickrell, and Anbo Wang, "Grating-assisted operating-point tuning for fiber optic Fabry-Perot interferometric sensors," Proceedings of SPIE Vol. 5272, pp. 41-48 (1899), 2004.

9. A. Wang, H. Xiao, J. Wang, Zhiyong Wang, W. Zhao and R. G. May, "Self-Calibrated Interferometric-IntensityBased Optical Fiber Sensors,” J. Lightwave Technol., Vol. 19, pp.1495-1501, 2001.

10. M. D. Giovanni, Flat and Corrugated Diaphragm Design Handbook. New York: Mercel Dekker, 1982.

11. J. Zhou, S. Dasgupta, H. Kobayashi, J. M. Wolff, H. E. Jackson, and J. T. Boyd, "Optically Interrogated MEMS Pressure Sensors for Propulsion Applications,” Optical Engineering, Vol. 40, pp. 598-604, April, 2001. 


\title{
Miniature fiber-optic pressure sensor for turbine engine
}

\author{
Yizheng Zhu*a , Gary Pickrell ${ }^{\mathrm{a}}$, Xingwei Wang ${ }^{\mathrm{a}}$, Juncheng Xü ${ }^{\mathrm{a}}$, Bing Yu ${ }^{\mathrm{a}}$, Ming $\mathrm{Han}^{\mathrm{a}}$, \\ Kristie Cooper ${ }^{\mathrm{a}}$, Anbo Wang ${ }^{\mathrm{a}}$, Aditya Ringshia ${ }^{\mathrm{b}}$, Wing $\mathrm{Ng}^{\mathrm{b}}$ \\ ${ }^{\mathrm{a}}$ Center for Photonics Technology, \\ The Bradley Department of Electrical and Computer Engineering, \\ ${ }^{\mathrm{b}}$ Department of Mechanical Engineering, \\ Virginia Polytechnic Institute and State University, Blacksburg, VA, 24061
}

\begin{abstract}
In this paper, we present a miniature fiber optic pressure sensor. The sensor is extrinsic Fabry-Perot interferometer (EFPI) based with its FP cavity directly fabricated on the tip of the fiber by fusion splicing and chemical etching. The processes are simple, with no other materials but silica fibers involved. The sensor has the same dimension as the fiber itself, only $125 \mu \mathrm{m}$ in diameter. The length of the FP cavity and the interference pattern will change in response to ambient pressure variation. The signal is demodulated by tracing the spectrum shift. Sensitivity can be adjusted for different applications with low or high pressure range. Prototype sensors were fabricated and tested for static response. Dynamic measurements were performed in a turbine engine. Theoretical and experimental analysis of the sensor response are also presented.
\end{abstract}

Keywords: fiber optic sensor, miniature, pressure sensor

\section{INTRODUCTION}

Pressure sensors have been widely used in a large number of applications from automotive and aerospace industries to healthcare. Miniature sensors are preferred in many situations because of the minimized impact on the measured system. Significant efforts have been exerted to fabricate these small sensors, either electrically or optically interrogated. Compared to capacitive or piezoresistive sensors, ${ }^{1-2}$ interferometric optical fiber sensors offer the advantages of high resolution, high sensitivity, intrinsic electrical passivity and immunity to electromagnetic interference. Miniature fiber optic sensors based on Fabry-Perot interferometry have been reported using various approaches $^{3-5}$. In this paper, we demonstrate a novel miniature pressure sensor fabricated directly on the tip of a fiber, using only fusion splicing, cleaving and wet chemical etching, all of which are simple and cost-effective processes.

Current fiber-optic sensors usually employ several different materials in fabrication. The proposed sensor is made of silica fibers only and thus has an all-fused-silica structure. Thermal expansion mismatch between different materials is eliminated in this sensor, whose high temperature capability is only limited by silica itself. Temperature dependence is also minimized because of the extremely small coefficient of thermal expansion (CTE) of fused silica. Therefore this miniature sensor has great potential to operate reliably at elevated temperatures. It may find applications where high temperature and/or small size are important.

The paper is organized as follows: section 2 introduces the fabrication steps of the miniature sensor; the system setup and results of static tests are discussed in section 3; then section 4 presents and analyzes the dynamic pressure measurement results in a turbine engine.

*yizhu1@vt.edu; phone 1-540-231-8359; fax 1-540-231-2158; http://www.ee.vt.edu/ photonics 


\section{SENSOR DESIGN AND FABRICATION}

\subsection{Sensor Fabrication}

Three silica fibers are used: single-mode (SM) fiber, 62.5/125 $\mu \mathrm{m}$ graded-index multimode (MM) fiber and 105/125 $\mu \mathrm{m}$ step-index MM fiber. The $62.5 / 125 \mu \mathrm{m}$ fiber is germanium-doped in the core with pure fused silica cladding, while the $105 / 125 \mu \mathrm{m}$ fiber has pure fused silica core and fluorine-doped cladding. Hydrofluoric acid (HF, 50\%) is used for wet chemical etching where doped silica will be etched much faster than updoped region ${ }^{6}$.

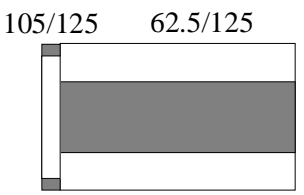

(a)

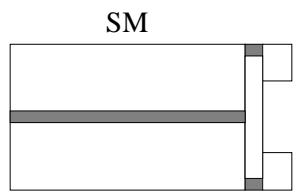

(b)

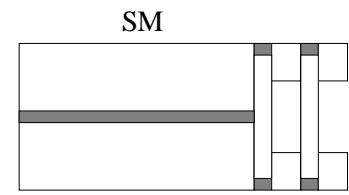

(c)

Fig. 1. Sensor fabrication steps

Figure 1 shows the fabrication process. (a) A $105 / 125 \mu \mathrm{m}$ fiber is fusion-spliced with $62.5 / 125 \mu \mathrm{m}$ fiber and cleaved under microscope to leave a thin layer of $105 / 125 \mu \mathrm{m}$ fiber. This undoped silica layer acts as both the etch stop and the diaphragm in the following steps. (b) A part in (a) is spliced with SM fiber and cleaved. The length of the 62.5/125 $\mu \mathrm{m}$ fiber will be approximately the length of the FP cavity. Immersing the tip in HF to remove the core of the 62.5/125 $\mu \mathrm{m}$ fiber. The etching rate for the doped $62.5 \mu \mathrm{m}$-core is about $17 \mu \mathrm{m} / \mathrm{min}$ in $50 \% \mathrm{HF}$ while that of undoped silica is only about $1 \mu \mathrm{m} / \mathrm{min}$. Therefore the etching "stops" at the barrier layer, generating a flat bottom and protecting the core of SM fiber from being etched. Otherwise, etching of the SM fiber core will reduce its reflection intensity. The cladding of the $62.5 / 125 \mu \mathrm{m}$ fiber is also pure fused silica and remains almost intact, left as the wall of the fabricated pit. (c) Splice the pit with a part in (a) to form the FP cavity. Reduced power and duration of the splicing arc are crucial to prevent the structure from being melted and collapsed by excess heat. Cleave the $62.5 / 125 \mu \mathrm{m}$ fiber and etch in HF. After the core of $62.5 / 125 \mu \mathrm{m}$ fiber is removed, the $105 / 125 \mu \mathrm{m}$ layer will be left as the diaphragm. Its thickness can be further reduced to increase the sensitivity of the sensor. The final structure is made of pure fused silica.

\section{STATIC PRESSURE TEST}

The sensor was tested for static pressure response using the system shown in Fig. 2. For faster data acquisition and better resolution, we used Micron Optics' Si720 Component Testing System (CTS) which is essentially a spectrometer with a build-in tunable laser source and a detector. Its spectral range spans from $1520 \mathrm{~nm}$ to $1570 \mathrm{~nm}$ with a wavelength resolution of $2.5 \mathrm{pm}$. Light from the CTS travels to the sensor through a circulator and is reflected back into the CTS. The sensor is sealed in a tube and pressurized by a pressure calibrator (Pressure Systems, Inc., 9035). A computer controls the pressure calibrator and retrieves spectrum data from the CTS.

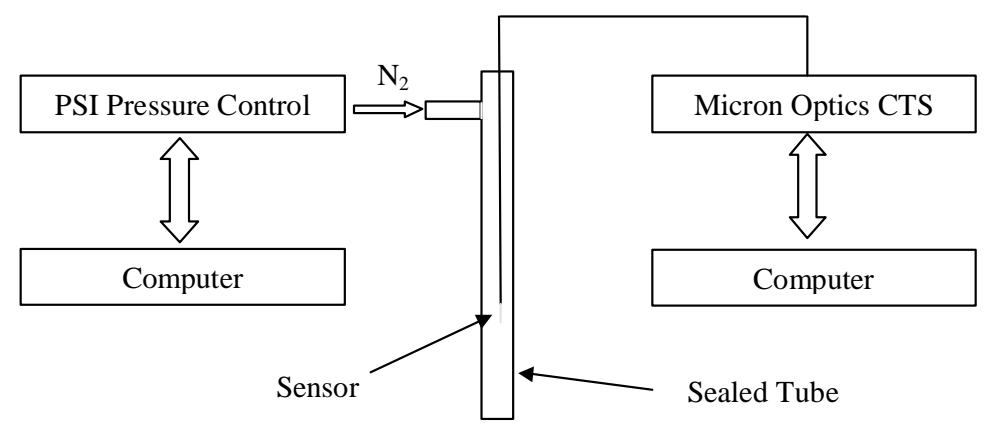

Fig. 2. Test system for sensor static response 
Figure 3 shows a typical CTS spectrum of the sensor, where the interference fringes are clearly seen. When ambient pressure varies, the spectrum will shift accordingly. The change of the sensor's cavity length can be determined by tracing the positions of interference minima/maxima (valley/peak). The cavity length then results by multiplying the position with the fringe order, as in Eq. (2).

$$
4 \pi L / \lambda+\pi=2 N \pi \Rightarrow L=(2 N-1) \lambda / 4
$$

where $\mathrm{L}$ is cavity length, $\lambda$ is valley/peak wavelength and $\mathrm{N}$ is fringe order. $\mathrm{N}$ and $\lambda$ are calculated from the spectrum.

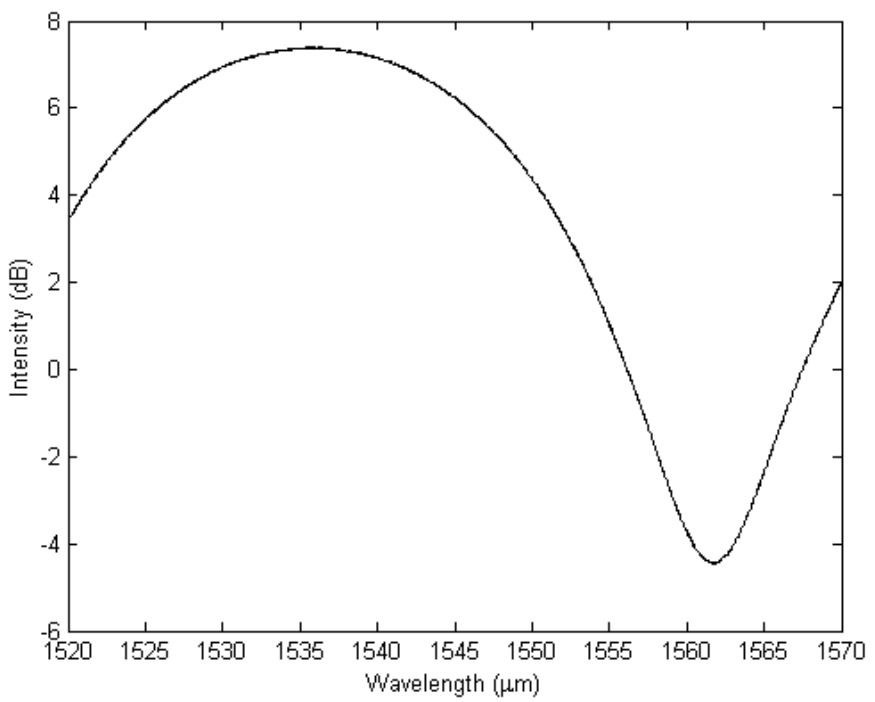

Fig. 3. Typical sensor spectrum by CTS

One of the sensors was pressurized at room temperature from 30 psi to $110 \mathrm{psi}$ for three times. The sensor's response is shown in Fig. 4, with excellent repeatability.

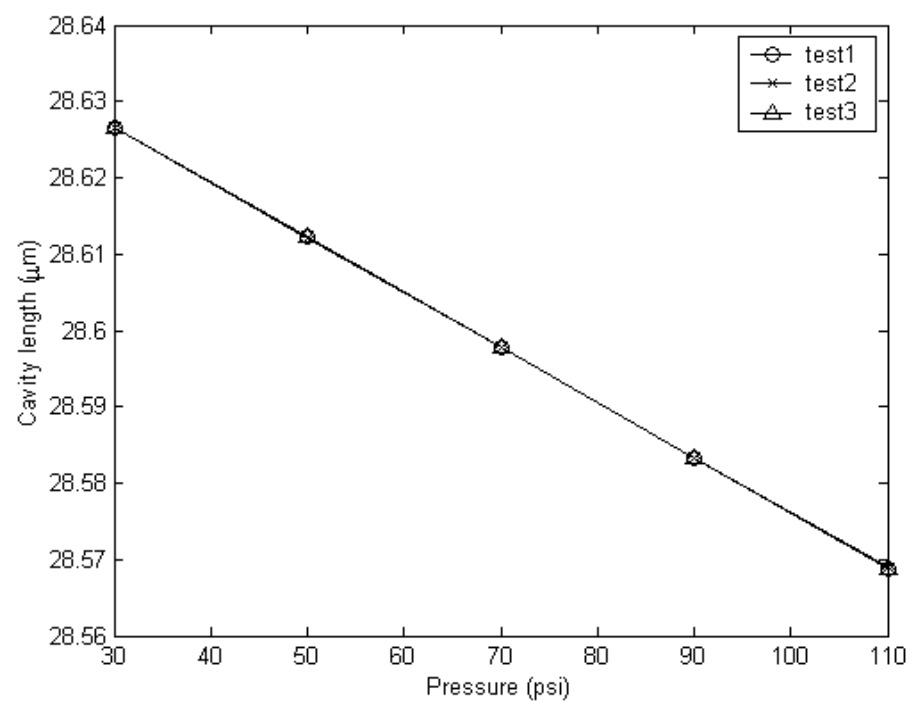

Fig. 4. Pressure test from 30psi to 110 psi for three times 


\section{DYNAMIC PRESSURE MEASUREMENT}

\subsection{Self-Calibrated Interferometric Intensity-Based (SCIIB) System}

The sensors were installed in a turbine engine for dynamic measurements. However, the system based-on spectral detection is limited in frequency response, usually unable to achieve beyond a few tens of Hertz and therefore unsuitable for dynamic pressure measurements. Instead, intensity-based systems using photodiodes as detectors are widely employed for such purposes. In our test, a self-calibrated interferometric intensity-based (SCIIB) system $^{7}$ was used. It is constructed as in Fig. 5.

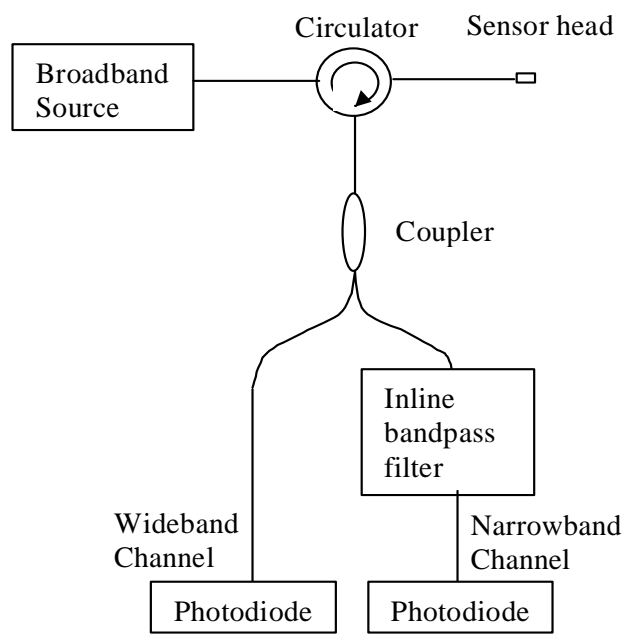

Fig. 5. Schematic diagram of a SCIIB system

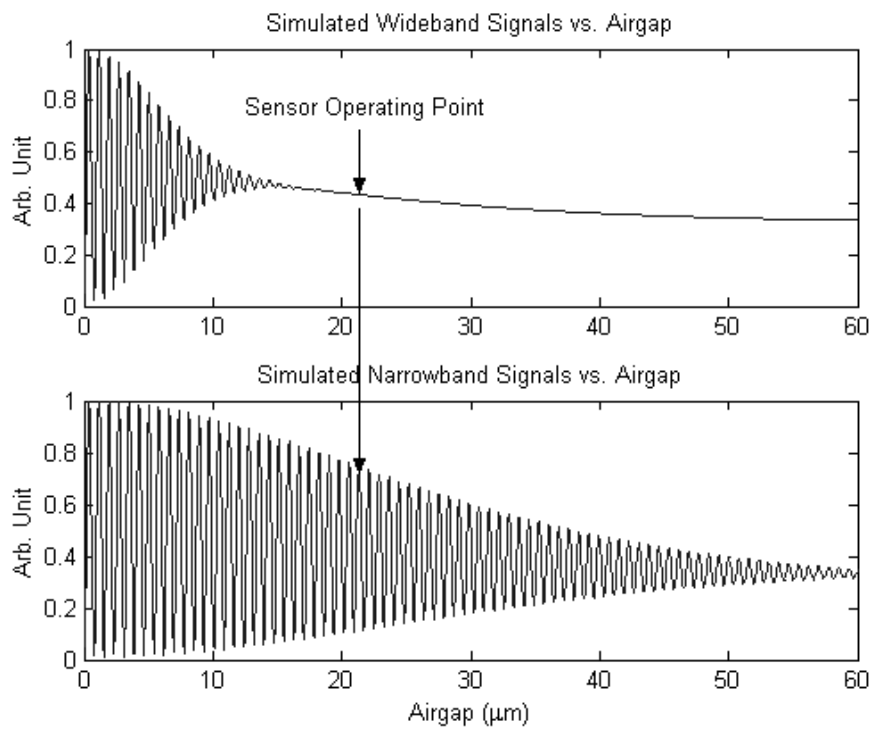

Fig. 6. Simulated wideband and narrowband signals of a SCIIB system 
The light from a broadband source propagates to the sensor head through a circulator. The reflected signal is divided into two channels by a coupler, wideband and narrowband. The latter is further filtered by an inline band-pass filter. Then the power in the two channels is detected by photodiodes. Figure 6 shows the simulated signal for both channels. The wideband channel has a shorter coherence length because of its broader bandwidth and hence tends to stabilize more quickly than narrowband channel. The initial cavity length of the sensor is chosen to be larger than the coherence length of the wideband channel. So when the cavity length changes around its initial value, the wideband channel will very much remain stable but the narrowband channel will still oscillate.

The ratio of the two channels can cancel out the power fluctuation due to source drift, fiber bending, etc. and improve the system stability and indicates the sensor response. SCIIB has advantages in dynamic pressure measurement, with a much higher frequency response compared to systems based on spectrum detection.

\subsection{Sensor packaging and installation}

Sensors were packaged in a metal fitting which was mounted into the wall of the engine inlet. A photo of the packaged sensors is shown in Fig. 7. Since the sensor size is so small, each fitting can house several of them. In the photo two sensors were packaged in the fitting. The fiber protruded the fitting about $1 \mathrm{~mm}$ in order to place the sensor at the same level as the inner wall of the inlet, as shown in a cross-section view of the sensor mounting in Fig. 8. Figure 9 and 10 show the sensor mounted on the wall from outside and inside, respectively. Along with the miniature sensors, a Kulite pressure sensor was also mounted on the engine.

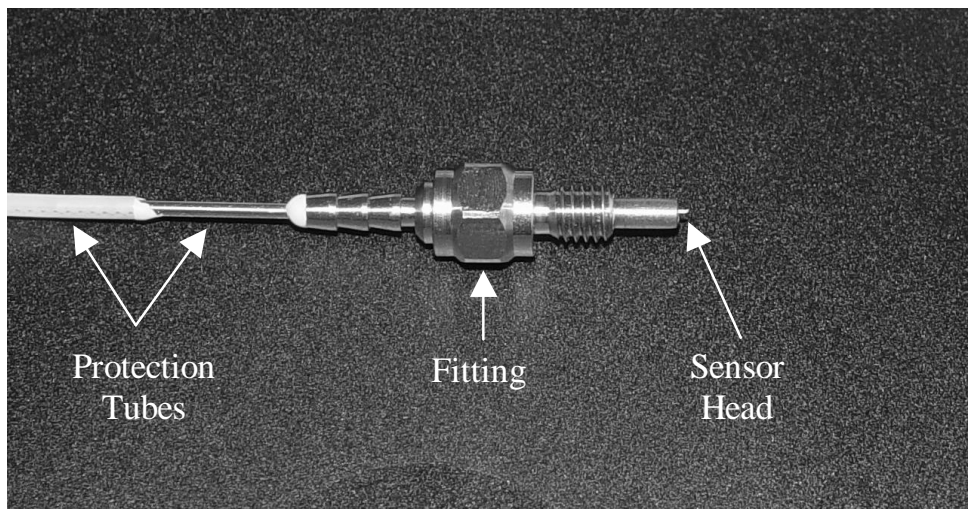

Fig. 7. A packaged miniature sensor

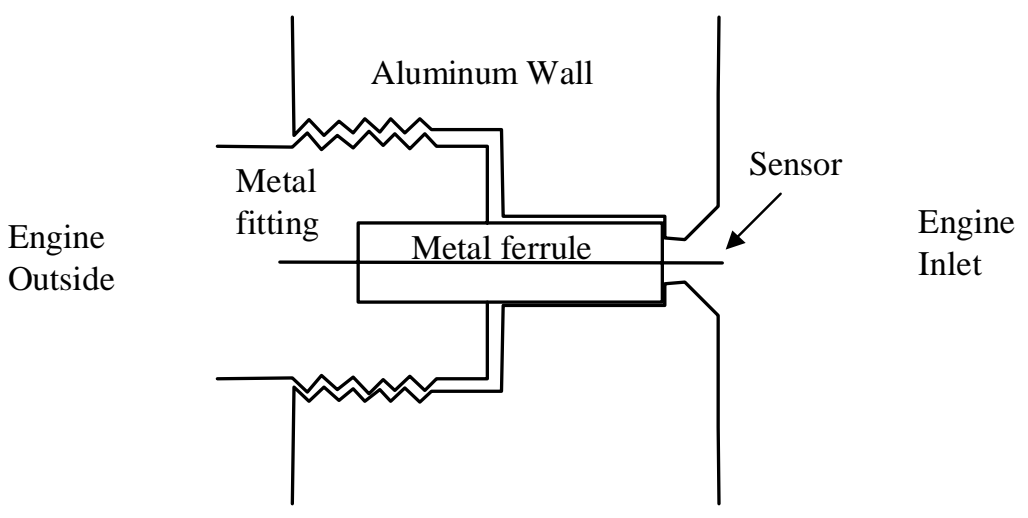

Fig. 8. A cross-section view of the sensor installation 


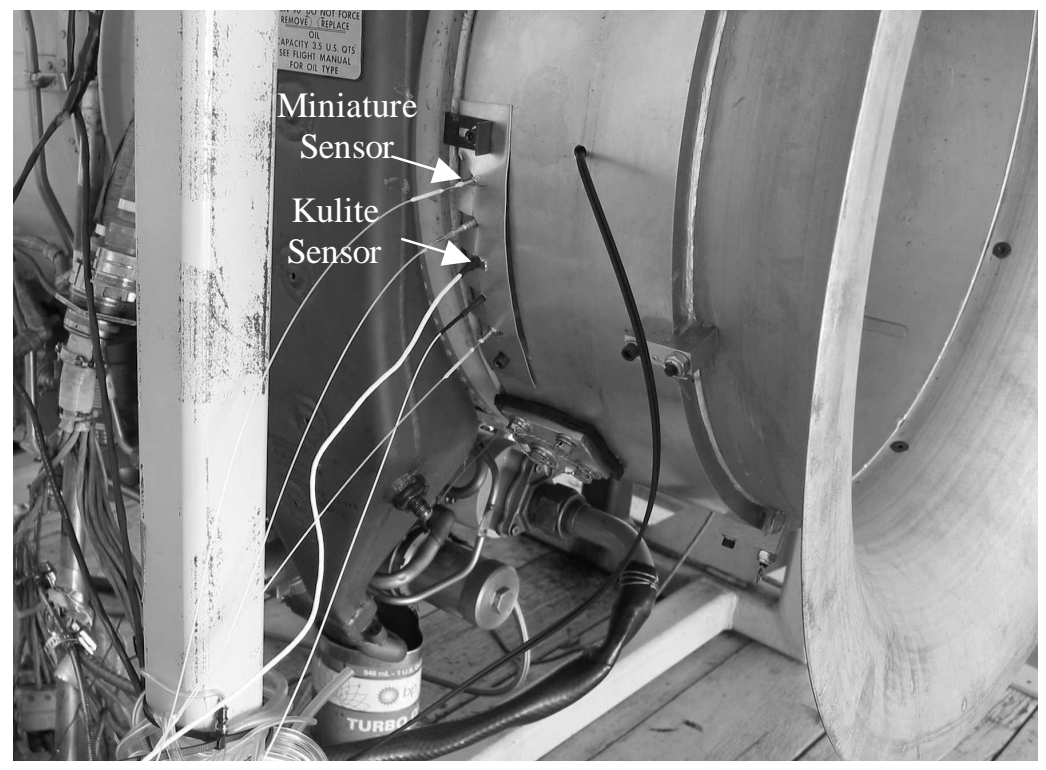

Fig. 9. The outside view of the engine with sensor mounted in the wall

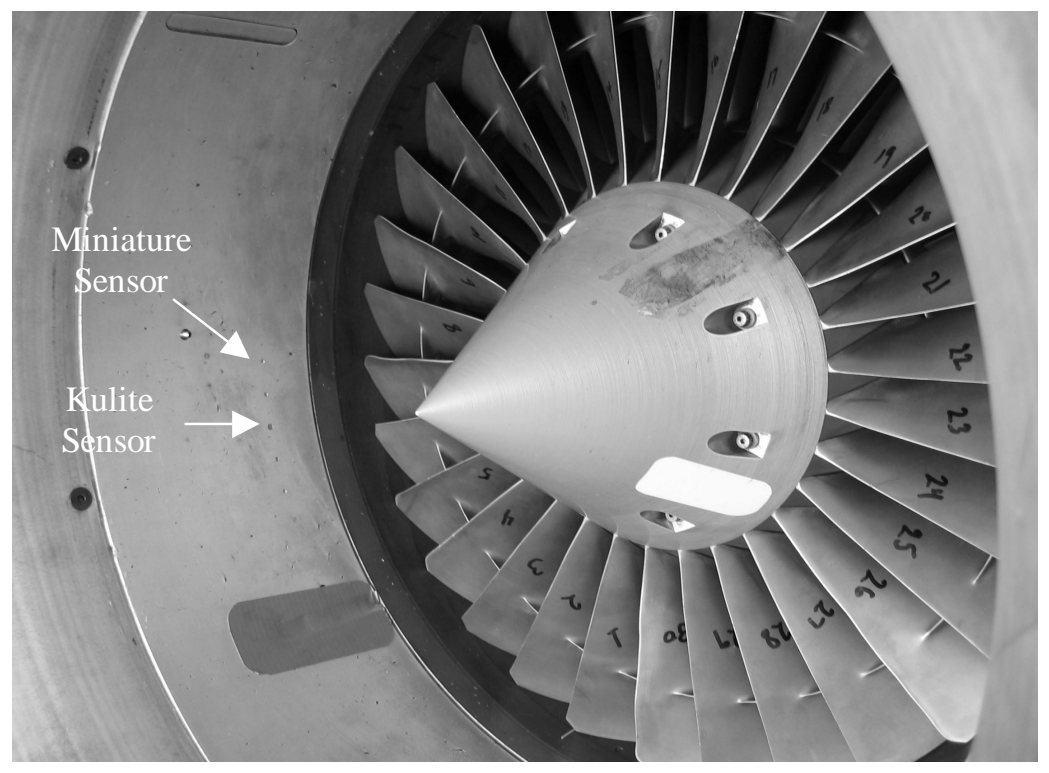

Fig. 10. The outside view of the engine with sensor mounted in the wall

\subsection{Test resutls}

In the test, the outputs of the photodiodes were recorded by a oscilloscope and sent to a computer through GPIB interface. Figure 11 show the pressure response of both the miniature sensor and the Kulite sensor. It can be seen that there is a time delay between the signals due to the different mounting positions. Figure 12 shows the Fourier transform of the signals. The fundamental harmonic is about $6 \mathrm{kHz}$, indicating the frequency of the blades' passing by. The frequencies and amplitudes of the all the harmonics are listed in Table 1 for both sensors. 

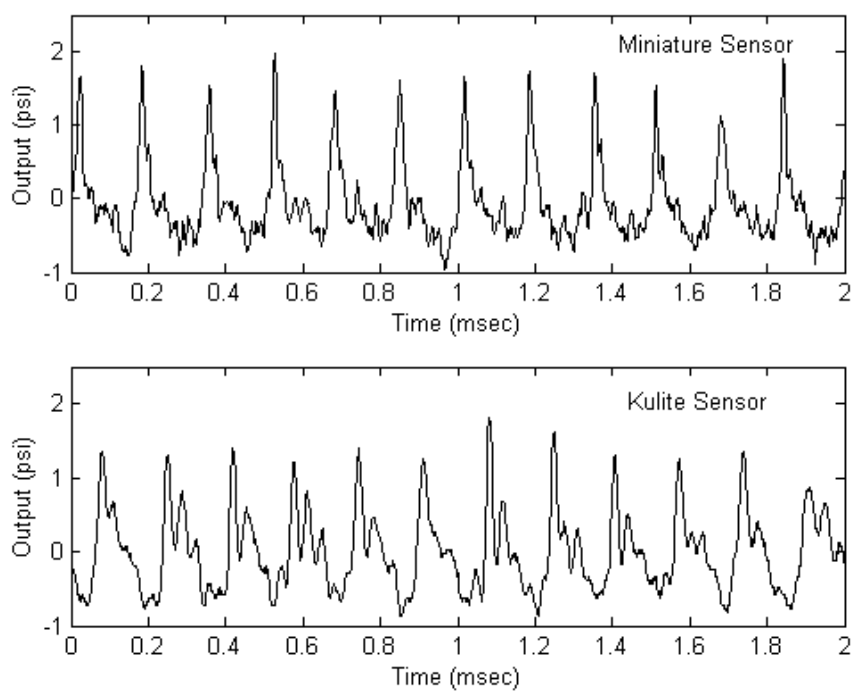

Fig. 11. FFT of the signals for both miniature sensor and Kulite sensor
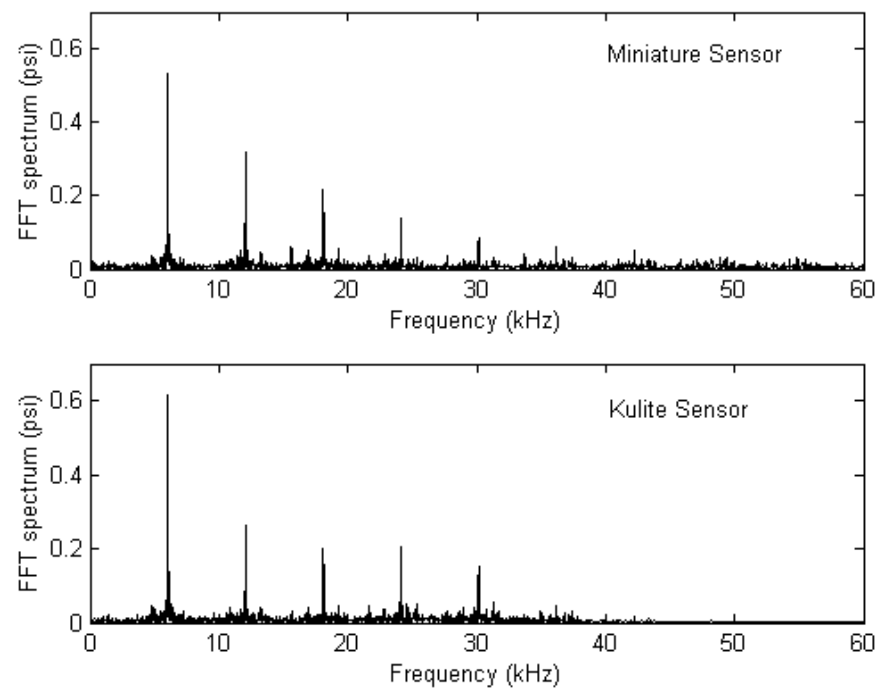

Fig. 12. Signals for both miniature sensor and Kulite sensor

Table 1. Frequencies and amplitudes of $1^{\text {st }}-6^{\text {th }}$ order harmonics for miniature sensor and Kulite sensor

\begin{tabular}{|c|c|c|c|c|}
\hline \multirow{2}{*}{ Peak \# } & \multicolumn{2}{|c|}{ Frequency $(\mathrm{kHz})$} & \multicolumn{2}{c|}{ Amplitude (psi) } \\
\cline { 2 - 5 } & Mini-sensor & Kulite & Mini-sensor & Kulite \\
\hline 1 & 6.030 & 6.030 & 0.533 & 0.614 \\
\hline 2 & 12.060 & 12.060 & 0.317 & 0.265 \\
\hline 3 & 18.085 & 18.090 & 0.216 & 0.201 \\
\hline 4 & 24.115 & 24.120 & 0.137 & 0.205 \\
\hline 5 & 30.145 & 30.145 & 0.084 & 0.151 \\
\hline 6 & 36.180 & 36.175 & 0.062 & 0.048 \\
\hline
\end{tabular}




\section{CONCLUSIONS}

In summary, this paper presents the fabrication and tests of a miniature fiber-optic sensor. The sensor showed excellent repeatability in the static test. For the dynamic measurements in a turbine engine, it showed comparable results with a semiconductor Kulite pressure sensor.

The sensor has a structure built by silica only, eliminating the thermal expansion mismatch and expected to be able to work at elevated temperature. Future work would involve investigation of the sensor's high temperature capability. The sensor has great potential to work at the hot section of the engine where conventional semiconductor pressure transducers are unable to apply.

\section{ACKNOWLEDGMENTS}

This research was sponsored by the U.S. Department of Energy under grant DE-FC36-01G011050. The authors would like to thank Mr. Hugh Hill and Mr. Frank Caldwell for their help in the engine field test, and to thank Micron Optics for the partial donation of the CTS used in this research.

\section{REFERENCES}

1. D. Goustouridis, P. Normand, and D. Tsoukalas, "Ultraminiature silicon capacitive pressure-sensing elements obtained by silicon fusion bonding", Sens. Actuators A, vol. A68, pp. 269-274, 1998.

2. P. Melvås, E. Kälvesten, P. Enoksson, and G. Stemme, "A free-hanging strain-gauge for ultraminiaturized pressure sensors", Sens. Acuators A, vol. 97-98, pp. 75-82, 2002.

3. T. Katsumata, Y. Haga, K. Minami, and M. Esashi, "Micromachined $125 \mu \mathrm{m}$ diameter ultra miniature fiber-optic pressure sensor for catheter," Trans. IEE Jpn., Part E, vol. 120-E, pp. 58-63, 2000.

4. E. Cibula, D. Donlagic, and C. Stropnik, "Miniature fiber optic pressure sensor for medical applications," in Proc. of IEEE Sensors 2002, First IEEE Int. Conf. on Sensors, Piscataway, NJ, 2002, vol. 1, pp.711-714.

5. D. C. Abeysinghe, S. Dasgupta, J. T. Boyd, and H. E. Jackson, "A novel MEMS pressure sensor fabricated on a optical fiber," IEEE Photon. Technol. Lett., vol. 13, pp. 993-995, Sep. 2001.

6. T. Pangaribuan, K. Yamada, S. Jiang, H. Ohsawa, and M. Ohtsu, "Reproducible fabrication technique of nanometric tip diameter fibre probe for photon scanning tunneling microscope," Jpn. J. Appl. Phys., vol. 31, pp. 1302-1304, 1992.

7. A. Wang, H. Xiao, J. Wang, Z. Wang, W. Zhao, and R. G. May, "Self-calibrated interferometric-intensity-based optical fiber sensors," J. Lightwave Technol., vol. 19, pp. 1495-1501, 2001. 


\title{
Fiber-optic high-temperature thermometer using sapphire fiber
}

\author{
Yizheng Zhu*, Zhengyu Huang, Ming Han, Fabin Shen, Gary Pickrell, Anbo Wang \\ Center for Photonics Technology, \\ The Bradley Department of Electrical and Computer Engineering, \\ Virginia Polytechnic Institute and State University, Blacksburg, VA, 24061
}

\begin{abstract}
Sapphire (single crystal alumina) has superior optical and mechanical properties. With a very high melting point of about $2050^{\circ} \mathrm{C}$, sapphire fiber is an excellent candidate in optical fiber sensing area for high-temperature measurements. This paper presents a new type of sapphire-fiber-based extrinsic Fabry-Perot interferometric (EFPI) temperature sensor. The spectral interference pattern is generated by a sapphire diaphragm placed in front of the sapphire fiber. The sensing element is interrogated by a white-light source. Temperature is demodulated from the spectral change of interference pattern. Prototype sensor is tested at high temperature up to $1545^{\circ} \mathrm{C}$. Both theoretical and experimental analysis are presented. Preliminary data shows the sensor is very promising for measuring ultra-high temperature.
\end{abstract}

Keywords: fiber optic sensor, sapphire fiber, temperature sensor, interferometric, white-light

\section{INTRODUCTION}

Fiber-optic high-temperature sensors have been used in a wide range of industries. Silica-fiber-based thermometers are usually limited to below $1000^{\circ} \mathrm{C}$ due to their degraded properties and diffusion of germanium dopant at elevated temperature ${ }^{1}$. Owing to their excellent mechanical and optical properties at very high temperature, single-crystal optical fibers have been investigated by many researchers for high temperature applications. Based on these high performance fibers, a variety of high-temperature sensors have been developed using different technologies. Among them, radiationbased $^{2-4}$ and fluorescence-based ${ }^{5,6}$ thermometers are two types which have been studied for years. The former uses blackbody radiation (intensity or spectral shape) as the measrand while the latter is based on the temperature dependence of the fluorescence decay time. Optical fibers are used for light collection and guidance in both cases.

Fiber-optic Fabry-Perot (FP) interferometric sensors are also popular in temperature measurements ${ }^{1,7,8}$. However, due to the large fiber diameter and large refractive-index difference between the core (fiber itself) and cladding (mostly air), single-crystal fibers are highly multimoded and thus much more difficult than singlemode fiber to generate interference fringes, which, in this case, is very sensitive to the smoothness, the flatness, and especially the parallelism of the two surfaces and therefore needs careful alignment ${ }^{9}$. In this paper, we use a sapphire wafer as the FP interferometer. Since high quality surface and parallelism can be readily achieved in current wafer lapping/polishing industry, fringes can be easily generated even for highly multimoded sapphire fiber.

The paper is organized as following: sensor principles and experimental setup are described in section 2; then the algorithm for spectral processing is discussed in section 3; Preliminary test results, including temperature response and system resolution, are presented and analyzed in section 4.

*yizhu1@vt.edu; phone 1-540-231-8359; fax 1-540-231-2158; http://www.ee.vt.edu/ photonics 


\section{PRINCIPLE AND SYSTEM SETUP}

\subsection{Principle}

The principle of the sensor is illustrated in Fig. 1. A wafer is placed in front of a fiber and constitutes an extrinsic FabryPerot interferometer, serving as the sensing element. Light from the source travels through the fiber and gets reflected at both surfaces of the wafer. These reflections will be coupled back into the fiber and interfere with each other, producing a modulated spectrum, whose pattern is determined by the Optical Path Difference (OPD) between them. Both the thickness and the refractive index of the diaphragm are temperature-dependent. Therefore the OPD and the spectrum pattern will be sensitive to ambient temperature variation. A spectrometer is used to detect the reflected spectrum, from which the temperature information can be demodulated.

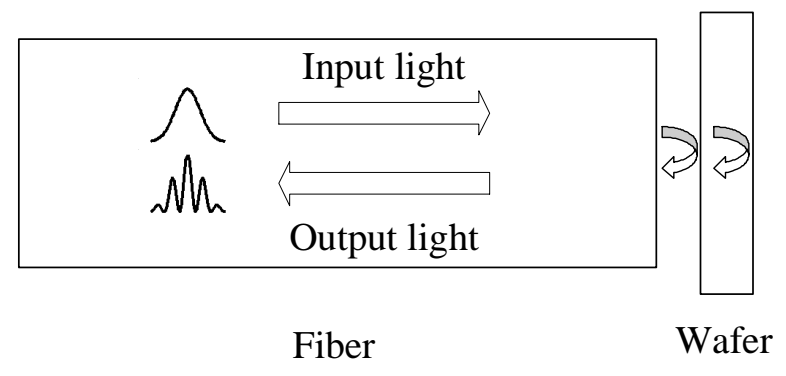

Fig. 1. Principle of wafer-based temperature sensor

The OPD of the wafer-based interferometer can be expressed as

$$
\begin{aligned}
\mathrm{OPD} & =2 \mathrm{nd}+\frac{\lambda}{2} \\
& =2 \mathrm{OT}+\frac{\lambda}{2}
\end{aligned}
$$

where $\mathrm{n}$ is the refractive index of the material, $\mathrm{d}$ is the thickness, OT is the optical thickness of the wafer and equals to the product of $n$ and $d$. An extra phase shift of $\lambda / 2$ is caused by the reflection at the air/wafer interface. Let $\alpha$ be the coefficient of thermal expansion (CTE) of the material and $\beta$ the thermal coefficient of refractive index, we have

$$
\begin{aligned}
\operatorname{OPD}\left(\mathrm{T}_{0}+\Delta \mathrm{T}\right) & =2 \mathrm{~d}\left(\mathrm{~T}_{0}+\Delta \mathrm{T}\right) \mathrm{n}\left(\mathrm{T}_{0}+\Delta \mathrm{T}\right)+\frac{\lambda}{2} \\
& =2\left(\mathrm{~d}_{0}+\alpha \mathrm{d}_{0} \Delta \mathrm{T}\right) \cdot\left(\mathrm{n}_{0}+\beta \Delta \mathrm{T}\right)+\frac{\lambda}{2} \\
& =2 \mathrm{n}_{0} \mathrm{~d}_{0}\left[1+\left(\alpha+\frac{\beta}{\mathrm{n}_{0}}\right) \Delta \mathrm{T}+\frac{\alpha \beta}{\mathrm{n}_{0}} \Delta \mathrm{T}^{2}\right]+\frac{\lambda}{2}
\end{aligned}
$$

Neglecting the high-order term, the change of OPD, $\triangle \mathrm{OPD}$, is related to $\Delta \mathrm{T}$ as

$$
\begin{aligned}
\Delta \mathrm{OPD} & =\mathrm{OPD}\left(\mathrm{T}_{0}+\Delta \mathrm{T}\right)-\mathrm{OPD}\left(\mathrm{T}_{0}\right) \\
& =2 \mathrm{n}_{0} \mathrm{~d}_{0}\left(\alpha+\frac{\beta}{\mathrm{n}_{0}}\right) \Delta \mathrm{T}
\end{aligned}
$$

Since $\alpha$ and $\beta$ are both positive for sapphire, we will expect an increase of the OPD/OT with temperature. 


\subsection{System setup}

The sensor is interrogated by a white-light sensing system, a diagram of which is shown in Fig. 2, consisting of a LED source, a multimode (MM) 3-dB coupler and an OceanOptics S2000 miniature spectrometer. Figure 3 shows an enlarged view of the sensor head. A $99.8 \%$ alumina tube is used as supporting structure, to which both a $59 \mu \mathrm{m}$-thick sapphire wafer and a $75 \mu \mathrm{m}$ (diameter) sapphire fiber are bonded by high temperature alumina adhesive. The sapphire fiber is coupled with a $100 / 140 \mu \mathrm{m}$ MM silica fiber which is connected to the system.

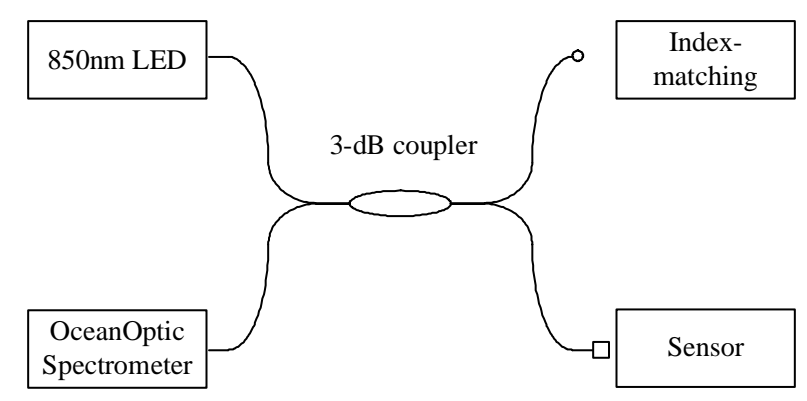

Fig. 2. White-light interferometric sensing system

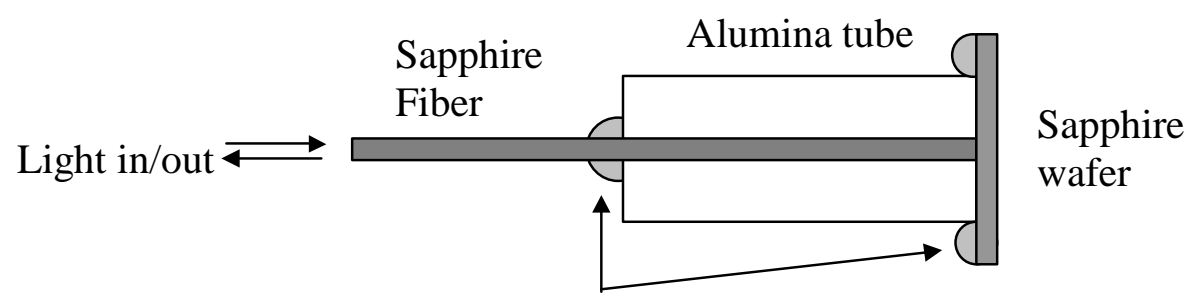

Alumina adhesive

Fig. 3. Sensor head structure

\section{SPECTRUM PROCESSING}

Light from the LED travels through the 3-dB coupler to the sensor head and gets reflected. The reflected signal propagates back to the spectrometer, from which the spectrum data is retrieved and processed by a computer. When temperature varies, the interference signal from the wafer will also change. Figure 4 shows the spectrum from the sensor head at room temperature, where the interference signals are clearly seen.

The reflected signal consists of several components. In general, the total light intensity received at the spectrometer can be written as

$$
\begin{aligned}
\mathrm{I}_{\mathrm{t}}(\lambda) & =\mathrm{I}_{\mathrm{B}}(\lambda)+\mathrm{I}_{\mathrm{D}}(\lambda)+\mathrm{I}_{\mathrm{LED}}(\lambda)\left(\mathrm{r}_{\text {couple }}+\mathrm{r}_{\text {end }}+\mathrm{r}_{\mathrm{s} 1}+\mathrm{r}_{\mathrm{s} 2}-2 \sqrt{\mathrm{r}_{\mathrm{s} 1} \mathrm{r}_{\mathrm{s} 2}} \cos \frac{2 \mathrm{n}(\mathrm{T}) \mathrm{d}(\mathrm{T})}{\lambda}\right) \\
& =\mathrm{I}_{\mathrm{B}}(\lambda)+\mathrm{I}_{\mathrm{D}}(\lambda)+\mathrm{I}_{\mathrm{LED}}(\lambda)\left(\mathrm{r}_{\text {couple }}+\mathrm{r}_{\text {end }}+\mathrm{r}_{\mathrm{s} 1}+\mathrm{r}_{\mathrm{s} 2}\right)-2 \mathrm{I}_{\mathrm{LED}}(\lambda) \sqrt{\mathrm{r}_{\mathrm{s} 1} \mathrm{r}_{\mathrm{s} 2}} \cos \frac{2 \mathrm{n}(\mathrm{T}) \mathrm{d}(\mathrm{T})}{\lambda}
\end{aligned}
$$




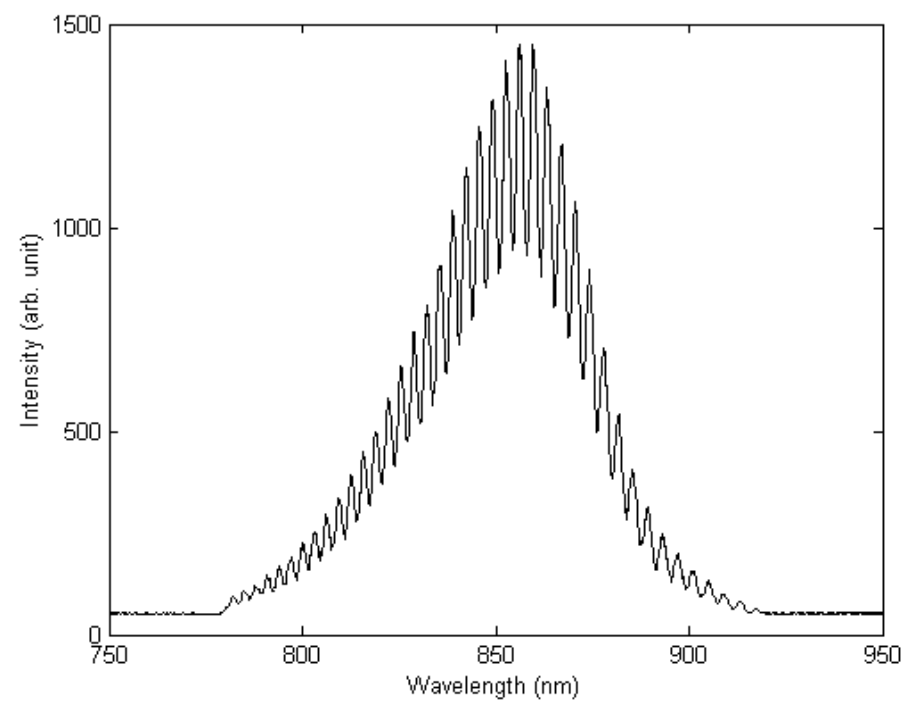

Fig. 4. Sensor spectrum at room temperature

where $\mathrm{I}_{\mathrm{B}}(\lambda), \mathrm{I}_{\mathrm{D}}(\lambda)$ and $\mathrm{I}_{\mathrm{LED}}(\lambda)$ is the light intensity of blackbody radiation, dark current and LED. $\mathrm{r}_{\text {couple }}, \mathrm{r}_{\text {end }}, \mathrm{r}_{\mathrm{s} 1}$ and $\mathrm{r}_{\mathrm{s} 2}$ are reflections at every surfaces, the coupling point of silica fiber and sapphire fiber, the end face of the sapphire fiber, the first and second surfaces of the sapphire wafer, respectively, $\mathrm{n}$ is the refractive index of sapphire and $\mathrm{d}$ is the thickness of the wafer. In Eq. 4, the first three terms stands for the light intensity from blackbody radiation, dark current and reflections at every surfaces, all of which are slow-varying compared to the interference signal and thus will locate around the zero frequency after a Fourier transform of the spectrum. On the other hand, the last term, which contains the cosine, is a high frequency component and will be separated from the first three items by FFT.

A band-pass filter can be used to filter all the low frequency components out, leaving only the last item, $2 \mathrm{I}_{\mathrm{LED}}(\lambda) \sqrt{\mathrm{r}_{\mathrm{s} 1} \mathrm{r}_{\mathrm{s} 2}} \cos \frac{2 \mathrm{n}(\mathrm{T}) \mathrm{d}(\mathrm{T})}{\lambda}$, which is a cosine term modulated by the LED spectrum profile $\mathrm{I}_{\mathrm{LED}}(\lambda) . \mathrm{r}_{\mathrm{s} 1}$ and $\mathrm{r}_{\mathrm{s} 2}$ are constants and do not have impact. This LED modulation profile can be extracted by envelope detection. It then can be used to normalize the modulated signal, resulting in a pure cosine term,

$$
\begin{aligned}
\mathrm{I}(\lambda) & =-\cos \frac{2 \mathrm{n}(\mathrm{T}) \mathrm{d}(\mathrm{T})}{\lambda} \\
& =-\cos \left(\frac{2 \times \text { Optical Thickness }}{\lambda}\right)
\end{aligned}
$$

which corresponds only to the properties of the sapphire wafer. The peaks and valleys of this signal can be expressed as

$$
2 \times \text { Optical Thickness }=m \pi \lambda_{\mathrm{m}}
$$

where $\mathrm{m}$ is the fringe order and $\lambda_{\mathrm{m}}$ is the corresponding peak/valley position. By tracing the change of $\lambda_{\mathrm{m}}$ with temperature, a dependence of optical thickness on temperature can be determined. 


\section{RESULTS}

For temperature test, the sensor is placed in a high temperature furnace along with a B-type thermocouple, the tip of which is right next to the sapphire wafer. The furnace was operated from about $230^{\circ} \mathrm{C}$ to $1545^{\circ} \mathrm{C}$ at a ramping rate of $3^{\circ} \mathrm{C} / \mathrm{min}$. Plotted in Fig. 6 is the optical thickness change with respect to the temperature measured by the B-type thermocouple. The data was fitted using a cubic polynomial, as shown in Eq. 7.

$$
\mathrm{T}=4.8548(\mathrm{OT}-106.5)^{3}-36.383(\mathrm{OT}-106.5)^{2}+458.70(\mathrm{OT}-106.5)+1038.4
$$

This curve converts an optical thickness value from the sensor to a temperature value, difference between which and the thermocouple measurement is plotted in Fig. 7. The deviation varies between $-5^{\circ} \mathrm{C}$ and $+5^{\circ} \mathrm{C}$, corresponding to about $\pm 0.38 \%$ full scale accuracy.

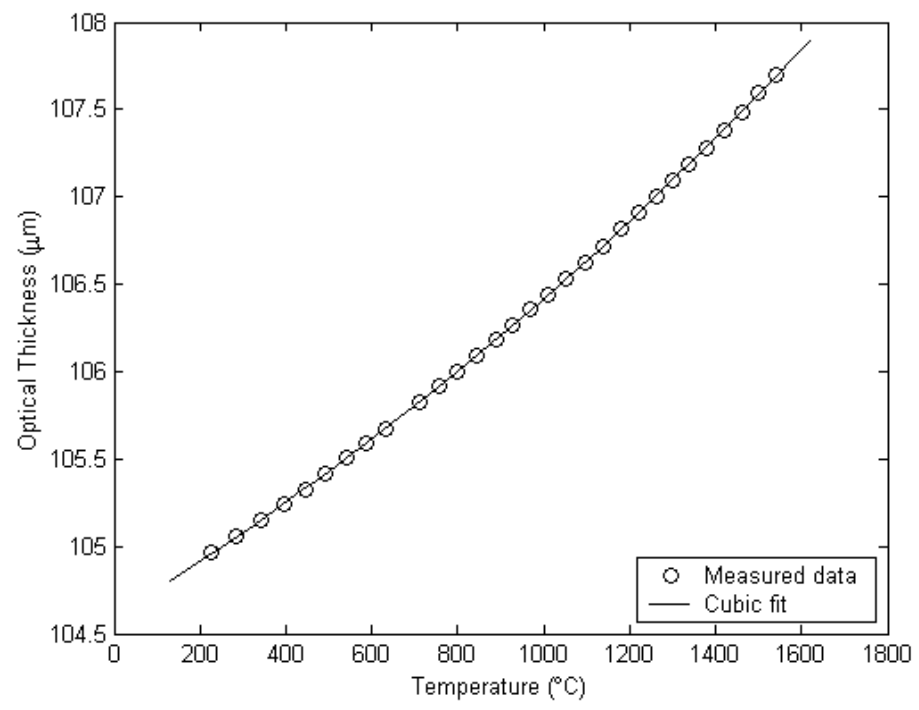

Fig. 6. Optical thickness vs. temperature

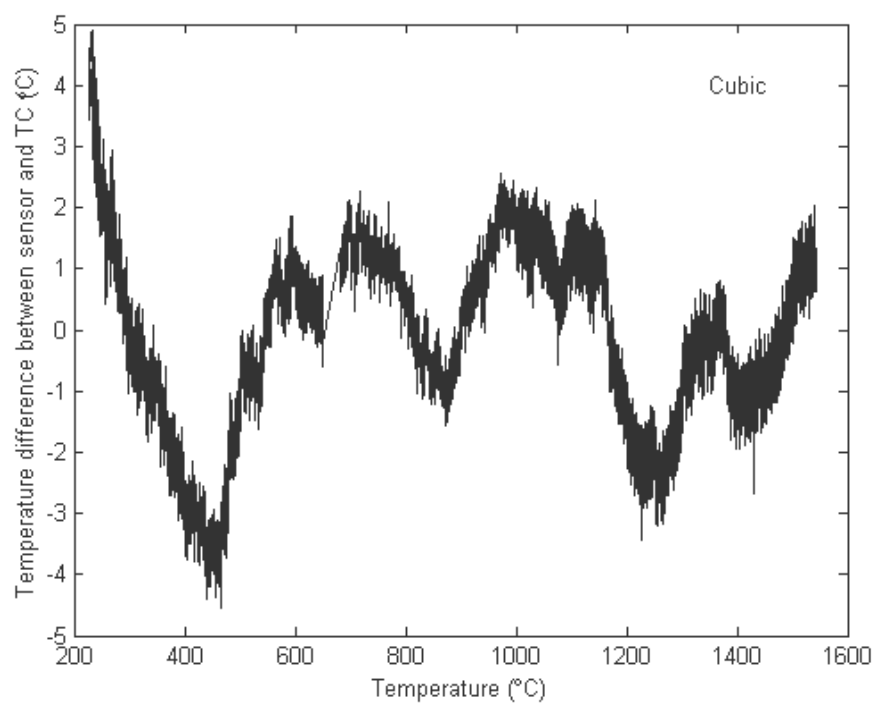

Fig. 7. Temperature difference between the sensor (cubic fitting) and the thermocouple 
A $4^{\text {th }}$ order polynomial fitting would give the following calibration curve

$$
\begin{aligned}
\mathrm{T}= & -3.7604(\mathrm{OT}-106.5)^{4}+2.2943(\mathrm{OT}-106.5)^{3} \\
& -30.682(\mathrm{OT}-106.5)^{2}+460.81(\mathrm{OT}-106.5)+1037.3
\end{aligned}
$$

Figure 8 shows the temperature difference between the sensor and the thermocouple using Eq. 8 . The deviation reduces to between $-3^{\circ} \mathrm{C}$ and $+3^{\circ} \mathrm{C}$ or $0.23 \%$ full scale accuracy. Further increase of the order of the polynomial would not give better result.

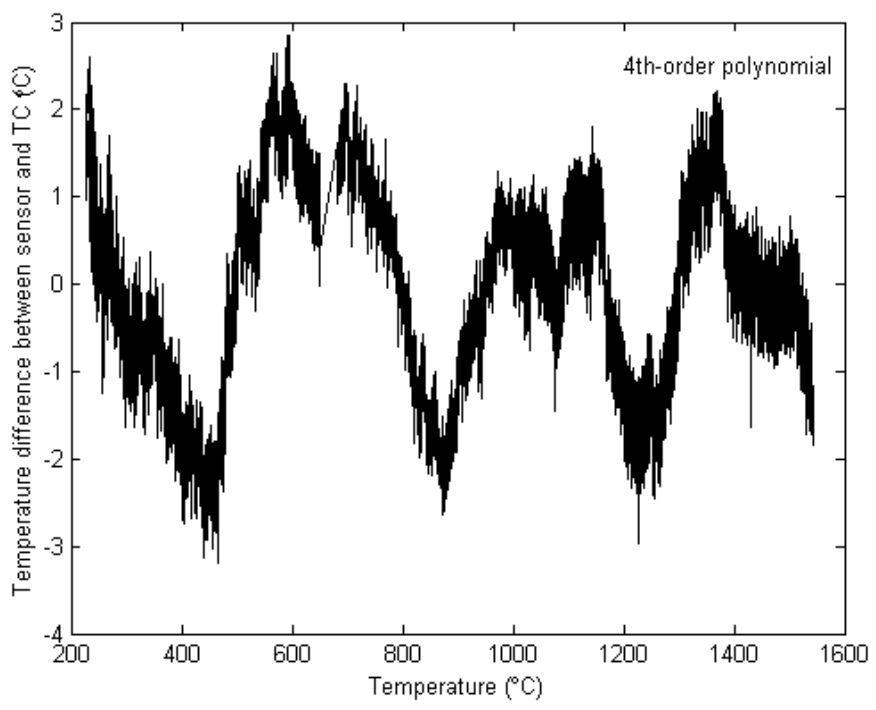

Fig. 8. Temperature difference between the sensor $\left(4^{\text {th }}\right.$ order polynomial $)$ and the thermocouple

Figure 9 shows a system resolution test at room temperature. The sensor was kept in the furnace at room temperature and the optical thickness was continuously recorded for 5 minutes. The standard deviation $\sigma$ is about $0.33 \mathrm{~nm}$. The sensitivity of the sensor at room temperature is about $1.34 \mathrm{~nm} /{ }^{\circ} \mathrm{C}$. So the $2 \sigma$-resolution is about $2 * 0.33 \mathrm{~nm} / 1.34 \mathrm{~nm} /{ }^{\circ} \mathrm{C}=$ $0.5^{\circ} \mathrm{C}$. Instead of processing only one spectrum at a time, recording more spectrum and taking average can reduce the noise and further improve resolution. A 4-time average can reduce the standard deviation to $0.165 \mathrm{~nm}$ or a resolution of $0.25^{\circ} \mathrm{C}$. Increasing the averaging time to 9 will further reduce this value to $0.105 \mathrm{~nm}$, corresponding to about $0.16^{\circ} \mathrm{C}$ temperature resolution.

Figure 10 plots the standard deviation of 5-minute data for different averaging times. X-axis is plotted as square root of the averaging times. For averages which is more than 10 times, the standard deviation could not further reduce but reach a limit around $0.11 \mathrm{~nm}$. The temperature variation inside the furnace should account for the reason of this limit. This was verified by measurements using thermocouple.

\section{CONCLUSIONS}

We have demonstrated a fiber optic interferometric sensor for high-temperature measurement. In the sensor a sapphire wafer is used to generate interference fringe and a sapphire fiber is used to transmit light. The sensor was tested up to $1545^{\circ} \mathrm{C}$ and demonstrated the excellent mechanical and optical performance of sapphire at elevated temperature. The change of optical thickness of the wafer with temperature is fitted to a cubic curve, which gives a $\pm 3.8 \%$ full scale accuracy. When fitted by a $4^{\text {th }}$ order polynomial, the accuracy can be improved to about $0.23 \%$ full scale. The sensor has great potential to work at higher temperature. 


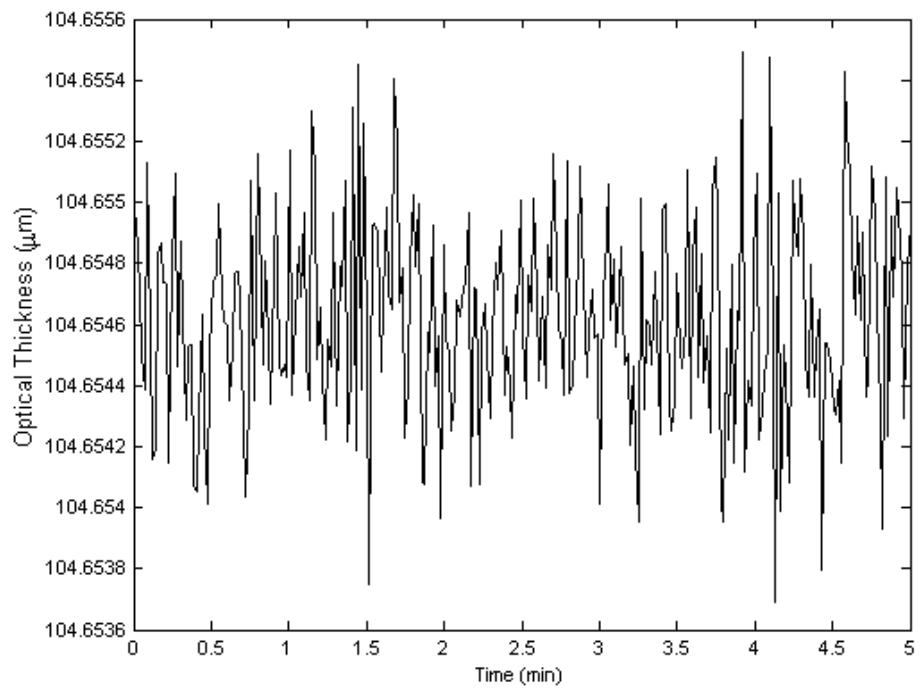

Fig. 9. System resolution test at room temperature

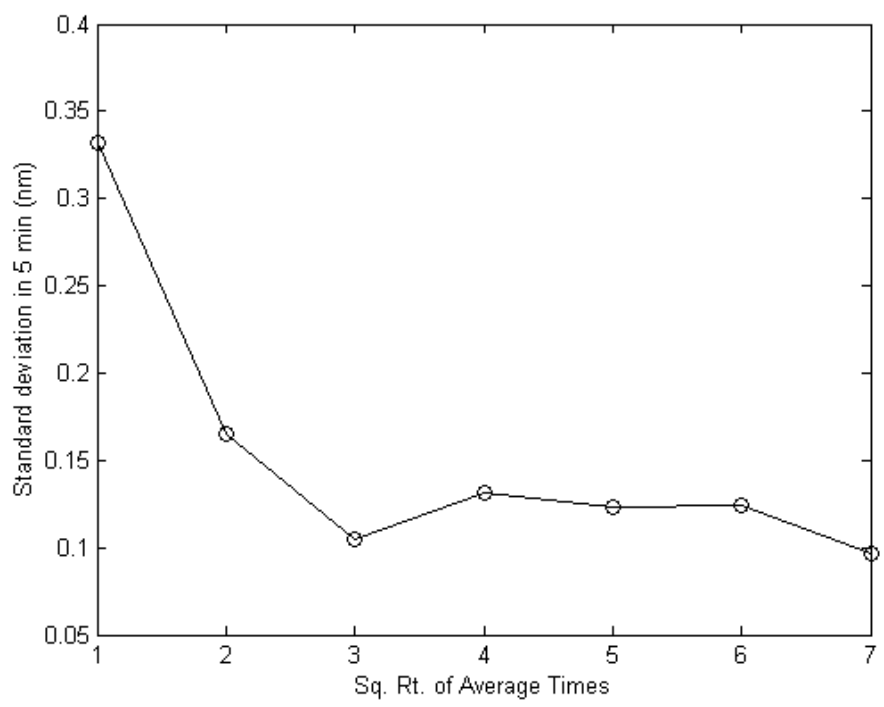

Fig. 10. Effect of averaging on system resolution

\section{ACKNOWLEDGEMENT}

This research was sponsored by the U.S. Department of Energy under grant DE-FC36-01G011050. 


\section{REFERENCE}

1. A. Wang, S. Gollapudi, K. A. Murphey, R. G. May, and R. O. Claus, "Sapphire-fiber-based intrinsic Fabry-Perot interferometer," Opt. Lett. 17, 1021-1223 (1992)

2. R. R. Dils, "High-temperature optical fiber thermometer," J. Appl. Phys. 54, 1198-1201 (1983)

3. R. R. Dils, J. Geist, and M. L. Reilly, "Measurement of the silver freezing point with an optical fiber thermometer: Proof of concept," J. Appl. Phys. 59, 1005-1012 (1986)

4. L. Tong, Y. Shen, L. Ye, and Z. Ding, "A zirconia single-crystal fiber-optic sensor for contact measurement of temperatures above $2000^{\circ}$ C," Meas. Sci. Technol. 10, 607-611 (1999)

5. Z. Y. Zhang, K. T. V. Grattan, and B. T. Maggitt, "Thulium-doped fiber optic decay-time temperature sensors: Characterization of high temperature performance," Rev. Sci. Instrum. 71, 1614-1620 (2000)

6. J. L. Kenndey, and N. Djeu, "Operation of Yb:YAG fiber-optic temperature sensor up to $1600^{\circ} \mathrm{C}$," Sensors Actuators A 100, 1870191 (2002)

7. A. Wang, S. Gollapudi, R. G. May, K. A. Murphey, and R. O. Claus, "Advances in sapphire-fiber-based intrinsic interferometric sensors," Opt. Lett. 17, 1544-1546 (1992)

8. A. Wang, G. Z. Wang, K. A. Murphey, and R. O. Claus, "Birefringence-balanced polarimetric optical fiber sensor for high-temperature measurements," Opt. Lett. 17, 1391-1393 (1991)

9. H. Xiao, J. Deng, G. Pickrell, R. G. May, and A. Wang, "Single-crystal sapphire fiber-based strain sensor for hightemperature applications," J. Lightwave Technol. 21, 2276-2283 (2003) 


\title{
UV-induced intrinsic Fabry-Perot interferometric fiber sensors
}

\author{
Fabin Shen", Wei Peng, Kristie Cooper, Gary Pickrell, Anbo Wang \\ Center for Photonics Technology, Bradley Department of Electrical and Computer Engineering, \\ Virginia Polytechnic Institute and State University, Blacksburg, VA 24061
}

\begin{abstract}
In this paper, we present a novel point-wise laser writing method that utilizes a focused ultraviolet (UV) laser beam and metallic masks to write local Fresnel reflectors and intrinsic Fabry-Perot interferometric (IFPI) sensors in photosensitive fibers. These UV-induced IFPI sensors have features of low reflectance and low power loss and have the potential to be densely multiplexed. We also present a sweeping laser based measurement system that measures the interference spectra and estimates the optical path distances (OPD) of IFPI sensors. We also demonstrated IFPI sensors for temperature, strain and pressure measurement. Laboratory test results show that these UV-induced IFPI sensors can have a resolution of $0.1^{\circ} \mathrm{C}$ for temperature measurement and 0.5 micro-strain for strain measurement, and can be used in a temperature environment as high as $600^{\circ} \mathrm{C}$.
\end{abstract}

Keywords: Fiber sensors, UV irradiation, intrinsic Fabry-Perot interferometer, temperature, strain, pressure.

\section{INTRODUCTION}

The phenomenon of photosensitivity to UV irradiation in germanium-doped silica fiber has been widely used to make a variety of fiber components for communications and optical fiber sensors. ${ }^{[1][2]}$

We can use this phenomenon directly to change the refractive index of a segment of photosensitive fiber by exposing it to UV, and thus local Fresnel reflectors inside the fiber can be fabricated. J. A. Greene et al. introduced UV-induced broadband Fresnel reflectors in hydrogen-loaded $\mathrm{GeO}_{2}$-doped optical fiber. ${ }^{[3][4]}$ They also demonstrated optical time domain reflectometry (OTDR)-based IFPI strain and temperature sensors by measuring the reflectance at a certain wavelength or counting the fringe numbers due to the parameter changes ${ }^{[4]}$. However, neither of these measurement methods was satisfying for practical measurement.

Recently, we successfully fabricated IFPI sensors by irradiating commercially available $\mathrm{GeO}_{2}$ doped photosensitive fiber with a slit-masked UV beam and demonstrated a measuring system based on interference spectrum measurement. We also developed a signal processing method that uses FIR filtering, fringe order determination and multiple peak tracking to retrieve the optical path length of the Fabry-Perot (FP) cavity. Sensors for physical parameters sensing, including temperature, strain and pressure were investigated.

The contents of the paper are arranged as follows. In section 2, the principle of UV-induced IFPI sensors and the fabrication procedure are introduced. In section 3, a sweeping laser based measurement system, which measures the interference spectrum and estimates the OPD of IFPI sensor, is introduced.. In section 4, experimental results for temperature, strain and pressure sensing are presented. In section 5, conclusions are given.

\section{UV-INDUCED IFPI SENSOR}

\subsection{Principle of Operation}

The basic structure and fabrication method of the UV-induced IFPI sensor are conceptually explained in Fig 1. A segment of photosensitive single-mode fiber is placed behind a masking slit and illuminated on the side by a UV laser beam. Due to the photosensitivity of the fiber material, the refractive index of the exposed spot is permanently changed.

*fashen1@vt.edu; phone 1540 231-2155; fax 1540 231-2158 
This narrow segment of fiber with increased refractive index can then function as a Fresnel reflector partially reflecting the light propagating in the fiber. A pair of such reflectors can therefore form an intrinsic Fabry-Perot interferometer.

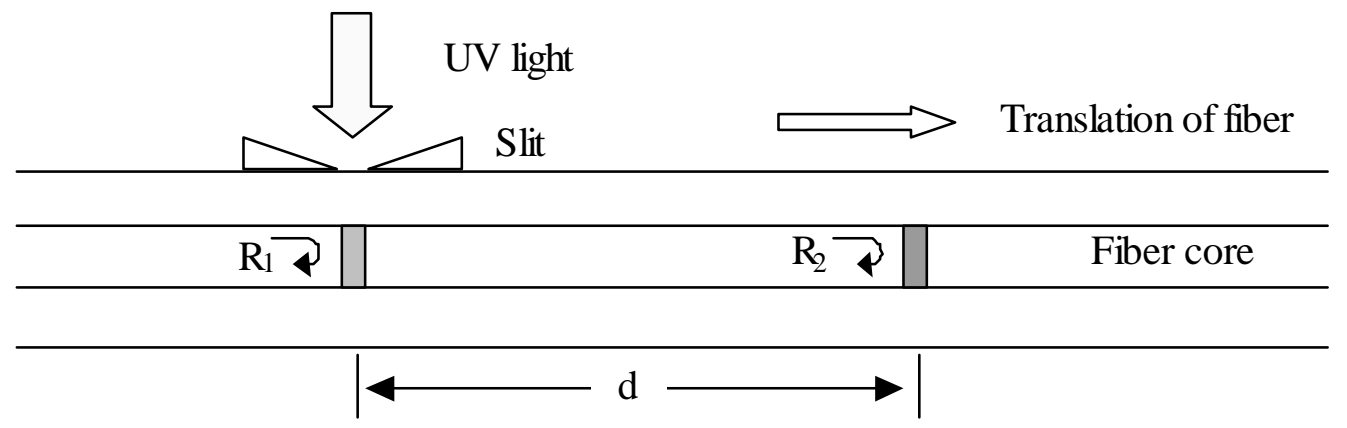

Fig. 1. Structure of UV-induced IFPI sensor.

The optical path distance (OPD) between the two reflectors is

$$
L=2 n_{e f f} d
$$

where $d$ is the distance between the two reflectors and $n_{\text {eff }}$ is the effective refractive index of the fiber at a certain wavelength.

Due to the low reflectance at each reflector, the interference between the two reflections at wavelength $\lambda$ is approximately

$$
I=I_{0}\left[R_{1}+R_{2}+2 \sqrt{R_{1} R_{2}} \cos \left(\frac{2 \pi L}{\lambda}+\phi\right)\right]
$$

where

I Reflected light power measured at wavelength $\lambda$.

$I_{0} \quad$ Power of the light source at wavelength $\lambda$.

$R_{1}, R_{2}$ Reflectivity of the reflectors at wavelength $\lambda$.

$\phi \quad$ Constant phase difference term due to the phase changes on reflection interface.

$\lambda \quad$ Wavelength of light source.

Normalized interference at wavelength $\lambda$ can be written as

$$
\frac{I}{I_{0}}=R_{1}+R_{2}+2 \sqrt{R_{1} R_{2}} \cos \left(\frac{2 \pi L}{\lambda}+\phi\right)=A+B \cdot \cos (k L+\phi)
$$

where

$$
\begin{array}{ll}
A=R_{1}+R_{2} & \text { DC term. } \\
B=2 \sqrt{R_{1} R_{2}} & \text { Amplitude of the sinusoid. } \\
k=2 \pi / \lambda & \text { Wave number. }
\end{array}
$$

If the width of the exposed segment is controlled to be on the order of micrometers, a broadband Fresnel reflector can be achieved by controlling the laser exposure conditions. For a broadband Fresnel reflector, the reflection can be considered to be wavelength independent. Thus, $\mathrm{R}_{1}$ and $\mathrm{R}_{2}$, and therefore $\mathrm{A}$ and $\mathrm{B}$, can be considered to be constant within a certain wavelength range. 
When a normalized interference spectrum is measured in a certain wavenumber range, the measured normalized interference spectrum for a single IFPI sensor can be written as

$$
x_{n}=A+B \cos \left(k_{n} L+\phi\right)+v_{n}
$$

where $x_{n}, k_{n}, v_{n}$ are respectively normalized intensity, wavenumber and noise of the $\mathrm{n}$-th wavenumber sample.

Although $\mathrm{n}_{\text {eff }}$ is not the same at all wavelengths for dispersive silica fiber, it can be reasonably regarded as a constant in a small window of wavelength range. Therefore, in each sample of interference spectrum, $L$ can be regarded as a constant for a given cavity length $d, x_{n}$ can be regarded as a sinusoid with constant frequency and phase. It is evident that $L$ and $\phi$ can be derived by estimating the frequency and phase of the sinusoid described in (4).

\subsection{Sensor Fabrication}

An IFPI sensor fabrication system has been developed at the Center for Photonics Technology at Virginia Tech. The system consists of an UV excimer laser, optical components including a UV-grade mirror and lens, a metallic shadow mask, 3-D translation stages and a monitoring system. The diagram of the setup of the sensor fabrication system is shown in Fig. 2.

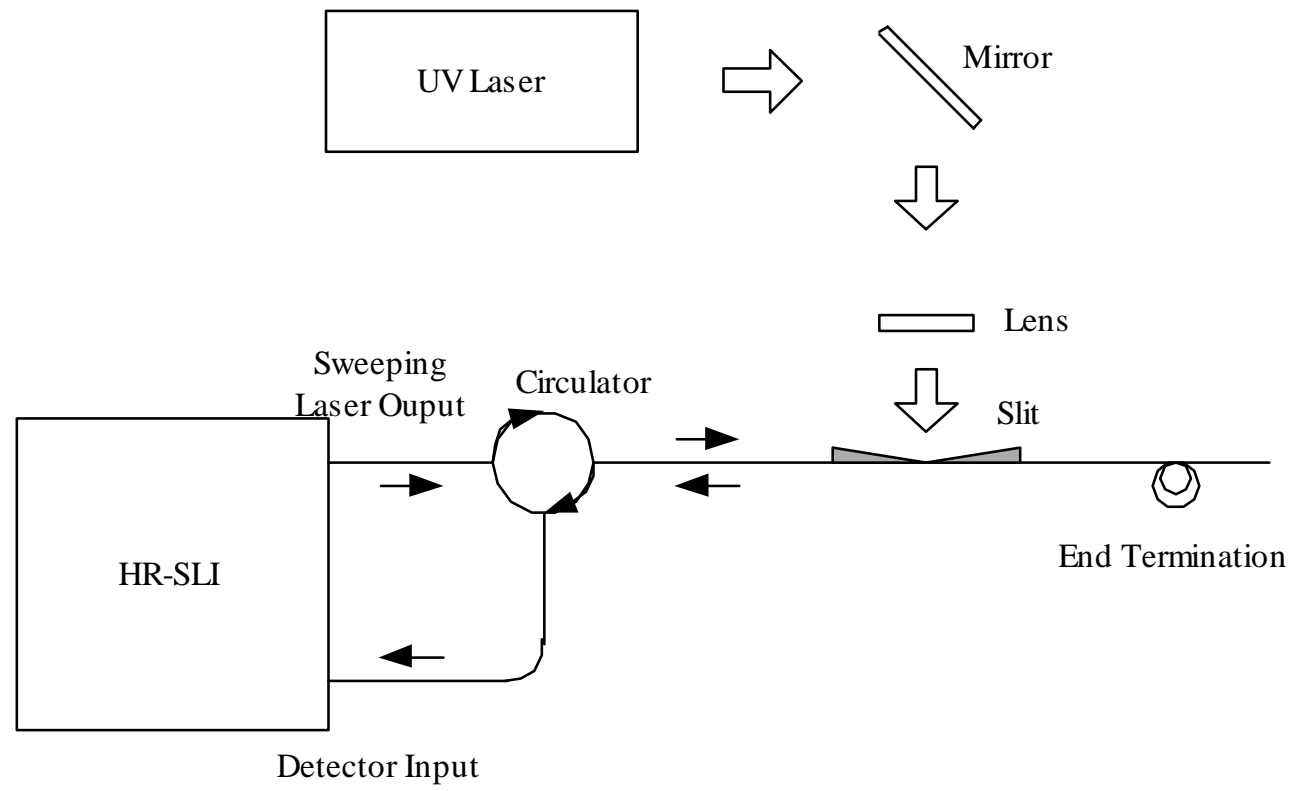

Fig. 2. Diagram of IFPI sensor fabrication system.

A $\mathrm{KrF}$ excimer laser (MPB Communication Inc, MSX-250) with a $248 \mathrm{~nm}$ wavelength is used as the UV source. A UV-grade mirror and a UV-grade lens are used to focus the laser beam onto the side of the photosensitive fiber. A pair of metallic plates is used to form a narrow slit that just allows a small portion of laser beam to pass though. The two plates are mounted onto 3-D stages. The slit width can be controlled by changing the positions of the two plates. The photosensitive fiber is placed behind the slit and in contact with the metallic plates to reduce the influence of laser beam diffraction at the narrow slit. The fiber is also mounted onto a 3-D translation stage so that the position of the laser writing point can be translated along the fiber axis direction. A high resolution swept laser interrogator (HR-SLI) (Micron Optics, SI720), which function as both a sweeping laser source and an optical spectrum analyzer, together with an optical circulator, which couples the reflected light to the optical detector, are used to monitor the sensor fabrication procedure. The operation principle of the monitoring and measuring system will be explained in section 3. Finally, a microscope is used to observe the laser writing point so that the slit width can be roughly estimated. 
The sensor fabrication procedure can be described as follows. Photosensitive fiber, usually a piece of photosensitive fiber spliced between conventional Corning SMF-28 single mode fibers, is placed behind the slit, which is close to the focus point of the lens. The end reflection of the fiber is eliminated by either using a fiber termination or curling the fiber into a coil with a small diameter about several millimeters. At this point the signal detected by the HR-SLI is the background reflection caused by Raleigh backscattering of the fiber and the local reflection of the optical test system itself. The background reflection is very low, usually less than $-60 \mathrm{~dB}$. When the first reflector is written, the Fresnel reflection at this point, which is usually as high as about $-55 \mathrm{~dB} \sim-50 \mathrm{~dB}$, can be observed. When the second reflector is written, the interference fringes of the two reflections can be observed on the HR-SLI. The contrast of the fringes is determined by ratio of the reflectivity at the two reflectors, $R_{1}$ and $R_{2}$ respectively in (2).

The UV power density irradiated on the fiber is controlled to be around $500 \mathrm{~mJ} / \mathrm{cm}^{2}$. The pulse duration is $20 \mathrm{~ns}$. The repetition rate is set to $5 \mathrm{~Hz}$. Usually, after tens to hundreds of pulses, significant refractive index change, normally $0.005 \sim 0.01$, can be achieved. Two kinds of commercially available photosensitive fibers, Thorlab GF1 and Newport SFBG-15, have been used to make IFPI sensors and both of them provide satisfactory photosensitivity.

A photograph of a fabricated IFPI sensor is shown in Fig 3. The laser witting points can clearly be seen due to the refractive index change of the fiber and the flaw on the side of the fiber caused by UV-irradiation. The distance between the two writing points in Fig. 3 is about $1 \mathrm{~mm}$.

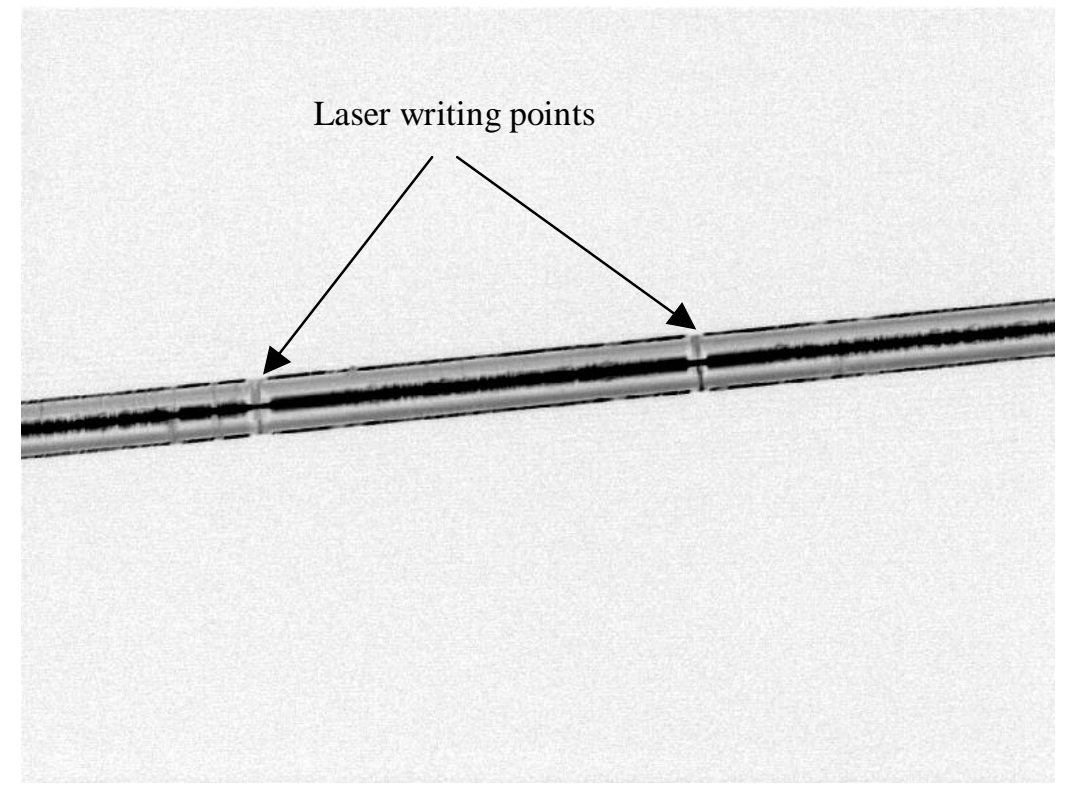

Fig. 3. Photograph of a UV-induced IFPI sensor

UV-induced IFPI sensors have the features of low reflectance and low power loss, and therefore are good candidates for dense multiplexing. They can be used in quasi-distributed sensing applications including multiple point temperature measuring, structure health monitoring etc.

\section{SPECTRUM-BASED MEASUREMENT SYSTEM}

Unlike the OTDR-based measurement system discussed in [4], we use a spectrum-based measurement system for UVinduced IFPI sensors. The interference spectrum of IFPI sensor is measured, and the signal is demodulated by means of an interference spectrum fringe analysis method.

The reflection of a UV-induced IFPI sensor is rather low. It is difficult to measure the interference spectrum by conventional methods that utilize a low-coherence light source and passive optical spectrum analyzer. In our measuring system, a Micron Optics Si720, which contains a sweeping laser source, detectors, intensity and wavelength referencing 
units and supporting electronic circuits, is used as the optical spectrum measurement equipment. A diagram of the measurement system for IFPI sensors is shown is Fig. 4.

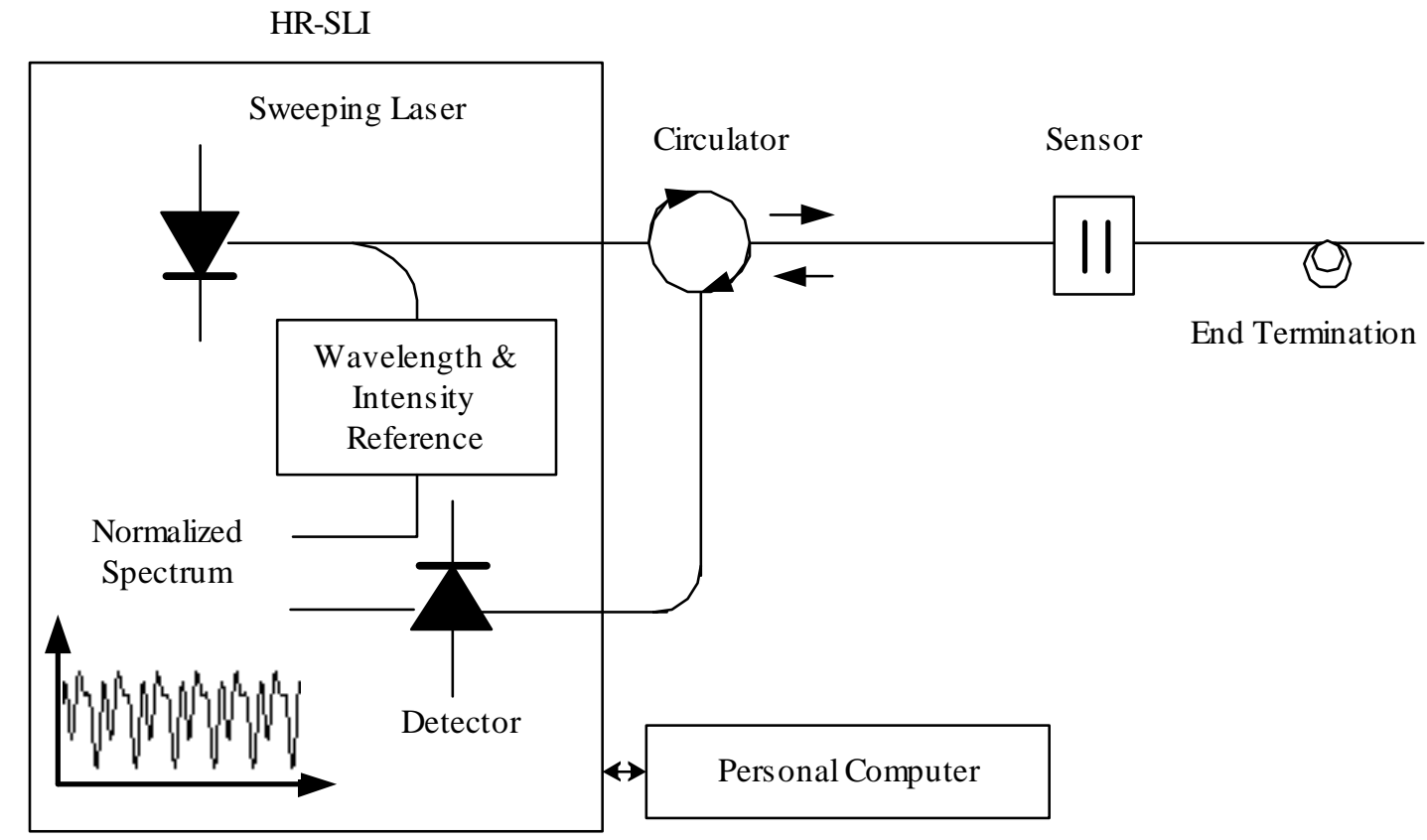

Fig. 4. Diagram of the measurement system.

Narrowband light from a sweeping laser is launched into an optical circulator, which transmits the light to the IFPI sensors. At the sensor, part of the light is reflected back to the circulator. The circulator then couples the reflected light to an optical detector. The power of the reflected light, $I$ in (2)(3), is measured by the detector. A portion of the light is tapped from the light source inside the HR-SLI. The intensity and wavelength of the tapped light are measured as both an intensity and wavelength reference. Therefore, $I_{0}$ and $\lambda$, then the normalized interference $I / I_{0}$ at $\lambda$ in (2)(3) can be measured. The laser is operated to finish a sweep in 200 milliseconds. In total, 20,000 samples are measured in the $1520-1570 \mathrm{~nm}$ wavelength range with a wavelength step of 2.5 picometers during one sweeping cycle of the laser source.

The measured interference spectrum is transferred to a personal computer via a GPIB interface. A Matlab program running on the host computer was written to retrieve the spectrum from the HR-SLI and perform the signal processing tasks. The OPD of the FP cavity can be estimated by estimating the frequency and phase information of the interference spectrum.

We developed a signal processing algorithm to estimate the OPD of FP cavity based on the white-light interferometric fringe analysis approaches described in [5][6]. Some additional features, such as FIR digital filtering, fringe order determination and multiple peak tracking, were added into the algorithm. The performance of the algorithm is illustrated through the experimental result shown in Fig. 6.

\section{EXPERIMENTAL RESULTS}

From (1), it can be derived that

$$
\Delta L=2\left(\Delta n_{\text {eff }} d+n_{\text {eff }} \Delta d\right)=L\left(\frac{\Delta n_{\text {eff }}}{n_{\text {eff }}}+\frac{\Delta d}{d}\right)
$$


It is evident that the IFPI sensor can be used to measure any quantity that changes either the refractive index $n_{\text {eff }}$ or fiber dimension $d$ or both. Some of the common parameters include temperature, strain and pressure. The sensor structures and experimental results for temperature, strain and pressure measurement will be discussed below.

\subsection{Temperature Sensor}

When the intrinsic FP cavity is put into a temperature-varying environment, the OPD of the cavity will change due to the thermo-optic effect and the thermal expansion of the silica fiber. The dimension change of the fiber due to the temperature change is

$$
\frac{\Delta d}{d}=\alpha_{T} \Delta T
$$

where $\alpha_{T}$ is the well known coefficient of thermal expansion (CTE). For silica, $\alpha_{T}=0.5 \mathrm{e}-6\left(1 /{ }^{\circ} \mathrm{C}\right)$. The refractive index change due to the temperature change is

$$
\Delta n_{e f f}=\frac{\partial n_{e f f}}{\partial T} \Delta T
$$

The total OPD change $\Delta L$ can be written as

$$
\Delta L=L\left(\frac{\partial n_{e f f}}{\partial T} / n_{e f f}+\alpha_{T}\right) \Delta T=L\left(\sigma_{T}+\alpha_{T}\right) \Delta T
$$

where $\sigma_{T}=\frac{\partial n_{\text {eff }}}{\partial T} / n_{\text {eff }}$ is the thermo-optic coefficient . For silica fiber, it is on the order of $10^{-5}\left(1 /{ }^{\circ} \mathrm{C}\right)$, which is much larger than $\alpha_{T}$. That is to say, for temperature dependence, the thermo-optic effect is dominant.

Fig. 5 shows the temperature response of an IFPI sensor. The sensor part was put into a miniature furnace. The measuring range is from room temperature to $650^{\circ} \mathrm{C}$. The temperature during the increasing and decreasing portions of the cycle was recorded. As shown in Fig. 5, the sensor responses to increasing and decreasing temperature are almost overlapping.

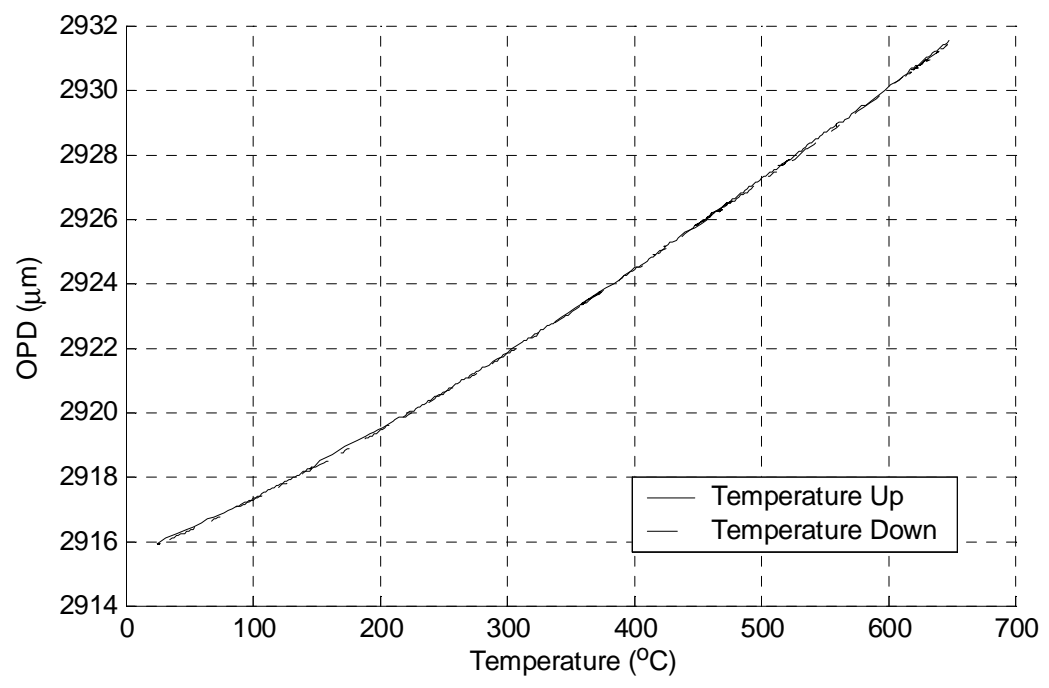

Fig. 5. Response of IFPI temperature sensor.

Fig 6. shows the resolution test results of the IFPI sensor and the signal processing algorithm. The sensor was kept at $25 \pm 0.1^{\circ} \mathrm{C}$ for about 4 hours. The upper graph shows the response of the sensor, the lower graph shows the error 
distribution of the measurement. The standard deviation of OPD is less than $0.5 \mathrm{ppm}$ of the original OPD. The corresponding temperature resolution is better than $0.1^{\circ} \mathrm{C}$.
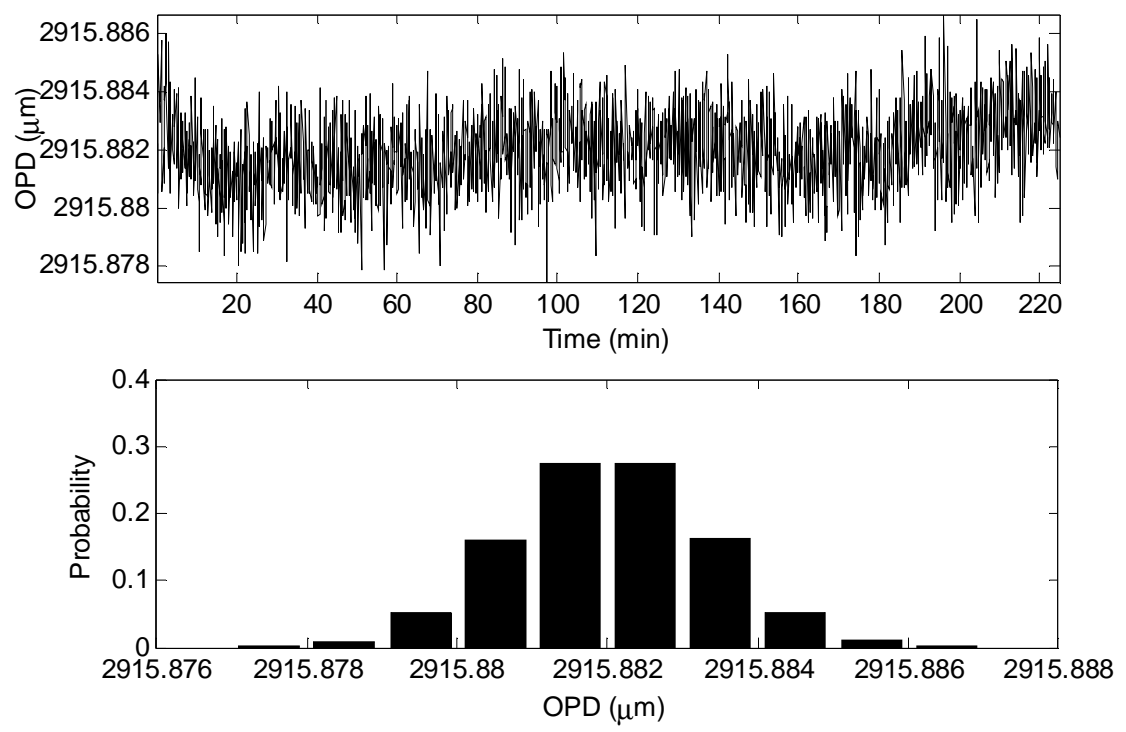

Fig. 6. Resolution test of IFPI temperature sensor.

The photosensitivity of fiber can be classified to three categories, Type I, Type IIA and Type II ${ }^{[2][7]}$. For Type IIA and Type II, large refractive index changes can be achieved by either a long duration irradiation or fewer laser pulses with a high energy density. Type IIA refractive index changes can withstand a high temperature of $500^{\circ} \mathrm{C}$. Type II refractive index change can withstand a high temperature of $800^{\circ} \mathrm{C}{ }^{[7]}$. In our experiment, IFPI sensors had been proven to be capable at $600^{\circ} \mathrm{C}$ or even higher without significant degradation.

\subsection{Strain Sensor}

The UV-induced IFPI sensor can also be used for strain sensing. The OPD change is due to the dimension change and the stress-optic effect in the silica fiber. The refractive index change caused by the strain is

$$
\Delta n_{\text {eff }}=-\frac{n_{\text {eff }}^{3}}{2}\left(p_{12}-v\left(p_{11}+p_{12}\right)\right) \varepsilon
$$

where $p_{11}, p_{12}$ are components of strain-optic tensor, $v$ is Poisson's ratio and $\varepsilon=\Delta d / d$ is the strain. Therefore, from (16), the total OPD change can be written as

$$
\Delta L=L\left(1-P_{e}\right) \mathcal{E}
$$

where

$$
P_{e}=\frac{n_{e f f}^{2}}{2}\left(p_{12}-v\left(p_{11}+p_{12}\right)\right)
$$

is known as an effective strain-optic constant. For a typical optical fiber, $p_{11}=0.113, p_{12}=0.252, v=0.16$, $n_{\text {eff }}=1.468, P_{e}=0.21$.

In our experiment, we used the setup shown in Fig. 7 to measure the strain response of the IFPI sensors. The strain caused by the deadweight can be written as

$$
\varepsilon=\frac{P}{G}
$$


where $\mathrm{G}$ is Young's modulus of silica fiber $(73.4 \mathrm{GPa})$ and $\mathrm{P}$ is the tensile stress. P can be written as

$$
P=\frac{m g}{\pi r^{2}}
$$

where $m$ is mass of the deadweight, $g$ is the acceleration of gravity and $r$ is the radius of the fiber.

The testing result is shown in Fig. 8. A tensile stress increasing and decreasing cycle was measured. Testing results matched the model that (19) - (22) described. The standard deviation of the OPD at each measurement point was less than $0.5 \mathrm{ppm}$ of original OPD; $0.5 \mu$-strain resolution can be expected.

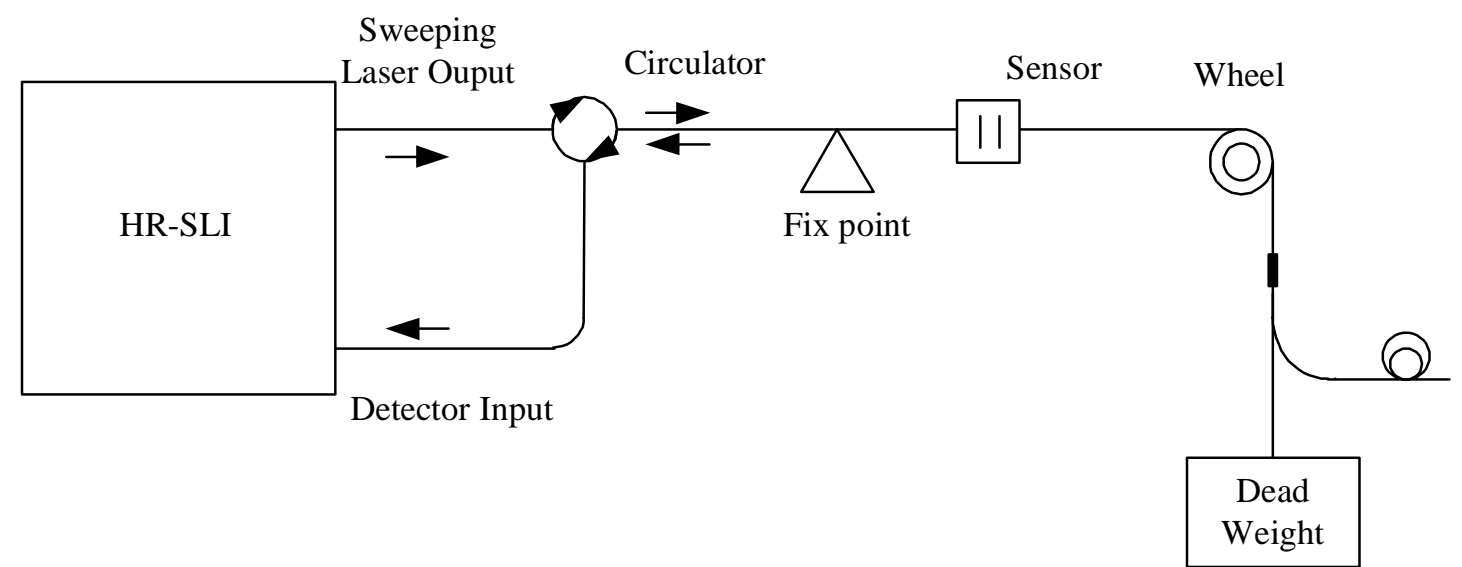

Fig. 7. Experiment setup for strain sensing of IFPI sensor.

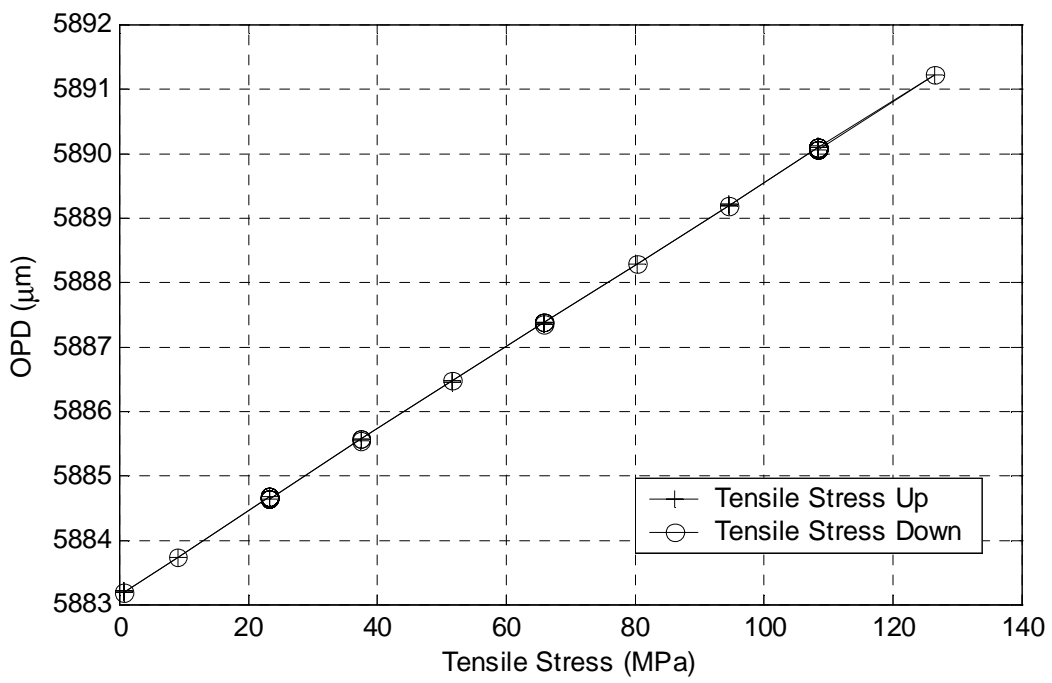

Fig. 8. Response of IFPI strain sensor.

\subsection{Pressure Sensor}

By adding an additional structure, the UV-induced sensors can also be used for pressure sensing. A pressure sensor with an outer glass tube is shown in Fig. 9. The fiber inside the tube was bonded to the tube by melting the glass with a $\mathrm{CO}_{2}$ laser. A similar structure with an extrinsic FP cavity was discussed in [8]. 


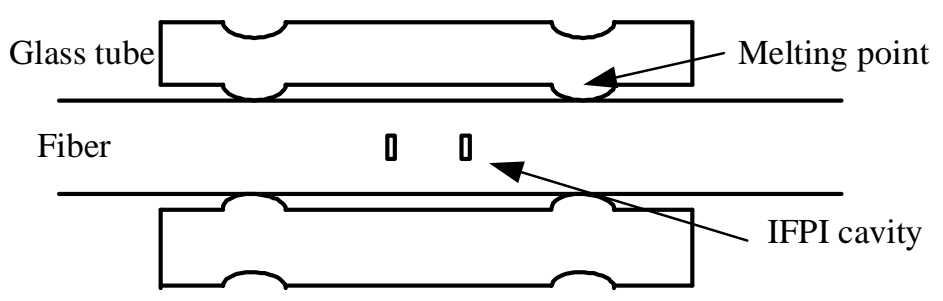

Fig. 9. Structure of IFPI pressure sensor.

If the Young's modulus of the fiber and the glass tube are the same, then the OPD change can be approximated as

$$
\Delta L=L\left(1-P_{e}\right) \frac{P\left(r_{o}^{2}-r_{i}^{2}\right)}{G r_{o}^{2}}(1-2 v)
$$

where

$P_{e} \quad$ Effective strain-optic constant.

$r_{o}, r_{i} \quad$ Outside and inside diameter of the glass tube.

$v \quad$ Poisson's ratio.

$G \quad$ Young's modulus of the glass tube and the glass fiber.

$P \quad$ Pressure around the sensor.

In the sensor, a fused silica tube with $360 \mu \mathrm{m}$ outside diameter and $130 \mu \mathrm{m}$ inside diameter was used. The sensor was tested in a pressure test chamber at pressures from ambient to 4000psi. Fig. 10 shows the response of the sensor to the pressure cycle.

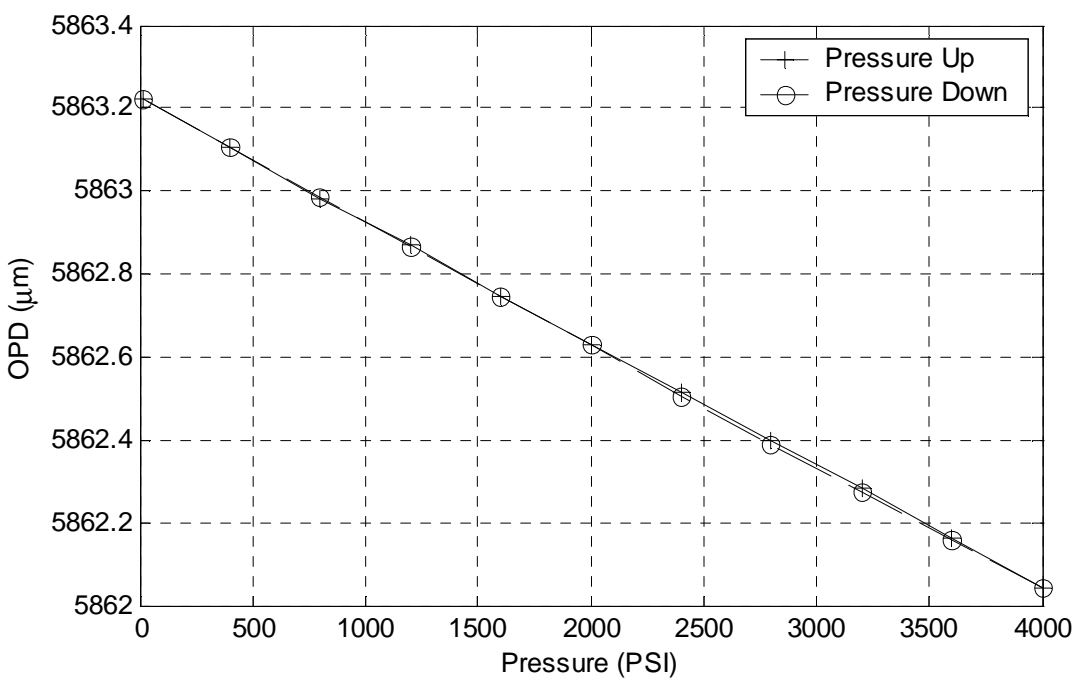

Fig. 10. Response of IFPI pressure sensor.

\section{CONCLUSIONS}

By irradiating photosensitive fiber with a UV beam, we can make local Fresnel reflectors inside the fiber core. A pair of inline Fresnel reflectors will form an IFPI sensor. We described a point-by-point method for fabricating IFPI sensors by using a focused UV-beam and metallic mask. We presented a spectrum-based measurement system to measure the interference spectrum and to estimate the OPD of IFPI sensors. Experimental results showed that the UV-induced IFPI sensors could be used for temperature, strain and pressure sensing. 


\section{ACKNOWLEDGMENTS}

This research was mainly sponsored by the U.S. Department of Energy under grant DE-FC36-01G011050 and partially sponsored by Prime Photonics Inc. The authors would also like to thank Micron Optics Inc. for the partial donation of the HR-SLI si-720 used in this research.

\section{REFERENCES}

1. Kenneth O. Hill and Gerald Meltz, "Fiber Bragg grating technology fundamentals and overview." J. Lightwave Technol. Vol 15(8), pp. 1263-1276, 1997.

2. Andreas Othonos, "Fiber Bragg gratings", Review of Scientific Instruments, vol. 68(12), pp. 4309-4341, 1997.

3. J. A. Greene, K. A. Murphy, A. J. Plante, V. Bhatia and M. Sen, "Broadband photoinduced Fresnel reflectors", Lasers and Electro-Optics Society Annual Meeting, LEOS '94 vol. 2, pp. 157, 1994.

4. J. A. Greene, T. A. Tran, K. A. Murphy, A. J. Plante, V. Bhatia, M. Sen and R. O. Claus, "Photoinduced Fresnel Reflectors for Point-wise and Distributed Sensing Applications", Proc. SPIE vol. 2444, pp. 64, 1995.

5. Bing Qi, Gary R. Pickrell,Juncheng Xu, Po Zhang, Yuhong Duan, Wei Peng, Zhenyu Huang, Wei Huo, Hai Xiao, Russell G. May, and Anbo Wang, "Novel data processing techniques for dispersive white light interferometer ", Opt. Eng. 42, pp. 3165-3171, 2003.

6. J. Schwider and Liang Zhou, "Dispersive interferometric profilometer", Opt. Lett. 19(13), pp. 995-997, 1994.

7. Andreas Othonos and Kyriacos Kalli, "Fiber Bragg Gratings: Fundamentals and Applications in Telecommunications and Sensing", pp. 11-12, Artech House, 1999.

8. Anbo Wang, Hai Xiao, J. Wang, Zhiyong Wang, W. Zhao, R. G. May, "Self-Calibrated Interferometric-IntensityBased Optical Fiber Sensors", J. Lightwave Technol., Vol 19(10), pp. 1945-1501, 2001. 


\title{
Novel Fabry-Perot fiber optic sensor with multiple applications
}

\author{
Xiaopei Chen*, Fabin Shen, Anbo Wang, Zhuang Wang, Yan Zhang \\ Center for Photonics Technology, Bradley Department of Electrical and Computer Engineering, \\ Virginia Polytechnic Institute and State University, Blacksburg, VA 24061-0111
}

\begin{abstract}
A novel Intrinsic Fabry-Perot fiber-optic sensor is presented in this paper. The sensors were made through two simple steps: wet chemical etch and fusion splice. Micro air-gaps were generated inside the fibers and functioned as reflective mirrors. This procedure not only provides a simple and cost effective technology for fabricating intrinsic Fabry-Perot Interferometric (IFPI) fiber sensors, but also provides two possible IFPI structures. Both of the fiber cavity between the air-gaps or the air-gap and cleaved fiber end can be used as sensing elements.. With these two structures, this sensor can be used to measure the temperature, strain, pressure, refractive index of chemicals and the thin film thickness by itself. Multi-point measurements can also be achieved by multiplexing. Furthermore, it also can be multiplexed with other sensors such as Long Period Gratings (LPG) to provide compensations for other perturbation sensing. Theoretical and experimental studies of two sensor structures are described. Experimental results show that high resolution and high sensitivity can be obtained with appropriate signal processing.
\end{abstract}

Keywords: Fabry-Perot interferometer, intrinsic, fiber optic sensor, wet chemical etching, fusion splicing, multiple applications

\section{INTRODUCTION}

Among various fiber optic sensors, Fabry-Perot interferometric (FPI) sensors have distinct advantages because of their high sensitivity, simple structure, polarization independence and multiplex capability. Fiber optic sensors based upon Fabry-Perot interferometer can be classified as extrinsic Fabry-Perot interferometric (EFPI) sensors and intrinsic FabryPerot interferometric (IFPI) sensors. In the EFPI sensor, the fiber propagates light to and from another fiber or a nonfiber element. While in the IFPI sensor, the fiber guides light and experiences perturbation of the interest. IFPI sensors are "all-fiber sensors", which can reduce or eliminate the bonding problems experienced with extrinsic sensors. IFPI sensors are also more versatile for installation and are more robust. But IFPI sensors are usually more difficult to fabricate or need more expensive equipments to fabricate. So from a commercial viewpoint, intrinsic sensors are significantly more expensive.

To fabricate IFPI sensors, most concentrations are focus on producing thin-film mirrors on the cleaved fiber end-face through vacuum deposition, magnetron sputtering or electron-beam evaporation ${ }^{[1],[2]}$. However, the thin-film mirrors are easily damaged or deteriorated in quality by the splicing electric arc. Another procedure to fabricate IFPI sensor is to utilize only splicing technique that fuses two fibers with different core diameter as a reflective mirror ${ }^{[3]}$. But the fiber end-face need extremely carefully polished, rather than simply cleaved, in order to avoid ample power losses ${ }^{[3]}$.

We propose a simple, novel process to fabricate IFPI sensors by combining wet chemical etching and fusion splicing technology. This process is not only a cost effective technology but also has potential for batch production. Two possible structures can be selected by this process to provide multiple functions. It can be used to measure the temperature, strain, pressure, refractive index of the chemicals and thin film thickness by itself. It can also be multiplexed for multiple-points measurement or as compensation element for other perturbation measurement.

\section{SENSOR FABRICATION}

Microfabrication techniques have been developed that allow differential etching of the core with respect to the cladding of the fiber. With suitable buffered acid solution, micro-pits can be obtained on the cleaved fiber end faces. These etched fibers then fusion splice with other un-etched fibers to generate micro-air-gaps inside the fibers. The air-gap can 
be used as a reflective mirror to form Fabry-Perot interferometers with a cleaved fiber end-face or with another microair-gap.

The core of SMF-28 (Corning) fiber is made out of germania doped silica, while its cladding is made out of pure silica, when we use a buffered acid solution $\left(\mathrm{NH}_{4} \mathrm{~F}\right.$ : HF: $\left.\mathrm{H}_{2} \mathrm{O}\right)$ with volumes ratio of $\mathrm{NH}_{4} \mathrm{~F}$ to $\mathrm{HF}$ lower than $1.7: 1^{[4]}$, the etching rate of fiber core is faster than its cladding. This differential etching results in a small pit on the fiber end-face, whose base diameter corresponds to the single mode core of the fiber. In Fig. 1, we demonstrate the differential etching process and the results. When this etched fiber is fusion spliced with another well cleaved fiber, a micro air-gap can be obtained. The fusion splicing process must undertake under low splicing power and short splicing time. The micro airgap can be used as a reflective mirror in the fiber.

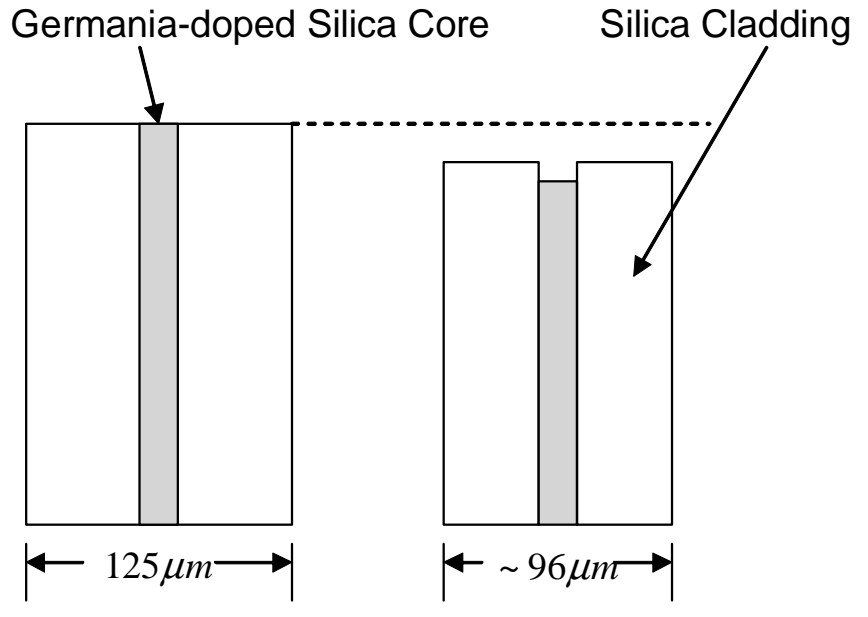

(a) (b)

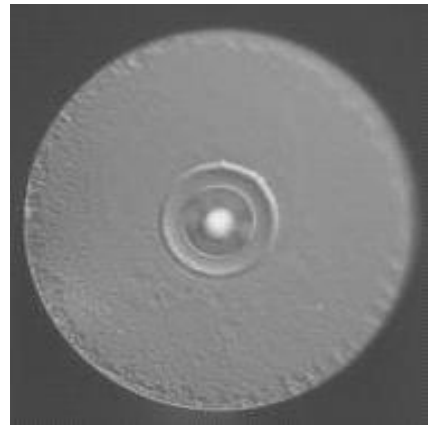

(c)

Fig. 1. Differential wet chemical etching

(a) Before etching; (b) After etching; (c) End-face of etched fiber

Two structures can be developed from this fabrication process. In one of the structures, the Fabry-Perot interferometric cavity is formed between a micro air-gap and a well cleaved fiber end-face. This structure is shown in Fig. 2. The fiber cavity length is determined by the distance between the air-gap and the fiber end-face. The advantage of this structure is its simplicity and potential use in chemical and biological applications. The other structure is to use two identical micro air-gaps to form a Fabry-Perot interferometric cavity. These two micro-air-gaps function as two reflective mirrors of the interferometer. The fiber cavity length is determined by the distance between the two air-gaps, as shown in Fig. 3 . Compared to the first structure, the advantage of this structure is the capability of multiplexing with other sensors.

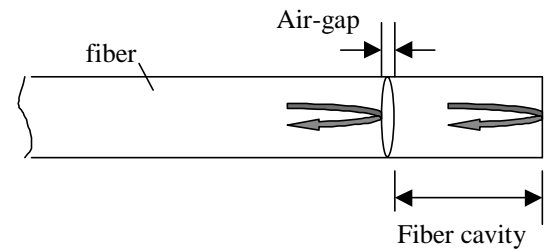

Fig. 2. $1^{\text {st }}$ structure of the sensor

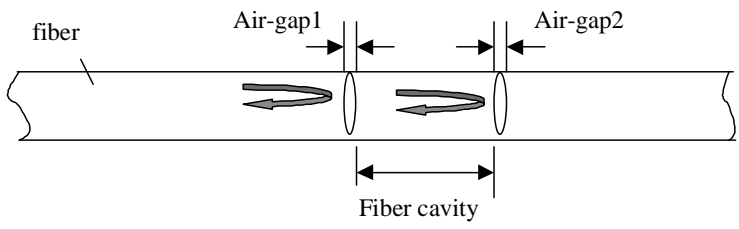

Fig. 3. $2^{\text {nd }}$ structure of the sensor 


\section{THERORETICAL ANALYSIS}

When the light is guided into the sensor, the light reflected from the end surface of the air-gap or from the cleaved fiber end face will generate constructive (i.e., reflections are in phase with each other) or destructive (i.e., reflections are out of phase with each other) interference. If the refractive index and/or length of fiber cavity are changed with perturbation, the reflective power will sweep through the maximum and minimum interference effects. These signals can be processed to determine the amount of perturbation.

Multiple cavities (including air-cavity and fiber-cavity) are formed in both structures of this sensor because of multiple reflective surfaces, especially in the $2^{\text {nd }}$ structure. For simplicity, we model the $1^{\text {st }}$ structure as the case of three reflective surfaces, as shown in Fig. 4. Three reflective surfaces have its reflectance $\left(R_{1}, R_{2}, R_{3}\right)$. The cavity lengths are $\mathrm{L}_{1}, \mathrm{~L}_{2}$ and $\mathrm{L}_{3}=\mathrm{L}_{1}+\mathrm{L}_{2}$. We assume that the energy of light from the source is 1 .

The phase shift can be obtained from

$$
\varphi=\frac{4 \pi n L \cos i}{\lambda}+\varphi_{0},
$$

where $i$ is the incident angle of the light and we assume it is $90^{\circ} . n$ is the refractive index of the fiber. $\mathrm{L}$ is the length of the fiber cavity and $\varphi_{0}$ is original phase.

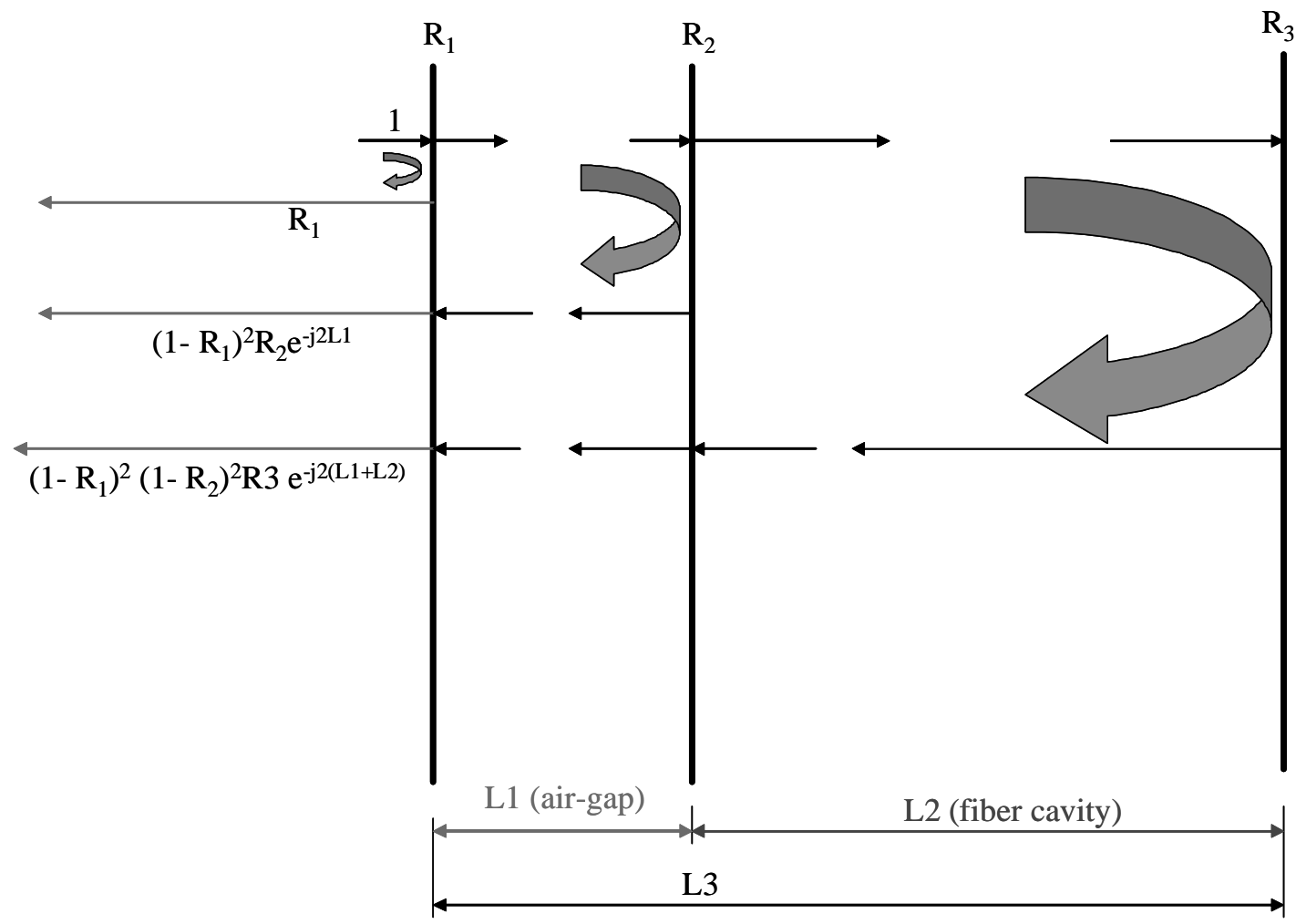

Fig. 4. Theoretical model for multiple cavities

For low-finesse FP cavity, multiple reflections can be neglected. Three different rays reflected back. They are:

Ray 1 with normalized energy: $R_{1}$;

Ray 2 with normalized: $\left(1-R_{1}\right)^{2} R_{2} e^{-j 2 \varphi_{1}}$ 
Ray 3 with normalized: $\left(1-R_{1}\right)^{2}\left(1-R_{2}\right)^{2} R_{3} e^{-j 2\left(\varphi_{1}+\varphi_{2}\right)}$

We sum them together, the total energy received by the detector can be

$$
E=R_{1}+\left(1-R_{1}\right)^{2} R_{2} e^{-j 2 \varphi_{1}}+\left(1-R_{1}\right)^{2}\left(1-R_{2}\right)^{2} R_{3} e^{-j 2\left(\varphi_{1}+\varphi_{2}\right)}
$$

and the normalized intensity $I$ is:

$$
I=E \times E^{*}
$$

Since the air-gap is very small and build-in inside the fiber, it is difficult to know its size. In order to know the effect of all parameters on the interference spectrum, we developed a graphic-user-interface (GUI) to make it easy to change every parameter related to the sensors, such as reflectance, fiber cavity length. The interface of the GUI for IFPI sensor is shown in Fig. 5. As an example, in Fig. 5, we use $68.66 \mu \mathrm{m}$ as fiber cavity length. In simulation, we set $L_{2}=68.66 \mu \mathrm{m}$, and when $L_{1}=1.548 \mu \mathrm{m}$ (air-gap size), refractive index are $0.02792,0.02792$ and 0.04 respectively, the spectrum from the simulation is become the same with the actual spectrum shown in Fig. 6. In this way, we can approximately know all the values that are hard to measure.

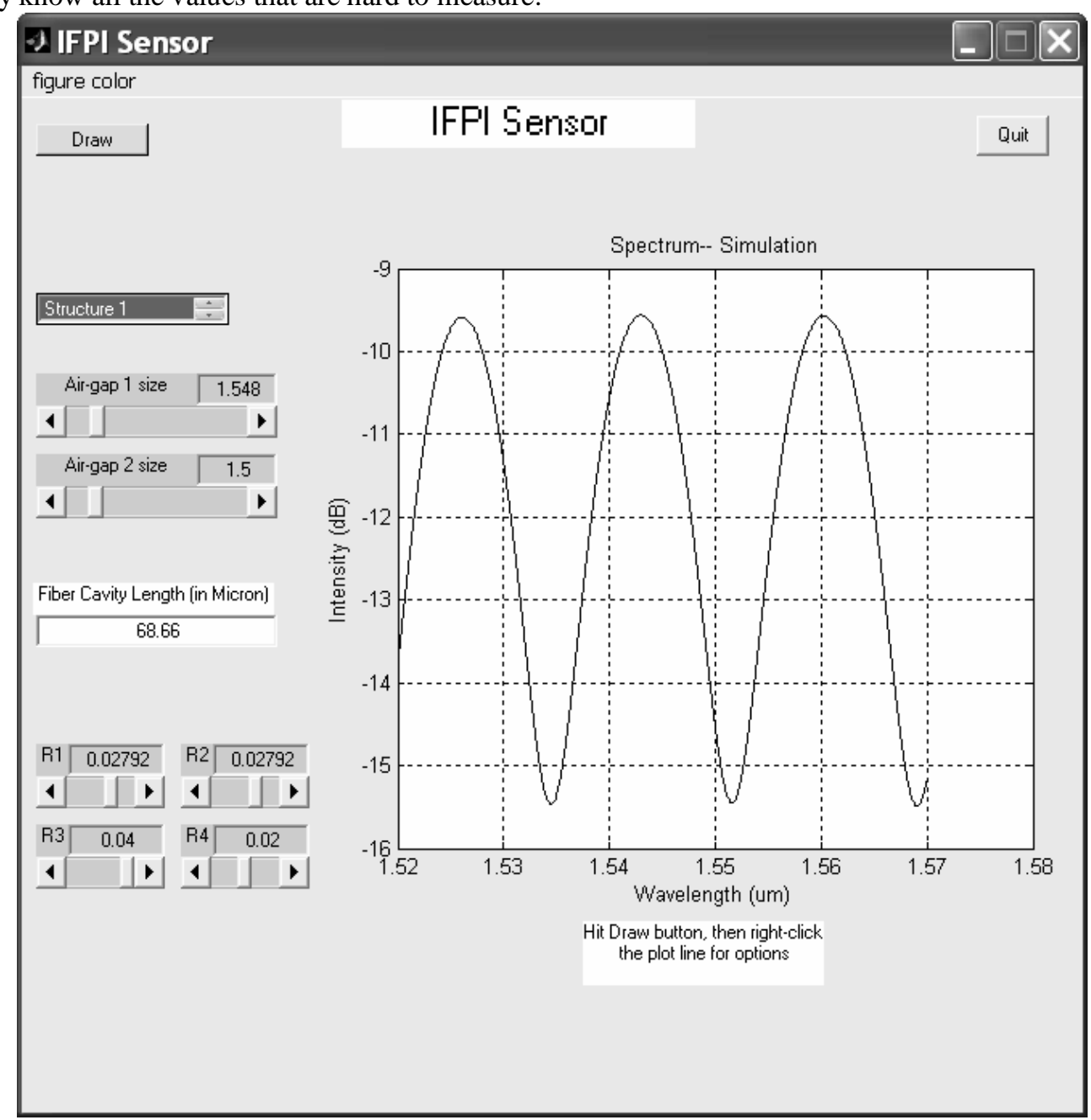

Fig. 5. Simulation based on theoretical model 


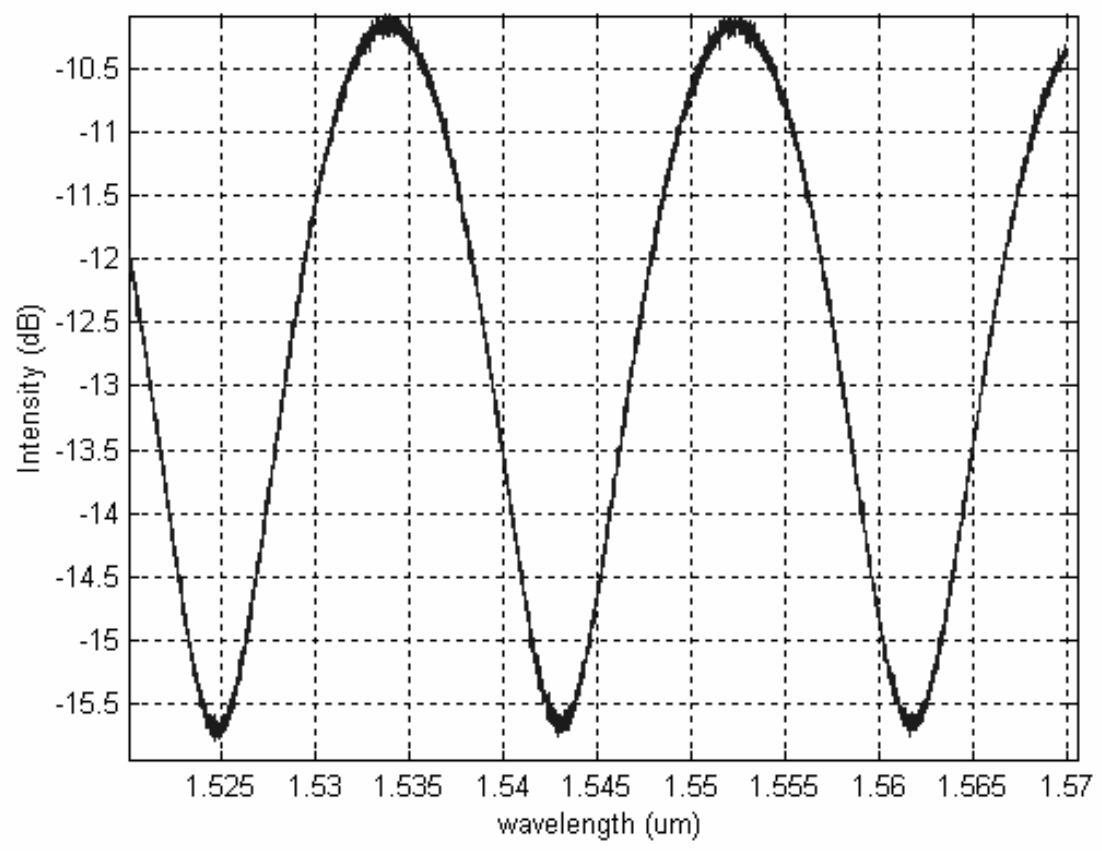

Fig. 6. Interference fringes captured from testing system

\section{APPLICATIONS}

Real time-component testing system (RT-CTS) developed by Micron Optics, Inc. was used to monitor the interference fringes. Particular signal processing software developed by CPT, Virginia Tech. University provides the capacity to filter out the desired signal in frequency domain. The principle diagram of the RT-CTS system is shown in Fig. 7.

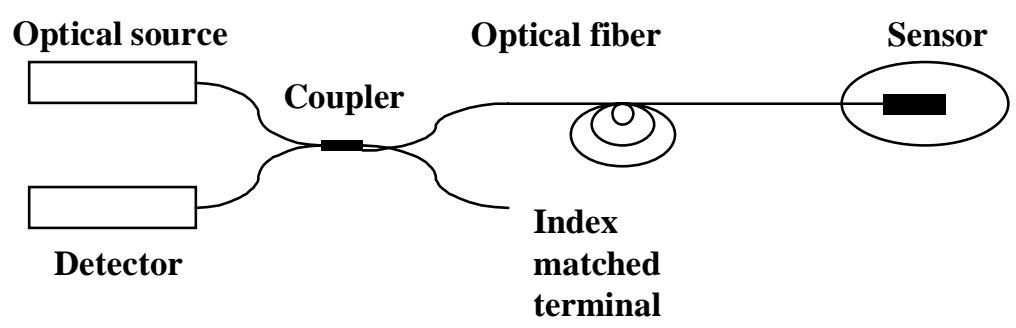

Fig. 7. Principle diagram of the sensor testing system

Eq. (1) indicates that any changes on refractive index $n$ and fiber cavity length $L$ can induce an optical phase change, therefore, induce an optical path difference (OPD) between measurements before and after the perturbation is applied. So that certain perturbations can be measured by monitoring OPD as long as $n$ and $L$ changed. Based on this particular structure of IFPI sensors, they are capable to measure temperature, strain, high pressure, acoustic, refractive index and thin film thickness. Also they can be used to multiplex with themselves, other type of IFPI sensors, EFPI sensors and fiber grating sensors by structure 2 to do multiple points measurement and to compensate the temperature change.

We now present some of applications, such as temperature measurement, multiple points strain measurement and temperature compensation for thin film thickness measurement. Some measurements use structure 1 and some use structure 2 , and some use both of them. 


\subsection{Temperature measurement}

We used both structures of this sensor to measure the temperature changes. Their performance as temperature sensors were demonstrated from room temperature to $700^{\circ} \mathrm{C}$.

For the temperature measurement can be determined by

$$
O P D=L\left(\frac{d n}{d T}+n \frac{d L}{d T}\right) \Delta T
$$

where OPD is optical path difference, $\mathrm{dn} / \mathrm{dT}$ is the thermooptic coefficient, and $\mathrm{dL} / \mathrm{dT}$ is the thermal expansion coefficient of the sensing fiber, $\Delta T$ is the temperature change per period of fringe. For Corning SMF-28, $n=1.4488$ at wavelength of $1550 \mathrm{~nm}$,

$$
\begin{aligned}
& d L / d T \approx 0.55 \times 10^{-6} /{ }^{\circ} \mathrm{C}, \\
& d n / d T \approx 9.2 \times 10^{-6} /{ }^{\circ} \mathrm{C} .
\end{aligned}
$$

We use a sensor with fiber cavity length of $623.0656 \mu \mathrm{m}$ to measure the temperature. Our measurement showed that the optical length change $4.41 \mathrm{~nm} /{ }^{\circ} \mathrm{C}$. In Fig. 8, temperature was increased for four times and in Fig. 9, temperature decreased from $700^{\circ} \mathrm{C}$ to $50^{\circ} \mathrm{C}$ for four times. These two figures show that this sensor has high repeatability in temperature measurement.

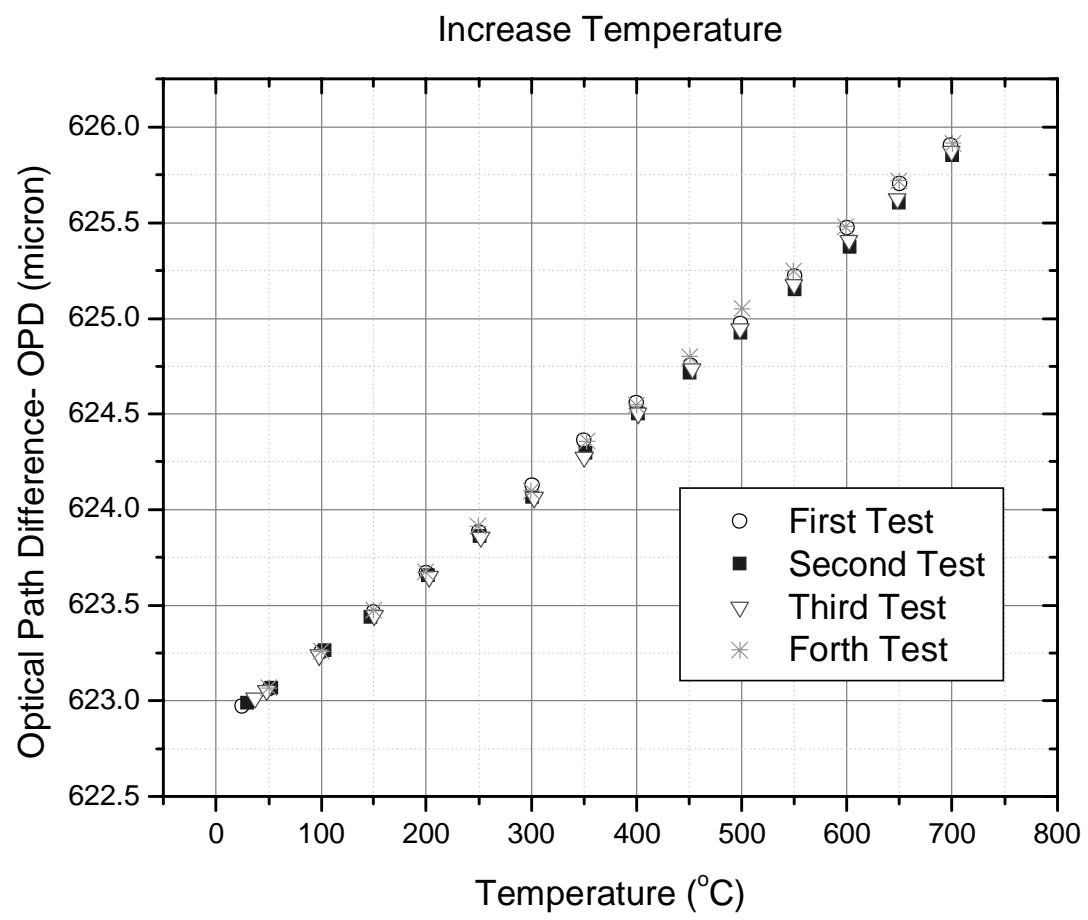

Fig. 8. OPD vs. temperature when increase temperature 


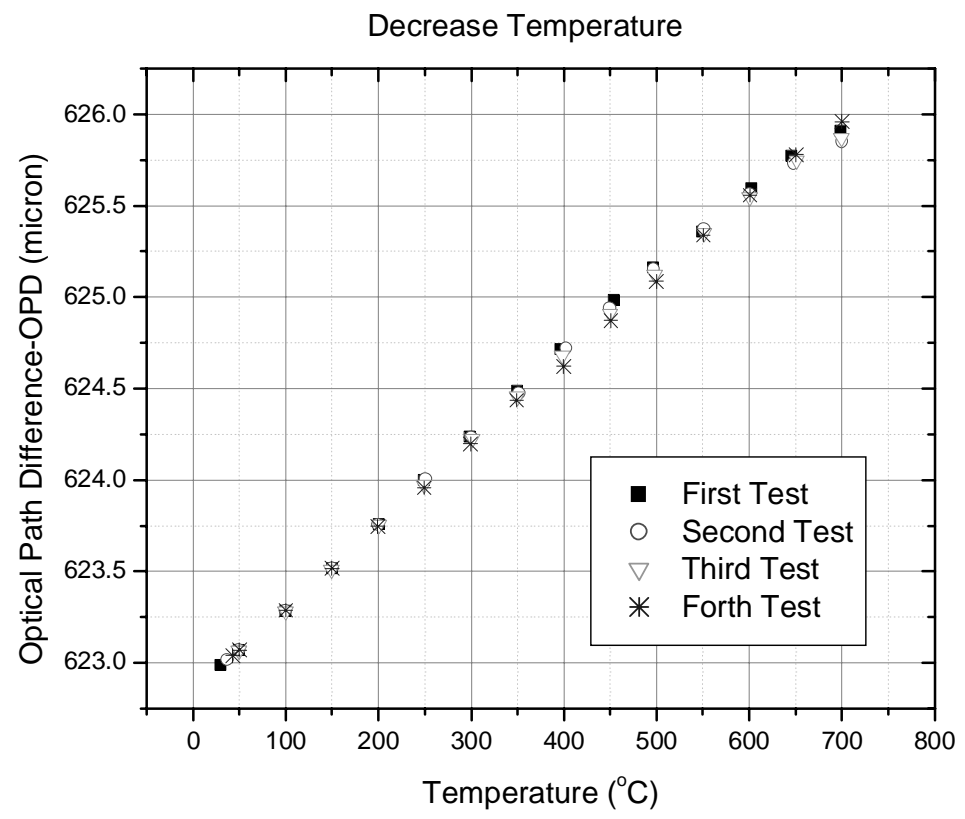

Fig. 9. OPD vs. temperature when decrease temperature

Compared to other two types of IFPI sensors, thin-film IFPI sensors ${ }^{[1]}$ and different core diameter IFPI sensors ${ }^{[3]}$, this structure can perform well in detecting temperature in larger range and more robust.

\subsection{Multiple points strain measurement}

For strain measurement, we use the $2^{\text {nd }}$ structure of the sensor to cascade two sensors to make multi-point strain measurement. We used a Foil Gauge as a reference to calibrate the optical sensors. The strain test setup is shown as in Fig. 10.

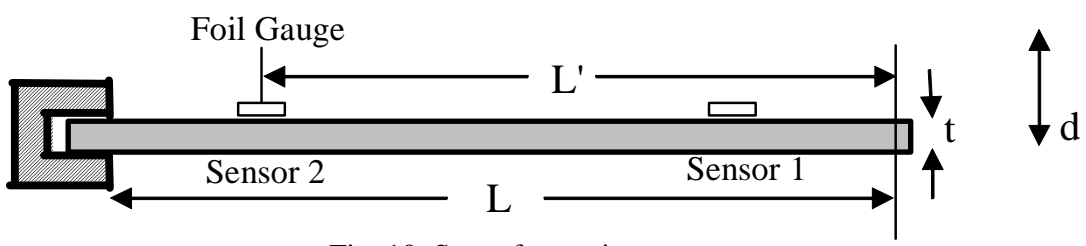

Fig. 10. Setup for strain measurement

Theoretically, the stain is

$$
\text { TheoryStrain }=\frac{3 L^{\prime} t d}{2 L^{3}},
$$

where $t$ is the thickness of the substrate to support the sensors and the $d$ is the height when one side of the substrate can be lifted up to cause the strain change. $\mathrm{L}$ is the total length of the substrate and $\mathrm{L}^{\prime}$ is the distance between the substrate end and where foil gauge was placed.

If we measure the optical path changes to get the strain change information, we can follow the following equations:

$$
\frac{\Delta O P D}{O P D_{0}}=\frac{\Delta n}{n_{0}}+\frac{\Delta l}{l_{0}}=0.737 \frac{\Delta l}{l_{0}}
$$




$$
\text { OpticalStrain }=\frac{1}{0.737} \frac{\triangle O P D}{O P D_{0}}
$$

Fig. 11 and Fig. 12 show that the optical sensors' test results agreed with the results from foil strain test system. And Fig.13 shows that the experimental results agree with the theoretical results.

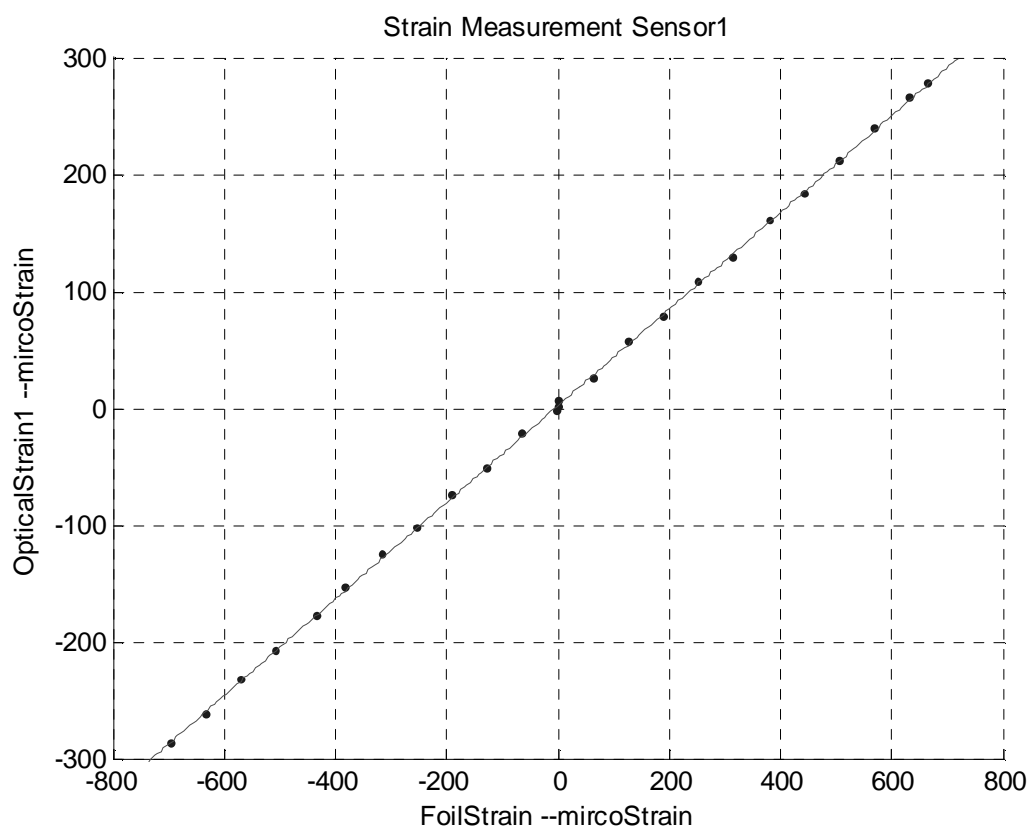

Fig. 11. Strain test result of the $1^{\text {st }}$ sensor

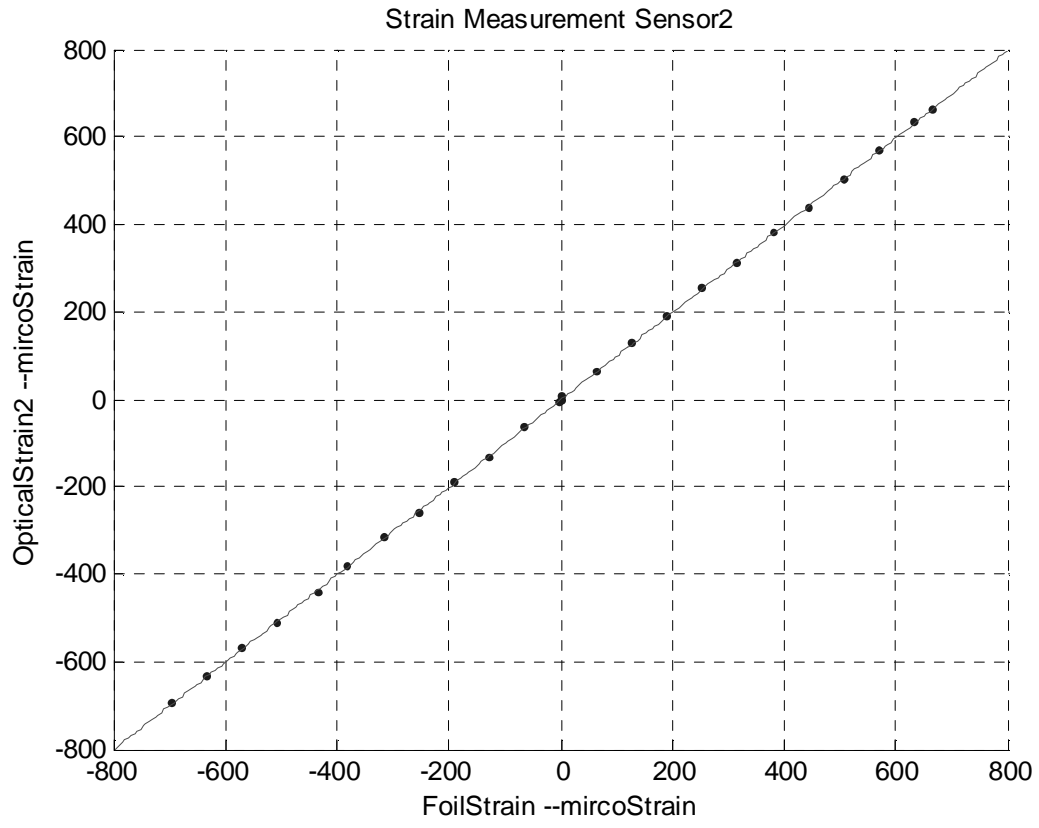

Fig.12. Strain test result of the 2nd sensor 


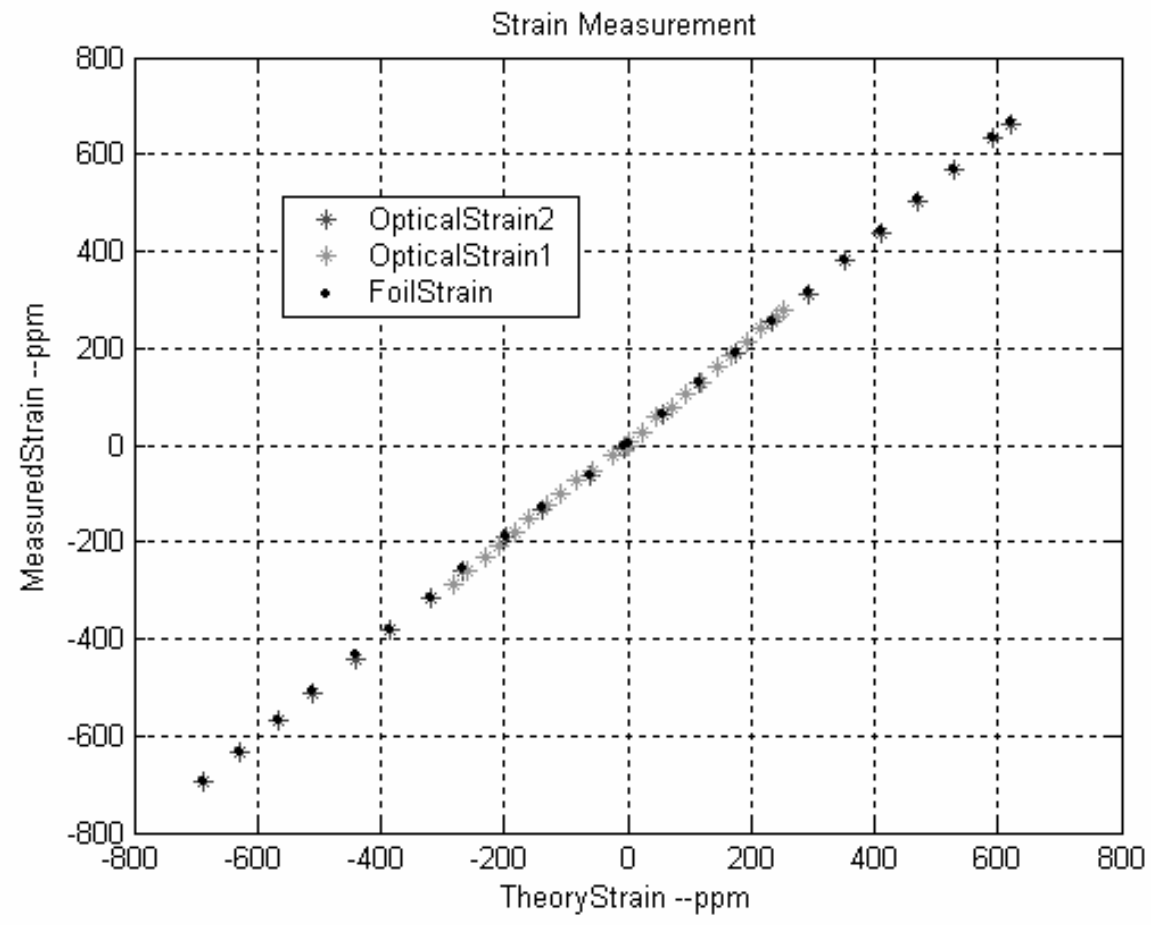

Fig.13. Comparison between the optical sensors and the foil strain test

\subsection{Temperature compensation for thin film thickness measurement}

In order to evaluate the growth of the thin film on the surface of the fiber end, it was necessary to design the system to be robust against noise factors presented during the measurement so that the thickness measurement would not be adversely affected. Through a set of special designed experiments, we found that the temperature is the major source of vibration. A temperature compensation technology was developed to reduce the temperature effect. Fig.14 shows the structure of the thin-film sensor with temperature compensation. The original thin-film sensor (structure 1) was multiplexed with another temperature sensor (structure 2).

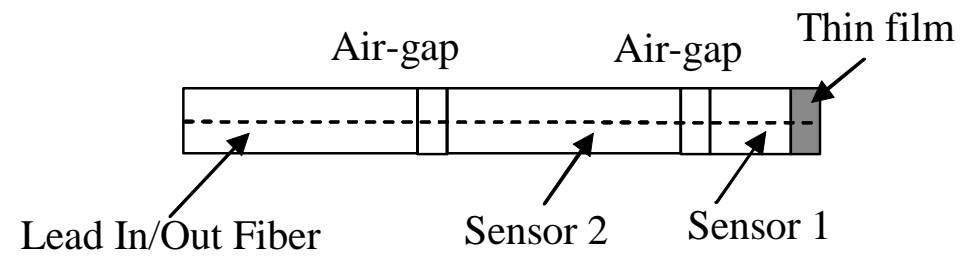

Fig. 14. Structure of the temperature compensation thin-film sensor

From analysis of variance (ANOVA) method in statistics, we found that before temperature compensation, 79\% vibration in the thin film thickness measurement is caused by temperature instability. After temperature compensation, most of the temperature effect has been eliminated. In Fig. 15, we show the results generated by the statistic software (Qualtek-4). Y-axis in Fig. 15 represents the percentage of deviation from mean (in percentage) and x-axis represents the number of experiments. 


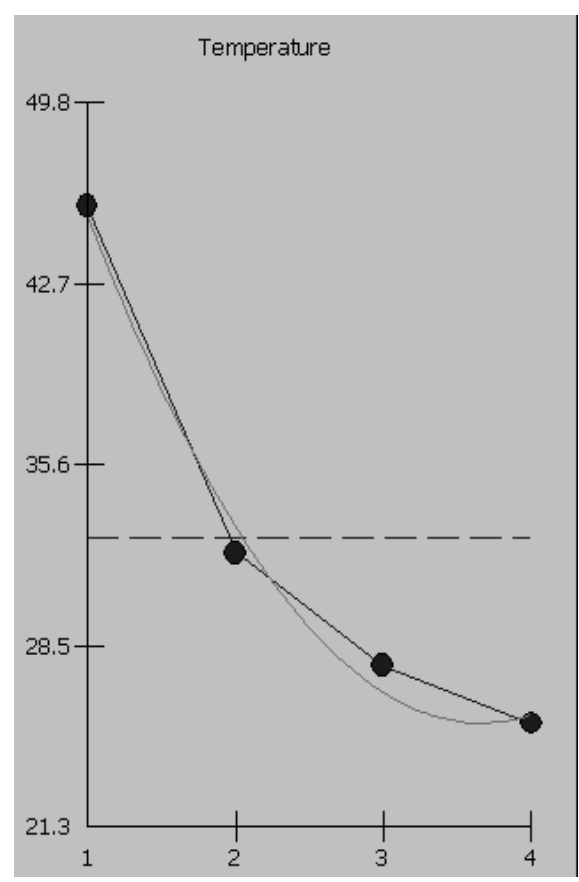

(a)

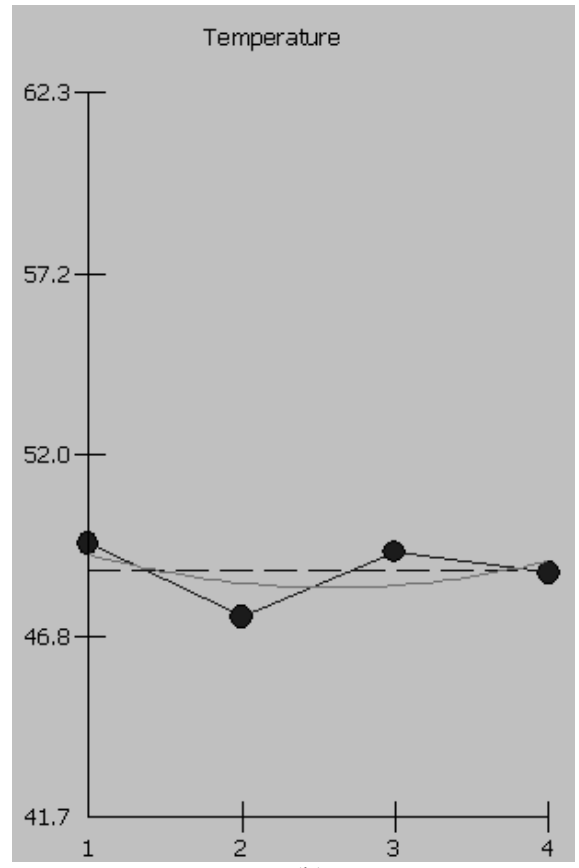

(b)

Fig. 15. Temperature contribution to thin film thickness measurement (a) Before temperature compensation; (b) After temperature compensation

\section{CONCLUSIONS}

We have presented a novel process to fabricate IFPI sensors. This process is based on wet chemical etching and fusion splicing. It is simple, cost effective, robust, and with potential of batch production. The measurement results demonstrate that this type of sensor, with two possible structures, has multiple applications and advantages over other IFPI sensors, especially in temperature measurement. We will continue to working on developing more applications for this sensor structure. The works undergoing include multiplexing this sensor with LPG and multiplexing this sensor with a miniature diaphragm based pressure sensor to provide temperature compensation.

\section{ACKNOWLEDGEMENTS}

The authors would like to thank the U.S. Department of Energy (contract number: DE-FC36-01GO11050) for supporting this project. Also, the authors appreciate the partial donation of RT-CTS from Micron Optics.

\section{REFERENCES}

[1] C.E.Lee, and H.F. Taylor, "Optical-fiber Fabry-Perot embedded sensor", Optical Letters, Vol. 14, No. 21, November 1991, pp1225-1227

[2] Harmeet Singh and James S. Sirkis, "Simultaneously Measuring Temperature and Strain Using Optical Fiber Microcavities", Journal of Lightwave Technology, Vol.15, No.4, April 1997, pp647-653 
[3] Woo-Hu Tsai, Chun-Jung Lin, "A Novel Structure for the Intrinsic Fabry-Perot Fiber-Optic Temperature Sensor", Journal of Lightwave Technology, Vol.19, No.5, May 2001, pp682-686

[4] A. Klini, David, E. Bourillot, S. Emonin, P. Papadopoulos, J. P. Goudonnet, and G. Kotrotsios, "Reproducible Optical Fiber Tips for Photon Scanning Tunneling Microscopy with Very Small $\left(<5^{\circ}\right)$ Cone Angle", Journal of Lightwave Technology, Vol.16, No.7, July 1998

*xichen5@ vt.edu; phone 1540 231-8359; fax: 1540 231-2158;www.ee.vt.edu/ photonics/ 


\title{
Miniature Fiber-Optic Pressure Sensor
}

\author{
Yizheng Zhu, Student Member, IEEE, and Anbo Wang, Senior Member, IEEE
}

\begin{abstract}
This letter presents a miniature fiber-optic pressure sensor fabricated directly on the tip of a fiber. The sensor measures only $125 \mu \mathrm{m}$ in diameter. A Fabry-Pérot cavity and a thin silica diaphragm are fabricated by simple techniques involving only fusion splicing, cleaving, and wet chemical etching. Interference pattern of the sensor is analyzed and issues in sensor design are discussed. The sensor has been tested for static pressure response, showing excellent repeatability and no hysteresis. Its all-fused-silica structure lends it great potential for high-temperature pressure measurements. Pressure testing results at $530{ }^{\circ} \mathrm{C}$ are given. The sensor may also find uses in medical applications.
\end{abstract}

Index Terms-Fabry-Pérot (F-P) interferometer, fiber-optic sensor, high temperature, miniature, pressure sensor.

\section{INTRODUCTION}

$\mathbf{P}$ RESSURE sensors have been widely used in a large number of applications from automotive and aerospace industries to healthcare. Miniature sensors are preferred in many situations because of the minimized impact on the measured system. Significant efforts have been exerted to fabricate these small sensors, either electrically or optically interrogated. Compared to electrical sensors which usually employ capacitive or piezoresistive detection [1], [2], interferometric optical fiber sensors have the advantages of high resolution, high sensitivity, intrinsic electrical passivity, and immunity to electromagnetic interference. Miniature fiber-optic sensors based on Fabry-Pérot (F-P) interferometry have been reported using various approaches [3]-[5].

In this letter, we demonstrate a novel miniature fiber-optic pressure sensor fabricated directly on the tip of a fiber, using only fusion splicing, cleaving, and wet chemical etching, all of which are simple and cost-effective processes.

While current miniature fiber-optic sensors usually involve several different materials in fabrication, the proposed sensor is made of commercial silica fibers only, resulting in an allfused-silica structure with excellent mechanical properties. It eliminates the problem of thermal expansion mismatch between different materials, extending the sensor's high-temperature capability to the limit determined only by silica itself. Therefore, this miniature sensor has great potential to operate reliably at elevated temperatures. It may find applications where high temperature and/or small size are important.

Manuscript received June 4, 2004; revised September 8, 2004. This work was supported by the U.S. Department of Energy under Grant DE-FC36-01G011050.

The authors are with the Center for Photonics Technology, Bradley Department of Electrical and Computer Engineering, Virginia Polytechnic Institute and State University, Blacksburg, VA 24061 USA (e-mail: yizhu1@vt.edu; awang@vt.edu).

Digital Object Identifier 10.1109/LPT.2004.839002

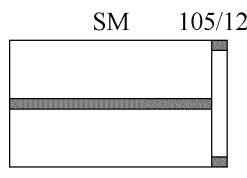

(a)

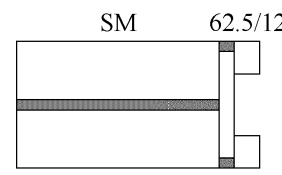

(b)

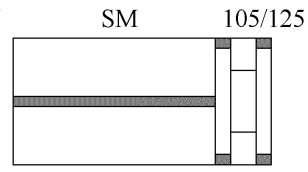

(c)
Fig. 1. Fabrication steps. (a) Etch barrier splicing/cleaving. (b) Cavity splicing/cleaving/etching. (c) Diaphragm splicing/cleaving/etching. (Gray area indicates doped region).

\section{SENSOR DESIGN AND FABRICATION}

Three types of commercial silica fibers are used: single-mode (SM) fiber (Corning, Inc.), 62.5/125- $\mu \mathrm{m}$ graded-index multimode fiber (Thorlabs, Inc.), and $105 / 125-\mu \mathrm{m}$ step-index multimode fiber (Fiberguide Industries, Inc.). The 62.5- $\mu \mathrm{m}$ fiber is germanium-doped in the core with undoped fused silica cladding, while the $105-\mu \mathrm{m}$ fiber has undoped fused silica core and fluorine-doped cladding. Hydrofluoric acid (HF, 50\%) is used for wet chemical etching where doped silica will be etched much faster than updoped region [6].

Fig. 1 shows the fabrication processes. In Fig. 1(a), an SM fiber is spliced with $105-\mu \mathrm{m}$ fiber. Through a $40 \times$ microscope, the splicing point between the two fibers can be clearly seen and a cleave is then made just beside it to leave a thin layer of $105-\mu \mathrm{m}$ fiber on top of the SM fiber. The thickness of this layer is usually $10 \sim 15 \mu \mathrm{m}$ which, however, is not important since this undoped silica layer acts only as an etch stop in the following step. Different thickness will slightly affect the light loss but will not alter the sensor's interference pattern. In Fig. 1(b), a $62.5-\mu \mathrm{m}$ fiber is spliced with this part and cleaved to a certain length for the fabrication of the F-P cavity. In our case, $20 \sim 30 \mu \mathrm{m}$ is the best value in terms of easiness in both fabrication and signal processing. Immersing the tip in 50\% HF to remove the core of the $62.5-\mu \mathrm{m}$ fiber. The etching rate for the doped core is $17 \mu \mathrm{m} / \mathrm{min}$ while that of undoped silica is only 1 $\mu \mathrm{m} / \mathrm{min}$. Therefore, the etching "stops" at the barrier layer, generating a flat bottom and protecting the core of SM fiber from being etched. Otherwise, etching of the SM fiber core will reduce its reflection intensity. The cladding of the $62.5-\mu \mathrm{m}$ fiber is also undoped fused silica and remains almost intact, left as the wall of the pit. In Fig. 1(c), we splice the pit with 105- $\mu \mathrm{m}$ fiber to form the F-P cavity. Instead of regular splices in previous steps, reduced power and duration of the splicing arc are necessary to prevent the structure from being over-melted. Fiber gap and overlap also need careful control to minimize the deformation of the cavity when the two fibers are pushed together for splice. We cleave the $105-\mu \mathrm{m}$ fiber to a thin layer, which is then etched to reduce its thickness and serves as the diaphragm. The final structure is made of undoped fused silica. 


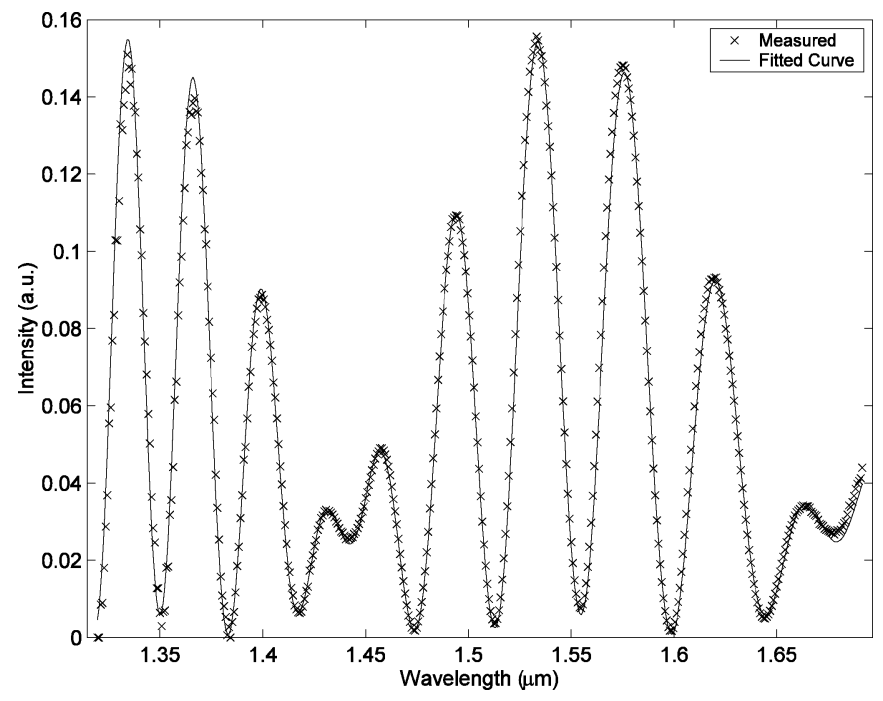

Fig. 2. Interference pattern from the sensor head.

The sensor head will generate three reflections, one from the cavity bottom and the other two from the two surfaces of the diaphragm. They will interfere with each other and the total reflected light intensity can be written as

$$
\begin{aligned}
I(\lambda)= & \left|A_{1}-A_{2} \exp \left(-\frac{j 4 \pi L}{\lambda}\right)+A_{3} \exp \left(-\frac{j 4 \pi\left(L+n_{\lambda} d\right)}{\lambda}\right)\right|^{2} \\
= & A_{1}^{2}+A_{2}^{2}+A_{3}^{2}-2 A_{1} A_{2} \cos \left(\frac{4 \pi L}{\lambda}\right) \\
& -2 A_{2} A_{3} \cos \left(\frac{4 \pi n_{\lambda} d}{\lambda}\right) \\
& +2 A_{3} A_{1} \cos \left(\frac{4 \pi\left(L+n_{\lambda} d\right)}{\lambda}\right)
\end{aligned}
$$

where $A_{1}, A_{2}$, and $A_{3}$ are the amplitudes of the light from the cavity bottom, the inner and outer surfaces of the diaphragm, respectively, $L$ is the cavity length, $d$ is the diaphragm thickness, $n_{\lambda}$ is the refractive index of silica, and $\lambda$ is the wavelength. The negative sign of $A_{2}$ results from a phase change of $\pi$ at the inner surface of the diaphragm where light incidents from air to silica.

A 1550-nm light emitting diode (LED) and an optical spectrum analyzer (OSA) are used to get the interference pattern. At 1 atmospheric pressure $(1 \mathrm{~atm}, 13.7 \mathrm{psi}, 1 \mathrm{psi}=6.89 \times$ $10^{3} \mathrm{~Pa}$ ) and room temperature, the signal spectrum from 1320 to $1690 \mathrm{~nm}$ is normalized to LED spectrum and plotted in Fig. 2. Equation (1) is applied to fit the data. The fitted curve matches very well with the measured spectrum with $L=25.6548 \mu \mathrm{m}$ and $d=3.4860 \mu \mathrm{m}$. Interference of the F-P cavity $\left(A_{1}\right.$ and $\left.A_{2}\right)$ is modulated by a low frequency envelope which is due to the third reflection $\left(A_{3}\right)$ and corresponds to the thin thickness of the diaphragm.

In Fig. 2, average signal intensity and visibility reach minimal at about $1440 \mathrm{~nm}$, where the phase difference between the two reflections from the diaphragm is $(2 \pi \times 2 \mathrm{nd} / \lambda+\pi)=(4 \times$ $1.444 \times 3.486 / 1.44+1) \pi \approx 15 \pi$. So they are out of phase and very much cancel each other out, leaving only the first beam going back into the fiber. This should be avoided at the operating wavelength. On the other hand, at $1550 \mathrm{~nm}$, the two reflections are in phase (phase difference: $14 \pi$ ) and strengthen each other, maximizing the power level while maintaining good visibility as well. This is preferred and achieved by monitoring the signal power to reach its maximum during etching.

Ambient pressure change will cause diaphragm to deflect, reducing the cavity length and blue-shifting the interference spectrum. For the same sensor in Fig. 2, curve-fitting of the shifted spectrum at 190 psi gives $L=25.6021 \mu \mathrm{m}$ and $d=$ $3.4861 \mu \mathrm{m}$. As expected, $d$ remains the same while $L$ is reduced by $52.6 \mathrm{~nm}$, giving a sensitivity of $S=52.6 \mathrm{~nm} /(190-$ $13.7) \mathrm{psi}=0.3 \mathrm{~nm} / \mathrm{psi}$.

To compare with the experimental results, the theoretical model can be applied which states for an edge-clamped circular diaphragm, its deflection $\Delta L$ will follow

$$
\Delta L=\frac{3\left(1-\nu^{2}\right) R^{4} p}{16 E d^{3}}
$$

where $\nu$ and $E$ are Poisson's ratio and Young's modulus of fused silica, respectively, $R$ is the diaphragm radius, $d$ is the thickness, and $p$ is the pressure change. The sensitivity of the sensor is $S=\Delta L / p$. For this silica sensor, $E=73 \mathrm{Gpa}$, $\nu=0.17, R$ is estimated to be $33 \mu \mathrm{m}$, larger than the core radius due to etching. Using the sensitivity given above $(0.3 \mathrm{~nm} / \mathrm{psi})$, $d$ is estimated to be $4.08 \mu \mathrm{m}, 0.6 \mu \mathrm{m}$ thicker than the fitted value. It is believed that the difference largely comes from the nonuniformity of the diaphragm thickness as a result of imperfect cleaving, with other errors from radius estimation and nonideal circular shape.

\section{Sensor Testing And Results}

Rather than fitting the entire spectrum, simpler detection of spectral shift can be achieved with higher resolution by tracing the positions of interference minima-maxima (valley-peak) [7]. A local parabolic curve fitting is carried out for data around the valley-peak to determine its position. The cavity length then results in multiplying the position with the fringe order, as

$$
\frac{4 \pi L}{\lambda}+\pi=2 N \pi \Rightarrow L=\frac{(2 N-1) \lambda}{4}
$$

where $L$ is cavity length, $\lambda$ is valley-peak wavelength, and $N$ is fringe order. $N$ and $\lambda$ are calculated from the spectrum.

The low frequency envelope induced by $A_{3}$ will slightly change the valley-peak position. Thus, the calculated cavity length will have a small deviation from the actual value. The pressure dependence of refractive index of air will also slightly modify $A_{3}$ and add to this effect. Nevertheless, since this impact is repeatable, the calculated cavity length will have a repeatable relation with the ambient pressure and, thus, can still be used as an index to it. A calibration is needed to specify this relation.

Fig. 3 shows the experimental setup for static pressure test. For faster data acquisition and better resolution, the LED and OSA are replaced by Micron Optics' Si720 Component Testing System (CTS) which is essentially a spectrometer with a build-in tunable laser source and a detector. It has a spectral range from 1520 to $1570 \mathrm{~nm}$ and wavelength resolution of $2.5 \mathrm{pm}$. Light from the CTS travels to the sensor through a circulator and is reflected back into the CTS. The sensor is 


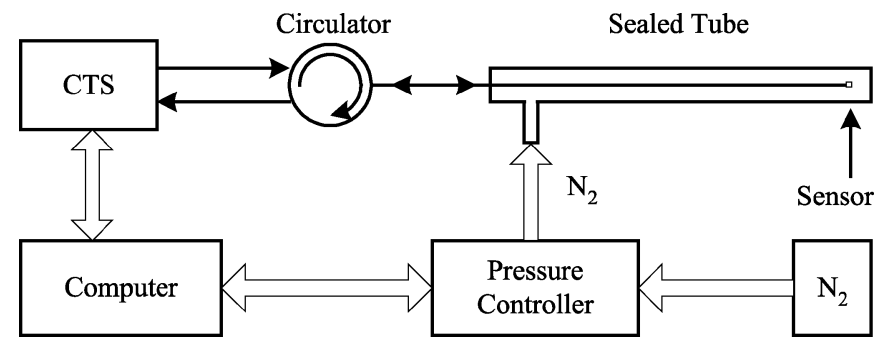

Fig. 3. System setup for static pressure test.

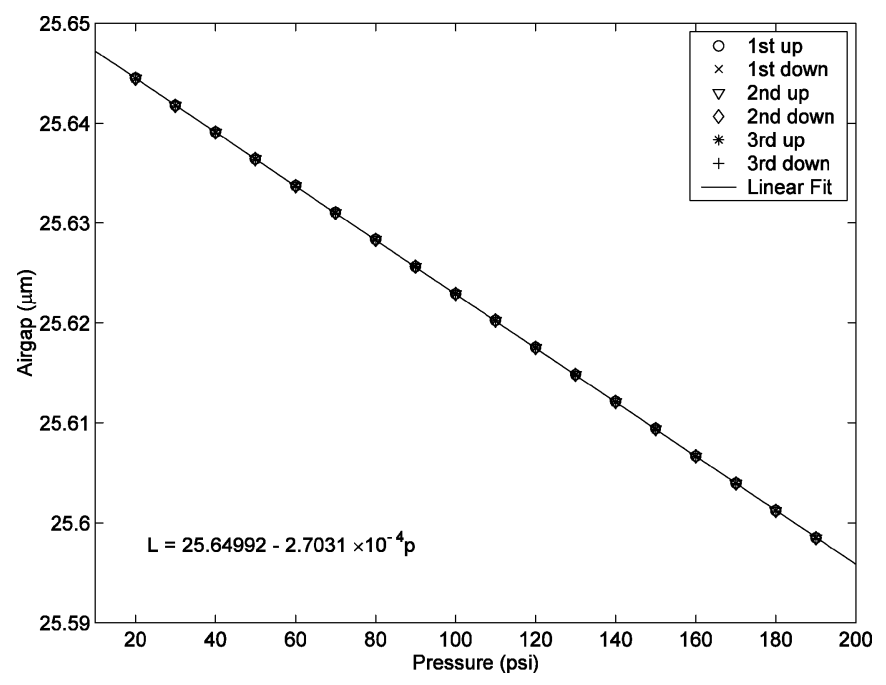

Fig. 4. Sensor pressure response from 20 to 190 psi for three cycles.

sealed in a tube and pressurized by a pressure calibrator (Pressure Systems, Inc., 9035). A computer controls the pressure calibrator and retrieves spectrum data from the CTS.

Plotted in Fig. 4 is the sensor's pressure response at room temperature for three pressure cycles, each of which is from 20 to $190 \mathrm{psi}$ and then back down to $20 \mathrm{psi}$. The repeatability is about $0.2 \% \mathrm{FS}$ without hysteresis observed, indicating the excellent mechanical properties of the structure.

A test is also conducted to explore the sensor's high-temperature capability. A second sensor is tested at $530{ }^{\circ} \mathrm{C}$ from 25 to 150 psi with the sensor end of the tube in Fig. 3 placed in a furnace. The results are plotted in Fig. 5 with a repeatability about $1 \% \mathrm{FS}$, a little worse than that at room temperature. The reason is believed to be the slight creeping of stressed silica due to its increased viscosity at elevated temperature. Therefore, the mechanical properties of fused silica at high temperature will set the limit for sensor's capability, details on which will be investigated in future research.

\section{SUMMARY AND DISCUSSION}

We have proposed and demonstrated a novel miniature fiber-optic pressure sensor fabricated directly on the tip of a fiber. Preliminary tests prove its potential for high-temperature applications.

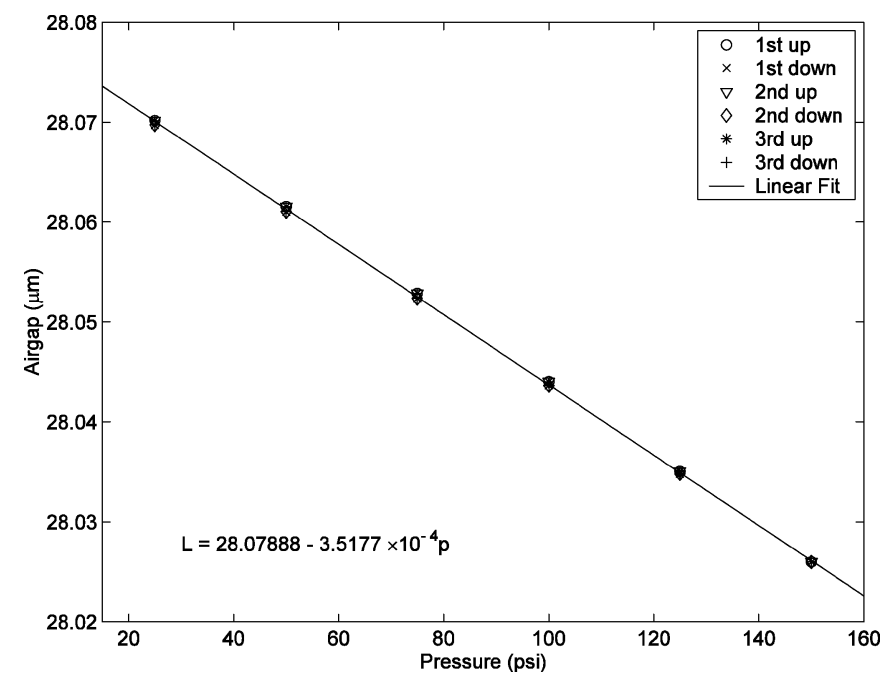

Fig. 5. High-temperature pressure test at $530{ }^{\circ} \mathrm{C}$ from 25 to $150 \mathrm{psi}$.

As for sensor's temperature sensitivity, thermal expansion is a major source. The CTE of silica is $0.55 \mathrm{ppm} /{ }^{\circ} \mathrm{C}$. For a $25-\mu \mathrm{m}$-long cavity and temperature change of $500{ }^{\circ} \mathrm{C}$, the expansion is about $7 \mathrm{~nm}$, or $13 \%$ compared to the cavity length change of $52.6 \mathrm{~nm}$ for the sensor in Fig. 2. A temperature variation of $50{ }^{\circ} \mathrm{C}$ will reduce it to $1.3 \%$. Therefore, this error could be reduced or even neglected for applications where temperature change is limited and/or pressure change is large. Otherwise, temperature compensation may be necessary for high accuracy measurement. A possible solution is to use a temperature sensor along with the pressure sensor to correct the thermal expansion based on sensor's temperature sensitivity.

\section{ACKNOWLEDGMENT}

The authors would like to thank Micron Optics for the partial donation of the CTS used in this research.

\section{REFERENCES}

[1] D. Goustouridis, P. Normand, and D. Tsoukalas, "Ultraminiature silicon capacitive pressure-sensing elements obtained by silicon fusion bonding," Sens. Actuators A, vol. A68, pp. 269-274, 1998.

[2] P. Melvås, E. Kälvesten, P. Enoksson, and G. Stemme, "A free-hanging strain-gauge for ultraminiaturized pressure sensors," Sens. Acuators A, vol. 97-98, pp. 75-82, 2002.

[3] T. Katsumata, Y. Haga, K. Minami, and M. Esashi, "Micromachined 125 $\mu \mathrm{m}$ diameter ultra miniature fiber-optic pressure sensor for catheter," Trans. Inst. Electr. Eng. Jpn. E, vol. 120-E, pp. 58-63, 2000.

[4] E. Cibula, D. Donlagic, and C. Stropnik, "Miniature fiber optic pressure sensor for medical applications," in Proc. IEEE Sensors 2002, 1st IEEE Int. Conf. Sensors, vol. 1, Piscataway, NJ, 2002, pp. 711-714.

[5] D. C. Abeysinghe, S. Dasgupta, J. T. Boyd, and H. E. Jackson, "A novel MEMS pressure sensor fabricated on a optical fiber," IEEE Photon. Technol. Lett., vol. 13, no. 9, pp. 993-995, Sep. 2001.

[6] T. Pangaribuan, K. Yamada, S. Jiang, H. Ohsawa, and M. Ohtsu, "Reproducible fabrication technique of nanometric tip diameter fiber probe for photon scanning tunneling microscope," Jpn. J. Appl. Phys., vol. 31, pp. 1302-1304, 1992.

[7] B. Qi, G. R. Pickrell, J. Xu, P. Zhang, Y. Duan, and W. Peng et al., "Novel data processing techniques for dispersive white light interferometer," Opt. Eng., vol. 42, pp. 3165-3171, 2003. 


\title{
Sapphire-fiber-based white-light interferometric sensor for high-temperature measurements
}

\author{
Yizheng Zhu, Zhengyu Huang, Fabin Shen, and Anbo Wang \\ Center for Photonics Technology, The Bradley Department of Electrical and Computer Engineering, \\ Virginia Polytechnic Institute and State University, Blacksburg, Virginia 24061
}

Received October 5, 2004

\begin{abstract}
We present a fiber-optic temperature sensor with a single-crystal sapphire fiber as the light guide and a sapphire wafer as the sensing element. Temperature is determined by measurement of the thermal dependence of the wafer's optical thickness by use of white-light interferometry. We applied digital signal processing techniques to analyze the sensor's spectrum. A prototype sensor was tested to $1600{ }^{\circ} \mathrm{C}$ and demonstrated excellent reproducibility. An accuracy of $\pm 0.2 \%$ full scale was obtained. The sensor is simple, small, and flexible, with potential advantages of batch fabrication and easy calibration. () 2005 Optical Society of America

OCIS codes: $060.2370,120.6780$.
\end{abstract}

Over the past two decades, fiber-optic hightemperature sensors have seen many applications in a wide range of industries. A variety of such sensors have been developed based on different technologies. As silica-fiber-based temperature sensors usually cannot exceed $1000{ }^{\circ} \mathrm{C}$ because of degraded mechanical strength and thermal diffusion of the germanium dopant, ${ }^{1}$ single-crystal optical fibers have received extensive attention owing to their compact structures and excellent mechanical and optical properties at elevated temperatures. Radiation-based temperature sensors that use sapphire fibers as light pipes to collect and transmit blackbody radiation were developed more than two decades ago ${ }^{2,3}$ and are currently the sensor of choice in semiconductor industries for monitoring rapid thermal processing. Tong et al. demonstrated the operation of such a sensor up to $2300{ }^{\circ} \mathrm{C}$, using zirconia single-crystal fiber. ${ }^{4}$ In fluorescence-based sensors a doped fiber is excited by a pulsed laser to generate fluorescence whose decay time is temperature dependent and is used as the measurand. The use of silica fibers limits most sensors of this type to relatively low temperatures. Kennedy and Djeu reported a maximum operating temperature of $1600^{\circ} \mathrm{C}$ by an Yb-doped singlecrystal YAG optical fiber. ${ }^{5}$ Fiber-optic Fabry-Perot (FP) interferometric sensors have the advantages of high accuracy, high resolution, and simple configuration. Various principles based on this structure have been demonstrated for high-temperature sensing. Wang et al. proposed both intrinsic ${ }^{1,6}$ and extrinsic ${ }^{7}$ FP temperature sensors that use sapphire fiber.

Single-crystal fibers are highly multimode owing to their large diameters and large numerical apertures. Multimode-fiber-illuminated extrinsic FP interferometers cannot easily generate good interference. The fringes are highly sensitive to the smoothness, the flatness, the distance between, and especially the parallelism of the two surfaces. ${ }^{8}$ It was reported that an angle of the order of $10^{-2} \mathrm{deg}$ could significantly reduce fringe visibility. ${ }^{9}$ In the research reported in this Letter we use a sapphire wafer as an extrinsic
FP interferometer. Because high surface quality and excellent parallelism can readily be achieved in the current wafer lapping-polishing industry, fringes can easily be generated, even for highly multimode sapphire fiber and large cavity lengths (thick wafers). The choice of relatively large thickness is important, as it offers great convenience for signal processing, as we shall see below.

Figure 1 shows schematics of the system and the sensor. A $59-\mu \mathrm{m}$-thick $C$-plane sapphire wafer of $1 \mathrm{~mm} \times 1 \mathrm{~mm}$ size is placed in front of a 15-cm-long 75- $\mu \mathrm{m}$-thick sapphire fiber (MicroMaterials, Inc.) by a $99.8 \%$ alumina tube (OD, $0.71 \mathrm{~mm}$ ) and hightemperature adhesive. The sapphire fiber also has the $C$ axis along its length. Light from an 850-nm LED is launched into the $100-140-\mu \mathrm{m}$ multimode fiber and propagates to the sapphire wafer through a $3-\mathrm{dB}$ coupler. The sapphire fiber is fusion spliced to the $100-140-\mu \mathrm{m}$ fiber. The interference spectrum generated by the reflections at the two surfaces of the wafer is coupled back into the system and detected by a miniature spectrometer (OceanOptics, Inc., USB2000). Both the refractive index and the thickness of the wafer increase with temperature, changing the optical path difference of the interferometer and encoding the spectrum with temperature information. For temperature tests, the sensor head, including 4-cm-long sapphire fiber, was placed into a furnace (DelTech, Inc.) along with a B-type thermocouple (Omega, Inc.) as a reference.

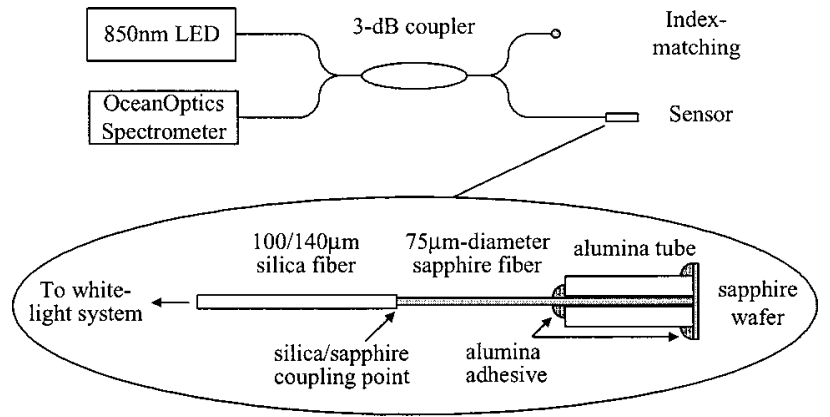

Fig. 1. Schematics of the system and of the sensor. 
The total light intensity received at the spectrometer can be written as

$$
\begin{aligned}
I_{\text {total }}(\lambda)= & I_{B}(\lambda)+I_{D}(\lambda)+I_{\mathrm{LED}}(\lambda)\left(r_{\text {couple }}+r_{\text {end }}\right) \\
& +I_{\mathrm{LED}}(\lambda)\left[r_{s 1}+r_{s 2}-2 V\left(r_{s 1} r_{s 2}\right)^{1 / 2}\right. \\
& \left.\times \cos \frac{4 n(T) d(T) \pi}{\lambda}\right]
\end{aligned}
$$

where $I_{B}(\lambda), I_{D}(\lambda)$, and $I_{\mathrm{LED}}(\lambda)$ are the light intensity of the blackbody radiation, the dark current of the spectrometer, and the LED, respectively; $r_{\text {couple }}, r_{\text {end }}$, $r_{s 1}$, and $r_{s 2}$ are reflectance at every surface along the light path: the silica-sapphire fiber coupling point, the end face of the sapphire fiber, and the first and second surfaces of the sapphire wafer, respectively; $n$ is the refractive index of sapphire, $d$ is the thickness of the wafer, $V$ is the fringe visibility, $\lambda$ is the wavelength, and $T$ is the temperature. Figure 2 shows the spectra at $25^{\circ} \mathrm{C}$ and $1597{ }^{\circ} \mathrm{C}$. The background intensity is significantly raised at $1597^{\circ} \mathrm{C}$ by the blackbody radiation collected by the sapphire fiber.

In Eq. (1), $I_{B}(\lambda), I_{D}(\lambda)$, and $I_{\mathrm{LED}}(\lambda)$ are all slowly varying. Consequently, in the fast Fourier transform of the spectrum, all but the interference signal (cosine term) will be located about the zero frequency. The cosine term, however, is a high-frequency component and separates from the others, as shown in Fig. 3. A bandpass filter is then used to filter all the lowfrequency components out, leaving only the last term, $-2 V\left(r_{s 1} r_{s 2}\right)^{1 / 2} I_{\mathrm{LED}}(\lambda) \cos [4 n(T) d(T) \pi / \lambda]$. As the LED profile $I_{\mathrm{LED}}(\lambda)$ may drift with time or vary during propagation, it should be removed for more stable measurement. This is an envelope-detection problem and can be conveniently solved by the Hilbert transform. It is well known that ${ }^{10}$ if a modulation signal $m(t)$ is low pass and if carrier $\cos (t)$ is at a higher frequency and their spectra do not overlap, the Hilbert transform of their product $p(t)$ follows as

$$
H[p(t)]=H[m(t) \cos (t)]=m(t) H[\cos (t)]=m(t) \sin (t),
$$

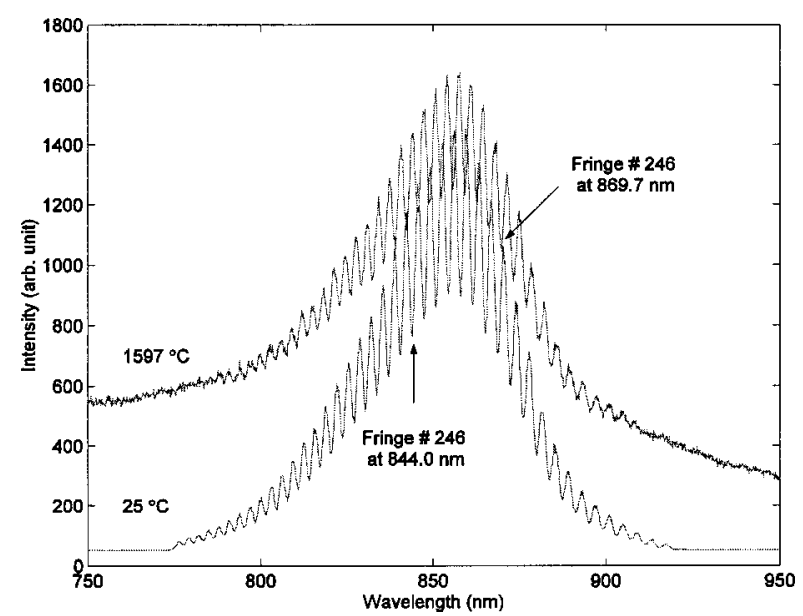

Fig. 2. Sensor spectrum at two temperatures.

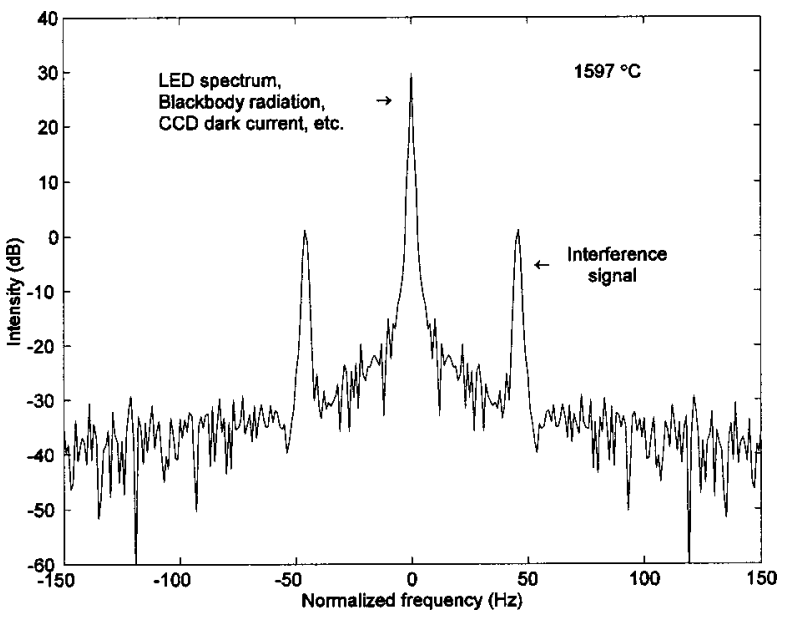

Fig. 3. Fast Fourier transform of the spectrum.

where $H($ ) denotes the Hilbert transform. Therefore $m(t)$ can be extracted as follows:

$$
m(t)=\left\{p^{2}(t)+H^{2}[p(t)]\right\}^{1 / 2} .
$$

Then $\cos (t)$ is obtained by normalization. In our case, $m(t)$ is $2 V\left(r_{s 1} r_{s 2}\right)^{1 / 2} I_{\mathrm{LED}}(\lambda)$. As we have chosen the thickness of the wafer to be relatively large, the cosine term has higher frequency and is located far away from the $I_{\mathrm{LED}}(\lambda)$ spectrum; they have practically no overlap. Therefore $2 V\left(r_{s 1} r_{s 2}\right)^{1 / 2} I_{\mathrm{LED}}(\lambda)$ can be extracted and the normalization results, $-\cos [4 n(T) d(T) \pi / \lambda]$, from which the optical thickness (OT) of the sapphire wafer, $n(T) d(T)$, can be determined by the positions of interference valleys and peaks, ${ }^{11}$ as follows:

$$
\frac{4 \mathrm{OT} \pi}{\lambda}=2 \pi N \Rightarrow \mathrm{OT}=\frac{N \lambda}{2},
$$

where $\lambda$ is the position of the valley or peak and $N$ is the fringe order, both of which are calculated from the spectrum. Considering the negative sign of the cosine term, integer $N$ corresponds to a valley, whereas a half-integer results in a peak. The OT, and hence the spectrum, changes with temperature, as indicated in Fig. 2, where the position of the same valley (interference minimum \#246) is redshifted from $844.0 \mathrm{~nm}$ at $25^{\circ} \mathrm{C}$ to $869.7 \mathrm{~nm}$ at $1597^{\circ} \mathrm{C}$. The system continuously retrieves the spectrum and calculates the positions to trace the shift and monitor changes of the OT. The system can operate now at a frequency of $25 \mathrm{~Hz}$, limited primarily by the $20-\mathrm{ms}$ integration time of the spectrometer and the processing time of the software.

The large cavity length greatly simplifies signal processing in that it not only separates the interference spectrum from blackbody radiation and other irrelevant signals but also makes it easy to remove the LED profile by envelope detection.

In the test the furnace was run three times from $230{ }^{\circ} \mathrm{C}$ to $1600{ }^{\circ} \mathrm{C}$ at a ramping rate of $3{ }^{\circ} \mathrm{C} / \mathrm{min}$. The change in optical thickness with respect to temperature measured by the B-type thermocouple is 
plotted in Fig. 4. By fitting all the data using a fourth-order polynomial, we obtained a calibration curve with which to convert each measured optical thickness of the wafer to a temperature, which is taken as the temperature measured by the sensor. Then the accuracy of the sensor can be estimated by comparison of the temperatures measured by the sensor and the thermocouple. Their difference is plotted in Fig. 5. It varies from $-3{ }^{\circ} \mathrm{C}$ to $3{ }^{\circ} \mathrm{C}$, corresponding to $\pm 0.2 \%$ full-scale accuracy. This variation may be due largely to the accuracy of the thermocouple, which has a specification of $\pm 0.5 \%$ full scale over $800^{\circ} \mathrm{C}$.

In summary, we have demonstrated a sapphirefiber-based extrinsic Fabry-Perot interferometric sensor for high-temperature measurements. The sensor is simple and small. Fringes were readily generated by use of a sapphire wafer as the sensing interferometer. Because each large wafer can be diced into small identical pieces, this method is expected to be readily reproducible and has the potential for batch fabrication and easy calibration. The sensor is interrogated by spectral-domain white-light interferometry. The interference signal is extracted by digital signal processing, which removes the blackbody radiation and other sources of error from the spectrum. A prototype sensor was tested from $230{ }^{\circ} \mathrm{C}$ to $1600{ }^{\circ} \mathrm{C}$ with excellent reproducibility. An accuracy of $\pm 0.2 \%$ full scale was obtained. The test temperature is limited by the furnace used. Both the sensor and the signal processing algorithm work well throughout the temperature range, showing the system's capability of operating at even higher tempera-

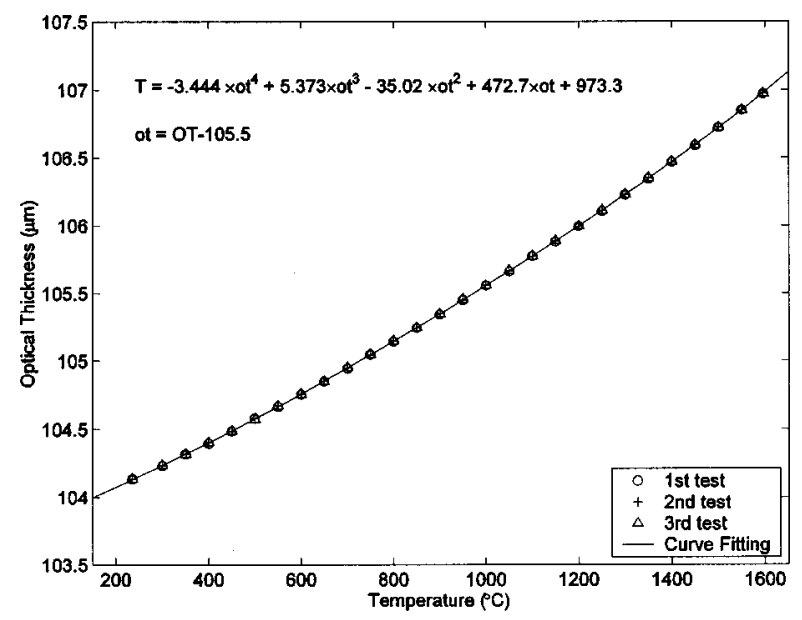

Fig. 4. OT versus temperature for three tests.

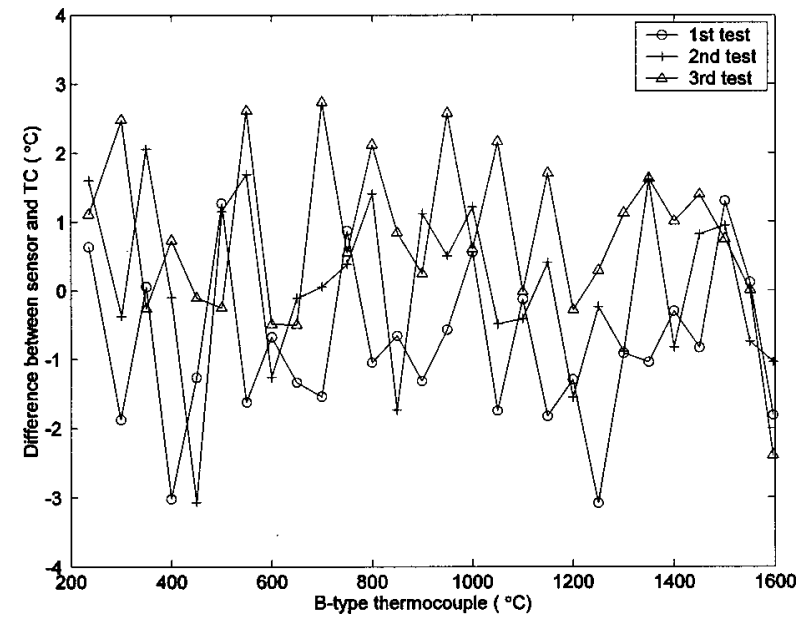

Fig. 5. Difference in temperature between the sensor and the thermocouple (TC) after calibration.

tures. The sensor is expected to work close to sapphire's melting point, which is greater than $2000{ }^{\circ} \mathrm{C}$. Other crystal fibers with higher melting points could be employed to extend the limit further.

This research was sponsored by the U.S. Department of Energy under grant DE-FC36-01G011050. Y. Zhu's e-mail address is yizhu1@vt.edu.

\section{References}

1. A. Wang, S. Gollapudi, K. A. Murphey, R. G. May, and R. O. Claus, Opt. Lett. 17, 1021 (1992).

2. R. R. Dils, J. Appl. Phys. 54, 1198 (1983).

3. R. R. Dils, J. Geist, and M. L. Reilly, J. Appl. Phys. 59, 1005 (1986).

4. L. Tong, Y. Shen, L. Ye, and Z. Ding, Meas. Sci. Technol. 10, 607 (1999).

5. J. L. Kenndey and N. Djeu, Sens. Actuators, A 100, 1870191 (2002).

6. A. Wang, S. Gollapudi, R. G. May, K. A. Murphey, and R. O. Claus, Opt. Lett. 17, 1544 (1992).

7. A. Wang, S. Gollapudi, R. G. May, K. A. Murphey, and R. O. Claus, Scanning Microsc. Suppl. 4, 147 (1995).

8. R. E. Wagner and C. R. Sandahl, Appl. Opt. 21, 1381 (1982).

9. F. Pérennès, P. C. Beard, and T. N. Mills, Appl. Opt. 38, 7026 (1999).

10. W. Tranter, K. Shanmugan, T. Rappaport, and K. Kosbar, Principles of Communication Systems: Simulation with Wireless Applications (Prentice-Hall, Englewood Cliffs, N.J., 2003).

11. B. Qi, G. R. Pickrell, J. Xu, P. Zhang, Y. Duan, W. Peng, Z. Huang, W. Huo, H. Xiao, R. G. May, and A. Wang, Opt. Eng. 42, 3165 (2003). 


\title{
A Novel Temperature-Insensitive Optical Fiber Pressure Sensor for Harsh Environments
}

\author{
Juncheng Xu, Gary Pickrell, Xingwei Wang, Wei Peng, Kristie Cooper, and Anbo Wang
}

\begin{abstract}
A novel diaphragm-based miniature optical fiber pressure sensor has been shown to work at temperatures up to $700{ }^{\circ} \mathrm{C}$ with a sensitivity of $2.93 \mathrm{~nm} / \mathrm{psi}$ and a resolution of $0.01 \mathrm{psi}$ (68.9 $\mathrm{Pa}$ ). A passive temperature compensation scheme was used to reduce the temperature dependence to $0.0076 \mathrm{psi} /{ }^{\circ} \mathrm{C}\left(52.4 \mathrm{~Pa} /{ }^{\circ} \mathrm{C}\right)$. The sensor exhibited a linear response in the available testing range from 0 to $200 \mathrm{psi}(1.38 \mathrm{MPa})$, and being composed entirely of fused silica, the sensor's structure is very reliable, corrosion resistant, and immune to electromagnetic interference.
\end{abstract}

Index Terms-Diaphragm, high temperature, optical fiber, pressure sensor.

\section{INTRODUCTION}

$\mathbf{P}$ RESSURE measurements in high-temperature environments are required in a variety of applications, such as gas turbine engines, power plants, and material-processing systems. The widely used semiconductor pressure sensors have several major drawbacks. These include a limited maximum operating temperature of $482{ }^{\circ} \mathrm{C}$, poor reliability at high temperatures, severe sensitivity to temperature changes, and susceptibility to electromagnetic interference. In the past decade, a number of fiber-optic pressure sensors have been developed. Compared with hollow cylinder-based pressure sensors for high-pressure measurement [1], [2], diaphragm-based configurations are more suitable for pressure measurements requiring high sensitivity [3], [4]. However, these diaphragm-based pressure sensors are still not suitable for applications above $500{ }^{\circ} \mathrm{C}$. Also, the large coefficient of thermal expansion (CTE) mismatch will cause severe stress between different materials used in sensor construction. This stress will degrade the sensor performance or lead to a failure. Even if the same material is used to fabricate the sensor elements, the bonding adhesive used, especially if epoxy-based, is still a major concern for the sensor's performance [5], [6]. For example, epoxy will exhibit a time-dependent viscoelastic dimensional change and will decompose at high temperatures. Also, the bonding adhesive having a different CTE from the sensor elements will cause

Manuscript received October 25, 2004; revised December 30, 2004. This work was supported by the U.S. Department of Energy (DOE) under Contract DE-FC36-01GO11050.

J. Xu, G. Pickrell, X. Wang, K. Cooper, and A. Wang are with the Center for Photonics Technology, Department of Electrical and Computer Engineering, Virginia Polytechnic Institute and State University, Blacksburg, VA 24061 USA (e-mail: juxu1@vt.edu; pickrell@vt.edu; xiwang@vt.edu; klcooper@vt.edu; awang@vt.edu).

W. Peng is with the Department of Chemistry and Biochemistry, Arizona State University, Tempe, AZ 85287-1604 USA (e-mail: wei.peng@asu.edu), and also with the Center for Photonics Technology, Department of Electrical and Computer Engineering, Virginia Polytechnic Institute and State University, Blacksburg, VA 24061 USA.

Digital Object Identifier 10.1109/LPT.2005.844013

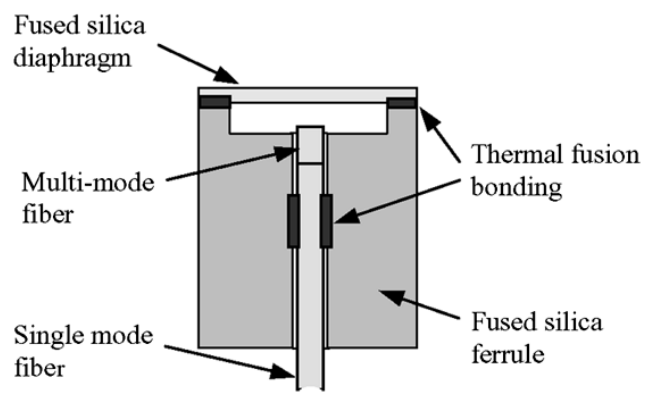

Fig. 1. Structure of the optical fiber pressure sensor.

a large temperature dependence in the pressure measurement or cause the sensor to fail. Although anodic bonding [7], [8] is adhesive-free bonding, it cannot be used for bonding fused silica glass, which has a higher softening point and much lower CTE than other glass and is the most compatible material to silica optical fiber.

In this letter, we present a novel diaphragm-ferrule-based miniature optical fiber pressure sensor. The sensor head structure is composed entirely of fused silica, which allows high-temperature operation up to at least $700^{\circ} \mathrm{C}$. The problem with CTE mismatches is eliminated with this sensor design, and with the same structure, sensors with various sensitivities can be fabricated by changing only the diaphragm thickness or the ferrule pit diameter.

The structure of the pressure sensor is shown in Fig. 1. The length of the Fabry-Pérot (F-P) cavity (air gap) will decrease with deflection of the diaphragm as a result of the applied pressure. Light is injected into the optical fiber and reflected partially (4\%) by the end face of the fiber and the inside surface of the diaphragm. Then the two reflections propagate back through the same fiber and generate interference fringes, which are demodulated to determine the air-gap thickness.

\section{SENSOR FABRICATION}

A pit with a diameter of $1.5 \mathrm{~mm}$ was mechanically machined in one end of the 1.8-mm outside diameter (O.D.) silica glass ferrule. A $125-\mu \mathrm{m}$-thick silica wafer was attached to the ferrule top by a hermetic circular laser-induced fusion bond [Fig. 2(a)]. Since the wafer was heated locally by the $\mathrm{CO}_{2}$ laser (Synrad 48-2) at the edges, the wafer surface near the center was not affected, even when coated with a metal reflective film.

A silica single-mode fiber (SMF) spliced to a piece of silica multimode fiber (MMF) was inserted in the central bore of the 8-mm-long ferrule to form an F-P cavity between the fiber end face and the inside surface of the diaphragm and was connected to a white light interferometer system [9]. The cavity length (air gap) was preadjusted by a one-dimensional translation stage 


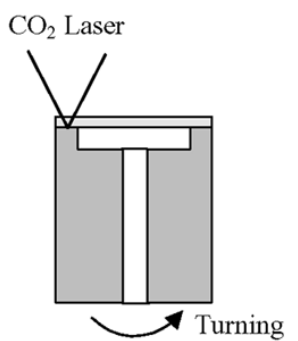

(a) (b)

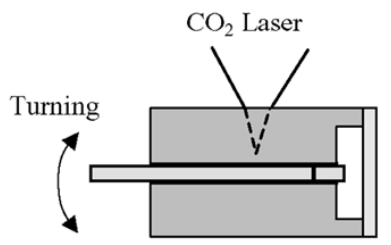

Fig. 2. Schematics of both laser fusion bonding processes.

holding the fiber tail, and monitored on-line by the white light system. The $\mathrm{CO}_{2}$ laser beam was defocused and directed to the side of the rotating ferrule and fiber [Fig. 2(b)]. The ferrule and fiber were rotated clockwise and then counterclockwise alternately, to avoid twisting the fiber tail. Part of the cladding of the SMF was fusion bonded with the ferrule to realize a hermetic seal. A portion of the air was driven out during the high temperature fusion bonding, such that after cooling, the sensor cavity experienced a partial vacuum. This reduced the temperature dependence significantly since the gases inside the cavity will expand as the temperature is increased, which in turn would cause the diaphragm to deflect outward.

The splice between the SMF and the MMF will introduce an optical loss, but typically less than $0.1 \mathrm{~dB}$. The $\mathrm{MMF}(\approx 0.5 \mathrm{~mm})$ and part of the SMF $(\approx 2 \mathrm{~mm})$ were free to expand or shrink when the temperature changes. The lengths of the SMF and MMF can be optimized to minimize temperature sensitivity. Since the SMF has a thicker cladding than the MMF, its core will be affected less by the heat. The MMF has a higher CTE than both the SMF and the ferrule. When temperature increases, the MMF expansion can compensate for the ferrule axial expansion.

Since no adhesive is used to bond the sensor parts, the sensor is expected to be reliable and can endure high temperatures, near the operational limit of the optical fiber itself. Also, this all-silica configuration will be resistant to corrosion.

The fabrication is relatively safe because no chemical processes are involved. In addition, once the diaphragm thickness and the ferrule pit are determined, the sensor's pressure response is highly repeatable. The variation in sensitivity is within $0.3 \mathrm{~nm} / \mathrm{psi}$ based on the ten sensors prepared and tested.

\section{SENSOR DESIGN AND EXPERIMENT RESUlTS}

Since the diaphragm is flat and of uniform thickness, its center deflection $y$ under applied pressure $P$ can be calculated by [10]

$$
y=\frac{3\left(1-\mu^{2}\right) P}{16 E h^{3}} r^{4}
$$

where $\mu$ is the Poisson's ratio, $h$ and $r$ are the thickness and effective radius of the diaphragm, and $E$ is the Young's module of the diaphragm. But this equation is valid only when the deflection is no more than $30 \%$ of the thickness of the diaphragm [10], which means $y_{\max } \leq 0.3 h$. The pit diameter is limited by the ferrule size, which is determined by measurement requirements. Therefore, when the pit diameter is selected and the pressure measurement range is known, the minimum thickness of

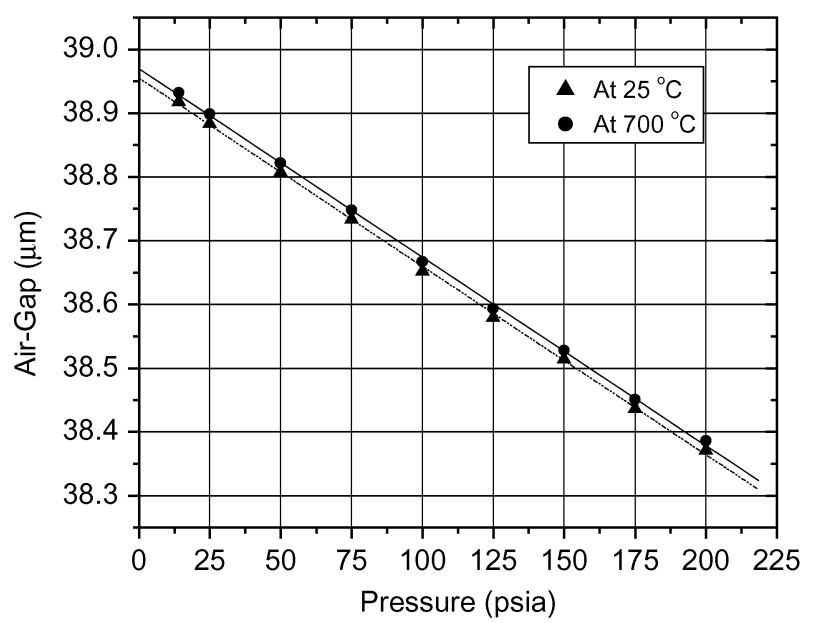

Fig. 3. Sensor pressure response at $25^{\circ} \mathrm{C}$ and $700^{\circ} \mathrm{C}$.

the diaphragm is given by (2), which will lead to the highest sensitivity

$$
h_{\min }=r\left(5\left(1-\mu^{2}\right) P_{\max } / 8 E\right)^{\frac{1}{4}} .
$$

By choosing different diaphragm thicknesses from 40 to $250 \mu \mathrm{m}$ and pit diameters from 0.8 to $1.6 \mathrm{~mm}$ (if ferrule O.D. $=1.8 \mathrm{~mm}$ ), the sensor can be designed for a wide range of pressure measurements from $10(68.9 \mathrm{kPa})$ to $15 \mathrm{kpsi}$ (103.4 MPa) with sensitivities up to $109 \mathrm{~nm} / \mathrm{psi}$.

The fused silica ferrule has a CTE of approximately $\alpha_{h}=$ $5.5 \times 10^{-7} /{ }^{\circ} \mathrm{C}$. The single-mode optical fiber used was a communication grade fiber (Corning SMF28), with a CTE of $\alpha_{s}=$ $5.6 \times 10^{-7} /{ }^{\circ} \mathrm{C}$, which is very close to that of the ferrule. The MMF was Corning 50/125 $\mu \mathrm{m}$ fiber with a CTE of about $\alpha_{m}=$ $7.7 \times 10^{-7} /{ }^{\circ} \mathrm{C}$. In general, the more germanium doping in the optical fiber, the higher the CTE. The total temperature dependence of the sensor can be expressed as

$$
\frac{\Delta L}{\Delta T}=\left(\alpha_{h}-\alpha_{m}\right) L_{m}+\left(\alpha_{h}-\alpha_{s}\right) L_{s}+\alpha_{h} L+Y P_{r} / T_{r}
$$

where $L$ is the air-gap thickness, $L_{m}$ is the MMF length, $L s$ is the SMF length, $Y$ is the sensor sensitivity (nanometer/psi), and $\operatorname{Pr}$ is the residual air pressure at temperature $\operatorname{Tr}$. By optimizing these parameters, the sensor temperature dependence can be minimized for a specific temperature range over which the CTEs are valid.

The tunable laser of a component test system (CTS, Micron Optics) was coupled into the sensor through a $2 \times 2$ coupler and the reflected light was routed back through the same coupler to the receiver of the CTS. A real-time scan of the tunable laser produced the spectrum information from 1520 to $1570 \mathrm{~nm}$ with a 2.5-pm resolution. A phase trace method [11] was used to process the optical signal in order to demodulate the air-gap thickness. The air-gap measurement exhibits a resolution of $0.03 \mathrm{~nm}$. Each sensor can be calibrated by using a pressure gauge calibration system (Pressure Systems, Model 9035). Based on the current work, the process is simple and takes approximately $2 \mathrm{~h}$. The sensor static pressure response is shown in Fig. 3 and the sensor temperature dependence is shown in Fig. 4. The sensor showed a linear response to static pressure 0-200 psi at both room temperature and $700{ }^{\circ} \mathrm{C}$ with only a 


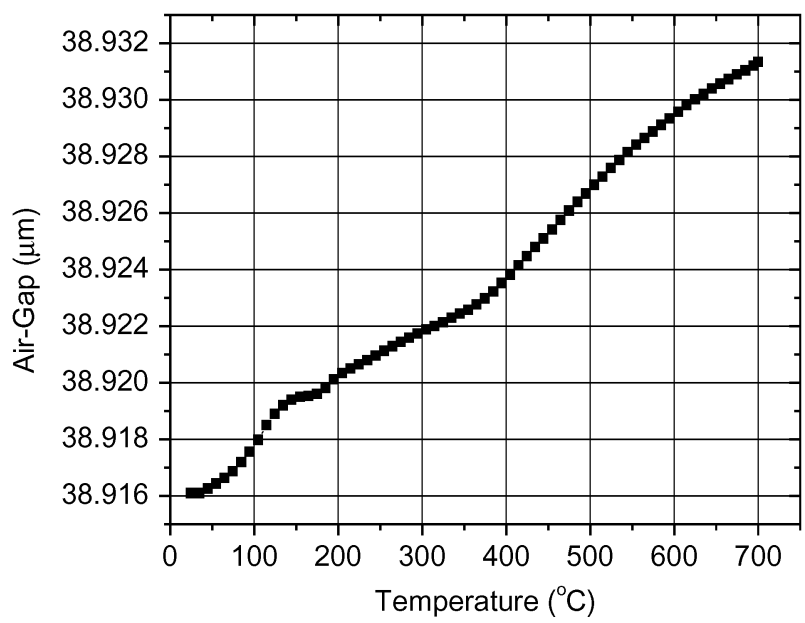

Fig. 4. Sensor temperature dependence from $25^{\circ} \mathrm{C}$ to $700{ }^{\circ} \mathrm{C}$ at atmospheric pressure.

slight shift. The sensor pressure sensitivity was $2.93 \mathrm{~nm} / \mathrm{psi}$ and the sensor system had a resolution of $0.01 \mathrm{psi}(68.9 \mathrm{~Pa})$. The temperature dependence was approximately $15.11 \mathrm{~nm} / 675^{\circ} \mathrm{C}$, so the temperature dependence of the pressure measurement was about $0.0076 \mathrm{psi} /{ }^{\circ} \mathrm{C}$. The sensor pressure sensitivity is consistent with the theoretical prediction.

\section{CONCLUSION}

A novel high-temperature fiber-optic pressure sensor has been developed, which exhibits a very low temperature dependence. Simple fabrication processes have been developed to produce hermetic seals with negligible CTE mismatches. The sensor can operate at temperatures up to $700{ }^{\circ} \mathrm{C}$ and with a sensitivity of $2.93 \mathrm{~nm} / \mathrm{psi}$, a resolution of $0.01 \mathrm{psi}(68.9 \mathrm{~Pa})$, and temperature dependence $0.0076 \mathrm{psi} /{ }^{\circ} \mathrm{C}\left(52.4 \mathrm{~Pa} /{ }^{\circ} \mathrm{C}\right)$. Further work, such as dynamic pressure measurement at elevated temperature, is in progress.

\section{ACKNOWLEDGMENT}

The authors would like to thank J. Ivasauskas of Prime Photonics, LC for designing the ferrule holding fixture and M. Gredell, D. Thorsen, and Dr. R. May, also of Prime Photonics, for useful discussions.

\section{REFERENCES}

[1] A. Wang, H. Xiao, J. Wang, Z. Wang, W. Zhao, and R. G. May, "Self-calibrated interferometric-intensity-based optical fiber sensors," J. Lightw. Technol., vol. 19, no. 10, pp. 1495-1501, Oct. 2001.

[2] K. A. Murphy, M. F. Gunther, A. M. Vengsarkar, and R. O. Claus, "Quadrature phase shifted extrinsic Fabry-Pérot optical fiber sensors," Opt. Lett., vol. 16, pp. 273-275, Feb. 1991.

[3] Y. J. Rao, D. A. Jackson, R. Jones, and C. Shannon, "Development of prototype fiber-based Fizeau pressure sensors with temperature compensation," J. Lightw. Technol., vol. 12, no. 9, pp. 1685-1695, Sep. 1994.

[4] M. I. Belovlov, M. M. Bubnov, and S. L. Semjonov, "High sensitive fiber interferometric pressure sensor," Lasers Electro-Opt. Europe, p. 192, Sep. 1996.

[5] B. Yu, D. W. Kim, J. Deng, H. Xiao, and A. Wang, "Fiber Fabry-Pérot sensors for detection of partial discharges in power transformers," Appl. Opt., vol. 42, pp. 3241-3250, Jun. 2003.

[6] M. J. Gander, W. N. MacPherson, J. S. Barton, R. L. Reuben, J. D. C. Jones, R. Stevens, K. S. Chana, S. J. Anderson, and T. V. Jones, "Embedded micromachined fiber-optic Fabry-Pérot pressure sensors in aerodynamics applications," IEEE Sensors J., vol. 3, no. 1, pp. 102-107, Feb. 2003.

[7] J. Zhou, S. Dasgupta, H. Kobayashi, J. M. Wolff, H. E. Jackson, and J. T. Boyd, "Optically interrogated MEMS pressure sensors for propulsion applications," Opt. Eng., vol. 40, pp. 598-604, Apr. 2001.

[8] D. C. Abeysinghe, S. Dasgupta, H. E. Jackson, and J. T. Boyd, "Novel MEMS pressure and temperature sensors fabricated on optical fibers," J. Micromech. Microeng., vol. 12, pp. 229-135, May 2002.

[9] J. Xu, G. R. Pickrell, Z. Huang, B. Qi, P. Zhang, Y. Duan, and A. Wang, "Double-tubing encapsulated fiber optic temperature sensor," in AIP Conf. Proc. 8th Temperature Symp., vol. 684, Sep. 2003, pp. 1021-1026.

[10] M. D. Giovanni, Flat and Corrugated Diaphragm Design Handbook. New York: Marcel Dekker, 1982.

[11] B. Qi, G. R. Pickrell, J. Xu, P. Zhang, Y. Duan, W. Peng, Z. Huang, W. Huo, H. Xiao, R. G. May, and A. Wang, "Novel data processing techniques for dispersive white light interferometer," Opt. Eng., vol. 42, pp. 3165-3171, Nov. 2003. 


\title{
Tunable-optical-filter-based white-light interferometry for sensing
}

\author{
Bing Yu, Anbo Wang, Gary Pickrell, and Juncheng Xu \\ Center for Photonics Technology, Bradley Department of Electrical and Computer Engineering, \\ Virginia Polytechnic Institute and State University, Blacksburg, Virginia 24061
}

Received January 10, 2005

\begin{abstract}
We describe tunable-optical-filter-based white-light interferometry for sensor interrogation. By introducing a tunable optical filter into a white-light interferometry system, one can interrogate an interferometer with either quadrature demodulation or spectral-domain detection at low cost. To demonstrate the feasibility of effectively demodulating various types of interferometric sensor, experiments have been performed using an extrinsic Fabry-Perot tunable filter to interrogate two extrinsic Fabry-Perot interferometric temperature sensors and a diaphragm-based pressure sensor. (C) 2005 Optical Society of America

OCIS codes: $120.2440,120.3180,060.2370$.
\end{abstract}

White-light interferometry (WLI), also called lowcoherence interferometry, is a technique in which a broadband light source is used to illuminate an interferometer. Compared with its counterpart, laser interferometry, WLI possesses considerable advantages, such as the ability to provide absolute and unambiguous measurements, greatly reduced effects of wavelength instabilities, higher spatial density of sensors in a multiplexing array, reduced Rayleigh backscattering and spurious interference, insensitivity to optical feedback, and low cost. In the past two decades WLI has been a powerful optical evaluation tool widely used in optical coherence-domain reflectometry, ${ }_{3}$ optical coherence tomography, ${ }^{2}$ surface profiling, ${ }^{3}$ and optical sensing. ${ }^{4,5}$

Scanning WLI (S-WLI) and spectral-domain WLI (SD-WLI) are two major kinds of WLI. A conventional S-WLI system is generally composed of a sensing interferometer and a local receiving interferometer. ${ }^{5}$ The sensing interferometer is used to convert the measurand's perturbation into phase modulation of the light waves through variation of the optical path difference (OPD) between its sensing and reference arms. It usually has an OPD larger than the coherence length of the light source such that interference fringes cannot be observed. Recovering the OPD of the sensing interferometer requires that the OPD of the local receiving interferometer be scanned, creating a fringe pattern. When the OPDs of the two interferometers are the same, the system has the maximum fringe peak, which is termed the central fringe, or centroid. A great number of algorithms to locate the centroid are available. In general, S-WLI has a large dynamic range but also a large system size, low mechanical stability, and relatively low resolution. A variation of S-WLI is widely used in time-domain optical coherence tomography, ${ }^{2}$ surface metrology, ${ }^{3}$ and some Mach-Zehnder or Michelson sensors, in which the optical length of the reference arm is directly scanned. The major advantage of this configuration is that only one interferometer is required. However, it is hard, if not impossible, to apply S-WLI to FabryPerot and Sagnac interferometers because of difficulty of access to the reference beam. Another issue is that the two arms may experience different environmental fluctuations.

SD-WLI is based on detection of the output spectrum of a sensing interferometer with a spectrometer. ${ }^{6}$ Once the output spectrum is obtained, fringe tracking or a Fourier transform may be used to recover the OPD of the interferometer. SD-WLI is applicable to all types of interferometer, is insensitive to source fluctuations, is accurate and reliable, and requires no mechanical scanning. Speed and price are two major issues with SD-WLI. Commercial spectrometers in general are slow and expensive, especially in the $1.0-1.7-\mu \mathrm{m}$ band.

In this Letter we describe an alternative WLI technique, which is termed tunable-optical-filter-based WLI (TOF-WLI). By introducing a tunable optical filter into a WLI system, one can interrogate an interferometer with either quadrature demodulation or spectral-domain detection at low cost. Demonstrative experiments that use TOF-WLI as an effective solution for various types of interferometric sensor are also presented.

Figure 1 shows a general configuration of a TOFWLI measurement system. It consists of a broadband light source, a circulator, a sensing interferometer, a tunable optical filter, receiving electronics, a computer, and optical fibers to connect all the optical parts. Obviously, one may readily obtain the output spectrum of the sensing interferometer by scanning the tunable optical filter (TOF). The system can work similarly to SD-WLI, and most of the developed signal-processing algorithms, such as fringe peak tracking and Fourier transformation, can be directly applied. In a scan mode, a scan range of 50-100 nm and a filter bandwidth of $0.5-2 \mathrm{~nm}$ are usually preferred for high resolution and a high signal-to-noise ratio.

When small OPD changes or dynamic perturbations are to be measured, linear or quadrature operation has to be considered for the highest sensitivity and largest signal bandwidth provided by the linear region (a small region on an interference fringe near its quadrature point, or $Q$ point). Quadrature demodulation is an important tool in laser interferom- 


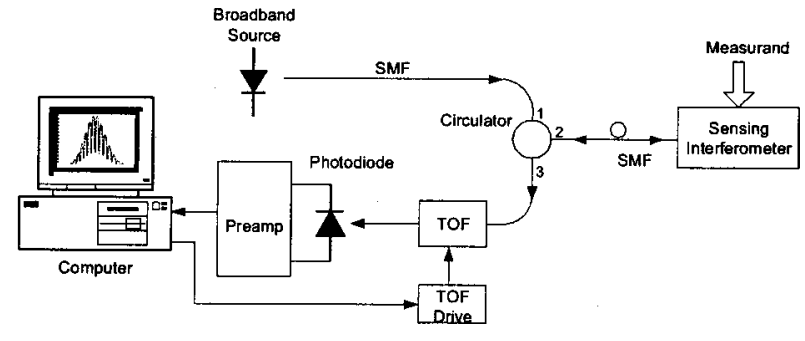

Fig. 1. TOF-WLI measurement system. SMFs, singlemode fibers.

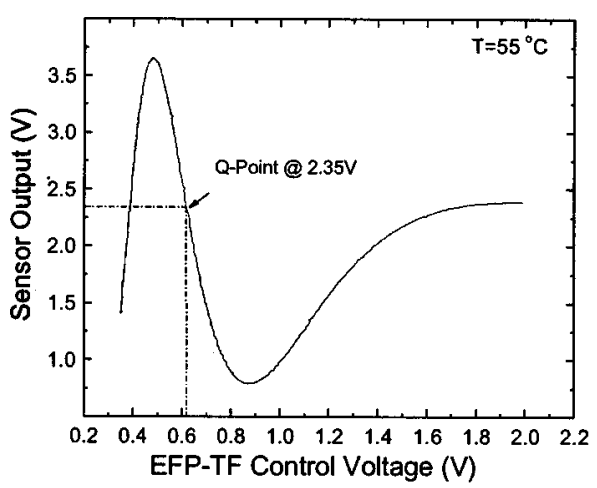

(a)

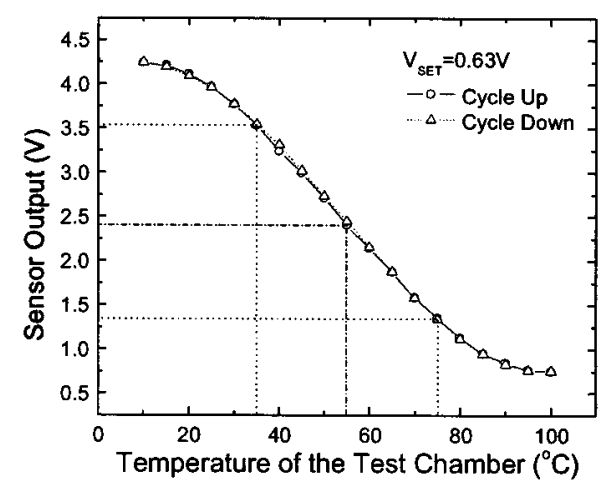

(b)

Fig. 2. Low-finesse EFPI temperature sensor: (a) $Q$-point searching, (b) lock mode.

etry. The biggest challenge is that $Q$ points drift as a result of fabrication tolerances and random environmental fluctuations. The optical phase difference between the sensing and the reference beams is given by $\phi=2 \pi \mathrm{OPD} / \lambda$, where $\lambda$ is the wavelength. In this system, a tuning of $\Delta \lambda=[\Delta(\mathrm{OPD}) / \mathrm{OPD}] \lambda$ is required for compensation of phase change $\Delta \phi$ caused by an environmentally induced drift of magnitude $\Delta(\mathrm{OPD})$. As most interferometers have an initial OPD $\gg \Delta(\mathrm{OPD})$, a small wavelength tuning may be used to compensate for a large fluctuation. Tunable lasers have sometimes been used for this purpose. ${ }^{7}$ However, linear operation has not been provided by current WLI techniques owing to their poor fringe contrast and lack of $Q$-point stabilization. A unique feature of the TOF-WLI is that it does provide this capability, with reasonably good fringe contrast and potentially low cost. In addition, TOF-WLI retains all the advantages of WLI, is an effective solution for various types of interferometer, can be tailored for both static and dynamic signals, and may dramatically reduce the cost per sensor to a fraction of that of a current system. In a linear mode, depending on the source power density, a TOF bandwidth of $5-8 \mathrm{~nm}$ may be required for both a good signal-to-noise ratio and a good fringe contrast.

The optical intensity arriving at the photodiode, with TOF central wavelength $\lambda_{0}$, can be expressed as

$$
I\left(\mathrm{OPD}, \lambda_{0}\right)=\alpha_{0} I_{0} \int_{0}^{+\infty} f_{s}(\lambda) f_{\mathrm{TOF}}\left(\lambda, \lambda_{0}\right) X(\phi) \mathrm{d} \lambda
$$

where $I_{0}$ is the total output intensity of the broadband light source, $\alpha_{0}$ describes the total loss of all optical parts and fibers in the signal path, $f_{S}(\lambda)$ is the spectral distribution of the source, $f_{\mathrm{TOF}}\left(\lambda, \lambda_{0}\right)$ is the transmission spectrum of the TOF at $\lambda_{0}$, and $X(\phi)$ is the transfer function of the interferometer at wavelength $\lambda$. In a scan mode the OPD can be treated as a constant, whereas, in a linear mode, TOF wavelength $\lambda_{0}$ is fixed. Equation (1) is the starting point for the calculation of the system's sensitivity, fringe contrast, and linear range.

The key component in a TOF-WLI is the TOF, which must have a large tuning range, preferably above $100 \mathrm{~nm}$, low insertion loss, polarization independence, and ease of fabrication and control, thus

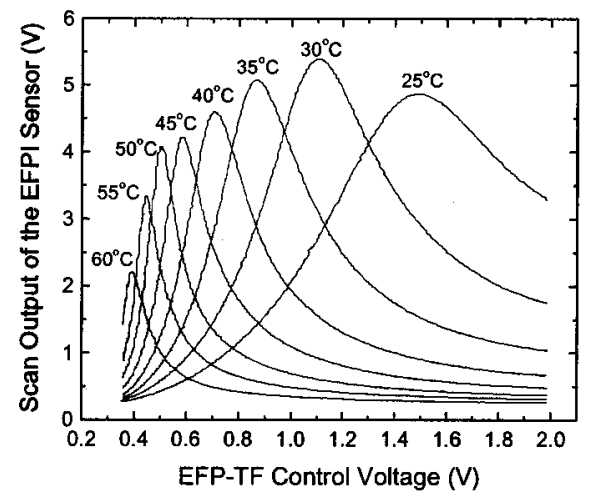

(a)

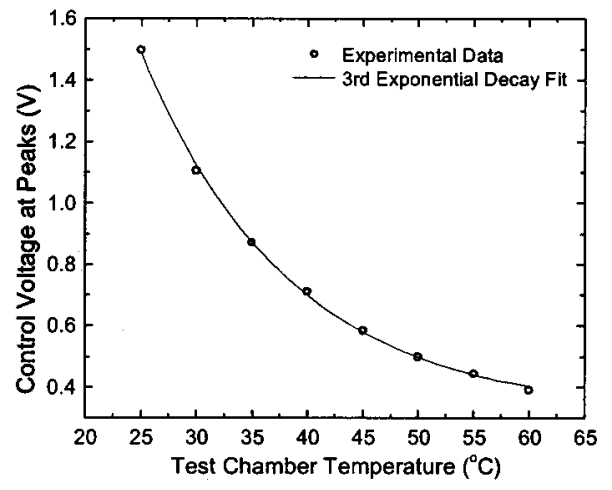

(b)

Fig. 3. High-finesse EFPI temperature sensor: (a) peak tracking, (b) temperature-voltage curve. 
resulting in low cost. All other parts required for the system are commercially available at commodity prices. Previously, an extrinsic Fabry-Perot tunable filter $^{8}$ (EFP-TF) that was specially designed for this application was reported. To demonstrate its feasibility for the interrogation of various fiber-optic sensors we fabricated an EFP-TF with a FWHM of $7.8 \mathrm{~nm}$ and a free spectral range of $110 \mathrm{~nm}$ and built a TOFWLI system that uses a $1550-\mathrm{nm}$ superluminescent LED with a FWHM of $62 \mathrm{~nm}$ and an output power of $1.0 \mathrm{~mW}$.

In the first experiment a low-finesse extrinsic Fabry-Perot interferometric (EFPI) temperature sensor with a borosilicate tube was used in the linear mode. ${ }^{9}$ The sensor was tested inside a Testequity 1000 Series temperature chamber. By scanning the TOF at a chamber temperature of $55^{\circ} \mathrm{C}$ we found the $Q$ point of the sensor at a control voltage of $0.63 \mathrm{~V}$, as shown in Fig. 2(a). Locking the control voltage to this point but cycling the temperature chamber from $10^{\circ} \mathrm{C}$ to $100^{\circ} \mathrm{C}$ and then back to $10^{\circ} \mathrm{C}$ yielded a sensor output as shown in Fig. 2(b). Obviously this EFPI sensor has a linear operation range of approximately $35^{\circ} \mathrm{C}$ to $75^{\circ} \mathrm{C}$ with a sensitivity of $54.8 \mathrm{mV} /{ }^{\circ} \mathrm{C}$ and a dynamic range-to-resolution ratio of $\sim 5000: 1$.

In a second experiment a high-finesse EFPI temperature sensor was fabricated and tested in the same chamber. The major difference from the previous experiment was that here transmissive mode detection, instead of a reflective mode, and fringe peak tracking, ${ }^{10}$ instead of a linear mode, were used. Figure 3(a) shows the scan outputs of the sensor at chamber temperatures of $25^{\circ} \mathrm{C}$ to $60^{\circ} \mathrm{C}$ in $5^{\circ} \mathrm{C}$ intervals. The peak intensity variations from scan to scan were caused by changes in source power distribution, whereas the changes in width were due mainly to the nonlinear response of the EFP-TF to its control voltage. By plotting the control voltages at the peaks versus the chamber temperature we obtained a temperature-voltage curve with a third-order exponential decay fit, which was, once again, due to the same relationship between the EFP-TF and its control voltage. In this approach, a dynamic range-toresolution ratio of 2200:1 was obtained, which was limited by the free spectral range of the sensor, the tuning range, and the control voltage noise of the EFP-TF as well as by the available superluminescent light-emitting diode's bandwidth.

In the last experiment a field test of diaphragmbased Fabry-Perot interferometric (DFPI) sensors for dynamic pressure measurements at the inlet of a jet turbine engine was performed at the Virginia Tech Airport. ${ }^{11}$ Three DFPI sensors with diaphragms of $50-\mu \mathrm{m}$ thickness and $1.5-\mathrm{mm}$ effective diameters were mounted on the engine with a Kulite XT-190$300 \mathrm{~A}$ pressure sensor as a reference. The Kulite sensor, with a model 1822 differential amplifier, had a total sensitivity of $33.3 \mathrm{mV} / \mathrm{psi}(4.83 \mathrm{mV} / \mathrm{kPa})$. A TOF-WLI system operating in a linear mode was employed. Figure 4 shows typical frequency-domain

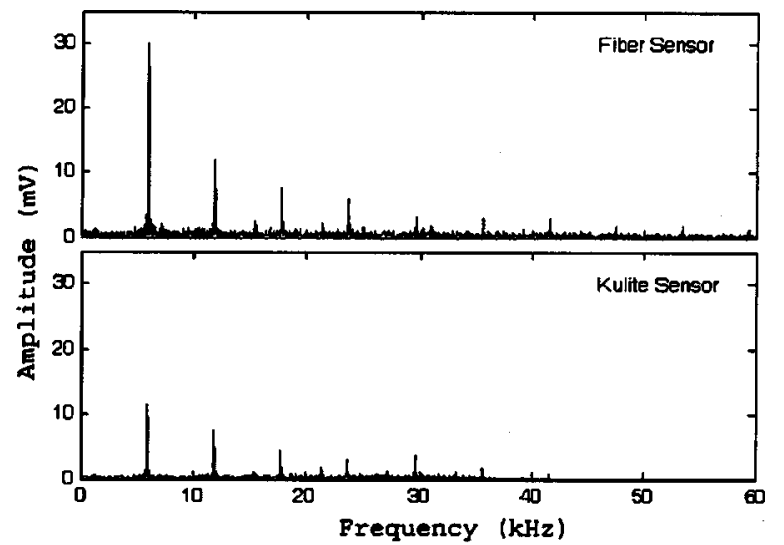

Fig. 4. Frequency-domain signals from turbine engine pressure sensors.

pressure signals recorded by one of the three DFPI sensors and by the Kulite sensor. Apparently the signals matched well at all major frequency components, and the DFPI sensors generally had a sensitivity of $87 \mathrm{mV} / \mathrm{psi}$ and a resolution of $0.023 \mathrm{psi}$ (159 Pa).

In conclusion, we have described a TOF-WLI that features a simple structure, flexibility of operation, large signal bandwidth, large dynamic range-toresolution ratio, applicability to various types of interferometer for both static and dynamic signals, and potentially low cost. Therefore this system appears to have great potential in applications such as sensor interrogation, surface profiling, and biomedical imaging. Experimental results of the use of an EFP-TFbased WLI for low- and high-finesse EFPI temperature sensors and a DFPI pressure sensor have demonstrated its feasibility for sensor interrogation.

This research is supported by U.S. Department of Energy contract DE-FC36-01GO11050. The authors thank Wing Ng for providing the engine test facilities. B. Yu's e-mail address is biyu@vt.edu.

\section{References}

1. R. C. Youngquist, S. Carr, and D. E. N. Davies, Opt. Lett. 12, 158 (1987).

2. J. M. Schmitt, IEEE J. Quantum Electron. 5, 1205 (1999).

3. L. Deck and P. D. Groot, Appl. Opt. 33, 7334 (1994).

4. C. E. Lee and H. F. Taylor, J. Lightwave Technol. 9, 129 (1991).

5. Y.-J. Rao and D. A. Jackson, Meas. Sci. Technol. 7, 981 (1996).

6. S. H. Yun, G. J. Tearney, B. E. Bouma, B. H. Park, and J. F. de Boer, Opt. Express 11, 3598 (2003).

7. P. C. Beard and T. N. Mills, Appl. Opt. 35, 663 (1996).

8. B. Yu, G. Pickrell, and A. Wang, IEEE Photonics Technol. Lett. 16, 2296 (2004).

9. A. Wang, H. Xiao, J. Wang, Z. Wang, W. Zhao, and R. May, J. Lightwave Technol. 19, 1495 (2001).

10. V. Bhatia, M. B. Sen, K. A. Murphy, and R. O. Clause, Electron. Lett. 32, 247 (1996).

11. J. Xu, G. R. Pickrell, B. Yu, M. Han, Y. Zhu, X. Wang, K. L. Cooper, and A. Wang, Proc. SPIE 5590, 1 (2004). 


\section{Diaphragm-based extrinsic Fabry-Perot interferometric optical fiber sensor for acoustic wave detection under high background pressure}

\author{
Ming Han \\ Xingwei Wang \\ Juncheng $\mathrm{Xu}$ \\ Kristie L. Cooper \\ Anbo Wang \\ Virginia Polytechnic Institute and State \\ University \\ Center for Photonics Technology \\ Blacksburg, Virginia 24061-0287
}

\begin{abstract}
A new structure for diaphragm-based extrinsic Fabry-Perot interferometric (EFPI) optical fiber sensors is presented. This structure introduces a through hole in a conventional diaphragm-based EFPI sensor and significantly reduces the effect of operating point drift due to the background pressure and fluctuations. This structure also potentially has high temperature stability. (c) 2005 Society of Photo-Optical Instrumentation Engineers. [DOI: 10.1117/1.1924689]
\end{abstract}

Subject terms: fiber optic sensors; Fabry-Perot interferometers.

Paper L040816 received Nov. 1, 2004; revised manuscript received Mar. 24, 2005; accepted for publication Apr. 7, 2005; appeared online Apr. 11, 2005; published online Jun. 9, 2005.

\section{Introduction}

Currently, fiber optic extrinsic Fabry-Perot interferometric (EFPI) sensors have been studied extensively for acoustic signal detection due to their inherent advantages including small size, light weight, high sensitivity, high frequency response, electrical nonconductivity, immunity to EMI noise, and single-point measurement. ${ }^{1}$ Among them is a diaphragm-based EFPI sensor comprised of a fused silica diaphragm and a single-mode fiber (SMF) end., ${ }^{2,3}$ The vibration of the diaphragm caused by acoustic waves operates the interferometer in the linear range of one of its interference fringes. The sensors are designed to be sensitive to pressure changes in order to measure acoustic waves. However, small fluctuations of background pressure could change the operating point of the sensor in an unpredictable way. Several approaches, such as multiwavelength interrogation $^{4,5}$ or spectral interrogation ${ }^{5}$ and Q-point control using a bandpass filter, ${ }^{3}$ have been employed to compensate the operating point drift, while the price is a significant increase in system complexity and cost. In this letter, we present a new structure for diaphragm-based EFPI optical fiber sensors to eliminate operating point dependence on background pressure fluctuations.

\section{Principle of Operation}

The sensor structure is shown in the inset of Fig. 1. A SMF zirconia ferrule with outer diameter $D=2.5 \mathrm{~mm}$ is inserted into the ferrule sleeve, leaving a small distance $L$ $=0.2 \mathrm{~mm}$ between the ends of the ferrule and the sleeve. A fused silica diaphragm of thickness $170 \mu \mathrm{m}$ is bonded on the end of the sleeve. The sleeve has a 0.3-mm-wide slot initially designed for the easy insertion of the ferrule. In this structure, the slot, together with the diaphragm and ferrule, forms a $0.2-\times 0.3-\mathrm{mm}$ through hole connecting the air cavity with the outside environment. The cleaved lead-in fiber is then inserted into the ferrule, forming an air gap $d$ between the fiber end and the inside end of the diaphragm. The other side of the diaphragm is roughened by sandpaper to eliminate undesirable reflections. The air gap can be properly adjusted using a white light system. The fiber, ferrule, ferrule sleeve, and diaphragm are all bonded together by epoxy.

Since the "through hole" area $S_{h}$ is much smaller than that of the diaphragm $S_{d}$, the acoustic energy diffracted into the air cavity through the hole is negligible. Suppose the acoustic energy diffracted into the air cavity is proportional to the area of the hole $S_{h}$. In our case, $S_{h}$ $=0.06 \mathrm{~mm}^{2}, S_{d}=\pi(D / 2)^{2}=4.91 \mathrm{~mm}^{2}, S_{h} / S_{d}=0.012$. The acoustic energy diffracted into the cavity is only $1.2 \%$ of the energy applied on the diaphragm. The sensitivity to acoustic waves does not decrease much compared to a sensor without a through hole. However, for background pressure, which is characterized as a static pressure with slow fluctuations, the pressure inside and outside the air cavity will be quickly balanced by the through hole and will not change the air gap. Thus the sensor is expected to be insensitive to the background pressure while maintaining its sensitivity to the acoustic waves. In addition, such through structure offers potential temperature stability, because it eliminates the effect of the trapped air expansion induced pressure, which is an issue in conventional hermetic sensors. Also, when an epoxy-free bonding method, such as laser bonding, is used, the sensor can survive hightemperature environments. ${ }^{6}$

\section{Experimental Results}

A static pressure test was first performed. The experimental setup is shown in Fig. 1. The optical light from a highresolution swept laser interrogator (HR-SLI) (Model V5.1, Micron Optics, Inc.) was coupled into the sensor through a 3-dB SMF coupler. The reflected signal from the sensor was collected by the same coupler and measured by the detector built in the HR-SLI. The sensor was sealed in a chamber connected to a high-accuracy pressure controller. Figure 2 shows the air gap of the sensor changes $12 \mathrm{~nm}$ when the pressure in the chamber increases from 20 to 80 psi. If we define the linear operation range of the sensor as the air gap change that causes a phase change of $40 \%$ of a full fringe period, then the linear range of the sensor at operating wavelength $1550 \mathrm{~nm}$ is $228 \mathrm{~nm}$. The air gap change due to a pressure change from 20 to 80 psi is only $5.25 \%$ of the linear operation range, which is negligible in most practical applications. Also, this can be further re- 


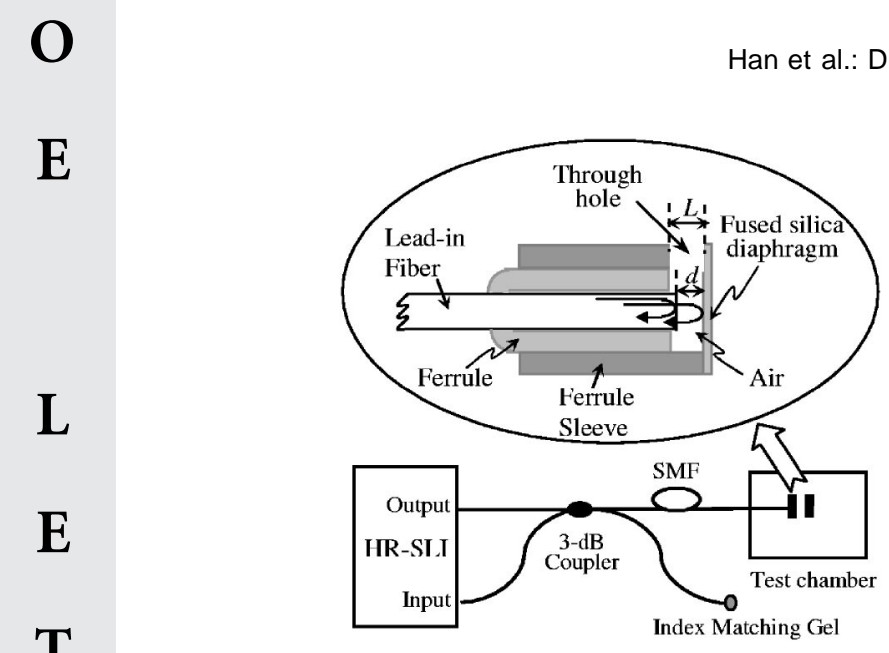

Fig. 1 Experimental setup for sensor test. The inset shows the structure of the sensor head.

duced by choosing a fused silica ferrule and sleeve, which are of the same material as the fiber, and using epoxy-free bonding.

The sensor was then applied to the acoustic wave test system. The setup is the same as Fig. 1 except that a tunable laser was used as the light source and a high-speed InGaAs detector together with an electrical current amplifier was used to transfer the optical signals from the sensor into electrical ones. A digital oscilloscope was used to display and store the acoustic signal captured by the sensor. An ultrasonic air transducer was sealed into the same chamber to apply acoustic waves to the sensor. Meanwhile the background pressure was controlled by the pressure controller. The wavelength of the laser was tuned to 1546 $\mathrm{nm}$ and maintained there during the test. Figure 3(a) shows the $46-\mathrm{kHz}$ voltage signal applied to the transducer. The waveform should be the same as the acoustic signal. Figure 3(b) shows the sensor signal displayed on the oscilloscope, which indicates the sensor is capable of faithfully detecting the acoustic signals. The experiment was repeated at different background pressures up to $80 \mathrm{psi}$. In all cases the sensor worked well with no need to adjust the operating point.

For a diaphragm-based sensor without a through hole, the sensitivity of the diaphragm is given by $\delta=2.524$

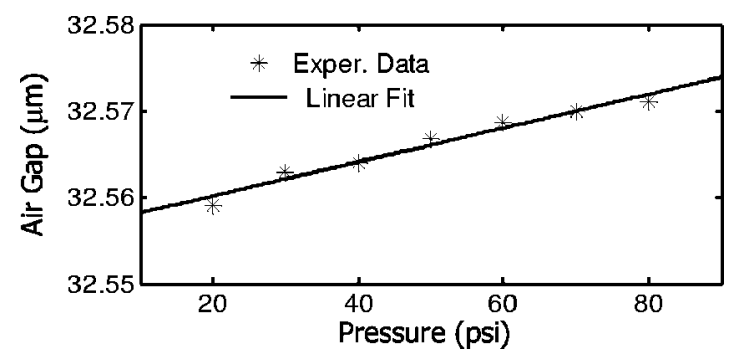

Fig. 2 Static pressure test: air gap change of the sensor versus pressure applied.

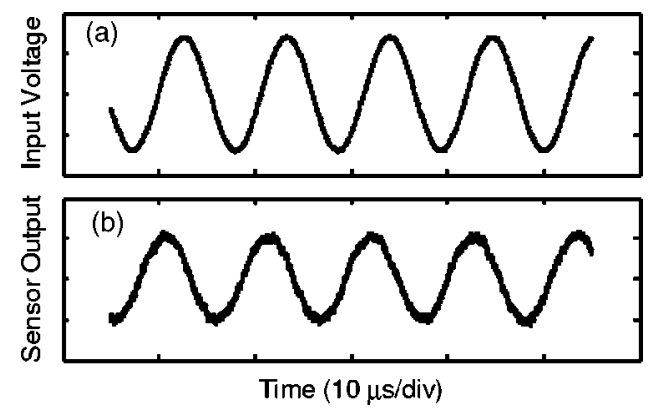

Fig. 3 Acoustic wave test at background pressure: $P=40$ psi. (a) Signal applied to the acoustic transducer. (b) Signal from the sensor.

$\times 10^{-6} a^{4} / h^{3}[\mathrm{~nm} / \mathrm{kPa}],{ }^{3}$ where $a$ is the diaphragm radius and $h$ is the diaphragm thickness, both in micrometers. Assume $a=D / 2=1.25 \mathrm{~mm}$ and $h=170 \mu \mathrm{m}$, which are the same parameters as the diaphragm used in the sensor with the through hole; then the air gap change is calculated to be $518.86 \mathrm{~nm}$ when the pressure is increased from 20 to 80 psi, which is 2.28 times the linear operating range of the sensor. In comparison, the through hole in the proposed sensor reduces the pressure-dependent air gap change to $2.31 \%$ of this value, or $12 \mathrm{~nm}$.

\section{Conclusions}

In summary, a new diaphragm-based EFPI sensor structure for acoustic wave detection has been presented. The sensitivity of the sensor to background pressure is significantly reduced, eliminating the need for operating point control procedures in some applications, and thus reducing the system cost. This structure also provides the potential advantage of temperature insensitivity and high-temperature capability if fused silica ferrules and diaphragms and epoxyfree bonding are used.

\section{References}

1. K. A. Murphy, M. F. Gunther, A. Wang, and R. O. Claus, "Detection of acoustic emission location using optical fiber sensors," in Smart Structures and Materials 1994: Smart Sensing, Processing, and Instrumentation, J. S. Sirkis, Ed., Proc. SPIE 2191, 282-290 (1994).

2. J. Deng, H. Xiao, W. Huo, M. Luo, R. May, A. Wang, and Y. Liu, "Optical fiber sensor-based detection of partial discharges in power transformers," Opt. Laser Technol. 33, 305-311 (2001).

3. B. Yu, D. Kim, J. Deng, H. Xiao, and A. Wang, "Fiber Fabry-Perot sensors for detection of partial discharges in power transformers," Appl. Opt. 42, 3242-3250 (2003).

4. N. Furstenau, M. Schmidt, H. Horack, W. Goetze, and W. Schmidt, "Extrinsic Fabry-Perot interferometer vibration and acoustic sensor systems for airport ground traffic monitoring," IEE Proc.: Optoelectron. 144, 134-144 (1997).

5. W. Pulliam, P. Russler, R. Mlcak, K. Murphy, and C. Kozikowski, "Micromachined, SiC fiber optic pressure sensors for high temperature aerospace applications," in Industrial Sensing Systems, A. Wang and E. Udd, Eds, Proc. SPIE 4202, 21-30 (2000).

6. J. Xu, G. R. Pickrell, B. Yu, M. Han, Y. Zhu, X. Wang, K. L. Cooper, and A. Wang, "Epoxy-free high-temperature fiber optic pressure sensors for gas turbine engine applications," Proc. SPIE 5590, 1-10 (2004). 


\section{Wavefront splitting intrinsic Fabry-Perot fiber optic sensor}

\author{
Zhengyu Huang \\ Xiaopei Chen \\ Yizheng Zhu \\ Anbo Wang \\ Virginia Polytechnic Institute and State University \\ Center for Photonics Technology \\ Department of Electrical and Computer Engineering \\ Blacksburg, Virginia 24061-0111
}

Abstract. We present the principle, fabrication, and characterization of a novel wavefront splitting intrinsic Fabry-Perot fiber temperature sensor. The sensor is made by splicing a section of fused silica tubing to the tip of a single-mode fiber. The completed sensor has the same diameter as the fiber and the sensor length is less than $0.5 \mathrm{~mm}$. (c) 2005 Society of Photo-Optical Instrumentation Engineers.

[DOI: 10.1117/1.1978847]

Subject terms: fiber sensors; Fabry-Perot; splice.

Paper L040815RR received Nov. 29, 2004; revised manuscript received Apr. 26, 2005; accepted for publication May 16, 2005; published online Jul. 22, 2005.

\section{Introduction}

Intrinsic Fabry-Perot interferometer (IFPI) fiber sensors are well known for their ability to measure temperature, strain, pressure, and ultrasound perturbations due to their excellent sensitivity, rapid response, immunity to electromagnetic interference, and potential of multiplexing. ${ }^{1}$

The interferometers can be divided into two major categories: amplitude splitting interferometers and wavefront splitting interferometers. ${ }^{2}$ Most of the IFPI sensors are based on the amplitude splitting principle. The amplitude splitting scheme varies from using different mode-fielddiameter single-mode fibers, internal mirrors to fiber micro cavities, ${ }^{1,3-5}$ to name just a few. However, fiber polishing, ${ }^{1}$ dielectric mirror deposition, and arc splicing ${ }^{3}$ impose stringent control in the sensor fabrication.

We report here an IFPI fiber sensor that is based on a wavefront splitting principle. Only splicing and cleaving of a section of fused silica tubing to the single-mode fiber (SMF) is used. The fabrication process is significantly simplified and equivalent performance to the traditional IFPI sensor is achieved. The theoretical analysis, sensor fabrication, as well as sensor performance are presented.

\section{Principles of Operation}

The wavefront split IFPI structure is illustrated in Fig. 1. A piece of fused silica tubing is spliced to the SMF, and then cleaved close to the splice point. The wavefront of the guiding $\mathrm{LP}_{01}$ mode of the $\mathrm{SMF}$ is split into two components at the fiber-tubing interface. The two components are reflected by the fiber core-air interface and cleaved tubing end, respectively. The Fabry-Perot cavity length is the optical length of the fused silica tubing. The interference spectrum is obtained by a sensor interrogator, and the cavity length is calculated by a high-precision white-light algorithm. ${ }^{6}$
The reflectivity $R$ is given by the Fresnel formula ${ }^{2}$

$R=\left(\frac{n_{\text {glass }}-n_{\text {air }}}{n_{\text {glass }}+n_{\text {air }}}\right)^{2}$

where $n_{\text {glass }}$ and $n_{\text {air }}$ are refractive indices of fused silica and air.

The electric fields from the cleaved SMF reflection, fiber core-air reflection, and fused silica tubing reflection are shown in Fig. 1 and given by

$$
\begin{aligned}
& E_{R}(r, \varphi)=A_{0} \sqrt{R} e^{\frac{* r^{2}}{w^{2}}} \\
& E_{\text {core }}(r, \varphi)=\left\{\begin{array}{ll}
E_{R}(r, \varphi), & r \leqslant a \\
0, & r>a
\end{array}\right. \text { and } \\
& E_{\text {tube }}(r, \varphi)= \begin{cases}E_{R}(r, \varphi), & r \geqslant a \\
0, & r<a\end{cases}
\end{aligned}
$$

where $A_{0}$ is the magnitude of the incident electric field, $w$ is the mode-field radius of the single-mode fiber, and $a$ is the inner radius of the fused silica tube. Gaussian field distribution approximation ${ }^{7}$ is used in Eq. (2).

Only those of the truncated reflection components coupled into the fundamental mode of the SMF can reach the detector. The coupling coefficients are

$$
\begin{gathered}
\eta_{\text {core }}=\frac{\int_{0}^{2 \pi} \int_{0}^{a} E_{\text {core }} E_{R}^{*} r \mathrm{~d} r \mathrm{~d} \varphi}{\int_{0}^{2 \pi} \int_{0}^{+\infty} E_{R} E_{R}^{*} r \mathrm{~d} r \mathrm{~d} \varphi} \text { and } \\
\eta_{\text {tube }}=\frac{\int_{0}^{2 \pi} \int_{a}^{+\infty} E_{\text {tube }} E_{R}^{*} r \mathrm{~d} r \mathrm{~d} \varphi}{\int_{0}^{2 \pi} \int_{0}^{+\infty} E_{R} E_{R}^{*} r \mathrm{~d} r \mathrm{~d} \varphi} .
\end{gathered}
$$

Thus the normalized reflection power of the two components is given by $\eta^{2}$.

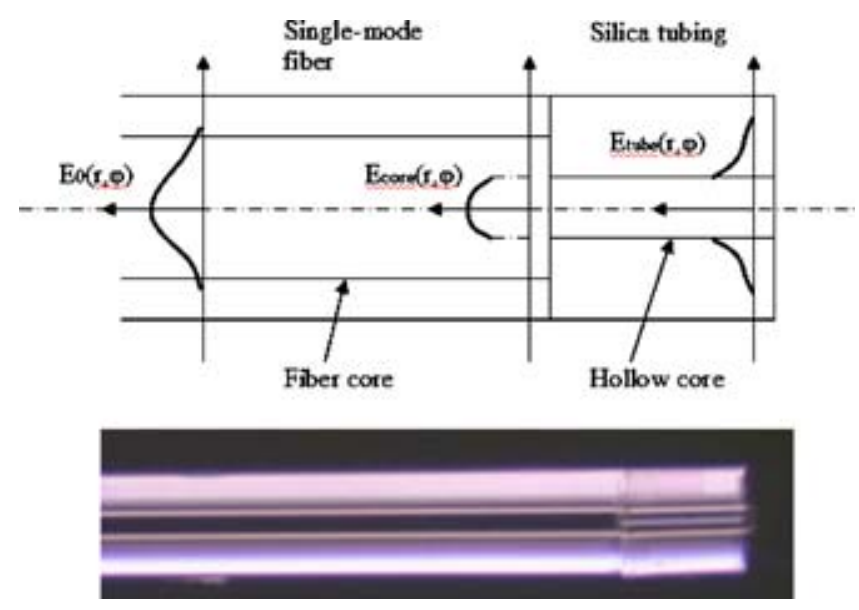

Fig. 1 Split wavefront IFPI sensor head structure. 


\section{Sensor Fabrication}

The objective of IFPI fabrication is to obtain large signalto-noise ratio and high fringe visibility. In the fabrication process, the fused silica tubing is first spliced to an SMF. The fiber tubing is then put on a fiber cleaver and placed under a microscope. The splicing point can be easily identified under the microscope. The tubing is then cleaved at a distance to the splicing point. Corning SMF28 and fused silica tubing from Polymicro Technologies are used. The fiber splicer and cleaver used are Type-36 Fiber Splicer (Sumitomo Electric) and CT-04B High Precision Fiber Cleaver (Fujikura). The microscope is from Olympus (SZ40). A Si720 system (Micron Optics) is used as sensor interrogator.

It is found that fiber core-air reflection decreases monochromatically when increasing either the fusion splicing arc power or the arc duration as shown in Fig. 2 (the arc power $P$ and arc duration $D$ are normalized to $P=15$ and $D=2.0$, which is used in the standard SMF splicing configuration). This is because the silica tubing inner diameter shrinks with the increasing amount of arc generated heat, which results in a decreased effective reflection area. Therefore to fabricate a good IFPI sensor, low arc power and short arc duration that does not degrade the mechanical strength of the sensor is adopted. Silica tubing with a 5- $\mu \mathrm{m}$ inner diameter is chosen because it provides the closest magnitudes of reflections on both interfaces, therefore high fringe visibility. The experimental data is compared with theoretical prediction in Fig. 3, which proves the coupling model a valid assumption. The tubing should be well cleaved, because a tilted end face will increase the coupling loss of the reflection to the single-mode fiber and reduce the fringe visibility. The silica tubing length should be controlled within a $500-\mu \mathrm{m}$ range. The light in the tubing will diverge because it is no longer confined in a waveguide structure. Longer cavity length would result in larger divergence and less reflected light coupled into the single-mode fiber.

Since the fiber splicing process is highly repeatable and controlling the IFPI length within $500 \mu \mathrm{m}$ is not difficult

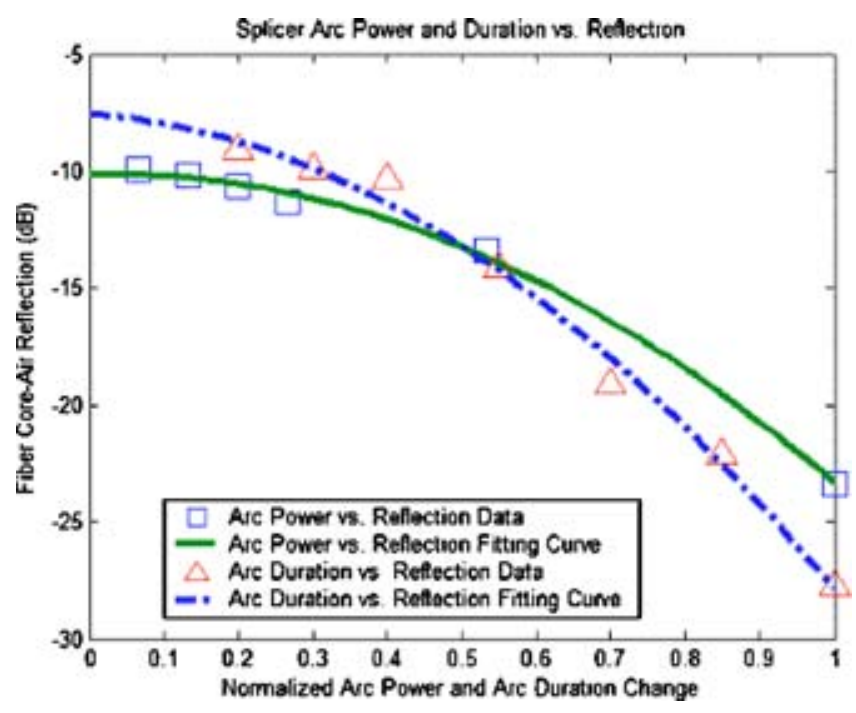

Fig. 2 IFPI fiber core-air reflection vs splicing arc power and duration (normalized to cleaved SMF reflection).

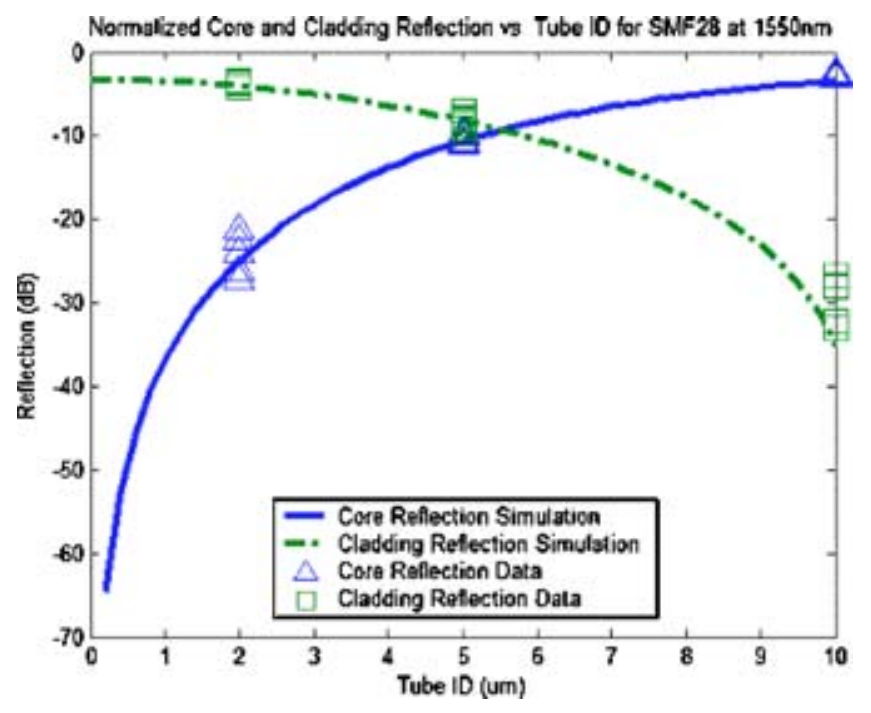

Fig. 3 IFPI core and cladding reflection (normalized to cleaved SMF reflection).

with a microscope, the chance of successful IFPI fabrication is only dependent on the cleaving quality of the tube. Ten out of 13 sensors that we have made demonstrate over 10-dB peak-to-peak fringe as shown in Fig. 4. With a finetuned fiber cleaver, the successful rate of IFPI fabrication is expected to exceed $75 \%$.

\section{Sensor Performance}

The sensor is co-positioned with a thermocouple (K-type, Omega) in a double-bore ceramic tube and then placed in a furnace (Thermolyne 48000). The function of the ceramic tube is to dampen the temperature fluctuation. The temperature is increased with $50^{\circ} \mathrm{C}$ per step and $1 \mathrm{~h}$ at each step from room temperature to $600^{\circ} \mathrm{C}$ back to room temperature for four cycles. The mean value of sensor's optical path difference (OPD) at each temperature step is shown in Fig. 4. The maximum deviation between the experimental OPD curve and the four-time averaged OPD curve is $\pm 0.23 \mathrm{~nm}$.
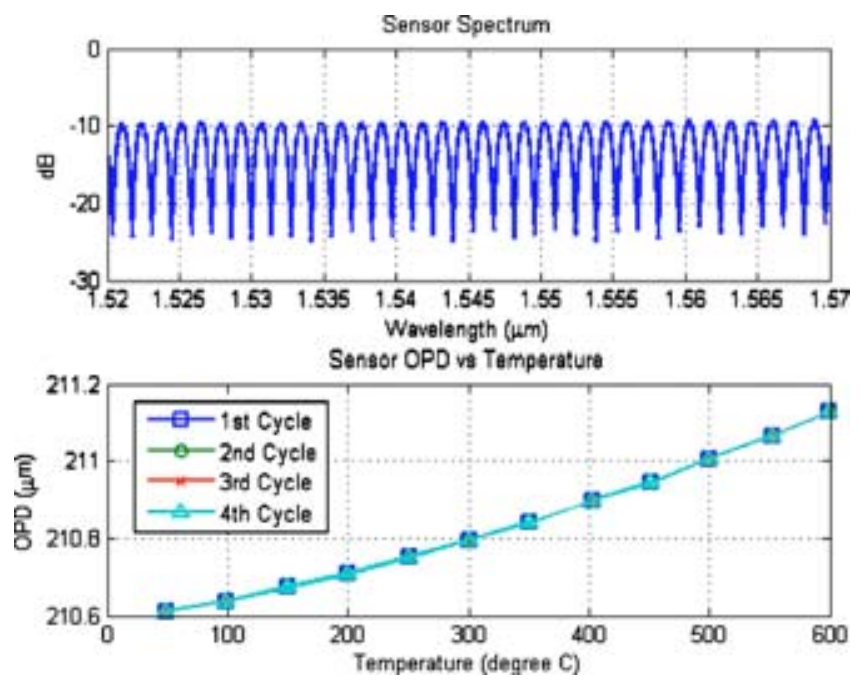

Fig. 4 IFPI spectrum and temperature performance (normalized to cleaved SMF reflection). 


\section{OE LETTERS}

The total OPD change from $50^{\circ} \mathrm{C}$ to $600^{\circ} \mathrm{C}$ is $517.0 \mathrm{~nm}$. Thus the sensor repeatability is approximately $\pm 0.24^{\circ} \mathrm{C}$ and $\pm 0.046 \%$ of the dynamic range. The resolution of the sensor system is usually interpreted by its standard deviation of temperature measurements. It is common to use twice the standard deviation (STD) as the direct measure of resolution. The sensor is inserted into a ceramic tube and placed in an environmental chamber (Test Equity $1000 \mathrm{Se}-$ ries Temperature Chamber) at $25^{\circ} \mathrm{C}$ for $5 \mathrm{~h}$, and the OPD is sampled every $5 \mathrm{~s}$. The STD is $0.13 \mathrm{~nm}$. The resolution of the sensor system is $0.26 \mathrm{~nm}$, which corresponds to $0.27^{\circ} \mathrm{C}$ and $0.05 \%$ of its dynamic range. The nonlinear behavior of the sensor is caused by the temperature sensitivity of both the refractive index of silica glass and the length of the cavity.

\section{Conclusion}

In this letter, we presented the principle of the proposed wavefront splitting IFPI followed by discussion on fabrication optimization. The IFPI is made with a $5-\mu \mathrm{m}$ ID fused silica tube. Short arc duration and low arc power is used in the sensor fabrication. The sensor is measured to $600^{\circ} \mathrm{C}$ for four cycles and demonstrated $0.27^{\circ} \mathrm{C}$ resolution and $\pm 0.24{ }^{\circ} \mathrm{C}$ repeatability.

\section{References}

1. W.-H. Tsai and C.-J. Lin, "A novel structure for the intrinsic FabryPerot fiber-optic temperature sensor," J. Lightwave Technol. 19(5), 682-686 (May 2001).

2. M. Born and E. Wolf, Principles of Optics, Cambridge University Press, 7th ed., Cambridge, UK (1999).

3. C. E. Lee, W. N. Gibler, R. A. Atkins, and H. F. Taylor, "In-line fiber Fabry-Perot interferometer with high-reflectance internal mirrors," J. Lightwave Technol. 10(10), 1376-1379 (Oct. 1992).

4. Y. L. Lo, J. S. Sirkis, and C. C. Chang, "Passive signal processing of in-line fiber etalon sensor for high strain-rate loading," J. Lightwave Technol. 15(8), 1578-1586 (1997).

5. H. Singh and J. S. Sirkis, "Simultaneously measuring temperature and strain using optical fiber microcavities," J. Lightwave Technol. 15(4), 647-653 (1997).

6. Q. Bing, G. Pickrell, J. Xu, Z. Huang, et al., "Novel data processing techniques for dispersive white light interferometer," Opt. Eng. 43(11), 3165-3171 (2003).

7. D. Marcuse, "Loss analysis of single-mode fiber splices," Bell Syst. Tech. J. 56(5), 703-718 (1977). 


\title{
Measurement of the frequency response of a diaphragm-based pressure sensor by use of a pulsed excimer laser
}

\author{
Fabin Shen, Juncheng Xu, and Anbo Wang \\ Center for Photonics Technology, Bradley Department of Electrical and Computer Engineering, Virginia Polytechnic \\ Institute and State University, Blacksburg, Virginia 24061 \\ Received February 25, 2005 \\ We present a novel method for measuring the frequency response of a diaphragm-based optical fiber Fabry- \\ Perot interferometric pressure sensor. The impulse response of the sensor to the radiation pressure gener- \\ ated by an excimer laser pulse is measured. The Fourier transform of the impulse response yields the fre- \\ quency response of the pressure sensor. Experimental results show that it is a convenient and efficient \\ method for measurement of the frequency response of diaphragm-based pressure sensors. (C) 2005 Optical \\ Society of America \\ OCIS codes: $060.2370,140.2180,120.2230$.
}

Frequency response is one of the most important parameters of pressure sensors. The methods with which the frequency responses of pressure sensors are measured may be classified into two categories, namely, frequency-domain methods and time-domain methods. ${ }^{1}$ Frequency-domain methods usually use pressure transducers to generate periodic pressure signals with narrow frequency bands and measure the sensor frequency response at these frequencies. High-frequency sensors require the pressure transducers to generate a reference pressure that can be adjusted over a wide range of amplitudes and frequencies. However, most commercially available pressure transducers are limited in bandwidth, so only the frequency responses in a certain frequency range can be measured in most cases. Time-domain methods usually use shock tubes or fast opening valves to generate a step or pulse shaped pressure and obtain the frequency response from the timedomain response by means of a Fourier transform. ${ }^{1-4}$ Time-domain methods are highly recommended if a good record of a step or pulse pressure signal can be obtained. Depending on the type of calibrator and gas medium used, changes in the rise times of pressure of the order of milliseconds or microseconds can be achieved. ${ }^{4}$ The dynamic pressure calibrations of pressure sensors at high frequencies are limited because of the relatively long rise time of the pressure change.

In this Letter we present a novel method for generating a pressure impulse by using the radiation pressure generated by a laser pulse with high energy. When a light beam is incident onto the surface of a medium, radiation pressure is generated as a result of the transfer of momentum from the incident photons. For normal incidence, the radiation pressure can be given as

$$
P_{r}=\frac{F}{A}=\frac{\eta P / c}{A}=\frac{\eta D}{c},
$$

where $F$ is the force, $\mathrm{A}$ is the interaction area, $P$ is the power of the light, $\eta$ is the coefficient of the momen- tum transfer that is due to absorption and reflection of the photons, $D$ is the power density, and $c$ is the velocity of light. 5,6

In our experiment we used a $248 \mathrm{~nm}$ excimer laser (MPB MSX250) to generate light pulses. The laser beam was focused by an UV-grade spherical lens and irradiated onto the surface of a diaphragm-based pressure sensor. Laser pulses with $20 \mathrm{~ns}$ duration and $20 \mathrm{~mJ}$ energy each were focused to an area of $5 \mathrm{~mm}^{2}$. The corresponding power density of the light pulse was

$$
D=\frac{E}{A T}=\frac{20 \mathrm{~mJ}}{5 \mathrm{~mm}^{2} \times 20 \mathrm{~ns}}=0.2 \times 10^{12}\left[\mathrm{~W} / \mathrm{m}^{2}\right],
$$

where $E$ and $T$ are the energy and the duration of the laser pulse.

The optical fiber's Fabry-Perot (FP) pressure sensor comprises a fused-silica diaphragm, a fused-silica ferrule with an O.D. of $1.8 \mathrm{~mm}$, and a 9-125 $\mu \mathrm{m}$ lead-in single-mode silica fiber, which are bonded together by a laser-induced fusion bonding method. ${ }^{7}$ An air-gap FP cavity is formed by the cleaved end face of the fiber and the inner surface of the diaphragm. The length of the air gap is $27.8 \mu \mathrm{m}$ under ambient atmospheric pressure. The structure of the diaphragmbased pressure sensor and the setup of the experiment are shown in Fig. 1.

The diaphragm of the pressure sensor is an UVgrade Corning 7980 fused-silica glass wafer with a thickness of $h=60 \mu \mathrm{m}$, a refractive index of $n=1.508$, and an absorption coefficient of $\alpha<0.002 \mathrm{~cm}^{-1}$ at $248 \mathrm{~nm} .{ }^{8,9}$ Compared with the reflections at the airglass interfaces of the diaphragm, each of which is $[(n-1) /(n+1)]^{2} \approx 0.04$, the absorption of the diaphragm, which is less than $0.002 \mathrm{~cm}^{-1} \times 60 \mu \mathrm{m}$ $=1.2 \times 10^{-5}$, can be neglected. Therefore the momentum transferred from the photons comes mainly from the reflection of the incident light. The reflected power is determined by the interference between the reflections at the two air-glass interfaces. The dia- 


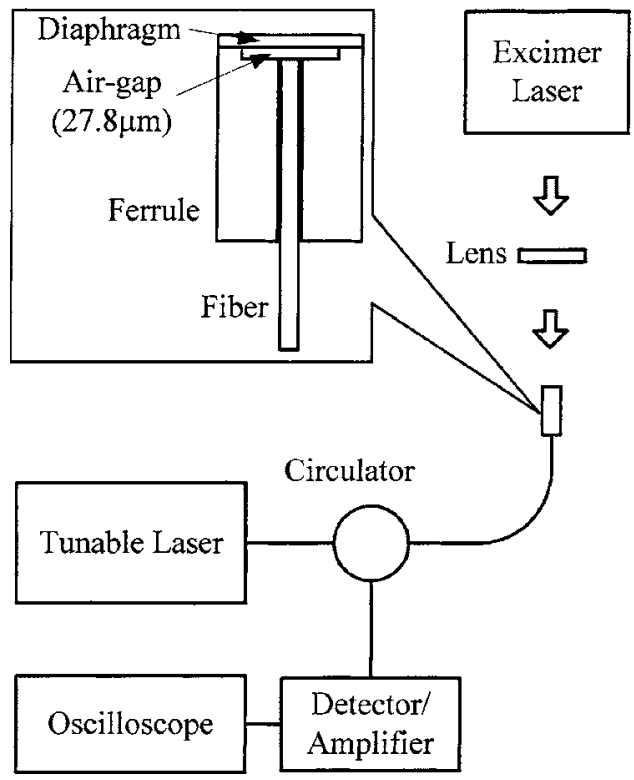

Fig. 1. Sensor structure and experimental setup for frequency-response measurement.

phragm itself is a FP cavity with an optical path difference of $\Delta L=2 n h=2 \times 1.508 \times 60 \approx 181 \mu \mathrm{m}$. The FWHM spectral width of the excimer laser is $\Delta \lambda$ $\approx 0.3 \mathrm{~nm}$, so the coherence length is estimated to be $L_{c}=\lambda^{2} / \Delta \lambda=248^{2} / 0.3 \mathrm{~nm} \approx 205 \mu \mathrm{m}$. Because $\Delta L$ is close to $L_{c}$, the contrast of the interference between the two reflections is low. Therefore the light reflected by the diaphragm can be approximated as the summation of the light reflected at the air-glass interfaces. Thus $\eta$ can be predicted as $\eta=2\left(R_{1}+R_{2}\right)$ $\approx 0.16$, where $R_{1}=R_{2} \approx 0.04$ are the reflection coefficients of the two air-glass interfaces and 2 means the doubling of momentum transfer because of the reverse in direction caused by the reflection. If $L_{c}$ is much larger than $\Delta L$, then the interference between the two reflections has to be taken into consideration. One can change the optical path difference of the diaphragm's FP cavity by tilting the diaphragm at a small angle such that the reflected light power and $\eta$ can be adjusted.

The sensitivity of the diaphragm is predicted to $\mathrm{be}^{10-12}$

$$
S=\frac{3\left(1-\mu^{2}\right)}{16 E} \frac{r^{4}}{h^{3}} \approx 2.1 \times 10^{-3}[\mathrm{~nm} / \mathrm{Pa}]
$$

where $\mu=0.16$ is Poisson's ratio, $E=72.7 \mathrm{GPa}$ is Young's modulus, ${ }^{8} r=650 \mu \mathrm{m}$ is the effective radius, and $h=60 \mu \mathrm{m}$ is the thickness of the diaphragm. For the radiation pressure generated in Eq. (1), which is predicted to be $P_{r}=0.16 \times 0.2 \times 10^{12} / 3 \times 10^{8}$ $\approx 1.1 \times 10^{2} \mathrm{~Pa}$, the deflection of the diaphragm is expected to be $0.23 \mathrm{~nm}$, which is detectable for the measurement system shown in Fig. 1.

Based on the diaphragm deflection analysis in Ref. 10 , the lowest-order resonant frequency of the fusedsilica diaphragm is predicted to be $\mathrm{e}^{11,12}$

$$
\begin{aligned}
f_{0} & =2.742 \times 10^{9} \frac{h}{r^{2}}[\mathrm{~Hz}]=2.742 \times 10^{9} \frac{60}{650^{2}}[\mathrm{~Hz}] \\
& \approx 389.4 k[\mathrm{~Hz}]
\end{aligned}
$$

where $h$ and $r$ are in micrometers.

A continuous-wave tunable laser (New Focus TLB6328 ) with a $1520-1570 \mathrm{~nm}$ tunable range was used as the light source of the optical fiber pressure sensor measurement system. It was tuned to the quadrature of the FP interferometric pressure sensor to yield maximum sensitivity. Light from the tunable laser was launched into the pressure sensor through an optical circulator. The reflected light from the sensor was coupled to a photodiode detector. An oscilloscope was used to monitor the amplified output of the detector and to capture the time-domain response of the sensor when a radiation pressure pulse was applied. The repetition rate of the excimer laser pulses was 5 $\mathrm{Hz}$.

The time-domain response of the sensor to a radiation pressure pulse and its Fourier transform are shown in Fig. 2. Figure 2(a) shows the time-domain response of the sensor to a radiation pressure pulse. Figure 2(b) shows the Fourier transform of the timedomain response. The resonant frequency of the sensor $f_{0}$ occurs at $400 \mathrm{kHz}$. The gain in the frequency region lower than $f_{0}$ is $\sim 15 \mathrm{~dB}$ higher than that of the frequency region higher than $f_{0}$. Because the $6 \mathrm{~dB}$ bandwidth of the amplifier was $1 \mathrm{MHz}$, no high-order resonant frequency was observed.

The sensitivity of the sensor at the quadrature for static pressure was tuned to $20 \mathrm{mV} / \mathrm{nm}$. The maximum peak-to-peak value of the captured waveform in Fig. 2(a) was $15.9 \mathrm{mV}$. The maximum deflection of the diaphragm was estimated to be $0.4 \mathrm{~nm}$, which is larger than the predicted value of $0.23 \mathrm{~nm}$. The difference between the values comes from the overshoot of the time-domain response and the estimation errors of radiation pressure $P_{r}$ in Eq. (1) and sensitivity $S$ in Eq. (3).

The frequency response of the sensor can be given as
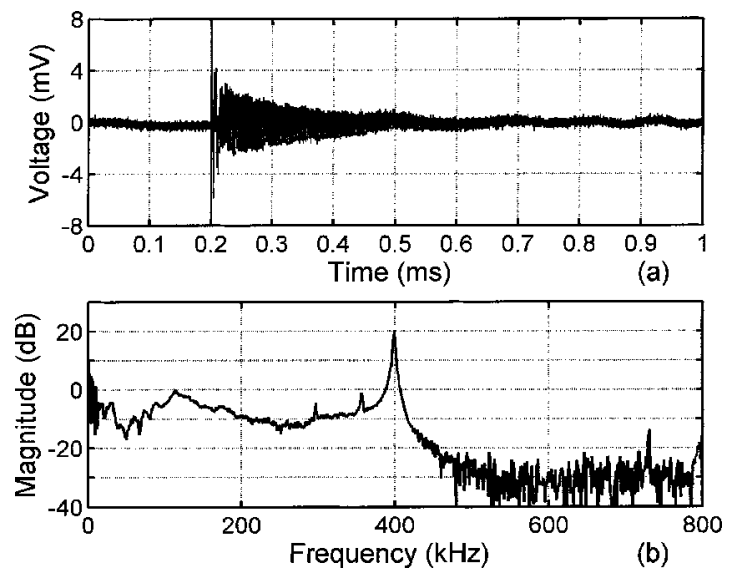

Fig. 2. Time-domain response of a pressure sensor to the radiation pressure pulse and its Fourier transform: (a) time-domain response, (b) Fourier transform. 


$$
S(j \omega)=R(j \omega) / E(j \omega),
$$

where $R(j \omega)$ is the Fourier transform of the measured time-domain response of the sensor and $E(j \omega)$ is the Fourier transform of the excitation of the radiation pressure pulse. Because the duration of the laser pulse was $T=20 \mathrm{~ns}$, the pulse can be considered to be a Dirac impulse for the pressure sensor whose frequency response is significantly slower than $1 / T$ $=50 \mathrm{MHz}$. Thus $E(j \omega)$ can be approximated to be 1 in the frequency range of the sensor. Therefore the time-domain response to the pressure pulse can be considered to be the impulse response of the sensor, and its Fourier transform simply gives the frequency response of the sensor, as Fig. 2(b) shows. The frequency response of the sensor can be monitored directly by use of the fast-Fourier-transform function of an oscilloscope.

The high power density of the excimer laser pulse may damage the surface of the fused-silica diaphragm, whose damage threshold is $\sim 5 \mathrm{~J} / \mathrm{cm}^{2}$ for a 20 ns pulse. ${ }^{9}$ Therefore the power density of the excimer laser pulse has to be controlled to be lower than the damage threshold. One can control the power density by controlling the offset between the sensor position and the focal plane of the lens. A dielectric or metal mirror coated onto the diaphragm surface of the pressure sensor will help to protect the sensor and to increase the radiation pressure at the same time.

In conclusion, we have presented a novel, convenient, and efficient method with which to measure the frequency responses of diaphragm-based pressure sensors. The frequency response is obtained as the Fourier transform of the response of the sensor to the radiation pressure excitation generated by an excimer laser pulse. Although the experimental result is demonstrated only for an optical fiber pressure sensor, the radiation pressure excitation method for frequency-response measurement can be applied to various kinds of pressure sensor.

This research was partially supported by the U.S. Department of Energy under grant DE-FC3601G011050. The authors are grateful to the anonymous reviewers for the comments to improve this paper. F. Shen's e-mail address is fashenl@vt.edu.

\section{References}

1. J. Zakrzewski and K. Wróbel, IEEE Trans. Instrum. Meas. 51, 1358 (2002).

2. V. E. Bean, Metrologia 30, 737 (1993-1994).

3. J. P. Damion, Metrologia 30, 743 (1993-1994).

4. J. Lally and D. Cummiskey, Sensors 20 (4), 15 (2003).

5. P. Lorrain and D. R. Corson, Electromagnetic Fields and Waves, 2nd ed. (Freeman, 1970), p. 543.

6. M. Mansuripur, Opt. Express 12, 5375 (2004).

7. J. Xu, G. Pickrell, X. Wang, W. Peng, K. Cooper, and A. Wang, IEEE Photon. Technol. Lett. 17, 870 (2005).

8. Corning 7980 specifications: http://www.corning.com/ semiconductoroptics/products_services/pdf/ h0607_hpfskrf_productsheet.pdf.

9. D. N. Nikogosyan, Properties of Optical and LaserRelated Material: a Handbook (Wiley, 1997), p. 169.

10. M. D. Giovanni, Flat and Corrugated Diaphragm Design Handbook (Marcel Dekker, 1982).

11. J. Xu, G. Pickrell, B. Yu, M. Han, Y. Zhu, X. Wang, K. L. Cooper, and A. Wang, Proc. SPIE 5590, 1 (2004).

12. B. Yu, D. W. Kim, J. Deng, H. Xiao, and A. Wang, Appl. Opt. 42, 3241 (2003). 


\title{
Frequency-estimation-based signal-processing algorithm for white-light optical fiber Fabry-Perot interferometers
}

\author{
Fabin Shen and Anbo Wang
}

\begin{abstract}
A novel signal-processing algorithm based on frequency estimation of the spectrogram of single-mode optical fiber Fabry-Perot interferometric sensors under white-light illumination is described. The frequency-estimation approach is based on linear regression of the instantaneous phase of an analytical signal, which can be obtained by preprocessing the original spectrogram with a bandpass filter. This method can be used for a relatively large cavity length without the need for spectrogram normalization to the spectrum of the light source and can be extended directly to a multiplexed sensor system. Experimental results show that the method can yield both absolute measurement with high resolution and a large dynamic range. Performance analysis shows that the method is tolerant of background noise and variations of the source spectrum. (C) 2005 Optical Society of America
\end{abstract}

OCIS codes: $\quad 060.2370,120.3180,120.2650$.

\section{Introduction}

Fiber optic white-light interferometry has proved to be a practical method for measuring absolute optical path difference (OPD) with high accuracy and a large dynamic range. ${ }^{1-8}$ In a white-light measurement system, light from a low-coherence source, such as a light-emitting diode (LED), a superluminescent LED, or a broadband lamp, is transmitted to a single or multiplexed fiber sensors. A fringe pattern is produced by the light transmitted by or reflected from the sensor and recorded either spectrally by an optical spectrum analyzer (OSA) or temporally by a photodiode in a scanning interferometric system. ${ }^{1,2}$

When the interferogram of an interferometer is measured, the OPD of the interferometer can be estimated from the fringe patterns of the interference by fringe analysis. The fringe analysis approach most often used for white-light interferometers is the fringe peak tracking method, in which the peak position of a fringe or the fractional sample points between fringes in the interferogram are identified to determine the fringe order and to estimate the OPD

The authors are with the Center for Photonics Technology, Bradley Department of Electrical and Computer Engineering, Virginia Polytechnic Institute and State University, Blacksburg, Virginia 24061. F. Shen's e-mail address is fashen1@vt.edu.

Received 15 February 2005; revised manuscript received 7 April 2005; accepted 8 April 2005.

0003-6935/05/255206-09\$15.00/0

(C) 2005 Optical Society of America of the sensor. ${ }^{1-5}$ Although this fringe analysis method has been successfully used in many single interferometric sensor systems, it requires a high signal-tonoise ratio (SNR) to determine the fringe orders correctly and detect the peak positions accurately and cannot be directly applied to multiplexed sensor systems.

The most often used multiplexing scheme for fiber white-light interferometers is the path-matched differential interferometric coherence division multiplexing technique, in which the OPD of a receiving interferometer is scanned to match the OPDs of the interferometers interrogated. The multiplexed interferometers must have different OPDs that are larger than the coherence length of the light source. The path-matched differential interferometric fringe patterns from different sensors fall into different temporal ranges during the scanning of the receiving interferometer and can be separated in time or spatial domain. ${ }^{6-8}$

If a spectrogram of multiple interferometers is measured by an OSA, the fringe patterns from different interferometers will be mixed with one another. However, if the OPDs of the multiplexed interferometers are different, the measured spectrogram will have multiple frequency components that can be separated in the frequency domain.

In this paper we present a phase linear regressionbased frequency-estimation approach to the measurement of absolute OPDs of multiplexed Fabry-Perot (FP) interferometers from a measured spectrogram. We analyze the frequency components in a spectro- 


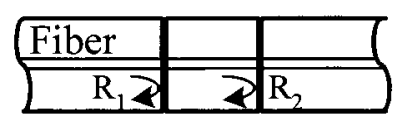

(a)

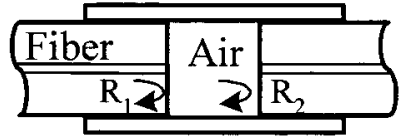

(b)
Fig. 1. FP interferometric sensors: (a) IFPI sensor, (b) EFPI sensor.

gram, use a finite impulse-response (FIR) filter to select the frequency component that corresponds to an individual FP interferometer, and estimate the frequency by linear regression of the instantaneous phase of the analytical signal. The OPD estimations are given for both known and unknown initial phases of FP interferometers.

The contents of this paper are arranged as follows: In Section 2, the frequency components of a spectrogram are analyzed, the analytical signal is introduced, and the frequency-estimation method based on instantaneous phase linear regression is presented. In Section 3, experimental results for multiplexed FP interferometers are demonstrated. The performance of the signal-processing method at several SNR levels is analyzed in Section 4, followed by a statement of our conclusions in Section 5 .

\section{Principles of Operation}

\section{A. Fabry-Perot Interferometers}

There are two main categories of fiber FP interferometric sensors, namely, intrinsic FP interferometers (IFPIs) and extrinsic FP interferometers (EFPIs). An IFPI sensor contains two internal partial reflection mirrors. The fiber between the reflectors serves as both a sensing element and a light waveguide. Lee and Taylor ${ }^{9,10}$ demonstrated some IFPI sensors by building dielectric mirrors into optical fiber, as shown in Fig. 1(a). An EFPI sensor contains a lead-in fiber with a partial mirror at the end face, a cavity of air or another transparent medium, and a reflector on the other end, which may also be a piece of fiber. Researchers at Virginia Tech developed some EFPI sensors constructed by forming an air gap between end faces of two uncoated fibers inserted into an alignment tube, as shown in Fig. 1(b). ${ }^{11,12}$ Sirkis et al. introduced in-line fiber etalons by fusion splicing two fibers with a section of hollow tube of the same outside diameter. ${ }^{13}$

The optical path length between the light reflected from the two partial mirrors in Figs. 1(a) and 1(b) is

$$
L=2 n_{e} d,
$$

where $n_{e}$ is the refractive index of the cavity medium and $d$ is the geometrical distance between the two reflectors.

When the light is launched into the FP interferometer, the light reflected from the two reflectors will interfere. Assuming that the reflection coefficients at the two reflectors, $R_{1}$ and $R_{2}$, are very small, the FP interferometer can be approximated to a two-beam or Fizeau interferometer with all the multipath reflections neglected. The electric field of the reflected light can be given as

$$
E=E_{1}+E_{2}=\eta_{1} R_{1} E_{0}+\eta_{2} R_{2} E_{0} \exp [j(k L+\phi)],
$$

where $E_{0}$ is the electric field of the incident light, $k$ is the wave number, $L$ is the OPD in Eq. (1), $\eta_{1}$ and $\eta_{2}$ are the coefficients of coupling efficiency of the light reflected into the lead-in fiber, and $\phi$ is a phase term that refers to reflection and light propagation. ${ }^{14}$

The intensity of the reflected light can be given as

$$
\begin{aligned}
I & =|E|^{2} \\
& =\left|E_{0}\right|^{2}\left[\eta_{1}{ }^{2} R_{1}{ }^{2}+\eta_{2}{ }^{2} R_{2}{ }^{2}+2 \eta_{1} \eta_{2} R_{1} R_{2} \cos (k L+\phi)\right] \\
& =I_{0}[A+B \cos (k L+\phi)],
\end{aligned}
$$

where $I_{0}$ is the intensity of the incident light at wave number $k$.

When light from a low-coherence light source is reflected by the FP cavity and measured by an OSA, the spectrogram of the interference can be given as

$$
I\left(k_{n}\right)=A I_{0}\left(k_{n}\right)+B I_{0}\left(k_{n}\right) \cos \left(k_{n} L+\phi\right)+v\left(k_{n}\right),
$$

where $I_{0}\left(k_{n}\right)$ are samples of the spectrum of incident light and $v\left(k_{n}\right)$ are the measurement noises. The coefficients $A, B, L$, and $\phi$ are assumed to be wavelength independent, which is true for most cases.

The measured spectrogram contains a background source spectrum $A I_{0}\left(k_{n}\right)$, an amplitude-modulated (AM) signal $B I_{0}\left(k_{n}\right) \cos \left(k_{n} L+\phi\right)$ with a carrier of frequency $L$ in the wave-number domain, and a noise term $v\left(k_{n}\right)$.

The goal of signal processing in white-light interferometry is to determine $L$ from the measured spectrum $I\left(k_{n}\right)$. This is equivalent to estimating carrier frequency $L$ from AM signal $B I_{0}\left(k_{n}\right) \cos \left(k_{n} L+\phi\right)$ corrupted by background noises $A I_{0}\left(k_{n}\right)$ and $v\left(k_{n}\right)$.

One may store a background spectrum of the light source $I_{0}\left(k_{n}\right)$ and obtain a normalized interference spectrogram:

$$
I_{N}\left(k_{n}\right)=\frac{I\left(k_{n}\right)}{I_{0}\left(k_{n}\right)}=A+B \cos \left(k_{n} L+\phi\right)+\xi\left(k_{n}\right) .
$$

Then the task becomes the classic problem of estimating the frequency of a sinusoid. However, unless the stored background source spectrum can be updated frequently, the normalization in Eq. (5) may give an error when the real light source spectrum, which is dependent on its driving current and ambient temperature, does not match the stored source spectrum.

A normalization method that can handle the variations of the light source spectrum was given in Refs. 5 and 6 . Two auxiliary spectral curves, $I_{\max }(k)$ and 

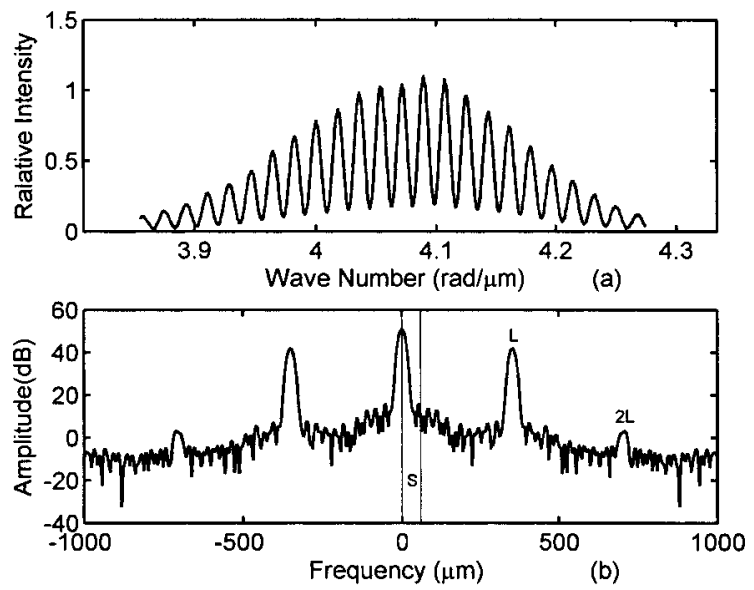

Fig. 2. (a) Spectrogram and (b) result of fast Fourier transformation of an EFPI sensor.

$I_{\text {min }}(k)$, which are the envelopes of the measured spectrogram, can be interpolated from the maxima and minima of the spectrogram and used to calculate the cosine term and eliminate the source spectrum in Eq. (4). However, this method also depends on the accuracy of extremum detection, which is difficult when there is a low SNR or when multiple sinusoids exist. It also requires that the number of extrema be large enough to yield a good interpolation, which means that the cavity length should be long enough.

We present a signal-processing approach that does not need normalization explicitly. The approach is based on frequency estimation of analytical signals, as discussed below.

\section{B. Analytical Signal}

The spectrogram of an EFPI cavity and its discrete Fourier-transformation result are shown in Fig. 2. The spectrogram has a variable of $k$ in radians per micrometer; its frequency component has units of micrometers.

The source spectrum, $I_{0}(k)$, falls into the lowfrequency region with spectral range $S$. The AM signal, $I_{0}(k) \cos (k L+\phi)$, shifts the spectrum of $I_{0}(k)$ to frequencies of $\pm L$. If $L$ is selected to be $L>2 S$ during the sensor fabrication, then the spectra of $I_{0}(k)$ and $I_{0}(k) \cos (k L+\phi)$ will not overlap, as Fig. 2 shows. We can use a bandpass filter to separate them to select the AM signal, $I_{0}(k) \cos (k L+\phi)$.

The analytical signal model of the AM signal can be expressed as

$$
I(k)=I_{0}(k) \exp [j(k L+\phi)] .
$$

We can use either of two methods to obtain this analytical signal from Eq. (4). One method is to design a single-band filter, which can be implemented by a double-band filter followed by a Hilbert transform, to select the single band of the AM signal, as shown in

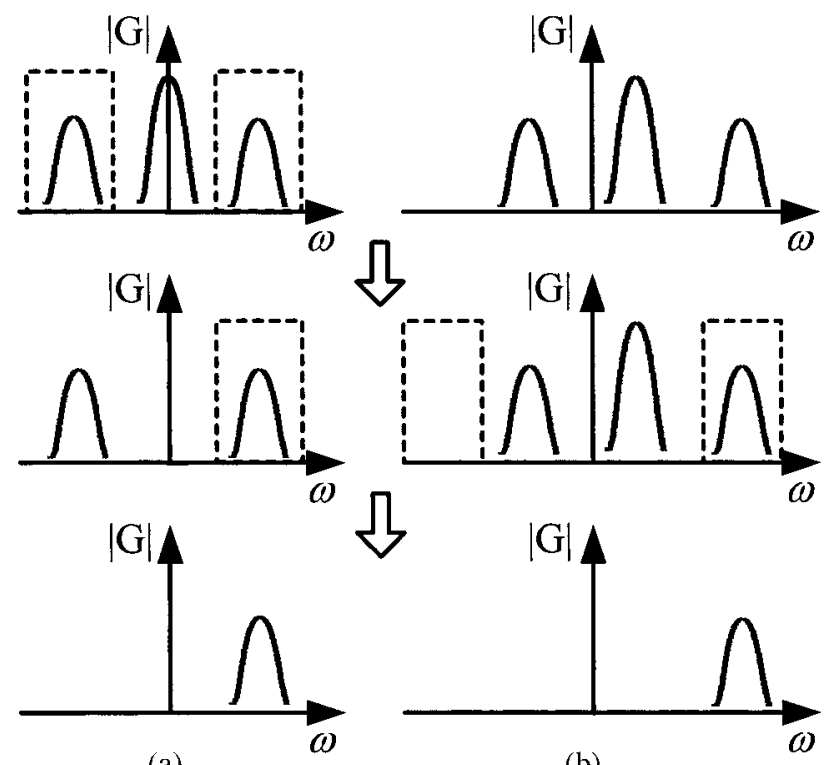

(a)

(b)

Fig. 3. Methods for obtaining analytical signals: (a) single-band filter, (b) frequency shift.

Fig. 3(a). The filtering and Hilbert transformation can be calculated efficiently by fast Fourier transformation. The other method is first to shift the frequency components by multiplying Eq. (4) by $\exp \left(j k L_{0}\right)$ and filtering the signal with a double-band filter. Then an analytical signal with central frequency $L+L_{0}$ can be obtained, as shown in Fig. 3(b). Schematics of these two methods are shown in Fig. 3.

We use a digital FIR bandpass filter whose passband center frequency is close to $L$ to select the AM signal in Eq. (4). It is well known that a FIR filter is a linear phase filter. It introduces a pure delay into the signal without giving any other distortion to the signal in the passband. The pure delay of samples caused by the FIR filter is

$$
n_{d}=\left(M_{f}-1\right) / 2 \text {, }
$$

where $M_{f}$ is the order of the FIR filter. One can simply shift the filtered signal backward by $n_{d}$ samples to compensate for the phase change caused by the FIR filter.

If the wave number is uniformly spaced in steps of $k_{s}$ from starting wave number $k_{0}$, then, after filtering and backward shifting, the analytical signal can be written as

$$
\begin{aligned}
\bar{x}_{n} & =\bar{A}_{n} \exp \left[j\left(k_{n} L+\phi\right)\right]+\bar{v}_{n} \\
& =\bar{A}_{n} \exp \left[j\left(k_{s} L n+k_{0} L+\phi\right)\right]+\bar{v}_{n} \\
(n & =1,2, \ldots N),
\end{aligned}
$$

where $\bar{A}_{n}$ is the amplitude after filtering, $k_{0}$ is the starting wave number, $k_{s}$ is the wave-number step, and $\bar{v}_{n}$ is the residual noise after filtering. Carrier 
frequency $L$ or normalized angular carrier frequency

$$
\omega=k_{s} L
$$

can be estimated by the method of phase linear regression discussed below.

\section{Frequency Estimation}

A frequency estimation based on linear regression of the instantaneous signal phase was given in Ref. 15 . The frequency and phase estimation of sinusoids from a sequence of signal samples is given as

$$
x_{n}=A \exp [j(\omega n+\phi)]+v_{n},
$$

where $A, \omega$, and $\phi$ are unknown parameters to be estimated and $v_{n}$ is additive Gaussian white noise.

The difference between Eqs. (8) and (10) is that amplitude $\bar{A}_{n}$ in Eq. (8) is wave-number dependent, whereas amplitude $A$ in Eq. (10) is constant. However, the derivations of the unwrapped phases of Eqs. (8) and (10) are the same as shown in Eq. (11). Thus the frequency-estimation method in Ref. 15 can be extended to the AM analytical signal model.

The unwrapped phase of the sinusoids in Eq. (8) can be given as

$$
\phi_{n}=\left[\angle \bar{x}_{n}\right]_{2 \pi}=k_{n} L+\phi+\zeta_{n} \quad(n=[1, \ldots, N]),
$$

where $\left[\angle \bar{x}_{n}\right]_{2 \pi}$ means $\tan ^{-1}\left[\operatorname{Im}\left(\bar{x}_{n}\right) / \operatorname{Re}\left(\bar{x}_{n}\right)\right]+2 m \pi, \zeta_{n}$ is the equivalent phase noise of additive noise $\bar{v}_{n}$ in Eq. (8), and $N$ is the length of the unwrapped phase sequence.

The parameters $L$ and $\phi$ can be estimated by the method of least-squares estimation or linear regression to minimize the square error:

$$
S=\Sigma\left[\phi_{n}-k_{n} \hat{L}-\hat{\phi}\right]^{2} .
$$

The solution is

$$
\left[\begin{array}{l}
\hat{L} \\
\hat{\phi}
\end{array}\right]=\left(A^{T} A\right)^{-1} A^{T} \Phi,
$$

where

$$
\begin{aligned}
A & =\left[\begin{array}{cccc}
k_{1} & k_{2} & \ldots & k_{N} \\
1 & 1 & \ldots & 1
\end{array}\right]^{T}, \\
\Phi & =\left[\begin{array}{llll}
\phi_{1} & \phi_{2} & \ldots & \phi_{N}
\end{array}\right]^{T} .
\end{aligned}
$$

Estimation $\hat{L}$ usually does not have high precision, especially when the SNR is not high. A simulation result of the variance of the normalized frequency estimation for unknown frequency and phase is given in Subsection 4.A below. In practice, phase term $\phi$ in Eqs. (4) and (8), which is a constant, can be known $a$ priori by either accurate modeling or calibration in advance. We can use it to get a higher frequencyestimation precision than that of an unknown phase. One can obtain averages of multiple estimations $\hat{L}$ and $\hat{\phi}$ from Eq. (13) and store them as calibrated values of $\bar{L}$ and $\bar{\phi}$. The modulus of $\bar{\phi}$ with $2 \pi, \tilde{\phi}$ $=[\bar{\phi}]_{2 \pi}$, whose value falls within $[-\pi, \pi]$, can be used as a good calibrated approximation of $\phi$.

If the estimation error $\Delta L=\hat{L}-L$ is less than $\lambda / 2$, where $\lambda$ is the wavelength of light, the phase error caused by $\Delta L$,

$$
\Delta \phi_{n}=k_{n} \Delta L,
$$

will be in the range $[-\pi, \pi]$. Therefore, for the estimation of $\hat{L}$ and $\hat{\phi}$ from Eq. (13), we can select

$$
\Delta \phi=[\hat{\phi}-\phi]
$$

or

$$
[\hat{\phi}-\tilde{\phi}],
$$

whose value falls within $[-\pi, \pi]$, as an estimation of phase error caused by $\Delta L$. Then we can estimate $\Delta L$ by minimizing the variance

$$
S=\Sigma\left[\Delta \phi-k_{n} \Delta \hat{L}\right]^{2}
$$

with the solution

$$
\Delta \hat{L}=\left(C^{T} C\right)^{-1} C^{T} D
$$

where

$$
\begin{aligned}
& C=\left[\begin{array}{llll}
k_{1} & k_{2} & \ldots & k_{N}
\end{array}\right]^{T}, \\
& D=\left[\begin{array}{llll}
\Delta \phi & \Delta \phi & \ldots & \Delta \phi
\end{array}\right]^{T} .
\end{aligned}
$$

We can use the estimation of Eq. (17) as compensation for the frequency estimation of Eq. (13). The compensated frequency estimation for known $\phi$ is

$$
\tilde{L}=\hat{L}+\Delta \hat{L} \text {. }
$$

It will have higher precision than that of the unknown $\phi$. The simulation results of frequency estimation for unknown and known phases, $\hat{L}$ in Eq. (13) and $\tilde{L}$ in Eq. (18), at different SNR levels, are shown in Subsection 4.A below.

It needs to be noted that the signal-processing approach can be extended to a multiplexing system in which the measured spectrogram has multiple frequency components that correspond to different interferometers. The spectrogram of multiplexed interferometers can be given as 


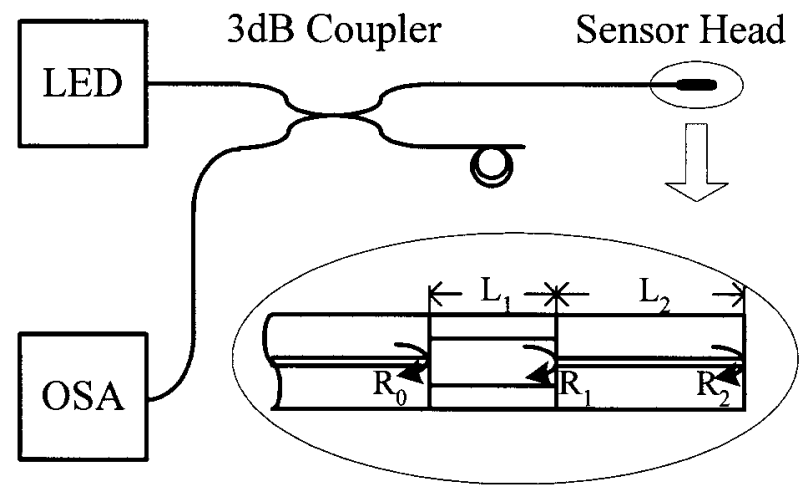

Fig. 4. Schematic of the experimental setup.

$$
I\left(k_{n}\right)=A I_{0}\left(k_{n}\right)+\sum_{i=1}^{M} B_{i} I_{0}\left(k_{n}\right) \cos \left(2 \pi k_{n} L_{i}+\phi_{i}\right)+v\left(k_{n}\right),
$$

where $M$ is the number of frequency components. We can use bandpass filters with different central frequencies to select and estimate each frequency component separately.

The signal-processing method for multiple frequency components with known phases is summarized as follows:

(a) If needed, manipulate the measured spectrogram to be equally spaced in wave numbers.

(b) Use a FIR bandpass filter to select a single frequency component and obtain a single-band analytical signal as described in Eq. (8).

(c) Obtain the unwrapped phase of the analytical signal.

(d) Obtain an estimation of $\hat{L}$ and $\hat{\phi}$ from Eq. (13).

(e) Obtain the phase difference between $\hat{\phi}$ and the known phase from Eq. (15).

(f) Obtain a compensated frequency estimation from Eqs. (17) and (18).

(g) Repeat steps (b)-(f) until all frequencies are estimated.

The experimental results of this method for multiplexed FP interferometers are demonstrated in Section 3 below.

\section{Experimental Results}

We use a simple setup to evaluate the signalprocessing algorithm for multiplexed FP interferometers. The setup of the experiment is shown schematically in Fig. 4.

We construct the sensor head by splicing a piece of hollow tube between single-mode fibers as described in Ref. 13. The interfaces between the silica fibers and the air in the hollow tube serve as partial reflection mirrors, which give reflections $R_{0}$ and $R_{1}$. The cleaved end face of the single-mode fiber serves as another mirror that gives reflection $R_{2}$. Each pair of these reflections will interfere and construct a FP interferometer. Thus the sensor head contains an EFPI air gap cavity formed by $R_{0}$ and $R_{1}$, an IFPI silica fiber cavity by $R_{1}$ and $R_{2}$, and an extra FP cavity by $R_{0}$ and $R_{2}$.

Light from a low-coherence light source is launched to the sensor head through a single-mode fiber and a $3 \mathrm{~dB}$ directional coupler. The LED has a central wavelength of $1.55 \mu \mathrm{m}$ and a FWHM bandwidth of $80 \mathrm{~nm}$. The light reflected from the sensor head is coupled to an OSA (Ando AQ-6315A) by the coupler, while we eliminate the reflection from the other arm by curling the fiber into several turns of small diameter. The OSA has a wavelength accuracy of $\pm 0.05 \mathrm{~nm}$.

The electric fields of these reflections coupled back to the lead-in fiber can be given as

$$
\begin{aligned}
E= & E_{1}+E_{2}+E_{3} \\
= & \eta_{0} R_{0} E_{0}+\eta_{1} R_{1} E_{0} \exp \left[j\left(k L_{1}+\phi_{1}\right)\right] \\
& +\eta_{2} R_{2} E_{0} \exp \left[j\left(k L_{1}+\phi_{1}\right)\right] \exp \left[j\left(k L_{2}+\phi_{2}\right)\right],
\end{aligned}
$$

where $\eta_{1}, \eta_{2}$, and $\eta_{3}$ are the coupling coefficients of $R_{1}$, $R_{2}$, and $R_{3}$ into the lead-in fiber and $L_{1}$ and $L_{2}$ are the OPDs of the air gap and the silica fiber FP cavities, respectively. All the multipath reflections are neglected owing to the low reflections $R_{0}, R_{1}$, and $R_{2}$.

The intensity of these reflections at wave number $k$ can be given as

$$
\begin{aligned}
I= & |E|^{2} \\
= & I_{0}\left[A+B \cos \left(k L_{1}+\phi_{1}\right)+C \cos \left(k L_{2}+\phi_{2}\right)\right. \\
& \left.+D \cos \left(k L_{3}+\phi_{3}\right)\right],
\end{aligned}
$$

where

$$
\begin{aligned}
& I_{0}=\left|E_{0}\right|^{2}, \\
& A=\eta_{0}^{2} R_{0}^{2}+\eta_{1}^{2} R_{1}^{2}+\eta_{2}^{2} R_{2}^{2}, \\
& B=2 \eta_{0} \eta_{1} R_{0} R_{1}, \\
& C=2 \eta_{1} \eta_{2} R_{1} R_{2}, \\
& D=2 \eta_{0} \eta_{2} R_{0} R_{2}, \\
& L_{3}=L_{1}+L_{2}, \\
& \phi_{3}=\phi_{1}+\phi_{2} .
\end{aligned}
$$

The measured spectrogram of the sensors can be expressed as Eq. (19) with three sinusoidal components corresponding to the three FP cavities. A measured spectrogram and its discrete Fourier transformation result are shown in Figs. 5(a) and 5 (b), respectively. The OPDs of the air gap FP cavity and the glass fiber FP cavity, $L_{1}$ and $L_{2}$, respectively, were selected to be 384 and $1315 \mu \mathrm{m}$. The frequencies of the sinusoids corresponding to $L_{1}$ and $L_{2}$ were located separately in the frequency domain. 

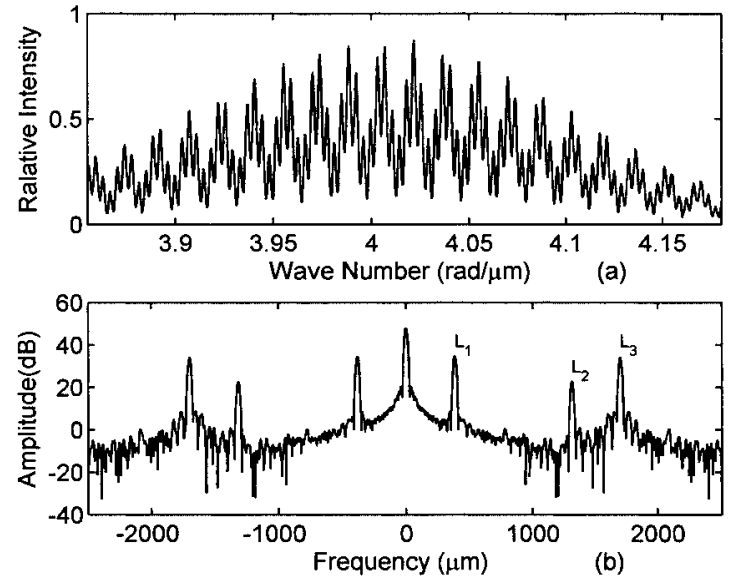

Fig. 5. (a) Spectrogram and (b) result of fast Fourier transformation of multiplexed FP sensors.

We investigated the temperature dependence of these two FP cavities to verify the performance of the signal-processing algorithm discussed in Section 2. We estimated $L_{1}$ and $L_{2}$ separately, following steps (b)-(f). Two Hanning-windowed FIR bandpass filters were used to select the frequency components corresponding to $L_{1}$ and $L_{2}$.

When the sensor head is put into a temperaturevarying environment, the OPDs of the FP interferometers will change owing to thermal expansions of the cavity lengths and thermo-optic effects of the media between the reflectors. The OPD change of a FP interferometer can be given as

$$
\Delta L=2\left(\Delta n_{e} d+n_{e} \Delta d\right)=L\left(\frac{\Delta n_{e}}{n_{e}}+\frac{\Delta d}{d}\right) .
$$

For the air gap FP cavity, one can assume that the refractive index of air is not temperature dependent. The OPD change of $L_{1}$ is due only to the thermal expansion of the silica hollow tube:

$$
\Delta L_{1}=L_{1} \frac{\Delta d}{d}=L_{1} \alpha_{T} \Delta T
$$

where $\alpha_{T}$ is the coefficient of thermal expansion of the silica hollow tube and $\Delta T$ is the temperature change. For silica, $\alpha_{T} \approx 0.5 \times 10^{-6}\left(1 /{ }^{\circ} \mathrm{C}\right)$.

For the silica fiber FP cavity, the OPD change is due to both the thermo-optic effect and the thermal expansion of the silica fiber. The refractive-index change of the fiber caused by a temperature change is

$$
\Delta n_{e}=\frac{\partial n_{e}}{\partial T} \Delta T
$$

where $\partial n_{e} / \partial T$ is a thermo-optic coefficient, which is $\sim 1.0 \times 10^{-5} /{ }^{\circ} \mathrm{C}$ for silica fiber. Thus the total OPD change $\Delta L_{2}$ can be written as
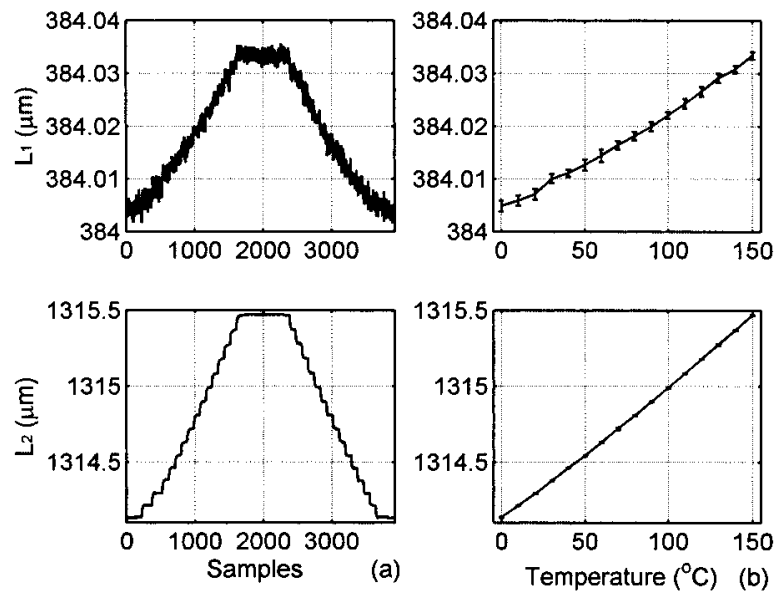

Fig. 6. Temperature responses of the multiplexed FP sensors: (a) OPDs during the temperature cycle, (b) OPDs versus temperature.

$$
\Delta L_{2}=L_{2}\left(\frac{\partial n_{e}}{\partial T} / n_{e}+\alpha_{T}\right) \Delta T=L_{2}\left(\sigma_{T}+\alpha_{T}\right) \Delta T
$$

where $\sigma_{T}$ and $\alpha_{T}$ are effective thermo-optic and thermal expansion coefficients of silica fiber, respectively. $\sigma_{T}$ is much larger than $\alpha_{T}$, which means that the thermo-optic effect is dominant for the temperature dependence of the OPD of the silica fiber cavity.

The sensor head was tested in a temperature chamber with a temperature range of $0^{\circ}-150{ }^{\circ} \mathrm{C}$ and temperature steps of $10{ }^{\circ} \mathrm{C}$. The time at each step during the increasing-decreasing cycle was $1 \mathrm{~h}$, except at $150{ }^{\circ} \mathrm{C}$, where it was $8 \mathrm{~h}$. The history curves and temperature dependences of $L_{1}$ and $L_{2}$ are shown in Figs. 6(a) and 6(b), respectively.

Figure 6(a) shows the measured OPDs of the two FP cavities during the temperature cycle. Figure 6(b) shows the temperature dependence of the OPDs. The standard deviations of the estimations of $L_{1}$ and $L_{2}$ at $150{ }^{\circ} \mathrm{C}$ are 0.8 and $3.0 \mathrm{~nm}$, respectively. The uncertainty of the OPD measurement, $\delta L / L$, is $\sim 2.2 \times 10^{-6}$ in both cases.

Experimental results show that the method can attain both absolute measurement of OPDs with high resolution and a large dynamic measurement range. For example, the estimation of the OPD of the silica fiber FP cavity has a $3.0 \mathrm{~nm}$ resolution in a dynamic range of $14 \mu \mathrm{m}$.

\section{Performance Analysis}

\section{A. Background Noise}

The background noises include $A I_{0}\left(k_{n}\right)$, which is a slowly varying process from the light source, and $v\left(k_{n}\right)$, which can be modeled as a Gaussian random process. $v\left(k_{n}\right)$ may include the detector's dark-current noise, the shot noise, and the thermal noise from the electric circuits.

The frequency estimation based on the phase linear regression in Eq. (13) is the optimal maximumlikelihood estimation for Eq. (11) when $\zeta_{n}$ is Gaussian 


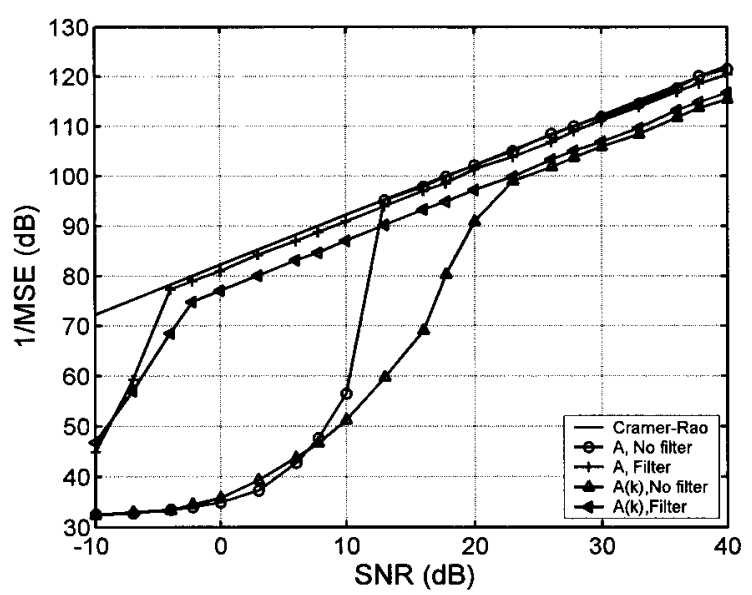

Fig. 7. Simulation results of frequency estimations for unknown phases.

white noise. ${ }^{16,17}$ When the SNR is moderately high, the estimator can attain the Cramer-Rao bound for a sinusoidal signal with constant amplitude, as described in Eq. (10). ${ }^{15,16}$ However, when the sinusoidal signal is amplitude modulated as described in Eq. (8), the average SNR is decreased; thus the estimator will have a larger estimation error for the AM signal than for that of pure sinusoids. It can also be expected that prefiltering with a bandpass filter will reject the noise outside the passband and increase the SNR greatly. Therefore prefiltering will greatly improve the performance of the estimator, especially for a low-SNR case.

The performance of the estimator [Eq. (13)] for an unknown phase was investigated for AM and pure sinusoidal signals at different SNR levels. The simulation results with and without prefiltering were compared. The simulation was based on a sequence of 1000 samples of a sinusoid whose normalized frequency is 0.024 . Figure 7 shows the relationship between the mean-square errors (MSEs) of the frequency estimation and SNR levels. It can be seen from Fig. 7 that estimator (13) can yield a good estimation for a high SNR but poor performance for a low SNR. Prefiltering will greatly improve performance at low SNR levels. The Cramer-Rao bound of the frequency estimation for Eq. (10) with an unknown phase ${ }^{15,17}$ is also plotted for comparison.

Frequency estimations for unknown phase and known phase, as described in Eqs. (13) and (18), are compared in Fig. 8. The simulation data were generated for a FP interferometer with an OPD of $360 \mu \mathrm{m}$ illuminated by a LED with a Gaussian shaped spectrum whose FWHM bandwidth is $80 \mathrm{~nm}$. It can be seen that the frequency-estimation error for a known phase can be much smaller than that of an unknown phase, which means that the frequency estimation in Eq. (18) can have higher precision than that of Eq. (13).

The frequency estimation for a known phase given by Eqs. (13), (17), and (18) is based on the assumption that the phase error caused by the frequency-estimation error is within $[-\pi, \pi]$. However, when the SNR is low, the frequency-

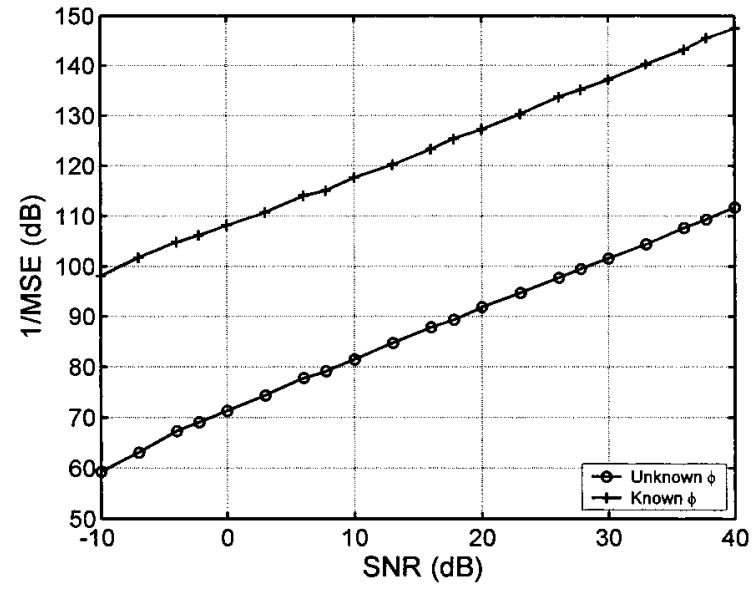

Fig. 8. Comparison of frequency-estimation errors for unknown and known phases.

estimation error given by Eq. (13) may be large; thus the phase error selected by Eq. (15) may have a $2 \pi$ ambiguity, which will lead to error jumping of the frequency estimation. This $2 \pi$ ambiguity is similar to that of the order-determination ambiguity problem in the fringe peak tracking-based signalprocessing approach. For example, to ensure a coarse estimation error $\Delta L=\hat{L}-L$ of less than $\lambda / 2$, from Eq. (5), for a wave-number step of $k_{s}=4.2$ $\times 10^{-4} \mathrm{rad} / \mu \mathrm{m}$, the frequency-estimation error should be less than $3.2 \times 10^{-4}$, which requires that $1 / \mathrm{MSE}$ in Fig. 7 be larger than $70 \mathrm{~dB}$. Thus it can be seen that the required SNR should be larger than $0 \mathrm{~dB}$ to yield a reasonable confidence interval to prevent a $2 \pi$ ambiguity problem.

\section{B. Amplitude Noise}

The frequency range of $I_{0}(k)$ is $S$, as Fig. 2 shows. We consider only the amplitude noise whose bandwidth is limited by $S$. In practice, the amplitude variation caused by the driving current and by the change in ambient temperature of the LED is usually of low frequency. The variation of the amplitude can thus be eliminated from Eq. (11) because only the phase of the analytical signal is obtained.

Figure 9 shows the experimental results of the EFPI cavity shown in Fig. 2 illuminated by (A) a LED of total power $50 \mu \mathrm{W}$, (B) the same LED, whose power of $60 \mu \mathrm{W}$ has been increased by an increase in driving current, and (C) a superluminescent LED of total power of $1 \mathrm{~mW}$. The source spectra of several illuminations are shown in Fig. 9(a); the OPD estimations are shown in Fig. 9(b). Although the source spectra are different, which means that the amplitudes of the analytical signal vary greatly, the OPD readings are consistent with a small variation of $2 \mathrm{~nm}$.

\section{Phase Noise}

The estimation [Eq. (13)] for the model in Eq. (11) is optimal when $\zeta_{n}$ is Gaussian white noise. However, 

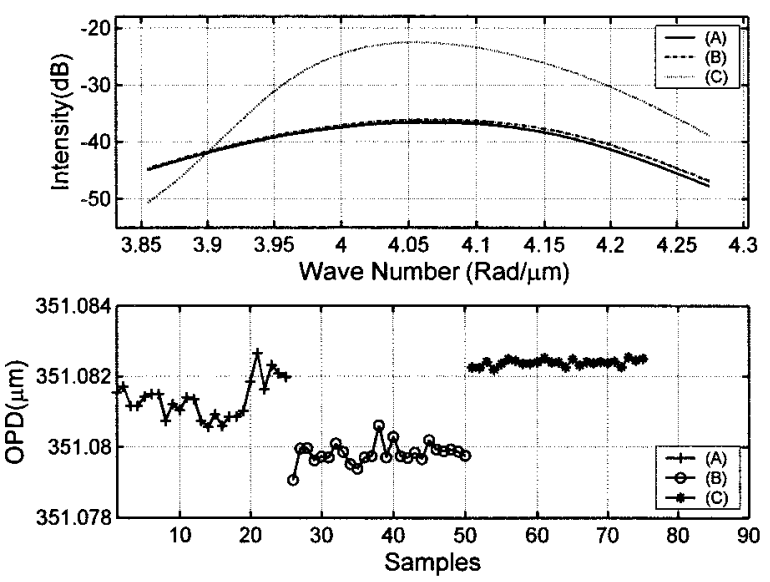

Fig. 9. OPD estimations under three illuminations: (a) source spectra, (b) OPD estimations.

phase noise $\zeta_{n}$ in a FP interferometer, which is wave number and geometric length dependent, is not really white. The dependency of phase noise can be given as

$$
\delta \phi=\delta k L+k \delta L=\delta k L+k \alpha L=(\delta k+k \alpha) L,
$$

where $\alpha$ is the ratio of change in the OPD. Wavenumber error $\delta k$ is caused by jittering of the light source and by the wavelength measurement uncertainty of the OSA. OPD error $\delta L$ is caused by the variation in the OPD during measurement of the spectrogram. Usually the wavelength uncertainty of an OSA is the main source of phase noise.

From Eq. (26) it is evident that phase noise $\delta \phi$ caused by $\delta k$ and $\delta L$ is proportional to $L$. Therefore the SNR level for large $L$ is reduced because of the larger phase noise. This is the reason why the frequency-estimation precision for a small $L$ is higher, as the experimental result in Section 3 shows. Another disadvantage of a large $L$ is that it lowers the interference contrast, which decreases the signal power and the SNR. There is also a higher probability of occurrence of $2 \pi$ ambiguity for large $L$ because a larger frequency-estimation error $\Delta L$ may occur. Therefore, if possible, one should select a shorter $L$ that satisfies the condition $L>2 S$, as discussed in Subsection 2.B, for both precision of estimation and avoidance of ambiguity.

\section{Two-Beam Approximation}

We use a two-beam approximation model [Eq. (3)] to describe a FP interferometer. Multipath reflections are neglected because they are low reflections. However, multipath reflections will give some harmonic frequency components to the measured spectrogram. The side peaks of the harmonics can be observed in Fig. 2.

Because a bandpass filter is applied to select the fundamental frequency components, the influence of these harmonics has been reduced to a minimum. If the frequency component of another interferometer coincides with the positions of these harmonics in a multiplexed sensor system, cross talk will occur. Thus, one should carefully design the OPDs of the sensors and locate the frequencies properly to prevent cross talk between interferometers. However, the power of the harmonics is rather low compared with the fundamental frequency component, $-28 \mathrm{~dB}$ for a $4 \%$ air-glass interface reflection, so the influence of cross talk can be reasonably neglected.

\section{Conclusions}

A frequency-estimation-based signal-processing algorithm for white-light optical fiber Fabry-Perot interferometers has been presented. The frequency estimation is based on linear regression of the instantaneous phase of the analytical signal, which can be obtained from the spectrogram of a FP interferometer. Experimental results for multiplexed FP interferometers show that the method can achieve both absolute optical path difference estimation with high resolution and a large dynamic range. We have analyzed the performance of the algorithm by considering various noises at different levels.

This research was supported in part by U.S. Department of Energy under grant DE-FC3601G011050 and U.S. National Science Foundation under grant CMS-0427951.

\section{References}

1. B. T. Meggit, "Fiber optic white light interferometric sensors," in Optical Fiber Sensor Technology, K. T. V. Grattan and B. T. Meggitt, eds. (Kluwer Academic, 2000), Vol. 4, 193-238.

2. S. Chen, A. W. Palmer, K. T. V. Grattan, and B. T. Meggitt, "Digital signal-processing techniques for electronically scanned optical-fiber white-light interferometry," Appl. Opt. 31, 6003-6010 (1992).

3. B. Qi, G. R. Pickrell, J. Xu, P. Zhang, Y. Duan, W. Peng, Z. Huang, W. Huo, H. Xiao, R. G. May, and A. Wang, "Novel data processing techniques for dispersive white light interferometer," Opt. Eng. 42, 3165 (2003).

4. R. Cortés, A. V. Khomenko, A. N. Starodumov, N. Arzate, and L. A. Zenteno, "Interferometric fiber-optic temperature sensor with spiral polarization couplers," Opt. Commun. 15, 268-272 (1998).

5. J. Tapia-Mercado, A. V. Khomenko, and A. Garcia-Weidner, "Precision and sensitivity optimization for white-light inteferometric fiber-optic sensors," J. Lightwave Technol. 19, 70-74 (2001).

6. J. L. Brooks, R. H. Wentworth, R. C. Youngquist, M. Tur, B. Y. Kim, and H. J. Shaw, "Coherence multiplexing of fiber-optic interferometric sensors," J. Lightwave Technol. 3, 1062-1072 (1985).

7. W. V. Sorin and D. M. Baney, "Multiplexed sensing using optical low-coherence reflectometry," IEEE Photon. Technol. Lett. 7, 917-919 (1995).

8. Y.-L. Lo, "Study of cross-talk of parallel Fabry Perot sensors in path-matching differential interferometry," Opt. Lasers Eng. 31, 401-410 (1999).

9. C. E. Lee and H. F. Taylor, "Interferometric optical fiber sensors using internal mirrors," Electron Lett. 24, 193-194 (1988).

10. C. E. Lee and H. F. Taylor, "In-line Fiber Fabry Perot interferometer with high reflectance internal mirrors," J. Lightwave Technol. 10, 1376-1379 (1992).

11. K. A. Murphy, M. F. Gunther, A. Wang, R. O. Claus, and A. M. Vengsarkar, "Extrinsic Fabry Perot optical fiber sensor," in 
Eighth Optical Fiber Sensors Conference (Institute of Electrical and Electronics Engineers, 1992), pp. 193-196.

12. A. Wang, H. Xiao, J. Wang, Z. Wang, W. Zhao, and R. G. May, "Self-calibrated interferometric-intensity-based optical fiber sensors," J. Lightwave Technol. 19, 1495-1501 (2001).

13. J. Sirkis, T. A. Berkoff, R. T. Jones, H. Singh, A. D. Kersey, E. J. Friebele, and M. A. Putnam, "In-line fiber etalon (ILFE) fiber-optic strain sensors," J. Lightwave Technol. 13, 12561268 (1995).

14. M. Han, Y. Zhang, F. Shen, G. R. Pickrell, and A. Wang, "Signal-processing algorithm for white-light optical fiber ex- trinsic Fabry-Perot interferometric sensors," Opt. Lett. 29, 1736-1378 (2004).

15. S. A. Tretter, "Estimating the frequency of a noisy sinusoid by linear regression," IEEE Trans. Inf. Theory IT-31, 832-835 (1985).

16. S. M. Kay, "A fast and accurate single frequency estimator," IEEE Trans. Acoust. Speech Signal Process. 37, 1987-1990 (1989).

17. D. C. Rife and R. R. Boorstyn, "Single-tone parameter estimation from discrete-time observations," IEEE Trans. Inf. Theory IT-20, 591-598 (1974). 


\title{
Miniature all-silica fiber optic pressure and acoustic sensors
}

\author{
Juncheng Xu, Xingwei Wang, Kristie L. Cooper, and Anbo Wang \\ Center for Photonics Technology, Bradley Department of Electrical and Computer Engineering, Virginia Polytechnic \\ Institute and State University, Blacksburg, Virginia 24061
}

\begin{abstract}
Received August 23, 2005; revised manuscript received August 23, 2005; accepted August 23, 2005
We present a miniature diaphragm-based Fabry-Perot (F-P) interferometric fiber optic sensor fabricated by novel techniques for pressure or acoustic wave measurement that is only $\sim 0.32 \mathrm{~mm}$ in diameter. By choosing different diaphragm thicknesses and effective diameters, we obtain a sensor measurement range from 5 to $10,000 \mathrm{psi}$ ( $1 \mathrm{psi}=51.72$ Torr) and a frequency response up to $2 \mathrm{MHz}$. In addition, the sensor's F-P cavity can be set from micrometers to millimeters with a precision of several nanometers. With the all-silica structure, the sensor is reliable, biocompatible, and immune to electromagnetic interference and has hightemperature sensing capability. (C) 2005 Optical Society of America

OCIS codes: $060.2370,120.2230,120.3180$.
\end{abstract}

Diaphragm-based Fabry-Perot (F-P) interferometric fiber optic sensors are widely used in pressure and acoustic wave measurements because of their high sensitivity and high-frequency response. A variety of diaphragm-based F-P interferometric sensors have been developed with diameters ranging from a few millimeters (ferrule-based) $)^{1,2}$ to only $125 \mu \mathrm{m}$ (fibertip based $)^{3-6}$ in recent years. The smaller ones attract more attention in applications for which restricted space is a consideration, but they are more difficult to fabricate and handle. Moreover, compared with larger ones, they have some unavoidable limitations. First, as the effective diameter of the diaphragm, limited by the fiber itself, is small, the diaphragm must be thin if it is to have considerable sensitivity according to the diaphragm deflection $\operatorname{model}^{7}$ [see Eq. (1) below], which presents a great challenge in sensor fabrication. Second, the F-P cavity (air gap) length cannot easily be adjusted during fabrication. Third, these sensors do not have temperature selfcompensation capability. Although some microelectromechanical system pressure sensors ${ }^{8-10}$ with diameters of a few hundred micrometers may overcome some of the drawbacks just mentioned, they will encounter high stress because of mismatches in coefficients of thermal expansion (CTEs) among the elements made from different materials, which may lead to a failure.

In this Letter we describe the novel fabrication of a diaphragm-based F-P interferometric pressure sensor, which offers high sensitivity in combination with miniature size, greatly relaxed restrictions on diaphragm thickness, precise F-P cavity length control, and self-temperature compensation capability. The sensor size can be chosen from 200 to $500 \mu \mathrm{m}$ to meet application requirements. In addition, the sensor's all-silica structure possesses many advantages, including excellent reliability (without CTE mismatches), high-temperature sensing capability, corrosion resistance, immunity to electromagnetic interference, and biocompatibility, making it suitable for both industrial and biomedical applications. The sensor structure is shown in Fig. 1. The length of the F-P cavity (air gap) will decrease with deflection of the diaphragm as a result of the applied pressure. Light is injected into the optical fiber and partially reflected (4\%) by the end face of the fiber and the inside surface of the diaphragm. Then the two reflections propagate back through the same fiber and generate interference fringes, which are demodulated to determine the air gap's thickness.

Compared with the sophisticated processes involved in making a conventional microelectromechanical system sensor, this sensor's fabrication is simple in that only cleaving, splicing, and etching are required. A piece of silica glass capillary tubing with an I.D. of $158 \mu \mathrm{m}$ and an O.D. of $323 \mu \mathrm{m}$ was cleaved to produce a flat end face. A cleaved silica optical fiber with a $330 \mu \mathrm{m}$ O.D. was spliced to the end of the tubing by a fiber fusion splicer (Sumitomo, Type-36) and was cleaved to retain a thin layer approximately 10-30 $\mu \mathrm{m}$ thick. A cleaved lead-in single-mode optical fiber (SMF-28) was inserted into the tubing, and a $\mathrm{CO}_{2}$ laser bonding technique was used to make a fusion bond. The lead-in fiber can also be a multimode fiber, depending on application requirements. Because the dimensional changes in the fiber and the tubing are slightly different during laser fusion, the sensor's air gap can be precisely controlled, with a precision of about $3 \mathrm{~nm} .{ }^{11}$ This property can provide excellent flexibility in sensor design, fabrication, and signal demodulation. For example, in a selfcalibrated interferometric-intensity-based sensor system $^{12}$ the air gap must be set in the linear range of a half-fringe. In a white-light interferometry

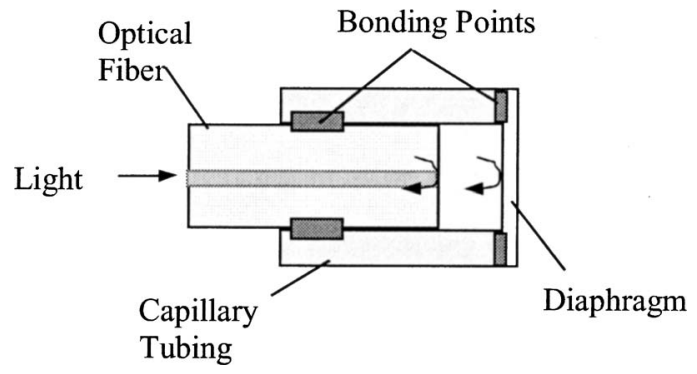

Fig. 1. Miniature diaphragm-based fiber optic pressure sensor. 


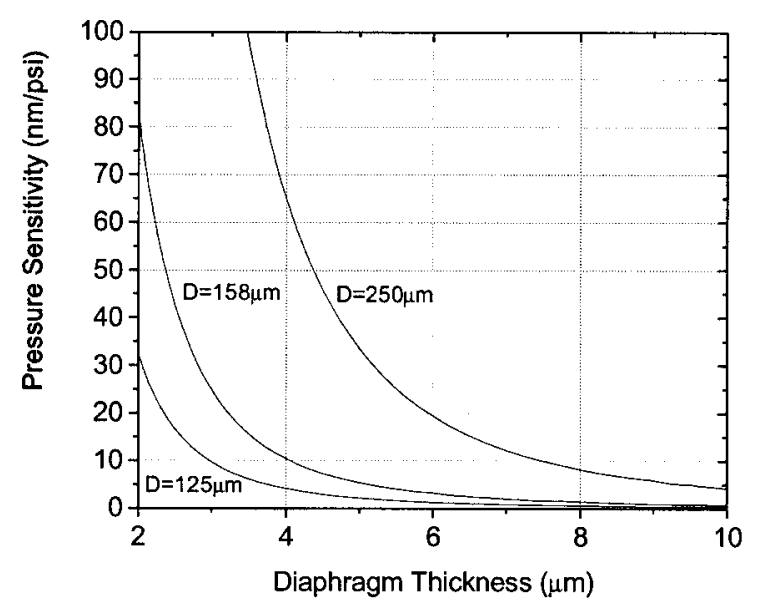

Fig. 2. Theoretical pressure sensitivities of the sensor for three diaphragm diameters $D$.

system $^{13}$ the air gap should be set such that the sensor can work efficiently in a range that has good interference fringes. As it is hard to get a cleaved diaphragm thinner than $10 \mu \mathrm{m}$, hydrofluoric acid (HF) etching was used to reduce the diaphragm's thickness to a few micrometers. Because the two surfaces of the diaphragm are not parallel after cleaving and also because HF etching will make the outside surface rough, the reflection from the outside surface can be neglected. Usually we define the ratio between the deflection and the pressure difference as the diaphragm's pressure sensitivity $(Y)$. Because only the diaphragm's center deflection is of interest, sensitivity $Y_{c}$ (for fused-silica material at $25^{\circ} \mathrm{C}$ ) is given by

$$
Y_{c}=\frac{3\left(1-\mu^{2}\right)}{16 E h^{3}}=1.71 \times 10^{-5} \frac{r^{4}}{h^{3}}[\mathrm{~nm} / \mathrm{psi}],
$$

where $r$ and $h$ are the radius and the thickness of the diaphragm, respectively, in micrometers, $E$ is Young's modulus, and $\mu$ is the Poisson ratio. Using Eq. (1), we derived the analytical prediction of the sensor's pressure sensitivity, which is shown in Fig. 2; the sensor's experimental static pressure response is shown in Fig. 3. The measured sensor pressure sensitivity is $3.98 \mathrm{~nm} / \mathrm{psi}$. Based on the diaphragm's initial thickness and the $\mathrm{HF}$ etching rate and time, the diaphragm's final thickness is approximately 5.5-6 $\mu \mathrm{m}$, which is consistent with the theoretical calculation. In the experiment we used a component test system (MicronOptics, Inc.) and a spectrum analysis algorithm $^{13}$ to calculate F-P cavity length $L$ :

$$
L=\frac{\lambda_{1} \lambda_{2}}{2\left(\lambda_{2}-\lambda_{1}\right)}
$$

where $\lambda_{1}$ and $\lambda_{2}$ are the wavelengths of two adjacent valley points in the spectrum. In addition, the onevalley tracking method ${ }^{14}$ improves the measurement resolution to be $\sim 1$ part in $10^{6}$ of the initial air gap length, or $\sim 0.02 \mathrm{~nm} .{ }^{15}$ Therefore the pressure measurement resolution is $\sim 0.005 \mathrm{psi}(34.5 \mathrm{~Pa}$ or 0.26 Torr). Additionally, one can exponentially increase the sensor's pressure sensitivity by etching the diaphragm to 2-4 $\mu \mathrm{m}$ thickness (Fig. 2).

Because the diaphragm has the capability of detecting dynamic pressure, the sensor is also suitable for acoustic applications such as medical imaging and diagnosis. Based on the properties of fused silica, the lowest natural frequency of the diaphragm can be theoretically calculated as follows, and the curves are shown in Fig. 4:

$$
f_{00}=\frac{\alpha_{00}}{4 \pi}\left[\frac{E}{3 w\left(1-\mu^{2}\right)}\right]^{1 / 2}\left(\frac{h}{r^{2}}\right)=2.742 \times 10^{9} \frac{h}{r^{2}}[\mathrm{~Hz}],
$$

where $\alpha_{00}$ is a constant related to the vibrating modes, which is 10.21 for the lowest natural frequency, $w$ is the mass density of the diaphragm, and $r, h, E$, and $\mu$ are the same as the parameters in Eq. (1)

To respond faithfully to dynamic pressures, the sensor's natural resonant frequency should be at least three to five times higher than the highest applied frequency. This sensor, which has a natural frequency of $\sim 2.5 \mathrm{MHz}$ according to the calculation, should thus be capable of detecting acoustic waves of $500-800 \mathrm{kHz}$. For specific applications the sensor can

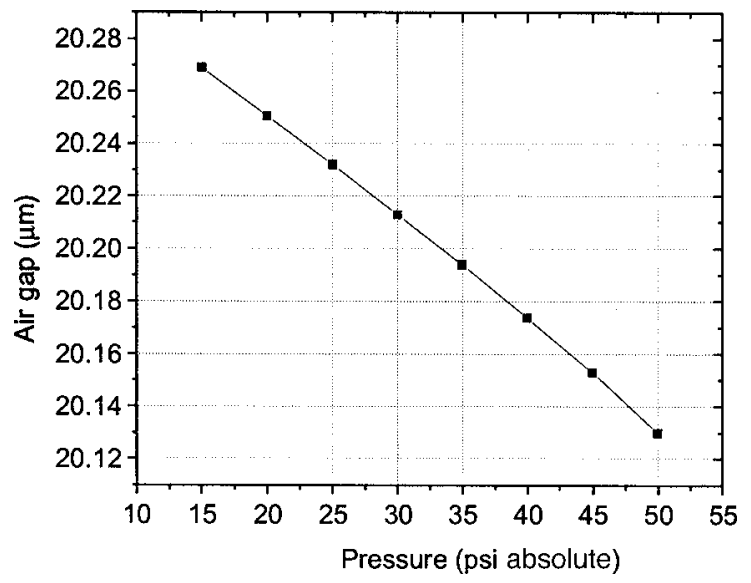

Fig. 3. Sensor's static pressure response (15-50 psi) at room temperature.

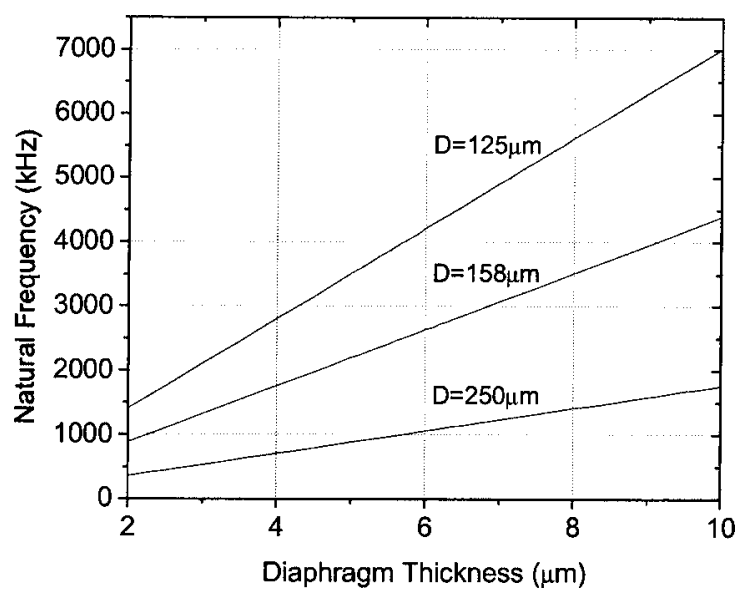

Fig. 4. Theoretical natural frequency of the sensor for three diaphragm diameters $D$. 


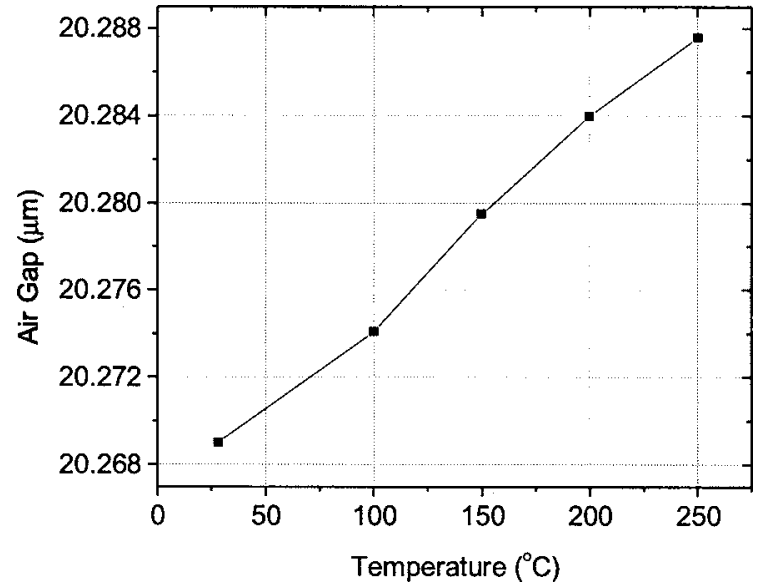

Fig. 5. Dependence of sensor on temperature at room pressure.

also work near its resonant frequency as a narrowband high-sensitivity acoustic sensor.

The sensor was connected to the self-calibrated interferometric-intensity-based system in an acoustic experiment. The acoustic source generated a 46.9 $\mathrm{kHz}$ acoustic wave with $\sim 0.1$ psi dynamic pressure. The sensor output signal showed a frequency of 46.9 $\mathrm{kHz}$, which was consistent with the acoustic source, and the signal-to-noise ratio of the signal was $\sim 19 \mathrm{~dB}$. For applications for which the acoustic frequency is roughly known, a narrowband filter and amplifier can be used to improve the system's performance considerably.

In addition, this sensor configuration has a passive temperature self-compensation capability because the optical fiber normally has a higher CTE than that of the fused silica tubing owing to the germanium doping inside the fiber, whose expansion in the cavity will compensate for that of the tubing when the temperature is increased. As shown in Fig. 5, the sensor's temperature dependence is $\sim 0.083 \mathrm{~nm} /{ }^{\circ} \mathrm{C}$, which will lead to a pressure measurement error of $\sim 0.02 \mathrm{psi} /{ }^{\circ} \mathrm{C}$. This temperature dependence was caused partly by the changes in inner pressure of the trapped air. Because multimode fiber (Corning 50/125) has a higher CTE than the SMF-28 single-mode fiber, by using a multimode lead-in fiber or splicing a short section of multimode fiber to the lead-in single-mode fiber, ${ }^{2}$ one can compensate for most of this sensor's temperature dependence. Because the cladding of the fiber is made from pure fused silica, the bonding between the cladding and the tubing will not introduce thermal expansion stress.
We have described a novel diaphragm-based miniature fiber optic pressure and acoustic sensor that preserves the advantages of ferrule-based sensors while offering miniature size, which could be attractive in applications in which the operating space is restricted, such as in biomedical uses. The precisely controlled F-P cavity length permits flexibility in choosing signal demodulation schemes. In addition, the all-silica sensor structure's capability for temperature self-compensation can operate in a wide temperature range, which is ideal for applications in harsh environments.

The authors thank Micron Optics for the partial donation of the component test system used in this research. We also thank the reviewers and editors for comments that improved this Letter. J. Xu's e-mail address is juxu1@vt.edu.

\section{References}

1. B. Yu, D. W. Kim, J. Deng, H. Xiao, and A. Wang, Appl. Opt. 42, 3241 (2003).

2. J. Xu, G. Pickrell, X. Wang, W. Peng, K. L Cooper, and A. Wang, IEEE Photon. Technol. Lett. 17, 870 (2005).

3. E. Cibula, D. Donlagic, and C. Stropnik, in Sensors; 2002 Proceedings (Institute of Electrical and Electronics Engineers, 2002), Vol. 1, p. 711.

4. K. Totsu, Y. Haga, and M. Esashi, in Transducers, Solid-State Sensors, Actuators and Microsystems, (2003), Vol. 1, p. 931.

5. Y. Zhu and A. Wang, IEEE Photon. Technol. Lett. 17, 447 (2005).

6. X. Wang, J. Xu, Y. Zhu, K. L. Cooper, and A. Wang, "All-fused-silica miniature optical fiber tip pressure sensor," Opt. Lett. (to be published).

7. M. D. Giovanni, Flat and Corrugated Diaphragm Design Handbook (Marcel Dekker, 1982).

8. D. C. Abeysinghe, S. Dasgupta, J. T. Boyd, and H. E. Jackson, IEEE Photon. Technol. Lett. 13, 993 (2001).

9. M. J. Gander, W. N. MacPherson, J. S. Barton, R. L. Reuben, J. D. C. Jones, R. Stevens, K. S. Chana, S. J. Anderson, and T. V. Jones, IEEE Sens. J. 3, 102 (2003).

10. J. Zhou, S. Dasgupta, H. Kobayashi, J. M. Wolff, H. E. Jackson, and J. T. Boyd, Opt. Eng. 40, 598 (2001).

11. J. Xu, G. R. Pickrell, K. L Cooper, P. Zhang, and A. Wang, presented at Conference on Lasers and ElectroOptics (CLEO 05), (Optical Society of America, 2005), paper CThL5.

12. A. Wang, H. Xiao, J. Wang, Z. Wang, W. Zhao, and R. G. May, J. Lightwave Technol. 19, 1495 (2001).

13. J. Xu, G. R. Pickrell, Z. Huang, B. Qi, P. Zhang, Y. Duan, and A. Wang, AIP Conf. Proc. 684, 1021 (2003).

14. B. Qi, G. R. Pickrell, J. Xu, P. Zhang, Y. Duan, W. Peng, Z. Huang, W. Huo, H. Xiao, R. G. May, and A. Wang, Opt. Eng. 42, 3165 (2003).

15. F. Shen, W. Peng, K. Cooper, G. Pickrell, and A. Wang, in Proc. SPIE 5590, 47 (2004). 


\section{High-temperature fiber optic cubic-zirconia pressure sensor}

\author{
Wei Peng, MEMBER SPIE* \\ Gary R. Pickrell, MEMBER SPIE \\ Anbo Wang, MEMBER SPIE \\ Virginia Polytechnic Institute and \\ State University \\ Center for Photonics Technology \\ Department of Electrical and \\ Computer Engineering \\ Blacksburg, Virginia 24061
}

\begin{abstract}
There is a critical need for pressure sensors that can operate reliably at high temperatures in many industrial segments such as in the combustion section of gas turbine engines for both transportation and power generation, coal gasifiers, coal fired boilers, etc. Optical-based sensors are particularly attractive for the measurement of a wide variety of physical and chemical parameters in high-temperature and highpressure industrial environments due to their small size and immunity to electromagnetic interference. A fiber optic pressure sensor utilizing single-crystal cubic zirconia as the sensing element is reported. The pressure response of this sensor has been measured at temperatures up to $1000^{\circ} \mathrm{C}$. Additional experimental results show that cubic zirconia could be used for pressure sensing at temperatures over $1000^{\circ} \mathrm{C}$. This study demonstrates the feasibility of using a novel cubic-zirconia sensor for pressure measurement at high temperatures. (c) 2005 Society of PhotoOptical Instrumentation Engineers. [DOI: 10.1117/1.2138013]
\end{abstract}

Subject terms: Fiber optics; sensors; pressure; high temperature; cubic zirconia.

Paper 040495RR received Jul. 22, 2004; revised manuscript received May 16, 2005; accepted for publication May 18, 2005; published online Dec. 8, 2005.

\section{Introduction}

Pressure measurement at high temperature is vitally important in many industrial segments, such as in the combustion sections of gas turbine engines for both transportation and power generation, in the primary and secondary stages of coal gasification processes, in coal fired boilers, to name only a few. In the past several decades, fiber optic sensor systems have shown distinct advantages in measuring or monitoring a wide variety of physical and chemical parameters due to numerous inherent properties. ${ }^{1}$ Conventional fused silica glass fiber optic sensors have shown excellent performance in pressure measurement applications. Qiao et al. ${ }^{2}$ and Zhao et al. ${ }^{3}$ demonstrated an optical fiber Bragg grating sensor and made simultaneous measurements of pressure and temperature in the oil and gas down hole environment; Abeysinghe et al. ${ }^{4}$ proposed a novel MEMS fiber optic sensor for pressure and temperature measurement. However, all these silica-based fiber optic sensors are strictly limited to low-temperature applications because the optical fiber experiences a significant degradation in physical and optical properties when the operational temperature exceeds $800{ }^{\circ} \mathrm{C}$. This is due to the onset of crystallization, which will affect the optical loss and mechanical properties; the diffusion of dopants from the central core or from the cladding regions, which will affect the modal properties and optical loss; and reaction of the silica fibers with various agents in the environment (including water), which will increase the optical attenuation, reduce the softening point,

\footnotetext{
*Current address: Arizona Applied NanoSensors, Department of Chemistry \& Biochemistry, Arizona State University, P.O. Box 871604, Tempe, AZ 85287. Tel: (480) 323-9386; Fax: (480) 965-2747; E-mail: wei.peng@asu.edu.

0091-3286/2005/\$22.00 @ 2005 SPIE
}

and reduce the mechanical strength. Optical crystals with high melting temperatures have been used for hightemperature measurement for many years. Dils of the National Bureau of Standards demonstrated a sapphire fiberbased system that used the blackbody radiation to determine the temperature. ${ }^{5}$ Wang et al. developed a hightemperature sapphire optical-fiber-based intrinsic FabryPerot interferometer. ${ }^{6,7}$ Tong et al. ${ }^{8,9}$ demonstrated a radiation-based zirconia single-crystal fiber optic sensor to measure temperature above $2000{ }^{\circ} \mathrm{C}$. But very little data has been reported for optical pressure measurement with high-temperature-capability crystals. Recently, Pulliam et al. ${ }^{90}$ developed a high-temperature fiber optic MEMS pressure sensor that combines a $\mathrm{SiC}$ diaphragm, a sapphire waveguide, and an optical fiber. But this sensor was only tested up to $600{ }^{\circ} \mathrm{C}$ and 210 psi.

In this work, a cubic-zirconia-based fiber optic pressure sensor has been investigated for use in harsh industrial environments, characterized by high temperature, high pressure, and/or corrosive agents. This paper reports on a novel single-crystal cubic-zirconia-based optical pressure sensor design and the results of preliminary laboratory testing. These results indicate that the single-crystal cubic-zirconia fiber optic sensor can be used as a pressure sensor at high temperature.

\section{Principle of Sensor Design}

The basic concept of this design is based on the photoelastic characteristics of the single-crystal cubic zirconia. Compared with single-crystal sapphire, which has been demonstrated for high-temperature measurement by several authors, cubic zirconia has a higher melting temperature, around $2500{ }^{\circ} \mathrm{C}$, depending upon the type of stabilizing agent used (generally calcia, yttria, or magnesia). Cubic zirconia is optically isotropic and exhibits no reported bi- 


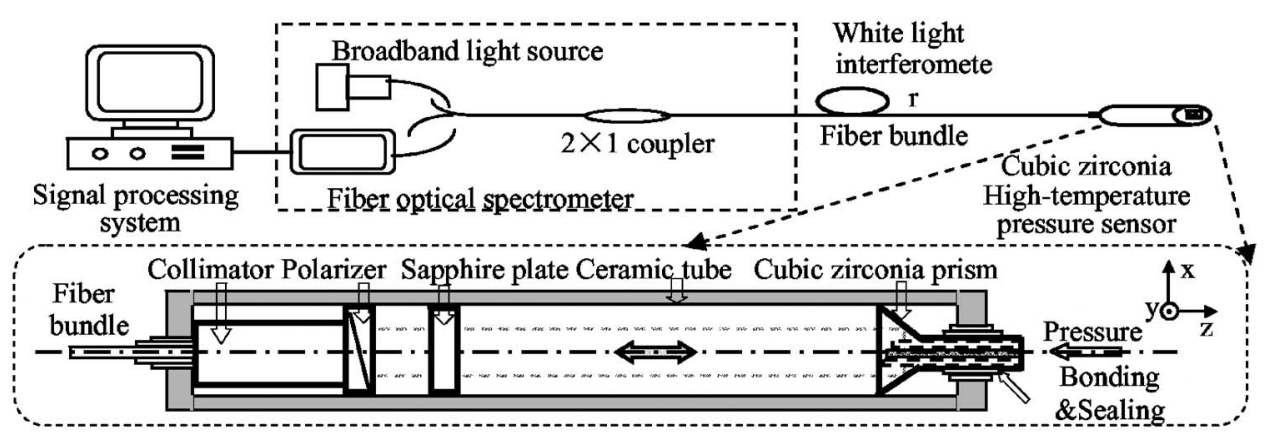

Fig. 1 Schematic of the cubic zirconia fiber optic pressure sensor system.

refringence as a function of temperature. Although singlecrystal cubic zirconia is optically isotropic when unstrained, when strained anisotropically, the optical characteraristics become anisotropic, ${ }^{11}$ which can be used to monitor pressure by detecting the related birefringence and thickness changes caused by the applied pressure.

The operating principle of this cubic-zirconia fiber optic pressure sensor is illustrated in Fig. 1. This sensor system is composed of a sensor probe, a white-light spectrometer, and a $2 \times 1$ multimode fiber bundle linking them together. A white-light interferometer was used due to its high resolution and stability. ${ }^{12}$ The broadband light from a lightemitting diode was launched into a multimode fiber bundle connected with a $2 \times 1$ coupler, through a collimator, a polarizor, and a sapphire plate, and then propagated to the sensor head. The reflected signal from the sensor head propagated along the same path back to the fiber bundle, and was detected by the spectrometer. The collimator was used to generate parallel light. The Nicole polarized prism functioned both as a polarizer and as an analyzer. The sapphire plate was used to add a birefringence signal for easy detection and analysis. The detected spectra were analyzed by a multimode white-light interferometer that demodulates the pressure from the detected light signal fringes.

As shown in Fig. 2, the pressure sensor head was designed as an optical crystal prism with two 45-deg reflection surfaces, which also functioned as the reflector for the optical signal detection. Two nonthrough parallel boreholes were drilled into this prism to allow the outside pressure into the prism. The light signal passed through the middle region of the two nonthrough bore holes twice. Both the

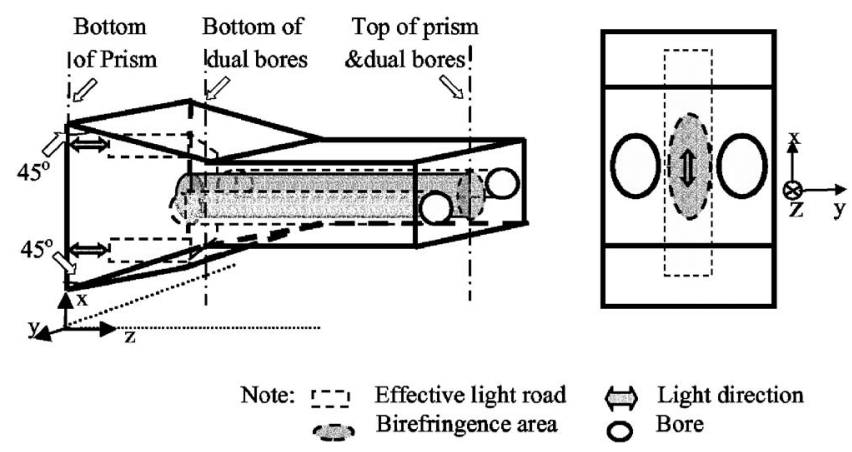

Fig. 2 Illustration of cubic zirconia sensor design: (a) 3-D structure and (b) top view. birefringence and thickness changed with the applied pressure. Therefore, the pressure can be measured by analyzing the detected light signals.

The reflected interference spectrum signal from the cubic-zirconia sensor head can be expressed as:

$I(\lambda)=2 K I_{o}(\lambda)\left\{1+\cos \left[\frac{4 \pi d(P) \Delta n(P)}{\lambda}\right]\right\}$,

where $I_{o}(\lambda)$ is the spectral power distribution as a function of the wavelength $\lambda$ of the broadband input light source, $K$ is a parameter representing the power loss of the optical system and can be treated as a constant, $d$ is the effective thickness of the cubic zirconia between the two bores in the block, and $\Delta n=n_{e}-n_{o}$ is the birefringence between the extraordinary ray and the ordinary ray determined by the crystallographic orientation of the atomic structure of the cubic zirconia. Both $d$ and $\Delta n$ are functions of the applied pressure $P$. Ideally, the interference fringes would form a perfect sinusoidal curve if plotted in wave number after it is normalized with respect to $I_{o}(\lambda)$ according to Eq. (1):

$$
\begin{aligned}
I(\lambda) & =2 K\left\{1+\cos \left[\frac{4 \pi d(P) \Delta n(P)}{\lambda}\right]\right\} \\
& =2 K\left\{1+\cos \left[\frac{4 \pi F(P)}{\lambda}\right]\right\}
\end{aligned}
$$

where $F(P)=d(P) \Delta n(P)$.

An algorithm was developed to measure the difference of optical path $F(P)$ between the two orthogonal linearly polarized light beams in the cubic zirconia block, which is uniquely related to the differential phase delay between these two orthogonal polarized light beams. From Eq. (2), the normalized reflected interferometric spectrum consists of a series of fringes, and assuming the wavelengths of two consecutive peaks or valleys as $\lambda_{m}$ and $\lambda_{m+1}$, the $F(P)$ can be expressed as:

$F(P)=\frac{\lambda_{m} \cdot \lambda_{m+1}}{2\left(\lambda_{m+1}-\lambda_{m}\right)}$.

The $F(P)$ values will be referred to as the equivalent gap distance to represent the optical path difference between the two orthogonal linearly polarized light beams, which contains the pressure information and can be calibrated with the applied standard pressures. Therefore, the single-crystal 


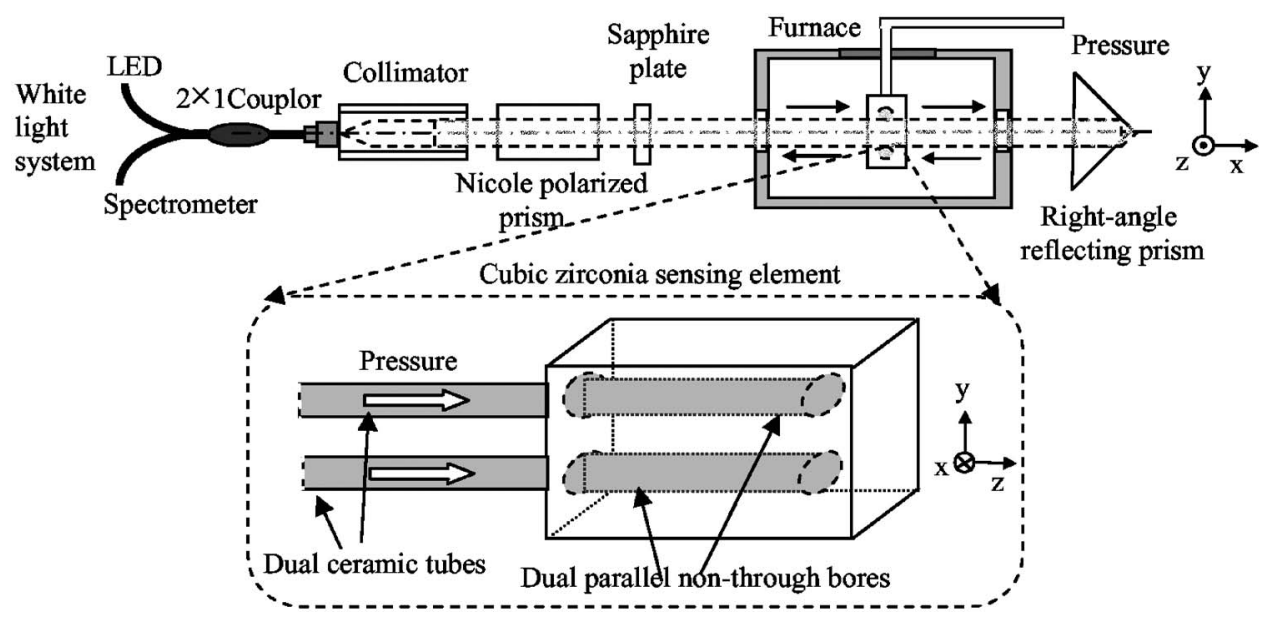

Fig. 3 Schematic of the dual-bore-based zirconia sensor modified to incorporate an external zirconia reflecting prism.

cubic zirconia, which is initially optically isotropic, has a pressure-induced birefringence due to the anisotropically applied pressure. This birefringence causes a differential phase delay in the two orthogonal polarizations propogating within the material. This differential phase delay is directly related to the magnitude of the applied pressure and, therefore, can be used to demodulate the pressure applied.

In addition to the common advantages of fiber optic sensors, this optical-crystal-based fiber optic pressure sensor also combines many other potential features together, such as suitability for high-temperature use, compact structure, simplicity of application, and potential for low-cost fabrication. Based on these factors, this system was investigated for pressure measurement in harsh industrial environments at high temperature.

\section{Experimental Procedure and Results}

As described above and shown in Fig. 2, fabrication of this zirconia sensor head, which has two 45-deg polished surfaces that act as the light reflectors, was limited by the current laboratory-scale optical material processing capability. But to demonstrate the function of this dual-bore pressure sensor, a simpler dual-bore sensor structure was fabricated that could work with a separate right-angle cubic-zirconia reflecting prism. Therefore, the only difference between the two structures was in the separation of the reflecting surfaces into an external prism instead of being an integral part of the dual-bore zirconia block. Preliminary experiments were performed by applying hydraulic pressure to this sensor block to demonstrate the pressure sensitivity of the designed structure. Additional experiments were performed using a mechanically induced pressure on the cubic-zirconia block to demonstrate pressure measurement over $1000{ }^{\circ} \mathrm{C}$. For both experiments, a multimode fiber optic white-light spectrometer was used as the optoelectronics signal detection system. A $2 \times 1$ multimode fiber bundle and a collimator were both from Multimode Fiber Optics, Inc. Also a 1/16-in.-thickness, 1-in.-diameter sapphire plate was used as a birefringence background in the test.

\subsection{Hydraulic Pressure Measurement on a Cubic- Zirconia Sensor Head}

The hydraulic pressure testing apparatus using the substitute dual-bore-based cubic-zirconia sensor is illustrated in Fig. 3. Figure 4 is the image of this dual-bore cubiczirconia block. Two 1/4-in. parallel nonthrough bores were drilled on a 1.32- $\times 0.71-\times 1.25$-in. cubic-zirconia block. The two surfaces of the block were finely polished to reduce scattering of the light signals. The bore distance was $3 / 8$ in.. The middle region between the two bores was the optically interrogated region for pressure sensing. As shown in Fig. 4, these two nonthrough holes were connected with two 1/4-in. o.d. $\sim 1 / 2$-in. id ceramic tubes and sealed by high-pressure Epoxy 907. The two ceramic tubes were connected to the stainless steel tubes that were connected with an APCS-30K-1 pressure system to supply the controllable hydraulic pressure, which is a computercontrolled high-performance pressure generator/controller manufactured by Advanced Pressure Products, Inc., and can

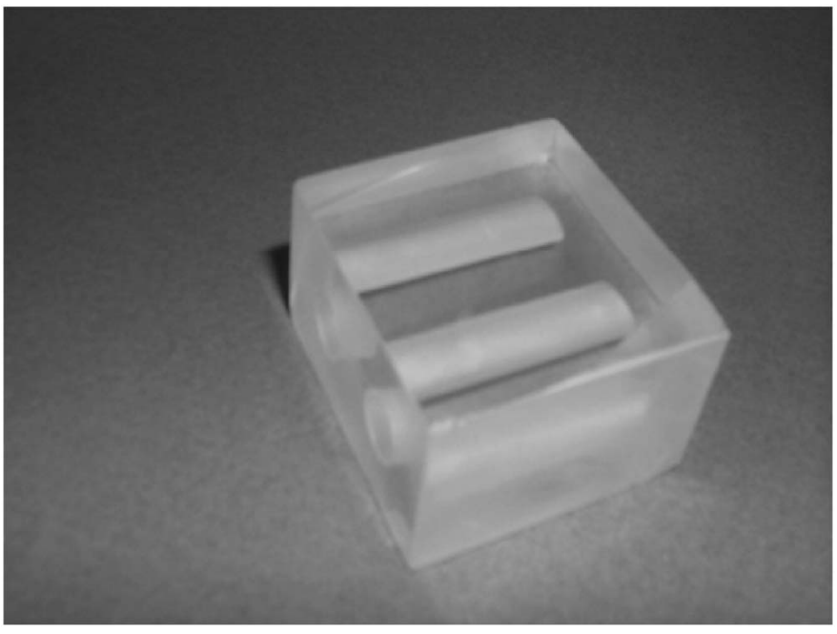

Fig. 4 Single-crystal cubic zirconia block used for hydraulic pressure measurement. 


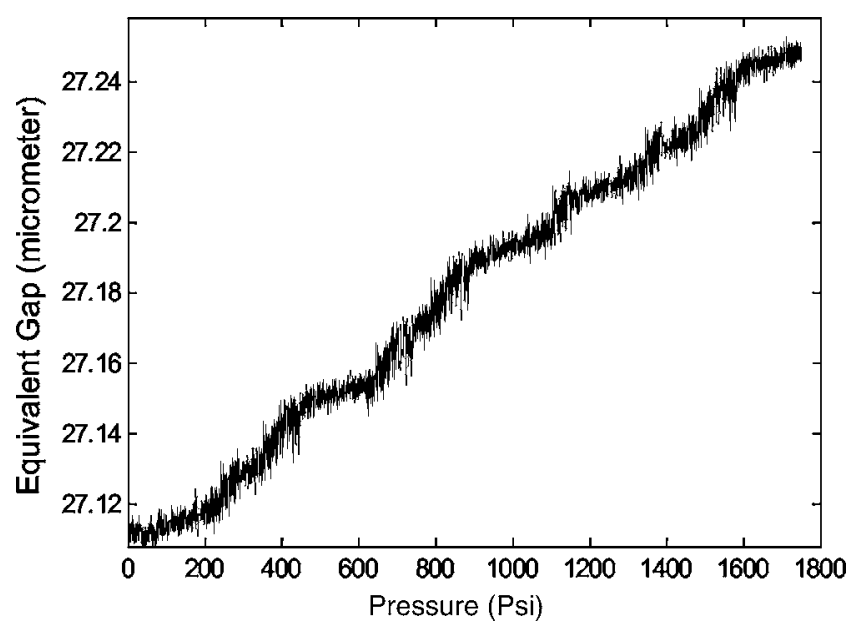

Fig. 5 Equivalent gap versus the applied hydraulic pressure.

supply a hydrostatic pressure up to 20,000 psi. The accuracy of the pressure output is $0.1 \%$ of the full scale.

The light path was adjusted carefully to ensure it could propagate through the middle region of the two bores in the zirconia block to produce stronger birefringence information caused by the applied hydraulic pressure. As shown in Fig. 5, the maximum equivalent gap change, which represents the largest optical path difference between the two orthogonal linearly polarized light beams generated by the largest pressure that the sensor is designed for, was around $130 \mathrm{~nm}$ for a pressure range from $0 \sim 1750$ psi. Figure 6 shows the pressure measurement result at room temperature $\left(23{ }^{\circ} \mathrm{C}\right)$. As shown in Fig. 4, to illustrate the temperature effects of this structure, this zirconia block was placed into a controllable furnace from Thermolyne Inc. with holes machined into the walls to let the light signal pass through. The temperature was maintained at $23^{\circ} \mathrm{C}, 55^{\circ} \mathrm{C}$, and $80{ }^{\circ} \mathrm{C}$, and the zirconia sensor was tested up to $800 \mathrm{psi}$ separately; these results are shown in Fig. 7. The deviations among these tests are around 100 psi. As illustrated in Fig. 8 , the stability of this cubic-zirconia-based pressure sensor

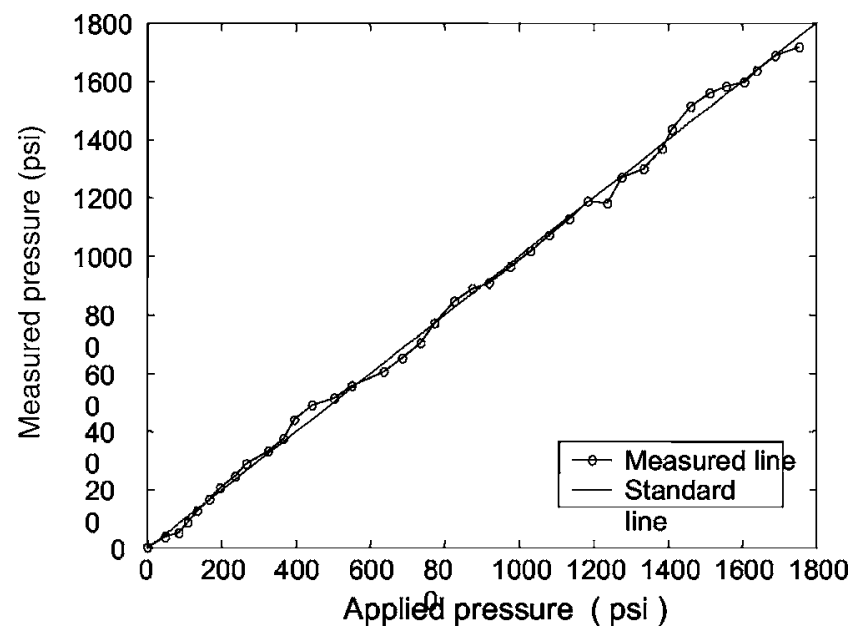

Fig. 6 Measured versus applied pressure at room temperature $\left(23^{\circ} \mathrm{C}\right)$.

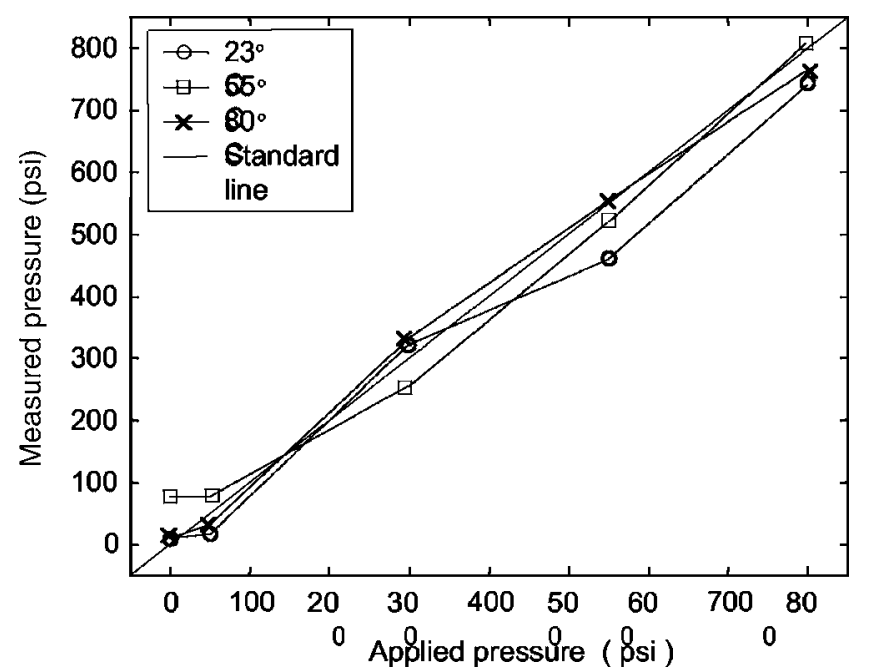

Fig. 7 Measured versus applied pressure as a function of temperature.

was tested by maintaining hydraulic pressure at $1000 \mathrm{psi}$ for $1 \mathrm{~h}$, which resulted in a maximum deviation of 100 psi.

Considering that the accuracy of the APP System is $20 \mathrm{psi}$, the repeatability and stability of this system was mainly affected by the stability of the sensor structure and the secondary-level temperature effect from the induced birefringence effect.

\subsection{High-Temperature Testing of Cubic-Zirconia Sensor by Mechanically Applied Pressure}

To demonstrate the possibility of applying the cubiczirconia material for pressure testing at high temperature, an experiment was performed using a mechanically applied pressure on the cubic-zirconia block, placed in a programmable furnace. As shown in Fig. 9, a 0.25- $\times 0.60-\times 1.9$ -in. zirconia block was fabricated and set up for this testing by considering the verticality, repeatability, and precision of the applied pressure. The cubic-zirconia piece was in-

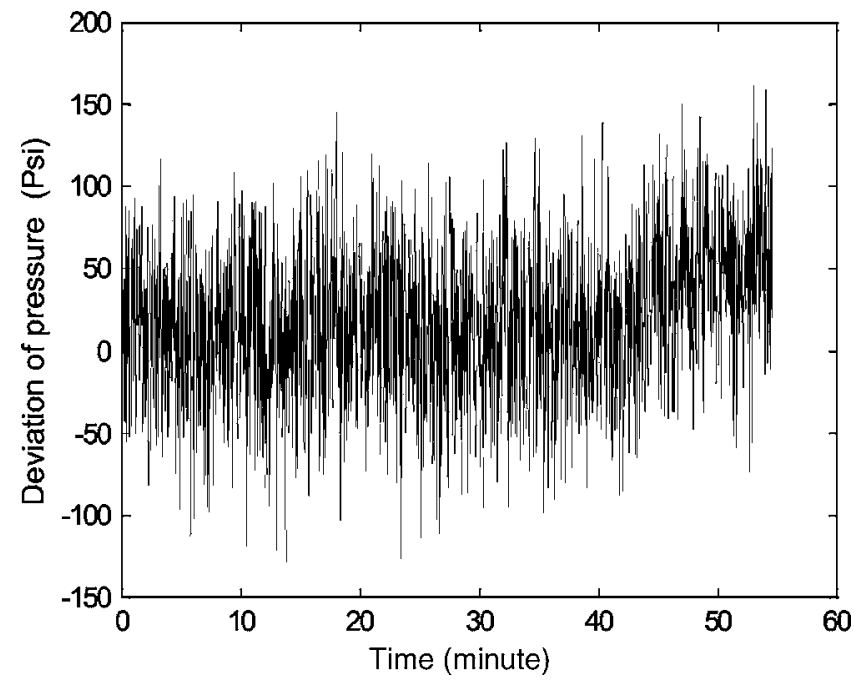

Fig. 8 Stability of the single-crystal cubic zirconia sensor based on white light interferometry. 


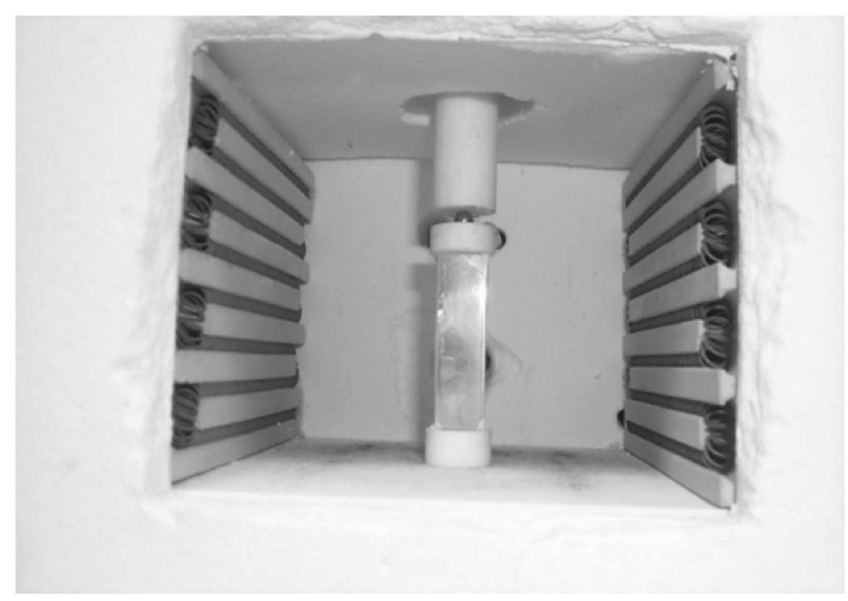

Fig. 9 Cubic zirconia block in high-temperature furnace for mechanical pressure testing.

serted into the furnace and mechanical pressure was applied from the top by adding different amounts of weights. The maximum applied weight was $14.3 \mathrm{~kg}$, corresponding to a maximum applied pressure on the single-crystal cubiczirconia sensor of 210 psi. As shown in Fig. 10, the photoelastic characteristic of the cubic-zirconia piece was calibrated at room temperature by recording the equivalent gap changes when weight was added; the equivalent gap change was $40 \mathrm{~nm}$ when the pressure change was 210 psi. The temperature of the furnace was increased from $23{ }^{\circ} \mathrm{C}$ to $1026{ }^{\circ} \mathrm{C}$; the corresponding test results are shown in Fig. 11.

Figure 12 shows the measured pressure deviation as a function of applied pressure at various temperatures. Although the system was only tested to $1026{ }^{\circ} \mathrm{C}$, the potential for higher temperature operation is apparent from the refractory nature of the cubic zirconia (with a melting temperature well above $2000{ }^{\circ} \mathrm{C}$ ). The stability of the testing setup and the repeatability of the applied mechanical pressure affect the observed measurement. At high temperature, the accuracy of the measurement was degraded by the presence of blackbody radiation. This was evidenced by a

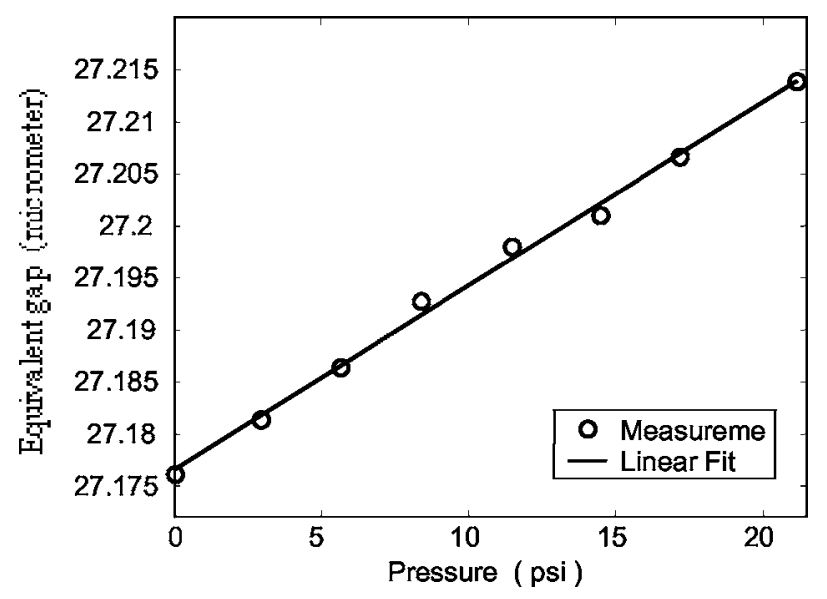

Fig. 10 Equivalent gap versus applied mechanical pressure at room temperature $\left(23^{\circ} \mathrm{C}\right)$.

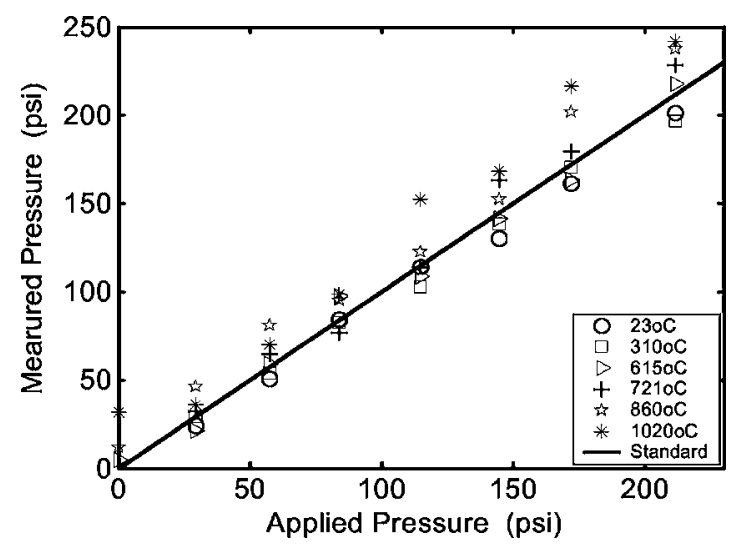

Fig. 11 Measured versus applied pressure for the single-crystal cubic zirconia sensor.

stronger background on the optical interference fringes with the increasing temperature. To eliminate the effects of the blackbody radiation, the optical source could be modulated and the observed signal filtered digitally. Since the blackbody radiation is essentially a de signal, it can be easily removed by optical source modulation and signal filtering techniques. As the temperature of testing increases, the blackbody radiation will become even more pronounced. This will be conducted in future work to improve the accuracy of the system, and even higher temperature operation will be demonstrated.

\section{Conclusion}

A single-crystal cubic-zirconia-based optical pressure sensor system has been reported in this paper, based on the change in birefringence of the initially isotropic cubic zirconia. Anisotropic application of pressure to the crystal causes a phase delay in the orthogonally polarized light beams, the magnitude of which is related to the magnitude of the applied pressure. The results of preliminary experiments have been presented for pressure measurement at high temperature. These results demonstrate the general functionality of the single-crystal cubic-zirconia-based optical sensor for pressure measurement at high temperature.

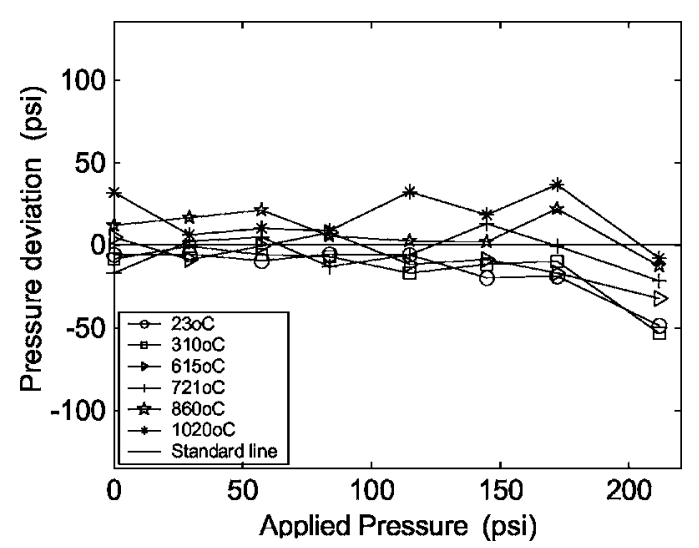

Fig. 12 Measured pressure deviation versus applied pressure at different temperatures. 


\section{Acknowledgments}

This research was sponsored by the U.S. Department of Energy under grant DE-FC36-01G011050 and Virginia Polytechnic Institute \& State University.

\section{References}

1. E. Udd, Fiber Optic Sensors-An Introduction for Engineers and Scientists, Chap. 1, pp. 1-9, Wiley, New York (1991).

2. X. Qiao and M. Fiddy, "Distributed optical fiber Bragg grating sensor for simultaneous measurement of pressure and temperature in the oil and gas downhole," Proc. SPIE 4870, 554-558 (2002).

3. Y. Zhao, Y. Liao, and S. Lai, "Simultaneous measurement of downhole high pressure and temperature with a bulk-modulus and FBG sensor," IEEE Photon. Technol. 14(11), 1584-1586 (2002).

4. D. Abeysinghe, S. Dasgupta, H. Jackson, and J. Boyd, "Novel MEMS pressure and temperature sensors fabricated on optical fibers," J. Micromech. Microeng. 12(3), 229-235 (2002).

5. R. R. Dils, "Optical fiber thermometer," U.S. Patent No. 4,576,486 (1986).

6. A. Wang, S. Gollapudi, R. May, K. Murphy, and R. Claus, "Advances in sapphire-fiber-based intrinsic interferometric sensors," Opt. Lett. 17(21), 1544-1546 (1992).

7. H. Xiao and A. Wang, "Absolute sapphire optical fiber sensor for high-temperature application," Proc. SPIE 3201, 36-42 (1999).

8. L. Tong, "Multi-doped zirconia ceramic probe for fiber-optic contact measurement of ultra-high temperatures in oxidative atmosphere," Proc. SPIE 4578, 384-391 (2001).

9. L. Tong, "Single-crystal Y2O3-ZrO2 rectangular waveguides for ultrahigh-temperature sensing applications," Appl. Opt. 41(19), 3804-3808 (2002)

10. W. Pulliam, P. Russell, and R. Fielder, "High-temperature, high bandwidth, fiber-optic, MEMS pressure sensor technology for turbine engine component testing," Proc. SPIE 4578, 229-238 (2001).

11. M. Born and E. Wolf, Principles of Optics, p. 704, Pergamon Press, Oxford, New York, (1965).

12. B. Qi, G. R. Pickrell, J. Xu, P. Zhang, Y. Duan, W. Peng, Z. Huang, R. G. May, and A. Wang, "Novel data processing techniques for dispersive white light interferometer," Opt. Eng. 42(11), 3165-3171 (2003).

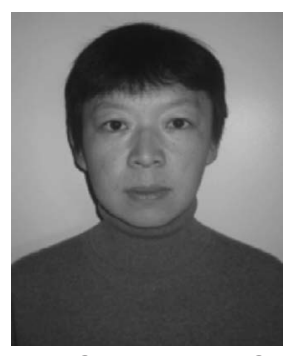

Wei Peng received her BS in optics from Shandong University, China, in 1991, and her PhD in optical engineering from Dalian University of Technology, China, in 1999. From March 2000 to April 2004, she worked for the Center for Photonics Technology in the Department of Electrical and Computer Engineering at Virginia Tech as a visiting scholar then a postdoctoral associate. She is currently working as a faculty research associate in the Arizona Applied NanoSensors Center in the College of Liberal Arts and Sciences at Arizona State University. She is an author or co-author of more than 30 research papers in various prestigious journals, and has six pending patents based on her research works in the United States. Her research interests include fiber optic physical, chemical, and biological sensors, laser spectroscopy, laser radar and optical metrology, and corresponding applications.

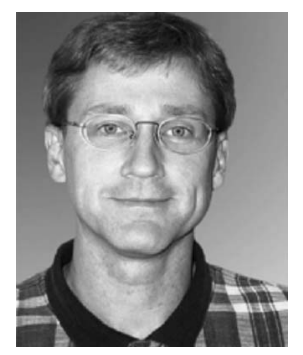

Gary R. Pickrell received his BS and MS degrees in ceramic engineering from Ohio State University in 1985 and 1987, respectively, and his $\mathrm{PhD}$ in materials engineering science from Virginia Tech in 1994. He is an assistant professor in the Materials Science and Engineering Department and associate director of the Center for Photonics Technology in the Electrical and Computer Engineering Department at Virginia Tech. His industrial experience includes various research, development, and technical management positions at Owens Illinois, Corning, Selee, and Porvair Advanced Materials. He has authored over 60 technical papers, is a member of the editorial board of the International Journal of Six Sigma and on the international editorial board of the Sensors journal, and has nine patents issued and six additional patents pending. His current research is focused on random hole optical fibers and optical fiber sensors, glass, ceramic, and various other aspects of materials science and engineering.

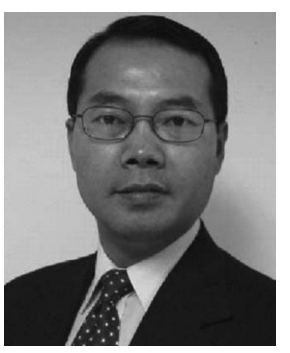

Anbo Wang received his $\mathrm{PhD}$ degree in applied optics from Dalian University of Technology, Dalian, China, in 1990. After spending three years as a research staff in the Fiber \& Electro-Optics Research Center in the Department of Electrical and Computer Engineering at Virginia Tech, he then joined the faculty of the same department as an assistant professor, where he is now a professor. $\mathrm{He}$ is the founding director of the Center for Photonics Technology. In the past, his research has been mainly focused on optical fiber-based physical, chemical, and biomedical sensors. Lately he expanded his interest to include optical metrology for testing on the constancy of fundamental physical constants. He is an author/co-author of 5 book chapters, 60 journal articles, 148 conference papers, and 56 patent disclosures with 22 licensed plus many invited presentations. In the past ten years, he has been responsible for more than 60 research programs in excess of $\$ 12$ million from various federal funding agencies and private industry. He has also served as a chair of numerous national and international conferences in photonics and sensors. 


\title{
High-temperature thermometer with fiber optic readout
}

\author{
Juncheng $\mathrm{Xu}^{1}$, Gary R. Pickrell, Kristie L Cooper, Xingwei Wang, and Anbo Wang \\ Center for Photonics Technology \\ Bradley Department of Electrical and Computer Engineering \\ Virginia Polytechnic Institute and State University, Blacksburg, VA 24061 USA
}

\begin{abstract}
Ultra-high temperature measurement is required in many harsh environment applications such as temperature monitoring in combustors and furnaces. This paper presents a novel thermometer, which is capable to measure temperatures above $1000^{\circ} \mathrm{C}$ with high resolution and accuracy. This sensor functions as a gas thermometer with a fiber optic readout, which will not suffer from blackbody radiation noise. The sensing part has high hardness, good electrical insulation, good wear resistance and does not react with most chemicals. The size of the sensor can be chosen flexibly to satisfy different application requirements. Since no electrical components are involved, the sensor is spark/explosion free and immune to electromagnetic interference (EMI). The sensor structure is simple, easy to fabricate and low cost. Some temperature measurement results in the laboratory are presented.
\end{abstract}

Keywords: fiber optic, gas thermometry, high temperature, sensor, thermometer.

\section{INTRODUCTION}

Temperature measurement is required in many industrial applications and some of them include harsh environments such as coal gasifiers, turbine combustors and glass melting furnaces. These operating environments are often with temperatures above $1000^{\circ} \mathrm{C}$, corrosion chemicals and electromagnetic noise, which challenge the current available high temperature sensors. Gas thermometers are an old temperature transducer ${ }^{[1]}$, which is often used as a teaching device or for calibration purposes because of their inconvenience in setup and in usage. However, all thermometry is based on the gas thermometer, which is still a basic measurement device offering high accuracy.

This paper presents a novel thermometer based on gas thermometry. The thermometer has a simple structure and can be used conveniently in real applications. An optical fiber sensor is used to readout the gas pressure that will be used to obtain the corresponding temperature. Since the fiber optic pressure sensor is at the low temperature end, the blackbody radiation will not interfere with the sensor signals. The remote monitoring is also easy to realize because of the low loss of the optical fiber. In addition, other types of pressure sensors can be used instead to meet different requirements, which offer a great flexibility in sensor design and usage.

\footnotetext{
1.juxu1@vt.edu
} 


\section{PRINCIPLE OF OPERATION}

The principle of this thermometer is based on gas thermometry. The basic schematic of gas thermometry is shown in Fig. 1. A fixed volume vessel contains a specific amount of gas and a capillary tube is connected with the vessel for pressure measurement. When the temperature of the vessel changes, the pressure of the gas will change correspondingly.

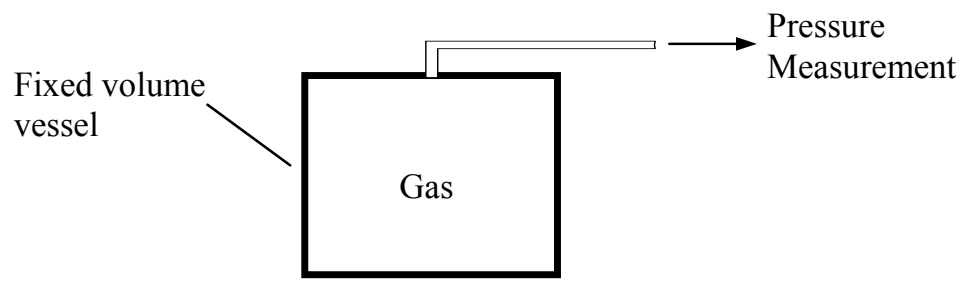

Fig. 1. Schematic of a constant-volume gas thermometer.

The equation of state of an ideal gas:

$$
\mathrm{PV}=\mathrm{nRT}
$$

where $\mathrm{P}$ is the gas pressure, $\mathrm{V}$ is the volume, $\mathrm{T}$ is the temperature in Kelvin, $\mathrm{n}$ is moles of gas, $\mathrm{R}$ is the gas constant.

For a fixed amount of gas we can get the following equation:

$$
\frac{P_{i} V_{i}}{T_{i}}=\frac{P_{f} V_{f}}{T_{f}}
$$

where $P_{i}, V_{i}, T_{i}$ and $P_{f}, V_{f}, T_{f}$ and are the initial and final pressure, volume and temperature respectively.

If assume $V_{\mathrm{i}}=V_{\mathrm{f}}$, then

$$
T_{f}=\frac{T_{i}}{P_{i}} P_{f}
$$

Since the Ti and Pi are known, which are typically the room temperature and atmospheric pressure respectively, once we know the final pressure, the temperature to be measured can be obtained.

The structure of the thermometer is shown in Fig. 2. The sensor functions as a gas thermometer, in which the temperature variations are converted to pressure changes based on the ideal gas law. The air pressure sealed in the inner chamber will increase when temperature increases, which will be measured by a fiber optic pressure sensor. No blackbody radiation will go into the fiber optic readout system and no complicated optical alignment is needed. The thermometer is just like a protection package. The size and configuration of the thermometer can be modified to meet different application requirements ${ }^{[2]}$. 


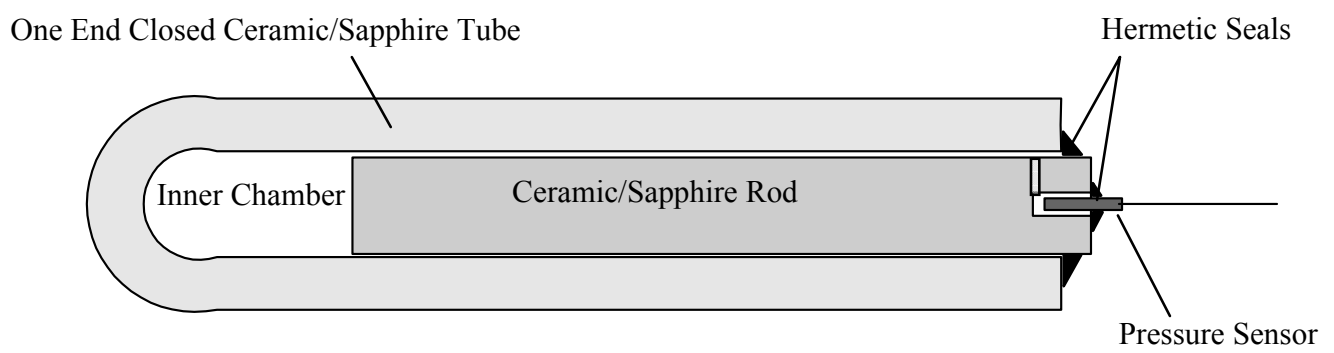

Fig. 2. Ceramic/sapphire vessel based thermometer with fiber optic readout

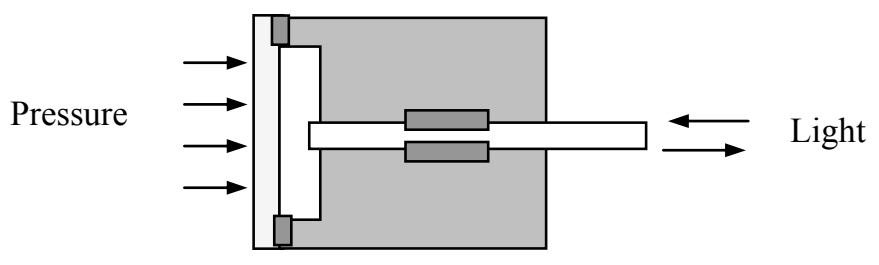

Fig. 3. Diaphragm based Fabry-Perot optical fiber pressure sensor

\section{SENSOR FABRICATION AND TEST}

A $45 \mathrm{~cm}$ long one end closed non-porous alumina tube is used as the thermometer front end and a $30 \mathrm{~cm}$ long alumina rod is inserted into the tube. The rod outside diameter is very close to the inside diameter of the tube. The back end of the rod was machined to make a pit and a side slot as shown in Fig. 2. The diaphragm-based fiber optic pressure sensor ${ }^{[3]}$ is shown in Fig. 3. Both the rod and the optical fiber sensor were sealed with the tube by epoxy. The pressure sensor used in the temperature transducer can be any type of sensor. The sensor selection depends on the requirement of measurement and the environment where the pressure sensor is used. For example, if the temperature near the pressure sensor end is still above $200^{\circ} \mathrm{C}$ or there is electromagnetic interference (EMI), then an optical fiber pressure sensor is preferred.

Since the air-gap change (diaphragm deflection) is linearly proportional to the change of the applied pressure ${ }^{[4]}$, we can get the following equation:

$$
\Delta G=\gamma \Delta P
$$

where $\mathrm{G}$ is the air-gap and $\mathrm{P}$ is the pressure,

$\gamma$ is a the pressure sensitivity $(\mathrm{nm} / \mathrm{psi})$ determined by diaphragm properties.

In temperature measurement test, the $20 \mathrm{~cm}$ long front end of the tube was inserted into a furnace (47900, Thermolyne) as shown in Fig. 4. The sensor signal interrogation system is shown in Fig. 5. A scan of the tunable laser produced the spectrum information from $1520 \mathrm{~nm}$ to $1570 \mathrm{~nm}$. The two reflections of the Fabry-Perot pressure sensor generated interference fringes, and a valley trace method ${ }^{[5]}$ was used to process the fringes to demodulate the Fabry-Perot cavity length (air-gap). The measurement results are shown in Fig. 6. 


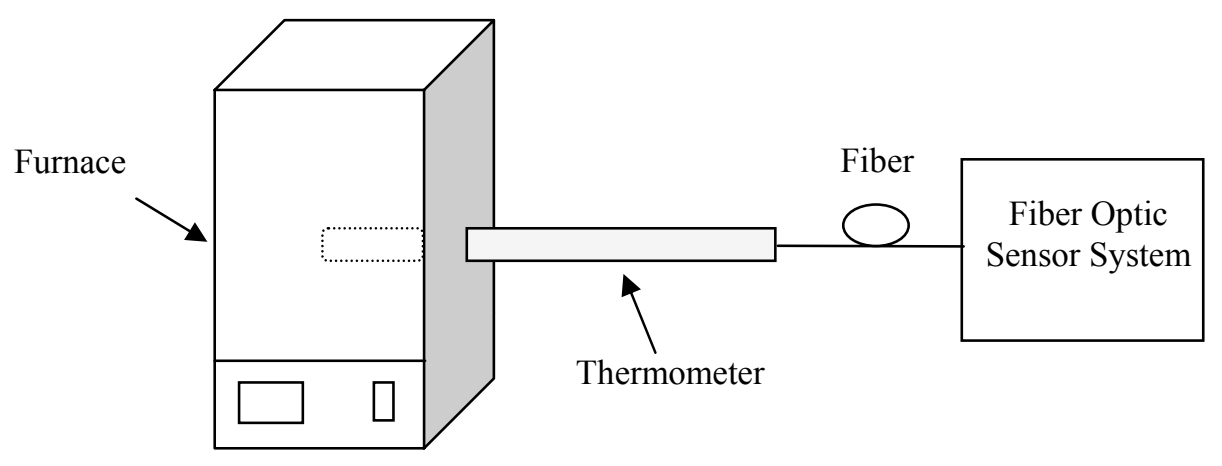

Fig. 4. Temperature measurement experiment setup

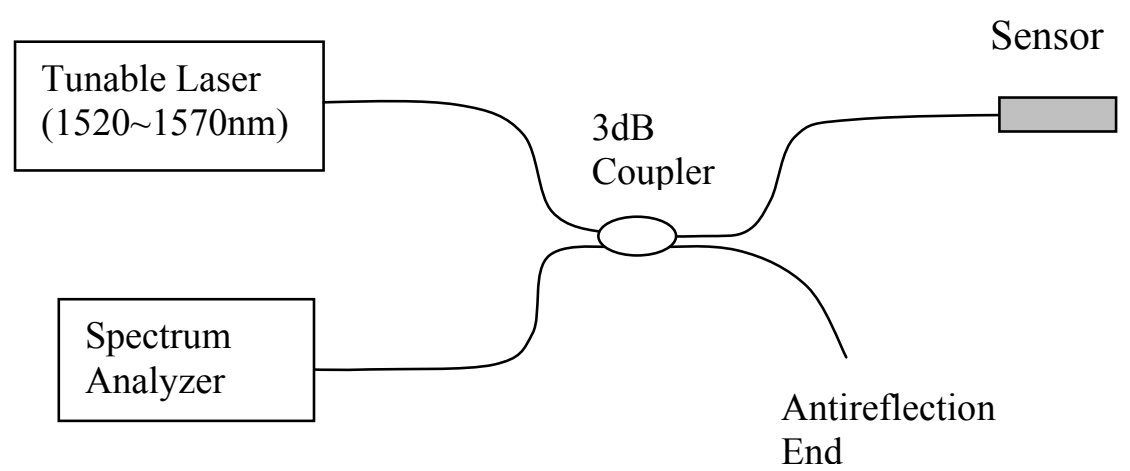

Fig. 5. Sensor signal interrogation system

The sensor interrogation system exhibited an air-gap measurement resolution of about $0.03 \mathrm{~nm}$ and the optical fiber pressure sensor had a total air-gap change of about $97.2 \mathrm{~nm}$ in the test. Thus, the temperature measurement resolution is about $0.3^{\circ} \mathrm{C}$. A pressure sensor with higher sensitivity or a system with higher resolution will improve the temperature resolution.

We observed a small non-linearity in the test results (see Fig. 6). To improve the thermometer performance, the constantvolume gas thermometry is not enough and some corrections and data processing should be made to achieve better results:

(1) The gas will not be exactly ideal, and the degree of non-ideality should be considered.

(2) Corrections should be made for thermal expansion or contraction of the vessel caused by the temperature changes.

(3) A small portion of air in the vessel may be still at low temperature.

(4) Non-linear curve fittings are needed.

Nevertheless, this thermometer can achieve high resolution and high accuracy. 


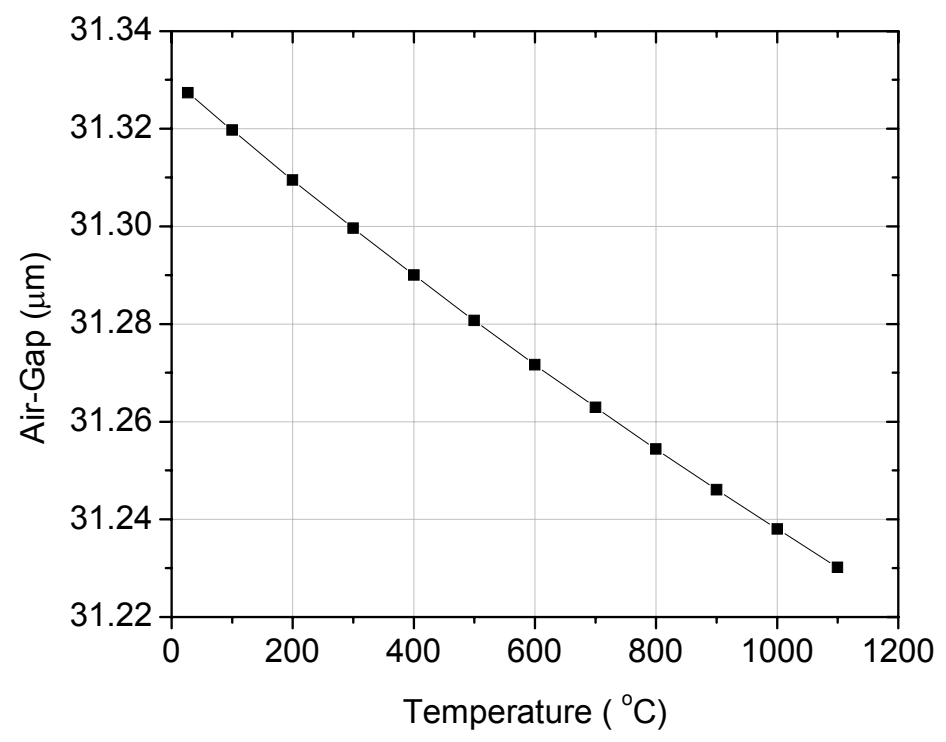

Fig. 6. The thermometer temperature test result

\section{CONCLUSIONS}

A novel high temperature thermometer has been described, which is based on gas thermometry and has a fiber optic readout. The thermometer is simple, easy to fabricate and flexible in size. Since no electrical parts are involved, it is safe for applications having EMI or spark sources. In addition, the sensor can be remotely monitored kilometers away without any power supply at the sensing point or link repeaters. Also, any other pressure sensor can be used to build the thermometer depending on the application requirements.

\section{REFERENCES}

1. R. P. Benedict, Fundamentals of Temperature, Pressure, and Flow Measurements, 3rd ed., Wiley, New York, 1984.

2. J. Xu and A. Wang, "A Novel Temperature Transducer for Harsh Environments", Virginia Tech Intellectual Property Disclosure VTIP No. 04-010.

3. J. Xu, G. Pickrell, X. Wang, W. Peng, K. L Cooper and A. Wang, "A Novel Temperature Insensitive Optical Fiber Pressure Sensor For Harsh Environments," IEEE Photon. Technol. Lett. Vol. 17, 870-872, 2005.

4. M. D. Giovanni, Flat and Corrugated Diaphragm Design Handbook. New York: Marcel Dekker, 1982.

5. B. Qi, G. R. Pickrell, J. Xu, P. Zhang, Y. Duan, W. Peng, Z. Huang, W. Huo, H. Xiao, R. G. May, and A. Wang, "Novel data processing techniques for dispersive white light interferometer," Opt. Eng., Vol. 42, pp. 3165-3171, Nov. 2003. 


\title{
Frequency-Division-Multiplexed Fabry-Perot Interferometric Fiber Sensors for Temperature Monitoring in a Selective Catalytic Reduction Unit
}

\author{
Fabin Shen ${ }^{*}$, Zhengyu Huang ${ }^{\mathrm{a}}$, Yizheng Zhu ${ }^{\mathrm{a}}$, Michael Coffey ${ }^{\mathrm{b}}$, Robert Frank ${ }^{\mathrm{b}}$, Gary Pickrell ${ }^{\mathrm{a}}$, and \\ Anbo Wang ${ }^{\mathrm{a}}$ \\ ${ }^{\mathrm{a}}$ Center for Photonics Technology, Bradley Department of Electrical and Computer Engineering, \\ Virginia Polytechnic Institute and State University, Blacksburg, VA 24061 \\ ${ }^{\mathrm{b}}$ EPRI I\&C Center/Kingston Fossil Plant, Harriman, TN 37748
}

\begin{abstract}
We present the quasi-distributed temperature measurement results in a selective catalytic reduction unit of a power plant by using a frequency-division-multiplexing optical fiber measurement system with eight intrinsic Fabry-Perot interferometric fiber sensors along a single fiber. The sensor was constructed by splicing a section of multimode fiber between single mode fibers. A high resolution swept laser interrogator was used to measure the spectrogram of the reflected light from the sensors, which contains multiple frequency components in wave number domain corresponding to sensors with different cavity lengths. The temperatures were measured by estimating the optical path length of each Fabry-peort interferometer. Field test results show that the proposed technology can potentially be used in applications of multi-point high temperature sensing.
\end{abstract}

Keywords: Fiber sensors, Fabry-Perot interferometer, frequency division multiplexing, temperature, selective catalytic reduction.

\section{INTRODUCTION}

Real-time multiple-point or distributed temperature measurement is important for the operation of a selective catalytic reduction (SCR) unit in a power plant. Most importantly, temperature profiling in an SCR devices ensures proper operation. Catalytic reactions occur only over a small temperature range, and variation in the temperature profile will reduce their effectiveness. Ultimately, failure to ensure proper operating temperature in an SCR results in increased pollution emitted into the environment. Temperature profile monitoring in an SCR unit also contributes to improvements in optimizing boiler operation, prevention of system failure, and reduction in maintenance cost.

The distributed sensing technology based on optical fiber sensors provides a promising solution for the temperature measurement inside a SCR unit. Intrinsic distributed sensing technology based on Raman scattering ${ }^{[1]}$ and Brillouin scattering ${ }^{[2]}$ usually use the flight of time of a light pulse to determine the measurement location and can measure the temperature along a fiber at any desired position. The pulse width of the light pulse has to be as narrow as nanoseconds to obtain a spatial resolution usually in meters. However, a narrow pulse will cause a low average power and thus a low signal to noise ratio. Therefore, an average of multiple measurements is usually needed to obtain reasonable measurement accuracy, which restricts the speed of real-time monitoring. An alternative method is the quasi-distributed sensing technology in which in-line fiber sensors are arranged along a single fiber to measure the temperatures at certain predefined locations. Such in-line sensors include fiber Bragg grating (FBG) sensors ${ }^{[3]}$ and fiber Fabry-Perot (FP) interferometric (FFPI) sensors ${ }^{[4-10]}$.

*fashen1@vt.edu; phone 1540 231-2155; fax 1540 231-2158

Sensors for Harsh Environments II, edited by Anbo Wang, Proc. of SPIE Vol. 5998, 59980H, (2005) - 0277-786X/05/\$15 - doi: 10.1117/12.633894 
FBG sensors are constructed by generating periodic refractive index change inside a photosensitive fiber by UV irradiation. A FBG is a narrow band reflector and its Bragg wavelength depends on the environment temperature. FBG sensors can be multiplexed for quasi-distributed temperature and strain sensing. However, the refractive index change in a FBG is usually in Type-I regime and can only withstand a temperature less than $250^{\circ} \mathrm{C} .{ }^{[3]}$ In a high temperature environment, the refractive index change in a FBG will be reduced to some extent or even totally erased. Thus traditional FBG sensors cannot be used inside a SCR unit whose temperature can be as high as $700^{\circ} \mathrm{C}$.

In-line Fabry-Perot (FP) fiber sensors with high temperature survivability have attracted considerable research interests in past decade. Lee et al demonstrated an intrinsic FP interferometer (IFPI) by building dielectric mirrors into optical fiber. ${ }^{[4]}$ Murphy et al developed an extrinsic FP interferometric (EFPI) sensor by constructing an air gap between end faces of two uncoated fibers inserted into an alignment tube. ${ }^{[5]}$ Sirkis et al introduced in-line fiber etalons by fusion splicing two fibers with a section of hollow core fiber of the same outside diameter. ${ }^{[6]}$ Chen et al introduced an IFPI sensor by constructing a pair of air-gap inside the fiber through hydrofluoric acid etching and fusion splicing. These FP sensors can be used for high temperature measurement. ${ }^{[7]}$ However, due to the relatively high return loss or insertion loss, the multiplexing capacity of these FP sensors is limited to a small number.

For the multiplexing of a large number of sensors along a single fiber, in-line sensors with low return losses and low insertion losses, such as UV-induced IFPI sensors, ${ }^{[8,9]}$ are desirable. Recently, we developed an intrinsic FP interferometric sensor with a singlemode-multimode-singlemode (SMS) structure by splicing a section of multimode mode fiber (MMF) between two single mode fibers (SMF). ${ }^{[10]}$ This sensor can be used for high temperature sensing and has relative low power loss and thus good multiplexing capability.

Different multiplexing techniques, including time-division-multiplexing, ${ }^{[8]}$ code-division-multiplexing, ${ }^{[11]}$ and coherence multiplexing ${ }^{[12,13]}$ schemes, have been reported for the multiplexing of FP sensors. Recently, we developed a frequency-division-multiplexing (FDM) scheme based on spectrum measurement for the multiplexing of in-line FP sensors. ${ }^{[14]}$ We used a frequency estimation based signal-processing algorithm to estimate the optical path imbalance of each FP sensor. ${ }^{[15]}$

In this paper, after an introduction of the SMS sensor and the FDM scheme, we present the temperature profile measurement result of a SCR unit in a power plant. A sensor array with 8 SMS sensors was deployed inside the SCR unit. The measurement results of 3 weeks were recorded. Test results show that the SMS sensors in a FDM scheme can be used for multi-point temperature measurement in a relative high temperature environment with a measurement resolution of $0.5^{\circ} \mathrm{C}$.

\section{PRINCIPLE OF OPERATION}

\subsection{SMS Fabry-Perot Interferometric Sensor}

The structure of the SMS sensor is shown in Fig. 1. Two Fresnel reflectors are constructed at the splicing interfaces due to the difference of refractive indices between MMF and SMF fibers. These two reflectors form a Fabry-Perot interferometric sensor with the MMF serving as both a waveguide and a sensing element.

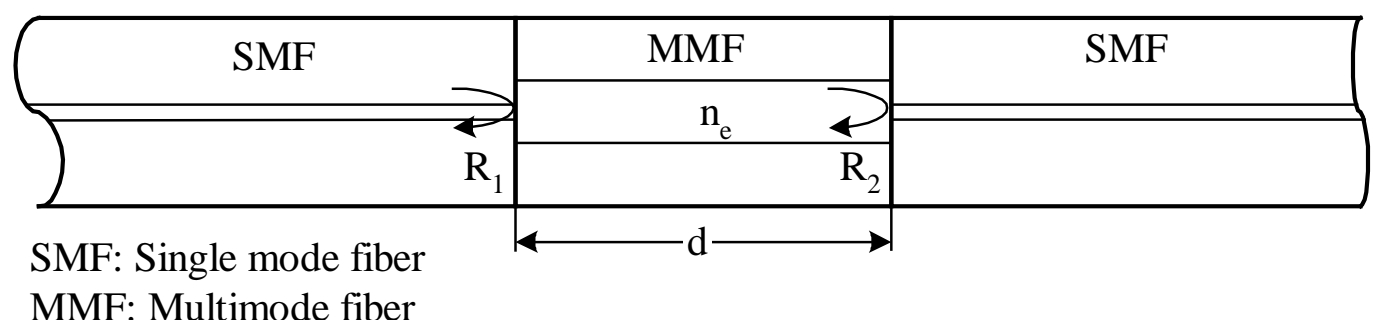

Fig. 1 Structure of the SMS Fabry-Perot interferometric sensor. 
The optical path difference (OPD) between the reflections at these two reflectors is

$$
L=2 n_{e} d
$$

where $n_{e}$ is the effective refractive index and $d$ is the length of the MMF. The reflectivity at the SMF/MMF interface is low, thus the Fabry-Perot interferometer can be approximated to a two-beam interferometer with all the multi-path reflections neglected. The normalized interference between the two reflections at wavelength $\lambda$ is

$$
x(\lambda)=\frac{I(\lambda)}{I_{0}(\lambda)}=R_{1}+R_{2}+2 \sqrt{R_{1} R_{2}} \cos \left(\frac{2 \pi L}{\lambda}+\phi\right)=A+B \cos \cos \left(\frac{2 \pi L}{\lambda}+\phi\right)
$$

where $I(\lambda)$ is reflected light power, $I_{0}(\lambda)$ is the incident light power, $R_{1}$ and $R_{2}$ are the reflectivity of the two reflectors and $\phi$ is a constant phase.

We can measure the normalized interference spectrum of the FP sensor at multiple wavelengths by using the measurement system given in Fig. 2. The measured interference spectrum can be given as

$$
x\left(k_{n}\right)=A+B \cos \left(k_{n} L+\phi\right)+v_{n} \quad(\mathrm{n}=1,2, \ldots, \mathrm{N})
$$

where $k_{n}=2 \pi / \lambda_{n}$ is the wave number at wavelength $\lambda_{n}, v_{n}$ is the measurement noise, and $\mathrm{N}$ is the sampling number.

It is evident $x\left(k_{n}\right)$ is a sinusoid with respect to $k_{n}$ with an amplitude of $\mathrm{B}$, a frequency of $L$, a phase of $\phi$ and a $\mathrm{dc}$ bias of the A. The OPD of the sensor, which is the frequency of $x\left(k_{n}\right)$, can be estimated by means of frequency estimation method.

\subsection{Frequency-Division-Multiplexing Scheme}

We developed a spectrum-based measurement system and a FDM scheme for the multiplexing of inline Fabry-Perot interferometric sensors along a single fiber. The diagram of the measurement system is shown in Fig. 2.

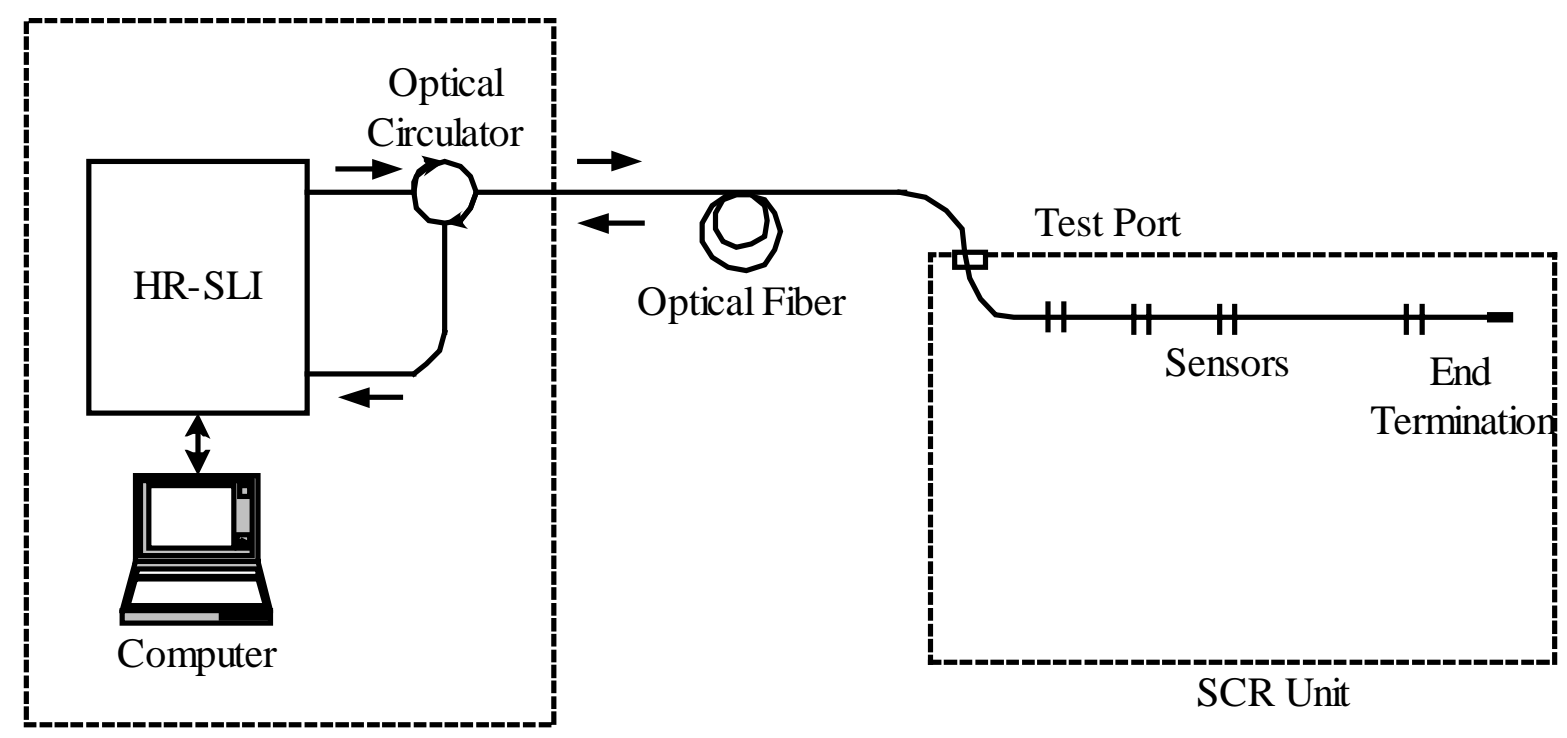

Control Room

HR-SLI: High Resolution Swept Laser Interrogator

Fig. 2. Diagram of the measurement system. 
We use a high resolution swept laser interrogator (HR-SLI, Micron Optics, si720), which contains a swept laser source, detectors, intensity and wavelength referencing units and supporting electronic circuits in a single standalone box, to measure the interference spectrum of IFPI sensors. Narrowband light from the swept laser is launched into an optical circulator, which transmits the light to the IFPI sensors. At each sensor, part of the light is reflected back to the circulator. The circulator then couples the reflected light to an optical detector. A portion of light from the laser source is tapped from the light source inside the HR-SLI. The intensity and wavelength of the tapped light are measured as both an intensity and wavelength reference. Normalized interference spectrum of IFPI sensors can be measured in each sweeping period of the laser source. The laser is operated to finish a sweep in $200 \mathrm{~ms}$. The wavelength sweeping rang is 1520-1570 nm with $2.5 \mathrm{pm}$ wavelength step. Totally. 20,000 samples are measured during one sweeping cycle of the laser source.

The normalized interference spectrum of multiple FP sensors is measured and can be given as

$$
x\left(k_{n}\right)=\sum_{\mathrm{j}=1}^{\mathrm{J}}\left[A_{j}+B_{j} \cos \left(k_{n} L_{j}+\phi_{j}\right)\right]+v_{n}=\sum_{j=1}^{J} A_{j}+\sum_{j=1}^{J} B_{j} \cos \left(k_{n} L_{j}+\phi_{j}\right)+v_{n}
$$

where $\mathrm{J}$ is the number of sensors multiplexed, $A_{j}, B_{j}, L_{j}, \phi_{j}$ are the dc bias, amplitude, frequency and phase of the sinusoidal signal from the $\mathrm{j}$-th sensor, respectively.

The FP sensors have different OPDs and thus the sinusoids in Eq. (4) have different frequencies, which means that the multiplexing of FP sensors is based on a frequency-division-multiplexing scheme. We use band pass filters to select each frequency and use a frequency estimation method to estimate the OPD of each sensor.

The swept laser source has a line width of $500 \mathrm{M} \mathrm{Hz}$. The coherence length of the light source, $L_{c}$, is about $0.6 \mathrm{~m}$. The distance between adjacent FP sensors is designed to be larger than $L_{c}$. Thus the interference between reflectors from different sensors cannot be observed.

\subsection{Calibration Curve}

When the FP sensor is put into a temperature-varying environment, the OPD of the FP cavity will change due to the thermo-optic effect and the thermal expansion of the MMF. The total OPD change $\Delta$ can be given as

$$
\Delta L=2\left(\Delta n_{e} d+n_{e} \Delta d\right)=L\left(\frac{\Delta n_{e}}{n_{e}}+\frac{\Delta d}{d}\right)
$$

The dimension change of the fiber due to the temperature change is

$$
\Delta d=\alpha_{T} \Delta T d,
$$

where $\alpha_{T}$ is the coefficient of thermal expansion (CTE). The refractive index change due to the temperature change is

$$
\Delta n_{e}=\frac{\partial n_{e}}{\partial T} \Delta T
$$

Thus the normalized OPD change can be given as

$$
\frac{\Delta L}{L}=\left(\frac{\partial n_{e}}{\partial T} / n_{e}+\alpha_{T}\right) \Delta T=\left(\sigma_{T}+\alpha_{T}\right) \Delta T
$$

where $\sigma_{T}=\frac{\partial n_{e}}{\partial T} / n_{e}$ is the effective thermal-optic coefficient. 
It is evident that the relationship between the normalized OPD change and the temperature change depends on $\sigma_{T}$ and $\alpha_{T}$, which are only related to the material of the MMF. Therefore, we can use a single calibration curve of the normalized OPD change for all the sensors multiplexed. Fig. 3 shows the calibration curve of the sensors. A SMS sensor with OPD of $408.34 \mu \mathrm{m}$ was tested to obtain the calibration curve. The OPD of the sensor at $25^{\circ} \mathrm{C}$ was used as a reference and normalized to 1 . At each temperature, an average of 300 measurements in 30 minutes was applied. A cubic polynomial fitting was used to obtain the calibration curve.

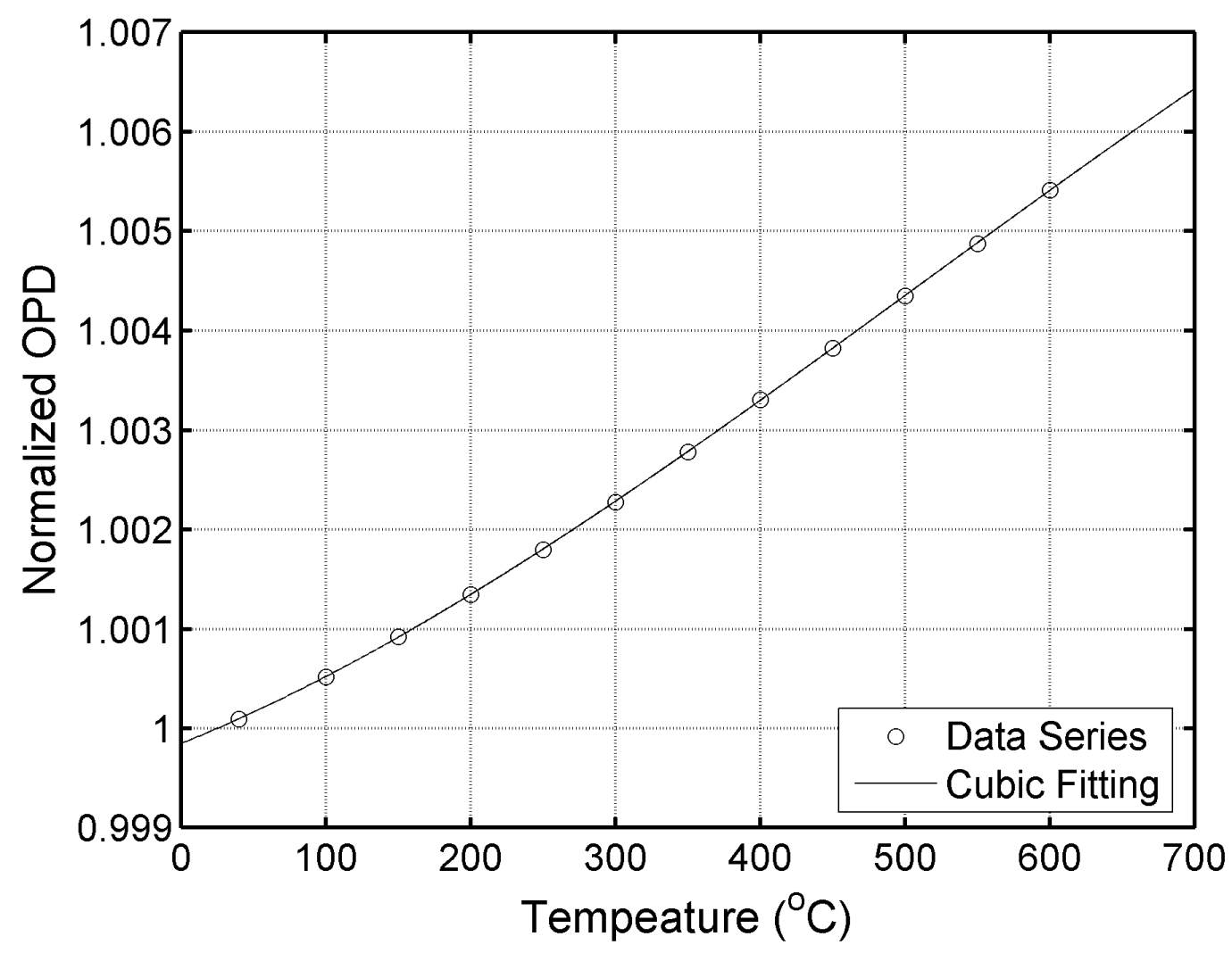

Fig. 3. Temperature response and calibration curve of the SMS sensor.

\section{SENSOR INSTALLATION}

The sensor sets were installed inside SCR unit 6 at the TVA Kinston Fossil Plant, located in Harriman, TN. This unit is approximately 50 years old and is rated at $200 \mathrm{MW}$. The SCR units were a recent addition to the plant and have reduced emitted NOx compounds by as much as $80 \%$.

Two multiplexed sensor arrays, each of which had eight sensors, were installed during an outage of the SCR unit. The sensors in each set were spliced into a single fiber with a total length of 10 meters. The distance between adjacent sensors was about 1 meter. Each sensor set was packaged into a stainless steel tube with an outside diameter of $1 \mathrm{~mm}$. Angle polished FC connectors were made ready for each sensor set. The diagram of the sensor set is shown in Fig. 4(a).

The SCR unit has a length of 12 meters and a width of 4 meters. We installed two $1 / 4$ " stainless steel tubes between the two test ports at one side of the SCR unit. These two tubes were used as the slots to support the sensor sets. The diagram of the tube slots is shown in Fig. 4(b). The ends of these two slot tubes were mounted onto the end caps of the test ports with Swagelok adapters. 
The two sensor sets were inserted into the two tube slots, respectively. Optical cables with a length of 25 meters each are used to connect the sensor sets and the measurement system in the control room. Due to the limitation of the detection scheme, only one sensor set can be monitored at one time as shown in Fig.2.

A multi-threaded win32 program running on the host computer was written in $\mathrm{C} / \mathrm{C}++$ to retrieve the spectrum from the HR-SLI and perform the signal processing tasks. OPD of the sensors were measured and normalized with the references stored in the computer. The temperature readings were determined by looking up the calibration curve shown in Fig. 3. The host computer in the control room was set to dialup the Internet automatically, so that the historic temperature readings can be remotely accessed.

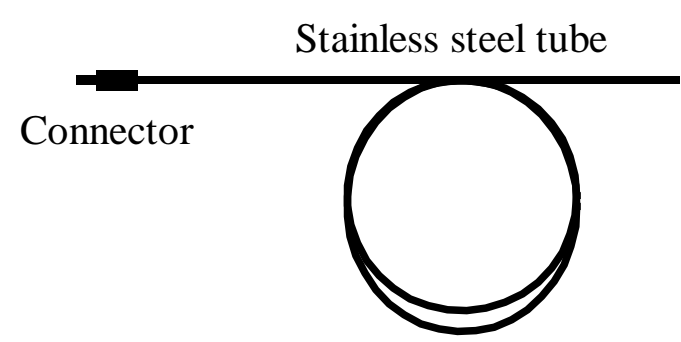

(a)

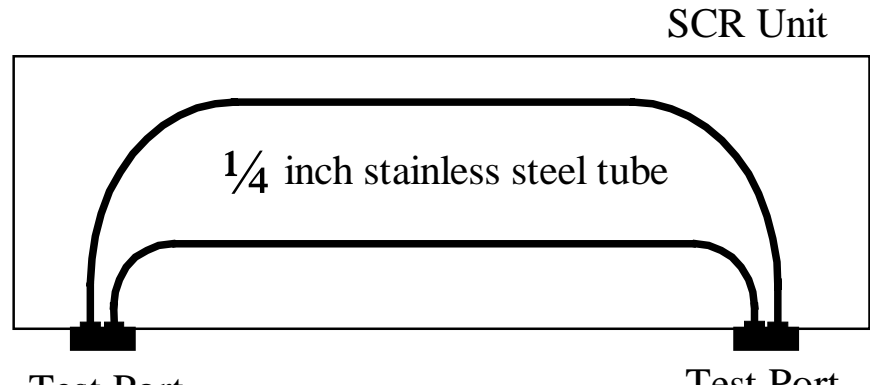

(b)
Test Port

Fig. 4. Sensor installation. (a). Sensor set protected by stainless steel tube with connector ready. (b) Slot tubes installed inside the SCR unit.

\section{TEST RESULTS}

The temperature readings of one sensor set were monitored for about three weeks, from April 23, 2005 to May 12, 2005. The test results of the sensor set are shown in Fig. 5 and Fig. 6. Fig. 5 shows a 2D plot of the historic curves of the temperature readings. Fig. 6 shows a 3D plot that presents the temperature profile more clearly.

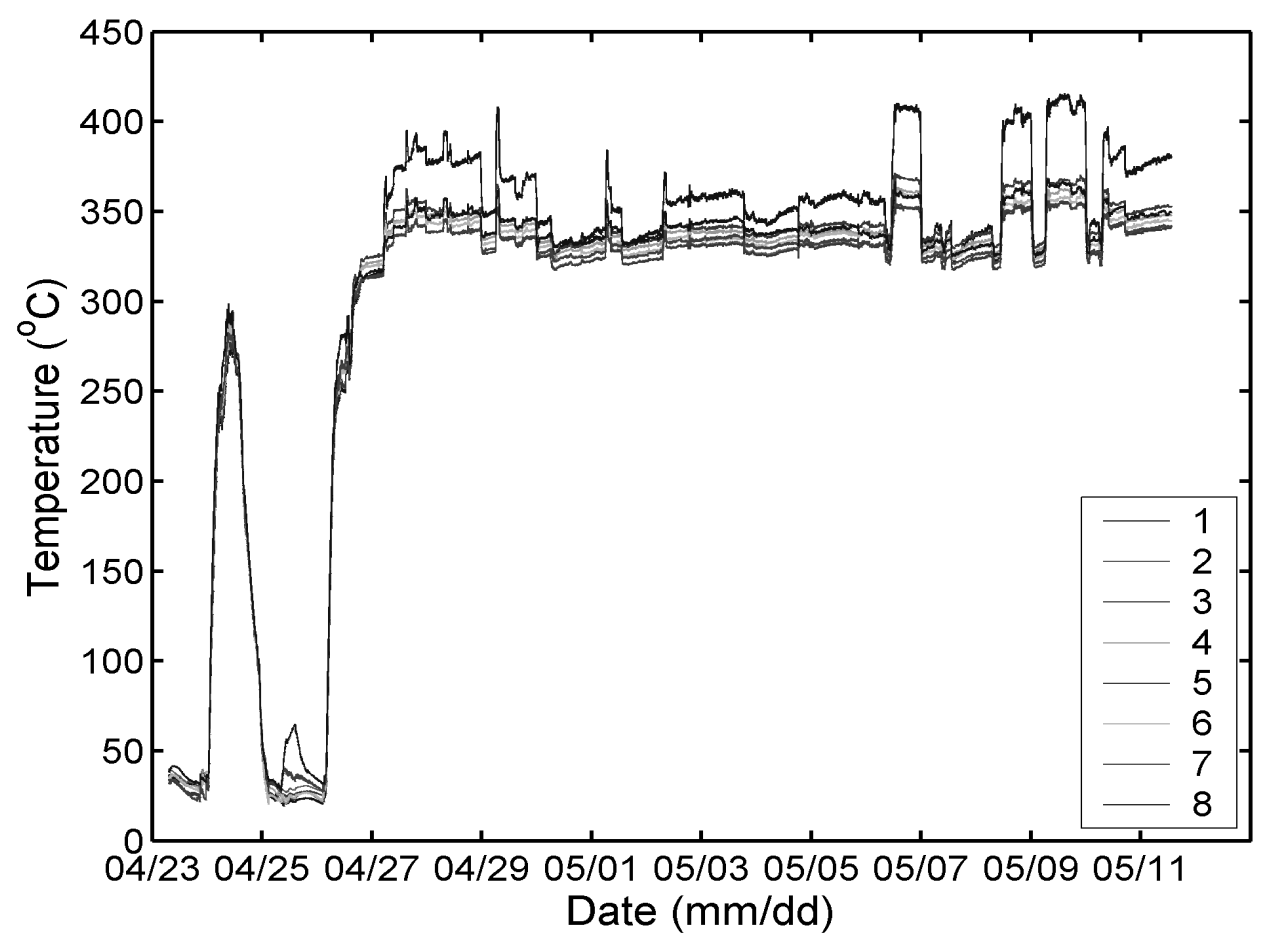

Fig. 5. Historic curves of the temperature measured by eight SMS sensors. 


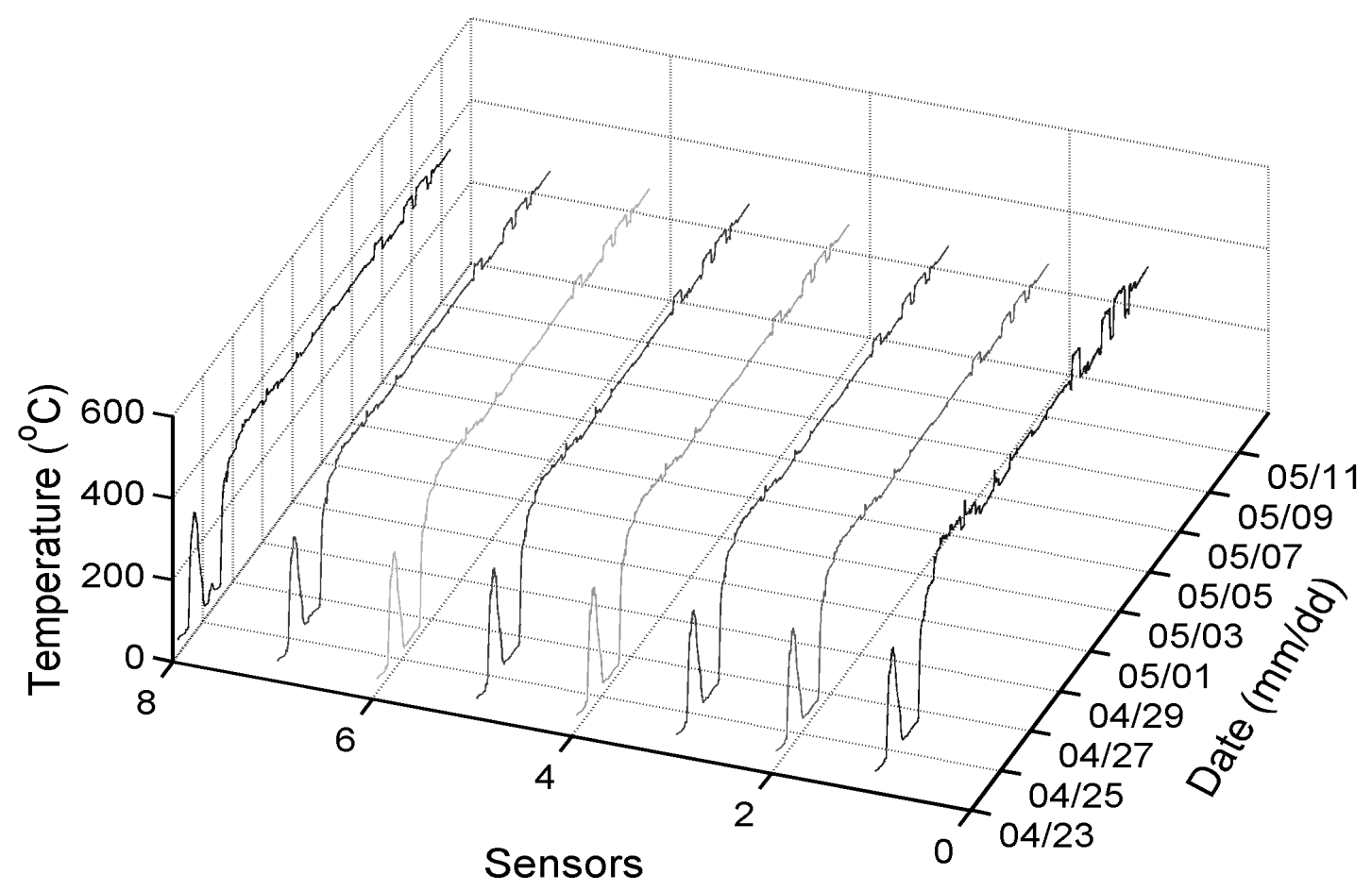

Fig. 6. Temperature measurement results in 3D plot.

The first sensor, which was the closest one to the test port, was near to the heat pipe on the wall of the SCR unit, and thus saw a higher temperature than other sensors. An average temperature of $340^{\circ} \mathrm{C}$ was recorded when the SCR unit was running in normal conditions. The temperature readings of the eight sensors were consistent and reflect the temperature variations inside the SCR unit. The measurement resolution is better than $0.5^{\circ} \mathrm{C}$. The broad range of temperature variation show a startup test, then normal startup conditions of the unit. Step changes over time reflect the changing load (change in MW output) of the unit demanded by the transmission system.

\section{CONCLUSIONS}

We presented a frequency-division-multiplexed SMS sensor array for multi-point temperature measurement inside a SCR unit of a power plant. Eight sensors were arranged along a single fiber and packaged in a stainless steel tube with a small diameter. The sensor set was installed inside a SCR unit of a power plant. The temperature profile of the SCR unit was monitored for 3 weeks. Test results show that the SMS fiber sensors can be multiplexed in a FDM scheme for quasi-distributed temperature measurement in a relative high temperature environment.

\section{ACKNOWLEDGMENTS}

This research was supported in part by the U.S. Department of Energy under grant DE-FC36-01G011050 and the U.S. National Science Foundation under grant CMS-0427951. We would like to thank the operation and support staff at the TVA Kingston facility, Earl Deskins Manager. We would also like to specifically thank Bill Oberg of the plant engineering staff for assistance in discussions regarding the temperature measurement, the installation of the sensor set, and providing access to house the test equipment. Lastly, we would also like to thank Edgar Brown of TVA telecom for providing the connection of the dialup line in the control room. 


\section{REFERENCES}

1. M. A. Farahani and T. Gogolla, "Spontaneous Raman Scattering in Optical Fibers with Modulated Probe Light for Distributed Temperature Raman Remote Sensing", J. Lightwave Technol. 17(8), 1379-1391, 1999.

2. T. Kurashima, T. Horiguchi, M. Tateda, "Distributed temperature sensing using stimulated Brillouin scattering in optical silica fibers," Opt. Lett. 15(18), 1038-1041, 1990.

3. Andreas Othonos, "Fiber Bragg gratings", Review of Scientific Instruments, vol. 68(12), pp. 4309-4341, 1997.

4. C. E. Lee and H. F. Taylor, "Interferometric optical fiber sensors using internal mirrors", Electron. Lett. 24, pp. 193, 1988.

5. K. A. Murphy, "Extrinsic Fabry Perot Optical Fiber Sensor", Proc. IEEE Optical Fiber Sensors, 8th, Monterey, CA, pp. 193, 1992.

6. J. Sirkis, T. A. Berkoff, R. T. Jones, H. Singh, A. D. Kersey, E. J. Friebele and M. A. Putnam, "In-line fiber etalon (ILFE) fiber-optic strain sensors", J. Lightwave Technol. 13, 1256-1268, 1995.

7. X. Chen, F. Shen, A. Wang, Z. Wang, Y. Zhang, "Novel Fabry-Perot fiber optic sensor with multiple applications," Proc. SPIE, Vol. 5590, p. 111-121, 2004.

8. J. A. Greene, T. A. Tran, K. A. Murphy, A. J. Plante, V. Bhatia, M. Sen and R. O. Claus, "Photoinduced Fresnel Reflectors for Point-wise and Distributed Sensing Applications", Proc. SPIE, Vol. 2444, pp. 64, 1995.

9. F. Shen, W. Peng, A. Wang, K. L. Cooper, G. R. Pickrell, "UV-Induced Intrinsic Fabry-Perot Interferometric Fiber Sensors," Proc. SPIE, Vol. 5590, pp. 47-56, 2004.

10. Zhengyu Huang, Yizheng Zhu, Xiaopei Chen, and Anbo Wang, "Intrinsic Fabry-Perot Fiber Sensor for Temperature and Strain Measurements," accepted by IEEE Photonics Technol. Lett.

11. S. H. Poland, J. P. Bengtsson, M. Bhatnagar, K. C. Ravikumar, M. J. de Vries and R. O. Claus, "Multi-measured multiplexed extrinsic Fabry-Perot interferometric sensors," Proc. Conf. on Smart Structures and Materials, SPIE '94, Orlando, FL, pp 58-66,1994.

12. M. E. Jones, J. L. Grace, V. Bhatia, K. A. Murphy, R. O. Claus, J. A. Greene and T. A. Tran, "Multiplexed absolute strain measurements using extrinsic Fabry-Perot interferometers," Proc. Conf on Smart Structures and Materials, SPIE 95 paper 2444-28, 1995.

13. M Singh, C J Tuck and G F Fernando, "Multiplexed optical fibre Fabry-Perot sensors for strain metrology," Smart Mater. Struct. 8, pp. 549-553, 1999.

14. Fabin Shen, Wei Peng, Anbo Wang, Kristie L. Cooper and Gary R. Pickrell, "UV-Induced Intrinsic Fabry-Perot Inteferometric Fiber Sensor and Frequency Division Multiplexing Scheme," manuscript in preparation.

15. Fabin Shen, and Anbo Wang, "Frequency-estimation-based signal-processing algorithm for white-light optical fiber Fabry-Perot interferometers," Appl. Opt., 44(25), 5206-5214, 2005. 


\title{
Miniature fiber optic pressure and temperature sensors
}

\author{
Juncheng $\mathrm{Xu}^{1}$, Xingwei Wang, Kristie L Cooper, Gary R. Pickrell, and Anbo Wang \\ Center for Photonics Technology \\ Bradley Department of Electrical and Computer Engineering \\ Virginia Polytechnic Institute and State University, Blacksburg, VA 24061 USA
}

\begin{abstract}
New miniature extrinsic Fabry-Perot interferometric (MEFPI) optical fiber sensors with a size of $125 \mu \mathrm{m}$ in diameter are presented, which are ideal for applications where the operation space is highly restricted. The temperature sensor can work up to $800^{\circ} \mathrm{C}$ with a sensitivity of $0.46 \mathrm{~nm} /{ }^{\circ} \mathrm{C}$. The pressure sensor exhibited a sensitivity of about $0.36 \mathrm{~nm} / \mathrm{psi}$. The sensitivities of the pressure and temperature sensor can be controlled with high precision during fabrication. In addition, their Fabry-Perot cavity lengths can be controlled with a resolution of several nanometers, which provides excellent flexibility in sensor design and signal demodulation. The sensors are composed entirely of fused silica, which is very reliable, biocompatible, corrosion resistant and immune to electromagnetic interference (EMI).
\end{abstract}

Keywords: fiber optic sensor, Fabry-Perot, pressure sensor, temperature sensor

\section{INTRODUCTION}

Miniature pressure and temperature sensors are widely used in industrial and biomedical applications where operation spaces are very limited or a high spatial resolution is required. Optical fiber sensors can be made very small, with diameters typically in the range of a few hundred microns. As one example, Fabry-Perot (FP) fiber optic sensors are easy to fabricate with low cost, and can be classified as intrinsic Fabry-Perot interferometers (IFPI) ${ }^{[1]}$ or extrinsic Fabry-Perot interferometers (EFPI) ${ }^{[2][3]}$. Generally, the sensing element of an IFPI sensor is a short section of fiber sandwiched between the two FP mirrors. For a conventional EFPI sensor, the lead-in and reflecting fibers and the tubing holding them are the sensing elements. IFPI sensors are often more sensitive to temperature changes and a little smaller than EFPI sensors while the latter make it easier to adjust the FP cavity length and often have higher pressure sensitivities. In addition to the sensors mentioned, an in-line fiber etalon (ILFE) sensor has also been reported ${ }^{[4]}$, in which the sensing element is a short section of hollow fiber.

We present novel miniature extrinsic Fabry-Perot interferometric (MEFPI) optical fiber pressure and temperature sensors, which have the same diameter as the fiber itself. The structure of the miniature sensor is shown in Fig. 1. The length of the FP cavity (air-gap) will change by the net result of length changes in the hollow fiber and reflecting fiber along with variations of temperature or pressure. Light is injected into the lead-in optical fiber and partially reflected $(\sim 4 \%)$ by the end faces of the two fibers. Then the two reflections propagate back through the same lead-in fiber and generate interference fringes, which will be demodulated to determine the air-gap thickness. The FP cavity length can be adjusted with high precision in a wide range, giving a great deal of flexibility in the choice of light source or the signal demodulation scheme selection.

\footnotetext{
${ }^{1}$ juxu1@vt.edu
} 


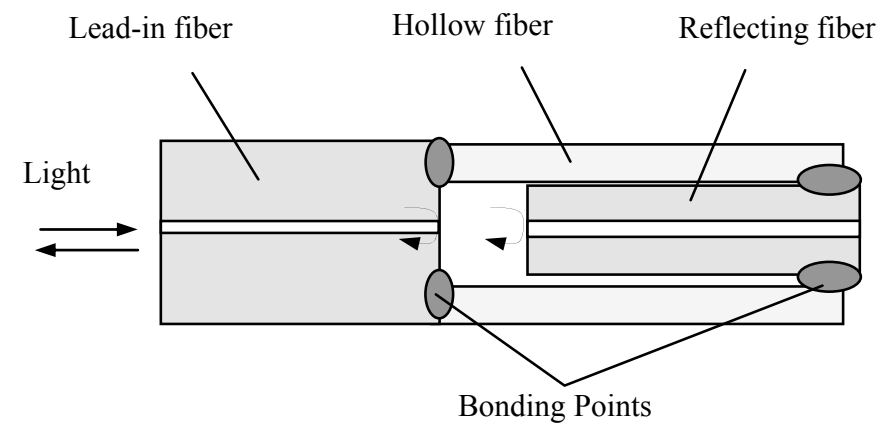

Fig. 1. Miniature fiber optic EFPI sensors

\section{SENSOR DESIGN AND FABRICATION}

A cleaved silica optical fiber $(125 \mu \mathrm{m}$ diameter) was spliced with an approximately $3.5 \mathrm{~mm}$ long hollow fiber with inside diameter (I.D.) of $75 \mu \mathrm{m}$ and outside diameter (O.D.) the same as the optical fiber by using a fusion splicer (Sumitomo, Type-36). Another optical fiber with O.D. of $60-65 \mu \mathrm{m}$ was inserted into the hollow fiber from the other end as a reflecting fiber to form a FP cavity between these two fiber end faces. The thinner fiber can be obtained by etching or drawing a standard $125 \mu \mathrm{m}$ O.D. optical fiber. The lead-in fiber was connected to a white light interferometer system. The cavity length was pre-adjusted by the splicer stage holding the reflecting fiber tail, and monitored on-line by the white light system. The air-gap can be set from zero to millimeters depending on the coherence length of the light source and application requirements. Because the fiber fusion splicer has precisely controlled alignment and movement, the sensor fabrication is simple, convenient and fast. Once the air-gap was adjusted to near the desired value, the electric arc was applied to bond the reflection fiber with the hollow fiber end. In addition, the sensor air-gap can be adjusted with a resolution as high as $3 \mathrm{~nm}$ by properly controlling the power, duration and number of electric arcs. This thermally induced air-gap control technique was recently reported ${ }^{[5]}$.

\section{Pressure Sensor}

The sensor's pressure sensitivity is determined by its gauge length. Generally, the hollow fiber was cleaved with a length near the desired gauge length to minimize the sensor size. In addition, compared with the fiber-tip-diaphragm based sensors ${ }^{[6][7]}$, this "shrinking cylinder" based pressure sensor is much easier to fabricate, resistant to scratches and has much larger pressure measurement range.

Since the hollow fiber is uniform along its axis, the air-gap change $\Delta \mathrm{G}$ under applied pressure $P$ can be calculated by ${ }^{[8]}$.

$$
\frac{\Delta G}{\Delta P}=\frac{L(1-2 \mu) D^{2}}{E\left(D^{2}-d^{2}\right)}
$$

where $d$ and $D$ are the I.D. and O.D. of the hollow fiber,

$L$ is the sensor gauge length,

$\mu$ is the Poisson's ratio,

$E$ is the Young's modulus of the hollow fiber.

Once the sensor gauge length is determined, the sensor's pressure sensitivity is fixed. The variation in sensitivity is within $0.05 \mathrm{~nm} / \mathrm{psi}$ based on the eight sensors prepared and tested. By choosing different gauge lengths from $0.2 \mathrm{~mm}$ to 
$15 \mathrm{~mm}$ the sensor can be designed for pressure measurement up to $15 \mathrm{kpsi}(103.4 \mathrm{MPa})$ with sensitivities up to 1.5 $\mathrm{nm} / \mathrm{psi}$.

The fused silica hollow fiber has a coefficient of thermal expansion (CTE) of approximately $\alpha_{\mathrm{h}}=5.5 \sim 5.8 \times 10^{-7} /{ }^{\circ} \mathrm{C}$. The single mode optical fiber used was a communication grade fiber (Corning SMF-28), with a CTE of $5.6 \times 10^{-7} /{ }^{\circ} \mathrm{C}$, which is very close to that of fused silica. The total temperature dependence of the hermetically sealed MEFPI sensor can be expressed as:

$$
\frac{\Delta G}{\Delta T}=\left(\alpha_{h}-\alpha_{r}\right) L_{r}+\alpha_{h} G+\frac{P}{T} S_{P}
$$

where $\mathrm{G}$ is the air-gap thickness, $\alpha_{\mathrm{r}}$ and $L_{r}$ is the reflecting fiber' CTE and length, $S_{P}$ is the sensor pressure sensitivity $(\mathrm{nm} / \mathrm{psi})$, and $P$ is the residue air pressure inside the hollow fiber at temperature $T(K)$. By optimizing these parameters, the sensor's temperature dependence can be minimized for a specific temperature range over which the CTEs are valid.

\section{Temperature Sensor}

For the MEFPI temperature sensor, the temperature sensitivity can be optimized by maximizing the CTE mismatch between the reflecting fiber and hollow fiber, or increasing the gauge length. We chose a multi-mode fiber as the reflecting fiber with a considerably higher CTE than the hollow fiber, which is about $7.0 \sim 7.7 \times 10^{-7} /{ }^{\circ} \mathrm{C}$. In general, the more germanium doping in the optical fiber, the higher the CTE. Moreover, fibers made of other materials can be used as the reflecting fiber such as borosilicate fiber, crystal fiber or even metal fiber.

\section{SENSOR INTERROGATION SYSTEM}

The basic configuration of the sensor interrogation system is shown in Fig. 2. The tunable laser of a Component Test System (CTS, Micron Optics) was coupled into the fiber sensor through a $2 \times 2$ coupler and the light was partially reflected $(\sim 4 \%)$ by the end faces of the lead-in and reflecting fiber. Both the reflections were routed back through the same coupler to the receiver of the CTS.

To obtain an interference spectrum with high fringe visibility, the air-gap must be within the coherence length of the light source used in the system. In this system, the coherence length is dependent on the spectral resolution of the CTS, the air-gap and the quality of the MEFPI sensor head. The interference spectrum measured by the spectrometer is given by ${ }^{[9]}$ :

$$
I(\lambda)=2 I_{s}(\lambda) \bullet\left(1+\gamma \cos \left(\frac{4 \pi G}{\lambda}+\varphi_{0}\right)\right)
$$

where $I_{s}(\lambda)$ is the spectral power distribution of the light source with wavelength $\lambda, \gamma$ is the visibility of the interference spectrum, $\varphi_{0}$ is the arbitrary initial phase difference, $G$ is the air-gap, which is determined by the physical parameters (such as pressure or temperature) to be measured. Normalizing equation (3) respect to the Gaussian spectrum of the light source, the normalized interference output can be expressed as

$$
I_{n}(\lambda)=2\left(1+\gamma \cos \left(\frac{4 \pi G}{\lambda}+\varphi_{0}\right)\right)
$$


The value of the air-gap $\mathrm{G}$ can be calculated from equation (4) and then be used to demodulate the physical parameter of interest. A typical spectrum from a MEFPI sensor is shown in Fig. 3.

A scan of the tunable laser produced the spectrum information from $1520 \mathrm{~nm}$ to $1570 \mathrm{~nm}$ with a $2.5 \mathrm{pm}$ resolution. The two reflections generated interference fringes and a valley trace method ${ }^{[10]}$ was used to process the spectrum curve to demodulate the air-gap.

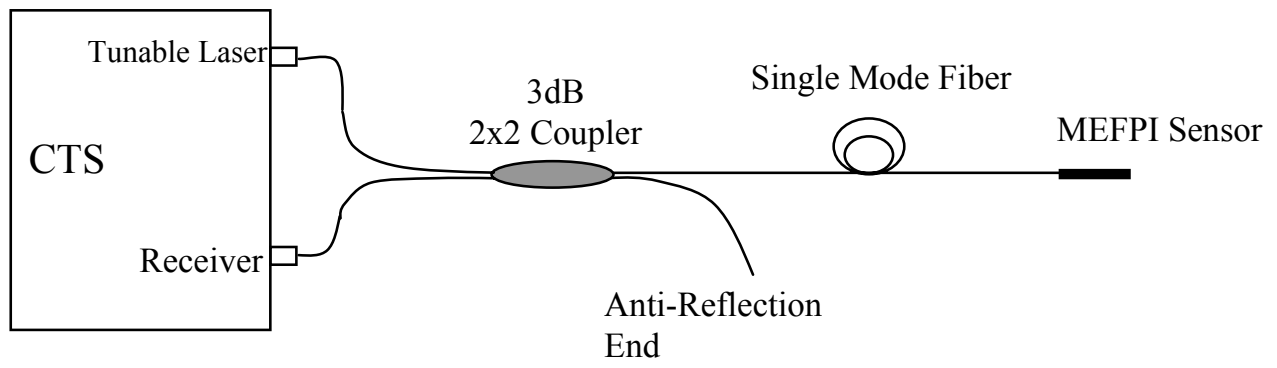

Fig. 2. Schematic of the sensor interrogation system.

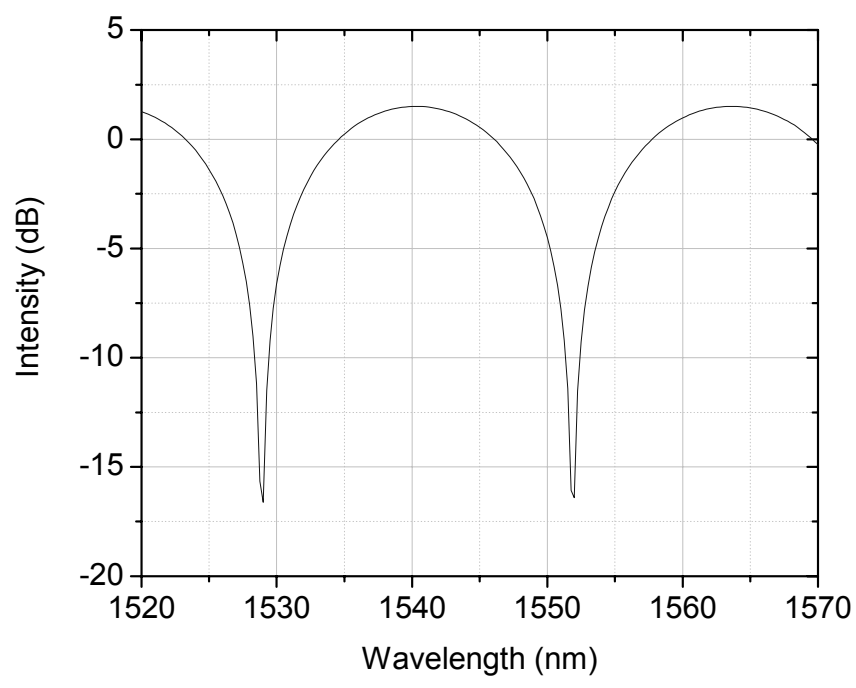

Fig.3. The typical spectrum from a MEFPI sensor

\section{EXPERIMENT RESULTS}

The air-gap measurement of the MEFPI sensors exhibited a resolution of about $0.02 \sim 0.04 \mathrm{~nm}$. The pressure sensor was calibrated by using a pressure gauge calibration system (Model 9035, Pressure Systems), while the temperature sensor was tested in a furnace (47900, Thermolyne Co.). The pressure sensor showed a linear response to static pressure $0-200$ psi at room temperature as shown in Fig. 4. The sensor pressure sensitivity was $0.36 \mathrm{~nm} / \mathrm{psi}$ and the sensor system exhibited a pressure measurement resolution of about $0.1 \mathrm{psi}(689 \mathrm{~Pa})$. The pressure sensor's temperature dependence was about $0.057 \mathrm{~nm} /{ }^{\circ} \mathrm{C}$ and the result is shown in Fig. 5. An enhanced temperature compensation method ${ }^{[11]}$ can be used to 
reduce the temperature dependence greatly. The temperature test result for the MEFPI temperature sensor is shown in Fig. 6. Its temperature sensitivity is about $0.46 \mathrm{~nm} /{ }^{\circ} \mathrm{C}$ and the measurement resolution is about $0.05^{\circ} \mathrm{C}$. When temperature increased, the sensor air-gap change was dominated by the elongation of the multi-mode fiber. If the sensor is to be used in an environment with large pressure variations, a pressure isolation scheme ${ }^{[12]}$ can be chosen in sensor fabrication.
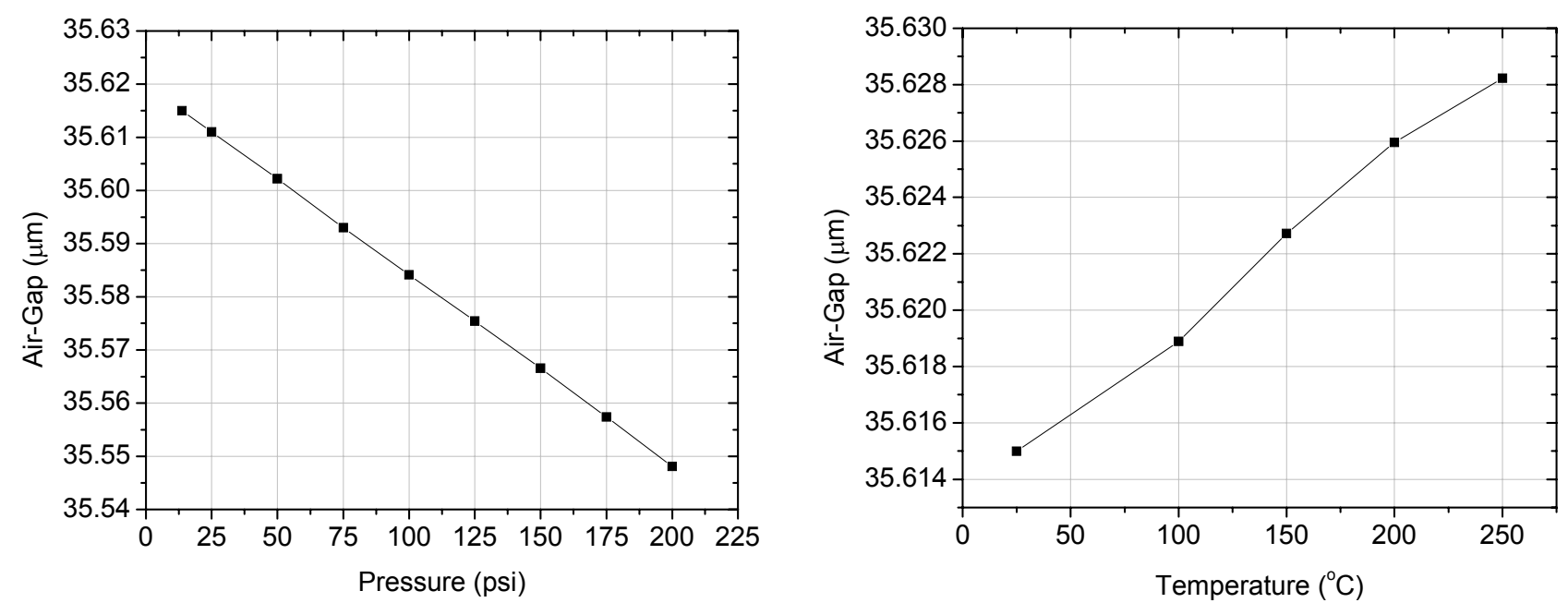

Fig. 4. Pressure sensor pressure response at $25^{\circ} \mathrm{C}$

Fig. 5. Pressure sensor temperature response at atmospheric pressure

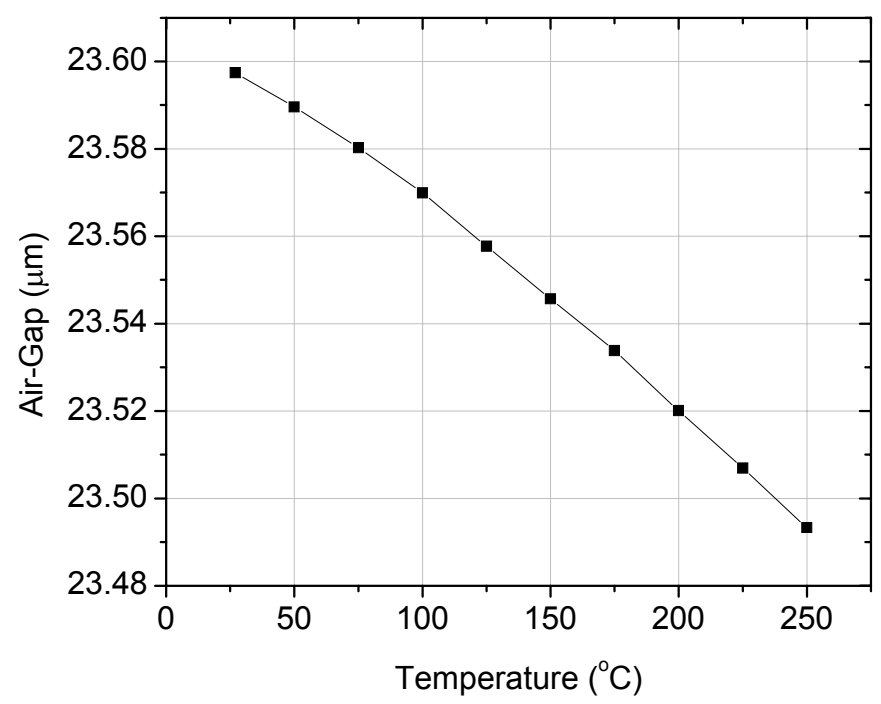

Fig. 6. Temperature sensor temperature response at atmospheric pressure. 


\section{CONCLUSIONS}

Novel miniature pressure and temperature fiber optic EFPI sensors have been described. These MEFPI sensors have the same diameter as the fiber itself and preserve advantages of conventional EFPI sensors. Simple fabrication processes have been developed to produce hermetic fusion bonds. The fabrication not only can control the sensor gauge length but also adjust the Fabry-Perot cavity with nanometers resolution. These all-silica sensors are ideal for both biomedical or industrial applications.

\section{ACKNOWLEDGEMENTS}

This work was supported by the US Department of Energy (DOE) under contract DE-FC36-01GO11050.

\section{REFERENCES}

[1] C. E. Lee, W. N. Gibler, R. A. Atkins, and H. F. Taylor, "In-Line Fiber Fabry -Perot Interferometer with HighReflectance Internal Mirrors,” J. Lightwave Technol., Vol. 10, pp.1376-1379, 1992.

[2] K. A. Murphy, M. F. Gunther, A. M. Vengsarkar, and R. O. Claus, "Quadrature phase shifted extrinsic Fabry-Perot optical fiber sensors," Opt. Lett., Vol. 16, pp. 273-275, Feb. 1991.

[3] A. Wang, H. Xiao, J. Wang, Z. Wang, W. Zhao, and R. G. May, "Self-Calibrated Interferometric-Intensity-Based Optical Fiber Sensors," J. Lightwave Technol., Vol. 19, pp. 1495-1501, Oct. 2001.

[4] J. S. Sirkis, D. D. Brennan, M. A. Putman, T. A. Berkoff, A. D. Kersey, E. J. Friebele, "In-line fiber etalon for strain measurement," Opt. Lett., Vol. 18, pp. 1973-1975, Nov. 1993.

[5] J. Xu, G. R. Pickrell, K. L Cooper, P. Zhang, and A. Wang, "Precise Cavity Length Control in Fiber Optic Extrinsic Fabry-Perot Interferometers," Conference on Lasers and Electro-Optics (CLEO), Baltimore, MD, May 2005.

[6] E. Cibula, D. Donlagic, and C. Stropnik, "Miniature fiber optic pressure sensor for medical applications," Sensors, 2002. Proceedings of IEEE, $1,711-714$, June 2002.

[7] K. Totsu, Y. Haga, and M. Esashi, "Vacuum sealed ultra miniature fiber-optic pressure sensor using white light interferometry," Transducers, Solid-State Sensors, Actuators and Microsystems, 12th International Conference, 1, 931-934, 2003.

[8] B. Qi, G. Pickrell, P. Zhang, Y. Duan, W. Peng, J. Xu, Z. Huang, J. Deng, H. Xiao, Z. Wang, W. Huo, R.G. May, and A. Wang, "Fiber optical pressure and temperature sensors for oil down hole applications," Proceedings of SPIE, 4578, 182-190, 2002.

[9] J. Dakin and B. Culshaw, “Optical Fiber Sensors: Principles and Components," Artech House, Inc., 1988.

[10]B. Qi, G. R. Pickrell, J. Xu, P. Zhang, Y. Duan, W. Peng, Z. Huang, W. Huo, H. Xiao, R. G. May, and A. Wang, "Novel data processing techniques for dispersive white light interferometer," Opt. Eng., Vol. 42, pp. 3165-3171, Nov. 2003.

[11] J. Xu, G. Pickrell, X. Wang, W. Peng, K. L Cooper and A. Wang, "A Novel Temperature Insensitive Optical Fiber Pressure Sensor For Harsh Environments," IEEE Photon. Technol. Lett. Vol. 17, 870-872 2005.

[12] J. Xu, G. R. Pickrell, Z. Huang, B. Qi, P. Zhang, Y. Duan, and A. Wang, "Double-Tubing Encapsulated Fiber Optic Temperature Sensor," in 8th Temperature Symposium, AIP Conference Proceedings, Vol. 684, pp. 1021-1026, Sept. 2003. 


\title{
High-Temperature Fiber-Tip Pressure Sensor
}

\author{
Yizheng Zhu, Student Member, IEEE, Kristie L. Cooper, Member, IEEE, Gary R. Pickrell, Member, IEEE, \\ and Anbo Wang, Senior Member, IEEE
}

\begin{abstract}
This paper presents a miniature fiber-optic hightemperature pressure sensor fabricated on the tip of a singlemode (SM) fiber by means of fusion splicing, cleaving, and wet chemical etching. A new approach was developed to simplify the fabrication and greatly improve the sensitivity. The sensor is made entirely of fused silica, whose high-temperature sensing capability is explored in detail for the first time. Two sensors were tested up to $611{ }^{\circ} \mathrm{C}$ and $710{ }^{\circ} \mathrm{C}$, respectively, showing excellent repeatability better than $0.62 \%$ and $1.4 \%$. The maximum operating temperature is limited by the mechanical creep of the fused silica diaphragm.
\end{abstract}

Index Terms-Fabry-Pérot (FP) interferometer, fiber-optic sensor, high temperature, miniature, pressure sensor.

\section{INTRODUCTION}

$\mathbf{H}$ IGH-TEMPERATURE pressure sensing is highly desired in such industries as automotive and aerospace. For example, in a jet engine compressor, pressure measurements would help to detect and control surge and stall to improve its performance and efficiency. However, the temperatures can reach as high as $600{ }^{\circ} \mathrm{C}$ for today's large engines. Such harsh environment applications impose great challenges on both the design and materials of the sensors and packaging, and generate a rising need for high-temperature pressure sensors.

Traditional semiconductor pressure sensors are micromachined on a silicon diaphragm with piezoresistors diffused into it. These sensors are generally limited to below $150{ }^{\circ} \mathrm{C}$ if used without an extra cooling mechanism due to the increasing p-n junction leakage. An improved silicon-on-insulator (SOI) structure isolates the piezoresistors from the silicon diaphragm by a layer of silicon dioxide and is operational up to about $500{ }^{\circ} \mathrm{C}$ where silicon starts to creep [1], [2].

Other high-temperature materials have also been explored to construct pressure sensors, nearly all of which inherit the diaphragm-based design and employ either piezoresistive or capacitive detection. Among these materials, silicon carbide (SiC) has received more attention as a long-recognized hightemperature semiconductor material for microelectromechanical systems (MEMSs). Okojie et al. proposed an $\alpha(6 \mathrm{H})-\mathrm{SiC}$ pressure sensor functional up to $500{ }^{\circ} \mathrm{C}$ [2]. Berg et al. demon-

Manuscript received February 1, 2005; revised August 11, 2005. This work was supported by the U.S. Department of Energy under Grant DE-FC36$01 \mathrm{G} 011050$.

The authors are with the Center for Photonics Technology, Bradley Department of Electrical and Computer Engineering, Virginia Polytechnic Institute and State University, Blacksburg, VA 24061 USA (e-mail: yizhu1@vt.edu; klcooper@vt.edu; pickrell@vt.edu; awang@vt.edu).

Digital Object Identifier 10.1109/JLT.2005.862444 strated the operation of a $\beta$-SiC pressure sensor in a combustion engine to about $300{ }^{\circ} \mathrm{C}$ [3]. Du et al. reported $400{ }^{\circ} \mathrm{C}$ test results of a capacitive sensor using $\beta$-SiC diaphragm as well. Ceramic [4], sapphire [5], and diamond [6] materials also have the potentials for high-temperature pressure sensing.

Compared to these electronic sensors, fiber-optic sensors are known for their immunity to electromagnetic interference (EMI), electrical passivity, high resolution, and high accuracy. Fiber-optic pressure sensors employing various techniques have been investigated extensively, yet, very few of them have been designed for high-temperature operation. Pulliam et al. proposed an $\mathrm{SiC}$ diaphragm-based interferometric pressure sensor promising to operate at high temperatures [7]. The key factor limiting fiber-optic pressure sensors' high-temperature capability is that their sensor head assemblies usually involve various materials other than fibers' fused silica, including silicon, low softening point glass (e.g., Pyrex), polymer, epoxy, or other adhesives. The thermal expansion mismatch between these materials can cause stress and degrade the structure's mechanical strength and stability at elevated temperatures. In addition, the common use of low-temperature materials (especially polymers and adhesives) will significantly affect the sensors' accuracy and repeatability.

Fused silica has excellent optical and mechanical properties, but it is rarely the choice for high-temperature sensing diaphragms mainly because of the difficulties in bonding, which used to require low-temperature adhesives. In this paper, we present an all-fused-silica pressure sensor with its diaphragm fabricated directly onto the fiber tip through fusion splicing, cleaving, and chemical etching. It eliminates thermal expansion mismatch and avoids any adhesives; it is, therefore, expected to be functional at high temperatures. Although its softening point is around $1600{ }^{\circ} \mathrm{C}$, fused silica will start creeping at much lower temperatures, setting the limit for this sensor's capability, the study of which is the primary goal of this research.

The authors have previously reported a sensor based on similar fabrication techniques [8]. In this paper, we introduce new processes to significantly reduce the diaphragm thickness and reach sensitivities more than one order of magnitude higher than the previous design in shorter time. The sensor's hightemperature performance is investigated in detail, which, to the best of our knowledge, is the first report on this topic. With a size no larger than the $125-\mu \mathrm{m}$ fiber diameter, the sensor is believed to be the smallest among high-temperature pressure sensors. Such a miniature size will allow more applications where minimal invasion is desired.

The paper is structured as follows. In Section II, the sensor fabrication is described and important issues in design are discussed. Section III then introduces the testing setup and signal 

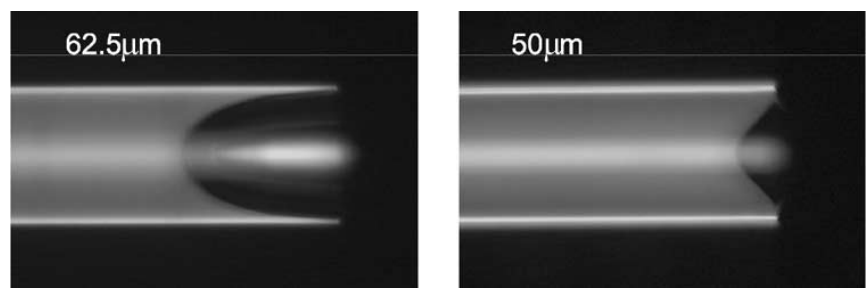

Fig. 1. MM fibers etched in $50 \% \mathrm{HF}$ for $5 \mathrm{~min}$.

processing techniques. Finally, the sensor's high-temperature test results are analyzed in Section IV.

\section{SEnsor Design And FAbrication}

\section{A. Fabrication}

The fabrication process utilizes the fact that germaniumdoped and undoped fused silica can be etched at different rates in buffered hydrofluoric acid (BHF) [9], [10]. For a Ge-doped multimode (MM) fiber, its core (doped) is etched much faster than the cladding (undoped), as shown in Fig. 1, where gradedindex MM fibers with 62.5- and 50- $\mu \mathrm{m}$ core were immersed in $50 \% \mathrm{HF}$ for $5 \mathrm{~min}$ to remove their cores yet keep the cladding, generating a hollow-core structure that forms the basis of the fabrication. The unevenly etched cores reflect the graded doping profile as different dopant concentrations results in different etching rates.

In $50 \% \mathrm{HF}$, the center of the $62.5-\mu \mathrm{m}$ fiber core will be etched at a rate of about $17 \mu \mathrm{m} / \mathrm{min}$, while the undoped fused silica has a much slower rate of $1 \mu \mathrm{m} / \mathrm{min}$.

The fabrication steps are shown in Fig. 2, involving three types of silica fibers, namely: 1) singlemode (SM) fiber; 2) $62.5 / 125 \mu \mathrm{m}$ graded-index MM fiber; and 3) $105 / 125 \mu \mathrm{m}$ step-index MM fiber. The 62.5- $\mu \mathrm{m}$ fiber has a Ge-doped core with undoped fused silica cladding, while the $105 / 125-\mu \mathrm{m}$ fiber has an undoped fused silica core and fluorine-doped cladding that can be quickly etched off and is of no use here. The 50\% HF is used for wet chemical etching.

The first step, as in Fig. 2(a), is to prepare a thin layer of undoped fused silica on the end of a doped fiber, achieved by fusion splicing the 105- and $62.5-\mu \mathrm{m}$ fiber and cleaving the $105-\mu \mathrm{m}$ fiber. Standard splicing parameters are used, and the cleaving is done through a $40 \times$ microscope. This thin layer usually can be made about $10 \mu \mathrm{m}$ thick or less, serving two purposes in the following steps.

The second step, as in Fig. 2(b), produces a cavity on the SM fiber tip. A complete assembly from step (a) is spliced with the SM fiber using standard parameters. The $62.5-\mu \mathrm{m}$ fiber is then cleaved to a certain length, followed by etching to remove the core and generate the cavity. The thin layer of fused silica serves as an etch stop to form a flat cavity bottom and protect the SM fiber core from being etched. Fig. 3(a) and (b) shows photos of the cavity bottom and edge after etch.

The final step, as in Fig. 2(c), is to fabricate a diaphragm to seal the cavity. Again, one complete assembly from step (a) is spliced to the cavity produced in step (b), but with special splicing required. The power and the duration of the splicing arc need to be significantly reduced to prevent overheating and cavity collapse. Then the $62.5-\mu \mathrm{m}$ fiber is again cleaved and etched, exposing the thin layer of fused silica, which, in this step, serves as the diaphragm. This diaphragm can be further etched to adjust its thickness and, therefore, the sensor's sensitivity. Fig. 3(c) shows a photo of a very thin diaphragm fabricated this way, where the concentric rings are the interference fringes of the two diaphragm surfaces under illumination, indicating a submicron thickness. The increasing fringe density from center to perimeter reveals a slightly concave surface as a result of the graded dopant profile in the $62.5-\mu \mathrm{m}$ fiber core whose center reaches the diaphragm first.

The final structure is primarily composed of undoped fused silica. Some residue of the $62.5-\mu \mathrm{m}$ core may remain around the cavity bottom, as seen in the photos in Fig. 2.

\section{B. Improvement}

This new process, especially the diaphragm, is a significant improvement over the former design described in [8], where the diaphragm was fabricated by splicing a $105-\mu \mathrm{m}$ fiber to the cavity, cleaving and etching it down to improve the sensitivity. Due to the nonsmooth fiber surface at the splicing point resulting from the low-power arc, as well as the existence of the cavity, the $105-\mu \mathrm{m}$ fiber cleave had to be made some distance away from the splicing point, usually $20-25 \mu \mathrm{m}$. This relatively large initial thickness took longer to etch, because it was all undoped fused silica. On the other hand, the thickness of the cavity wall imposed an upper bound on the etching time, limiting the fabrication flexibility to achieve a very thin thickness and very high sensitivity.

The current process, however, prefabricates the diaphragm in the first step where the standard splicing produces a smooth surface at the splicing point allowing cleaving less than $10 \mu \mathrm{m}$ away with high quality [Fig. 2(a)]. In the final step, the $62.5-\mu \mathrm{m}$ fiber serves just as a sacrificial layer to support this thin diaphragm during splicing but is removed later. Therefore, neither the length nor the quality of the final cleave of this $62.5-\mu \mathrm{m}$ fiber [Fig. 2(c)] is very critical. After a quick etching (usually $1-2 \mathrm{~min}$ ), the sacrificial layer ( $62.5 \mu \mathrm{m}$ core) is removed to expose the high-quality thin diaphragm. Then relatively shorter etching could further reduce its thickness. This way, the diaphragm deflection is an order of magnitude more sensitive than before, which improved from tenths of a nanometer per psi $\left(1 \mathrm{psi}=6.89 \times 10^{3} \mathrm{~Pa}\right)$ to a few nanometers per psi, or even higher. The diaphragm shown in Fig. 3(c) responds to pressure with about $20 \mathrm{~nm} / \mathrm{psi}$.

In addition to higher sensitivity and shorter etching time, the new process also offers the potential for batch processing in the first step by depositing silica dioxide to form the thin layer. This could possibly produce even thinner diaphragm and higher quality.

\section{Sensor Signal Analysis}

In this Fabry-Pérot (FP) interferometric sensor, light launched into the SM fiber will generate three reflections, one from the bottom of the cavity $\left(E_{1}\right)$, and the inner $\left(E_{2}\right)$ and outer 
(a)

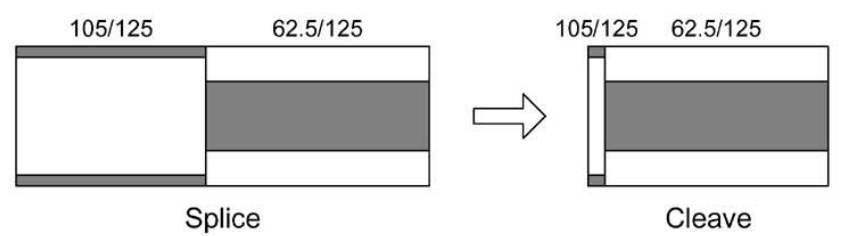

(b)

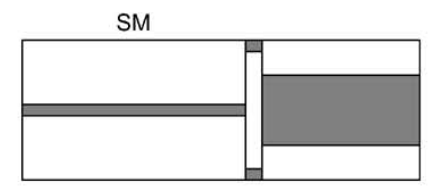

Splice

(c)

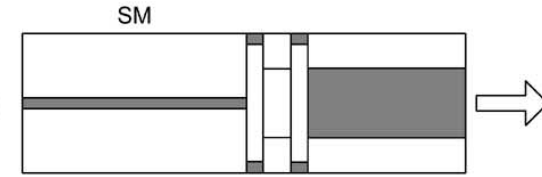

Splice

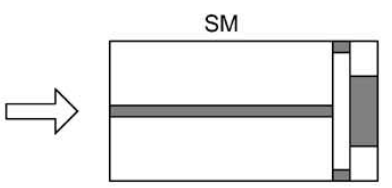

Cleave

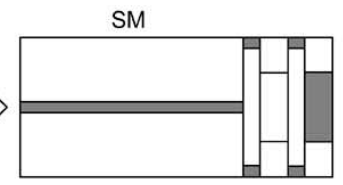

Cleave

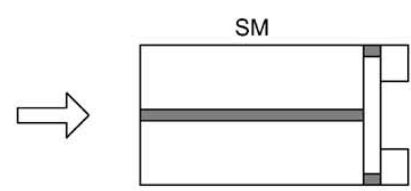

Etch

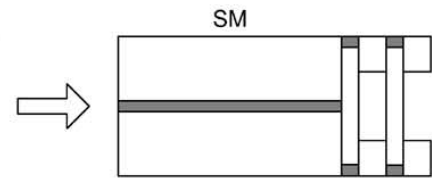

Etch
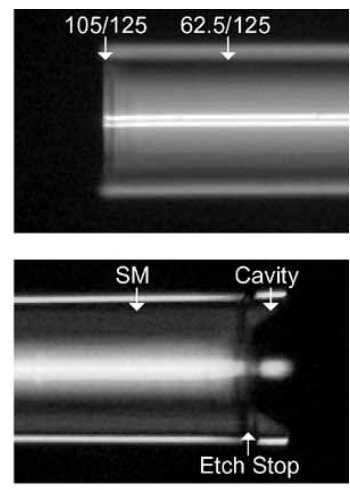

SM

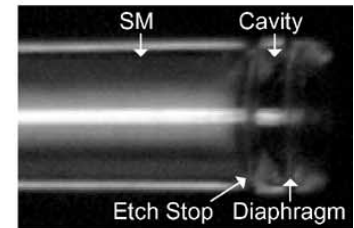

Fig. 2. Sensor fabrication processes.

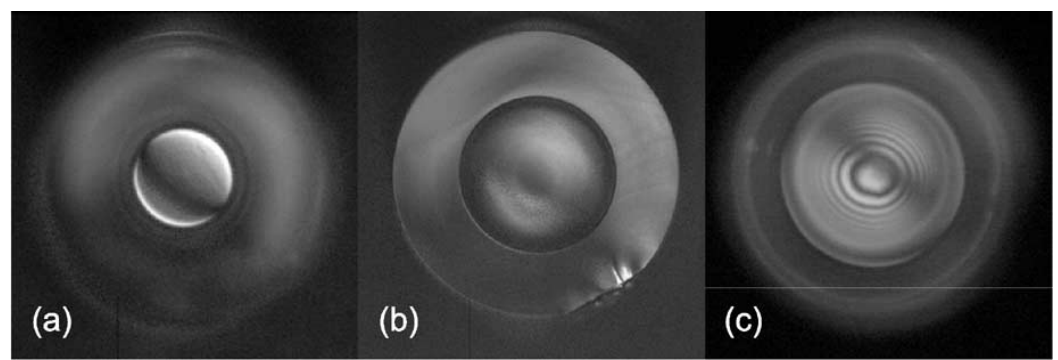

Fig. 3. Photos. (a) Cavity edge. (b) Cavity bottom. (c) Diaphragm.

$\left(E_{3}\right)$ surfaces of the diaphragm. The total reflected intensity can be written as

$$
\begin{aligned}
I(\lambda)= & \left|E_{1}+E_{2}+E_{3}\right|^{2} \\
= & \mid A_{1}+A_{2} \exp \left(-\frac{\mathrm{j} 4 \pi L}{\lambda}+\mathrm{j} \pi\right) \\
& +\left.A_{3} \exp \left(-\frac{\mathrm{j} 4 \pi(L+n d)}{\lambda}\right)\right|^{2} \\
= & A_{1}^{2}+A_{2}^{2}+A_{3}^{2}-2 A_{1} A_{2} \cos \left(\frac{4 \pi L}{\lambda}\right) \\
& -2 A_{2} A_{3} \cos \left(\frac{4 \pi n d}{\lambda}\right) \\
& +2 A_{3} A_{1} \cos \left(\frac{4 \pi(L+n d)}{\lambda}\right)
\end{aligned}
$$

where $A_{1}, A_{2}$, and $A_{3}$ are the amplitudes of $E_{1}, E_{2}$, and $E_{3}$, respectively; $L$ is the cavity length, $d$ is the diaphragm thickness, $n$ is the refractive index of fused silica, and $\lambda$ is the wavelength. $E_{2}$ has an extra phase shift of $\pi$ at the air-silica interface.
Unlike two-beam interference, $E_{3}$ can either strengthen or weaken $E_{2}$, hence, modulating the entire spectrum with a slowly varying envelope, as discussed in the following.

If the optical thickness of the diaphragm, $n d$, satisfies

$$
2 n d=N \lambda+\frac{\lambda}{2}, \quad N=0,1,2, \ldots
$$

$E_{2}$ and $E_{3}$ will have a phase difference of

$$
\frac{4 \pi n d}{\lambda}+\pi=(2 N+2) \pi, \quad N=0,1,2, \ldots
$$

and, therefore, add in phase and strengthen each other. Then (1) becomes

$$
I(\lambda)=A_{1}^{2}+\left(A_{2}+A_{3}\right)^{2}-2 A_{1}\left(A_{2}+A_{3}\right) \cos \left(\frac{4 \pi L}{\lambda}\right)
$$

with a maxima of

$$
\begin{aligned}
I_{\max }^{+} & =A_{1}^{2}+\left(A_{2}+A_{3}\right)^{2}+2 A_{1}\left(A_{2}+A_{3}\right) \\
& =\left(A_{1}+A_{2}+A_{3}\right)^{2} .
\end{aligned}
$$

Similarly, when

$2 n d=N \lambda \Rightarrow \frac{4 \pi n d}{\lambda}=(2 N+1) \pi, \quad N=0,1,2, \ldots$ 


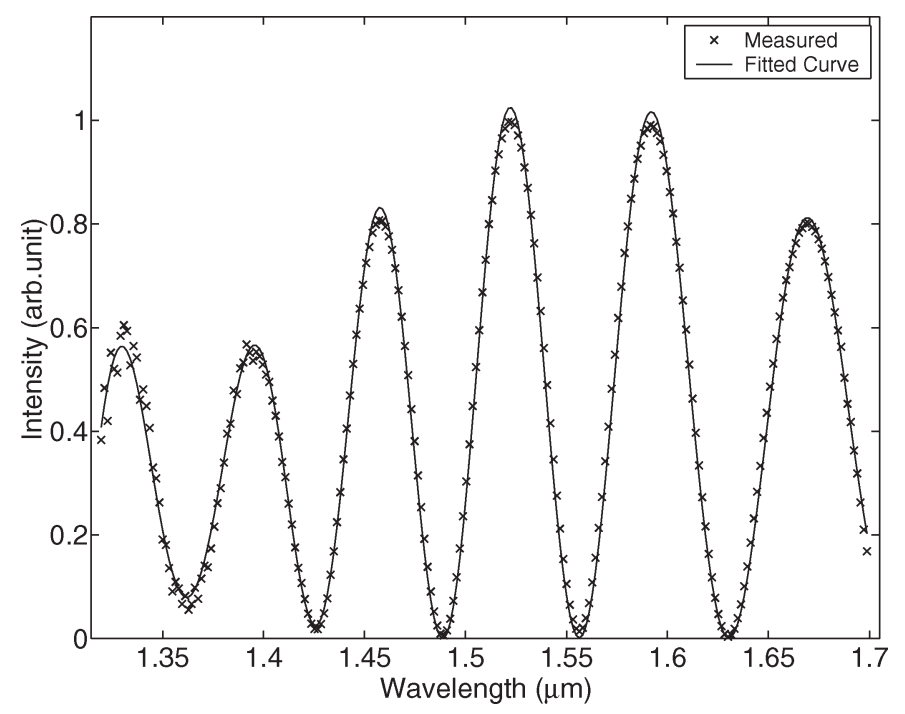

Fig. 4. Output spectrum of sensor 1 at room temperature and pressure.

$E_{2}$ and $E_{3}$ will be out of phase and weaken each other to reduce the total reflected energy, with a maximum power of only

$$
\begin{aligned}
I_{\max }^{-} & =A_{1}^{2}+\left(A_{2}-A_{3}\right)^{2}+2 A_{1}\left(A_{2}-A_{3}\right) \\
& =\left(A_{1}+A_{2}-A_{3}\right)^{2} .
\end{aligned}
$$

Although not necessarily required, the add-in-phase case is preferred for higher signal power and achieved by monitoring the signal strength during etching. Equation (2) results in a discrete "optimal" diaphragm thickness. For instance, if $N=3, \lambda=1.55 \mu \mathrm{m}$, and $n=1.444, d_{\mathrm{opt}}$ is determined to be $1.879 \mu \mathrm{m}$.

Fig. 4 plots the spectrum of sensor 1 at room temperature (RT) and pressure from 1320 to $1700 \mathrm{~nm}$ with its maximum power located around $1550 \mathrm{~nm}$ and its minimum around $1370 \mathrm{~nm}$, clearly showing the modulation by $E_{3}$. The spectrum is fitted using (1) with $A_{1}=0.495, A_{2}=0.371, A_{3}=0.158$, $L=16.343 \mu \mathrm{m}$, and $d=1.883 \mu \mathrm{m}$, very close to the optimal thickness calculated above.

\section{Sensitivity}

When ambient pressure varies, the diaphragm will deflect and the cavity length $L$ will change according to [11]

$$
\begin{aligned}
\Delta L & =\frac{3(1-\nu) R^{4}}{16 E d^{3}} \Delta p \\
& \triangleq S_{P} \Delta p
\end{aligned}
$$

where $\nu$ and $E$ are Poisson's ratio and Young's modulus of fused silica, respectively; $R$ is the radius of the diaphragm, $d$ is the diaphragm thickness, $\Delta p$ is the ambient pressure change, and $S_{P}$ is the sensor's pressure sensitivity. Fig. 5 shows the change in $S_{P}$ with $d$, where $E=73 \mathrm{GPa}, \nu=0.17$, and $R$ is estimated to be $33 \mu \mathrm{m}$, which is slightly larger than the radius of the core due to etching. $S_{P}$ increases quickly to a few nanometers per psi when $d$ decreases below $2 \mu \mathrm{m}$. The open

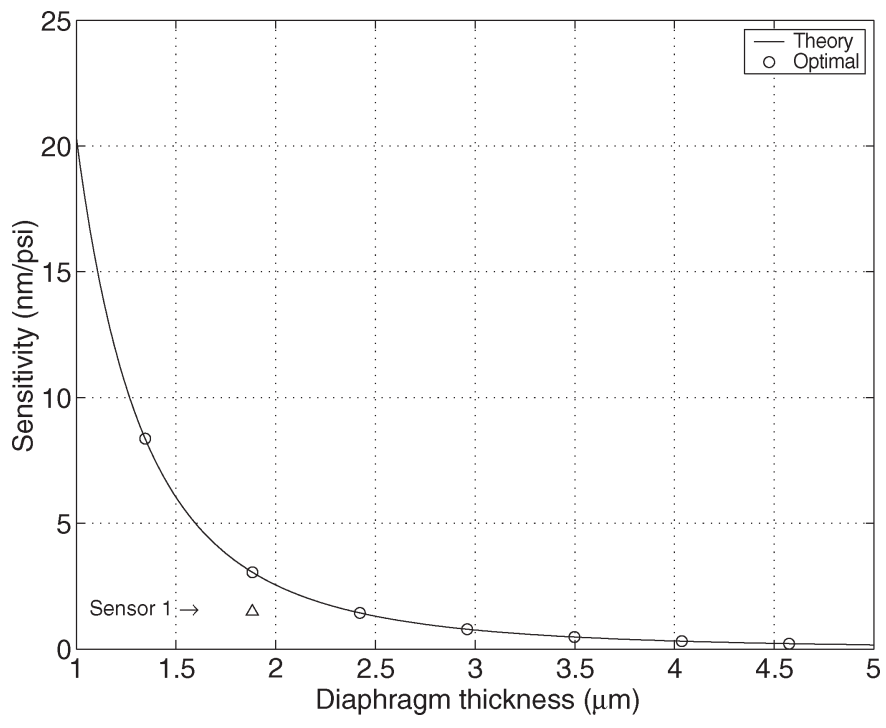

Fig. 5. Theoretical sensitivity for the sensor.

circles are the theoretical sensitivity at the "optimal" thickness derived by (2) for $1550 \mathrm{~nm}$.

For sensor 1's 1.883- $\mu \mathrm{m}$ diaphragm, its measured sensitivity, $1.49 \mathrm{~nm} / \mathrm{psi}$ as marked by the triangle in Fig. 5, is about half the theoretical value of $3.05 \mathrm{~nm} / \mathrm{psi}$. One error source is parameter accuracy. Another contribution may be the concave diaphragm surface mentioned above, which has an average thickness larger than its center where light beam mainly focuses, thus, reducing the sensitivity.

\section{Testing System And Signal Processing}

To investigate the sensor's high-temperature capability, spectrum detection was used to study how the cavity length responds to static pressure changes at different temperatures.

\section{A. Testing System}

Fig. 6 shows the test setup. The spectrometer is a Micron Optics si720 Component Testing System (CTS), which has a built-in tunable laser source and a detector, sweeping from 1520 to $1570 \mathrm{~nm}$ with a resolution of $2.5 \mathrm{pm}$ at $5 \mathrm{~Hz}$ to interrogate the sensor through a circulator. The sensor was sealed in a mullite tube and pressurized by a pressure calibrator (Pressure Systems, Inc., 9035). The sensor end of the tube was placed in a furnace (Thermolyne 48000) together with a K-type thermocouple for temperature reference. A computer controls the pressure calibrator and retrieves spectrum and temperature data from CTS and a temperature meter (OMEGA Dpi32-C24).

\section{B. Signal Demodulation}

Fig. 7 plots the sensor spectrum by CTS at different pressures in logarithmic scale. Increased pressure will reduce the cavity length and shift the spectra to shorter wavelengths. By monitoring the shift and the change in cavity length $L$, the pressure can be demodulated. 


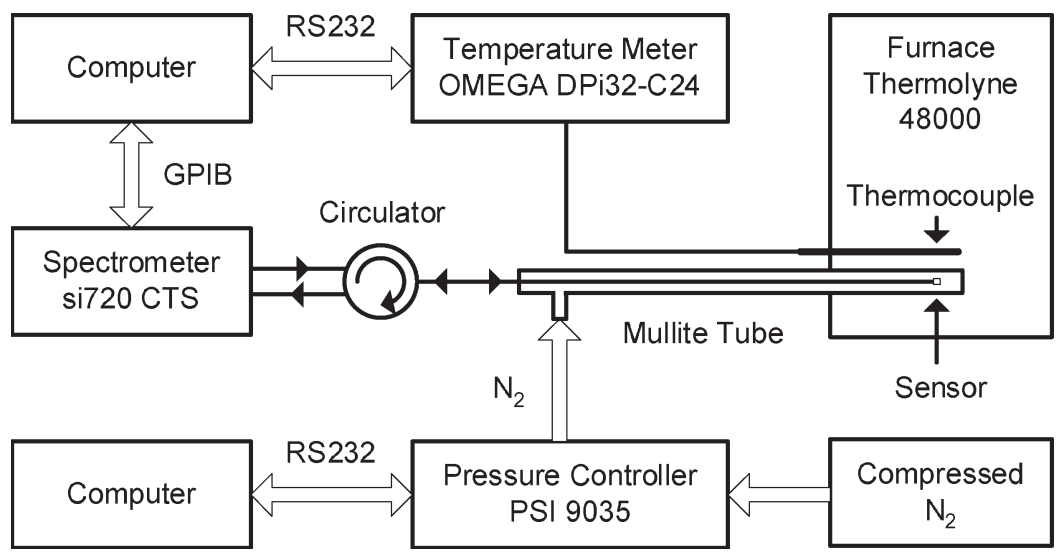

Fig. 6. System setup for high-temperature static pressure test.

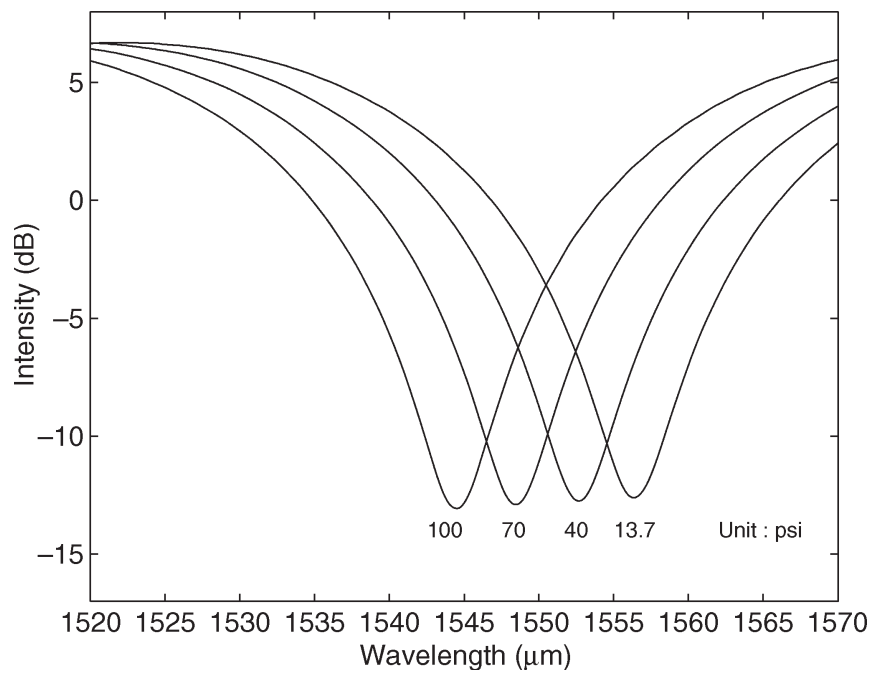

Fig. 7. Sensor spectra at $13.7,40,70$, and 100 psi.

Curve fitting the entire spectrum for $L$ is computationally ineffective and very sensitive to the initial conditions, therefore, impractical for signal demodulation. Tracing the interfering minima (valley) or maxima (peak) of the spectrum is a widely employed technique proven to be highly effective [12]. For example, if the position of a valley $\lambda_{v}$ has been determined, the cavity length can be calculated as

$$
\frac{4 \pi L_{\mathrm{calc}}}{\lambda_{v}}+\pi=2 N \pi \Rightarrow L_{\mathrm{calc}}=\frac{(2 N-1)}{4} \lambda_{v}
$$

where $N$ is the fringe order (integer for interference minima and half-integer for interference maxima). With $N$ predetermined from the spectrum, $L_{\text {calc }}$ is proportional to $\lambda_{v}$. In the algorithm, a low-pass filter is first used to remove the noise and then a local parabolic curve fitting is performed just around the valley to determine $\lambda_{v}$ with high resolution.

Equation (9) is accurate only for two-beam interference, while in this sensor, $E_{3}$ modulates the spectrum and $L_{\text {calc }}$ is not necessarily equal to the actual cavity length $L$. However, due to the thin diaphragm thickness, the envelope is only slowly varying and the spectrum is only slightly modulated. In addition, satisfaction of the optimal thickness condition of

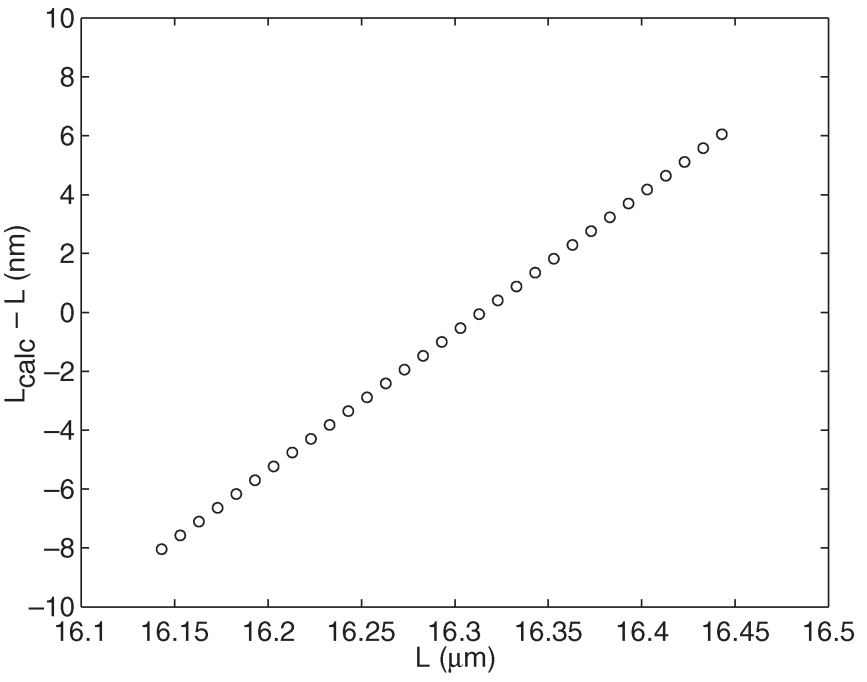

Fig. 8. Cavity length simulation: actual value $L$ versus calculated value $L_{\text {calc }}$.

(2), which is the case for sensor 1, will make the envelope even flatter at the working wavelength. These will reduce the error in using $L_{\text {calc }}$ as an estimator of $L$.

To examine more closely on how the $E_{3}$ modulation would deviate $L_{\text {calc }}$ from $L$, the previously generated fitting values for Fig. 4 were used to produce simulated sensor spectra. Changing $L$ while keeping $A_{1}, A_{2}, A_{3}$, and $d$ the same simulates the case where the sensor is under varying pressure. Then the valley-tracing algorithm was applied to these spectra to determine $L_{\text {calc }}$. The difference between $L_{\text {calc }}$ and $L$ is plotted in Fig. 8. For a $300-n m$ change in $L$ around its room ambient position, $L_{\text {calc }}$ lies within $\pm 8 \mathrm{~nm}$, confirmed as an excellent estimator of $L$.

In all the following discussions, if not otherwise mentioned, the cavity length will refer to $L_{\text {calc }}$.

\section{Resolution}

Fig. 9 shows a short duration stability test of the system, recording the cavity length for $5 \mathrm{~min}$ at RT with a standard deviation only about $0.002 \mathrm{~nm}$. This means that for the $1.49 \mathrm{~nm} / \mathrm{psi}$ sensitivity of sensor 1 , the system is able to resolve a pressure change about $0.002 \mathrm{~nm} / 1.49 \mathrm{~nm} / \mathrm{psi}=0.0013 \mathrm{psi}$, 

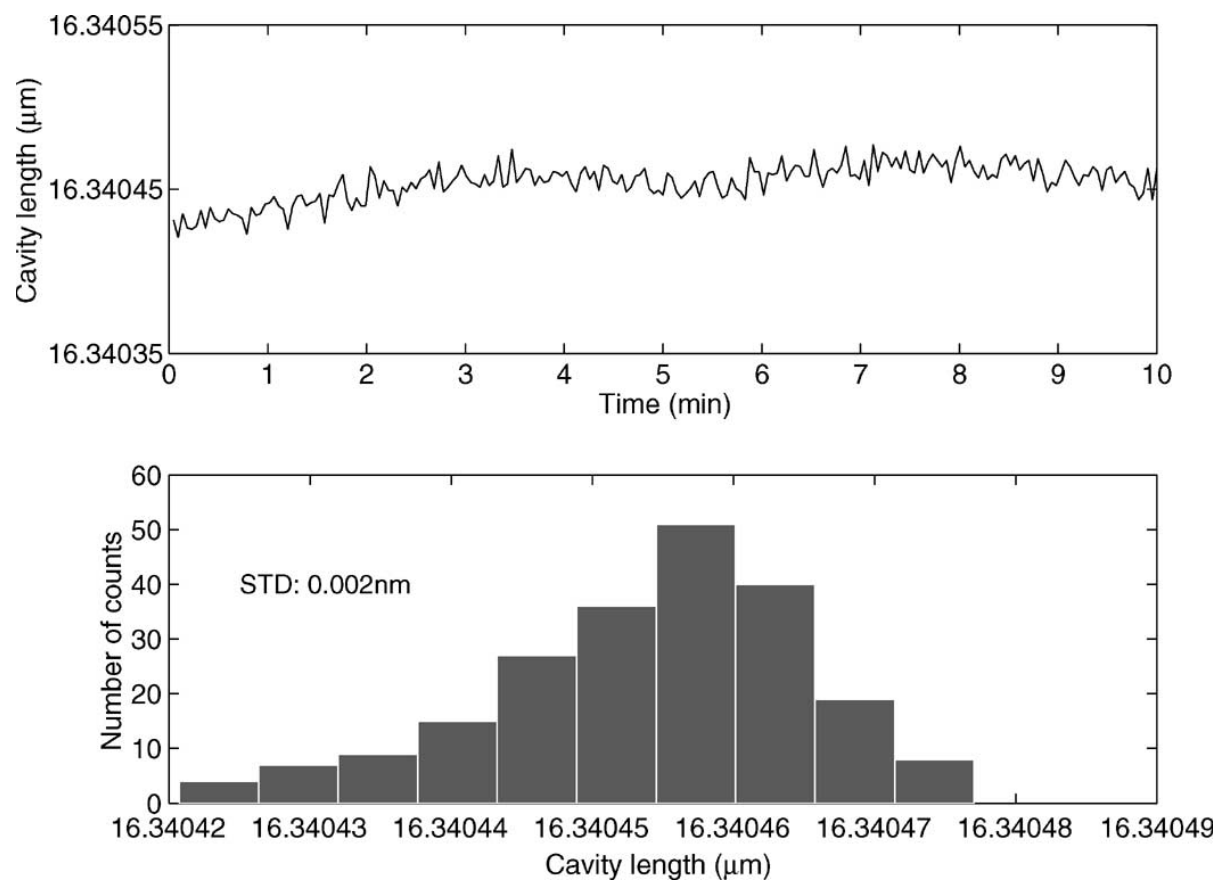

Fig. 9. System resolution test.

an excellent resolution owing to the high performance of both the algorithm and the CTS.

\section{High-Temperature Test Results}

Sensor 1 was tested at $24{ }^{\circ} \mathrm{C}, 308{ }^{\circ} \mathrm{C}, 412{ }^{\circ} \mathrm{C}, 513{ }^{\circ} \mathrm{C}$, $562{ }^{\circ} \mathrm{C}$, and $611^{\circ} \mathrm{C}$, with the decreasing temperature increment intended for a closer study focused on the sensor's hightemperature performance and the service limit of the fused silica diaphragm. At each temperature, the sensor was tested from 15 to 30 psi with a step of $0.5 \mathrm{psi}$ for three cycles. The full-scale cavity length change was $23.4 \mathrm{~nm}$.

For comparison, sensor 2 with a thicker diaphragm $(4.114 \mu \mathrm{m})$ and lower sensitivity $(0.17 \mathrm{~nm} / \mathrm{psi})$ was also tested, but at two more temperatures, $661{ }^{\circ} \mathrm{C}$ and $710{ }^{\circ} \mathrm{C}$. The applied pressure was from 20 to $160 \mathrm{psi}$ in steps of $10 \mathrm{psi}$, also for three cycles. The full range diaphragm deflection was $23.8 \mathrm{~nm}$, almost the same as sensor 1 .

\section{A. Pressure Response}

Fig. 10 shows the pressure test results for sensor 1 at all temperatures with all three cycles of data plotted together. For temperatures up to $561{ }^{\circ} \mathrm{C}$, the results of the three cycles almost perfectly overlap each other, while the $611{ }^{\circ} \mathrm{C}$ results are less repeatable due to the drift caused by creep. Sensor 2 exhibited similar curves. The results demonstrate the sensor's operability up to the $600{ }^{\circ} \mathrm{C}-700{ }^{\circ} \mathrm{C}$ range.

\section{B. Pressure Sensitivity}

Pressure responses at each temperature are linear fitted for both sensors. The slopes represent the pressure sensitivity $S_{P}$, plotted against temperature in Fig. 11, showing a decrease by

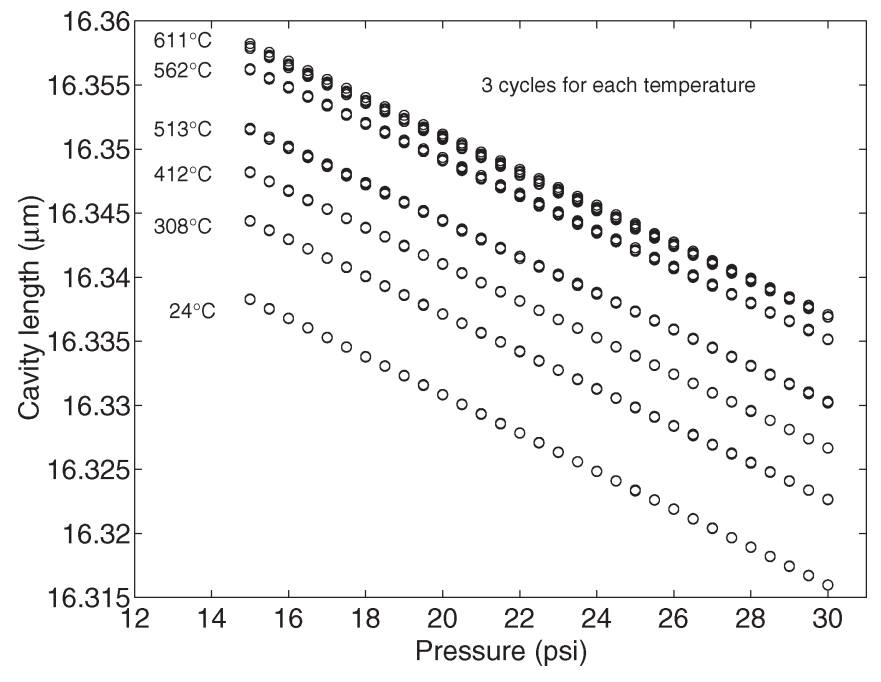

Fig. 10. Sensor testing results at different temperatures.

$5.9 \%$ from $24{ }^{\circ} \mathrm{C}$ to $611^{\circ} \mathrm{C}$ for sensor 1 , and $6.6 \%$ from $24{ }^{\circ} \mathrm{C}$ to $710^{\circ} \mathrm{C}$ for sensor 2 . This is the result of a slightly increasing Young's modulus and Poisson's ratio of fused silica with rising temperature, as reported by Fukuhara et al. [13] and reflected through (8).

\section{Repeatability}

The sensor becomes less repeatable at higher temperatures. The sensor repeatability was estimated as follows. At each temperature, the cavity length deviations from the linear fit were calculated for all data points in the three cycles. The ratio of the average deviation $\varepsilon_{\text {avg }}$ to the full-scale cavity length change $\Delta L_{\mathrm{FS}}, \varepsilon_{\mathrm{avg}} / \Delta L_{\mathrm{FS}}$, is defined as the relative repeatability and plotted in Fig. 12, which shows a deteriorating 


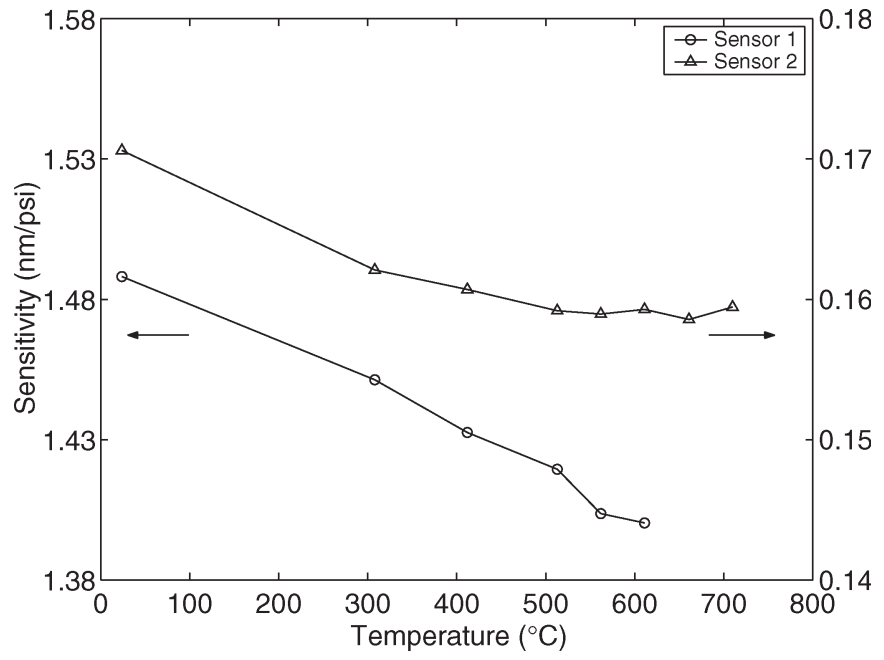

Fig. 11. Sensitivity versus temperature.

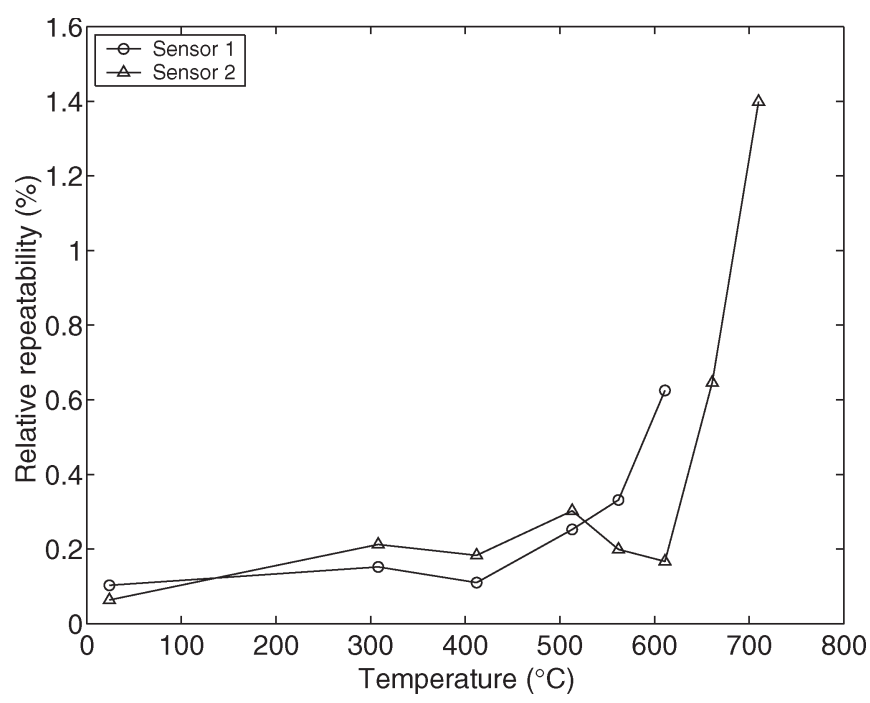

Fig. 12. Relative repeatability of the sensors at different temperatures.

repeatability, from $0.10 \%$ at $24{ }^{\circ} \mathrm{C}$ to $0.62 \%$ at $611{ }^{\circ} \mathrm{C}$ for sensor 1 and from $0.06 \%$ at $24{ }^{\circ} \mathrm{C}$ to $1.40 \%$ at $710{ }^{\circ} \mathrm{C}$ for sensor 2 , as the result of increasing material creep at higher temperatures.

For sensor 2, the deviation starts increasing significantly at around $600{ }^{\circ} \mathrm{C}$, about $50{ }^{\circ} \mathrm{C}$ higher than for sensor 1 . Although this is not much, it may be an indication that the thicker diaphragm exhibits less creep than a thinner one when subject to the same amount of deflection.

\section{Material Creep}

Material creep is the major factor limiting the sensor's hightemperature repeatability. Fig. 13 shows the test of sensor 1 at $611{ }^{\circ} \mathrm{C}$ when pressurized by a step change from ambient pressure (13.7 psi) to 50 psi for $10 \mathrm{~min}$ and then back to ambient pressure. At $50 \mathrm{psi}$, the cavity underwent a 1-nm drift, about $1.8 \% \mathrm{FS}$, in an exponential-like manner. After release, it slowly

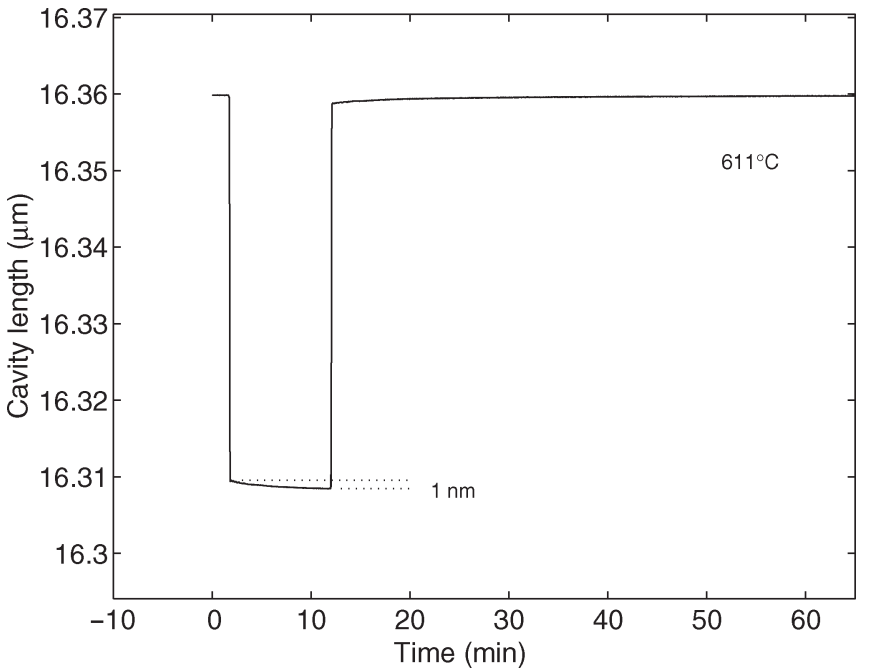

Fig. 13. Creep of sensor 1 at constant pressure and temperature.

returns to its original position, although the time constant is increased, indicating a delayed elastic response.

Creep is a complex function of temperature, pressure, duration, and, in the sensor case, possibly the diaphragm thickness as well. Even at the same temperature, the same sensor would experience a smaller cavity length change when subjected to a lower load or shorter period of time. Therefore, for different applications, the sensor parameters can be optimized for the best performance. The most important of these parameters will most likely include the thickness of the diaphragm and the sensitivity of the sensor.

\section{E. Temperature Cross Sensitivity}

As with most other high-temperature pressure sensors, this sensor also exhibits temperature dependence, of which one aspect, sensitivity, is related to the elastic properties of the diaphragm. A second aspect is the zero drift due to the thermal effect of the cavity length, as seen in Fig. 10.

Two factors were found to contribute to the cavity length increment with temperature. One is the thermal expansion of the cavity material and the other is the pressure change of the residual gas trapped inside the cavity during fabrication, denoted by $\Delta L_{\text {mat }}$ and $\Delta L_{P}$, respectively, both of which increase as temperature rises. The total temperature-induced cavity length change is

$$
\begin{aligned}
\Delta L_{T} & =\Delta L_{\mathrm{mat}}+\Delta L_{P} \\
& =\alpha L \Delta T+S_{P} \Delta p_{\mathrm{in}}
\end{aligned}
$$

where $\alpha$ is the coefficient of thermal expansion (CTE) of fused silica, $L$ is the cavity length, $\Delta T$ is the temperature change, $S_{P}$ is the sensor's pressure sensitivity, and $\Delta p_{\text {in }}$ is the pressure change of the gas inside the cavity.

$\Delta p_{\text {in }}$ is further related to $\Delta T$ by the ideal gas law, $p_{\text {in }} V=$ $N k T$, where $p$ is the gas pressure, $V$ is the cavity volume, $N$ is the number of molecules inside $V, k$ is the Boltzmann constant, and $T$ is the absolute temperature in Kelvin. Since the 


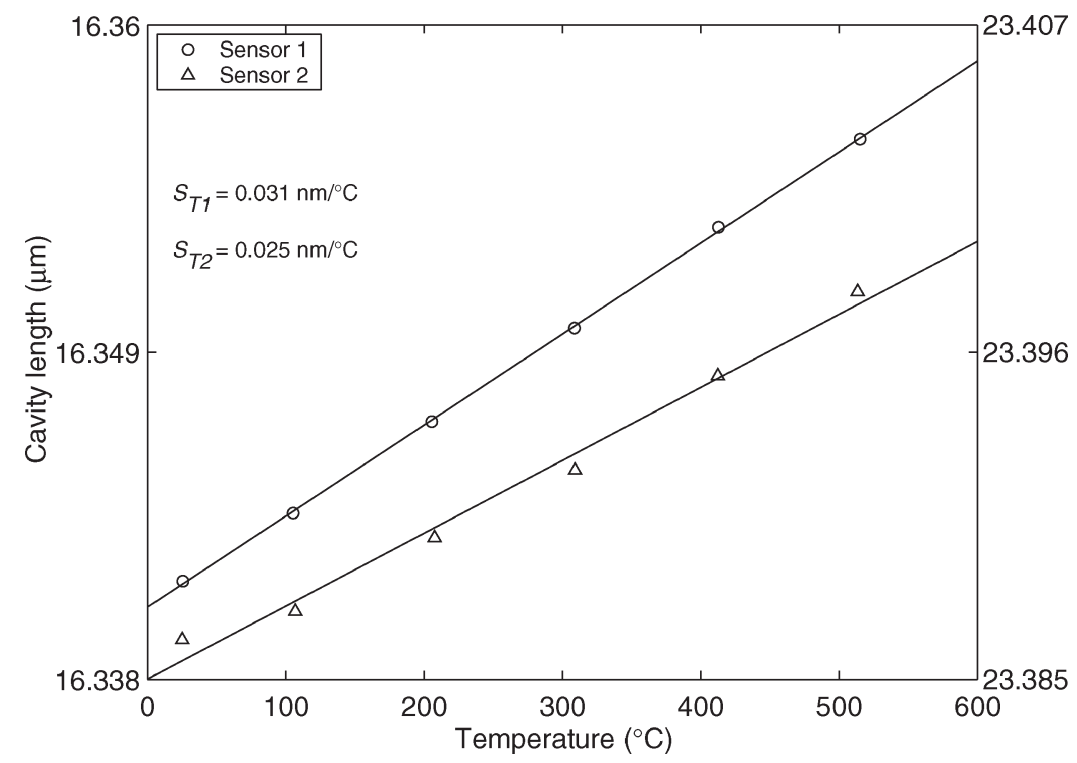

Fig. 14. Cavity length versus temperature for sensors 1 and 2.

diaphragm deflection $\Delta L$ is negligible compared to $L$, the volume $V$ can be regarded as a constant. With $N$ also unchanged for a sealed cavity, let $\beta=N k / V$ denote a proportional constant. Then we have $p_{\text {in }}=\beta T$ and $\Delta p_{\text {in }}=\beta \Delta T$. By substitution into (10), we have

$$
\begin{aligned}
\Delta L_{T} & =\alpha L \Delta T+\beta S_{P} \Delta T \\
& =\left(\alpha L+\beta S_{P}\right) \Delta T \\
& \triangleq S_{T} \Delta T
\end{aligned}
$$

where $S_{T}$ is defined as the temperature sensitivity of the sensor.

Measurements of $L$ from $24{ }^{\circ} \mathrm{C}$ to $513{ }^{\circ} \mathrm{C}$ are plotted in Fig. 14. Linearity is seen with fitted slope $S_{T}=0.031$ and $0.025 \mathrm{~nm} /{ }^{\circ} \mathrm{C}$ for sensors 1 and 2, respectively. This is because $\alpha$ is approximately a constant within this temperature range for fused silica, and $L$ and $S_{P}$ do not change much. With known values $L$ and $S_{P}$ for both sensors, $\alpha$ and $\beta$ can be solved as $0.98 \times 10^{-6} /{ }^{\circ} \mathrm{C}$ and $0.0095 \mathrm{psi} /{ }^{\circ} \mathrm{C}$. $\alpha$ is larger than the standard value for fused silica, $0.55 \times 10^{-6} /{ }^{\circ} \mathrm{C}$, partly as a result of residual doped core in the cavity that has a larger CTE. The effect of thermal expansion can be reduced by using a shorter cavity. From $\beta$, the pressure inside the cavity at RT can be derived as $p_{\text {in, } \mathrm{RT}}=\beta T=0.0095 \times 297=2.8 \mathrm{psi}$, about only one fifth of room pressure (13.7 psi), which is a clear indication that the amount of gas trapped inside the cavity is significantly reduced by the splicing arc.

Assuming that the temperature of the $\operatorname{arc}$ is $1700{ }^{\circ} \mathrm{C}$, slightly higher than the softening point of fused silica, $p_{\text {in }}$ is about 2 psi by the ideal gas law, which is roughly the theoretical limit by fusion splicing. Optimization of the splicing parameters may allow this limit to be approached.

If the sensor is to operate over a wide pressure range or a limited temperature range, the temperature cross sensitivity is relatively reduced. Otherwise, temperature compensation is generally necessary, as with almost all types of hightemperature pressure sensors.

\section{CONCLUSION AND Discussion}

We have demonstrated the high-temperature operation of a miniature fiber-optic pressure sensor up to $710{ }^{\circ} \mathrm{C}$. Pressureinduced cavity length change was accurately determined by tracing the spectrum shift. Sensor performance was analyzed in detail and is very promising for various high-temperature applications.

Future improvement is possible for the fabrication process. The fibers used are not optimized but chosen for their availability. Rather than graded-index $62.5-\mu \mathrm{m}$ fiber, step index would be preferred, with a higher dopant concentration for faster etching. The fluorine-doped $105-\mu \mathrm{m}$ fiber could be replaced by a $125-\mu \mathrm{m}$ fused silica fiber rod.

\section{ACKNOWLEDGMENT}

The authors thank Micron Optics for the partial donation of the CTS used in this research.

\section{REFERENCES}

[1] A. D. Kurtz, A. A. Ned, S. Goodman, and A. H. Epstein, "Latest ruggedized high temperature piezoresistive transducers," presented at the NASA Propulsion Measurement Sensor Development Workshop, Huntsville, AL, May 13-15, 2003.

[2] R. S. Okojie, A. A. Ned, and A. D. Kurtz, "Operation of $\alpha(6 \mathrm{H})-\mathrm{SiC}$ pressure sensor at $500{ }^{\circ}$ C," Sens. Actuators A, Phys., vol. 66, no. 1-3, pp. 200-204, Apr. 1998.

[3] J. von Berg, R. Ziermann, W. Reichert, E. Obermeier, M. Eickhoff, G. Krotz, U. Thoma, C. Cavalloni, and J. P. Nendza, "Measurement of the cylinder pressure in combustion engines with a piezoresistive $\beta$-SiCon-SOI pressure sensor," in Proc. High Temperature Electronics Conf., Albuquerque, NM, 1998, pp. 245-249.

[4] M. A. Fonseca, J. M. English, M. von Arx, and M. G. Allen, "Wireless micromachined ceramic pressure sensor for high-temperature application," J. Microelectromech. Syst., vol. 11, no. 4, pp. 337-343, Aug. 2002.

[5] M. Soeda, T. Kataoka, Y. Ishikura, S. Kimura, T. Masuda, Y. Yoshikawa, and M. Nagata, "Sapphire-based capacitive pressure sensor for high temperature and harsh environment application," in Proc. IEEE Sensors, Orlando, FL, 2002, vol. 2, pp. 950-953.

[6] K. C. Holmes, J. L. Davidson, W. P. Kang, and A. L. Sternberg, "Diamond microelectromechanical sensors for pressure and accelerating sensing," 
in Proc. Microelectromechanical Systems Conf., Berkeley, CA, 2001, pp. 45-49.

[7] W. Pulliam, P. Russler, R. Mlcak, K. Murphy, and C. Kozikowski, "Micromachined, $\mathrm{SiC}$ fiber optic pressure sensors for high-temperature aerospace applications," in Proc. SPIE, Boston, MA, 2000, Industrial Sensing Systems, pp. 21-30.

[8] Y. Zhu and A. Wang, "Miniature fiber-optic pressure sensor," IEEE Photon. Technol. Lett., vol. 17, no. 2, pp. 447-449, Feb. 2005.

[9] T. Pangaribuan, K. Yamada, S. Jiang, H. Ohsawa, and M. Ohtsu, "Reproducible fabrication technique of nanometric tip diameter fibre probe for photon scanning tunneling microscope," Jpn. J. Appl. Phys., vol. 31, no. 9A, pp. 1302-1304, 1992.

[10] E. Cibula, D. Donlagic, and C. Stropnik, "Miniature fiber optic pressure sensor for medical applications," in Proc. IEEE Sensors, 1st IEEE Int. Conf. Sensors, Orlando, FL, 2002, vol. 1, pp. 711-714.

[11] M. Di Giovanni, Flat and Corrugated Diaphragm Design Handbook. New York: Marcel Dekker, 1982.

[12] B. Qi, G. R. Pickrell, J. Xu, P. Zhang, Y. Duan, W. Peng et al., "Novel data processing techniques for dispersive white light interferometer," Opt. Eng., vol. 42, no. 11, pp. 3165-3171, Nov. 2003.

[13] M. Fukuhara and A. Sanpei, "High temperature-elastic moduli and internal dilational and shear frictions of fused quartz," Jpn. J. Appl. Phys., vol. 33, no. 5B, pp. 2890-2893, 1992.

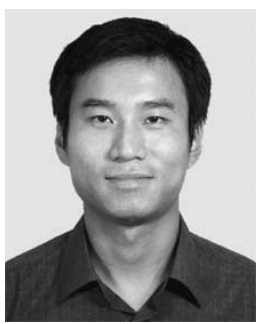

Yizheng Zhu (S'04) received the B.S. and M.S. degrees in electrical engineering from Tsinghua University, Beijing, China, in 1998 and 2000, respectively.

In 2001, he joined the Center for Photonics Technology at Virginia Polytechnic Institute and State University (Virginia Tech.), Blacksburg, as a Ph.D. student in the Department of Electrical and Computer Engineering. His current research is focused on the development of novel fiber-optic sensors and systems for physical, chemical, and biomedical sensing, as well as their field applications.

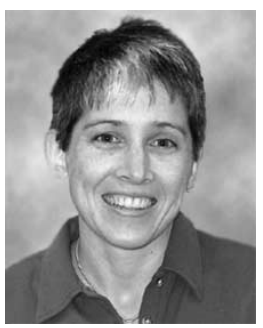

Kristie L. Cooper (S'96-M'01) received the B.S., M.S., and Ph.D. degrees in electrical engineering from the Virginia Polytechnic Institute and State University (Virginia Tech.), Blacksburg, in 1994, 1996, and 1999, respectively.

After spending five years as an Electrical Engineer at the Federal Aviation Administration Technical Center, she helped direct the design and development of self-assembled nanoscale materials for optical and optoelectronic thin films and devices at the Virginia Tech. Fiber and ElectroOptics Research Center and a startup R\&D company. In 2002, she joined the research faculty at Virginia Tech.'s Center for Photonics Technology. She has authored or coauthored more than 50 technical papers concerning nanoscale materials and sensing applications and has been responsible for over 30 sponsored research programs.

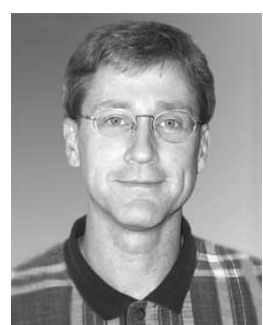

Gary R. Pickrell (M'04) received the B.S. and M.S degrees in ceramic engineering from the Ohio State University, Columbus, in 1985 and 1987, respectively, and the $\mathrm{Ph} . \mathrm{D}$. degree in materials engineering science from Virginia Polytechnic Institute and State University (Virginia Tech.), Blacksburg, in 1994.

$\mathrm{He}$ is an Assistant Professor in the Materials Science and Engineering Department of Virginia Tech. His industrial experience includes various research, development, and technical management positions at Owens Illinois, Corning, Selee, and Porvair Advanced Materials. He has authored over 60 technical papers, is a member of the Editorial Board of the International Journal of Six Sigma, is in the International Editorial Board of the Sensors Journal, and has nine patents issued and six additional patents pending. His current research is focused on random hole optical fibers and optical fiber sensors, glass, ceramic, and various other aspects of materials science and engineering.

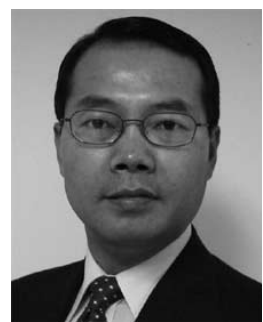

Anbo Wang (M'91-SM'92) received the Ph.D degree in applied optics from the Dalian University of Technology, Dalian, China, in 1990.

After spending three years as a Research Staff in the Fiber and Electro-Optics Research Center in the Department of Electrical and Computer Engineering at Virginia Polytechnic Institute and State University (Virginia Tech.), Blacksburg, he then joined the faculty of the same department as an Assistant Professor, where he is now a Professor and the Founding Director of the Center for Photonics Technology. His research interests include sensors and laser characterization and test on the constancy of physical constants. He is the author/coauthor of about 80 journal articles and 140 conference papers, and holds seven patents all licensed to industry.

Dr. Wang has chaired/cochaired numerous national or international conferences in photonics and sensors. 


\title{
Fast-Fourier-transform based numerical integration method for the Rayleigh-Sommerfeld diffraction formula
}

\author{
Fabin Shen and Anbo Wang
}

\begin{abstract}
The numerical calculation of the Rayleigh-Sommerfeld diffraction integral is investigated. The implementation of a fast-Fourier-transform (FFT) based direct integration (FFT-DI) method is presented, and Simpson's rule is used to improve the calculation accuracy. The sampling interval, the size of the computation window, and their influence on numerical accuracy and on computational complexity are discussed for the FFT-DI and the FFT-based angular spectrum (FFT-AS) methods. The performance of the FFT-DI method is verified by numerical simulation and compared with that of the FFT-AS method. (C) 2006 Optical Society of America
\end{abstract}

OCIS codes: $\quad 050.1960,000.4430,000.3860,350.5500$.

\section{Introduction}

The Helmholtz-Kirchhoff and the RayleighSommerfeld diffraction formulas have been widely used to analyze the propagation and diffraction of light in an isotropic, homogenous linear medium and have proved to be valid when the aperture size of the light field is much larger than the wavelength of light.1,2 The Rayleigh-Sommerfeld diffraction formula has been proved to yield exact evaluations of the light fields by methods of Fourier treatment ${ }^{3-5}$ and gives correct results for both far-field and near-field diffraction. ${ }^{6,7}$

Unfortunately, in most cases, these formulas cannot be solved analytically. Some approximations, such as Fresnel and Fraunhofer, are used to ease the difficulties in using these integral formulas for regions not close to the aperture. Also, paraxial approximations are often used for regions close to the optical axis. The validity of these approximations has been one issue in diffraction theories. ${ }^{8,9}$

The development of high-speed computers makes it possible to calculate these diffraction formulas without the need for Fresnel or Fraunhoffer approximations. In this paper we focus on the numerical calculation of the Rayleigh-Sommerfeld diffraction

The authors are with the Center for Photonics Technology, Bradley Department of Electrical and Computer Engineering, Virginia Polytechnic Institute and State University, Blacksburg, Virginia 24061. F. Shen's e-mail address is fashen1@vt.edu.

Received 15 March 2005; revised 9 September 2005; accepted 22 September 2005.

0003-6935/06/061102-09\$15.00/0

(C) 2006 Optical Society of America integral of an aperture under normal chromatic plane-wave illumination. The observation plane is parallel to the aperture plane, as Fig. 1 shows. The numerical solution of the Rayleigh-Sommerfeld integral can be calculated by either of two methods: angular spectrum (AS) and direct integration (DI). The AS method treats the propagation of light as a superposition of plane waves with different wave vectors and uses a Fourier transform to compute the light fields in the spatial-frequency domain. ${ }^{5,10,11} \mathrm{~A}$ fastFourier-transform (FFT) based AS (FFT-AS) method can have a high calculation speed and can be used for both parallel and arbitrarily oriented planes. ${ }^{12}$ The DI method computes the diffraction integrals in the spatial domain by means of numerical integration, which can be treated as a linear convolution and can be effectively calculated by means of a FFT and an inverse FFT (IFFT). ${ }^{13-15}$

Although numerical calculation of both the AS and the DI methods has been discussed extensively in the literature, $, 4,6,7,12-16$ there is still some confusion to be cleared up. First, the implementation of the diffraction integral by means of convolution and a FFT was not clearly presented. Proper implementation requires that the sampling intervals be properly selected and the discrete Fourier transformation be properly zero padded; improper implementation will cause erroneous results. Second, the selection of the sampling interval for a given diffraction problem was not clearly discussed. Li et al. suggested using an energy conservation criterion for validation of the sampling quality, but it is difficult to select a proper sampling interval in advance. ${ }^{15}$ Delen and Hooker used a small sampling interval (less than half of a 


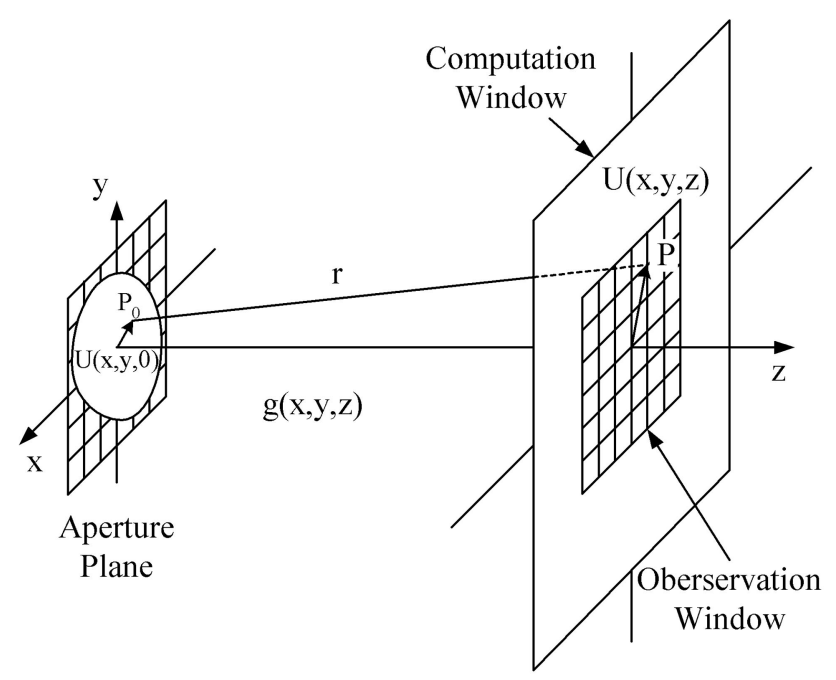

Observation Plane

Fig. 1. Illustration of the coordinate system of the RayleighSommerfeld diffraction theory.

wavelength) for the FFT-AS method but encountered a large computational load. ${ }^{16}$ An improper sampling interval will cause either a large aliasing error or a large computation load. Third, the selection of the computation window size in the FFT-AS method and its influence on numerical calculation accuracy, especially for an observation window that is not on the optical axis, was not clearly discussed. Some other confusion includes the applicable regions and computational complexity of the two methods. For example, although the FFT-AS method had been considered suitable for solving full scalar propagation problems, ${ }^{12}$ its expected numerical calculation accuracy and applicability to different regions have not been completely verified.

In this paper we present an implementation of a fast-Fourier-transform based DI (FFT-DI) method to calculate the Rayleigh-Sommerfeld diffraction integral and use Simpson's rule to improve the calculation accuracy. The selections of the sampling interval and the computation window and their influence on the calculation accuracy and computational load are discussed. The calculation accuracy, the computational speed, and the applicability regions of the FFT-DI and the FFT-AS methods are compared.

This paper is arranged as follows: In Section 2 a brief review of the AS method and the DI method is given. In Section 3 a method of implementing the FFT-DI method is described with improved accuracy by use of Simpson's rule. In Section 4 the sampling intervals, the computation window size, the calculation accuracy, and the computational load of the two methods are discussed. In Section 5 the simulation results for diffraction behind a circular aperture and a single slit are demonstrated; the calculation accuracy and computational speeds of the two methods are compared. In Section 6 our conclusions are given.

\section{Review}

The three-dimensional scalar Helmholtz equation for a linear homogenous isotropic medium,

$$
\frac{\partial^{2} U}{\partial x^{2}}+\frac{\partial^{2} U}{\partial y^{2}}+\frac{\partial^{2} U}{\partial z^{2}}+k^{2} U=0
$$

can be solved by either the AS method or the DI method described below.

\section{A. Angular Spectrum Method}

In the AS method the initial light field and its propagation are handled in the spatial-frequency domain. The propagation of the light can be given as

$$
A(\alpha, \beta, z)=A(\alpha, \beta, 0) G(\alpha, \beta, z),
$$

where $A(\alpha, \beta, z)$ is the Fourier transformation of the light field at distance $z$,

$$
\begin{aligned}
A(\alpha, \beta, z) & =F\{U(x, y, z)\} \\
& =\iint U(x, y, z) \exp (-j \alpha x-j \beta y) \mathrm{d} x \mathrm{~d} y,
\end{aligned}
$$

and

$$
G(\alpha, \beta, z)=\exp \left(j \sqrt{k^{2}-\alpha^{2}-\beta^{2}} z\right)
$$

is the optical transfer function of the linear homogenous isotropic medium. Then the light field $U(x, y, z)$ can be solved as the two-dimensional (2D) inverse Fourier transformation of $A(\alpha, \beta, z)$, given as

$$
\begin{aligned}
U(x, y, z)= & F^{-1}\{A(\alpha, \beta, z)\} \\
= & \frac{1}{4 \pi^{2}} \iint A(\alpha, \beta, 0) \\
& \times \exp \left(j \alpha x+j \beta y+j \sqrt{k^{2}-\alpha^{2}-\beta^{2}} z\right) \mathrm{d} \alpha \mathrm{d} \beta .
\end{aligned}
$$

The FFT-AS method for calculating $U(x, y, z)$ numerically has been reported and can be given as

$$
Q=\operatorname{IFFT} 2\left\{\operatorname{FFT} 2\left\{U\left(x_{m}, y_{n}, 0\right)\right\} \cdot \times G\left(\alpha_{m}, \beta_{n}, z\right)\right\},
$$

where $U\left(x_{m}, y_{n}, 0\right)$ and $G\left(\alpha_{m}, \beta_{n}, z\right)$ are samples of $U(x, y, 0)$ and $G(\alpha, \beta, z)$, FFT2 and IFFT2 denote a $2 \mathrm{D}$ FFT and a 2D IFFT, and $\cdot \times$ means element-byelement multiplication. ${ }^{5,10-12}$

\section{B. Direct Integration Method}

$G(\alpha, \beta, z)$ in Eq. (4) is the optical transfer function of the medium, and its inverse Fourier transformation 
will give the impulse response as

$$
\begin{aligned}
g(x, y, z)= & \frac{1}{4 \pi^{2}} \iint G(\alpha, \beta, z) \\
& \times \exp (j \alpha x+j \beta y) \mathrm{d} \alpha \mathrm{d} \beta \\
= & \frac{1}{2 \pi} \frac{\exp (j k r)}{r} \frac{z}{r}\left(\frac{1}{r}-j k\right),
\end{aligned}
$$

where $r=\sqrt{x^{2}+y^{2}+z^{2}} .10$ Thus $U(x, y, z)$ can be solved as the convolution of $U(x, y, 0)$ and $g(x, y, z)$ :

$$
\begin{aligned}
U(x, y, z)= & \iint_{A} U(\varsigma, \eta, 0) g(x-\varsigma, y-\eta, z) \mathrm{d} s \mathrm{~d} \eta \\
= & \iint_{A} U(\varsigma, \eta, 0) \frac{\exp (j k r)}{2 \pi r} \frac{z}{r} \\
& \times\left(\frac{1}{r}-j k\right) \mathrm{d} s \mathrm{~d} \eta,
\end{aligned}
$$

plane $\left(x_{m}, y_{n}, z\right)$, the integral can be calculated by numerical integration as a Riemann sum:

$$
\begin{aligned}
U\left(x_{m}, y_{n}, z\right)= & \sum_{i=1}^{N} \sum_{j=1}^{N} U\left(\varsigma_{i}, \eta_{j}, 0\right) g \\
& \times\left(x_{m}-\varsigma_{i}, y_{n}-\eta_{j}, z\right) \Delta \varsigma \Delta \eta,
\end{aligned}
$$

where $\Delta s$ and $\Delta \eta$ are sampling intervals on the aperture plane.

\section{Fast-Fourier-Transform Based Direct Integration Method}

The Riemann sum in Eq. (9) can be regarded as a discrete linear convolution of $U\left(s_{i}, \eta_{j}, 0\right)$ and $g\left(x_{m}, y_{n}, z\right)$, which can be calculated effectively by means of a FFT. We present a FFT-DI implementation for $N^{2}$ points located at sampling grids on the observation plane. The discrete convolution in Eq. (9) can be calculated as

$$
S=\operatorname{IFFT} 2[\operatorname{FFT} 2(U) \cdot \times \operatorname{FFT} 2(H)] \Delta \varsigma \Delta \eta,
$$

where

$$
\begin{aligned}
& U=\left[\begin{array}{cc}
U_{0} & \mathbf{0} \\
\mathbf{0} & \mathbf{0}
\end{array}\right]_{(2 N-1) \times(2 N-1)}=\left[\begin{array}{ccc:c}
U\left(s_{1}, \eta_{1}, 0\right) & \cdots & U\left(s_{1}, \eta_{N}, 0\right) & 0_{N \times(N-1)} \\
\vdots & \ddots & \vdots & U\left(s_{N}, \eta_{N}, 0\right) \\
U\left(s_{N}, \eta_{1}, 0\right) & \cdots & - & 0_{(N-1) \times(N-1)}
\end{array}\right], \\
& H=\left[\begin{array}{ccc}
g\left(X_{1}, Y_{1}, z\right) & \cdots & g\left(X_{1}, Y_{2 N-1}, z\right) \\
\vdots & \ddots & \vdots \\
g\left(X_{2 N-1}, Y_{1}, z\right) & \cdots & g\left(X_{2 N-1}, Y_{2 N-1}, z\right)
\end{array}\right]_{(2 N-1) \times(2 N-1)}, \\
& X_{j}=\left\{\begin{array}{ll}
x_{1}-\varsigma_{N+1-j} & j=1, \ldots, N-1 \\
x_{j-N+1}-\varsigma_{1} & j=N, \ldots, 2 N-1
\end{array},\right. \\
& Y_{j}=\left\{\begin{array}{ll}
y_{1}-\eta_{N+1-j} & j=1, \ldots, N-1 \\
y_{j-N+1}-\eta_{1} & j=N, \ldots, 2 N-1
\end{array} .\right.
\end{aligned}
$$

where $r=\sqrt{(x-\varsigma)^{2}+(y-\eta)^{2}+z^{2}}$. It is exactly the Rayleigh-Sommerfeld diffraction integral formula, which can be used for both near and far fields without any approximation.

In most cases, the diffraction integral in Eq. (8) has to be calculated by direct numerical integration. On the aperture plane, $U(\mathrm{~s}, \eta, 0)$ is sampled to $N \times N$ equidistant grids. For a point on the observation
The sampling grid of $U\left(s_{i}, \eta_{j}, 0\right)$ is zero padded to $(2 N-1) \times(2 N-1)$ as shown in Eq. (11) because Eq. (10) gives the circular convolution of $U$ and $H$. The result $S$ is a $(2 N-1) \times(2 N-1)$ complex matrix. The desired light fields in the observation plane can be given by the $N \times N$ lower right submatrix of $S$ :

$$
U\left(x_{m}, y_{n}, z\right)=S_{m+N, n+N} .
$$




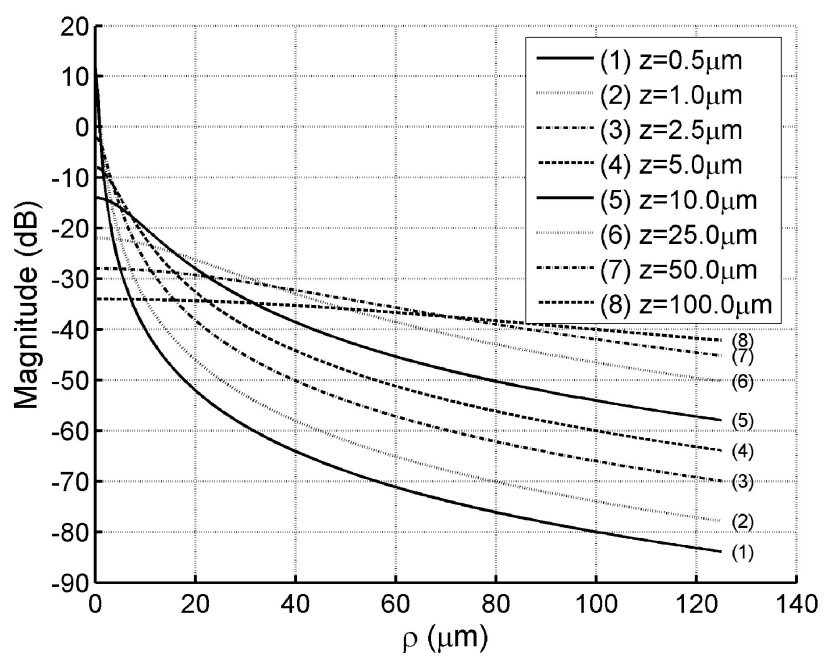

Fig. 2. Magnitude of $g(x, y, z)$ at several observation planes.

The accuracy of the FFT-DI method depends on the sampling intervals. Higher accuracy can be obtained when smaller sampling intervals are used. The error of the numerical integration in Eq. (9) can be estimated as

$$
E=O\left(\Delta \varsigma^{2}\right)+O\left(\Delta \eta^{2}\right)
$$

One can use Simpson's rule to improve the accuracy of numerical integration by introducing weight matrix $W$ into Eq. (11) as

$$
U=\left[\begin{array}{cc}
W \cdot \times U_{0} & \mathbf{O} \\
\mathbf{O} & \mathbf{O}
\end{array}\right]_{(2 N-1) \times(2 N-1)},
$$

where

$$
\begin{aligned}
& W=B^{T} B \\
& B=1 / 3\left[\begin{array}{llllllll}
1 & 4 & 2 & 4 & 2 & \ldots & 2 & 4
\end{array}\right]
\end{aligned}
$$

for an odd $N .{ }^{17}$ The error of Simpson's rule for $2 \mathrm{D}$ numerical integration can be estimated as

$$
E=O\left(\Delta s^{4}\right)+O\left(\Delta \eta^{4}\right)
$$

which is smaller than that of Eq. (16).

The FFT-DI method discussed above can also be extended to calculation of the Helmholtz-Kirchhoff integrals by use of different values of $g(x, y, z)$ in Eq. (9).

\section{Discussion}

\section{A. Sampling}

Both the FFT-AS and the FFT-DI methods need discrete $U(s, \eta, 0)$. If $U(s, \eta, 0)$ is band limited in the spatial-frequency domain as $\left.A(\alpha, \beta, 0)\right|_{|\alpha|>\alpha_{M} \text { or }|\beta|>\beta_{M}}$ $=0$, then, according to the Nyquist sampling theorem, the sampling intervals have to be less than

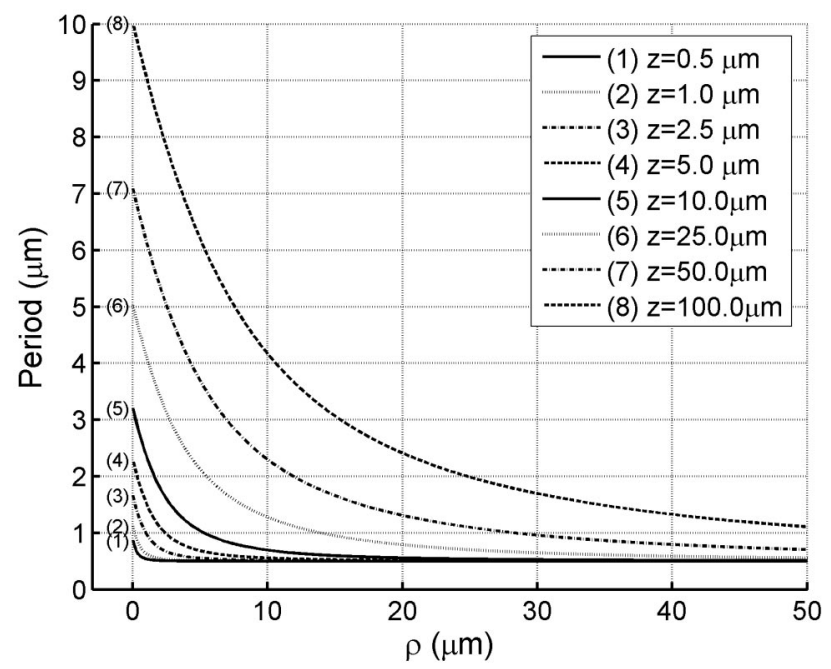

Fig. 3. Oscillating period of $g(x, y, z)$ at several observation planes.

$\left(2 \alpha_{M}\right)^{-1}$ and $\left(2 \beta_{M}\right)^{-1}$, respectively. However, most apertures are finite in spatial size, and their Fourier transformations are infinite in the spatial-frequency domain. Thus the frequency components greater than the sampling frequencies are truncated. When the sampling frequency is high enough, the total power of the truncated band is usually much lower than that of the low frequencies, and thus the truncation error can be neglected in most cases.

The FFT-DI method in Eq. (10) requires discrete $g(x, y, z)$. Because $g(x, y, z)$ is not band limited; the sampling of $g(x, y, z)$ is always aliased. However, $g(x, y, z)$ is of low frequencies for paraxial points and relatively long propagation distances. Thus, for a given diffraction problem, one can estimate the maximum frequency and select the appropriate sampling interval. The magnitude and the oscillation period of $g(x, y, z)$ at different observations planes are plotted in Figs. 2 and 3, respectively, with a simulation wavelength of $0.5 \mu \mathrm{m}$.

The attenuation of light fields with respect to the offset between the point and the optical axis at planes $z=0.5,1,2.5,5,10,25,50,100 \mu \mathrm{m}$ are plotted in Fig. 2. It is evident that more energy is diffracted to areas far from the optical axis as $z$ increases, but the attenuation rate gets smaller. This implies that more sampling points are required for small $z$ because of the rapid variations in the light field.

We can estimate the oscillation period of $g(x, y, z)$ by calculating the interval $\Delta \rho$ between points on the observation plane with $2 \pi$ phase difference, given as

$$
k \sqrt{(\rho+\Delta \rho)^{2}+z^{2}}-k \sqrt{\rho^{2}+z^{2}}=2 \pi,
$$

where $\rho=\sqrt{x^{2}+y^{2}}$ is the offset of a point from the optical axis. The solution is

$$
\Delta \rho=\sqrt{\lambda^{2}+\rho^{2}+2 \lambda \sqrt{\rho^{2}+z^{2}}}-\rho .
$$




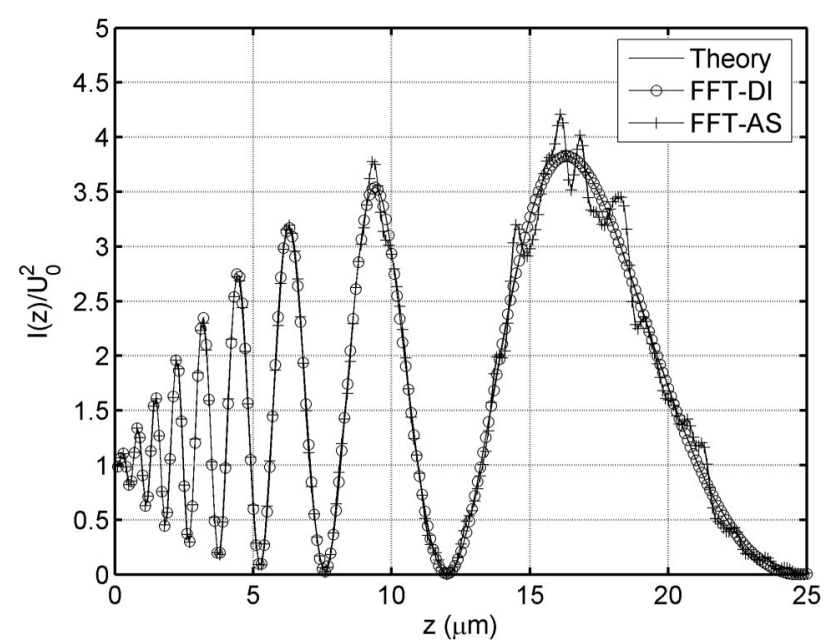

Fig. 4. Axial intensity distribution behind a circular aperture.

The relationship between $\Delta \rho$ and $\rho$ on different planes is shown in Fig. 3. When $z$ is large and $\rho$ is small, which is a paraxial case, the oscillation period of $g(x, y, z)$ is large and $g(x, y, z)$ is of low frequency. Thus a large sampling interval can be used without sacrificing calculation accuracy. However, when $\rho$ is large or $z$ is small, which is not the paraxial case, the oscillating period of $g(x, y, z)$ is asymptotic to $\lambda$, and the sampling intervals for $g(x, y, z)$ need to be smaller than half of the optical wavelength at least.

The minimum $\Delta \rho$ for a given $z$ and $\rho$ is a measure of the maximum frequency that $g(x, y, z)$ contains. Equation (22) gives a convenient rule for the selection of the sampling interval. For a light diffraction problem to be solved, the minimum propagation distance $z_{\min }$ and the maximum offset from the optical axis $\rho_{\max }$ can be substituted into Eq. (22) to yield a minimum $\Delta \rho_{\min }$. The sampling intervals on the $x-y$ plane should be less than $\Delta \rho_{\min } / 2$ to reduce the aliasing error.

\section{B. Accuracy}

The ranges of the integration in Eq. (5) and the resultant light fields are infinite. However, for a numerical calculation with the FFT-AS method, the size of the computation window is finite. The result of the IFFT in Eq. (6), $Q\left(x_{m}, y_{n}, z\right)$, is a replica of $U(x, y, z)$ in the spatial domain, which can be given as the summation of samples of $U(x, y, z)$ at all the grid points with equal distances of the computation window size:

$$
Q\left(x_{m}, y_{n}, z\right)=\sum_{i=-\infty}^{\infty} \sum_{j=-\infty}^{\infty} U\left(x_{m}+i X, y_{n}+j Y, z\right)
$$

where $X$ and $Y$ are dimensions of the computation window. ${ }^{18}$

It is evident that the calculation error of the FFT-AS method depends mainly on the light fields of grid points outside the computation window on the observation plane. Therefore the size of the computation window has to be large enough that the light fields outside the computation window can be neglected. An oversampling of the same computation

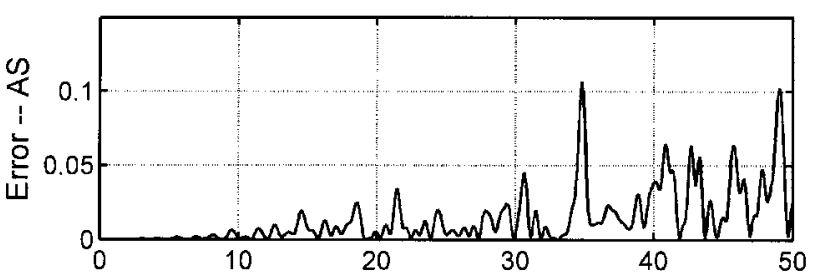

(a)

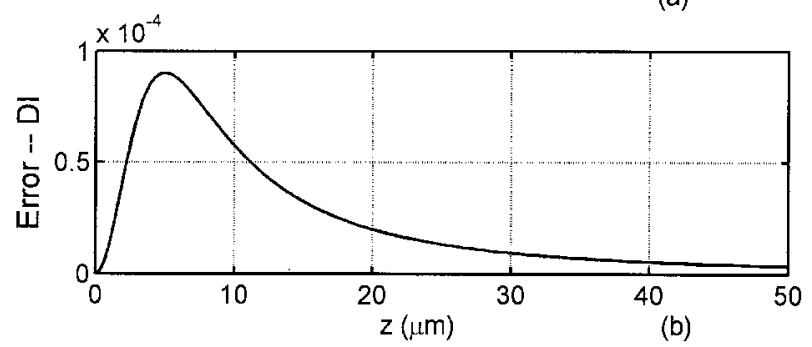

Fig. 5. Calculation errors of the FFT-AS method and the FFT-DI method: (a) FFT-AS and (b) FFT-DI methods.

window with small sampling intervals will not increase the accuracy. The accuracy of the DI method depends on the accuracy of the numerical integration in Eq. (9), which in turn depends on the sampling intervals, as shown in Eqs. (16) and (20). Small sampling intervals will yield high calculation accuracy. A simulation result for comparison of the accuracy of the FFT-AS method and the FFT-DI method is given in Subsections 5.A and 5.B below.

\section{Computation Window}

The rule for the selection of computation window size in the FFT-AS method is that all the light fields outside the window can be neglected for a given desired accuracy. In practice, owing to the diffraction of light, the size of the computation window has to be selected to be much larger than the aperture size, even if only a small observation window is desired. However, a large computation window will cause a large sampling array and thus a high computational load. Therefore the application of the FFT-AS method is limited to small apertures and near-field cases.

When the observation window is not on the optical axis, a large computation window centered on the optical axis has to be selected. This is not computationally efficient. Delen et al. tried to use a shifted Fourier transform to handle this problem with a smaller computation window by moving the center of the computation window to the optical axis. ${ }^{12}$ However, because the mathematical shift of the computation window does not change the real light field distribution, a small computation window will result in an incorrect calculation.

Here is a general guideline that we used for selection of the computation window in the FFT-AS method. For a given propagation distance $z$, the magnitude of $g(x, y, z)$ for light fields outside the computation window should be much smaller than that on the optical axis, given by

$$
|g(x, y, z)|<\varepsilon|g(0,0, z)|
$$



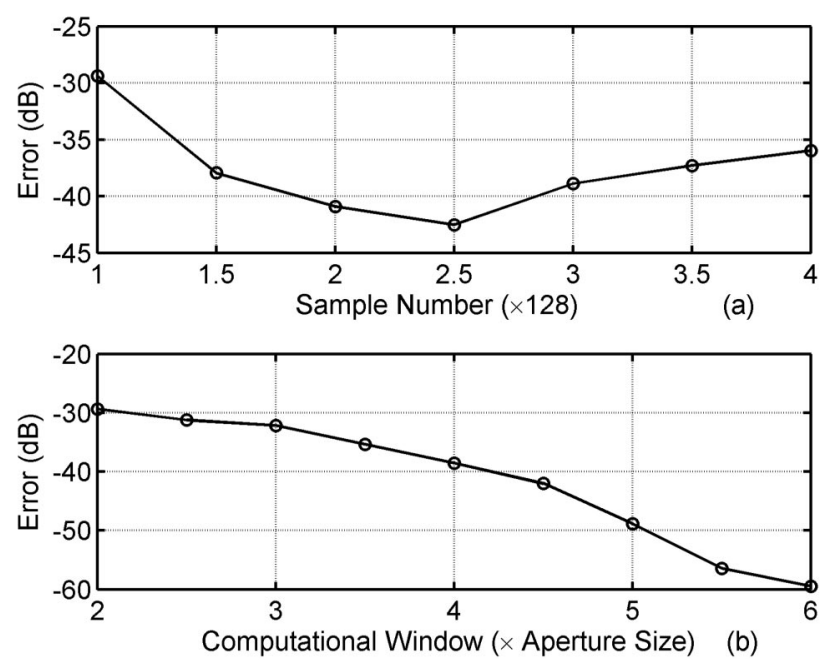

Fig. 6. Calculation errors of the FFT-AS method for the sampling numbers and computation window sizes shown.

where $\varepsilon$ is a small number. Inequality (24) can be rewritten as

$$
\left|\frac{1}{r^{2}}\left(\frac{1}{r}-j k\right)\right|<\varepsilon\left|\frac{1}{z^{2}}\left(\frac{1}{z}-j k\right)\right|,
$$

where $r=\sqrt{\rho^{2}+z^{2}}$. In practice, $z$ is usually much larger than several wavelengths; thus inequality (23) can be simplified to

$$
\frac{1}{r^{2}}<\varepsilon \frac{1}{z^{2}}
$$

and the minimum $\rho$ can thus be solved as

$$
\rho>z[(1 / \varepsilon)-1]^{1 / 2} .
$$

The minimum computation window can then be selected to be $(2 \rho+a) \times(2 \rho+b)$, where $a \times b$ is the size of the aperture.

The FFT-DI method, however, requires that the size of the computation window be the same as that of the aperture window. When the observation window is larger than the aperture, one can either enlarge the aperture window or divide the observation window into subwindows with smaller sizes. In Subsection 4.D below, we show that the latter method is more computationally effective.

\section{Computational Complexity}

The computational load of the FFT-AS method in Eq. (6) comes from (a) a FFT of $U$, (b) calculation of $G$, (c) element-by-element multiplication $A=\operatorname{FFT}(U)$ - $\times G$, and (d) IFFT ( $A$ ). Assuming that the array size is $N \times N$, the computational complexity is

$$
\begin{aligned}
C_{1} & =C_{a}+C_{b}+C_{c}+C_{d} \\
& =O\left(N^{2} \log _{2} N\right)+O\left(N^{2}\right)+O\left(N^{2}\right)+O\left(N^{2} \log _{2} N\right) .
\end{aligned}
$$

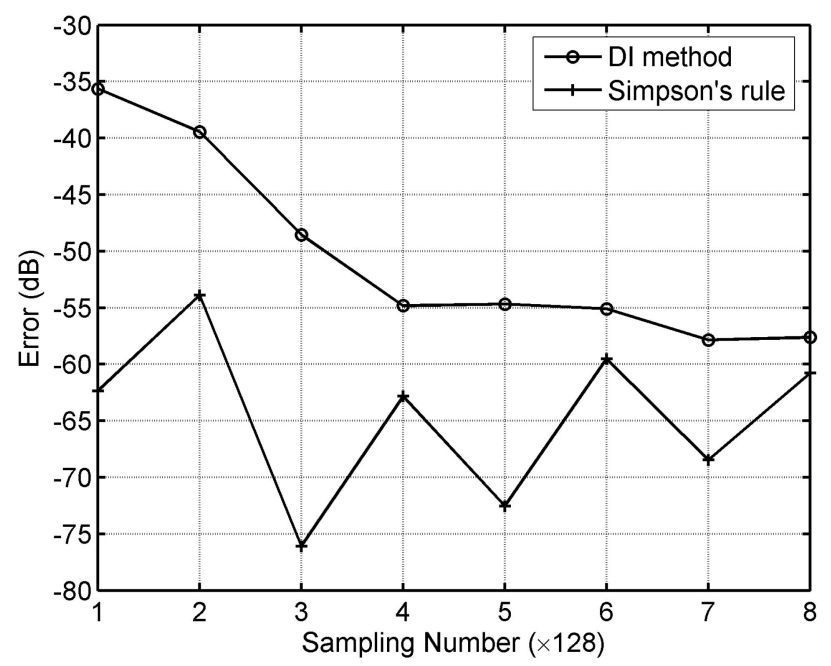

Fig. 7. Calculation errors of the FFT-DI method for several sampling numbers.

When $N^{2}$ points on the observation plane are arbitrarily located, the computational complexity of the traditional DI method is $O\left(N^{4}\right)$ (Ref. 12) because each point has a computational complexity of $O\left(N^{2}\right)$, as shown in Eq. (9). The FFT-DI method calculates the light fields on grid points and uses a FFT and an IFFT to improve the calculation speed. The computational load of the FFT-DI method in Eq. (10) comes from (a) $\operatorname{FFT}(U)$, (b) calculation of $H$, (c) $\operatorname{FFT}(H)$, (d) the element-by-element product of $\operatorname{FFT}(U)$ and FFT $(H)$, and (e) the IFFT of the product. For maximum efficiency of FFT and IFFT, $U$ and $H$ should be zero padded to have the size $2^{m} \times 2^{m}$, where $m$ is an integer. Assuming that the length of the FFT is $N_{F}$, the computational complexity of the FFT-DI method is

$$
\begin{aligned}
C_{2}= & C_{a}+C_{b}+C_{c}+C_{d}+C_{e} \\
= & O\left(N_{F}^{2} \log _{2} N_{F}\right)+O\left(N_{F}^{2}\right)+O\left(N_{F}^{2} \log _{2} N_{F}\right) \\
& +O\left(N_{F}{ }^{2}\right)+O\left(N_{F}{ }^{2} \log _{2} N_{F}\right),
\end{aligned}
$$

which is much lower than $O\left(N^{4}\right)$.

If the $N \times N$ sampling array in the FFT-DI method is zero padded to $2 N \times 2 N$, then the lengths of the FFTs in the FFT-DI method are the same as in the FFT-AS method when a computation window with $2 \times 2$ times the aperture size is used. From Eqs. (28) and (29), one can find that the FFT-DI method needs only one more 2D FFT than the FFT-AS method does, which means that the computational loads of the FFT-DI and FFT-AS methods are comparable.

In the FFT-DI method the size of the computation window is the same as that of the aperture window. One can divide a large observation window into smaller subwindows that have the same size as the aperture, such that the maximum computational efficiency of the FFT algorithm can be obtained. For example, if the desired observation window is $2 \times 2$ 

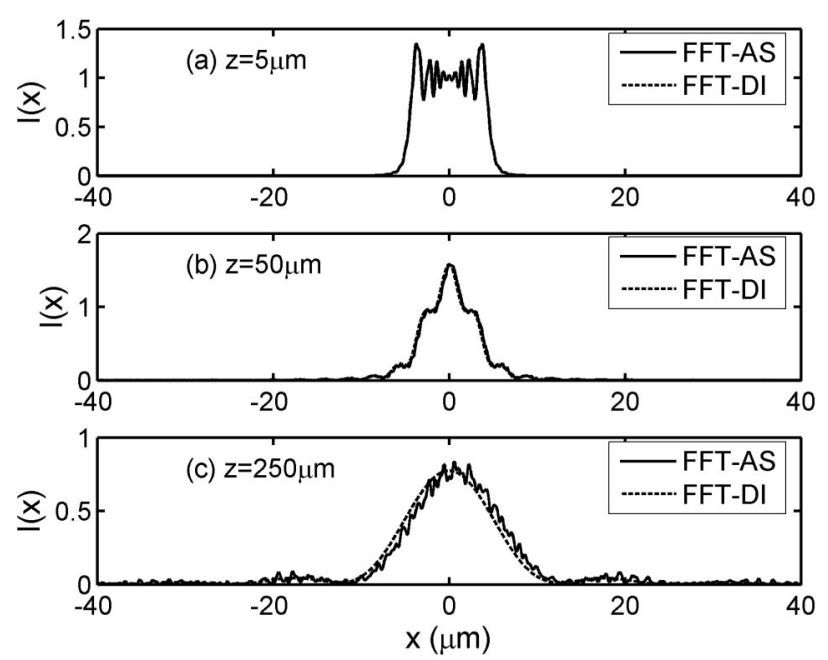

Fig. 8. Simulation results of diffraction pattern of a single slit.

times the aperture, one can divide it into four subwindows with $N \times N=\left(N_{1} / 2\right) \times\left(N_{1} / 2\right)$ arrays, where $N_{1}$ is the sampling number for the large computation window. The computational complexity for the FFT and the IFFT for the small windows is

$$
4 \times O\left(N^{2} \log _{2} N\right)=O\left[N_{1}^{2}\left(\log _{2} N_{1}-1\right)\right],
$$

which is less than that for the large computation windows, whose computational complexity is $O\left(N_{1}^{2} \log _{2} N_{1}\right)$.

\section{E. Two-Dimensional Diffraction}

The impulse response of a $2 \mathrm{D}$ linear isotropic homogenous medium is 7,11

$$
h(x, z)=\frac{j k z}{2 r} H_{1}^{(1)}(k r)
$$

where $H_{1}{ }^{(1)}(k r)$ is the first-order, first-kind Hankel function and $r=\sqrt{x^{2}+z^{2}}$. Thus the propagation of light can be represented as the convolution of $U(x, 0)$ and $h(x, z)$ :

$$
\begin{aligned}
U(x, z) & =\int_{A} U(\varsigma, 0) h(x-\varsigma, z) \mathrm{d} s \\
& =\int_{A} U(\varsigma, 0) \frac{j k z}{2 r} H_{1}^{(1)}(k r) \mathrm{d} s,
\end{aligned}
$$

where $r=\sqrt{(x-\varsigma)^{2}+z^{2}}$.

The FFT-DI method for 2D diffraction can be given as

$$
S=\operatorname{IFFT}[\operatorname{FFT}(U) \cdot \times \operatorname{FFT}(H)] \Delta s,
$$

where

$$
\begin{aligned}
& U=\left[U\left(\varsigma_{1}, 0\right) \ldots U\left(\varsigma_{N}, 0\right) 0 \ldots 0\right]_{2 N-1}, \\
& H=\frac{j k z}{2}\left[\frac{H_{1}^{(1)}\left(k r_{1}\right)}{r_{1}} \ldots \frac{H_{1}^{(1)}\left(k r_{2 N-1}\right)}{r_{2 N-1}}\right]_{2 N-1}, \\
& r_{j}= \begin{cases}\sqrt{\left(x_{1}-\varsigma_{N+1-j}\right)^{2}+z^{2}}, & j=1, \ldots, N-1 \\
\sqrt{\left(x_{j-N+1}-\varsigma_{1}\right)^{2}+z^{2}}, & j=N, \ldots, 2 N-1\end{cases}
\end{aligned}
$$

and $S(N: 2 N-1)$ gives the desired light fields. Similarly, Simpson's rule can also be applied to improve the accuracy of the calculation.

\section{Simulation Results}

\section{A. Circular Aperture}

It has been shown that the light fields on the optical axis behind a circular aperture under normal uniform plane-wave illumination have the following exact solution:

$$
U(z)=U_{0} z\left[\frac{\exp (j k z)}{z}-\frac{\exp \left(j k \sqrt{z^{2}+a^{2}}\right)}{\sqrt{z^{2}+a^{2}}}\right],
$$

where $U_{0}$ is the magnitude of the incident light and $a$ is the radius of the circular aperture. ${ }^{19,20}$

We used both the FFT-AS and the FFT-DI methods to calculate the light fields of axial points and compared the results with the theoretical solution in Eq. (36) to evaluate the accuracy of the two methods. The parameters used in the simulation are as follows: The wavelength of the chromatic light was $\lambda$ $=0.5 \mu \mathrm{m}$, the radius of the circular aperture was $a$ $=10 \lambda=5 \mu \mathrm{m}$, and the sampling interval was $0.1 \lambda$. For the FFT-AS method the computation window was set to $2 \times 2$ times the aperture window.

The simulation results of the axial intensity distribution are shown in Fig. 4. The FFT-DI method gives consistent simulation results for both small and large $z$. In contrast, the FFT-AS method gives consistent results only for small $z$. When $z$ is increased, because more light power is diffracted outside the computation window, a larger error occurs. The square errors of the calculations, $\left|U-U_{\mathrm{AS}}\right|^{2}$ and $\left|U-U_{\mathrm{DI}}\right|^{2}$, are plotted in Fig. 5, where $U$ is the theoretical value and $U_{\mathrm{AS}}$ and $U_{\mathrm{DI}}$ are the simulation results. The FFT-DI method has a much smaller calculation error than the FFT-AS method for a relatively large $z$. The error of the FFT-AS method tends to increase with $z$, whereas the error of the FFT-DI method shows a maximum at a certain $z$ and is asymptotic to zero when $z$ is large.

The errors of the FFT-AS method for several sampling intervals and computation window sizes are shown in Fig. 6. Figure 6(a) shows the errors of the FFT-AS method when the aperture is sampled from $128 \times 128$ to $512 \times 512$ with the computation window kept at the same size, which is $2 \times 2$ of the aperture window. The results show that oversampling on the aperture will not reduce the calculation error. Figure 
Table 1. Comparison of Calculation Speeds

\begin{tabular}{|c|c|c|c|c|}
\hline \multicolumn{2}{|c|}{ FFT-DI $^{a}$} & \multicolumn{2}{|c|}{ FFT-AS $^{b}$} & \multirow[b]{2}{*}{$\begin{array}{c}\text { Array } \\
\text { Size of FFT }\end{array}$} \\
\hline $\begin{array}{c}\text { Sampling } \\
\text { Grid of Aperture }\end{array}$ & $\begin{array}{l}\text { Execution } \\
\text { Time (s) }\end{array}$ & $\begin{array}{l}\text { Computation } \\
\text { Window }\end{array}$ & $\begin{array}{l}\text { Execution } \\
\text { Time (s) }\end{array}$ & \\
\hline $128 \times 128$ & 0.23 & $2 \times 2$ & 0.19 & $256 \times 256$ \\
\hline $256 \times 256$ & 0.95 & $4 \times 4$ & 0.70 & $512 \times 512$ \\
\hline $512 \times 512$ & 3.36 & $8 \times 8$ & 2.56 & $1024 \times 1024$ \\
\hline
\end{tabular}

${ }^{a}$ The computation window in the FFT-DI method has the same size as the aperture.

${ }^{b}$ The sampling grid of the aperture in FFT-AS method is $128 \times 128$.

6(b) shows the errors of the FFT-AS method when the computation window size is selected from $2 \times 2$ to $6 \times 6$ of the aperture size while the sampling numbers of the aperture are kept at $128 \times 128$. The results show that a large computation window will decrease the calculation error.

The errors of the FFT-DI method for several sampling numbers are shown in Fig. 7. The calculation error $\left|U-U_{\mathrm{DI}}\right|^{2}$ at $z=8 \mu \mathrm{m}$ for sampling numbers from $128 \times 128$ to $1024 \times 1024$ are plotted together with the calculation errors of Simpson's rule. It can be seen that the calculation error decreases when the sampling number increases. However, when the sampling interval is small enough, the rate of decrease in error becomes saturated. Simpson's rule can greatly reduce the calculation error and thus improve the calculation accuracy.

\section{B. Single Slit}

We verified the 2D diffraction formulas in Eqs. (30)(35) by calculating the diffraction patterns of a single infinite slit under uniform plane-wave illumination. The wavelength of the light was $0.5 \mu \mathrm{m}$. The width of the slit was $10 \mu \mathrm{m}$. The aperture plane was sampled to 1024 points along the $x$ axis. The observation window was set to $80 \mu \mathrm{m}$ width with the center on the optical axis. In the FFT-AS method the computation window was the same size as the observation window, which was eight times the size of the aperture. The diffraction patterns at observation planes of $z=5,50,250 \mu \mathrm{m}$, calculated by both the FFT-AS and the FFT-DI methods, are plotted in Fig. 8.

When $z$ is small $(5 \mu \mathrm{m})$, the differences between the calculation results from the FFT-AS and the FFT-DI methods are so small that they cannot be identified, as Fig. 8(a) shows. However, the error of the FFT-AS method increases when $z$ becomes larger $(50 \mu \mathrm{m})$, as Fig. 8(b) shows. When $z$ is large $(250 \mu \mathrm{m})$, the FFT-AS method fails to give an accurate result, as shown in Fig. 8(c).

\section{Calculation Speed}

In our simulation we used a Compaq personal computer with a Pentium 4, $1.4 \mathrm{GHz} \mathrm{CPU}$ and 512 Mbytes of RAM to investigate the speed of the FFT-AS and the FFT-DI methods. The simulation was based on the circular aperture diffraction as described in Subsection 5.A. The observation window was set to be $10 \mu \mathrm{m} \times 10 \mu \mathrm{m}$, the same size as the aperture window. The average execution time of 25 running cycles and the FFT array sizes of the FFT-DI and the FFT-AS methods are compared in Table 1. The simulation code was programmed and executed in Matlab v6.5 on a Windows XP operating system.

It is evident that the FFT-DI method can greatly reduce the computational load compared with the traditional DI method. The execution time of the FFT-DI method for an array size of $512 \times 512$ is $3.36 \mathrm{~s}$, which is much less than that of the traditional DI method reported in Ref. 16. The computational loads of the FFT-DI and the FFT-AS methods are comparable when the array sizes in these two methods are the same. The execution times of the FFT-DI method with a $1024 \times 1024$ sampling array and of the FFT-AS method with a computation window of $16 \times 16$ times the aperture window are 13.34 and $10.80 \mathrm{~s}$, respectively. The lengths of the FFT and the IFFT are $2048 \times 2048$ for both cases. The execution time of the FFT-DI method is only 1.24 times that of the FFT-AS method.

The FFT-DI method uses one more FFT than the FFT-AS method and thus needs one more array to save the FFT result. For example, for an array size of $1024 \times 1024$ with double precision, the FFT-AS needs 8 Mbytes of memory to save the result of one FFT, while the FFT-DI method needs 16 Mbytes of memory to save the results of the two FFTs.

\section{Conclusions}

Based on the investigation of the numerical calculation methods for the Rayleigh-Sommerfeld diffraction integral, a fast-Fourier-transform based DI method was implemented to lower the computational load of the numerical integration. Simpson's rule was introduced to improve the calculation accuracy. The parameter selections and the performance of the FFT-DI and the FFT-AS methods were discussed. The sampling of the light fields on the aperture plane needs to meet the requirements of the Nyquist sampling theorem in both methods. The accuracy of the FFT-AS method depends on the error caused by the finite size of the computation window. An oversampling in the aperture window will not increase the accuracy of the FFT-AS method. The accuracy of the FFT-DI method depends on the sampling intervals in the aperture plane. The size of the computation win- 
dow in FFT-DI method needs to be the same as that of the aperture window. The computational load of the FFT-DI method is comparable to that of the FFT-AS method but much lower than that of the traditional DI method. Simulation results have shown that the FFT-DI method is accurate and efficient and can be used universally, whereas the FFT-AS method should be restricted to small apertures and near-field cases.

This research was supported in part by the U.S. Department of Energy under grant DE-FC3601G011050 and by the National Science Foundation under grant CMS-0427951.

\section{References}

1. M. Born and E. Wolf, Principles of Optics, 6th ed. (Pergamon, 1980), Chap. 8.

2. E. Hecht, Optics, 2nd ed. (Addison-Wesley, 1987), Chap. 10.

3. N. Mukunda, "Consistency of Rayleigh's diffraction formulas with Kirchhoff's boundary conditions," J. Opt. Soc. Am. 52, 336-337 (1962).

4. E. Wolf and E. W. Marchand, "Comparison of the Kirchhoff and the Rayleigh-Sommerfeld theories of diffraction at an aperture," J. Opt. Soc. Am. 54, 587-594 (1964).

5. J. E. Harvey, "Fourier treatment of near-field scalar diffraction theory," Am. J. Phys. 47, 974-980 (1979).

6. J. C. Heurtley, "Scalar Rayleigh-Sommerfeld and Kirchhoff diffraction integrals: a comparison of exact evaluations for axial points," J. Opt. Soc. Am. 63, 1003-1008 (1973).

7. M. Totzeck, "Validity of the scalar Kirchhoff and RayleighSommerfeld diffraction theories in the near field of small phase objects," J. Opt. Soc. Am. A 8, 27-32 (1991).

8. A. M. Steane and H. N. Rutt, "Diffractions in the near field and the validity of the Fresnel approximation," J. Opt. Soc. Am. A 6, 1809-1814 (1989).

9. W. H. Southwell, "Validity of the Fresnel approximation in the near field," J. Opt. Soc. Am. 71, 7-14 (1981).

10. E. Lalor, "Conditions for the validity of the angular spectrum of plane waves,” J. Opt. Soc. Am. 58, 1235-1237 (1968).

11. J. J. Stamnes, "Focusing of two dimensional waves," J. Opt. Soc. Am. 71, 15-20 (1981).

12. N. Delen and B. Hooker, "Free-space beam propagation between arbitrarily oriented planes based on full diffraction theory: a fast Fourier transform approach," J. Opt. Soc. Am. A 15, 857-867 (1998).

13. J. A. Hudson, "Fresnel-Kirchhoff diffraction in optical systems: an approximate computational algorithm," Appl. Opt. 23, 2292-2295 (1984).

14. C. Kopp and P. Meyrueis, "Near-field Fresnel diffraction: improvement of a numerical propagator," Opt. Commun. 158, 7-10 (1998).

15. J. Li, Z. Fan, and Y. Fu, "The FFT calculation for Fresnel diffraction and energy conservation criterion of sampling quality," in Lasers in Material Processing and Manufacturing, S. Deng, T. Okada, K. Behler, and X. Wang, eds., Proc. SPIE 4915, 180-186 (2002).

16. N. Delen and B. Hooker, "Verification and comparison of a fast Fourier transform-based full diffraction method for tilted and offset planes," Appl. Opt. 40, 3525-2531 (2001).

17. C. Pozrikidis, Numerical Computation in Science and Engineering (Oxford U. Press, 1998), Chap. 7.

18. W. L. Briggs and V. E. Henson, The DFT: An Owner's Manual for the Discrete Fourier Transform (Society for Industrial and Applied Mathematics, 1995).

19. H. Osterberg and L. W. Smith, "Closed solutions of Rayleigh's integral for axial points,” J. Opt. Soc. Am. 51, 1050-1054 (1961).

20. A. Dubra and J. A. Ferrari, "Diffracted field by an arbitrary aperture,” Am. J. Phys. 67, 87-92 (1999). 


\title{
All-fused-silica miniature optical fiber tip pressure sensor
}

\author{
Xingwei Wang, Juncheng Xu, Yizheng Zhu, Kristie L. Cooper, and Anbo Wang \\ Center for Photonics Technology, Bradley Department of Electrical and Computer Engineering, \\ Virginia Polytechnic Institute and State University, Blacksburg, Virginia 24061
}

\begin{abstract}
Received September 6, 2005; revised November 5, 2005; accepted December 21, 2005; posted January 17, 2006 (Doc. ID 64655)
An all-fused-silica pressure sensor fabricated directly onto a fiber tip of $125 \mu \mathrm{m}$ diameter is described. Simple fabrication steps include only cleaving and fusion splicing. Because no chemical processes are involved, the fabrication is easy, safe, and cost effective. Issues in sensor design and loss analysis are discussed. The sensor has been tested for static pressure response, showing a sensitivity of $2.2 \mathrm{~nm} / \mathrm{psi}$, a resolution of $0.01 \mathrm{psi}(68.9 \mathrm{~Pa})$, a hysteresis of $0.025 \%$, and capability of operation at temperatures up to $600^{\circ} \mathrm{C}$. This miniature sensor may be suitable for medical diagnostics, environmental monitoring, and other industrial applications. (c) 2006 Optical Society of America OCIS codes: $060.2370,120.2230$.
\end{abstract}

Miniature pressure sensors have stimulated great interest in a large number of applications such as health care, automotive and aerospace industries, and down-hole monitoring in oil wells. Compared with currently available capacitive ${ }^{1}$ and piezoresistive $^{2}$ sensors, interferometric optical fiber sensors $^{3-5}$ offer the advantages of biocompatibility, high-temperature survivability, corrosion resistance, high resolution, high sensitivity, intrinsic electrical passivity, and immunity to electromagnetic interference.

However, fabrication of current miniature fiberoptic sensors usually involves several materials. The large mismatch in coefficient of thermal expansion will cause severe stress among the materials. Furthermore, the bonding adhesive, such as the commonly used epoxy, can decompose at high temperatures. ${ }^{6}$ When the sensor size is as little as micrometers, laser bonding ${ }^{7}$ is inconvenient.

In this Letter we present a novel miniature pressure sensor fabricated directly onto the tip of commercial silica fiber by use of only cleaving and fusion splicing steps. The all-fused-silica structure, combined with a thermal fusion bonding technique, solves the problem of thermal expansion mismatch among various materials and eliminates the difficulties associated with epoxy. Compared with current commercially available sensors, which can survive to only $485^{\circ} \mathrm{C}$ for a short period, our sensor has demonstrated high-temperature operation, at least $600^{\circ} \mathrm{C}$.

With respect to previously developed optical sensors, such as those that utilize techniques of wet etching, ${ }^{8,9}$ anodic bonding, ${ }^{10}$ and solgel bonding, the main improvement in this sensor is the fact that no chemical methods are involved. This not only ensures that the process is clean, safe, and easy but also greatly simplifies laboratory maintenance by eliminating the need for hazardous-waste disposal and fume-hood cleaning. This technique can be conveniently utilized by any laboratory including those without chemical processing facilities. Finally, the simpler method of fabrication and reduced material and tool requirements undoubtedly lower the total cost. This sensor, which exhibits micrometer size and high-temperature survivability, will be useful in applications for which high temperature and (or) small size is important, such as pressure measurement in turbine engines and boilers. ${ }^{11}$ In addition, the allfused-silica structure leads to potential biological and medical applications. ${ }^{12}$ Monitoring of arterial or venous blood pressure and coronary, pulmonary, and intracranial pressure measurements are only a few applications for which accurate and small disposable sensors are needed. Today, most invasive pressure measurements utilize a fluid-filled catheter that transfers pressure to an external transducer and is susceptible to many problems.

Figure 1 shows a schematic diagram of a sensor in which a Fabry-Perot interferometer is constructed that comprises the end face of an optical fiber and the surface of a diaphragm connected by a piece of hollow fiber. Applied pressure induces deflection of the diaphragm and changes the cavity length. Since the size of the diaphragm is of the order of micrometers, the effect of the pressure applied to the sidewalls can be neglected. Resolving the cavity change from the spectral shift can provide the pressure.

Commercially available single-mode fiber (SMF-28, Corning, Inc.) and silica hollow fiber (TSP-75/125, Polymicro) are utilized. To make the whole sensor compact, we selected an outside diameter of $125 \mu \mathrm{m}$ for the hollow fiber, the same as that of the SMF-28 fiber.

The fabrication consists of three basic steps: (a) Splice a piece of hollow fiber near the cleaved end of a standard fiber. (b) Cleave the hollow fiber near the junction according to the cavity length requirement. The cavity length can be cleaved down to the order of micrometers during inspection under a $40 \times$ microscope. (c) Splice another standard fiber to this part

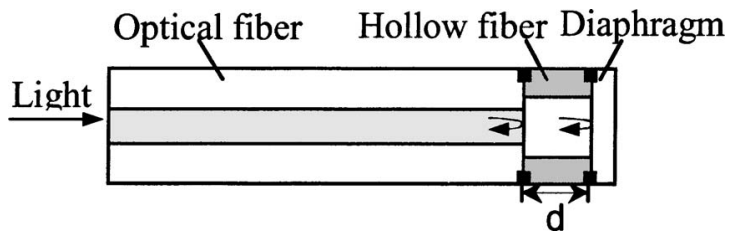

Fig. 1. Configuration of a fiber tip pressure sensor. 


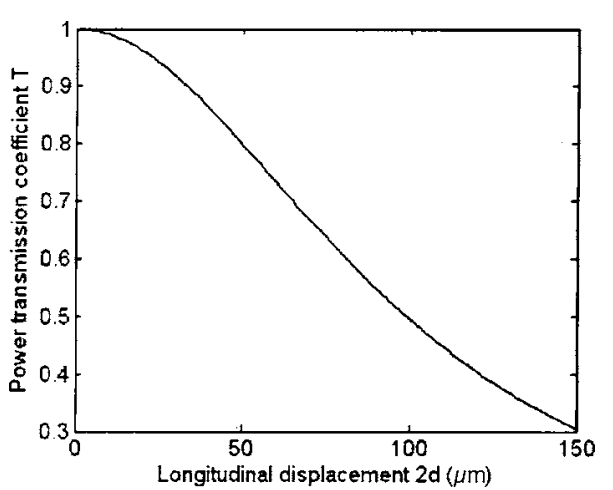

Fig. 2. Decrease in power transmission coefficient as the longitudinal displacement (cavity length) increases.

and cleave it to the desired length for use as a diaphragm. Each step utilizes an electric arc fusion splicer to align the components.

The reflection spectrum of the sensor is monitored by a component testing system (CTS; Model S1720, Micron Optics) that comprises a spectrometer with a laser light source and a detector. It has a spectral range of $1520-1570 \mathrm{~nm}$ with $2.5 \mathrm{pm}$ resolution.

Theoretically, three reflections are generated: from the end face of the lead-in single-mode fiber and from the two surfaces of the diaphragm. However, the interference between the reflections of the two diaphragm surfaces can be neglected because the diaphragm is thin. In addition, as the surface reflectivity is less than $4 \%$, the spectrum can be estimated as a two-beam interferometer.

During fabrication, different kinds of optical loss can be induced. Alignment losses owing to lateral and angular offset can be avoided by careful adjustment with a splicer. However, loss caused by the transmission of light inside the cavity, which can be regarded as a longitudinal misalignment of two fibers, is unavoidable. For longitudinal separation $L$, which is double cavity length $d$ in our case, transmission $T$ is $^{13}$

$$
\begin{aligned}
& T=\frac{4\left[4 Z^{2}+\left(w_{1}^{2} / w_{2}^{2}\right)\right]}{\left[4 Z^{2}+\left(w_{2}^{2}+w_{1}^{2} / w_{2}^{2}\right)\right]^{2}+4 Z^{2}\left(w_{2}^{2} / w_{1}^{2}\right)}, \\
& Z=\frac{L}{n_{3} k w_{1} w_{2}} .
\end{aligned}
$$

According to an empirical formula,${ }^{14}$ for step-index fibers

$$
\frac{w}{a}=0.65+\frac{1.619}{V^{3 / 2}}+\frac{2.879}{V^{6}},
$$

where $w, w_{1}$, and $w_{2}$ are the optimum widths of the Gaussian field profile, the sending fiber, and the receiving fiber, respectively; $a$ is the core diameter; $V$ $=2 \pi N A / \lambda=2 \pi\left(n_{1}{ }^{2}-n_{2}{ }^{2}\right)^{1 / 2} / \lambda$ is the normalized frequency; and $n_{1}, n_{2}$, and $n_{3}$ are indices of the fiber core, the fiber cladding, and air, respectively.

In our case, $n_{1}=1.45, n_{2}=1.447, n_{3}=1$, and $a$ $=9 \mu \mathrm{m}$. Figure 2 indicates that the power transmis- sion coefficient decreases as cavity length increases. As a result, the size of the cavity is designed to be approximately $20-50 \mu \mathrm{m}$.

When the applied pressure changes, the diaphragm is deformed accordingly. Because the diaphragm is flat and of uniform thickness, its center deflection $\Delta L$, under applied pressure $\Delta P$, can be calculated by

$$
\Delta L=\frac{3\left(1-\mu^{2}\right) R^{4}}{16 E h^{3}} \Delta P,
$$

where $R$ and $h$ are the radius and the thickness of the diaphragm, respectively, $E$ is Young's modulus, and $\mu$ is Poisson's ratio.

The effective pressure limit of the sensor is determined by the deflection limit, which is no more than $30 \%$ of the diaphragm's thickness. ${ }^{15}$ Under this condition, the sensitivity is uniform throughout the operating range and can be determined from Eq. (4). For example, with a radius of $\sim 37.5 \mu \mathrm{m}$ and a diaphragm thickness of $5.85 \mu \mathrm{m}$, the sensor has a pressure limit of $10,000 \mathrm{psi}(68,950 \mathrm{kPa})$, and the sensitivity is $0.17 \mathrm{~nm} / \mathrm{psi}$. With a $2.19 \mu \mathrm{m}$ thick diaphragm, the sensing limit is $200 \mathrm{psi}(1379 \mathrm{kPa})$ and the sensitivity is $3.2 \mathrm{~nm} / \mathrm{psi}$.

Figure 3 shows a diagram of a sensor testing setup. The sensor was sealed in a pressure chamber whose pressure was supplied and controlled by a pressure generator-controller (Pressure Systems, Inc., Model PSI 9035). The sensor's output was detected by the CTS.

Figure 4 is the sensor's pressure response at room temperature for three cycles, each from $20 \mathrm{psi}$ $(137.9 \mathrm{kPa})$ to $30 \mathrm{psi}(206.8 \mathrm{kPa})$ and then back down to $20 \mathrm{psi}$. The cavity length and the diaphragm thickness are 20.710 and $6 \mu \mathrm{m}$. Experimental results indicate that this sensor has a sensitivity of $2.2 \mathrm{~nm} / \mathrm{psi}$ with $0.01 \%$ resolution. Figure $4(\mathrm{~b})$ is an enlargement of Fig. 4(a), indicating a hysteresis of $0.025 \%$, which is caused by residual stress during sensor fabrication and can be leveled off by stress cycling or annealing.

Since the sensor is made entirely of fused silica and has the potential to operate at high temperature, a second sensor with $8 \mu \mathrm{m}$ diaphragm thickness was cycled from $100^{\circ} \mathrm{C}$ to $600^{\circ} \mathrm{C}$. As shown in Fig. 5, the temperature dependence is $0.05 \mathrm{psi} /{ }^{\circ} \mathrm{C}$ $\left(344.7 \mathrm{~Pa} /{ }^{\circ} \mathrm{C}\right)$. Thermal expansion of the material and the trapped air pressure changes are two main factors. For large pressure change or (and) limited temperature variation applications, this error can be

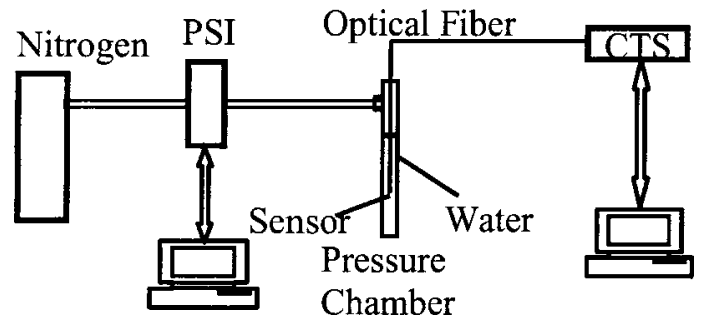

Fig. 3. System setup for static pressure test. PSI, pressure generator-controller. 


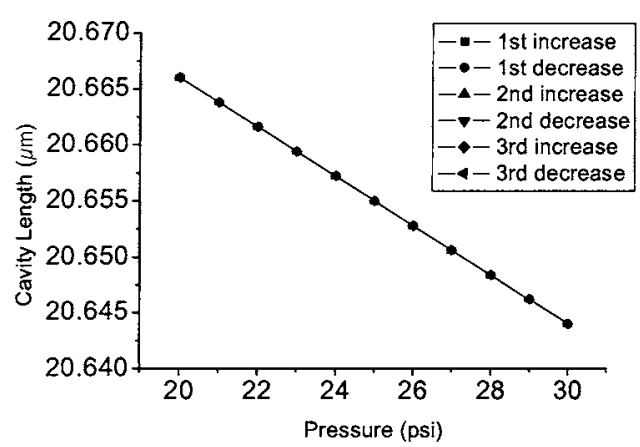

(a)

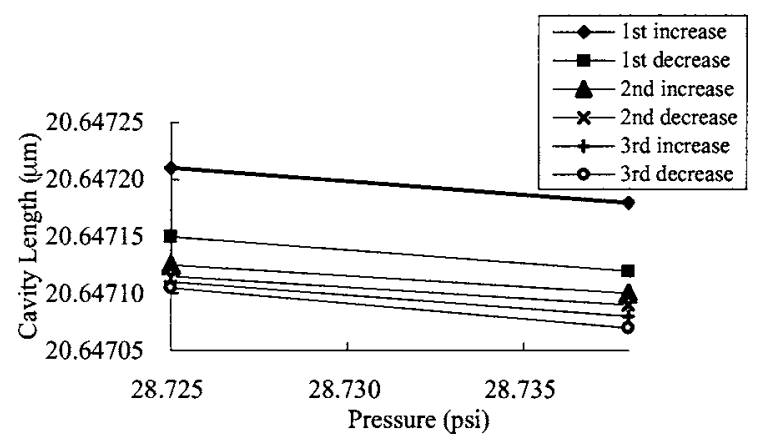

(b)

Fig. 4. (a) Sensor pressure response from 20 psi $(137.9 \mathrm{kPa})$ to $30 \mathrm{psi}(206.8 \mathrm{kPa})$ for three cycles and (b) enlargement from 28.725 to 28.738 psi, illustrating the small amount of hysteresis.

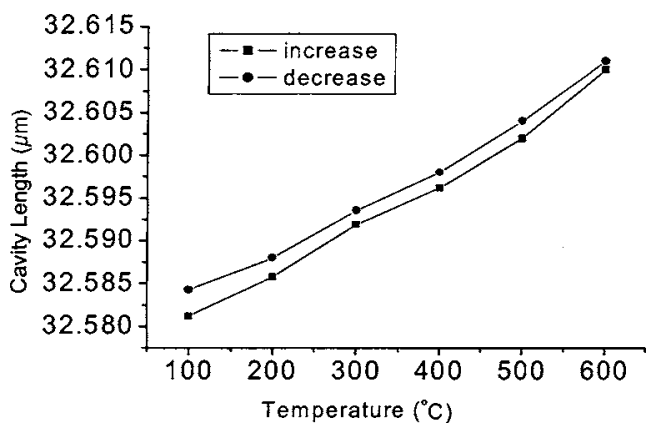

Fig. 5. Temperature dependence of the sensor.

neglected. Otherwise, temperature compensation techniques, such as combining a temperature sensor for calibration, are required.

A novel miniature all-fused-silica optical fiber tip pressure sensor has been developed that exhibits a
April 1, 2006 / Vol. 31, No. 7 / OPTICS LETTERS

micrometer scale size, simple structure, low cost, and immunity to electromagnetic interference. Fabrication involving no chemical processes provides safety and cost effectiveness. A sensitivity of $2.2 \mathrm{~nm} / \mathrm{psi}$, a resolution of $0.01 \mathrm{psi}(68.9 \mathrm{~Pa})$, and a hysteresis of $0.025 \%$ were demonstrated. Another tested sensor exhibited a temperature dependence of $0.05 \mathrm{psi} /{ }^{\circ} \mathrm{C}$ $\left(344.7 \mathrm{~Pa} /{ }^{\circ} \mathrm{C}\right)$. This miniature fiber tip pressure sensor may be suitable for medical diagnostics, environmental monitoring, and other industrial applications.

This work was supported by the U.S. Department of Energy under contract DE-FC36-01GO11050. Also, the authors thank Micron Optics for donation of part of the CTS. X. Wang's email address is xiwang @vt.edu.

\section{References}

1. D. Goustouridis, P. Normand, and D. Tsoukalas, Sens. Actuators A 68, 269 (1998).

2. P. Melvås, E. Kälvesten, P. Enoksson, and G. Stemme, Sens. Actuators A 97, 75 (2002).

3. J. Sirkis, T. A. Berkoff, R. T. Jones, H. Singh, A. D. Kersey, E. J. Friebele, and M. A. Putnam, J. Lightwave Technol. 13, 1256 (1995).

4. R. A. Wolthius, G. L. Mitchell, E. Saaski, J. C. Hartl, and M. A. Afromowitz, IEEE Trans. Biomed. Eng. 38, 974 (1991).

5. M. G. Xu and J. P. Dakin, in Proc. SPIE 1795, 2 (1992).

6. B. Yu, D. Kim, J. Deng, H. Xiao, and A. Wang, Appl. Opt. 42, 3241 (2003).

7. J. Xu, G. Pickrell, X. Wang, W. Peng, K. Cooper, and A. Wang, IEEE Photon. Technol. Lett. 17, 870 (2005).

8. Y. Zhu and A. Wang, IEEE Photon. Technol. Lett. 17, 447 (2005).

9. D. Donlagic and E. Cibula, Opt. Lett. 30, 2071 (2005).

10. J. Zhou, S. Dasgupta, H. Kobayashi, J. M. Wolff, H. E. Jackson, and J. T. Boyd, Opt. Eng. 40, 598 (2001).

11. X. Wang, J. Xu, Y. Zhu, B. Yu, M. Han, K. L. Cooper, G. Pickrell, and A. Wang, in Proc. SPIE 5998, 169 (2005).

12. X. Wang, J. Xu, Y. Zhu, B. Yu, M. Han, K. Cooper, G. Pickrell, and A. Wang, in Conference on Lasers and Electro-Optics / Quantum Electronics and Laser Science Conference (CLEO/QELS) 2005 (Optical Society of America, 2005; CD ROM).

13. D. Marcuse, Theory of Dielectric Optical Waveguides (Academic, 1974).

14. S. Nemoto and T. Makimoto, Opt. Quantum Electron. 11, 447 (1979).

15. M. D. Giovanni, Flat and Corrugated Diaphragm Design Handbook (Marcel Dekker, 1982). 


\title{
UV-induced intrinsic Fabry-Perot interferometric sensors and their multiplexing for temperature and strain sensing
}

\author{
Fabin Shen*, Zhuang Wang, Wei Peng, Kristie Cooper, Gary Pickrell, Anbo Wang \\ Center for Photonics Technology, Bradley Department of Electrical and Computer Engineering, \\ Virginia Polytechnic Institute and State University, Blacksburg, VA 24061
}

\begin{abstract}
We present UV-induced intrinsic Fabry-Perot interferometric (IFPI) fiber sensors and a frequency-division-multiplexing (FDM) scheme for quasi-distributed temperature and strain sensing. We present a spectrum-based measurement system with a swept laser source to measure the fringe patterns of IFPI sensors serially arranged along a single fiber. The FDM scheme is based on the multiplexing of sub-carrier frequencies generated by the frequency-modulation of a continuouswave light source. IFPI sensors with different optical path differences (OPD) will have different sub-carrier frequencies. We use band pass filters to select individual frequency component and use frequency-estimation based signal processing algorithms to determine the OPD of each sensor. Experimental results for multiplexed temperature and strain sensing are demonstrated. The performance of the multiplexing system is discussed.
\end{abstract}

Keywords: Optical fiber sensors, Fabry-Perot interferometer, ultraviolet, temperature, strain, distributed sensing, multiplexing, FDM.

\section{INTRODUCTION}

Distributed temperature and strain sensing is demanded by a wide range of applications, which include real-time monitoring of industrial processes, health monitoring of industrial and civil infrastructures etc. Many different kinds of electrical and optical sensors have been investigated to meet the requirements of such applications. Optical fiber distributed sensors, which have attracted tremendous research interest in the past decade, provide a promising solution for these applications. ${ }^{[1,2]}$

There are two main categories of optical fiber distributed sensing technologies, namely fully distributed sensing and quasi-distributing sensing. Fully distributed sensing techniques usually use Raleigh, Raman or Brillouin light scattering in a fiber to measure the temperature or strain at any location along the fiber with a certain spatial resolution. ${ }^{[1]}$ However, the spatial resolution and measurement accuracy in light scattering based measurement systems are usually limited due to the weak power of scattered light pulses. Quasi-distributed sensing technique use point fiber sensors and multiplexing techniques to measure the temperature or strain at the locations of interest. ${ }^{[2]}$ Multiple fiber sensors, such as fiber Bragg grating (FBG) sensors and inline fiber Fabry-Perot (FP) interferometric sensors, can be multiplexed along a single fiber. The multiplexing capacity of a quasi-distributed sensor network is usually limited to a small number because of the power loss at each sensor and the restriction of the multiplexing method.

The reflection spectrum change of a FBG in accordance with the variation of the environmental temperature and strain make FBG sensors a good candidate for quasi-distributed sensing. ${ }^{[3]}$ The inherent wavelength encoding nature of FBG makes it ideal to be interrogated in a wavelength division multiplexing (WDM) scheme. ${ }^{[4,5]}$ However, the number of FBGs in a WDM scheme is limited by the bandwidth of light source and the wavelength range reserved for each FBG sensor. The fabrication of FBG sensors with different central wavelengths also requires multiple phase masks or interferometric setups. A number of groups have been working to improve FBG sensors' multiplexing capability with time-division-multiplexing (TDM), FDM and hybrid multiplexing schemes. Froggatt et al demonstrated a FDM scheme for FBG sensors by using a frequency-modulated coherent light source and an air-gap reflector to determine the location of FBG sensors. ${ }^{[6]}$ Shlyagin et al exploited a twin FBG grating sensor configuration and a combination of wavelengthand frequency-division multiplexing. ${ }^{[7]}$

Inline fiber Fabry-Perot interferometric (FFPI) sensors have also been configured as a sensor array along a single fiber for quasi-distributed temperature and strain sensing. Several kinds of FFPI sensors have been reported including

*fashen1@ vt.edu; phone 1540 231-2155; fax 1540 231-2158

Smart Structures and Materials 2006: Sensors and Smart Structures Technologies for Civil,

Mechanical, and Aerospace Systems, edited by Masayoshi Tomizuka, et al., Proc. of SPIE Vol. 6174, 61740D, (2006) - 0277-786X/06/\$15 - doi: 10.1117/12.658979 
intrinsic Fabry-Perot interfermetric (IFPI) sensors and extrinsic Fabry-Perot interferometric (EFPI) sensors. An IFPI sensor contains two internal partial reflection mirrors. The fiber between the reflectors serves as both a sensing element and a light waveguide. Lee et al. demonstrated some IFPI sensors by building dielectric mirrors into optical fiber. ${ }^{[8]}$ An EFPI sensor contains a lead-in fiber with a partial mirror at the end-face, a cavity of air or other transparent medium and a reflector on the other end which may also be a piece of fiber. Researchers at Virginia Tech developed EFPI sensors by constructing an air gap between the end faces of two uncoated fibers in an alignment tube. ${ }^{\left[{ }^{910]}\right.}$ Sirkis et al introduced in-line fiber etalon based EFPI sensors by fusion splicing two fibers with a section of hollow tube of the same outside diameter. $^{[11]}$

FFPI sensors can be multiplexed in one of the multiplexing schemes such as TDM, FDM, and coherencemultiplexing (CM). ${ }^{[12-14]}$ However, the multiplexing of FFPI sensors has gained little success due to the relatively high power loss of the sensor. IFPI sensors with dielectric mirrors are difficult to fabricate and usually have high reflectivity. EFPI sensors formed by air-glass interfaces usually have high power loss at each sensor. Thus the multiplexing of FFPI sensors is usually limited to a small number.

UV-induced IFPI optical fiber sensors, which have low reflectance and low power loss, are good candidates for multiplexed sensors networks. J. A. Greene et al first introduced UV-induced broadband Fresnel reflectors in hydrogenloaded $\mathrm{GeO}_{2}$-doped optical fiber and demonstrated optical time domain reflectometry (OTDR)-based IFPI strain and temperature sensors by measuring the reflectance at a certain wavelength or counting the fringe numbers due to the parameter changes. ${ }^{[15]}$ We demonstrated a spectrum-based measurement method that can determine the OPD of the UVinduced IFPI sensors and demonstrated experimental results for temperature and strain measurement. ${ }^{[16]}$

In this paper, we present a FDM scheme for UV-induced IFPI sensors. Multiple sensors with different OPDs have different sub-carrier frequencies in the measured spectrum of IFPI sensors. The OPD of each sensor can be determined by estimating the frequency and the phase of the sub-carrier. We use a frequency estimation based digital signal processing algorithm to determine the OPD of the sensors. Digital filters are used to select individual frequency components and filter out the noise. We also demonstrate the experimental results of multiplexed IFPI sensors for temperature and strain measurement. The performance, crosstalk and multiplexing capacity of the multiplexed sensor system are analyzed. Experimental results showed that UV-induced IFPI sensors in a FDM scheme have good measurement accuracy for temperature and strain sensing and potentially have a large multiplexing capacity.

The contents of the paper are arranged as follows. In section 2, the principle of UV-induced IFPI sensors and the FDM multiplexing scheme are introduced. In section 3, the experimental results for temperature, strain sensing are presented. In section 4, conclusions are given.

\section{PRINCIPLE OF OPERATION}

\subsection{UV-induced IFPI sensors}

The photosensitivity of germanium-doped silica fiber has been widely used to make a variety of fiber components for communications and optical fiber sensors. When a short section of fiber is exposed to UV light, the reflective index of the fiber will be changed. Due to the mismatch of the refractive index, the UV-inscribed section will reflect part of the light and thus function as a partial mirror.

We have fabricated two types of UV-induced partial mirrors, namely Fresnel reflector and Bragg grating reflector. The Fresnel reflector is a broadband reflector fabricated by exposing the fiber directly to the UV-light through a narrow slit with a width of about 10-20 micrometers. The Bragg grating reflector can be fabricated by using a phase mask together with a narrow slit. When a FBG with only tens of periods is constructed, it also functions as a broadband reflector.

A pair of UV-induced partial mirrors inscribed in a fiber will construct an inline FP interferometer. The structure of the IFPI sensor is depicted in Fig. $1 .{ }^{[16]}$ Because that the reflectivity at these two partial mirrors are low, the FP interferometer can be approximated as a two-beam interferometer with the reflectivity given by

$$
R=R_{1}+R_{2}+2 \sqrt{R_{1} R_{2}} \cos (k L+\phi)=A+B \cos (k L+\phi)
$$

where $k=2 \pi / \lambda$ is the wave number and $\lambda$ is the wavelength of the incident light, $R$ is the reflectivity of the sensor, $R_{1}, R_{2}$ are reflectivities of the UV-induced reflectors, $L$ is the OPD between the two reflectors, and $\phi$ is a constant phase term due to the phase changes on reflection interfaces. 


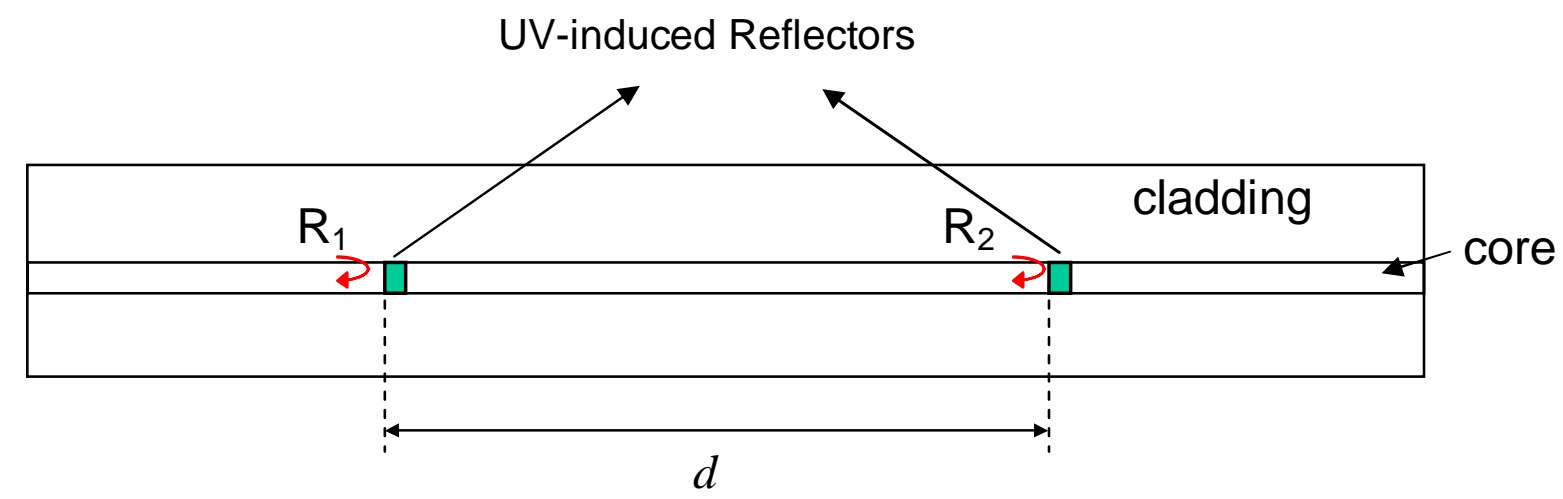

Fig. 1 Structure of a UV-induced IFPI sensor

When the reflectivity of the IFPI sensor is measured in a certain wave number range, the measurement series of reflectivity of an IFPI sensor at different wave numbers will contain a sinusoidal signal with a frequency of $L$, a amplitude of $B$, and a DC term of $A$. In Fresnel type reflectors, $R_{1}$ and $R_{2}$ are constant and the signal in Eq. (1) is a pure sinusoid. In FBG type reflectors, $R_{1}$ and $R_{2}$ are wavelength modulated and thus the sinusoidal signal in Eq. (1) is an amplitude-modulated signal with a carrier frequency of $L$. In both cases, the OPD of the sensor, $L$, can be determined by a frequency estimation approach.

The OPD between the two reflectors is

$$
L=2 n_{e} d
$$

where $n_{e}$ is the effective refractive index of the fiber and $d$ is the distance between the two reflectors. Both $n_{e}$ and $d$ will be changed when the temperature of the sensor is varied or when strain is applied to the sensor. Thus by determining the OPD of the sensor, we can perform temperature and strain measurement.

\subsection{Frequency-Division-Multiplexing}

Frequency-division-multiplexed optical fiber interferometric sensors have been reported by several research groups. Dandridge et al demonstrated a FDM scheme for a $\mathrm{J} \times \mathrm{K}$ sensor array by using multiple intensity-modulated laser sources. ${ }^{[17]}$ Sakai demonstrated an optical frequency domain reflectometry based FDM scheme with the sensor location determined by the frequencies. ${ }^{[18]}$ Wan et al demonstrated a frequency-modulated-continuous-wave (FMCW) based sub-carrier FDM scheme for FFPI sensors. ${ }^{[13]}$

We present a sub-carrier FDM scheme based on the reflection spectrum measurement of inline UV-induced IFPI sensors. The reflectivity of a UV-induced IFPI sensor is rather low and thus it is difficult to measure the reflection spectrum with conventional methods that utilize a low-coherence light source and passive optical spectrum analyzers. In our experiments, we use a high-resolution swept-laser interrogator (HRSLI, Micron Optics Si720), which contains a swept laser source, detectors, intensity and wavelength referencing units, and supporting electronic circuits in a standalone box, to measure the reflectivity of the IFPI sensors. The measurement system for multiplexed IFPI sensors is shown in Fig. 2.

Continuous-wave coherent light from a swept laser is launched into the sensor array. The wavelength of the laser is swept in a range of 1520-1570 nm with a step of $2.5 \mathrm{pm}$. The light reflected from the sensor is coupled to a detector by an optical circulator. A portion of the light from the laser source is tapped from the light source inside the HR-SLI and used as an intensity and wavelength reference. The normalized interference fringes of the IFPI sensors can be measured in each sweeping period of the laser source. The laser is operated to finish a sweep in $200 \mathrm{~ms}$. The average power injected to the fiber is about $1 \mathrm{~mW}$. The line width of the laser is about $500 \mathrm{M} \mathrm{Hz}$. 


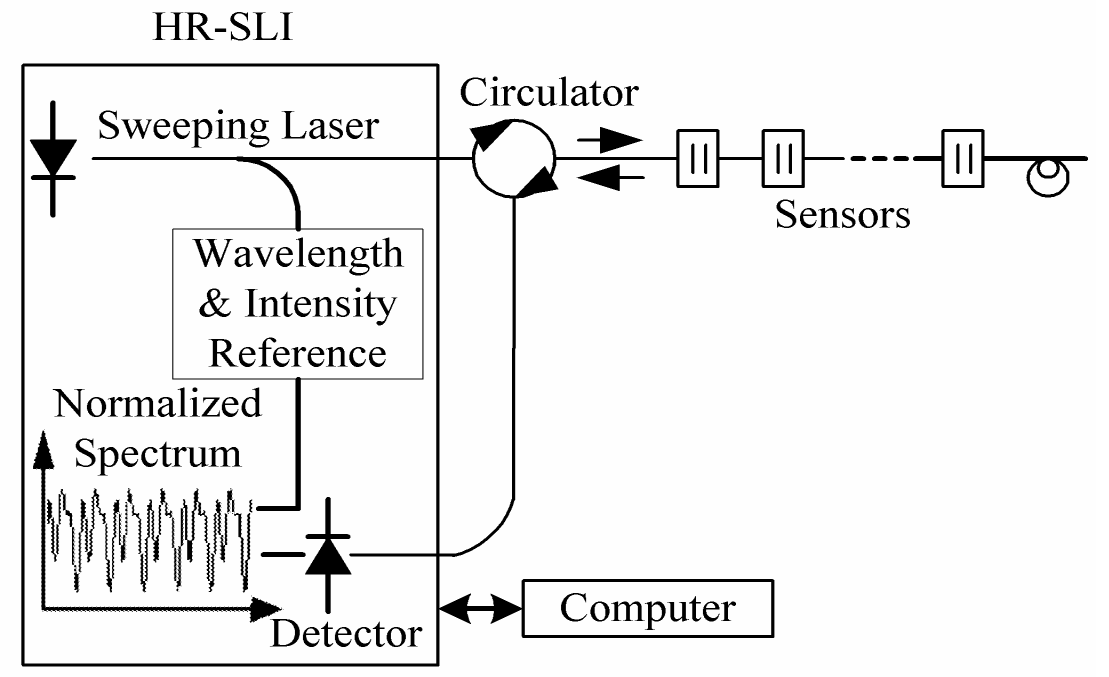

Fig. 2 Measurement system for frequency-division-multiplexed IFPI sensors.

IFPI sensors with different OPDs are arranged along a single fiber. The measured reflection spectrum of these sensors contains multiple sub-carrier frequency components corresponding to different OPDs. If the distance between two sensors is larger than the coherence length of the light source, then the detector in the HR-SLI will not see the interference between reflectors from different sensors. The measured reflection spectrum can be given as

$$
R\left(k_{n}\right)=\sum_{\mathrm{j}=1}^{\mathrm{J}}\left[A_{j}+B_{j} \cos \left(k_{n} L_{j}+\phi_{j}\right)\right]+v_{n}=\sum_{j=1}^{J} A_{j}+\sum_{j=1}^{J} B_{j} \cos \left(k_{n} L_{j}+\phi_{j}\right)+v_{n}
$$

where $J$ is the number of sensors, $k_{n}$ is the wave number at the n-th wavelength step, $L_{j}$ is the OPD of the j-th sensor, and $v_{\mathrm{n}}$ is the measurement noise at wave number $k_{n}$. By estimating the frequencies of the sub-carrier sinusoids from the measured signal, we can determine the OPDs of multiple IFPI sensors.

The task of the signal processing is to determine the frequencies from the measured signal in Eq. (3). It is a classical multi-tone frequency estimation problem. We use digital finite impulse response (FIR) digital filters to select each frequency component in the signal, and thus signal processing is simplified to a single-tone frequency estimation problem. ${ }^{[19,20]}$ We use a Hilbert transform based method for the frequency estimation of amplitude-modulated sinusoidal signal.

The measured interference spectrum is transferred to a personal computer via a GPIB interface for signal processing. A multi-threaded $\mathrm{C} / \mathrm{C}++$ win32 program running on the host computer was written to retrieve the spectrum from the HR-SLI and perform the signal processing tasks. The coefficients of FIR filters are stored in the computer. The C/C++ program running on the host computer can process the data acquisition and signal processing simultaneously at a speed of about 100 sensors per second.

\subsection{Discussions}

\section{A. Measurement resolution}

When the light source is linearly swept, the reflections of the two reflectors in one sensor will have different optical frequencies and generate a sub-carrier beat note. The frequency of the beat note is

$$
f_{b}=\frac{f_{s}}{T} \frac{L}{c}
$$

where $f_{s} / T$ is the frequency sweeping rate of the light source, and $\mathrm{c}$ is the speed of light. It is evident that the measurement resolution of $L$ is inversely proportional to the frequency sweeping rate for a given signal detection 
resolution of $f_{b}$. Conventional FMCW systems usually change the optical wavelength by modulating the driving current of a laser diode. The wavelength variation is usually in order of picometers. HR-SLI uses an optical tunable filter to change the wavelength of a fiber laser from $1520 \mathrm{~nm}$ to $1570 \mathrm{~nm}$ in a $200 \mathrm{~ms}$ period. The frequency sweeping rate of HR-SLI is much higher that conventional FMCW systems and thus a high measurement resolution of $L$ can be obtained. In our experiment, a relative measurement resolution of $1 \times 10^{-6}$ can be obtained.

\section{B. Multiplexing capacity}

The multiplexing capacity is determined by two factors, namely, the system power budget and the bandwidth budget in frequency domain.

The HR-SLI used in the measurement system has an average power output of $0 \mathrm{dBm}$ and a dynamic range of $80 \mathrm{~dB}$. The average reflected power of a UV-induced IFPI sensor is about $-50 \mathrm{dBm}$, thus the power budget for the measurement system is about $30 \mathrm{~dB}$. The insertion loss of a sensor can be as low as $0.1 \mathrm{~dB}$. Thus the multiplexing capacity is limited by the power budget to about 300 .

In order to separate an individual frequency from the others, each frequency component has to occupy certain bandwidth. The FIR filters discussed above also have some certain bandwidth. Thus the frequency spacing between adjacent frequencies cannot be very close. For a non-parametric frequency estimation method, the frequency resolution is $1 / \mathrm{N}$ due to the Rayleigh limit where $\mathrm{N}$ is the length of data sequence. In our experiment, the frequency spacing is set to (4 5)/N, which corresponds to an OPD difference of about 200 micrometers. The multiplexing capacity of the FDM scheme is determined by the ratio of the available bandwidth over the frequency spacing between sensors. The theoretically available bandwidth is 1 in normalized frequency domain based on the Nyquist sampling theorem. If the frequency spacing is set to $(4 \sim 5) / \mathrm{N}$, since $\mathrm{N}$ is a large number of 20,000 , then theoretically, thousands of sensors can be multiplexed.

Based on the discussion of the system power and bandwidth budgets, about 300 sensors could be practically multiplexed along a single fiber. The multiplexing number can be increased if the insertion loss of each sensor can be lowered and the bandwidth requirement of each sensor can be reduced. However, more precise control is needed to lower the insertion loss of each sensor and more efficient frequency estimation methods are desired to separate frequency components that are closely located.

\section{Crosstalk}

Multiplexed IFPI sensors in a serial topology will see some crosstalk between sensors. The signal of an IFPI sensor will be affected other sensors in the sensor array.

The reflectance of transmittance of $i$-th sensor is assumed to be $R_{i}$ and $T_{i}$ respectively. $T_{i}$ can be expressed as

$$
T_{i}=\eta_{i}\left(1-R_{i}\right)
$$

where $\eta_{i}$ is the insertion loss of the $\mathrm{i}$-th sensor. The measured reflectivity of $\mathrm{i}$-th sensor can be given as

$$
R_{m}=R_{i} \prod_{j=1}^{i-1} T_{j}^{2}=R_{i} \prod_{j=1}^{i-1} \eta_{j}^{2}\left(1-R_{j}\right)^{2} .
$$

Because $\mathrm{R}_{\mathrm{j}}$ is a very small value due to the low reflectivity of the IFPI sensor and $\eta_{j}$ is close to 1 due to the low insertion loss of each sensor, $R_{m}$ can be simplified to be

$$
R_{m} \approx R_{i} \prod_{j=1}^{i-1}\left(1-2 R_{j}\right) \approx R_{i}-2 R_{i} \sum_{j=1}^{i-1} R_{j} .
$$

The first term is the uncorrupted signal of i-th sensor and the second term is the crosstalk caused by the sensors in front of it. The ratio between them 


$$
r_{i}=2 \sum_{j=1}^{i-1} R_{j}
$$

is a measure of the crosstalk between sensors. The average reflectance of an UV-induced IFPI sensor is about $-50 \mathrm{~dB}$ $\left(1 \times 10^{-5}\right)$, thus when 100 sensors are multiplexed, the crosstalk is about than $2 \times 10^{-3}(-27 \mathrm{~dB})$, which is a very low value.

The multiplexed IFPI sensors occupy different frequency channels and the estimation of one frequency may be affected by the existence of other frequencies. This is another source of crosstalk between sensors. When the spacing between these frequencies is large enough that the side band of one frequency is very low, the crosstalk between different frequency components can be neglected. Practically, the difference between OPDs, which is designed to be about $200 \mu \mathrm{m}$, is large enough. When a Hamming windowed FIR filter is used, a $40 \mathrm{~dB}$ noise rejection level can be obtained.

\section{EXPERIMENTAL RESULTS}

\subsection{Multiplexing}

Fig. 3 shows the experimental results of 20 IFPI sensors with Fresnel type reflectors that are serially interrogated as shown in Fig. 2. Their OPDs were in the range of $3165 \mu \mathrm{m}$ to $7627 \mu \mathrm{m}$ with an approximate $235 \mu \mathrm{m}$ difference between adjacent OPDs.
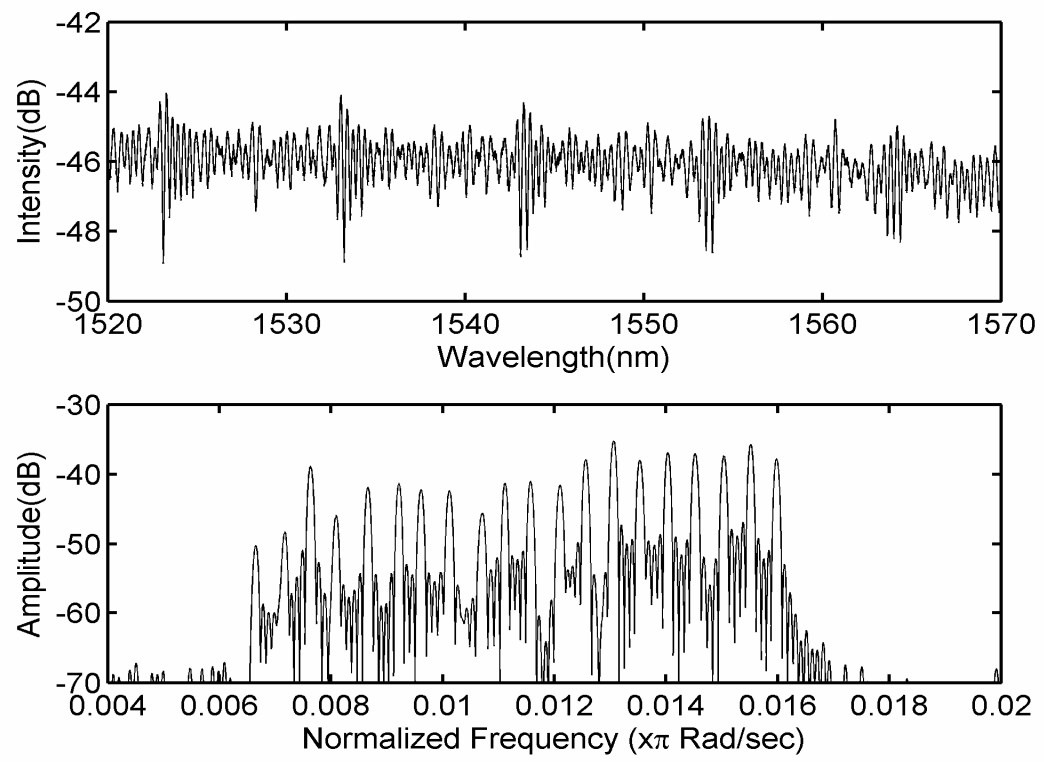

Fig. 3. Experimental result of multiplexed IFPI sensors.

The top graph shows the reflection spectrum measured by the HR-SLI. The bottom graph shows the fast Fourier transformation (FFT) result of the interpolated reflection spectrum. The horizontal axis of the bottom graph is the normalized frequency, which is the ratio of signal's frequency to the Nyquist frequency. Different peaks show different frequency components from different sensors.

\subsection{Temperature measurement}

When the intrinsic FP cavity is put into a temperature-varying environment, the OPD of the cavity will change due to the thermo-optic effect and the thermal expansion of the silica fiber. The OPD change of a sensor can be given as

$$
\Delta L=L\left(\alpha_{T}+\sigma_{T}\right) \Delta T
$$


where $\alpha_{T}$ is the coefficient of thermal expansion and $\sigma_{T}$ is the thermo-optic coefficient of silica fiber. It can be seen that the normalized OPD change, $\Delta L / L$, is only related to the properties of material and the temperature variation. Thus we can use a single calibration curve for multiple sensors with different OPDs. The temperature responses of 5 FBG type reflectors based IFPI sensors are plotted in Fig. 4. The second order polynomial fitting curve can be used as a calibration to determine the temperature readings. A measurement resolution of $0.1^{\circ} \mathrm{C}$ was obtained.

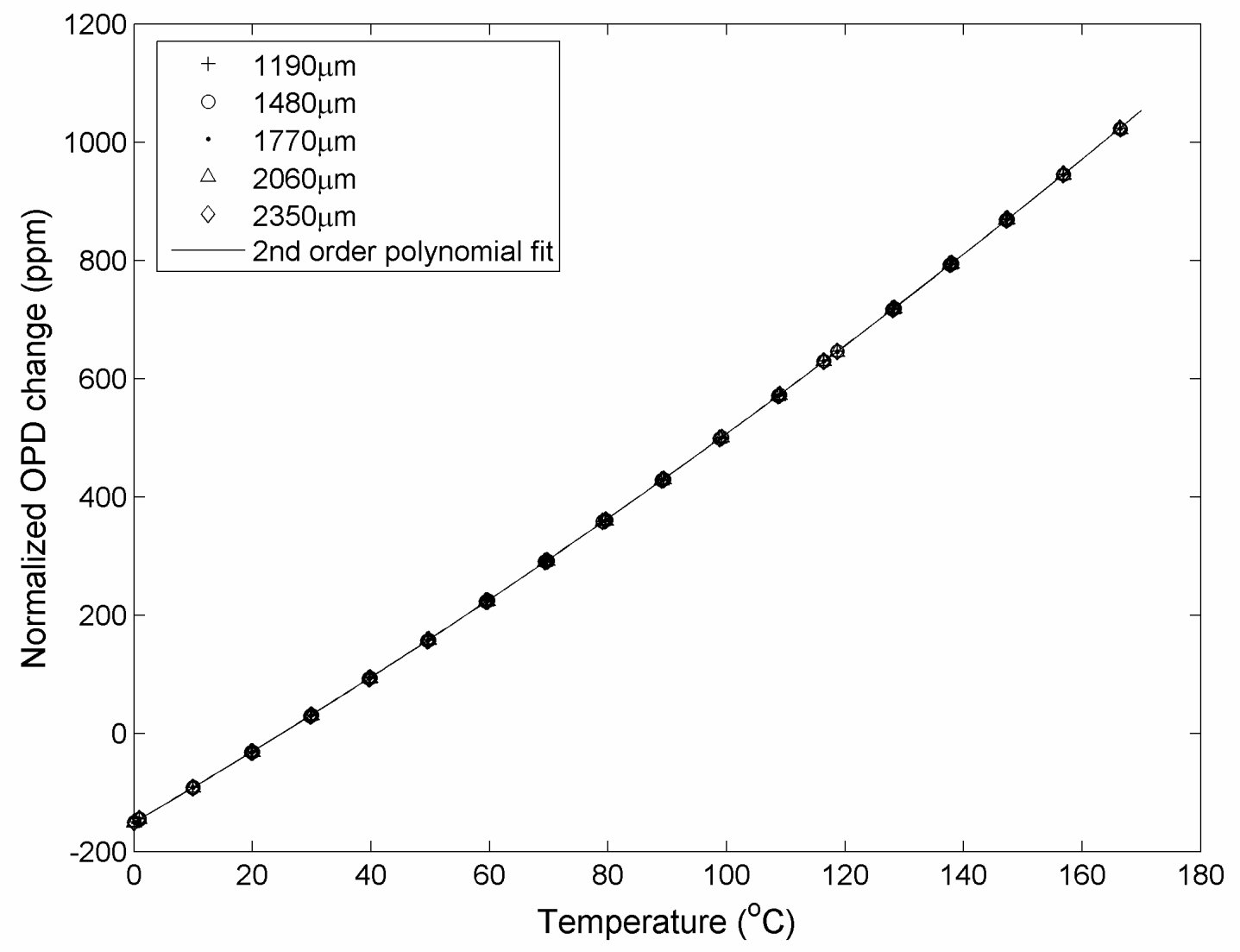

Fig. 4. Temperature responses of multiplexed IFPI sensors.

\subsection{Strain measurement}

The UV-induced IFPI sensor can also be used for strain sensing. The OPD change is due to the dimension change and the stress-optic effect of the silica fiber. The OPD change can be given as

$$
\Delta L=L\left(1-P_{e}\right) \mathcal{E}
$$

where $P_{e}=0.21$ is the effective strain-optic coefficient and $\varepsilon$ is the strain applied to the sensor. The experimental results for strain measurement are shown in Fig. 5. The sensors were bonded to an iron cantilever beam. The strains applied were read out by an electrical foil strange gauge. The OPD changes of IFPI sensors were recorded. The normalized OPD changes were plotted in Fig. 5. The linear fitting curve in Fig. 5 can be used as a calibration curve for strain measurement. A measurement resolution of $1.3 \mu \varepsilon$ was obtained. 


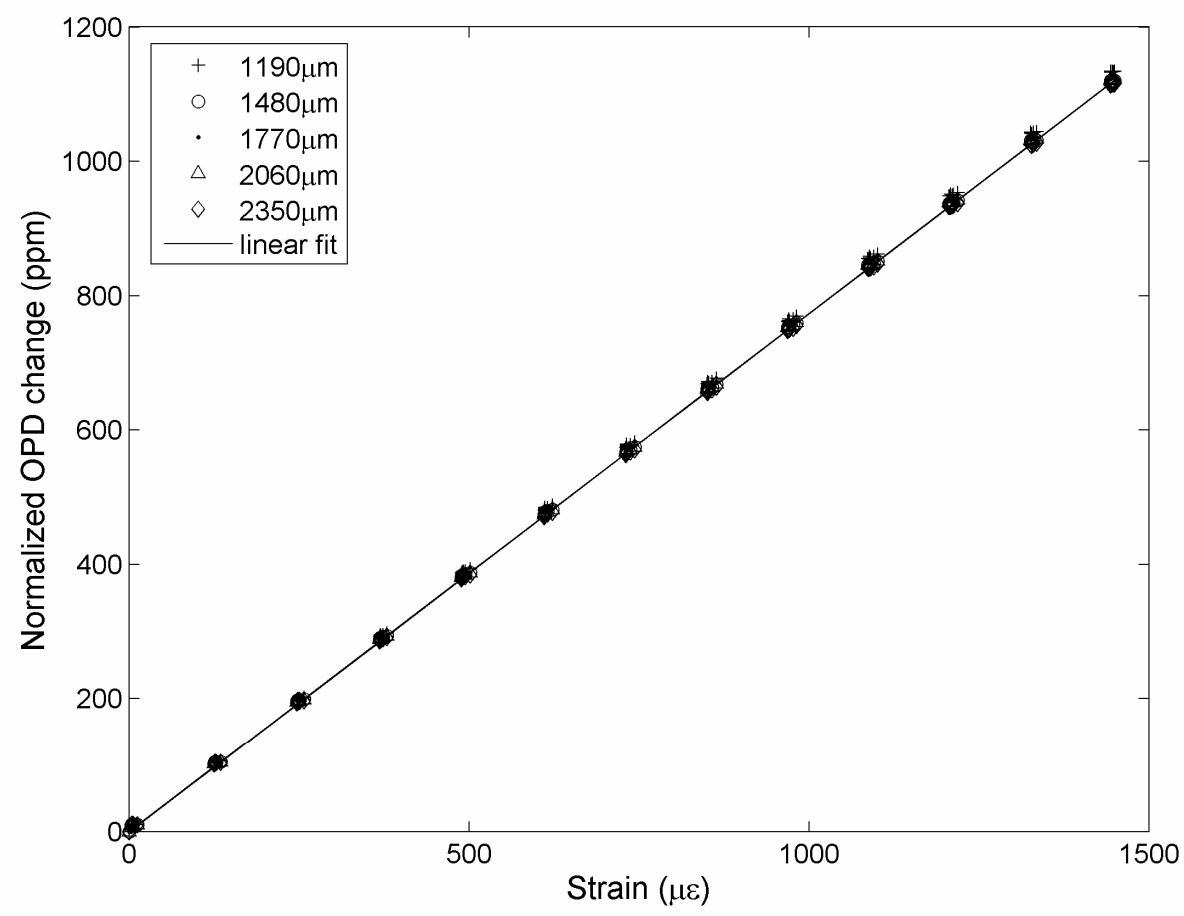

Fig. 5. Strain measurement results of multiplexed IFPI sensors.

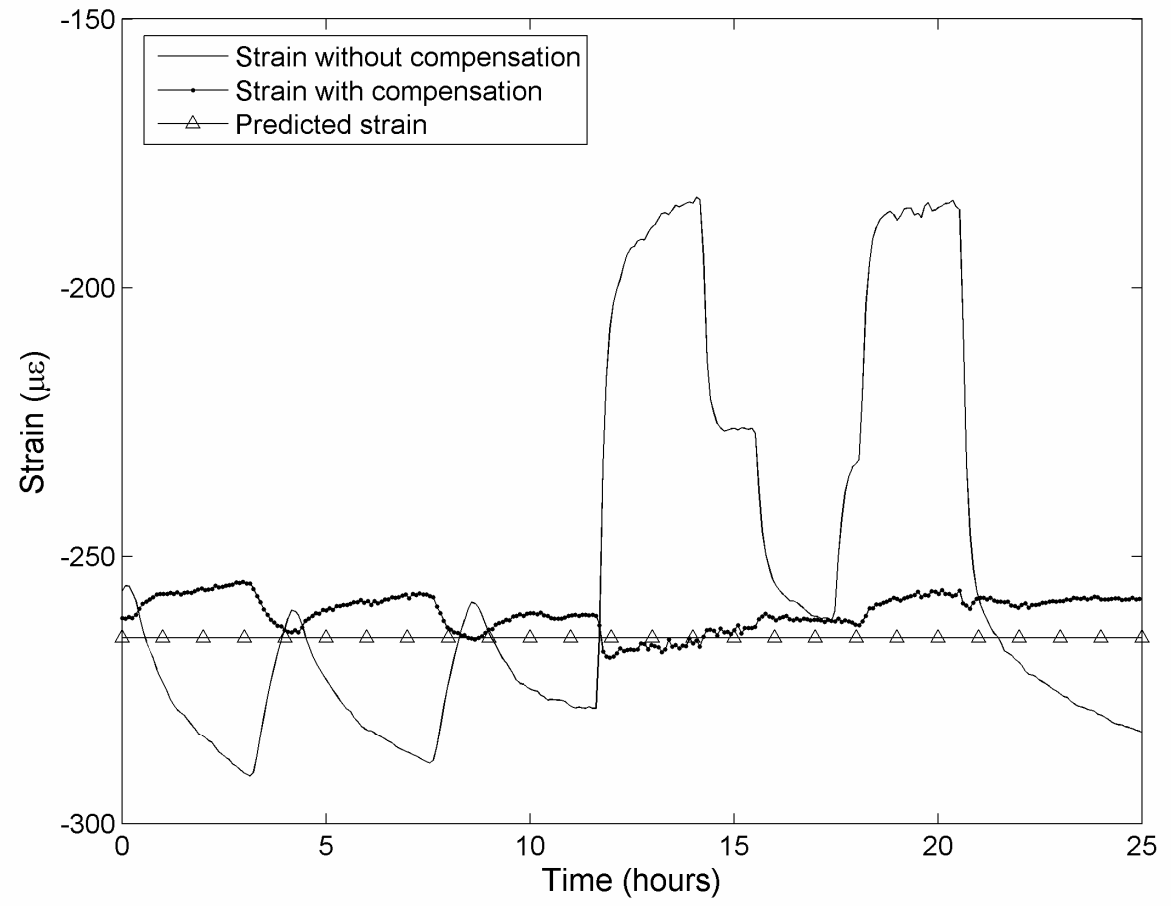

Fig. 6. Temperature compensation of strain measurement. 


\subsection{Temperature and strain discrimination}

The UV-induced IFPI sensor is sensitive to both temperature and strain. Therefore, unless the strain measurement is performed in a temperature-controlled environment or temperature measurement is performed without any external strain, the perturbation of strain and temperature has to be separated.

We demonstrate a temperature compensation method for strain measurement. We used a pair of sensors to separate the temperature and strain effect. One IFPI sensor was firmly bonded onto the surface of structures and acts as a strain sensor. The other IFPI sensor was loosely attached next to the strain sensor and acts as a temperature sensor that will not see strain change. From the reading of the temperature from the temperature sensor, we compensated the temperature effect of the strain sensor. The temperature compensation result is shown in Fig. 6. We installed 3 pairs of sensors on an iron beam. The strain of the iron beam was kept at a constant strain of $-270 \mu \varepsilon$. The temperature around the sensors was varied. The sensor's temperature and strain sensitivities were $17.1 \mathrm{ppm} /{ }^{\circ} \mathrm{C}$ and $0.78 \mathrm{ppm} / \mu \varepsilon$, respectively. Thus, the strain measurement was affected greatly by the temperature variation as Fig. 5 shows. After temperature compensation, the temperature effect was reduced by $85 \%$. The strain curve after temperature compensation is plotted in Fig. 6 and compared with the constant strain applied to the sensor.

\section{CONCLUSIONS}

In conclusion, we present UV-induced IFPI sensors and a FDM multiplexing scheme for temperature and strain sensing. We use a UV-inscribing technique to form Fresnel-type and FBG-type mirrors inside a fiber. Two such mirrors can construct an inline FFPI sensor. Multiple sensors can be arranged along a single fiber for quasi-distributed sensing. We use a FMCW technique to interrogate the IFPI sensors. The reflected light from different reflectors in an IFPI sensor will generate a sub-carrier sinusoidal beat note. The frequency of sub-carrier beat note is proportional to the OPD of the IFPI sensor. Signals from sensors with different OPDs will have different frequencies and thus can be demodulated by using a FDM multiplexing technique. We use digital filters and frequency estimation signal processing approaches to determine the OPDs of the IFPI sensors. We discuss the measurement resolution, multiplexing capacity and crosstalk of the FDM scheme. Potentially, a large number of UV-induced IFPI sensors can be multiplexed for quasi-distributed temperature and strain sensing by using the FDM scheme. We demonstrate the experimental results of multiplexed sensors for temperature and strain sensing and the discrimination of temperatures and strains.

\section{ACKNOWLEDGMENTS}

This research is mainly supported by the U.S. National Science Foundation under grant CMS-0427951 and partially supported by the U.S. Department of Energy under grant DE-FC36-01G011050. The authors would also like to thank Prime Photonics Inc. for the sponsorship and Micron Optics Inc. for the partial donation of the HR-SLI si-720 used in this research.

\section{REFERENCES}

1. J. P. Dakin, "Distributed optical fiber sensors", in Fiber Optic Smart Structures, E. Udd Eds. New York, NY, John Wiley \& Sons, Inc, 1995, Ch. 14, p.373-408.

2. R. Kist, "Point sensor multiplexing principles," in Optical Fiber Sensors: Systems and Applications, B. Culshaw, J. P. Dakin Eds., Norwood, MA, Artech House, 1988, vol. 2, ch. 14, pp. 511-574.

3. Kenneth O. Hill and Gerald Meltz, "Fiber Bragg grating technology fundamentals and overview." J. Lightwave Technol. Vol 15(8), pp. 1263-1276, 1997.

4. T. A. Berkoff, and A. D. Kersey, "Fiber Bragg Grating Array Sensor System Using a Bandpass Wavelength Division Multiplexer and Interferometric Detection," IEEE Photonoics Technol. Lett. 8(11). pp. 1522-1524, 1996.

5. A. Ezbiri, A. Munoz, S. E. Kanellopoulos and V. A. Handerek, "High resolution fibre Bragg grating sensor demodulation using a diffraction grating spectrometer and CCD detection," in IEE Colloquium on Optical Techniques for Smart Structures and Structural Monitoring, Feb. 1997, pp.5/1 - 5/6.

6. M. Froggatt and J. Moore, "Distributed measurement of static strain in an optical fiber with multiple Bragg gratings at nominally equal wavelengths," Appl. Opt., Vol. 37, No. 10, pp1741-46, 1998. 
7. M. G. Shlyagin, S. V. Miridonov, I. M. Borbon, V. V. Spirin, P. L. Swart, and A. A. Chtcherbakov, "Multiplexed twin Bragg grating interferometric sensor", Optical Fiber Sensors Conference Technical Digest, 2002. OFS 2002, 15th, pp. $191-194$

8. C. E. Lee and H. F. Taylor, "In-line Fiber Fabry Perot Interferometer With High Reflectance Internal Mirrors," J. Lightwave Technol., vol. 10, pp. 1376-1379, Oct. 1992.

9. K. A. Murphy, M. F. Gunther, A. Wang, R. O. Claus and A. M. Vengsarkar, "Extrinsic Fabry Perot Optical Fiber Sensor," Proc. OFS-8, pp. 193, Jan. 1992.

10. Anbo Wang, Hai Xiao, J. Wang, Zhiyong Wang, W. Zhao and R. G. May, "Self-Calibrated InterferometricIntensity-Based Optical Fiber Sensors," J. Lightwave Technol., Vol 19, pp. 1495-1501, Oct. 2001.

11. J. Sirkis, T. A. Berkoff, R. T. Jones, H. Singh, A. D. Kersey, E. J. Friebele and M. A. Putnam, "In-line fiber etalon (ILFE) fiber-optic strain sensors," J. Lightwave Technol., Vol.13, pp. 1256-1268, Jul. 1995.

12. Y. Chen and H. F. Taylor, "Multiplexed fiber Fabry-Perot temperature sensors system using white light interferometry", Opt. Lett. 27, 903-905 (2002).

13. X. Wan and H. F. Taylor, "Monitoring and Multiplexing Technique for Interferometric Fiber Optic Sensors with a Linearly Chirped Er Fiber Laser", Appl. Opt. 41, 7607-7611 (2002).

14. M. E. Jones, J. L. Grace, V. Bhatia, K. A. Murphy, R. O. Claus, J. A. Greene and T. A. Tran, "Multiplexed absolute strain measurements using extrinsic Fabry-Perot interferometers," Proc. Conf on Smart Structures and Materials, SPIE 95 paper 2444-28, 1995.

15. J. A. Greene, T. A. Tran, K. A. Murphy, A. J. Plante, V. Bhatia, M. Sen and R. O. Claus, "Photoinduced Fresnel Reflectors for Point-wise and Distributed Sensing Applications", Proc. SPIE vol. 2444, pp. 64, 1995.

16. F. Shen, W. Peng, K. L. Cooper, G. R. Pickrell, A. Wang, "UV-Induced Intrinsic Fabry-Perot Interferometric Fiber Sensors," Proc. SPIE, Vol. 5590, pp. 47-56, 2004.

17. A. Dandridge, A. Tveten, A. Kersey, A. Yurek, "Multiplexing of interferometric sensors using phase carrier techniques" J. Lightwave Technol. 5, pp. 947, 1987.

18. I. Sakai, "Frequency division multiplexing of fibre sensors using a frequency modulated source," Opt. \& Quantum Electron. 18, p. 279, 1986.

19. F. Shen and A. Wang, "Frequency-estimation-based signal-processing algorithm for white-light optical fiber FabryPerot interferometers", Applied Optics, Vol. 44, Issue 25, pp. 5206-5214, 2005.

20. F. Shen, Z. Huang, Y. Zhu, M. Coffey, R. Frank, G. Pickrell, and A. Wang, "Frequency-Division-Multiplexed Fabry-Perot Interferometric Fiber Sensors for Temperature Monitoring in a Selective Catalytic Reduction Unit", Proc. SPIE Vol. 5998, p. 136-143, 2005. 


\title{
Miniature Temperature-Insensitive Fabry-Pérot Fiber-Optic Pressure Sensor
}

\author{
Juncheng Xu, Xingwei Wang, Kristie L. Cooper, Gary R. Pickrell, and Anbo Wang
}

\begin{abstract}
We present a miniature fiber-optic pressure sensor with a diameter of only $125 \mu \mathrm{m}$. The sensor works as an extrinsic Fabry-Pérot interferometer and the cavity length can be controlled with a resolution of about several nanometers. Also the sensor sensitivity can be easily adjusted by controlling the sensor gauge length. A passive temperature compensation scheme was used to reduce most of the sensor's temperature dependence. Since the sensor is composed entirely of fused silica, it is reliable, biocompatible, corrosion resistant, and immune to electromagnetic interference and has high-temperature capability.
\end{abstract}

Index Terms-Fabry-Pérot (FP), fiber-optic sensor, miniature sensor, pressure sensor.

\section{INTRODUCTION}

$\mathbf{M}$ INIATURE fiber-optic sensors are more attractive compared to bulky sensors in many applications where restricted space or minimum measurement interference is a concern, for example, invasive blood pressure measurement [1], [2] and embedded material strain monitoring [3]. In the past 15 years, a number of Fabry-Pérot interferometric (FPI) fiber-optic sensors have been developed, which are classified as intrinsic Fabry-Pérot interferometers (IFPIs) [4] or extrinsic Fabry-Pérot interferometers (EFPIs) [5], [6]. Generally, the sensing element of an IFPI sensor is a short section of fiber sandwiched between two Fabry-Pérot (FP) mirrors. For a conventional EFPI sensor, the sensing elements are lead-in and reflecting fibers and a tubing holding them. IFPI sensors are often more sensitive to temperature changes and a little smaller than EFPI sensors while the latter make it easier to adjust the FP cavity length and often have higher pressure sensitivities. In addition to the sensors mentioned, an in-line fiber etalon sensor has also been reported [7], in which the sensing element is a short section of hollow fiber.

In this letter, we present a hollow fiber based miniature EFPI (MEFPI) fiber-optic pressure sensor, which has a diameter of only $125 \mu \mathrm{m}$. The structure of the pressure sensor is shown in Fig. 1. The length of the FP cavity (air gap) will decrease with the reduction in length of the hollow fiber as a result of the applied pressure. Light is injected into the lead-in optical fiber and partially reflected (4\%) by the end faces of the two fibers. Then the two reflections propagate back through the same lead-in

Manuscript received November 2, 2005; revised January 30, 2006. This work was supported by the U.S. Department of Energy (DOE) under Contract DE-FC36-01GO11050.

The authors are with the Center for Photonics Technology, Department of Electrical and Computer Engineering, Virginia Polytechnic Institute and State University, Blacksburg, VA 24061 USA (e-mail: juxu1@vt.edu).

Digital Object Identifier 10.1109/LPT.2006.873361

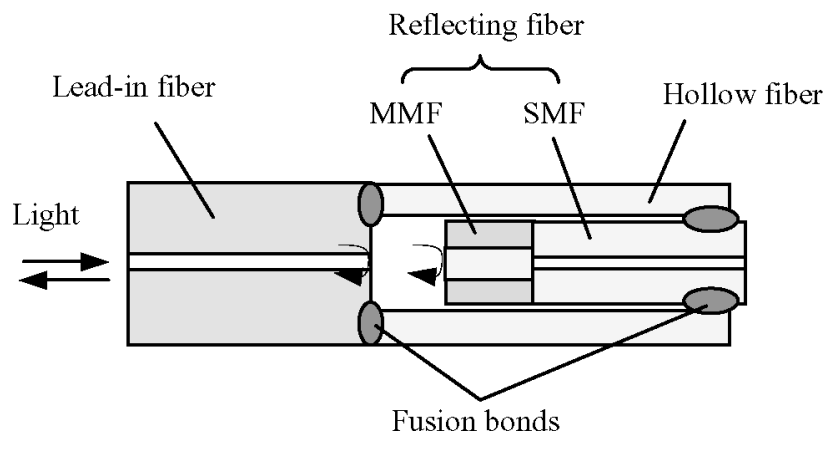

Fig. 1. MEFPI fiber-optic pressure sensor.

fiber and generate interference fringes, which will be demodulated to determine the air gap thickness. The FP cavity length can be adjusted with high precision in a wide range, giving a great deal of flexibility in the choice of light source or the signal demodulation scheme selection.

\section{SENSOR DESIGN AND FABRICATION}

A cleaved lead-in optical fiber [single-mode fiber (SMF-28)] was spliced with a 3.3-mm-long hollow fiber with inside diameter (I.D.) of $75 \mu \mathrm{m}$ and outside diameter (O.D.) about $125 \mu \mathrm{m}$ by using a optical fiber fusion splicer (Sumitomo, Type-36). Another cleaved optical fiber with O.D. of 60-65 $\mu \mathrm{m}$ was inserted into the hollow fiber from the other end as a reflecting fiber to form an FP cavity between these cleaved two fiber end faces. The thinner fiber can be obtained by etching or drawing a standard optical fiber (125- $\mu$ m O.D.). The lead-in fiber was connected to a white light interferometer system [8]. The air gap was preadjusted by the splicer stage holding the reflecting fiber tail, and monitored on-line by the white light system. The air gap can be set from zero to millimeters depending on the coherence length of the light source and application requirements. Because the stage of the fiber fusion splicer has precisely controlled alignment and movement, the sensor fabrication is simple, convenient, and fast. Once the air gap was adjusted to near the desired value, the electric arc was applied to bond the reflection fiber with the hollow fiber end. In addition, the sensor air gap can be adjusted with a resolution as high as nanometers by properly controlling the power, duration, and number of electric arcs as shown in Fig. 2. To our knowledge, this is the first time that the length of an optical fiber FP cavity was precisely controlled by electric arcs, which is a useful technique for sensor fabrication and investigation of FP interferometers.

The lead-in fiber can also be a multimode fiber (MMF) depending on application requirements, for example, more optical 


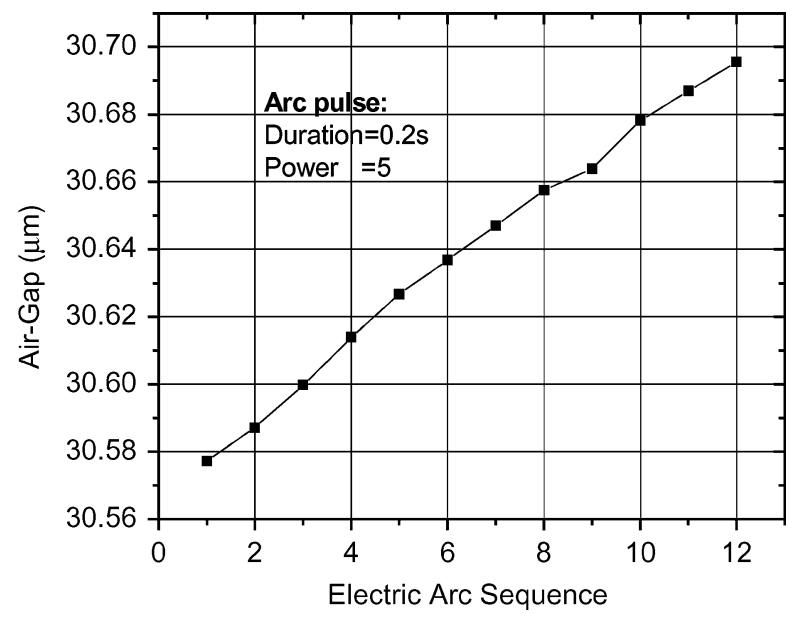

Fig. 2. FP cavity length controlled by arcs.

power needs to be coupled into the fiber. Since no adhesive is used to bond the sensor parts, this all-silica sensor is expected to be reliable, resistant to corrosion, and able to endure high temperature near the operational limit of the optical fiber itself. Also, the hair size structure is suitable for biomedical applications.

The sensor's pressure sensitivity is determined by its gauge length, which is highly repeatable because the gauge length is easily controlled with high precision by the splicer. The variation in sensitivity is within $0.05 \mathrm{~nm} / \mathrm{psi}$ based on ten sensors prepared and tested. Generally, the hollow fiber was cleaved with a length near the desired gauge length to minimize the sensor size. In addition, compared with the fiber-tip-diaphragm based sensors, this MEFPI pressure sensor is much easier to fabricate, resistant to scratches, and has much larger pressure measurement range.

Since the hollow fiber is uniform along its axis, its elastic deformation is similar to that of a larger tubing [9], therefore, the sensor air gap change $\Delta G$ under a pressure change $\Delta P$ can be calculated by

$$
\frac{\Delta G}{\Delta P}=\frac{L(1-2 \mu) D^{2}}{E\left(D^{2}-d^{2}\right)}
$$

where $d$ and $D$ are the I.D. and O.D. of the hollow fiber, $L$ is the sensor gauge length, $\mu$ is the Poisson's ratio, and $E$ is the Young's modulus of the hollow fiber material.

The fabricated sensor was expected to have a pressure sensitivity of about $0.321 \mathrm{~nm} / \mathrm{psi}$ according to (1). In addition, by choosing different gauge lengths from 0.2 to $15 \mathrm{~mm}$, the sensor can be designed for pressure measurement up to $15 \mathrm{kpsi}$ (103.4 MPa) with sensitivities up to $1.5 \mathrm{~nm} / \mathrm{psi}$.

For pressure measurement, the sensor is expected to be sensitive to pressure while possessing low temperature dependence. Since the pressure sensitivity of this hollow fiber-based sensor depends on its gauge length, the longer the gauge length the higher its sensitivity. However, its temperature cross sensitivity will increase accordingly as well. We found that if the reflecting fiber was SMF-28 only, the sensor air gap will enlarge when the temperature increases. To reduce this temperature dependence, a short piece of MMF (Corning, 50/125 $\mu \mathrm{m}$ ) was used in

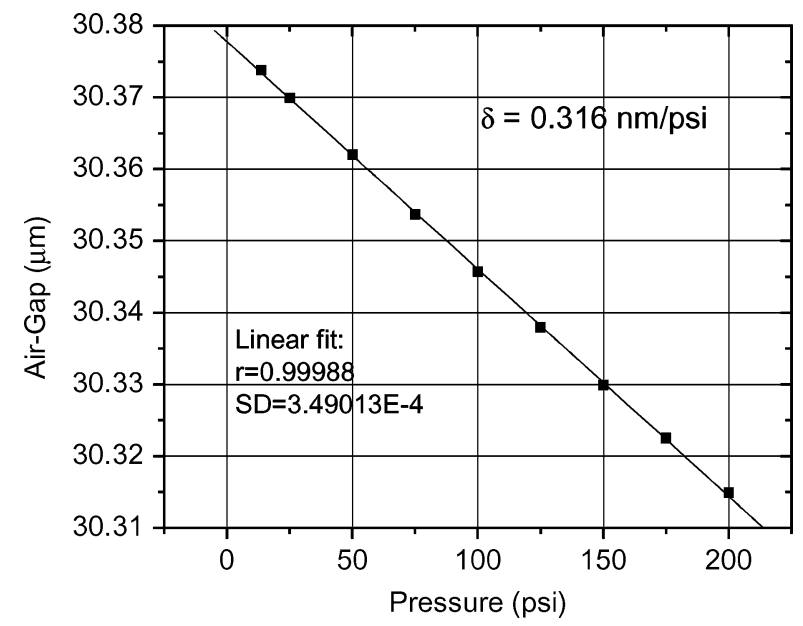

Fig. 3. Sensor pressure response at room temperature.

sensor fabrication for the temperature compensation because of its higher (coefficient of thermal expansion) CTE compared with SMF-28. As shown in Fig. 1, a short section of the MMF $(\approx 0.25 \mathrm{~mm})$ was spliced to the reflecting SMF $(\approx 3.0 \mathrm{~mm})$, which was free to expand or shrink when the temperature changes.

The fused silica hollow fiber, the single-mode optical fiber, and the MMF have CTEs of approximately $\alpha_{h}=5.5-5.9 \times$ $10^{-7} /{ }^{\circ} \mathrm{C}, \alpha_{s}=5.6 \times 10-7 /{ }^{\circ} \mathrm{C}$, and $\alpha_{m}=7.0-7.7 \times 10^{-7} /{ }^{\circ} \mathrm{C}$, respectively. The total temperature dependence of the hermetically sealed EFPI sensor can be expressed as [10]

$$
\frac{\Delta G}{\Delta T}=\left(\alpha_{h}-\alpha_{m}\right) L_{m}+\left(\alpha_{h}-\alpha_{s}\right) L_{s}+\alpha_{h} G+\frac{P}{T} S_{P}
$$

where $G$ is the air gap thickness, $L_{m}$ is the MMF length, $L_{s}$ is the SMF length, $S_{P}$ is the sensor sensitivity $(\mathrm{nm} / \mathrm{psi})$, and $P$ is the air pressure inside the hollow fiber at temperature $T(\mathrm{~K})$.

Theoretically, by optimizing these parameters, the sensor's temperature dependence can be reduced to zero for a specific temperature range over which the CTEs are valid.

\section{EXPERIMENT RESULTS}

The tunable laser of an optical sensing analyzer (si720, Micron Optics) was coupled into the sensor through a $2 \times 2$ coupler and the reflected light was routed back through the same coupler to the receiver of the analyzer. A scan of the tunable laser produced the spectrum information from 1520 to $1570 \mathrm{~nm}$ with a $2.5-\mathrm{pm}$ resolution. When the applied pressure changes, the spectrum fringes shifts accordingly. A fringe valley traced method [11] was used to process the fringes in order to demodulate the air gap thickness. The air gap measurement exhibits a resolution of $0.03 \mathrm{~nm}$ [12].

The pressure sensors were calibrated using a pressure gauge calibration system (Pressure Systems, Model 9035), while their temperature properties were tested in a furnace (Thermolyne, 47900 ). An MEFPI pressure sensor static pressure response is shown in Fig. 3. The sensor showed a linear response to static pressure 0-200 psi at room temperature. The sensor pressure sensitivity was about $0.316 \mathrm{~nm} / \mathrm{psi}$ and the sensor system had a resolution of about $0.1 \mathrm{psi}$ ( $689 \mathrm{~Pa}$ or $5 \mathrm{mmHg}$ ). The sensor's 


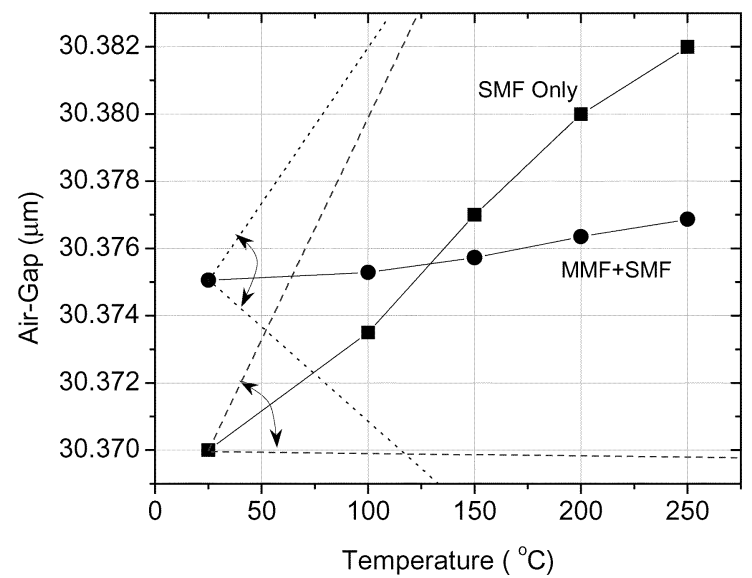

Fig. 4. Temperature dependences of two MEFPI sensors.

pressure sensitivity is consistent with the theoretical prediction and the small discrepancy may be caused by the measurement error of the sensor gauge length. The temperature test results of two different MEFPI sensors with the same pressure sensitivity are shown in Fig. 4. The dashed and dotted angle indicate the theoretical boundaries of the sensor temperature dependences. Compared with the SMF-only configuration, the temperature dependence of MMF + SMF one was reduced by about $85 \%$, which in terms of pressure measurement error was about $0.026 \mathrm{psi} /{ }^{\circ} \mathrm{C}\left(1.35 \mathrm{mmHg} /{ }^{\circ} \mathrm{C}\right)$. This all-silica sensor can work at temperatures near the limit of the optical fiber itself, however, the coating of SMF-28 cannot withstand temperatures that high. For applications above $250^{\circ} \mathrm{C}$, an optical fiber with high temperature coating, such as gold, is preferred.

\section{CONCLUSION}

We described a novel MEFPI pressure sensor. Simple fabrication processes have been developed to produce hermetic seals and permit adjustment of the sensor gauge length to control its pressure sensitivity. In addition, the length of the FP cavity can be controlled with a resolution of several nanometers. The sensor exhibited a very low temperature dependence and can operate at elevated temperatures. This hair size sensor could be suitable for biomedical applications.

\section{ACKNOWLEDGMENT}

The authors would like to thank Micron Optics for the partial donation of the optical sensing analyzer used in this research.

\section{REFERENCES}

[1] E. Cibula, D. Donlagic, and C. Stropnik, "Miniature fiber optic pressure sensor for medical applications," in Proc. IEEE Sensors, vol. 1, Jun. 2002, pp. 711-714.

[2] K. Totsu, Y. Haga, and M. Esashi, "Vacuum sealed ultra miniature fiberoptic pressure sensor using white light interferometry," in 12th Int. Conf. Transducers, Solid-State Sensors, Actuators and Microsystems, vol. 1, 2003, pp. 931-934.

[3] J. S. Sirkis and H. Singh, "Simultaneously measuring temperature and strain using optical fiber microcavities," J. Lightw. Technol., vol. 15, no. 4, pp. 647-653, Apr. 1997.

[4] C. E. Lee, W. N. Gibler, R. A. Atkins, and H. F. Taylor, "In-line fiber Fabry-Pérot interferometer with high-reflectance internal mirrors," $J$. Lightw. Technol., vol. 10, no. 10, pp. 1376-1379, Oct. 1992.

[5] K. A. Murphy, M. F. Gunther, A. M. Vengsarkar, and R. O. Claus, "Quadrature phase shifted extrinsic Fabry-Pérot optical fiber sensors," Opt. Lett., vol. 16, pp. 273-275, 1991.

[6] A. Wang, H. Xiao, J. Wang, Z. Wang, W. Zhao, and R. G. May, "Self-calibrated interferometric-intensity-based optical fiber sensors," J. Lightw. Technol., vol. 19, no. 10, pp. 1495-1501, Oct. 2001.

[7] J. S. Sirkis, D. D. Brennan, M. A. Putman, T. A. Berkoff, A. D. Kersey, and E. J. Friebele, "In-line fiber etalon for strain measurement," Opt. Lett., vol. 18, pp. 1973-1975, 1993.

[8] J. Xu, G. R. Pickrell, Z. Huang, B. Qi, P. Zhang, Y. Duan, and A. Wang, "Double-tubing encapsulated fiber optic temperature sensor," in AIP Conf. Proc. 8th Temperature Symp., vol. 684, 2003, pp. 1021-1026.

[9] B. Qi, G. Pickrell, P. Zhang, Y. Duan, W. Peng, J. Xu, Z. Huang, J. Deng, H. Xiao, Z. Wang, W. Huo, R. G. May, and A. Wang, "Fiber optical pressure and temperature sensors for oil down hole applications," Proc. SPIE, vol. 4578, pp. 182-190, 2002.

[10] J. Xu, G. Pickrell, X. Wang, W. Peng, K. L. Cooper, and A. Wang, "A novel temperature insensitive optical fiber pressure sensor for harsh environments," IEEE Photon. Technol. Lett., vol. 17, no. 4, pp. 870-872, Apr. 2005.

[11] B. Qi, G. R. Pickrell, J. Xu, P. Zhang, Y. Duan, W. Peng, Z. Huang, W. Huo, H. Xiao, R. G. May, and A. Wang, "Novel data processing techniques for dispersive white light interferometer," Opt. Eng., vol. 42, pp. 3165-3171, 2003.

[12] F. Shen, W. Peng, K. Cooper, G. Pickrell, and A. Wang, "UV-induced intrinsic Fabry-Pérot interferometric fiber sensors," Proc. SPIE 5590, pp. 47-56, 2004. 


\title{
Miniature optical fiber sensor for pressure measurements
}

\author{
Xingwei Wang, Juncheng Xu, Kristie L. Cooper, and Anbo Wang \\ Center for Photonics Technology, The Bradley Department of Electrical and Computer Engineering, \\ Virginia Polytechnic Institute and State University, Blacksburg, Virginia 24061 USA \\ 540-231-3488 \\ Email: xiwang@,vt.edu
}

\begin{abstract}
Fiber optic extrinsic Fabry-Perot interferometric (EFPI) sensors are widely used in dynamic pressure measurements because of their inherent advantages including small size, light weight, high sensitivity, high frequency response, electrical passivity, electromagnetic interference immunity (EMI), and single-point measurement. However, small fluctuations of background pressure could shift the operating point of the sensor in an unpredictable way, which introduces a nonlinear response in dynamic pressure measurements. In this paper, we present a novel open structure only $125 \mu \mathrm{m}$ in diameter to eliminate the operating point dependence on background pressure fluctuations. The essential element is a piece of hollow fiber, which connects a standard telecommunication fiber and another hollow fiber with a smaller inside diameter to form a Fabry-Perot cavity. The structure is open to the environment by a small hole, which allows gas exchange between the sensor cavity and the ambient atmosphere. On the other hand, the sensor is capable of responding to acoustic pressure because of the slow gas exchange through the small hole. In addition, this all fused silica structure features high temperature stability, good linearity, and high repeatability.
\end{abstract}

Keywords: Fiber optic sensors, miniature sensor, pressure sensor, Fabry-Perot

\section{INTRODUCTION}

Fiber optic extrinsic Febry-Perot interferometric (EFPI) sensors have been extensively studied recently due to their inherent advantages including small size, light weight, high sensitivity, high frequency response, electrical nonconductivity, immunity to electromagnetic interference (EMI) noise and single-point measurement [1]. Diaphragm based EFPI sensor is one of them. It comprises a fused silica diaphragm and a single mode fiber (SMF) end to form a FP cavity [2-4]. The vibration of the diaphragm caused by acoustic waves operates in the linear range of one of its interference fringes. Optimal parameters (thickness and cavity length) are designed to make the sensors sensitive to pressure changes in a specific range. However, this optimal operating point of the sensor can be changed in an unpredictable way by the small fluctuations of background pressure. To compensate this drift, several methods have been employed, including the multi-wavelength interrogation [5,6] or spectral interrogation [6], and Q-point control using a band-pass filter [7]. Nevertheless, these approaches can significantly increase of the system complexity and cost. In this paper, we present a novel open structure only $125 \mu \mathrm{m}$ in diameter to eliminate the operating point dependence on background pressure fluctuations

Photorefractive Fiber and Crystal Devices: Materials, Optical Properties, and Applications XII, edited by Francis T. S. Yu, Ruyan Guo, Shizhuo S. Yin, Proc. of SPIE Vol. 6314, 63141K, (2006)

0277-786X/06/\$15 - doi: 10.1117/12.680866

Proc. of SPIE Vol. $631463141 \mathrm{~K}-1$ 


\section{BRIEF DESCRIPTION}

This is a novel fiber optic Fabry-Perot structure with only $125 \mu \mathrm{m}$ in diameter. Its open structure has unique advantages in dynamic pressure measuring, chemical sensing and medical sensing. Also, the multiplexing structure makes it possible for distributed sensing and multi-parameter sensing.

The essential element is a piece of large inside diameter (ID) hollow fiber, which connects a standard telecommunication fiber end and a small ID hollow fiber to form a Fabry-Perot cavity. All three kinds of optical fiber are with the similar outside diameter, which is in the order of $125 \mu \mathrm{m}$. Light from the source is transmitted via the lead-in fiber. One portion of light is reflected by the lead-in fiber end. The reflectivity is about $4 \%$ for the fused silica fiber. The remaining portion of light is transmitted through the large ID hollow fiber. Since the diameter of this hollow fiber is so large that most light can reach the endface of the small ID hollow fiber. Here, the diameter is so small that the reflected light is strong enough to interfere with the first reflected light from the lead-in fiber end. At the same time, the structure is open to the environment by the small hole, which can be a path for the gas, chemical particles, or other measurands to go through. Thus, the basic open miniature Fabry Perot structure is formed.

Figure 1 gives the photograph of such structure.

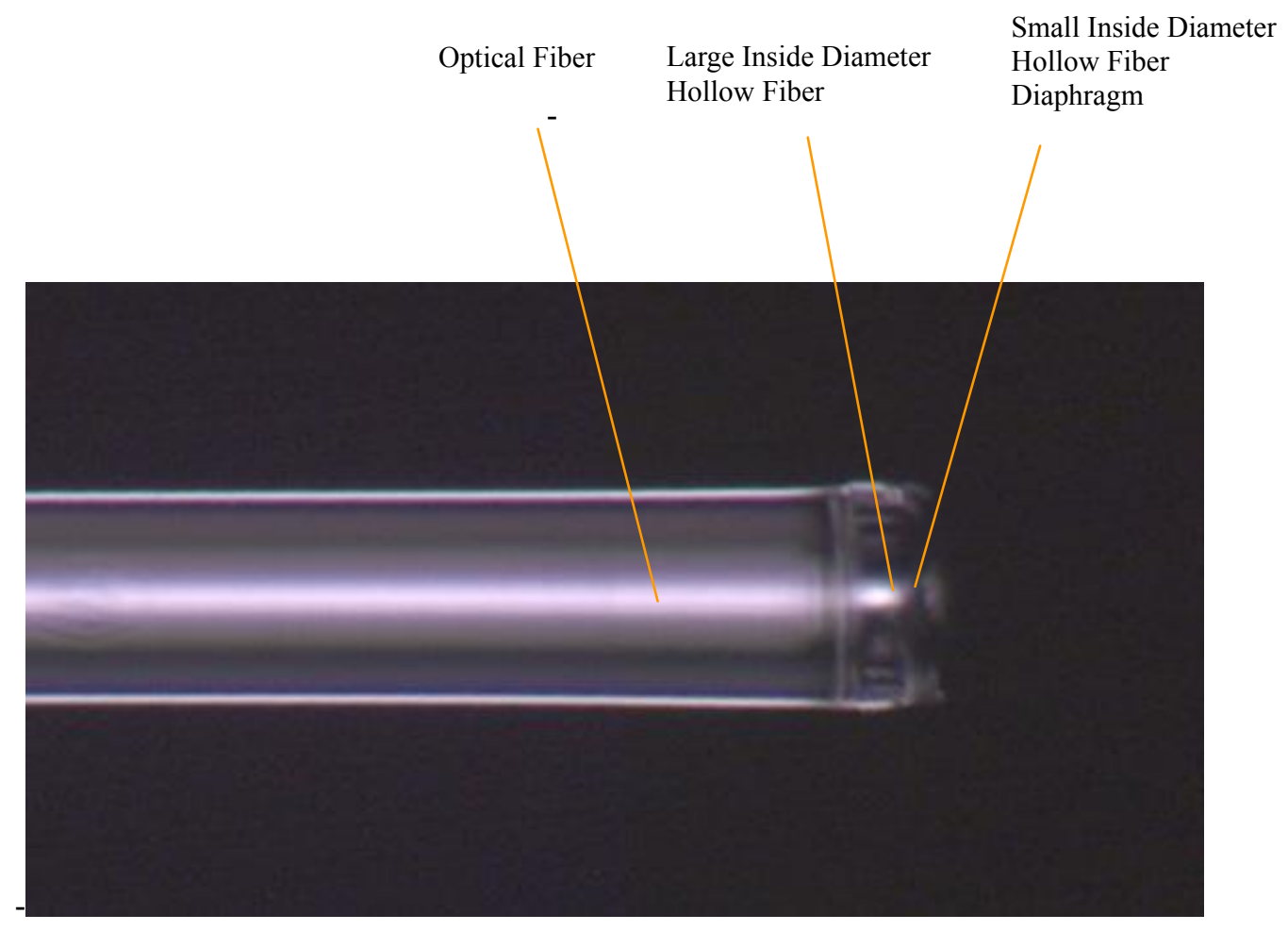

Fig.1 Photograph of the new structure 


\section{PRINCIPLE OF OPERATION}

When only static pressure is applied onto the open structure, the air will enter the cavity. After some time, the pressure inside and outside the cavity will balance with each other and the diaphragm will not deflect. On the other hand, the pressure on the side will compress the wall and elongate the cavity, which agrees with the Poisson's Theory. So, when the applied pressure is increased, the air gap will increase, which is shown in the experimental results part.

When dynamic pressure is applied, this Poisson effect is much smaller than that of the acoustic wave induced diaphragm vibration in F-P cavity changes. The structure can be used as a dynamic pressure sensor, which is insensitive to the background static pressure. Suppose the acoustic energy diffracted into the air cavity is proportional to the area of the hole, the acoustic energy diffracted inside the air cavity through the hole is negligible. Say, the hole is $2 \mu \mathrm{m}$ in diameter, while the whole diaphragm is $125 \mu \mathrm{m}$ in diameter. The percentage of energy diffracted into the cavity is

$$
\left(\frac{2}{125}\right)^{2}=0.000256=0.0256 \%
$$

The sensitivity to acoustic waves of the open structure sensor does not decrease much compared to a sensor without through hole. Thus the sensor is expected to be insensitive to the background pressure while maintaining its sensitivity to the acoustic waves.

Other small changes can be applied to this basic structure according to different specific applications. For example, the large ID hollow fiber can be replaced with those having side holes and the diaphragm can be a short section of a holley fiber. In this case, the diaphragm can be small ID hollow fiber, as mentioned in the previous paragraph, or a piece of commercial fiber cleaved to the required diameter thickness, or a piece of normal diaphragm, such as fused silica diaphragm. The measurands such as gas or chemical particles can get through the side holes in the large ID fiber or the holley fiber.

\section{FABRICATION PROCESS}

The fabrication of this kind of sensors are simple and straightforward. There are basically 5 steps as follows:

1. Splice a piece of large inside diameter hollow fiber at the cleaved end of a standard fiber.

2. Cleave the hollow fiber near the junction according to the requirement of the cavity length. The cavity length can be cleaved down to the order of micrometer under the inspection of a microscope or CCD.

3. Connect a piece of small diameter hollow fiber or normal diaphragm at the other end of the large diameter hollow fiber. The method can be splicing, agglutinating, heating, etc.

4. Control the small diameter hollow fiber's thickness to the order of sub-microns by dipping the fiber head into a kind of solution such as HF acid of suitable concentration.

5. If multiplexing structure is required, repeat step 1-4.

The whole process is under monitor by the power measurement device (for example, a white light system). The interior diameters of both hollow fibers can be selected according to different requirements. 


\section{NOVEL FEATURES}

This paper presents a novel Fabry-Perot structure. It has the following advantages:

1. Open structure: It breaks a new path to many new applications in such area as medicine, biology, chemistry and environment engineering.

2. Miniature size: The whole structure is of the fiber size, generally $125 \mu \mathrm{m}$ in diameter.

3. Reduced working point drift: For dynamic pressure sensing, this structure introduces a through hole in the conventional diaphragm-based EFPI sensors and significantly reduces the effect of working point drifting due to the background pressure and fluctuations.

4. Temperature independence: The open structure eliminates the effect of the trapped air expansion induced pressure.

5. Distributed and multiplexed sensing capabilities: With different cavity lengths, the sensor can be regarded as a distributed one.

6. Multi-parameter sensing capabilities: By coating the inside cavities with different reactants, the sensor can detect different chemicals simultaneously.

7. Simple and efficient fabrication: The process mainly includes cleaving and bonding steps, and lasts for less than one hour in general.

8. High temperature capability: When the diaphragm is sapphire, the structure can work under more than 2000 degree centigrade.

9. High signal strength and visibility: The cavity surfaces are smooth, flat and parallel, which guarantees the quality of the signal.

10. Low cost: The materials for the structure, namely, optical fiber and hollow fiber, are commercialized products. Also, the tools for the fabrication, say, cleaver and splicer, are common tools in a photonics lab. Furthermore, no working point control is needed, which can greatly cut down the cost.

11. Biological compatibility: The whole structure is made of fused silica with no impurity, which makes it ideal for medical and biological applications.

12. EMI immunity and electric passivity: Such optical structure is immune to the electromagnetic interference

\section{EXPERIMENT RESULTS}

As an example, we utilize a Fabry Perot sensor which has a hole in the diaphragm to test pressure.

Fig 2 shows the response of the sensor to the air pressure. Y-label indicates how the air gap of the sensor changes according to the pressure. The pressure is increased from $25 \mathrm{psi}$ to $150 \mathrm{psi}$, then decreased down to $25 \mathrm{psi}$. Repeat such process three times with $25 \mathrm{psi}$ as the step. The sensor's sensitivity is $0.4 \mathrm{~nm} / \mathrm{psi}$. 


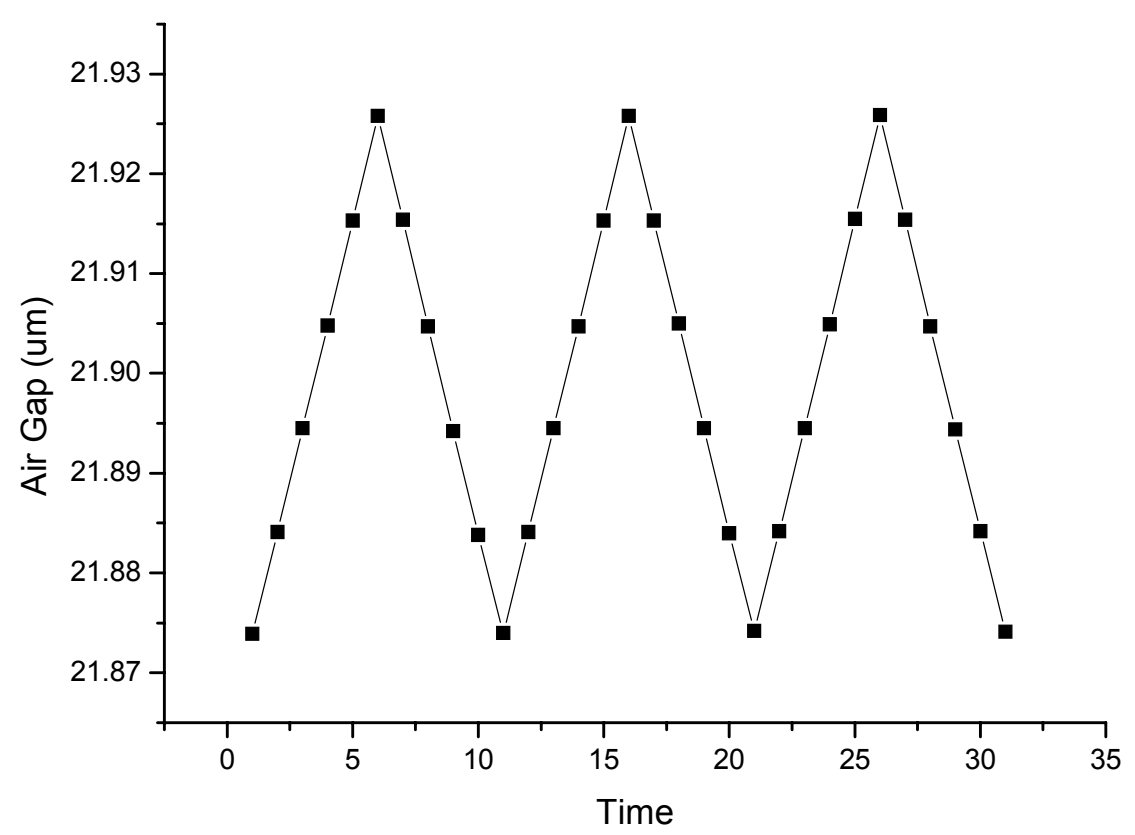

Fig 2. Air gap change according to the pressure from $25 \mathrm{psi}$ to $150 \mathrm{psi}$ with $25 \mathrm{psi}$ as the step

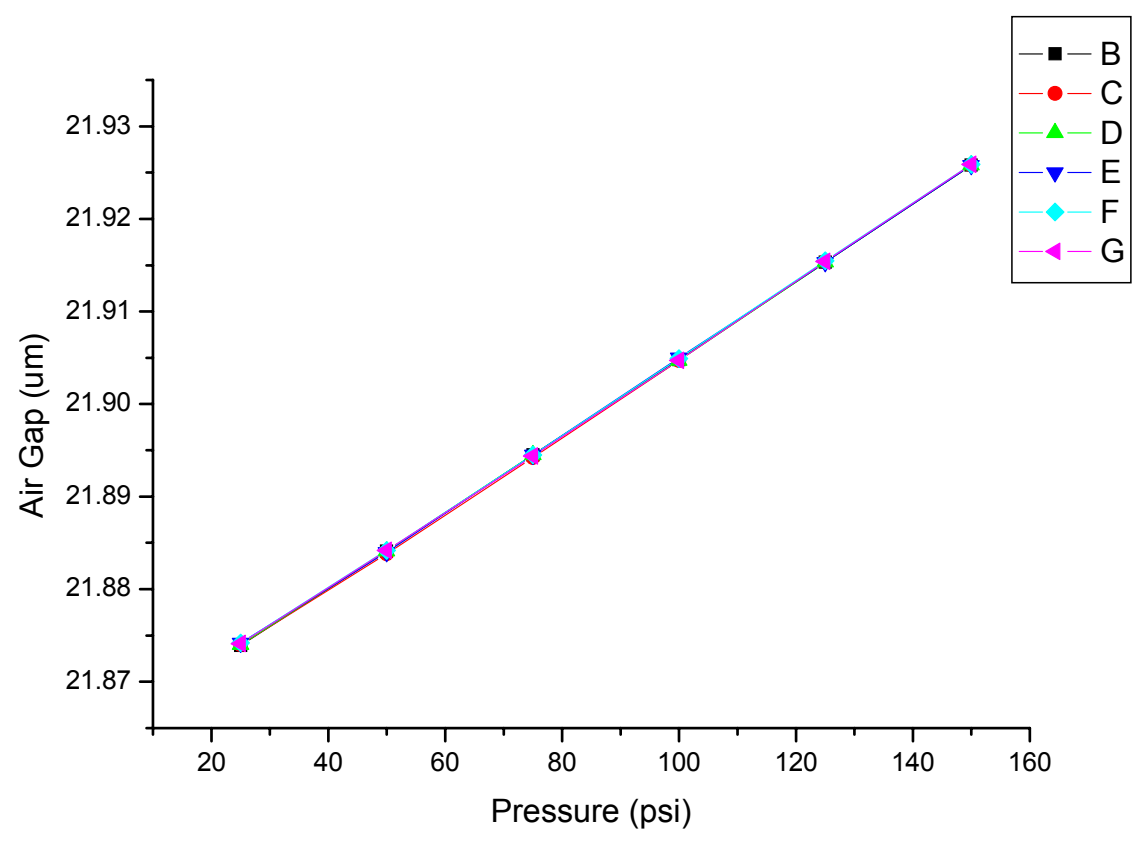

Fig 3. Linearity and repeatability of the sensor response 
Fig 3 shows that the sensor's response has very good linearity and repeatability.

Take Curve B as an example, the analysis is as following:

Linear Regression for Data1_B:

$\mathrm{Y}=\mathrm{A}+\mathrm{B} * \mathrm{X}$

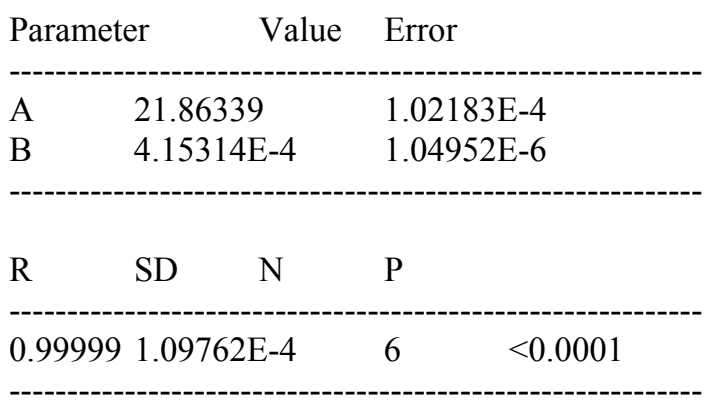

Also, compare curve $\mathrm{B}$ and curve $\mathrm{C}$ using the ALLOMETRIC1 function:

Fit results for DATA1_B

\begin{tabular}{lcccc}
\hline Parameter & Value & Error & LCL & UCL \\
a & 21.77776 & 0.01631 & 21.73247 & 21.82305 \\
b & 0.00129 & $1.71867 \mathrm{E}-4$ & $8.16998 \mathrm{E}-4$ & 0.00177 \\
& & & \\
$\mathrm{SSR}=0.000124278$ & & & \\
$\mathrm{DOF}=4$ & & & \\
$\mathrm{ChiSqr} / \mathrm{DOF}=3.10696 \mathrm{E}-005$ & &
\end{tabular}

Fit results for DATA1_C

\begin{tabular}{lcccc}
\hline Parameter & Value & Error & LCL & UCL \\
a & 21.77761 & 0.01681 & 21.73093 & 21.82429 \\
b & 0.00129 & $1.77137 \mathrm{E}-4$ & $8.03067 \mathrm{E}-4$ & 0.00179 \\
& & & \\
$\mathrm{SSR}=0.000132016$ & & & \\
$\mathrm{DOF}=4$ & & & \\
$\mathrm{ChiSqr} /$ DOF $=3.3004 \mathrm{E}-005$ & &
\end{tabular}

F-Test results from comparison of fits

$\mathrm{F}-$ Value $=4.114 \mathrm{E}-005, \mathrm{P}=0.999959$

At the 0.05 significance level the two datasets are NOT statistically different.

The preliminary results above show that the open structure can act as pressure sensor. 


\section{CONCLUSIONS}

In summary, a new structure of diaphragm based EFPI sensors for acoustic wave detection has been presented. The cost of the system is little. This structure also provides the potential advantage of temperature insensitivity and high temperature capability.

\section{REFERENCES}

1. 1. K. A. Murphy, M. F. Gunther, A. Wang, and R. O. Claus, "Detection of acoustic emission location using optical fiber sensors," in Smart Structures and Materials 1994: Smart Sensing, Processing, and Instrumentation, J. S. Sirkis, ed., Proc. SPIE 2191, 282-290 (1994).

2. J. Deng, H. Xiao, W. Huo, M. Luo, R. May, A. Wang, and Y. Liu, "Optical fiber sensor-based detection of partial discharges in power transformers," Opt. Laser Technol. 33, 305-311 (2001).

3. Bing Yu, Dae Woong Kim, Jiangdong Deng, Hai Xiao, and Anbo Wang, "Fiber Fabry-Perot sensors for detection of partial discharges in power transformers", Appl. Opt. 42, 3242-3250 (2003)

4. J. Xu, X. Wang, K. Cooper and A. Wang, "Miniature all-silica fiber optic pressure and acoustic sensors", Opt.Lett. 30, 3269-3271 (2005).

5. N. Furstenau, M. Schmidt, H. Horack, W. Goetze, and W. Schmidt, "Extrinsic Fabry-Perot interferometer vibration and acoustic sensor systems for airport ground traffic monitoring,"IEE Proc. Optoelectron. 144, 134-144 (1997).

6. W. Pulliam, P. Russler, R. Mlcak, K. Murphy, and C. Kozikowski, "Micromachined, SiC fiber optic pressure sensors for high temperature aerospace applications," in Industrial Sensing Systems, A. Wang and E. Udd, eds., Proc. SPIE 4202, 21-30 (2000).

7. Ming Han, Xingwei Wang, Juncheng Xu, Kristie L. Cooper, and Anbo Wang, "Diaphragm-based extrinsic FabryPerot interferometric optical fiber sensor for acoustic wave detection under high background pressure," Opt. Eng. 44, 060506 (2005). 